
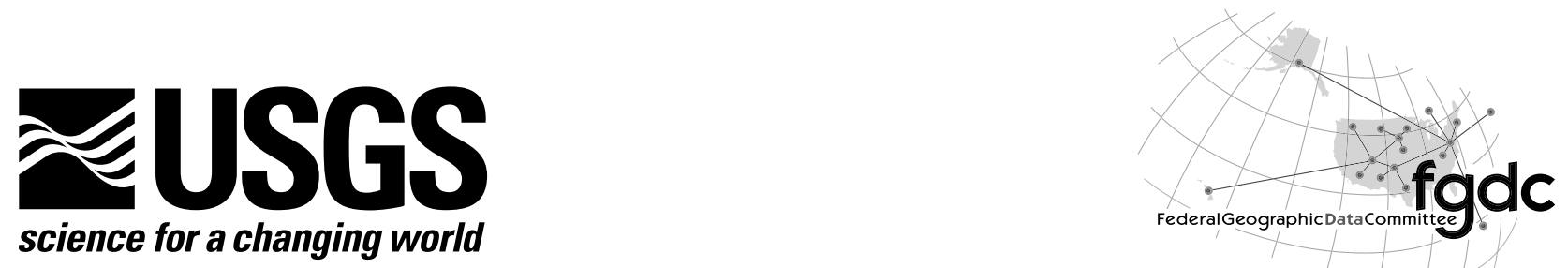

\title{
FGDC Digital Cartographic Standard for Geologic Map Symbolization (PostScript Implementation)
}

Prepared in cooperation with the Geologic Data Subcommittee of the Federal Geographic Data Committee

By the U.S. Geological Survey

Techniques and Methods 11-A2 


\section{Federal Geographic Data Committee}

Established by Office of Management and Budget Circular A-16, the Federal Geographic Data Committee (FGDC) promotes the coordinated development, use, sharing, and dissemination of geographic data.

The FGDC is composed of representatives from the Departments of Agriculture, Commerce, Defense, Energy, Housing and Urban Development, the Interior, State, and Transportation; the Environmental Protection Agency; the Federal Emergency Management Agency; the Library of Congress; the National Aeronautics and Space Administration; the National Archives and Records Administration; and the Tennessee Valley Authority. Additional Federal agencies participate on FGDC subcommittees and working groups. The Department of the Interior chairs the committee.

FGDC subcommittees work on issues related to data categories coordinated under the circular. Subcommittees establish and implement standards for data content, quality, and transfer; encourage the exchange of information and the transfer of data; and organize the collection of geographic data to reduce duplication of effort. Working groups are established for issues that transcend data categories.

For more information about the committee, or to be added to the committee's newsletter mailing list, please contact:

Federal Geographic Data Committee Secretariat c/o U.S. Geological Survey

590 National Center

Reston, Virginia 22092

Facsimile: (703) 648-5755

Internet (electronic mail): fgdc@fgdc.gov

World Wide Web: http://www.fgdc.gov/ 


\section{CONTENTS}

1. Introductory Material

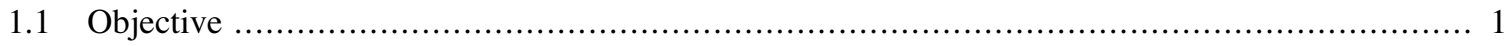

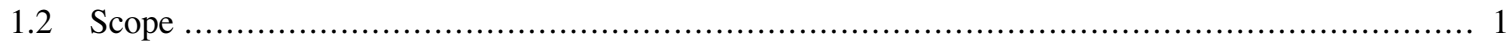

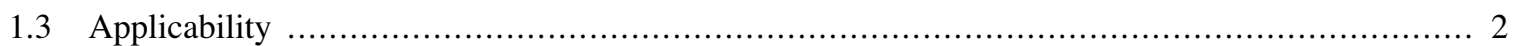

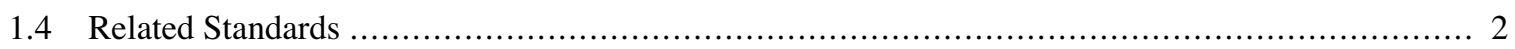

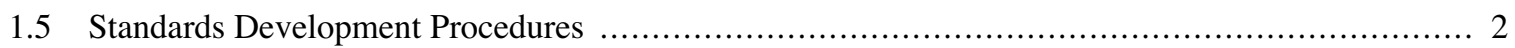

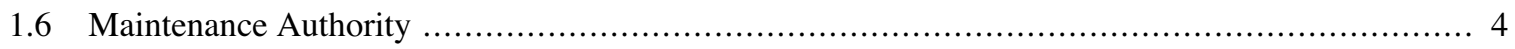

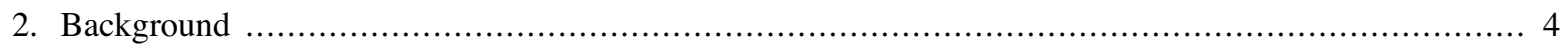

2.1 Relation to Previous U.S. Geological Survey Standards ...................................................... 4

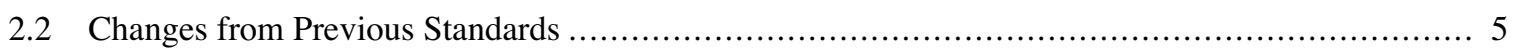

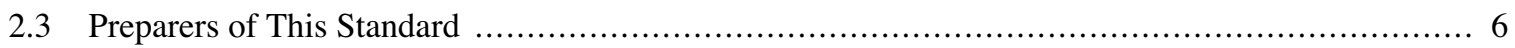

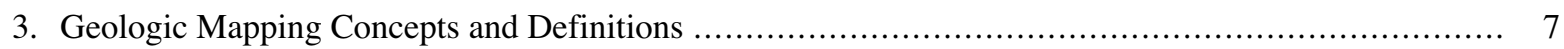

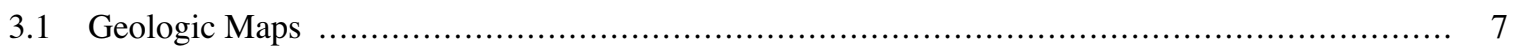

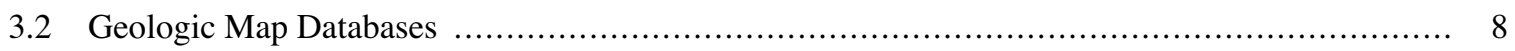

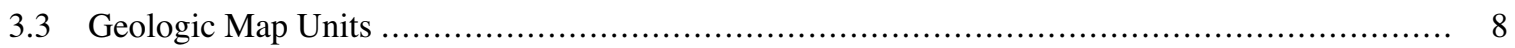

3.3.1 Geologic Time, the Ages of Rock Units, and Geologic Age Symbols ....................... 8

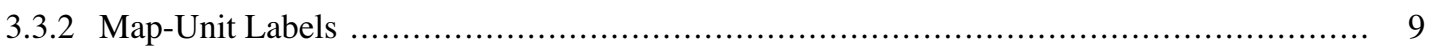

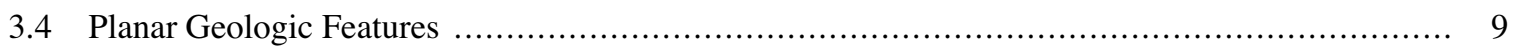

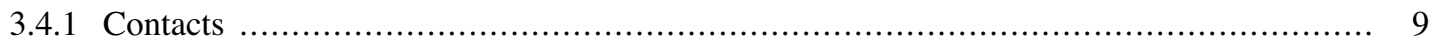

3.4.1.1 Discrete versus Gradational Contacts ….......................................... 10

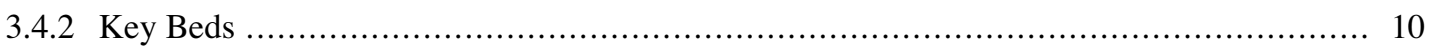

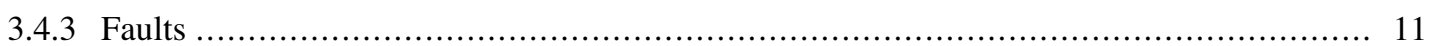

3.4.3.1 Discrete Faults versus Fault Zones ....................................................... 11

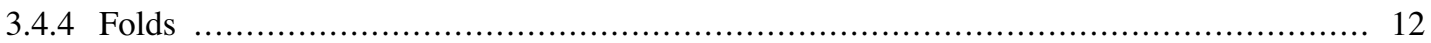

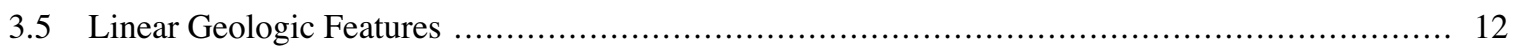

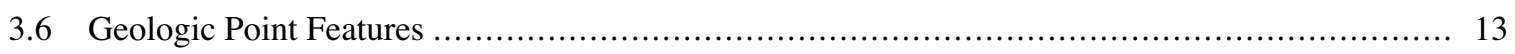

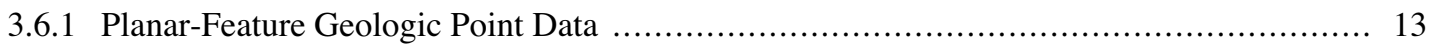

3.6.1.1 Point Symbols for Planar Features, and Their Placement Relative to Point of Observation ................................................................... 13

3.6.1.2 Specialized Planar-Feature Point Symbols for Multiple Observations at

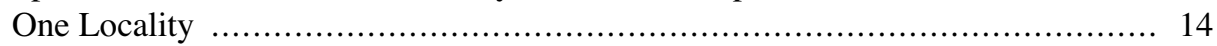

3.6.2 Linear-Feature Geologic Point Data ..................................................... 14

3.6.2.1 Point Symbols for Linear Features, and Their Placement Relative to Point

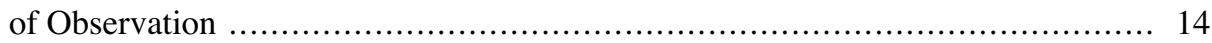

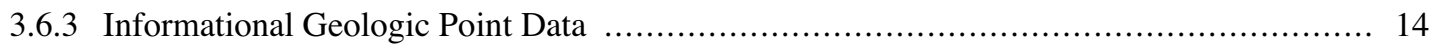

3.6.3.1 Locality-Information Point Data .................................................... 15

3.6.3.2 Line-Symbol Decorations .............................................................. 15

4. Scientific Confidence and Locational Accuracy of Geologic Features ....................................... 15

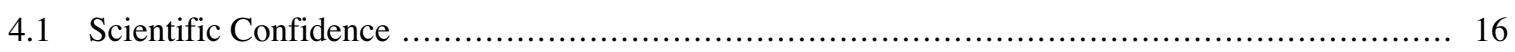

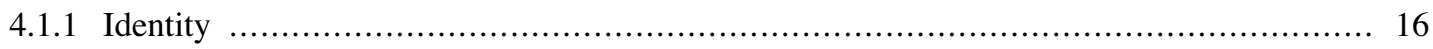

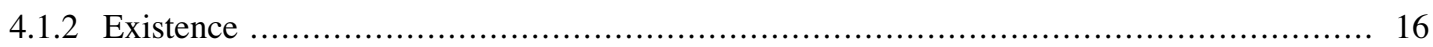

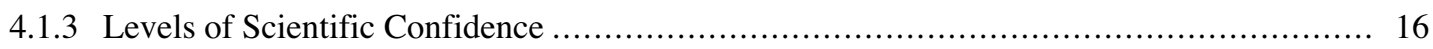

4.1.4 Cartographic Representation of Scientific Confidence .......................................... 17

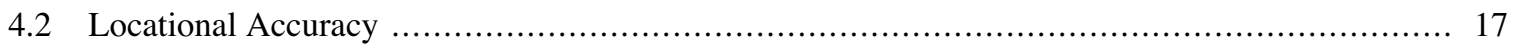

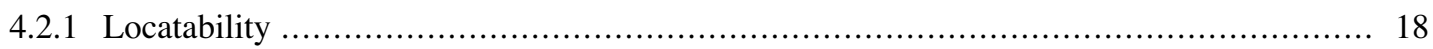




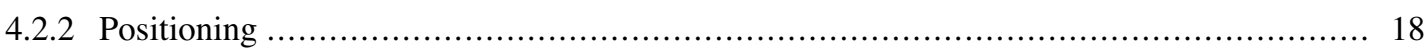

4.2.2.1 Specifying Positional Accuracy with the Zone of Confidence ....................... 19

4.2.2.2 Accommodating Different Values of the Zone of Confidence ....................... 22

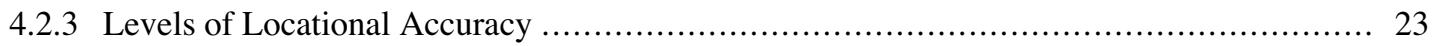

4.2.4 Cartographic Representation of Locational Accuracy ............................................... 23

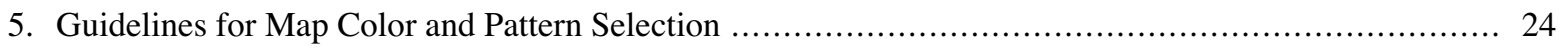

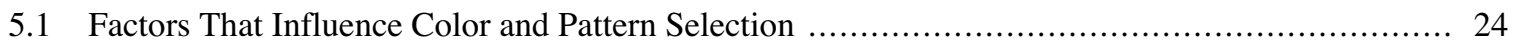

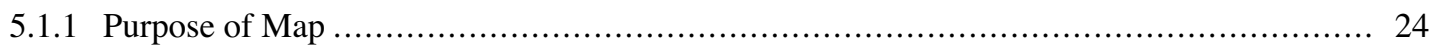

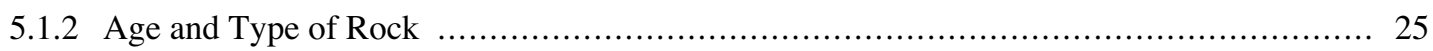

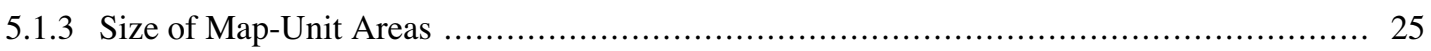

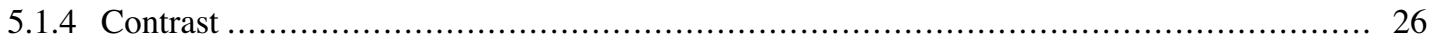

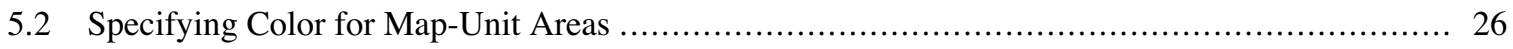

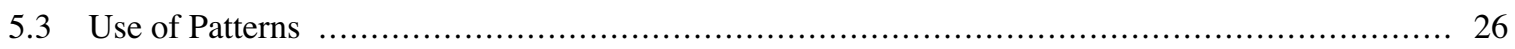

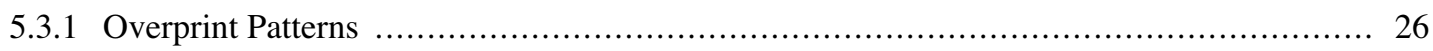

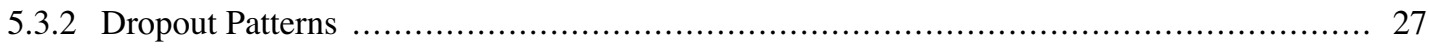

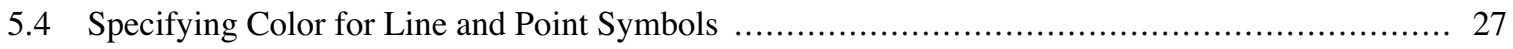

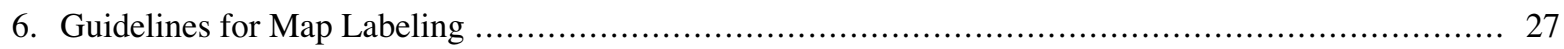

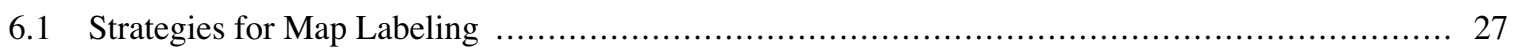

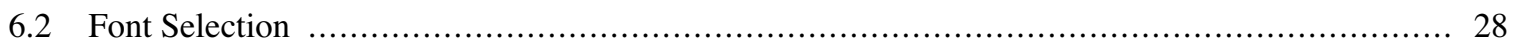

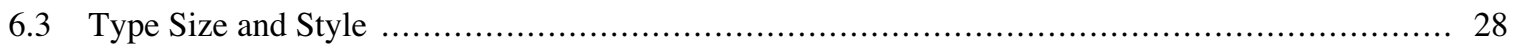

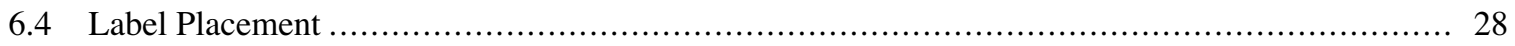

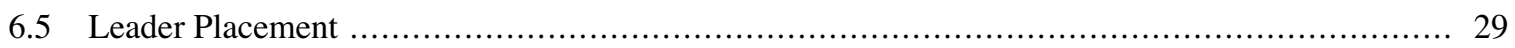

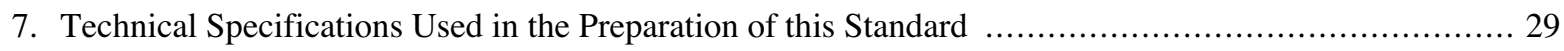

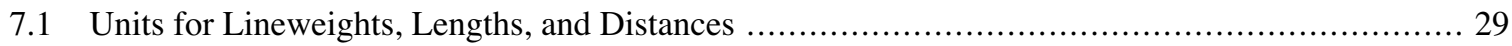

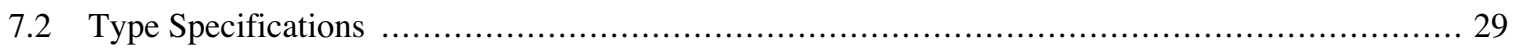

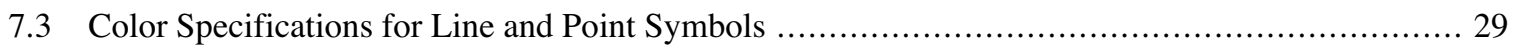

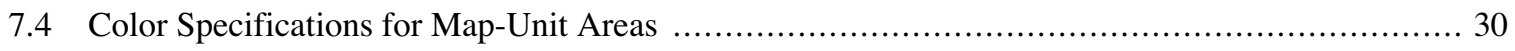

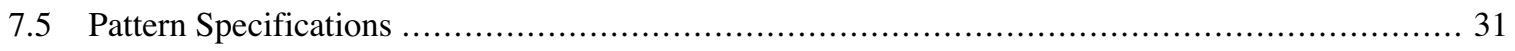

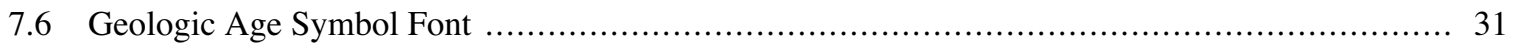

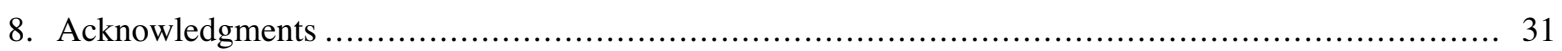

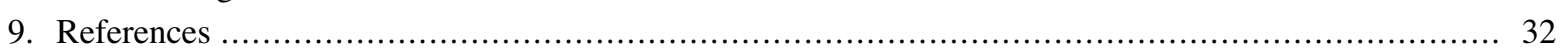

\section{FIGURES}

1. Diagram Showing Relation of New FGDC Standard Terminology to Historically Used Terminology and to Traditional Line Symbol Styles

2. Flowchart Showing Example of Logical Steps That Might Be Used to Determine Appropriate Line

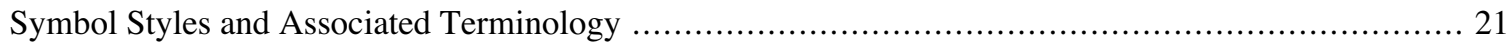

3. Figure Showing Examples of the Zone of Confidence for Planar, Linear, and Point Features .............. 22

\section{TABLES}

Page

1. Chart Showing Conversion Values from Inches (in) to Points (pts) to Millimeters (mm)................ A-iv

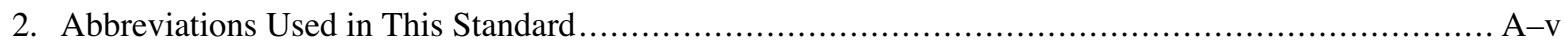

3. Spot Color Specifications Used in This Standard and Their Equivalent Colors in Other Color Models... A-v 


\section{APPENDIX A. GEOLOGIC MAP SYMBOLS, COLORS, AND PATTERNS}

Preface to Appendix A

Page

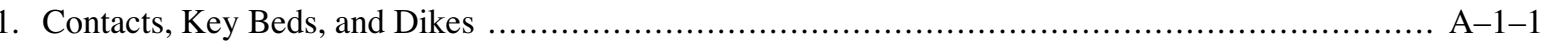

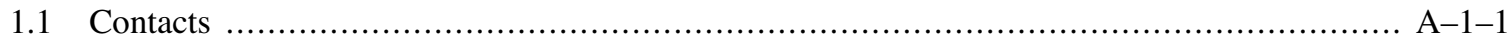

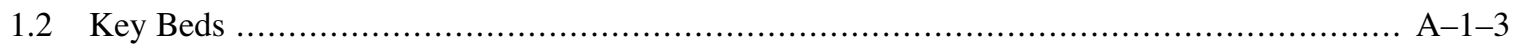

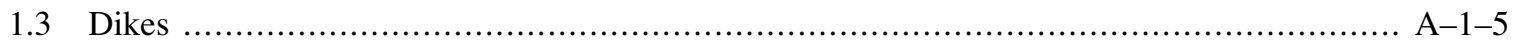

1.4 Line-Symbol Decorations and Notations for Contacts, Key Beds, and Dikes ................... A-1-6

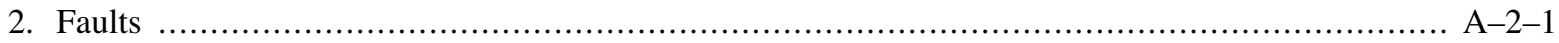

2.1 Faults (Generic; Vertical, Subvertical, or High-Angle; or Unknown or Unspecified

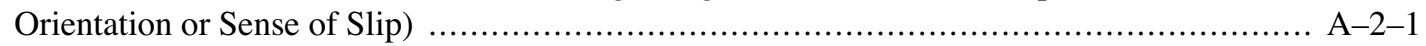

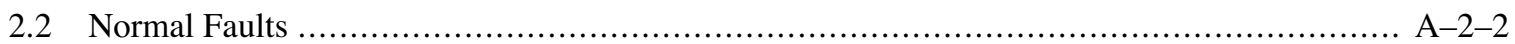

2.3 Low-Angle Faults (Unknown or Unspecified Sense of Slip) …............................... A-2-3

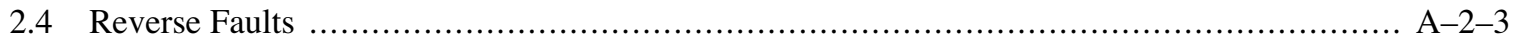

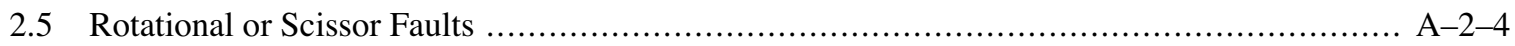

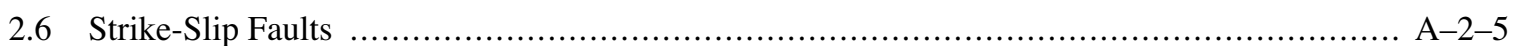

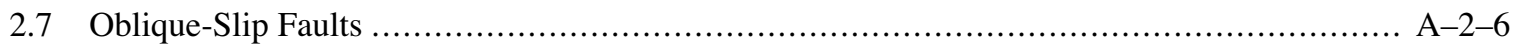

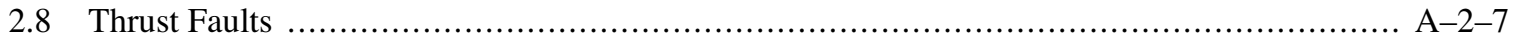

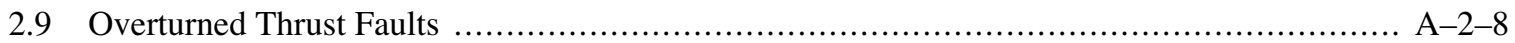

2.10 Detachment Faults (Sense of Slip Unspecified) ....................................................... A-2-9

2.11 Line-Symbol Decorations and Notations for Faults .............................................. A-2-11

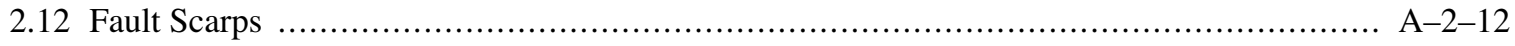

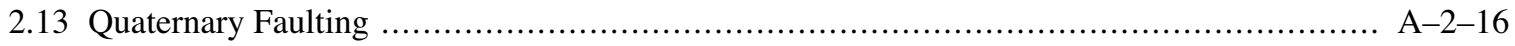

2.14 Shear Zones; Mylonite Zones; Fault-Breccia Zones ................................................ A-2-16

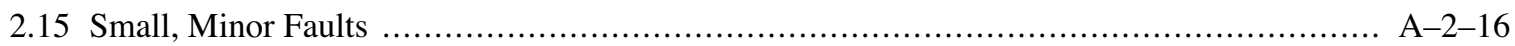

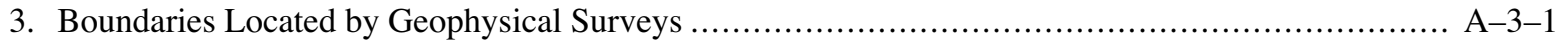

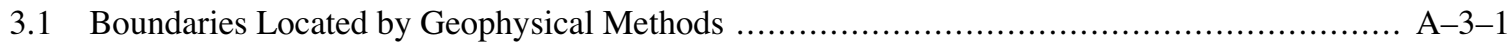

3.2 Faults Located by Geophysical Methods ................................................................. A-3

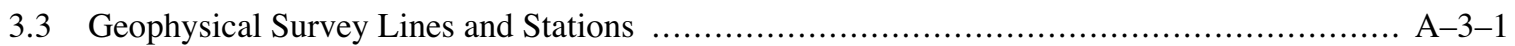

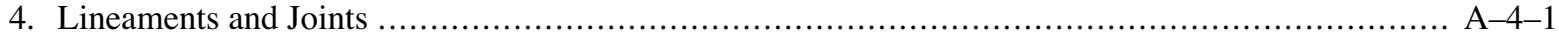

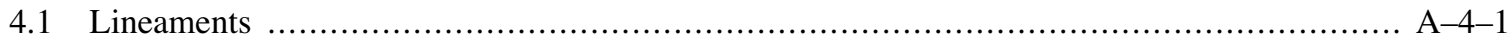

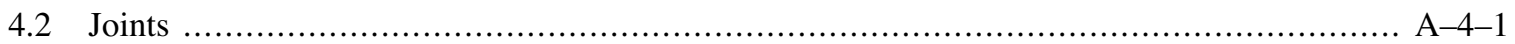

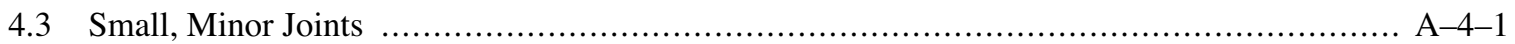

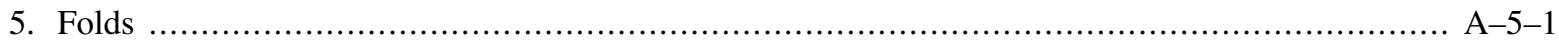

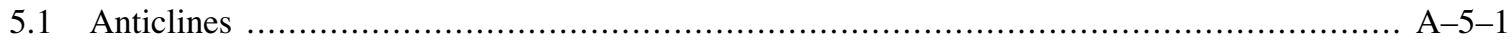

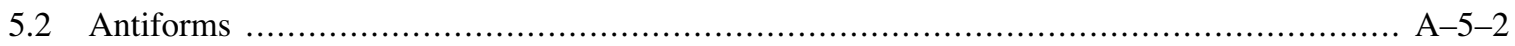

5.3 Asymmetric, Overturned, and Inverted Anticlines .............................................. A-5-3

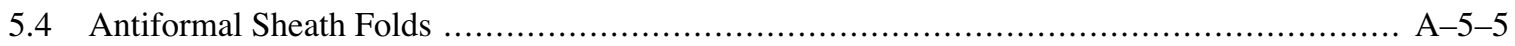

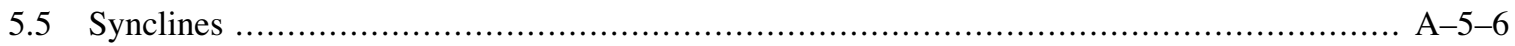

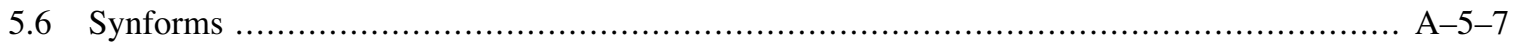

5.7 Asymmetric, Overturned, and Inverted Synclines .................................................... A-5-8

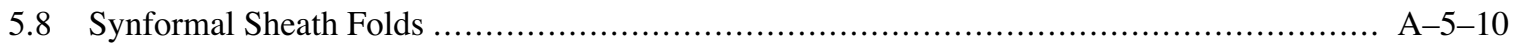

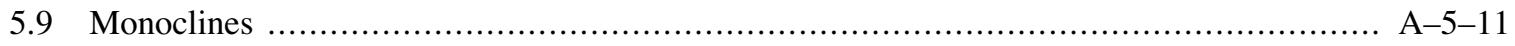

5.10 Line-Symbol Decorations And Notations For Folds …................................... A-5-13

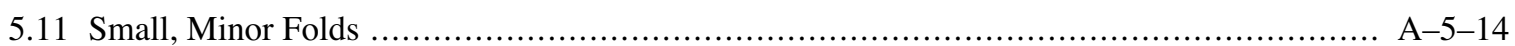

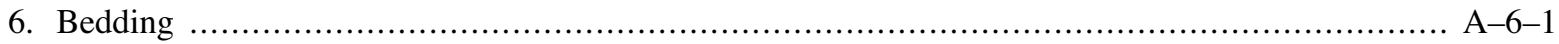


7. Cleavage

Page

8. Foliation A $-7-1$

8.1 Generic Foliation (Origin Not Known or Not Specified)

A $-8-1$

8.2 Primary Foliation or Layering (in Igneous Rocks)

A-8-1

8.3 Secondary Foliation (Caused by Metamorphism Or Tectonism)

A $-8-1$

9. Lineation

A $-8-3$

10. Paleontological Features

A-9-1

10.1 Fossil Locality.

A-10-1

10.2 Fossil Symbols

A-10-1

1. Geophysical and Structure Contours .......

A-10-1

12. Fluvial and Alluvial Features

A-11-1

13. Glacial and Glaciofluvial Features

A $-12-1$

14. Periglacial Features

A-13-1

15. Lacustrine and Marine Features ...............................

A-14-1

16. Eolian Features

A-15-1

17. Landslide and Mass-Wasting Features

A-16-1

18. Volcanic Features

A-17-1

19. Natural Resources

A-18-1

19.1 Veins and Mineralized Areas; Mineral Resource Areas; Metamorphic Facies Boundary ......

A-19-1

19.2 Areas of Extensively Disturbed Ground; Surface Workings; Subsurface Workings

19.3 Mining and Mineral Exploration (at Surface) .....

A-19-2

19.4 Mines and Subsurface Workings

A-19-3

19.5 Oil and Gas Fields; Wells Drilled for Hydrocarbon Exploration or Exploitation

20. Hazardous Waste Sites

21. Neotectonic and Earthquake-Hazard Features

22. Plate-Tectonic Features

A $-21-1$

23. Miscellaneous Uplift and Collapse Features

24. Terrestrial Impact Features

25. Planetary Geology Features

26. Geohydrologic Features

26.3 Water Gaging Stations

26.7 Miscellaneous Geohydrologic Features

27. Weather Stations

28. Transportation Features

29. Boundaries

30. Topographic And Hydrographic Features

30.1 Topographic, Bathymetric, And Glacier Contours 
31. Miscellaneous Map Elements A-31-1

32. Geologic Age Symbol Font ("FGDCGeoAge")

33. Suggested Ranges of Map-Unit Colors for Volcanic and Plutonic Rocks and for Stratigraphic Ages of Sedimentary and Metamorphic Rocks

33.1 Suggested Range of Map-Unit Colors for Volcanic and Plutonic Rocks

33.2 Suggested Range of Map-Unit Colors for Stratigraphic Ages of Sedimentary and Metamorphic Rocks A-33-1

34. State Location Maps A-34-1

34.1 Individual States; District of Columbia; Guam; Puerto Rico; U.S. Virgin Islands A-34-1

34.2 Conterminous States A-34-3

35. Bar Scales A-35-1

36. Mean Declination Arrows A-36-1

36.1 Magnetic North, East of True North A-36-1

36.2 Magnetic North, West of True North A-36-3

37. Lithologic Patterns A-37-1

37.1 Sedimentary-Rock Lithologic Patterns A-37-1

37.2 Metamorphic-Rock, Igneous-Rock, and Vein-Matter Lithologic Patterns A-37-3

38. Explanation for Pattern Chart A-38-1

39. Explanation for CMYK Color Chart A-39-1

\section{INDEX}

Index to Introductory Text and Appendix A I-1

\section{PLATES}

CMYK Color Chart In pocket

Pattern Chart In pocket 


\section{INTRODUCTORY MATERIAL}

\subsection{OBJECTIVE}

This document provides a single national standard for the digital cartographic representation of geologic map features. This standard is intended to support the Nation's producers and users of geologic map information by providing line symbols, point symbols, and colors and patterns that can be used to portray the various features on geologic maps. The objective of this standard is to aid in the production of geologic maps and related products, as well as to help provide geologic maps and products that are more consistent in both their appearance and their underlying database content.

A geologic map is a cartographic product that expresses information about the geology of a particular area. The map uses graphical elements such as line symbols, point symbols, and colored or patterned areas to portray complex geological information such as the composition, age, genesis, and extent of an area's geologic materials, as well as the geometry, orientation, and character of the geologic structures that have deformed them.

Geologic maps generally are intended for use by both the geoscience professional and the general public; however, designing and preparing a geologic map that will inform such an audience can be a daunting task because of the complexity of both the mapping concepts and the geologic information. The imperative for clear communication of geologic map information to a diverse audience was outlined early in the history of the U.S. Geological Survey (USGS) by then-Director John Wesley Powell, who stated that "the maps are designed not so much for the specialist as for the people, who justly look to the official geologist for a classification, nomenclature, and system of convention so simple and expressive as to render his work immediately available alike to the theoretic physicist or astronomer, the practical engineer or miner, and the skilled agriculturist or artisan" (Powell, 1888, p. 229).

The consistent, unambiguous expression of geologic map information is even more critical now because such information increasingly is compiled, stored, manipulated, and exchanged in digital files and geospatial databases. In the digital files, the cartographic representation of each feature on a geologic map must have a unique and explicit meaning, and it also must be compatible with the feature's attributes in the geologic map database. To that end, the preparers of this standard reviewed existing formal and informal USGS geologic map symbolization standards and adapted them for implementation with modern digital mapmaking systems and geospatial databases.

This standard attempts to facilitate geologic map communication and comprehension by providing clear and familiar symbology, thereby ensuring that the lines, points, and areas on the map convey the same meaning to all information producers and users. This standard also endeavors to clarify some of the concepts of geologic mapping, as well as to standardize some of the terminology used to describe the various features on a geologic map.

Although this standard is herein formalized, it is not intended to be used inflexibly or in a manner that will unduly restrict a geologist's ability to communicate the observations and interpretations gained from geologic mapping. On the contrary, this standard recognizes that, in certain situations, an existing symbol or its usage might need to be modified to fit a particular geologic situation or setting. Likewise, this standard recognizes that a new symbol or set of symbols may need to be created to more fully express local geologic conditions or to keep pace with evolving geologic mapping concepts and practices. Accordingly, such new or modified symbols, if found to be of wide applicability, will be incorporated into this standard through planned, periodic revisions.

\subsection{SCOPE}

This standard contains descriptions, examples, cartographic specifications, and notes on usage for a wide variety of symbols that may be used on typical, general-purpose geologic maps and related products such as cross sections. However, the standard also can be used for different kinds of special-purpose or derivative map products and databases that may be focused on a specific geoscience topic (for example, slope stability) or class of features (for example, a fault map). The standard is scale-independent, meaning that the symbols are appropriate for use with geologic mapping compiled or published at any scale. It is designed for use by anyone who either produces or uses geologic map information, whether in analog or digital form. 


\subsection{APPLICABILITY}

This document establishes standards that are applicable to all geologic map information (in other words, geologic maps and databases) published by the Federal Government and its Federally funded contractors and collaborators. Non-Federal agencies and private firms that produce geologic map information also are urged to adopt the standard.

The standard applies to all forms of geologic map publications, whether they are released as (1) hard-copy products, in either offset-print or plot-on-demand format, or (2) digital products, either as files for spatial analysis in Geographic Information Systems (GIS), as Portable Document Format (PDF) files in online publications, or as browse-graphic files for display on the World Wide Web. In particular, the standard applies to all geologic map products archived within the National Geologic Map Database (NGMDB), which is administered by the USGS: geologic map products submitted to and incorporated within the NGMDB will conform to this standard.

\subsection{RELATED STANDARDS}

The USGS traditionally has established nationally applicable cartographic standards for the production of geologic map information, both explicitly, through various formal and informal standards documents (see Section 2.1 below, entitled "Relation to Previous U.S. Geological Survey Standards"), and implicitly, through the cartographic content of its publications. This standard supersedes any existing USGS formal or informal cartographic standards for geologic maps.

During preparation of this standard, its relation to other standards or standards-development activities was assessed, and no significant conflicts were found. For example, the International Organization for Standardization (ISO) Standard 710, Parts 1-4, describes a general schema for graphical display of a selected set of geologic map symbols. Although similar to some that are included in this standard, they were found to have limited applicability. In addition, similar standards have been developed in other agencies of the Federal Government, including the U.S. Forest Service (in the geology component of their Terra database), the U.S. Army Corps of Engineers (in the geology component of their Spatial Data Standard for Facilities, Infrastructure, and the Environment [SDSFIE]), and the U.S. Bureau of Reclamation (in their Engineering Geology Office Manual). These were found to be somewhat specialized and limited in their coverage of geologic map features. Conversely, this standard provides comprehensive coverage of symbology for a broad range of geologic map features.

\subsection{STANDARDS DEVELOPMENT PROCEDURES}

This standards document represents only the latest milestone in a long history of geologic map standards development in the United States, which, within the USGS, began prior to 1881. As then-Director John Wesley Powell noted in 1888, in reference to geologic map standards under development at that time within the USGS, "While it is not professed that this [cartographic] system is final, or even unobjectionable, it represents the present state of knowledge and opinion" (Powell, 1888, p. 230). Although the present standards document draws heavily on previously established formal and informal cartographic standards of the USGS, it has undergone substantial revisions that reflect current geologic mapping practices and modern digital mapmaking methods. Accordingly, the standards-development procedures outlined in this section will address only the most recent development history of this standard (for a more complete historical background, see Section 2.1 below, entitled "Relation to Previous U.S. Geological Survey Standards").

This standards document was developed by members of the USGS Geologic Discipline's Western Publications Group and the National Geologic Map Database (NGMDB), with guidance and contributions from members of the Map Symbol Standards Committee (see below; see also, Section 2.3, entitled "Preparers of This Standard"). In addition, this standards document has benefited from the broad, modern-day perspective gained from the many thoughtful responses from reviewers of the Federal Geographic Data Committee's (FGDC) Public Review Draft of the standard (Federal Geographic Data Committee, 2000; see also, U.S. Geological Survey, 2000). The preparers of this standard gratefully acknowledge all current and prior participants and appreciate their invaluable contributions to the development of both this standards document and all preceding works.

In 1995, a proposed cartographic standard for geologic map information was informally released by the USGS 
as the "Cartographic and Digital Standard for Geologic Map Information" (U.S. Geological Survey, 1995a, 1995b). In 1996, this proposed standard was formally reviewed by geologists and cartographers from the USGS, as well as from the Association of American State Geologists (AASG), which represents the State geological surveys, and from the FGDC Geologic Data Subcommittee, which is composed of representatives from Federal agencies that produce or use geologic map information. That review (Soller, 1996) indicated the need for some revision to the proposed standard prior to its consideration by the FGDC for formal adoption as a Federal standard.

In 1996, plans were outlined to create a revised and updated Federal standard, and an early standardsdevelopment group was formed (see Section 2.3 below, entitled "Preparers of This Standard"). A proposal to develop the revised standard was submitted by the FGDC Geologic Data Subcommittee (see http://ngmdb.usgs.gov/fgdc_gds/mapsymbprop.php), and the FGDC accepted that proposal in 1997. Later that year, the standards-development group produced a preliminary version of the draft standard, which was circulated among selected USGS and State geological survey personnel for review. Comments were incorporated and, in 1999, the revised draft standard was submitted (as the "Working Draft") to the FGDC Geologic Data Subcommittee for consideration. Upon review and subsequent approval by the Subcommittee, the Working Draft was submitted to the FGDC Standards Working Group, which, in 2000, approved the document for public review as the "Public Review Draft" (see below), pending adoption of minor changes.

The Public Review Draft of this standard was finalized and then published in April 2000 (Federal Geographic Data Committee, 2000; see also, U.S. Geological Survey, 2000). In May 2000, the public was invited to review the draft standard and to provide comments and suggestions for revision (see http://ngmdb.usgs.gov/fgdc_gds/geolsymstd/prd/index.php). At the end of the 120-day public review period (May 19 through September 15, 2000), all comments and suggestions pertaining to the Public Review Draft were compiled, and a plan was developed to address the comments and make the necessary changes. Under this plan, a standing Map Symbol Standards Committee was formed to assist in the resolution of the public's review comments and suggestions, as well as in the long-term maintenance of the standard. Committee members were drawn from the geologic mapping community in the State geological surveys, academia, and the USGS (see Section 2.3 below, entitled "Preparers of This Standard").

Revisions to the standards document began in 2001. In July 2005, the revised standard was approved by the Map Symbol Standards Committee, and then it was submitted to the FGDC Geologic Data Subcommittee to begin the final approval process. After review and subsequent approval by the Geologic Data Subcommittee, as well as by the FGDC's Standards Working Group, Coordination Group, and Steering Committee, the final standard (this document) was formally approved as an FGDC standard in August 2006.

This standard will be managed as a "living" standard - that is, it will be maintained and revised as needed to reflect new mapping concepts or evolving usage conventions. The initial release of this FGDC-approved standard is available as an offset-printed document, supplemented by an online (PDF) version. However, all future updates to this standards document will be released online in PDF format only. To help maintain an up-to-date hard-copy version of the standards document, this initial offset-printed release has been designed in a "loose-leaf" format. Subsequent updates to this standards document may be downloaded as PDF files from the FGDC Geologic Data Subcommittee website (http://ngmdb.usgs.gov/fgdc_gds/) and then printed out and inserted where appropriate into a loose-leaf binder. These online updates will be the authoritative reference.

Because this standard is intended for use with digital applications, a PostScript implementation of the Public Review Draft was informally released as a USGS Open-File Report (U.S. Geological Survey, 2000). This early PostScript implementation enabled reviewers to directly apply the standard to geologic maps and illustrations prepared in desktop illustration and (or) publishing software. The PostScript implementation has been updated to reflect changes found in the now-approved standard and has been released as a USGS Techniques and Methods report (U.S. Geological Survey, 2006). Additionally, preliminary work on an ArcGIS implementation may be completed in the future and released as a USGS report. Information regarding these implementation efforts will be posted on the FGDC Geologic Data Subcommittee website (http://ngmdb.usgs.gov/fgdc_gds/).

Questions and comments about, or suggested additions to, this standard may be submitted by email to mapsymbol@flagmail.wr.usgs.gov or mailed to Geologic Map Symbol Standard, c/o David R. Soller, National Geologic Map Database, U.S. Geological Survey, 926A National Center, Reston, Virginia, 20192. 


\subsection{MAINTENANCE AUTHORITY}

On behalf of the FGDC, the USGS will maintain this Federal standard. The responsibility for coordinating Federal geologic mapping information is stipulated by Office of Management and Budget Circular A-16 (see http://www.whitehouse.gov/omb/circulars/a016/a016.html). The Geologic Mapping Act of 1992 (see http://ncgmp.usgs.gov/ncgmpabout/ngmact/ngmact1992 and subsequent reauthorizations) stipulates a requirement for standards development under the auspices of the National Geologic Map Database (NGMDB). Under this authority, the NGMDB will function on behalf of the USGS as coordinator of this maintenance activity (see http://ngmdb.usgs.gov/fgdc_gds/geolsymstd/maintenance.php). Maintenance will be conducted in cooperation with the AASG, which is the USGS's partner in the Geologic Mapping Act. The NGMDB will continue to rely on the Map Symbol Standards Committee to assist in its maintenance efforts. The Committee membership comes from the NGMDB, the USGS scientific staff and Publications Groups, the AASG, and the academic community (see Section 2.3 below, entitled "Preparers of This Standard"). The Committee will, as needed, review comments and suggestions for revisions, additions, and deletions to the standard.

\section{BACKGROUND}

\subsection{RELATION TO PREVIOUS U.S. GEOLOGICAL SURVEY STANDARDS}

Soon after the USGS was established in 1879, USGS geologists began to map and assess the Nation's lands, including many areas previously unexplored by Europeans. A new publication series, the Geologic Atlas (or "Folio") series, was created to publish many of these maps. Beginning prior to 1881, the USGS, then under the direction of John Wesley Powell, began to identify geologic and cartographic standards and conventions necessary to uniformly portray the geology in this series: "In providing for the publication of this large body of material, it seemed wise to adopt a common system of general nomenclature, a uniform color scheme for geographic geology, a system of conventional characters for diagrams, and a form for geologic and topographic charts and atlases" (Powell, 1882a, p. XL; see also, Powell, 1882b, for an elaboration on the proposed standards). Following an 1889 Conference on Map Publication, these standards were articulated in more detail and then were published (Powell, 1890).

The standards that were adopted by the USGS in the 1880s served as a strong foundation for the Nation's geological science. Paramount to systematized geologic mapping was the adoption of a standard rock stratigraphic nomenclature, a naming convention for geologic formations, and the subdivisions of geologic time. Another significant contribution was the adoption of a standardized color scheme for displaying geologic map units. This scheme used pure, single-ink colors, usually a different one for each geologic time period; to achieve this, a practical and informative system of overprint patterns also was developed, which served to differentiate the various mapped units within a single time period. Although this single-ink color scheme did not persist intact in the twentieth century because of the emergence of more modern printing technologies (for example, the combining of CMYK - cyan, magenta, yellow, and black - inks to produce a greater variety of colors), many of the overprint patterns that were developed then are still in use today.

In the following decades, as the geological sciences advanced, the concepts of geologic processes and historical geology became more complex, and new insights and refinements required more map symbols and precise scientific cartographic methods to convey details of geology. In 1920, the USGS published a manual on the preparation of illustrations (Ridgway, 1920). By that time, the need for standardization had become urgent: "More than 200 symbols have been used on maps to express 25 different kinds of data, a fact indicating at once a notable lack of uniformity and a need of standardization" (Ridgway, 1920, p. 20). The manual addressed various issues associated with geologic cartography, including standard symbology for geologic maps and cross sections (for example, geologic line and point symbols, water wells, oil and gas wells, coal seams, mine workings, and topographic and other base-category information) and stratigraphic columns (for example, lithologic patterns).

After 1920, and throughout much of the twentieth century, the maintenance of USGS standards for geologic map symbolization and cartography was an internal and somewhat informal process enacted through official USGS policy. For example, USGS Chief Geologist W.H. Bradley (written commun., 1956) adopted recommendations and a list of symbols from the Map Symbol Committee (E.N. Goddard, Chairman), and USGS Chief Geologist D.L. Peck (written commun., 1978) adopted recommendations from the committee for 
Standards for General Purpose Geologic Maps (J.C. Reed, Chairman).

In the mid-1970s, the USGS outlined the technical specifications for geologic symbology in its informal "Technical Cartographic Standards" volume (U.S. Geological Survey, ca. 1975). This informal standard, which was maintained until the mid-1980s, was available to USGS cartographers and editors as a set of green, loose-leaf notebooks that allowed pages to be replaced as the standard evolved. The technical specifications at that time were devised to serve the needs of cartographers who prepared maps for offset-print publication using hand-placed type, hand-scribed linework, and peelcoat color-separation techniques. This informal standard served the USGS well, but it was not available to other producers or users of geologic maps, nor was it formally recognized as a standard by the Nation's geoscience community. However, the cartographic details of this standard were clearly displayed on USGS geologic maps. And so, drawing from the cartographic content of USGS maps, others have published manuals on geologic map standards that have (unofficially) incorporated parts of this informal standard: for example, the American Geological Institute's "AGI Data Sheets for Geology in the Field, Laboratory, and Office" (Dietrich and others, 1982 [2nd ed.]; Dutro and others, 1989 [3rd ed.]) includes many symbols commonly shown on USGS geologic maps (see also, "Suggestions to Authors of the Reports of the United States Geological Survey" [7th ed.], Hansen, 1991).

Beginning about the mid-1980s, digital-cartographic and GIS (Geographic Information System) technologies rapidly evolved and became more widely available. The gradual adoption of digitally based mapmaking methods made clear the need to develop new cartographic standards that would satisfy the requirements of the latest technologies for the preparation of digital files, whether they are to be used for geospatial databases, for plot-on-demand or online map publications, or for the production of negatives for offset printing of maps.

In response to this steady increase in digital mapmaking and the accompanying concern about preparing consistent, high-quality, digitally produced geologic maps and geologic map databases, the USGS informally released in 1995 a proposed standard entitled "Cartographic and Digital Standard for Geologic Map Information" (U.S. Geological Survey, 1995a). As noted above, subsequent review of that document by the USGS, the AASG, and the FGDC Geologic Data Subcommittee (Soller, 1996) indicated the need for some revision prior to its consideration by the FGDC for formal adoption as a Federal standard, which led to the development of this standard (see discussion in Section 1.5 above, entitled "Standards Development Procedures").

\subsection{CHANGES FROM PREVIOUS STANDARDS}

In this new standard (contained in [normative] appendix A), descriptions, examples, cartographic specifications, and notes on usage are provided for a wide variety of symbols that may be used on typical digital geologic maps or related products such as cross sections. In the preparation of this standard, every effort was made to retain the original symbols and their specifications from the 1995 USGS proposed standard (U.S. Geological Survey, 1995a); however, many updates have been incorporated into this new version. The number of symbols has increased significantly, from about 800 to over 2300. Symbols are more logically grouped; some sections have been combined with others, and a few new sections have been added.

Many symbols, particularly lines, have been redesigned slightly so that they would more successfully translate to digital applications. For instance, in the old "Technical Cartographic Standards" volume (U.S. Geological Survey, ca. 1975), as well as in the 1995 USGS proposed standard (U.S. Geological Survey, 1995a), the lineweight for contacts was specified as .005 inches (.125 millimeters). However, experience has shown that .005 -inch lines do not always plot well when digitally output by high-resolution imagesetters. Therefore, the minimum lineweight for contacts, as well as for most other stroked-line symbol elements, has been increased to .006 inches (.15 millimeters) in this new standard. In addition, the dash and gap lengths for many line symbols have been adjusted so that their dash-gap templates can be more easily defined electronically.

A chart showing a wide range of CMYK colors ("CMYK Color Chart") has been included; an offset-print version of this chart has been in use at the USGS for many years, and the variety of colors has proved to be sufficient for portraying complex geology shown on most maps, regardless of the output medium. In addition, a chart that shows commonly used geologic patterns ("Pattern Chart") has been added; the patterns themselves are similar to what was in the old "Technical Cartographic Standards" volume (U.S. Geological Survey, ca. 1975), as well as in the 1995 USGS proposed standard (U.S. Geological Survey, 1995a), but most have undergone 
lineweight changes to facilitate digital output at high resolutions. The old pattern numbers have been revised and the patterns are now organized into seven geologically relevant series. A few new patterns have been added, and some have been eliminated. In addition, each pattern in the Pattern Chart, as well as each color in the CMYK Color Chart, has associated with it a generic lookup-table number that, if desired, may be used to access the pattern (or color) from within digital applications.

Also included in this new standard is a diagram showing suggested ranges of map-unit colors for stratigraphic ages of sedimentary and metamorphic rocks, as well as for volcanic and plutonic rocks. In addition, a new geologic age symbol font ("FGDCGeoAge") has been added. Three new sections that address map marginalia have been included: (1) quadrangle location maps for each of the 50 states (and District of Columbia, Guam, Puerto Rico, and U.S. Virgin Islands), as well as a map of the 48 conterminous states (so that quadrangle locations covering more than one state can be shown); (2) a variety of bar scales, as well as calculation tables that show how to convert between inches, miles, and kilometers; and (3) a series of mean declination arrows, showing magnetic north both east and west of true north.

A few new informational sections have been added to the introductory material in this standard. The section entitled "Guidelines for Map Color and Pattern Selection" provides useful information on color selection and the use of patterns. The section entitled "Guidelines for Map Labeling" provides recommendations on placement of text on a map.

The most significant update to this standard is the addition of two important sections to the introductory material. The section entitled "Geologic Mapping Concepts and Definitions" provides basic information about some of the fundamental concepts of geologic mapping, as well as defines and categorizes the various types of geologic map features. The section entitled "Scientific Confidence and Locational Accuracy of Geologic Features" clarifies the concepts of, and establishes new terminology for, the levels of scientific confidence and locational accuracy of geologic map features.

In response to reviewer's comments (Soller, 1996), much of the first part of the 1995 USGS proposed standard has been abandoned because it was either not pertinent to this standard (for example, the sections on geologic map content, metadata, and geocoding) or not widely applicable to the full range of mapping situations (for example, the specification of a "1.0 mm accuracy standard"). In addition, no attempt has been made in this new standard to provide detailed definitions for the geologic features represented by the various symbols. For such information, please refer to one of a number of reference books available; an excellent source is the American Geological Institute's Glossary of Geology (Jackson, 1997 [4th ed.]; Neuendorf and others, 2005 [5th ed.]).

\subsection{PREPARERS OF THIS STANDARD}

Principal contributors ${ }^{1}$ to the preparation of this FGDC Digital Cartographic Standard for Geologic Map Symbolization include the following individuals:

David R. Soller (USGS; Chief, National Geologic Map Database) -Coordinator, editor, and author, FGDC Digital Cartographic Standard for Geologic Map Symbolization; coordinator, Map Symbol Standards Committee.

Taryn A. Lindquist (USGS; Digital Map Specialist and Geologic Map Editor, Western Publications Group) -Editor, author, and compiler, FGDC Digital Cartographic Standard for Geologic Map Symbolization; designer, line symbols and point symbols, FGDC Digital Cartographic Standard for Geologic Map Symbolization.

Map Symbol Standards Committee: Thomas Berg (State Geologist, Ohio); Jay Parrish (State Geologist, Pennsylvania); Mark Jirsa (Minnesota Geological Survey); Robert Hatcher (University of Tennessee, Knoxville); Steven Reynolds (Arizona State University); and Byron Stone, Jack Reed, Jonathan Matti,

\footnotetext{
${ }^{1}$ Unless otherwise noted, persons listed as contributors to the "FGDC Digital Cartographic Standard for Geologic Map Symbolization" participated in the preparation of the following versions of the standard: Working Draft; Public Review Draft (Federal Geographic Data Committee, 2000) and its PostScript implementation (U.S. Geological Survey, 2000); and the now FGDC-approved standard (this document) and its PostScript implementation (U.S. Geological Survey, 2006).
} 
Taryn Lindquist, and David Soller (all USGS) - Referees and reviewers of public comments and subsequent revisions, Public Review Draft (Jonathan Matti is especially noted for his guidance on issues of scientific confidence and locational accuracy).

Sara Boore (USGS; Publication Graphics Specialist, Western Publications Group) - Book designer, FGDC Digital Cartographic Standard for Geologic Map Symbolization; designer, point symbols, line symbols, color charts, and patterns, FGDC Digital Cartographic Standard for Geologic Map Symbolization.

F. Craig Brunstein (USGS; Geologic Map Editor, Central Publications Group)-Technical reviewer, Working Draft.

Alessandro J. Donatich (USGS; Geologic Map Editor, Central Publications Group) - Technical reviewer, Working Draft.

Carolyn Donlin (USGS; Online Publications Specialist and Geologic Map Editor, Western Publications Group)-Preparer, online publication of Public Review Draft (PostScript implementation).

Michael F. Diggles (USGS; CD-ROM Publications Specialist and Online Publications Specialist, Western Publications Group) - Preparer, CD-ROM publications of PostScript implementations; preparer, online publication of FGDC-approved standard (PostScript implementation).

Kevin Ghequiere (USGS; Cartographer, Western Publications Group) - Designer, patterns, FGDC Digital Cartographic Standard for Geologic Map Symbolization.

Richard D. Koch (USGS; Digital Map Specialist, Western Publications Group) - Designer, geologic age symbol font, FGDC Digital Cartographic Standard for Geologic Map Symbolization.

Diane E. Lane (USGS; Geologic Map Editor, Central Publications Group) - Technical reviewer, Working Draft.

Susan E. Mayfield (USGS; Publication Graphics Specialist, Western Publications Group) - Designer, color charts and patterns, FGDC Digital Cartographic Standard for Geologic Map Symbolization.

Kathryn Nimz (USGS; Digital Map Specialist, Western Publications Group) - Designer, patterns, FGDC Digital Cartographic Standard for Geologic Map Symbolization.

Glenn Schumacher (USGS; Publication Graphics Specialist, Western Publications Group) - Designer, bar scales, mean declination arrows, and quadrangle location maps, FGDC Digital Cartographic Standard for Geologic Map Symbolization.

Stephen L. Scott (USGS; Publication Graphics Specialist, Western Publications Group) - Designer, point symbols and line symbols, FGDC Digital Cartographic Standard for Geologic Map Symbolization.

Will Stettner (USGS; Cartographer, Eastern Publications Group) - Technical reviewer, Working Draft.

José F. Vigil (USGS; Motion Graphics Specialist, Western Publications Group) - Designer, geologic age symbol font, FGDC Digital Cartographic Standard for Geologic Map Symbolization.

Jan L. Zigler (USGS; Geologic Map Editor, Western Publications Group) - Technical reviewer, Working Draft.

\section{GEOLOGIC MAPPING CONCEPTS AND DEFINITIONS}

\subsection{GEOLOGIC MAPS}

A geologic map is a cartographic product that portrays information about the geologic character of a specific geographic area. It is a two-dimensional representation of real-world, three-dimensional geologic features. To achieve this, a geologic map uses graphical elements to express detailed information about the different kinds of earth materials, the boundaries that separate them, and the geologic structures that have subsequently deformed them. For example, a typical general-purpose geologic map may consist of lines that trace contacts, faults, and folds; points that locate bedding attitudes, minor fold orientations, and sample localities; areas that represent geologic units, landslides, and areas of alteration; and labels that identify geologic map units, sample-locality 
numbers, and fault names. Thus, an appropriately symbolized and labeled geologic map can portray comprehensive information about the composition, age, and genesis of the geologic materials and the nature of their boundaries, as well as the character and three-dimensional geometry of the geologic structures that have deformed them. In addition, such geologic map information usually is drawn onto a base map that also uses graphical elements to represent the topography, drainage, and cultural features of an area, and so a geologic map also can depict the spatial relation of the various geologic features to the physical landscape. Other things that may be shown on a geologic map include information about the geomorphology, pedology, paleontology, rock alteration and mineralization, geophysics, geochemistry, or geochronology of an area.

\subsection{GEOLOGIC MAP DATABASES}

A geologic map database is a digitally compiled collection of spatial (geographically referenced) and descriptive geologic information about a specific geographic area. The information in the geologic map database consists of (1) the geographic location and the orientation, length, shape, and (or) area (in other words, the geometry) of each geologic feature or object (for example, an outcrop or a fault), and (2) many different types of descriptive geologic information about each feature or object.

A geologic map database also may contain extensive amounts of additional qualitative and quantitative geologic information. For example, a geologic map database may include geochemical analyses, radiometric ages, soilhorizon information, and geophysical contours, as well as information on the weathering of surface exposures of geologic features, the subsurface geometry of geologic map units, and the glacial landforms or other types of geomorphic features.

Fundamental data elements of a geologic map database are lines (for example, contacts and faults), points (for example, bedding attitudes and fossil localities), and areas or polygons (for example, map-unit areas and zones of alteration). In addition, each feature or object in the geologic map database has several associated feature attributes. The most basic feature attributes may simply identify the feature (for example, "thrust fault" or "overturned anticline") and express its scientific confidence and locational accuracy (for example, "identity certain" or "location inferred"). Other feature attributes may consist of detailed descriptions of each feature (for example, the lithologic characteristics of a map unit, the dip of a mapped fault, or the identification and age determination of a fossil specimen).

When a geologic map is generated as a cartographic product from a geologic map database, each geologic feature is represented by a specific geologic map symbol. The attributes in the database provide the information needed to symbolize each feature. In addition, annotation is added to the geologic map wherever necessary to identify the various features (for example, map-unit labels and fault names) and to provide essential quantitative information (for example, dip values and fossil-locality numbers).

\subsection{GEOLOGIC MAP UNITS}

A geologic map unit is a cartographic representation of a volume of geologic materials that share enough characteristics (for example, the composition, areal extent, age, and (or) genesis) to be considered a single entity (a single geologic unit). On a typical geologic map, most geologic units are represented by polygons that are filled with colors and (or) patterns. Geologic units can also be represented by lines (for example, dikes) or points (for example, blueschist blocks).

The formation, whether formal or informal, is the lithostratigraphic unit most commonly depicted on a geologic map. A formation can be subdivided into lower rank stratigraphic units (for example, members, tongues, lentils, or beds) or assembled with other formations to make up more generalized, higher rank stratigraphic units (for example, groups or supergroups), depending on the scale of the map or the focus of the geologist (see guidelines for the recognition and naming of geologic units by the North American Commission on Stratigraphic Nomenclature, 1983).

\subsubsection{Geologic Time, the Ages of Rock Units, and Geologic Age Symbols}

The USGS has published a scheme for the major divisions of geologic time, the age estimates of the boundaries, and the specialized geologic age symbols to be used on geologic maps (Hansen, 1991). This particular scheme was formally adopted after a 1980 meeting of the Geologic Names Committee of the USGS (Hansen, 1991). In 
addition, several other schemes of geologic time boundaries have been published (see, for example, Harland and others, 1982, 1989; Palmer, 1983; Snelling, 1985; Berggren and others, 1995; Gradstein and Ogg, 1996; Haq and van Eysinga, 1998; International Union of Geological Sciences, 1998; Palmer and Geissman, 1999), each of which is based on different assumptions, techniques, and (or) data. Any formally published age scheme may be used for a particular map, as long as which scheme was used is specified on the map and in the geologic map database.

\subsubsection{Map-Unit Labels}

A map-unit label is an alphanumeric symbol that identifies the geologic map unit on the map. The map-unit symbol is an abbreviated acronym that usually is made up of, in the following order, (1) either capital letters or geologic age symbols indicating the age of the geologic unit (see Appendix A, Section 32), and (2) lower case letters denoting the name or the lithologic characteristics of the geologic unit. In some cases, numerical subscripts are added to designate different subunits (for example, members or individual lava flows) within a geologic unit.

Map-unit labels are added to the geologic map wherever necessary to clearly identify the various geologic map units. In addition, map-unit labels are included among the feature attributes in the geologic map database, thereby designating each mapped area as belonging to a particular geologic map unit.

\subsection{PLANAR GEOLOGiC FEATURES}

A planar geologic feature is a two-dimensional geologic surface, which may be either a real-world, physical surface (for example, a contact between two geologic units) or a hypothetical surface (for example, an axial surface of a fold). The geometry of the geologic surface may be flat, curved, or crenulated, and its orientation may be horizontal, inclined, vertical, or overturned.

The intersection of a planar geologic feature with the ground surface forms a real or perceived (projected) linear trace. When these linear traces are mapped in the field and then plotted as lines on a base map, they become the most basic and fundamental elements of a typical geologic map: they may delineate simple map-unit areas, or they may define complex patterns of structural deformation. The various types of linear traces are portrayed on a geologic map by unique line symbols (Appendix A), each of which has a different width, pattern, ornamentation, or color; thus, a particular line symbol conveys specific information about the character and (or) geometry of each planar geologic feature.

\subsubsection{Contacts}

A contact is a planar surface that bounds a geologic unit (except where that bounding surface is a fault; see discussion below in Section 3.4.3, entitled "Faults"). A contact is intrinsic to the genesis of each geologic unit; that is, the contact delineates the stratigraphic position where, owing to changing environmental conditions or other genetic factors at the time of origin, the properties and characteristics of one geologic unit change, either abruptly or gradually, to those of another geologic unit.

Discussion of contacts in this standard primarily pertains to those that have been mapped in the field (for example, contacts that bound formations, members, beds, lava flows, or intrusions). Contacts can also exist between higher rank units, although these contacts typically are not mapped in the field; instead, they are concepts that may arise later when lower rank stratigraphic units are combined into higher rank stratigraphic units (see discussion of lithostratigraphic boundaries by the North American Commission on Stratigraphic Nomenclature, 1983, p. 856-58).

Contacts can be classified as one of a number of types, depending on the nature or origin of the contact and the geologic units that it separates. Examples of such contact types include the following: sedimentary (conformable; unconformable, etc.); alluvial; landslide; residual; igneous (intrusive, extrusive, pyroclastic); metamorphic; and high-strain (cataclastic, mylonitic, tectonic). If available, supplemental information about a contact's type is added as a feature attribute to the geologic map database; however, specialized line symbols usually are not used to represent these various contact types. In general, unless otherwise stated on the geologic map or in the geologic map database, contacts should be considered generic; that is, they have no particular type or identity. 
The geologic age of a contact also may be specified as a feature attribute in the geologic map database, but rarely is this characteristic symbolized on the geologic map; if desired, such information can be communicated by the addition of geologic point data or annotation placed along the trace of the contact. In addition, specific information collected about a contact's local surface exposure, orientation or character can be added as geologic point data and annotation placed along the trace of the contact where the observation was made.

\subsubsection{Discrete versus Gradational Contacts}

In the field, a contact between two geologic units is a transition zone whose width can range from very narrow to very broad. Examples of transition zones include the following:

- a single surface, as sharply delineated as a knife-edge, between two lithologically distinct geologic units;

- a single surface that zigzags between two intertonguing geologic units;

- a narrow zone, a few centimeters to a few decimeters wide, in which the lithologic character changes from one geologic unit to another;

- a diffuse zone, a few meters to many meters wide, in which the lithologic character of one geologic unit gives way gradually to that of another geologic unit.

Despite the differences inherent in each of these examples, contacts generally can be classified as either one of two types of transition zones: discrete or gradational. A precise definition of the width of a discrete versus a gradational contact, however, is difficult because of (1) different scales of mapping (for example, a contact that is gradational at a scale of 1:24,000 would probably be considered discrete at a scale of 1:100,000); (2) differing interpretations that can arise between geologists whose mapping primarily focuses on either sedimentary, igneous, or metamorphic rocks (for example, contact relations that are considered gradational by a geologist who maps sedimentary rocks may be viewed as discrete by a geologist who maps plutonic rocks); and (3) differences in individual biases that may arise from different geologic-mapping traditions in geologically dissimilar parts of the Nation. Because of these and other factors, this standard makes no attempt to delimit the precise width of a discrete or a gradational contact. Nevertheless, this standard provides the following general definitions:

A discrete contact is a map-unit boundary that is individually distinct; that is, the transition between geologic units is abrupt enough to be recognized and delineated easily on the map. A discrete contact may be a sharp, knife-edged surface, or it may be transitional across a zone as wide as a meter or more, depending on the scale of the map.

A gradational contact is a map-unit boundary that is diffuse; that is, the transition between geologic units is gradual enough that it cannot be recognized or delineated easily on the map. A gradational contact is so diffuse across the transition zone (the width of which will vary at different map scales) that delineation of its exact position can be difficult.

The discrete versus gradational character of a contact is specified as a feature attribute in the geologic map database. In addition, if the map scale allows, gradational contacts can be represented on the geologic map by a specialized line symbol (see Appendix A, Section 1). Unless otherwise stated on the map or in the geologic map database, however, a generic contact (that is, one not represented by a specialized line symbol) should be considered discrete at the scale of the map.

\subsubsection{Key Beds}

A key bed is an easily identifiable stratigraphic marker bed within a geologic unit. Although a key bed is a three-dimensional volume rather than a two-dimensional surface, commonly it is too thin to depict as a map-unit area at most map scales, and so it usually is classified as a planar geologic feature.

Key beds are identified on the basis of their lithologic character and, in most cases, their relation to the surrounding rock materials. Examples of various types of key beds include the following:

- a coal bed;

- a fossiliferous horizon; 
- a cross-cutting dike;

- a clay bed in a dominantly coarse-grained sedimentary sequence;

- a gravel bed in a dominantly fine-grained sedimentary sequence;

- a marine sedimentary bed in a dominantly nonmarine sedimentary sequence;

- a nonmarine sedimentary bed in a dominantly marine sedimentary sequence;

- a sandstone bed in a dominantly carbonate sedimentary sequence;

- a limestone bed in a dominantly dolomitic sedimentary sequence;

- a volcanic-ash bed or flow in a dominantly nonvolcanic sequence.

The type of key bed can be specified as a feature attribute in the geologic map database. In addition, some types of key beds are portrayed on the geologic map by specialized line symbols (see Appendix A, Section 1). In some cases, if the map scale allows, key beds are represented by colored or patterned areas. Map-unit labels are added to the geologic map to identify the various types of key beds shown on the map. In addition, map-unit labels are included among the feature attributes in the geologic map database to identify each key bed.

\subsubsection{Faults}

A fault is a planar surface of rupture along which geologic units have been fractured and then displaced. Faults can be geometrically complex structures that juxtapose map units over great distances, or they can be simple fracture planes along which the amount of offset is very small.

Discussion of faults in this standard primarily pertains to those that have been mapped in the field. Faults also can be required conceptually when lower rank stratigraphic units are grouped into higher rank units or tectonostratigraphic terranes, although these faults may not have been observed in the field.

Faults can be classified as one of a number of types, depending on the nature of their geometry and (or) sense of offset. Examples of fault types include the following: normal (low-angle, listric); reverse; thrust; overturned thrust; vertical; strike-slip (right-lateral, left-lateral); oblique-slip; detachment; or some combination of the above. Information about a fault's type is specified as a feature attribute in the geologic map database. When the map scale allows, such information also is represented on the geologic map by a specialized line symbol and (or) line-symbol decoration. A particularly robust set of specialized line symbols and line-symbol decorations has evolved to represent the various fault types (see Appendix A, Section 2). In general, unless otherwise stated on the map or in the geologic map database, faults that lack such specialized symbology should be considered generic; that is, their geometry or sense of offset either is not known or has not been specified.

The age of a fault also can be specified as a feature attribute in the geologic map database, but rarely is this characteristic symbolized on the geologic map; if desired, such information can be communicated through the addition of geologic point data or annotation placed along the trace of the fault. In addition, specific information collected about a fault's local orientation can be added as geologic point data and annotation placed along the trace of the fault where the observation was made.

Some faults are relatively minor structures whose traces are mapped within single geologic units until the faults can no longer be observed or they no longer exist. More commonly, faults are mapped as larger, thoroughgoing structures that can produce a significant amount of offset between one or more geologic units, so that the rupture surfaces form new map-unit boundaries. In addition, faulting sometimes can take place at the stratigraphic position where a contact would normally exist between two stratigraphically coherent geologic units. But because faulting is not a process intrinsic to a geologic units' genesis (in these cases, faulting has occurred through already-formed geologic units), these bounding surfaces do not meet the criteria to be called contacts (see discussion above in Section 3.4.1, entitled "Contacts"). Therefore, although they may form boundaries between geologic units, such structures are classified as "faults," not "fault contacts" or "faulted contacts."

\subsubsection{Discrete Faults versus Fault Zones}

In the field, a fault forms a zone of offset whose width can range from very narrow to very broad. Examples of 
such zones of offset include the following:

- a single offset-fracture surface, as sharply delineated as a knife-edge;

- a narrow zone of offset, a few centimeters to a few decimeters wide;

- a diffuse zone, a few meters to many meters or as much as a kilometer or more wide, within which offset has been distributed among a few or many shear planes.

Despite the differences inherent in each of these examples, faults generally can be described in either one of two ways: as a discrete fault or as a fault zone. A precise definition of the width of a discrete fault versus a fault zone, however, is difficult for a number of reasons (see related discussion above in Section 3.4.1.1, entitled "Discrete versus Gradational Contacts"), and this standard makes no attempt to do so. Nevertheless, this standard provides the following general definitions:

A discrete fault is a zone of offset that is individually distinct; that is, the zone is narrow enough to be recognized and delineated easily on the map. A discrete fault may be a sharp, knife-edged surface of offset, or it may be a zone of offset as wide as a meter or more, depending on the scale of the map.

A fault zone (also called a shear zone) is a diffuse zone within which offset has been distributed among a few or many shear planes, commonly resulting in a zone of crushed and sheared or ductily deformed rock. In some cases, a fault zone can be mapped as an area bounded by discrete fault planes.

The character of a fault (discrete fault versus fault zone) is specified as a feature attribute in the geologic map database. In addition, a fault zone can be portrayed either by a specialized line symbol or, if the map scale allows, by a colored or patterned area (see Appendix A, Section 2). Unless otherwise stated on the map or in the geologic map database, a generic fault (that is, one not portrayed as an area or by a specialized line symbol) should be considered discrete at the scale of the map.

\subsubsection{Folds}

In its simplest sense, a fold is a geologic structure that results when a flat-lying or otherwise undeformed geologic surface is warped and deformed into an undulating geologic surface. In reality, many fold structures further deform bodies of rock that may already be highly deformed and (or) metamorphosed. Thus, folds may form simple, symmetric structures, or they may form complex, multidimensional and multigenerational fold systems.

The fold axis or hinge line of a fold is a hypothetical line that traces the locus of maximum curvature of the fold structure. The axial surface or axial plane of a fold is a hypothetical planar surface that connects the fold axes or hinge lines of folded strata.

Folds can be classified as one of a number of fold types, depending on the geometry of the fold's axial surface and the geometry and the relative ages of the folded strata. Examples of fold types include the following: anticline, syncline, monocline; antiform, synform; symmetrical, asymmetrical, overturned, inverted, isoclinal, recumbent, and plunging.

Information about a fold's type is specified as a feature attribute in the geologic map database. In addition, such information is portrayed on the geologic map by specialized line symbols and line-symbol decorations (see Appendix A, Section 5). On a geologic map, a fold is mapped as a line where the trace of its axial surface intersects the ground surface. In some cases, the trace of a fold's crest line (highest point on a fold's crest) or trough line (lowest point in a fold's trough) can also be mapped.

The age of a fold also can be specified as a feature attribute in the geologic map database, but rarely is this characteristic symbolized on the geologic map; if desired, such information can be communicated through the addition of geologic point data or annotation placed along the trace of the fold. In addition, specific data collected about a fold's local orientation can be added as geologic point data and annotation placed along the trace of the fold where the observation was made.

\subsection{LINEAR GEOLOGIC FEATURES}

A linear geologic feature is a one-dimensional geologic or geomorphic line, which may be either a real-world, 
physical line (for example, a moraine, lineament, or outcrop-scale lineation) or a hypothetical line (for example, a hinge line of a fold or a paleocurrent direction). The geometry of the line may be straight, curved, or crenulated, and its orientation may be horizontal, inclined, or vertical.

The orientations of linear geologic features are mapped in the field and then plotted as lines on a base map. Information about the various types of linear geologic features is specified as a feature attribute in the geologic map database. In addition, such information is represented on a geologic map by a unique line symbol (Appendix A), each of which has a different width, pattern, ornamentation, or color; thus, a particular line symbol conveys specific information about the character and (or) geometry of each linear geologic feature.

\subsection{GEOLOGIC POINT FEATURES}

A geologic point feature consists of geologic or geomorphic information that has been collected at a particular point of observation in the field (except when that point feature is a line-symbol decoration; see discussion below in Section 3.6.3.2, entitled "Line-Symbol Decorations"). In some field situations, more than one observation can be taken at a single locality.

Geologic point data may pertain to a planar feature (for example, the orientation of bedded strata), a linear feature (for example, the plunge of a fold axis), or a single locality (for example, a fossil locality). Geologic point data also can be added as line-symbol decorations (for example, anticline arrows) that provide supplemental information about a particular part of a line on a geologic map.

Geologic point data are recorded in the field and then plotted as points on a base map. Information about the various types of geologic point data is specified as a feature attribute in the geologic map database. In addition, such information usually is represented on a geologic map by specialized point symbols and associated annotation (Appendix A).

\subsubsection{Planar-Feature Geologic Point Data}

Planar-feature geologic point data consist of quantitative information about the character and the orientation of a geologic surface, which may be a physical surface (for example, a fault plane or bedded strata) or a hypothetical surface (for example, an axial surface of a fold or a plane of foliation). The geologic surface may be horizontal, inclined, vertical, or overturned.

Two measurements, the strike and the dip, define the orientation of a geologic surface in three-dimensional space:

- the strike of a surface is the azimuthal direction of a hypothetical line formed by the intersection of the surface with an imaginary horizontal surface, as measured in the direction that the observer is facing when the surface dips down to the right (this method of directional measurement follows the right-hand rule convention);

- the dip of a surface is the angle of departure of that surface downward from horizontal, as measured perpendicular to the line of strike.

Information about the type of observation, as well as the values of strike and dip, is specified as feature attributes in the geologic map database. Such information also is represented on the geologic map by specialized point symbols and associated annotation: the strike value and the direction of dip are implicit in the orientation of the point symbol; the dip value is added as annotation.

\subsubsection{Point Symbols for Planar Features, and Their Placement Relative to Point of Observation}

The point symbols for inclined or overturned planar features typically are made up of two parts: a long shaft oriented in the strike direction, and a short tick (or ornamentation such as a triangle) pointing in the downdip direction. The point symbol is placed on the map so that the intersection of its long shaft and short tick (or ornamentation) is at the point of observation. When data have been collected about the local orientation of a planar feature that has been represented on the map by a line symbol (for example, the dip of a contact or a fault), the point symbol is placed directly on the line symbol at the point of observation.

The point symbols for vertical planar features are similar to those for inclined surfaces, except that two short 
ticks (or ornamentations), not one, point away from the long shaft. The point symbol is placed on the map so that the intersection of its long shaft and short ticks (or ornamentations) is at the point of observation.

The point symbols for horizontal planar features, which display no directional information, are simply placed on the map at the point of observation.

\subsubsection{Specialized Planar-Feature Point Symbols for Multiple Observations at One Locality}

In situations where more than one observation has been taken at a single locality, point symbols for planar features can be combined with other point symbols at the point of observation. In these cases, specialized point symbols may be used to avoid the overprinting of information. These specialized point symbols have the short ticks (or ornamentations such as triangles) moved down near the end of the long shafts; the symbols are joined at their endpoints (opposite the ticks or ornamentations) at the point of observation.

\subsubsection{Linear-Feature Geologic Point Data}

Linear-feature geologic point data consist of quantitative information about the orientation of a geologic or geomorphic linear feature, which may be a physical line (for example, a fault-plane groove or slickenline) or a hypothetical line (for example, the intersection of two surfaces of deformation). The geologic or geomorphic linear feature may be horizontal, inclined, or vertical.

Two measurements, the bearing and the plunge, define the orientation of a geologic or geomorphic line in three-dimensional space:

- the bearing of a line is the azimuthal direction of the trend of that line, as measured in its direction of plunge;

- the plunge of a line is the angle of departure of that line downward from horizontal.

Information about the type of observation, as well as the values of bearing and plunge, is specified as attributes in the geologic map database. Such information also is represented on the geologic map by specialized point symbols and associated annotation: the bearing value and the direction of plunge are implicit in the orientation of the point symbol; the plunge value is added as annotation.

\subsubsection{Point Symbols for Linear Features, and Their Placement Relative to Point of Observation}

The point symbols for inclined linear features typically are made up of two parts: a shaft oriented in the bearing direction, and an arrowhead pointing in the plunge direction. The symbol is placed on the map so that the end of its shaft opposite the arrowhead is at the point of observation. When data have been collected about the local orientation of a linear feature that has been represented on the map by a line symbol (for example, a lineation on a fault), the point symbol is placed directly on the line symbol at the point of observation.

The point symbols for horizontal linear features are similar to those for inclined linear features, except that arrowheads are at both ends of the long shaft. The symbol is placed on the map so that the middle of its shaft is at the point of observation.

The point symbols for vertical linear features, which display no directional information, are simply placed on the map at the point of observation.

In situations where more than one observation has been taken at a single locality, point symbols for linear features can be combined with other point symbols at the point of observation. When a single linear-feature observation and a single planar-feature observation are taken at a single locality, the symbols are combined so that the end of the arrow that represents the linear feature is placed at the intersection of the planar-feature point symbol's long shaft and short tick (or ornamentation). When more than two such observations are taken at a single locality, the point symbols for linear features are joined at their endpoints with the specialized point symbols for planar features (see Section 3.6.1.2 above, entitled "Specialized Planar-Feature Point Symbols for Multiple Observations at One Locality") at the point of observation.

\subsubsection{Informational Geologic Point Data}

Informational geologic point data consist of geologic information that is supplemental to a typical geologic map or its features. Informational geologic point data are divided into two types: locality-information point data, and 
line-symbol decorations.

\subsubsection{Locality-Information Point Data}

Locality-information point data record information collected at a particular locality (for example, fossil localities or sample localities). The type of data collected at the locality is specified as a feature attribute in the geologic map database. In addition, such information commonly is represented on the geologic map by a specialized point symbol placed at the point of observation. Sample numbers or other identifying labels are added as annotation near the point symbols.

\subsubsection{Line-Symbol Decorations}

Line-symbol decorations are specialized point symbols that convey qualitative information about the character of a particular line or line segment (for example, anticline arrows or ball-and-bar symbols). The type of linesymbol decoration is specified as a feature attribute in the geologic map database. Line-symbol decorations are not placed at a specific point of observation because they do not represent information collected at a particular locality; instead, they should be placed at a strategic location (or locations) along the trace of a line symbol in order to clearly communicate information about the nature of that line.

\section{SCIENTIFIC CONFIDENCE AND LOCATIONAL ACCURACY OF GEOLOGIC FEATURES}

Another important concept in geologic mapping is a geologist's level of confidence in the interpretation of features observed in the field. Many factors can adversely affect a geologist's level of confidence when mapping, and field situations often arise in which the interpretation of a feature may be in question, as indicated by the following examples:

- a planar feature is well-exposed in outcrop, but it is not easily identifiable as either a contact or a fault;

- a contact is clearly exposed in a roadcut, but its trace cannot be followed away from that roadcut;

- a fault's trace is obscured by vegetation, and so both its location and its sense of offset cannot be definitively determined;

- a fault's trace is completely concealed beneath valley fill.

As these examples show, uncertainties can exist in either the scientific interpretation or the mapped location of a feature (or in both). Therefore, not only is it important to communicate to the map user the level of confidence in each geologic map feature, but also which type of uncertainty (scientific and (or) locational) may be associated with that feature.

Traditionally, a system of solid, dashed, dotted, or queried line symbol styles (see, for example, Ridgway, 1920, plate 2) has been used on geologic maps to show levels of locational accuracy of planar and linear geologic features observed in the field. This convention followed USGS Director Powell's 1888 policy, which stipulated that "fault lines (particularly when they are formation boundaries) shall be indicated when actually traced by somewhat heavy full lines in black; and when not actually traced, by similar broken lines" (Powell, 1890, p. 76). More guidance was provided in 1956 by USGS Chief Geologist W.H. Bradley, who, in a memorandum to USGS personnel regarding geologic map standards, stated, "The accuracy of location of faults and contacts should be shown by appropriate symbols ... Solid lines should be used to indicate accurate locations of features that are geologically identifiable within the plottable limits of the map ... Features that are only approximately located should be shown by long dashed lines; those that are indefinite or inferred, by short dashed lines; and those that are concealed, by dotted lines" (W.H. Bradley, written commun., 1956). To further encourage the use of such symbology, Bradley added, "The use of many dashed contacts or faults on a map is not to be construed as a detraction from the quality of the map, and for many maps, it may be undesirable or impossible to achieve sufficiently accurate locations to permit use of solid lines. The quality of the map is not impaired so long as the reader can interpret the accuracy of location" (W.H. Bradley, written commun., 1956).

In conjunction with these traditional line symbol styles, geologists at various times have used terms such as "known," "probable," "certain," "uncertain," "accurately located," "approximately located," "inferred," 
"projected," "concealed," and "queried" to express the levels of confidence of planar and linear geologic features. However, these terms and their associated line symbol styles have not been used consistently from region to region or from map to map. Also, it has not been always clear whether they reflect uncertainty in a feature's scientific interpretation, its mapped location, or both.

To facilitate the communication of geologic map information, this standard clarifies the concepts of, and establishes the attributes for, the levels of scientific confidence and locational accuracy of geologic map features. In addition, to facilitate the cartographic representation of geologic map information, this standard establishes new terminology that expresses both these concepts.

\subsection{SCIENTIFIC CONFIDENCE}

Scientific confidence expresses a geologist's level of certainty regarding the nature, origin, geometry, identity, and even the existence of a geologic feature. The characteristics of the geologic materials and structures, the number of outcrops, and the availability of subsurface or geophysical data directly affect the level of scientific confidence in any area. Experience and resources available to a geologist also affect scientific confidence. These fundamental characteristics of geologic features can be grouped into two distinct but related concepts, identity and existence.

\subsubsection{Identity}

Identity expresses whether or not the observations and data support the stated nature, origin, or geometry of a mapped geologic feature (for example, a contact versus a fault, or a normal fault versus a thrust fault). The concept of identity is communicated in the following two ways:

- in the geologic map database, the attribute describing the confidence in a feature's identity is specified as either certain or questionable;

- on the geologic map, the confidence in a feature's identity is communicated in the symbol explanation and (or) the map unit description (see Section 4.1.3 below, entitled "Levels of Scientific Confidence") and also, for some types of geologic map features, conveyed cartographically (see Section 4.1.4 below, entitled "Cartographic Representation of Scientific Confidence").

\subsubsection{Existence}

Existence expresses whether or not the observations and data support the continuity or existence of a concealed or an otherwise unseen geologic feature (for example, a postulated fault or a subsurface fault). The concept of existence is communicated in the following two ways:

- in the geologic map database, the attribute describing the confidence in a feature's existence is specified as either certain or questionable;

- on the geologic map, the confidence in a feature's existence is communicated in the symbol explanation and (or) the map unit description (see Section 4.1.3 below, entitled "Levels of Scientific Confidence") and also, for some types of geologic map features, conveyed cartographically (see Section 4.1.4 below, entitled "Cartographic Representation of Scientific Confidence").

\subsubsection{Levels of Scientific Confidence}

A geologic map must communicate to the map user the level of scientific confidence associated with each mapped feature (both its identity and its existence). In a geologic map database, this information is contained in two attribute fields, identity (certain, questionable), and existence (certain, questionable). To facilitate the communication of the two concepts of identity and existence on a geologic map, this standard sets forth the following new terminology, which expresses clearly yet concisely the levels of scientific confidence of geologic features (see Figure 1 for the relation of this new terminology to historically used terminology):

"Identity and existence certain" Both the identity and the existence of a feature can be determined using relevant observations and scientific judgment; therefore, one can be reasonably confident in the scientific credibility of this interpretation. These criteria are met, for example, when a geologist reasons, "I am 
certain that the planar feature I see in this outcrop is a fault." This is the default condition for all geologic map features unless otherwise stated on the geologic map or in the geologic map database.

"Identity or existence questionable" Either the identity or the existence of a feature cannot be determined using relevant observations and scientific judgment; therefore, one cannot be reasonably confident in the scientific credibility of this interpretation. These criteria are met, for example, when a geologist reasons, "I can see some kind of planar feature in this outcrop, but I cannot be certain if it is a contact or a fault," or, "My interpretation requires that a thrust fault be present to account for incongruities in the stratigraphy of these rocks, but I can't be certain because I haven't yet seen one here."

This new terminology is intended to be used when choosing a particular style of symbol to represent a feature on a geologic map (Fig. 2), as well as when describing that feature in the symbol explanation (see Preface to Appendix A) and (or) the map unit description. If a feature is symbolized or described as "identity or existence questionable," the map user should consult the geologic map database for more complete information.

\subsubsection{Cartographic Representation of Scientific Confidence}

For most types of geologic map features, queries are used to communicate the lack of scientific confidence in a feature. A queried line symbol indicates that either the identity or the existence of a planar or linear feature may be in question (Figs. 1,2; see also, Appendix A); the map user should consult the geologic map database for more complete information. In contrast, a line symbol without a query most likely indicates that both the identity and the existence of a planar or linear feature are certain, unless otherwise stated in the geologic map database.

For geologic point data, queries are not added to point symbols to indicate that the scientific confidence of a feature may be in question. However, a limited amount of specialized symbology has evolved to express the scientific confidence of certain types of geologic point information; for example, to indicate that the direction of stratigraphic top is known, a small ball may be added to bedding and foliation symbols (see Appendix A, Sections 6 and 8, respectively). In addition, queries may be added to dip or plunge values, both on the geologic map and in the geologic map database, if those measurements are questionable.

A queried map-unit label indicates that either the identity or existence of the geologic map unit may be in question.

\subsection{LOCATIONAL ACCURACY}

Locational accuracy is based on the relation between a mapped feature's location in the field and its position on the base map. Information about the locational accuracy of mapped features is important to all disciplines, even those in which mapped features commonly are directly observable and can be positioned with a significant degree of accuracy (for example, roads or utilities). It is especially critical in the natural sciences, however, because many mapped features are either interpretive or not directly observable.

The process of locating a feature in the field and then positioning it on a base map is complex, and the locational accuracy of a mapped feature is not easily described or quantified. To evaluate the locational accuracy of a mapped feature, a geologist must consider the following three factors:

- the nature of the feature and its degree of exposure (for example, a contact may be gradational or sharp, and either poorly exposed or well-exposed);

- the quality of the base map (for example, whether the cultural or topographic features on the base map are positioned accurately, according to the geologist's observations);

- the confidence in accurately positioning the feature relative to the base-map information.

Together, these factors determine a geologist's confidence in the locational accuracy of the features on the map. Locational accuracy is expressed by two distinct but related concepts, locatability and positioning. 


\subsubsection{Locatability}

Locatability expresses whether or not a geologist can clearly observe a feature in the field, as indicated by the following examples:

- a planar or linear feature is observable in several outcrops along its trace;

- a planar or linear feature is not defined by a distinctive physical trace and so is not observable beneath either vegetation, a thin veneer of unmapped geologic material (colluvium, eolian deposits, or residual soil), or man-made features, therefore its location must be inferred by indirect means;

- a planar or linear feature is not observable because it is concealed by an overlying geologic map unit, water, or ice, although it may be observable nearby (for example, a thrust fault is visible on both sides of a glacial valley, but its location within the valley is concealed by glacial deposits), and so its location must be projected beneath the overlying map unit.

As the above examples show, uncertainty in a feature's locatability can arise in a number of geologic situations. The concept of locatability is communicated in the following two ways:

- in the geologic map database, the attribute describing the confidence in a feature's locatability is specified as either observable, inferred, or concealed;

- on the geologic map, the confidence in a feature's locatability is communicated in the symbol explanation and (or) the map unit description (see Section 4.2.3 below, entitled "Levels of Locational Accuracy") and also, for some types of geologic map features, conveyed cartographically (see Section 4.2.4 below, entitled "Cartographic Representation of Locational Accuracy").

\subsubsection{Positioning}

Positioning expresses the degree of confidence with which a feature is plotted on the base map. Commonly, a feature can be accurately plotted on the map because the base-map information is accurate, detailed, and distinctive. However, in some field situations, a feature cannot be confidently plotted on the base map, as indicated by the following examples:

- a feature is observable, but its position on the map cannot be plotted accurately because topographic contours, drainage lines, or cultural information on the base map is insufficiently detailed for the feature to be confidently located relative to the various base-map features (for example, a contact is observable in outcrop, but its location in relatively featureless terrain prevents its position from being plotted accurately on the base map);

- a feature is observable, and its geographic coordinates can be determined in the field by either triangulation or a Global Positioning System (GPS) device or in the laboratory by using a georeferenced aerial photographic stereopair; however, the geographic relation between these coordinates and the topographic or cultural setting shown on the base map is not compatible (for example, a feature was mapped on a hillside, but the GPS-derived coordinates, when plotted on the base map, place its position in a valley bottom).

In such situations, either a feature can be plotted relative to the indistinct or incompatible base-map features, or the locations of topographic contours or other base-map features can be adjusted (the latter approach is not encouraged unless it is done systematically and is well-documented). In either case, the inherent uncertainty in a feature's positioning must be communicated to the map user, both on the geologic map and in the geologic map database (see discussion in Section 4.2.2.1 below, entitled "Specifying Positional Accuracy with the Zone of Confidence").

In the USGS, stringent policies for the accuracy with which an observable feature can be positioned on the base map have been put forth in the past. For example, Chief Geologist W.H. Bradley's 1956 memorandum to the staff advocated a geologic map accuracy standard based on the United States National Map Accuracy Standards (NMAS) for topographic and other types of base maps. The geologic map adaptation of the NMAS stipulated that "features that ... can be located from exposures or other evidence [should be positioned] within 1/25 inch [on the map] of their true map position" (W.H. Bradley, written commun., 1956; see also, U.S. Geological Survey, 1995a, Part 1, p. 1.0-4). These earlier efforts to quantify the positional accuracy of geologic features 
were not widely adopted by the geoscience community, likely in part because of (1) the difficulty in translating to geologic mapping a concept designed for topographic and other types of base maps, (2) the impracticality of requiring that all geologic map information meet the same accuracy criteria uniformly across the Nation, in all types of geologic and topographic settings, and (3) the need to convert ground distance to publication-scale cartographic units before evaluating if a feature is plotted accurately on a base map.

In contrast, this standard advocates a more flexible and conceptually simpler approach in which the accuracy criteria can be defined for each project so that the specified positional accuracy takes into account the character of the geologic setting and other factors (see below). In addition, if the geologic map adaptation of the NMAS ( $1 / 25$ inch on the map) has been used when mapping, this value can be specified (1/25 inch on the map must first be converted to ground units).

\subsubsection{Specifying Positional Accuracy with the Zone of Confidence}

When a feature is drawn or digitized onto a base map, a geologist commonly has some sense of confidence regarding whether or not the feature is positioned accurately, depending on the quality of the base map and the ability to position features on that base map. This positioning confidence can be characterized as the likelihood that the feature actually occurs within a certain, roughly defined distance from where it is positioned on the base map. This hypothetical distance, which extends outward from a feature's position on the map, is herein defined as the zone of confidence, and its numerical value quantifies a feature's positional accuracy as follows:

- for planar and linear geologic features, the zone of confidence borders the feature along both sides, forming what is described in GIS terminology as a buffer zone, and its numerical value is specified as the approximate distance in ground units (feet or meters) from the feature to the edge of the buffer zone (Fig. 3);

- for geologic point features, the zone of confidence is concentric around the feature, forming a circle, and its numerical value is the approximate radius of that circle (Fig. 3).

For any geologic map or mapped area, the numerical value of the zone of confidence will depend on a number of factors: the area's geology, landscape terrain, vegetation cover, and (or) cultural features; the scale of mapping; the quality and nature of the base map used; and (or) a particular project's allotted field-mapping time or other logistical constraints. Because this standard recognizes that the factors affecting the value of the zone of confidence will vary from region to region (and from map to map), and because different agencies have differing mapping needs and mandates, a single, universally applicable value for the zone of confidence is not herein established. Instead, this standard advocates that the responsibility for setting the value of the zone of confidence for a particular geologic map or mapped area lies with each geoscience organization and each mapping geologist.

In the geologic map database, the attributes describing positioning confidence, which are expressed in terms of the zone of confidence, are as follows:

- a numerical value for the zone of confidence is specified (for example, 5 meters);

- a feature's positioning is specified as being either "within zone of confidence" or "may not be within zone of confidence" (note that this standard does not stipulate that a feature whose positioning is specified as "may not be within zone of confidence" must necessarily be located outside the zone of confidence, but simply that it may be).

On the geologic map, positioning confidence is communicated in the symbol explanation and (or) the map unit description (see Section 4.2.3 below, entitled "Levels of Locational Accuracy") and also, for some types of geologic map features, conveyed cartographically (see Section 4.2.4 below, entitled "Cartographic Representation of Locational Accuracy"). In addition, the numerical value of the zone of confidence is indicated, either in a general statement (if one value applies to the entire mapped area) or shown in an index map (if different values apply to different mapped areas; see Section 4.2.2.2 below, entitled "Accommodating Different Values of the Zone of Confidence"). Likewise, if the geologic map adaptation of the NMAS (1/25 inch on the map, converted to ground units) has been used during field mapping as a measure of positioning confidence, or if a zone of confidence was not used during field mapping or map compilation, this also is indicated. 


\begin{tabular}{|c|c|c|c|c|c|c|}
\hline \multirow{2}{*}{ Symbol style ${ }^{1}$} & \multirow{2}{*}{$\begin{array}{c}\text { Examples of historically } \\
\text { used terminology }\end{array}$} & \multirow{2}{*}{$\begin{array}{l}\text { Newly revised FGDC } \\
\text { standard terminology }\end{array}$} & \multicolumn{2}{|c|}{ Scientific confidence } & \multicolumn{2}{|c|}{ Locational confidence } \\
\hline & & & Identity ... & Existence ... & Location (in field) ... & Position (on map) ... \\
\hline & $\begin{array}{l}\text { certain; known; } \\
\text { accurately located }\end{array}$ & $\begin{array}{l}\text { identity and existence } \\
\text { certain, location } \\
\text { accurate }^{2}\end{array}$ & certain & certain & observable & $\begin{array}{l}\text { within zone of confi- } \\
\text { dence }^{6}\end{array}$ \\
\hline$?$ & $\begin{array}{l}\text { [not available for newly } \\
\text { defined symbol] }\end{array}$ & $\begin{array}{l}\text { identity or existence } \\
\text { questionable, location } \\
\text { accurate }\end{array}$ & may be questionable & may be questionable & observable & $\begin{array}{l}\text { within zone of confi- } \\
\text { dence }\end{array}$ \\
\hline & approximately located & $\begin{array}{l}\text { identity and existence } \\
\text { certain, location } \\
\text { approximate }^{3}\end{array}$ & certain & certain & observable & $\begin{array}{l}\text { may not be within } \\
\text { zone of confidence }\end{array}$ \\
\hline$-?-$ & $\begin{array}{l}\text { approximately located, } \\
\text { queried }\end{array}$ & $\begin{array}{l}\text { identity or existence } \\
\text { questionable, location } \\
\text { approximate }\end{array}$ & may be questionable & may be questionable & observable & $\begin{array}{l}\text { may not be within } \\
\text { zone of confidence }\end{array}$ \\
\hline & $\begin{array}{l}\text { inferred; probable; } \\
\text { projected }\end{array}$ & $\begin{array}{l}\text { identity and existence } \\
\text { certain, location } \\
\text { inferred }^{4}\end{array}$ & certain & certain & $\begin{array}{l}\text { inferred (between } \\
\text { outcrops or beneath } \\
\text { rubble or vegetation) }\end{array}$ & $\begin{array}{l}\text { may not be within } \\
\text { zone of confidence }\end{array}$ \\
\hline$---?---?---?---$ & inferred, queried & $\begin{array}{l}\text { identity or existence } \\
\text { questionable, location } \\
\text { inferred }\end{array}$ & may be questionable & may be questionable & $\begin{array}{l}\text { inferred (between } \\
\text { outcrops or beneath } \\
\text { rubble or vegetation) }\end{array}$ & $\begin{array}{l}\text { may not be within } \\
\text { zone of confidence }\end{array}$ \\
\hline & concealed; projected & $\begin{array}{l}\text { identity and existence } \\
\text { certain, location } \\
\text { concealed }^{5}\end{array}$ & certain & certain & $\begin{array}{l}\text { concealed (beneath } \\
\text { overlying map unit, } \\
\text { ice, or water) }\end{array}$ & $\begin{array}{l}\text { may not be within } \\
\text { zone of confidence }\end{array}$ \\
\hline - & concealed, queried & $\begin{array}{l}\text { identity or existence } \\
\text { questionable, location } \\
\text { concealed }\end{array}$ & may be questionable & may be questionable & $\begin{array}{l}\text { concealed (beneath } \\
\text { overlying map unit, } \\
\text { ice, or water) }\end{array}$ & $\begin{array}{l}\text { may not be within } \\
\text { zone of confidence }\end{array}$ \\
\hline
\end{tabular}

${ }^{1}$ Queries are added to symbols to indicate that a feature's scientific confidence (that is, either its identity or its existence) may be in question.

${ }^{2}$ The term "location accurate" is used when a feature is observable, and its plotted position on the map is within the zone of confidence.

${ }^{3}$ The term "location approximate" is used when a feature is observable, but its plotted position on the map may not be within the zone of confidence.

${ }^{4}$ The term "location inferred" is used when a feature's location must be inferred between outcrops or beneath rubble or vegetation, and so its plotted position on the map may not be within the zone of confidence.

${ }^{5}$ The term "location concealed" is used when a feature is concealed beneath an overlying map unit, ice, or water, and so its plotted position on the map may not be within the zone of confidence.

${ }^{6}$ The zone of confidence for a particular map or mapped area is specified by the mapping geologists and their agencies.

Figure 1. Diagram showing relation of new FGDC standard terminology to historically used terminology and to traditional line symbol styles. 
TYPE OF PLANAR GEOLOGIC FEATURE

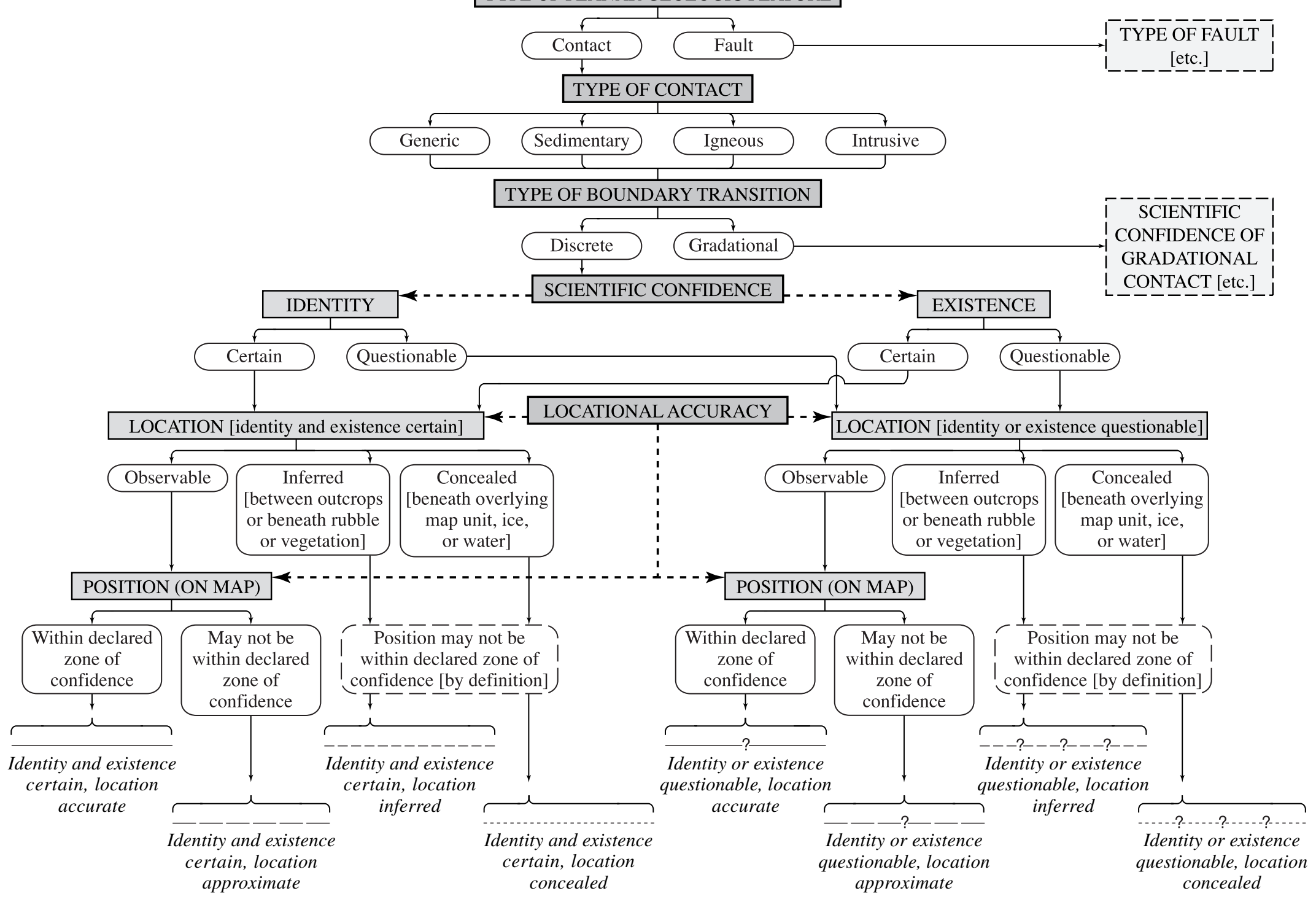

Figure 2. Flowchart showing example of logical steps that might be used to determine appropriate line symbol styles and associated terminology (in italics). 


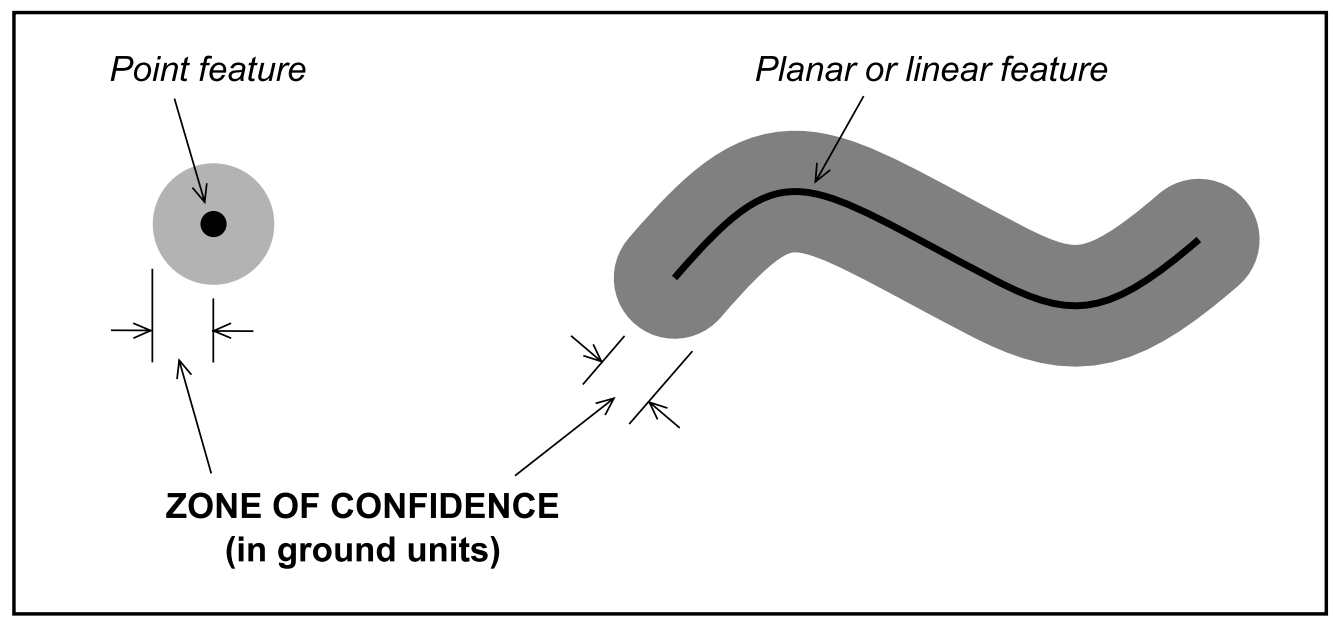

Figure 3. Figure showing examples of the zone of confidence for planar, linear, and point features. The region within which a point feature can be considered to be accurately positioned (on a base map) is a circle (light-shaded area above) around the point, and the value of the zone of confidence is the radius of that circle, in ground units. For a planar or linear feature, the region is a buffer zone (dark-shaded area above) surrounding the line, and the value of the zone of confidence is the distance from the line to the edge of the buffer zone, in ground units.

- a planar or linear feature is observable in only a few outcrops along its trace, but its physical characteristics permit locating it between outcrops by indirect methods;

\subsubsection{Accommodating Different Values of the Zone of Confidence}

For many geologic maps or mapped areas, especially those that are defined by latitude and longitude (for example, quadrangle maps) or political boundaries (for example, state or county maps), one map may contain areas of vastly contrasting geology, topography, vegetation cover, and (or) societal infrastructure, and so different positional accuracy criteria can exist within a single map. For example, a geologic map may include both a mountain range underlain by well-bedded sedimentary rocks and a broad alluvial valley underlain by mostly surficial deposits. In the mountains, clear distinction among the sedimentary rocks, as well as their high relief, may provide a geologist with a significantly higher sense of confidence in the position of contacts than in the adjacent valley, where few topographic landmarks or contours exist and where contacts may be gradational and obscured by vegetation and soil cover. In geologic settings as diverse as these, the levels of confidence in positional accuracy will be different, and so a geologist has the following two choices:

- express the differences in positioning confidence solely by differences in symbology (for example, specify one zone of confidence value for both areas, which might result in mostly solid-line contacts in the mountains and mostly dashed- or dotted-line contacts in the valley);

- express the differences in positioning confidence by specifying different values of the zone of confidence for each area (for example, specify the zone of confidence value as 5 meters in the mountains and 15 meters in the valley).

The choice might depend on the magnitude of the difference between the areas, or on the geologist's level of confidence in the positional accuracy of features across the map area.

Map compilations represent another example where different positional accuracy criteria can exist within a single map. A map compilation is made up of several source maps or mapped areas, each of which may have 
had a different value specified for the zone of confidence (or perhaps no value had been specified). These variations in the specified value of the zone of confidence should be preserved in the map compilation as well.

In situations in which the numerical values of the zone of confidence are different for different areas across the geologic map, the differences must be communicated to the map user. In the geologic map database, variations in the value of the zone of confidence can be readily accommodated because each feature is assigned (as an attribute in the database) the value of the zone of confidence that has been specified for a particular area. On the geologic map, areas that have different values of the zone of confidence should be shown in an index map.

\subsubsection{Levels of Locational Accuracy}

A geologic map must communicate to the map user the level of locational accuracy associated with each mapped feature (both its locatability in the field and its positioning on the base map). In the geologic map database, this information is contained in the following three attribute fields: (1) locatability (observable, inferred, concealed); (2) positioning (within zone of confidence, may not be within zone of confidence); and (3) the numerical value of the zone of confidence (for example, 5 meters).

To facilitate the communication of the two concepts of locatability and positioning on a geologic map, this standard sets forth the following revised terminology, which expresses clearly yet concisely the levels of locational accuracy of geologic features (see Figure 1 for the relation of this revised terminology to historically used terminology):

"Location accurate"

A feature is observable, and its plotted position on the map is within the declared zone of confidence. These criteria are met, for example, when a geologist reasons, "I can clearly see this contact in outcrop, and I can accurately plot its position on the map." This is the default condition for all geologic map features unless otherwise stated on the geologic map or in the geologic map database.

"Location approximate" A feature is observable, but its plotted position on the map may not be within the declared zone of confidence. These criteria are met, for example, when a geologist reasons, "I can see this contact in outcrop, but I can't tell exactly where it is located because I am surrounded by trees," or, "I can see this contact in outcrop, but the poor quality of my base map prohibits me from accurately plotting its position," or, "I can see that the width of the gradational contact between these two map units exceeds my value of the zone of confidence, and so, although my base map is of high quality, my confidence in the accuracy of its plotted position is not high."

"Location inferred" A feature is not directly observable between outcrops or beneath rubble or vegetation, so its location must be inferred by indirect means; by definition, its plotted position on the map may not be within the declared zone of confidence. These criteria are met, for example, when a geologist reasons, "I can see by the change in debris materials visible around these gopher holes that a contact runs through here, but I can't locate it very precisely."

"Location concealed" A feature is not observable because it is completely concealed beneath an overlying map unit or body of water or ice (although it may be observable nearby); by definition, its plotted position on the map may not be within the declared zone of confidence. These criteria are met, for example, when a geologist reasons, "I can see that a contact is present on both sides of this lake, but I can't tell where it is located beneath the water."

This revised terminology is intended to be used when choosing a particular style of symbol to represent a feature on a geologic map (Fig. 2), as well as when describing that feature in the symbol explanation (see Preface to Appendix A) and (or) the map unit description.

\subsubsection{Cartographic Representation of Locational Accuracy}

A system of solid, dashed, dotted, and queried line symbols has long been used on geologic maps to convey the uncertainty of planar and linear geologic features (Fig. 1), but it has not always been clear whether these line 
symbol styles reflect uncertainty in a feature's scientific interpretation, its mapped location, or both. This standard clarifies the use of these line symbols (Figs. 1,2) by applying its revised terminology for locational accuracy (see Section 4.2.3 above, entitled "Levels of Locational Accuracy") to the following line symbol styles ${ }^{2}$ :

- a solid, continuous line symbol indicates that the location of a feature is accurate; that is, its location in the field either is readily observable in outcrop or is revealed by the characteristic geomorphic expression of its trace, without extensive cover of thin overlying surficial deposits, and is verifiable by shallow excavations; in addition, it can be accurately plotted because base-map information is accurate, detailed, and distinctive, and so its position on the base map is within the declared zone of confidence.

- a long-dashed line symbol indicates that the location of a feature is approximate; that is, its location in the field either is readily observable in outcrop or is revealed by the characteristic geomorphic expression of its trace, without extensive cover of thin overlying surficial deposits, and is verifiable by shallow excavations; however, it cannot be accurately plotted because base-map information is inaccurate, indistinct, or incompatible with the location of the geologic feature, and so its position on the base map may not be within the declared zone of confidence.

- a short-dashed line symbol indicates that the location of a feature is inferred; that is, its location in the field generally is obscured by overlying (unmapped) surficial deposits, debris materials, or vegetation that may cover exposures and the geomorphic expression of its trace, and has therefore been projected between few outcrops; by definition, its position on the base map may not be within the declared zone of confidence.

- a dotted ${ }^{3}$ line symbol indicates that the location of a feature is concealed; that is, its location in the field is covered by a mapped overlying geologic unit or a mapped body of ice or water; by definition, its position on the base map may not be within the declared zone of confidence.

These types of line symbol styles (solid, long-dashed, short-dashed, and dotted) are intended to convey the various levels of locational accuracy of planar geologic features and certain types of linear geologic features.

The locational accuracy of a geologic map unit is not expressed by a specialized symbol but, instead, by the style of line symbols representing the planar features (contacts and faults) that bound it.

In most cases, specialized point symbols are not used to indicate that the locational accuracy of a geologic point feature may be in question. One exception is the specialized symbols that are used to portray bedding attitudes that have been determined using aerial photographs (see Appendix A, Section 6); however, these symbols also may be used to indicate that the scientific confidence (the measurement of dip) is in question, and so, when these types of symbols are used on a geologic map, the map user should consult the geologic map database for more complete information.

\section{GUIDELINES FOR MAP COLOR AND PATTERN SELECTION}

The goal in color design is to enhance the legibility of the map, as well as to lend meaning to the data presented by helping to focus attention on a particular map feature or group of features. Colors and patterns should not, however, be so visually dominant as to distract from the purpose of the map. A well-balanced color design can greatly improve the presentation of scientific information.

\subsection{FACTORS THAT INFLUENCE COLOR AND PATTERN SELECTION}

\subsubsection{Purpose of Map}

Color is used differently on different types of maps. For example, on geologic maps, color is primarily determined by age and type of rock, although other rules may apply for terrane maps or maps that portray only a

\footnotetext{
${ }^{2}$ Note that this standard restricts the use of queries to represent the lack of scientific confidence only (see discussion in Section 4.1.4 above, entitled "Cartographic Representation of Scientific Confidence").

${ }^{3}$ In reality, dotted line symbols that are thinner than a certain lineweight are difficult to produce with some software applications; therefore, this standard substitutes a very-short-dashed line symbol as the cartographic standard (see Figures 1,2; see also, Appendix A).
} 
limited range of ages or types of rocks. In addition, some map units, because of their geologic or economic importance, may need to be emphasized by selected colors.

Geophysical maps use several color schemes, depending on the purpose of the data being shown; usually a range of colors from dark to light is used. One such scheme is a graduated set of hues of similar value (for example, purple and magenta to orange and red). Another is a rainbow of hues in which the values alternate between full color and lightly screened color.

On slope-stability maps, the brightest colors are used on areas of highest instability. Similarly, on volcanic- or earthquake-hazard maps, areas of greatest hazard usually are shown in red, whereas areas of lowest hazard are shown in yellow or green.

Data on hydrologic maps are frequently shown in two or three colors. On maps showing depth to water table, color ranges from light blue at the shallowest depths to dark blue at the greatest depths. On maps showing dissolved-solids concentrations, color ranges from dark blue where concentration is lowest to dark red where concentration is highest.

\subsubsection{Age and Type of Rock}

Whenever possible, colors for ages and rock types on geologic maps should follow the scheme presented in the diagram showing "Suggested Ranges of Map-Unit Colors for Volcanic and Plutonic Rocks and for Stratigraphic Ages of Sedimentary and Metamorphic Rocks" (see Appendix A, Section 33). However, it may not always be feasible to show map units in the suggested color; in these cases, other characteristics should be emphasized with color.

On surficial maps, for example, it may be desirable to show all glacial deposits in one color, landslide deposits in another, lacustrine deposits in another, and alluvial deposits in yet another. On terrane maps, color may be used to show lithotectonic relations between various groups of rocks.

On maps that are mostly one age group, it is best to distinguish sedimentary rocks from volcanic rocks (usually shown in reds or other bright colors) and plutonic rocks (usually shown in pinks). On maps that are mostly one type of rock, differentiation between different rock sequences can be shown through the use of different colors.

On maps that cover a broad range of ages and rock types, relations between rocks within one age group can be shown by using similar colors, whereas relations between the same type of rock in different age groups can be shown by using patterns (for example, all volcanic rocks may have the same " $v "$ pattern). Patterns should be used sparingly, however, as their use can create an overly busy appearance; use them only when the complexity of the map requires the diversity achieved by the use of patterns.

Although it is preferable to follow the aforementioned guidelines, some rock types defy such guidelines because they traditionally have been shown in a particular color. For example, serpentinite and other ultramafic rocks characteristically are shown in purple; limestone usually is shown in bright blue; and glacial till often is shown in light green.

\subsubsection{Size of Map-Unit Areas}

In general, small map-unit areas should be shown in darker colors and large areas should be shown in lighter colors. An exception to this may be in situations when numerous small bands of map units are shown; in this case it may be best to alternate light and dark colors. In the case of map units that consist of both large and small areas, add labels and leaders to the smaller map units to avoid confusion. For guidelines and recommendations on the placement of map-unit labels and leaders, see Section 6 below, entitled "Guidelines for Map Labeling."

Because it is more difficult to clearly distinguish color in small areas, it is very important to choose as unique a color as possible for map units that are present only in small areas. The minimum size of map-unit area that can show color is about two square millimeters; anything smaller will need to be labeled. In addition, exercise caution when using patterns in small areas because small areas may fail to show enough of the pattern to adequately identify a map unit; about one square centimeter is the minimum size to clearly show patterns. If there can be any ambiguity in a map-unit area's identification, it is safest to add a label and leader. 


\subsubsection{Contrast}

Adequate contrast enhances readability. A key factor is not so much the difference in hue, such as blue or green, but the difference in intensity. Contrast should not, however, be so great as to be glaring, but it should be significant enough for easy legibility. Map units that need to be emphasized should be assigned colors that stand out and contrast well with the colors of less important units. In addition, greater contrast is required for small areas, whereas a more subtle contrast is sufficient for larger areas.

\subsection{SPECIFYING COLOR FOR MAP-UNIT AREAS}

To maintain control of color output, color on maps and illustrations should always be specified using processcolor (CMYK, cyan/magenta/yellow/black) inks, regardless of the intended output medium. If another non-ink color scheme such as RGB (red/green/blue) or HSV (hue/saturation/value) is used, then the output device (be it printer, plotter, or imagesetter) will automatically convert the non-CMYK values to CMYK during output, and unwanted color shifts often will take place. To aid in the selection of color fill for map units, a chart showing a wide variety of CMYK colors ("CMYK Color Chart") has been included herein.

Color values must be high enough to provide adequate contrast but not so great that they prevent the map-unit labels, structure symbols, and topographic base from showing clearly. Except in small areas, magenta and cyan should be used in intensities of $50 \%$ or less. A greater intensity of cyan might obscure drainage features (commonly shown in cyan), and a greater intensity of magenta might obscure magenta fold axes and dikes. As a general rule, use a combination of CMYK color values that, when added together, totals 100 or less (for example, $30 \%$ cyan $/ 40 \%$ magenta/ $20 \%$ yellow; $30 / 40 / 20=30+40+20=90$ ), especially in larger areas.

To maintain enough contrast between two colors, keep at least a $20 \%$ difference between the values of one of the CMYK colors (for example, 30\% cyan/8\% magenta/20\% yellow and 30\% cyan/8\% magenta/40\% yellow).

Avoid using $8 \%$ yellow because it is too light and cannot easily be distinguished from white. In addition, it may be wise to avoid using $13 \%$ or $20 \%$ cyan, as these colors may look like a body of water.

On maps that are to be offset printed, it may be best to use a solid (100\%) single-ink color such as cyan, magenta, or yellow in very small map-unit areas to avoid misregistration problems. For example, $100 \%$ cyan may be used to show small limestone blocks in melange, or $100 \%$ magenta may be used to show thin rhyolite intrusions.

\subsection{USE OF PATTERNS}

Patterns can be printed either in black, in color, or as a dropout. Ideally, patterns should be used sparingly and only when necessary for clarification, as they can add unnecessary complexity to a map. To select appropriate patterns for a map, both the type of rock and the size and (or) orientation of map-unit areas must be considered. To aid in the selection of patterns for map units, a chart showing a wide variety of geologic patterns ("Pattern Chart") has been included herein.

Although some flexibility exists in the use of patterns, some patterns are traditionally and exclusively used for certain rock types: for example, "+" patterns are used for plutonic rocks, and irregular " $v "$ patterns represent volcanic rocks. For map units that are present only in small areas, a tight, random pattern will fit more of the pattern elements into a particular area. Exercise caution, however, when choosing metamorphic patterns that display a strong directionality, as their use may imply a general orientation of metamorphic fabric that in reality is much more varied than the pattern may indicate.

\subsubsection{Overprint Patterns}

Color overprint patterns are usually specified in either cyan or magenta, but sometimes a spot color such as red is used. For offset printing, it is best to specify only one color for overprint patterns, as using more than one color can cause misregistration problems. Color overprint patterns can be screened to reduce their intensity.

Black overprint patterns are less effective than color in most situations, as they can conceal base-map information or interfere with type or structure symbols. Thus, it may be best to restrict the use of $100 \%$ black patterns to small, uncluttered areas; if a map-unit label is needed, it can be placed outside the area and leadered in. Black overprint patterns also can be screened to reduce their intensity. 


\subsubsection{Dropout Patterns}

Dropout patterns cause to be transparent one or more of the CMYK colors that combine to make a map-unit color, thus allowing the remaining color(s) to show through. Their use can be especially effective on a map that has a large amount of labeling or many structure symbols.

For offset printing, only one color should be dropped out, as dropping out more than one might lead to misregistration problems; in general, the most dominant color (the one with the highest value) other than yellow should be the one dropped out. For output to a single-pass inkjet plotter, a dropout pattern may be applied to all of the CMYK colors that make up a map-unit color; the dropout pattern would then show as white. Be aware, however, that doing so may cause that map unit to stand out more than is desired.

\subsection{SPECIFYING COLOR FOR LINE AND POINT SYMBOLS}

Color commonly is specified for many line and point symbols because it highlights these features. Whenever possible, color for line and point symbols should be specified as either $100 \%$ cyan or $100 \%$ magenta, two of the standard four process-color (CMYK, cyan/magenta/yellow/black) inks that are used for offset printing and in most inkjet plotters (other non-ink color schemes such as RGB or HSV should be avoided so that unwanted color shifts during output are prevented). In some cases, however, it may not be practical or preferable to specify cyan or magenta; for example, mineral resource assessment areas traditionally have been outlined in red.

Although it is possible to make a non-process color such as red from two or more process-color inks, this should be avoided if the map is to be offset printed because of the difficulties in registering large, CMYK-separated negatives. For maps that are to be offset printed, a Pantone color (single-ink spot color) should be specified. Each Pantone color is imageset onto a separate piece of film, thereby avoiding misregistration problems caused when a color is converted to CMYK and then is color separated onto more than one piece of film.

\section{GUIDELINES FOR MAP LABELING}

Map-unit labels are the most common labels on geologic maps. Other labels may include base-map information, feature names, and data items such as dip values, gold concentrations, well depths, radiometric ages, and sample locality numbers.

Before the advent of digital technologies for mapmaking, labels were either drawn by hand or applied using stick-up type. Nowadays, using digital mapmaking techniques, labels (and leaders) can be automatically plotted from information in a database; however, this often results in labels overprinting other map features, requiring them to be interactively repositioned or deleted. Regardless of the method employed, effective label placement is an important factor in producing a useful map.

\subsection{STRATEGIES FOR MAP LABELING}

Enough features on the map should be labeled so that the reader can identify all the various map elements; no unlabeled feature should leave the reader guessing. Labels (and leaders) should not, however, create an overly "busy" or cluttered appearance, which makes recognition of map patterns, shapes, and map-element distribution difficult to discern. For a map to be easily read, labels and leaders should be placed where they are clear and legible, taking care to avoid overprinting of linework, symbols, or other labels. In addition, they should not obscure base-map features that are mentioned in the text or that may be useful in locating places on the map.

Commonly, color or pattern can be used to identify an unlabeled map-unit area if a nearby area of the same map unit is labeled. Therefore, the color and pattern selection is critical when deciding whether or not to label a particular map-unit area, and so it is important to complete the color and pattern design of the map before attempting to place and move map-unit labels, especially for complex maps or those that have many map units.

There are no precise rules for which and how many of the map-unit areas on a map should be labeled, but the following are some general guidelines. If a map unit has a unique and clearly distinguishable color or pattern, it is not necessary to label every area of that map unit. Color and pattern can carry the identification of a group of areas of the same unit as long as some of them are labeled. Use judgment when deciding whether the color for that map unit is distinctive enough and (or) whether a particular unlabeled map-unit area can be visually or 
logically associated with any nearby labeled areas of the same unit. In small map-unit areas, however, even the most distinctive color or pattern may be difficult to discern. If there might be any doubt, add a label and leader.

At least one area of every map unit within a "normal field of view" should be labeled. This field of view is the area in focus when the map is viewed at a comfortable, readable distance. In uncluttered areas of the map or in areas of relatively simple geology, this field of view might have a radius of about two or three inches; in geologically complex or cluttered areas, however, it may be much smaller. The reader should not need to search across the map trying to find a labeled map-unit area that has a color that matches an unlabeled map-unit area.

In addition, maps that are to be downloaded from the Web will be sent to a plotter of unknown type, and there is no guarantee that colors that appear distinct when plotted on your plotter will also be distinguishable when plotted on other plotters. The more map-unit areas that are labeled, the less chance of ambiguity and confusion.

\subsection{FONT SELECTION}

For most type on a map (for example, unit labels, dip values, and fault names), a sans-serif font such as Helvetica (or FGDCGeoAge; see Appendix A, Section 32) should be used. Other sans-serif fonts such as Univers or Arial also may be used, but consider that not all fonts will plot correctly on all output devices. Also consider that combining FGDCGeoAge with Univers or Arial will result in odd-looking character strings because the character size and kerning (spacing of letters) of FGDCGeoAge is based on that of Helvetica; therefore, using Helvetica with FGDCGeoAge is recommended. For base-map information, use a combination of sans-serif (for example, Helvetica or Univers) and serif (for example, Times or Times New Roman) fonts; the general rule is to follow the styles used on a published topographic map sheet.

When placing labels digitally, it is important to use the same font that will be used for final publication because the size and kerning of characters are different for different fonts, even those having the same point size. If labels are placed carefully in tight areas using one font, but then another font is used for final publication, the labels may overprint linework or other features because the new font may have longer character heights and string lengths. Therefore, for best results, choose fonts early in a project, and then stay with that choice throughout the project. In addition, the use of PostScript fonts may result in more consistent final output for both print and digital publications.

\subsection{TYPE SIZE AND STYLE}

The ideal size for map-unit labels is $8 \mathrm{pt}$, although labels as small as $6 \mathrm{pt}$ may be substituted in places where space is tight. Fractional font sizes may be used if needed, and different sizes can be mixed on the same map. If unit labels contain subscripts or superscripts, the minimum unit-label size should be $7 \mathrm{pt}$; then the size for the subscript or superscript character would be $5 \mathrm{pt}$, two point sizes smaller.

Other sizes and styles are used to label different features. In general, use 8 pt type (all caps) for names of faults and major structures, for sample locality numbers and radiometric ages, and for fault $(\mathrm{U} / \mathrm{D}, \mathrm{A} / \mathrm{T})$ and contact (Y/O) ornamentation. Use $6 \mathrm{pt}$ italic type for dip or plunge values. Use $12 \mathrm{pt}$ italic type for cross-section labels. For labels of larger features, type size and (or) kerning (letter spacing) may be increased to improve legibility.

\subsection{LABEL PLACEMENT}

Map-unit labels and dip values should always be oriented horizontally. They should not overprint other map elements such as linework, point symbols, or any other dip values and labels, nor should they obscure base-map features that are referenced in text or are needed to orient the map in the field. Single labels can be used to identify more than one map-unit area; use multiple leaders where necessary.

Map-unit labels should not be placed in dark-colored map-unit areas or in densely patterned areas, both of which would make the labels hard to read; instead, move labels outside such areas and add leaders. If a label must be placed in a dark-colored or densely patterned map-unit area, it may be necessary to mask out the color or pattern around the label to help make it more legible.

Labels for linear map features should be aligned along those features. Other labels should have a logical or comfortable orientation relative to the map. In rare cases it might be desirable to have labels run parallel to lines of latitude, but in general they should be oriented horizontally. 


\subsection{LEADER PLACEMENT}

Leaders should be drawn as straight lines, not bent or curved. They should cross map-unit area boundaries at as high an angle as possible, and they should not stop at the boundary but should extend well into the map-unit area. Leaders should not cross through other map-unit areas to reach a particular map unit unless absolutely necessary. Multiple leaders emanating from a single label should not be joined at their "label" ends.

\section{TECHNICAL SPECIFICATIONS USED IN THE PREPARATION OF THIS STANDARD}

This new standard (contained in Appendix A) consists of geologic line and point symbols, geologic map-unit colors and patterns, a geologic age symbol font, and related map marginalia. This section provides some technical discussion regarding preparation of the standard and its implementations.

\subsection{UNITS FOR LINEWEIGHTS, LENGTHS, AND DISTANCES}

In previous standards, lineweights were specified in thousandths-of-an-inch, which corresponded to the widths of the engraving tools used to scribe the linework. Most lengths and distances also were given in inches. In this standard, the cartographic specifications are given in millimeters, in accordance with the Federal standard for metrification.

When preparing this standard, the old thousandths-of-an-inch specifications were converted to millimeters (Table 1), and then most were rounded to the nearest $.05 \mathrm{~mm}$ or $.025 \mathrm{~mm}$, for ease of use. Whenever possible, cartographic specifications for lengths and distances were given in whole- or half-integer values. However, when designing the symbol graphics in this standard document, as well as the symbols in its PostScript implementation, lineweights, lengths, and distances were specified electronically as points, and the exact conversion values (from inches to points; see Table 1) were retained.

As an example of the unit-conversion process, consider the symbol for faults, which in previous standards had a lineweight of $.015^{\prime \prime}$ specified. This original lineweight was converted to millimeters $\left(.015^{\prime \prime}=.381 \mathrm{~mm}\right.$; Table 1$)$ and then rounded to $.375 \mathrm{~mm}$, which is the value given as the cartographic specification in this standard (see p. A-2-1, Appendix A). However, when preparing the fault symbol for inclusion in this standard document (and in its PostScript implementation), the exact .015" lineweight was retained and directly converted to points $\left(.015^{\prime \prime}=1.08 \mathrm{pt}\right.$; Table 1$)$, and so the symbol lineweight was defined electronically as $1.08 \mathrm{pt}$.

Complications from unit conversion can arise not just when designing line symbols but also when creating point symbols and patterns, as most symbols are made of stroked lines. When creating symbols for a particular application, the user should choose the unit of measure most easily used in an application and then use the conversion table (Table 1) to convert to those units.

\subsection{TYPE SPECIFICATIONS}

Most type in this standard is specified as either Helvetica (sans-serif) or Times (serif), two fonts that are commonly used and widely available (see Table 2 for abbreviations for type faces used in this standard); type sizes are given in points. Other fonts such as Univers, Arial, or Times New Roman may be substituted, but consider that they may not be installed on all common output devices and thus may not plot correctly.

Geologic age characters have been specified as FGDCGeoAge, a specialized sans-serif font designed by the U.S. Geological Survey (see Appendix A, Section 32). The character size and kerning (spacing of letters) of FGDCGeoAge is based on that of Helvetica; therefore, using Helvetica with FGDCGeoAge is recommended.

\subsection{COLOR SPECIFICATIONS FOR LINE AND POINT SYMBOLS}

Color has been specified as the cartographic standard for many line and point symbols in this standard, either because of adherence to a long-established color convention or because using color for features such as folds and dikes may help them to stand out better from other full-black linework such as contacts and faults. In most cases, another color or black may be substituted if the color specified as the standard would not be visible when printed over an underlying map-unit color. 
Whenever possible, color has been specified as either cyan or magenta, two of the four process-color (CMYK, cyan/magenta/yellow/black) inks that are used both in inkjet plotters and for offset printing. However, in some cases it was not practical or preferable to specify cyan or magenta as the standard; for example, mineral resource assessment areas traditionally have been outlined in red (see p. A-19-1, Appendix A).

Although it is possible to make a non-process color such as red from two or more process-color inks, this should be avoided if the map is to be offset printed because of the difficulties in registering large, CMYK-separated negatives. Thus, in some cases a spot color (a single-ink, non-CMYK color) has been specified as the cartographic standard.

As a simple, general way of specifying spot colors, generic color names (for example, "red" and "green") have been used in this standard. Specifying color as these generic color names, however, may not be appropriate for use with certain output media. Therefore, the user must choose a method of specifying color that is appropriate for a particular output device; Table 3 shows suggestions for conversions of spot colors to other color models.

For maps that are to be offset printed, a Pantone color (single-ink spot color) should be specified (Table 3). Each Pantone color is imageset onto a separate piece of film, thereby avoiding misregistration problems caused when a color is converted to CMYK and then is color separated onto more than one piece of film. For output to an inkjet plotter, however, specifying a spot color as one of the generic color names is satisfactory because, during the plotter's RIP ${ }^{4}$ of the file, the color will automatically be converted to the proper amounts of CMYK inks that will combine to make the CMYK equivalent of that color. Misregistration is not a problem with single-pass inkjet-plotter output.

If simple, graphical map elements are to be published as part of a web page on the World Wide Web, it may be best to choose colors from a "Web-safe" color palette ${ }^{5}$ to avoid unwanted dithering on monitors that display only 256 colors (Weinman, 1996). As an aid in doing so, an attempt was made to provide "Web-safe" color equivalents of the Pantone spot colors used in this standard (Table 3). These "Web-safe" color equivalents are made up of the RGB (red/green/blue) values that are as close as possible to the directly converted RGBequivalent colors (Table 3). Note, however, that it was impossible to exactly reproduce the directly converted RGB-equivalent colors because, to make "Web-safe" colors, there are only six possible RGB values (000, 051, $102,153,204$, and 255) from which to choose.

\subsection{COLOR SPECIFICATIONS FOR MAP-UNIT AREAS}

To aid in the selection of color fill for geologic map units, a chart showing a wide variety of CMYK colors ("CMYK Color Chart") has been included in this standard. The CMYK Color Chart was designed in Adobe Illustrator 8.0.1 to closely replicate the colors on the offset-printed color chart entitled "Printing Colors and Screens in Use by the U.S. Geological Survey for Geologic and Hydrologic Maps" [yellow/magenta/cyan version], which has been in use for many years at the USGS. The new color chart contains the same colors that were in the original offset-printed USGS chart; however, the old color codes indicating the YMC (yellow/magenta/cyan) values have been updated to show CMYK (cyan/magenta/yellow, with $\mathrm{K}=0$ ) values, to conform to industry standards. In addition, each color in the CMYK Color Chart has associated with it a generic lookup-table number that, if desired, may be used to access the color from within digital applications.

In addition, a diagram showing "Suggested Ranges of Map-Unit Colors for Volcanic and Plutonic Rocks and for Stratigraphic Ages of Sedimentary and Metamorphic Rocks" (see Appendix A, Section 33) has been included in this standard. This diagram was designed in Adobe Illustrator 8.0.1 to reproduce a similar diagram in the old USGS Technical Cartographic Standards volume (U.S. Geological Survey, ca. 1975). In this new version, however, the range of colors was modified slightly, a few new colors were added, and the old color codes were updated to show CMYK (cyan/magenta/yellow, with $\mathrm{K}=0$ ) values.

\footnotetext{
${ }^{4} \mathrm{RIP}=$ raster-image processing, a process that runs on all plotters, printers, and imagesetters and converts data (in either raster or vector format) to printer dots to produce an image.

${ }^{5}$ Industry opinions on using "Web-safe" colors (8-bit, 216 colors) are changing, owing to the large number of monitors now in use that can display more than 256 colors; Chris MacGregor (in Dennis, 1999) stated that using non-"Web-safe" colors may be acceptable to use in detailed areas, although she still recommends using "Web-safe" colors in large areas.
} 


\subsection{PATTERN SPECIFICATIONS}

The old USGS Technical Cartographic Standards volume (U.S. Geological Survey, ca. 1975) contained no cartographic specifications (lineweights, dot sizes, or size and spacing of pattern elements) for its patterns. The volume dates back to a time when maps were conventionally prepared using hand-scribed linework and peelcoats. In those days, patterns were preprinted onto large sheets of film, which were photomechanically combined with the various peelcoats to make the CMYK negatives.

For this standard, the patterns (see "Pattern Chart") were recreated by scanning the old pattern sheets and then tracing the pattern elements in Adobe Illustrator 8.0.1. For most patterns, black, cyan, and magenta versions, as well as dropout versions, were created; yellow versions were not created because yellow patterns are not visible over color fill. Also, red and (or) brown versions were created if red or brown patterns were specified as the cartographic standard for a particular feature. Glacial and hydrologic patterns were created only in cyan and black, as it is unlikely that magenta or other colors would be used for these types of patterns.

To facilitate digital output, lineweights and dot sizes were in many cases increased. A few pattern tiles were scaled to accommodate the increased lineweights, and some of the lined patterns were dropped because an increased lineweight would fill in the pattern and because an increase in scale would cause the pattern to be too similar to other patterns. The lineweights and dot sizes for the color and dropout versions were increased even more than for the black versions, to help them show more clearly on maps.

All patterns were renumbered, and suffixes indicating color were added so that all versions of the same pattern are referenced by the same number. In addition, each pattern in the Pattern Chart has associated with it a generic lookup-table number that, if desired, may be used to access the pattern from within digital applications.

\subsection{GEOLOGIC AGE SYMBOL FONT}

A digital font named FGDCGeoAge (see Appendix A, Section 32) has been created, in which 16 special geologic age characters have been substituted into positions of normal keyboard characters. These characters can be typed either directly or with the Shift key; no Option, Control, or Alt keys are needed to type these characters (they are all in lower-order ASCII positions that have character ID numbers below 128), allowing the same character positioning to work on different computer platforms without interfering with special control key sequences.

\section{ACKNOWLEDGMENTS}

This standard owes its existence mostly to the well-established history and traditions of geologic map cartography by the USGS. In particular, the editors, authors, and compilers of this standard wish to thank the many cartographers, editors, and geologists who contributed to the informal USGS "Technical Cartographic Standards" volume (U.S. Geological Survey, ca. 1975), as well as Mitchell Reynolds, James Queen, Richard Taylor, and others who were responsible for preparing the earlier (1995) USGS proposed standard (U.S. Geological Survey, 1995a,b), from which this standard has evolved. We especially wish to thank the many members of the USGS Geologic Discipline's Western Publications Group who have made substantial contributions to the design and preparation of this standard (see Section 2.3 above, entitled "Preparers of this Standard").

We also want to thank the numerous geologists, cartographers, graphics specialists, GIS specialists, editors, and others who provided invaluable comments and suggestions for revisions to this standard during their review(s) of the previous versions of this standard: the 1995 USGS proposed standard (see Soller, 1996); the preliminary, beta version of this standard; the Working Draft of this standard; and, most importantly, the Public Review Draft of this standard. In addition, we gratefully acknowledge the intellectual contributions of members of the standing Map Symbol Standards Committee (see section 2.3 above, entitled "Preparers of This Standard"), and especially Jonathan Matti (USGS) for his guidance on issues of scientific confidence and locational accuracy. We also thank Jack Reed, Ron Wahl, Will Stettner, and Nancy Stamm (all USGS) for providing insight and access to USGS historical documents and standard cartographic practices.

Finally, we thank the National Geologic Map Database project, as well as the USGS National Cooperative Geologic Mapping Program, for providing financial support to this project. 


\section{REFERENCES}

Berggren, W.A., Kent, D.V., Aubry, M-P, and Hardenbol, Jan, eds., 1995, Geochronology, time scales, and global stratigraphic correlation: SEPM (Society for Sedimentary Geology), Special Publication 54, 386 p.

Dennis, Anita, ed., 1999, 101 hot tips: Publish, v. 14, no, 9, p. 62.

Dietrich, R.V., Dutro, J.T., Jr., and Foose, R.M, compilers, 1982, AGI data sheets for geology in the field, laboratory, and office (2nd ed.): Alexandria, Va., American Geological Institute.

Dutro, J.T., Jr., Dietrich, R.V., and Foose, R.M, compilers, 1989, AGI data sheets for geology in the field, laboratory, and office (3rd ed.): Alexandria, Va., American Geological Institute.

Federal Geographic Data Committee [prepared for the Federal Geographic Data Committee by the U.S. Geological Survey], 2000, Public Review Draft - Digital Cartographic Standard for Geologic Map Symbolization: Reston, Va., Federal Geographic Data Committee, 172 p., 2 plates.

Gradstein, F.M., and Ogg, J.G., 1996, A Phanerozoic time scale: Episodes, v. 19, nos. 1-2, p. 3-5.

Hansen, W.R., ed., 1991, Suggestions to authors of the reports of the United States Geological Survey (7th ed.): Washington, D.C., U.S. Geological Survey, 289 p.

Haq, B.U., and van Eysinga, F.W.B., 1998, Geological time table (5th ed.): New York, Elsevier Science Publishing Co., 1 sheet.

Harland, W.B., and others, 1982, A geologic time scale: Cambridge, England, Cambridge University Press, 131 p.

Harland, W.B., and others, 1989, A geologic time scale: Cambridge, England, Cambridge University Press, 263 p.

International Union of Geological Sciences (Juergen Remane, comp.), 1998, International Stratigraphic Chart: International Union of Geological Sciences, 1 sheet and explanatory text.

Jackson, Julia A., ed., 1997, Glossary of Geology (4th ed.): Alexandria, Va., American Geological Institute, 769 p.

Neuendorf, Klaus K.E., Mehl, James P., Jr., and Jackson, Julia A., eds., 2005, Glossary of Geology (5th ed.): Alexandria, Va., American Geological Institute, 779 p.

North American Commission on Stratigraphic Nomenclature, 1983, North American stratigraphic code: American Association of Petroleum Geologists Bulletin, v. 67, no. 5, p. 841-75.

Palmer, A.R., 1983, The Decade of North American Geology 1983 Geologic Time Scale: Geology, v. 11, no. 9, p. 503-4.

Palmer, A.R., and Geissman, J., 1999, 1999 Geologic Time Scale: Geological Society of America [http://www.geosociety.org/science/timescale/timescl.htm].

Powell, J.W., 1882a, Second Annual Report of the United States Geological Survey 1880-'81: Washington, D.C., U.S. Government Printing Office, 588 p.

Powell, J.W., 1882b, Sur la nomenclature générale, sur le coloriage et les signes conventionnels des Cartes géologiques, in Congrès Géologique International, Compte Rendu de la 2me Session, Bologne, 1881: Imprimerie Fava et Garagnani, Bologne, p. 627-41.

Powell, J.W., 1888, Methods of geologic cartography in use by the United States Geological Survey, in Congrès Géologique International, Compte Rendu de la 3me Session, Berlin, 1885: A.W. Schade's Buchdruckerei, Berlin, p. 221-40.

Powell, J.W., 1890, Tenth Annual Report of the United States Geological Survey 1888-'89, Part I - Geology: Washington, D.C., U.S. Government Printing Office, 774 p. 
Ridgway, J.L., 1920, The preparation of illustrations for reports of the United States Geological Survey: Washington, D.C., U.S. Government Printing Office, 101 p.

Snelling, N.J., ed., 1985, The chronology of the geological record: Geological Society of London, p. 261-6.

Soller, D.R., 1996, Review of USGS Open-File Report 95-525 ("Cartographic and digital standard for geologic map information") and plans for development of Federal draft standards for geologic map information: U.S. Geological Survey Open-File Report 96-725, 12 p. [http://pubs.usgs.gov/of/1996/of96-725/].

U.S. Geological Survey, ca. 1975, Technical cartographic standards volume: unpublished U.S. Geological Survey internal standards document.

U.S. Geological Survey, 1995a, Cartographic and digital standard for geologic map information: U.S. Geological Survey Open-File Report 95-525, 257 p.

U.S. Geological Survey, 1995b, Digital files of geologic map symbols with cartographic specifications: U.S. Geological Survey Open-File Report 95-526, 2 disks.

U.S. Geological Survey [prepared in cooperation with the Geologic Data Subcommittee of the Federal Geographic Data Committee], 2000, Public Review Draft - Digital Cartographic Standard for Geologic Map Symbolization (PostScript Implementation): U.S. Geological Survey Open-File Report 99-430 [CD-ROM; also, online at http://pubs.usgs.gov/of/1999/of99-430/].

U.S. Geological Survey [prepared in cooperation with the Geologic Data Subcommittee of the Federal Geographic Data Committee], 2006, FGDC Digital Cartographic Standard for Geologic Map Symbolization (PostScript Implementation): U.S. Geological Survey Techniques and Methods 11-A2 [CD-ROM; also, online at http://pubs.usgs.gov/tm/2006/11A02/].

Weinman, Lynda, 1996, Designing Web graphics: Indianapolis, Ind., New Riders Publishing, p. 49-72. 


\section{APPENDIX A. GEOLOGIC MAP SYMBOLS, COLORS, AND PATTERNS}

This [normative] appendix contains the geologic map symbols and their descriptions, their cartographic specifications, and notes on their usage. Also included are the CMYK Color Chart and the Pattern Chart (enclosed in sleeve on inside back cover), which contain colors and patterns for use on geologic maps.

\section{CONTENTS OF APPENDIX A}

\section{PREFACE TO APPENDIX A}

1. Contacts, Key Beds, and Dikes

2. Faults

3. Boundaries Located by Geophysical Surveys

4. Lineaments and Joints

5. Folds

6. Bedding

7. Cleavage

8. Foliation

9. Lineation

10. Paleontological Features

11. Geophysical and Structure Contours

12. Fluvial and Alluvial Features

13. Glacial and Glaciofluvial Features

14. Periglacial Features

15 Lacustrine and Marine Features

16. Eolian Features

17. Landslide and Mass-Wasting Features

18. Volcanic Features

19. Natural Resources

20. Hazardous Waste Sites
21. Neotectonic and Earthquake-Hazard Features

22. Plate-Tectonic Features

23. Miscellaneous Uplift and Collapse Features

24. Terrestrial Impact Features

25. Planetary Geology Features

26. Geohydrologic Features

27. Weather Stations

28. Transportation Features

29. Boundaries

30. Topographic and Hydrographic Features

31. Miscellaneous Map Elements

32. Geologic Age Symbol Font ("FGDCGeoAge")

33. Suggested Ranges of Map-Unit Colors for Volcanic and Plutonic Rocks and for Stratigraphic Ages of Sedimentary and Metamorphic Rocks

34. State Location Maps

35. Bar Scales

36. Mean Declination Arrows

37. Lithologic Patterns

38. Explanation for Pattern Chart

39. Explanation for CMYK Color Chart 


\section{PREFACE TO APPENDIX A}

In this preface to Appendix A, we include some general guidelines for using the "FGDC Digital Cartographic Standard for Geologic Map Symbolization," as well as some basic information on the newly implemented standard for the scientific confidence and the locational accuracy of geologic features. For more specific information, please refer to the following sections in the accompanying introductory text: "Geologic Mapping Concepts and Definitions" (Section 3); "Scientific Confidence and Locational Accuracy of Geologic Features" (Section 4); "Guidelines for Map Color and Pattern Selection" (Section 5); "Guidelines for Map Labeling" (Section 6); and "Technical Specifications Used in the Preparation of this Standard" (Section 7).

\section{ABOUT THIS VERSION OF THE STANDARD}

This now formally approved version of the standard incorporates revisions that reflect reviewers' responses to the "Public Review Draft — Digital Cartographic Standard for Geologic Map Symbolization" (Federal Geographic Data Committee, 2000). We thank all the reviewers for their contributions, and we are pleased that most of the comments and suggestions could be accommodated (see http://ngmdb.usgs.gov/fgdc_gds/geolsymstd/development.php). Note, however, that we were able to fully address suggestions for the inclusion of new symbols only if examples of the proposed symbol additions were provided.

We intend this standard to be a "living standard" — that is, we recognize that an existing symbol's usage may need to be modified, or a new symbol or set of symbols created, to more fully express local geologic conditions or to keep pace with evolving geologic mapping concepts and practices. Accordingly, such new or modified symbols, if found to be of wide applicability, will be incorporated into this standard through planned, periodic revisions. You are invited to submit comments and suggestions for updates or other improvements to this standard by email to mapsymbol@flagmail.wr.usgs.gov.

In response to reviewers' comments, we tested various cartographic specifications for dashed and ornamented line symbols in several different software packages (Adobe Illustrator v.8.0.1, ArcInfo v.7x, and ArcGIS v.8x) to ensure that symbols would render correctly and consistently. As a result, we found it necessary to modify the cartographic specifications (dash/gap lengths and ornament spacings) of many line symbols in this standard from the specifications found in previous versions of the standard.

\section{HOW TO USE THIS STANDARD}

The contents of this standard are not intended to be used inflexibly or in a manner that will limit one's ability to communicate the observations and interpretations gained from geologic mapping. On the contrary, we recognize that, in certain situations, a symbol or its usage might need to be modified in order to better represent a particular feature on a geologic map or cross section.

To that end, we emphasize that this standard allows the use of any symbol that doesn't conflict with others in the standard, provided that it is clearly explained on the map and in the database. In addition, modifying the size, color, and (or) lineweight of an existing symbol to suit the needs of a particular map or output device also is permitted, provided that the modified symbol's appearance is not too similar to another symbol on the map. Be aware, however, that reducing lineweights below $.125 \mathrm{~mm}(.005$ inch) may cause symbols to plot incorrectly if output at higher resolutions (1800 dpi or higher). For more information, please refer to Section 7 ("Technical Specifications Used in the Preparation of this Standard") in the accompanying introductory text.

To facilitate the use of this standard, we include in this preface tables showing conversion values from inches to points to millimeters (Table 1), abbreviations used (Table 2), and spot color specifications and their equivalent colors in other color models (Table 3). We also offer the following illustrations of a few key terms and concepts used when preparing this standard:

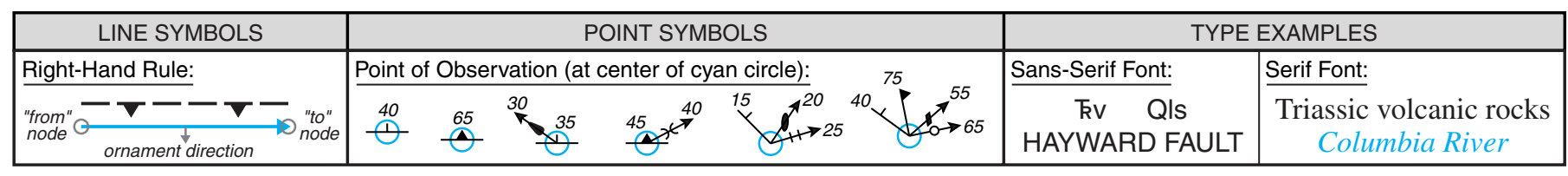

\section{THE NEWLY IMPLEMENTED STANDARD FOR THE SCIENTIFIC CONFIDENCE AND LOCATIONAL ACCURACY OF GEOLOGIC MAP FEATURES}

In response to reviewers' comments, we have implemented a new standard (concepts and terminology) for the scientific confidence and the locational accuracy of geologic map features (note that, at this time, we have applied these new concepts only to line features). Scientific confidence expresses a geologist's level of certainty about the identity or perhaps even the existence of a feature. Locational accuracy is based on the relation between a feature's location in the field and its position on the base map. For a more detailed discussion of these concepts and their associated terminology, please refer to Section 4 ("Scientific Confidence and Locational Accuracy of Geologic Features") in the accompanying introductory text.

\section{FEATURE ATTRIBUTES FOR SCIENTIFIC CONFIDENCE AND LOCATIONAL ACCURACY}

The following is a list of the feature attributes (in italics) that are used to express these concepts:

Scientific Confidence:

Identity — 'certain' or 'questionable'

Existence — 'certain' or 'questionable'

Locational Accuracy:

Locatability — 'observable, 'inferred' (between outcrops or beneath rubble or vegetation), or 'concealed' (beneath overlying map unit, ice, or water)

Zone of Confidence - [value, in ground units]; [unit of measurement]

Positioning Confidence - 'within zone of confidence' or 'may not be within zone of confidence' 


\section{LEVELS OF SCIENTIFIC CONFIDENCE AND LOCATIONAL ACCURACY}

Discrete levels of scientific confidence and locational accuracy have been developed to use as a terminology that can clearly yet concisely communicate the identity, existence, locatability, and positioning of geologic map features. These levels are directly derived from, or are closely associated with, the feature attributes (for more information, see Figures 1 and 2 in the accompanying introductory text). The following diagram shows how the various levels of scientific confidence and locational accuracy relate to the feature attributes; it also shows examples (in italics) of geologic situations to which the levels may be applied.

\begin{tabular}{|c|c|c|}
\hline \multicolumn{3}{|r|}{ SCIENTIFIC CONFIDENCE } \\
\hline \multicolumn{2}{|c|}{ Feature Attributes for Scientific Confidence } & Levels of Scientific Confidence \\
\hline $\begin{array}{l}\text { Identity: } \\
\text { - certain } \\
\text { - questionable }\end{array}$ & $\begin{array}{l}\text { Existence: } \\
\text { - certain } \\
\text { - questionable }\end{array}$ & $\begin{array}{l}\text { Identity and existence certain ("I am certain that the planar feature I see in this outcrop is } \\
\text { a fault") } \\
\text { Identity or existence questionable ("I can see some kind of planar feature in this outcrop, } \\
\text { but I cannot be certain if it is a contact or a fault") }\end{array}$ \\
\hline \multicolumn{3}{|r|}{ LOCATIONAL ACCURACY } \\
\hline $\begin{array}{l}\text { Locatability: } \\
\text { - observable } \\
\text { - inferred } \\
\text { - concealed }\end{array}$ & $\begin{array}{l}\text { Zone of Confidence: } \\
\text { - [value, in ground units] } \\
\text { - [unit of measurement] } \\
\text { Positioning Confidence: } \\
\text { - within zone of confidence } \\
\text { - may not be within zone of } \\
\quad \text { confidence }\end{array}$ & $\begin{array}{l}\text { Location accurate ("I can clearly see this contact in outcrop, and I can accurately plot its } \\
\text { position on the map") } \\
\text { Location approximate ("I can see this contact in outcrop, but I can't tell exactly where it is } \\
\text { located because I am surrounded by trees") } \\
\text { Location inferred ("I can see by the change in debris materials visible around these } \\
\text { gopher holes that a contact runs through here, but I can't locate it precisely") } \\
\text { Location concealed ("I can see that a contact is present on both sides of this lake, but I } \\
\text { can't tell where it is located beneath the water") }\end{array}$ \\
\hline
\end{tabular}

The levels of scientific confidence and locational accuracy have been used to identify and describe the line symbols in this standard. The following example is extracted from Appendix A (see p. A-1-1).

\begin{tabular}{|c|c|c|c|}
\hline DESCRIPTION & SYMBOL & DESCRIPTION & SYMBOL \\
\hline $\begin{array}{l}\text { Contact-Identity and existence certain, loca- } \\
\text { tion accurate }\end{array}$ & & $\begin{array}{l}\text { Contact-Identity and existence certain, loca- } \\
\text { tion inferred }\end{array}$ & ---------- \\
\hline $\begin{array}{l}\text { Contact-Identity and existence certain, loca- } \\
\text { tion approximate }\end{array}$ & ----- & $\begin{array}{l}\text { Contact-Identity and existence certain, loca- } \\
\text { tion concealed }\end{array}$ & \\
\hline
\end{tabular}

In the symbol explanation on a published map, the levels of scientific confidence and locational accuracy also are used to identify and describe the various types and styles of line symbols that appear on the map; however, not every style of a particular line symbol needs to be listed individually in the explanation, as the following two examples show.

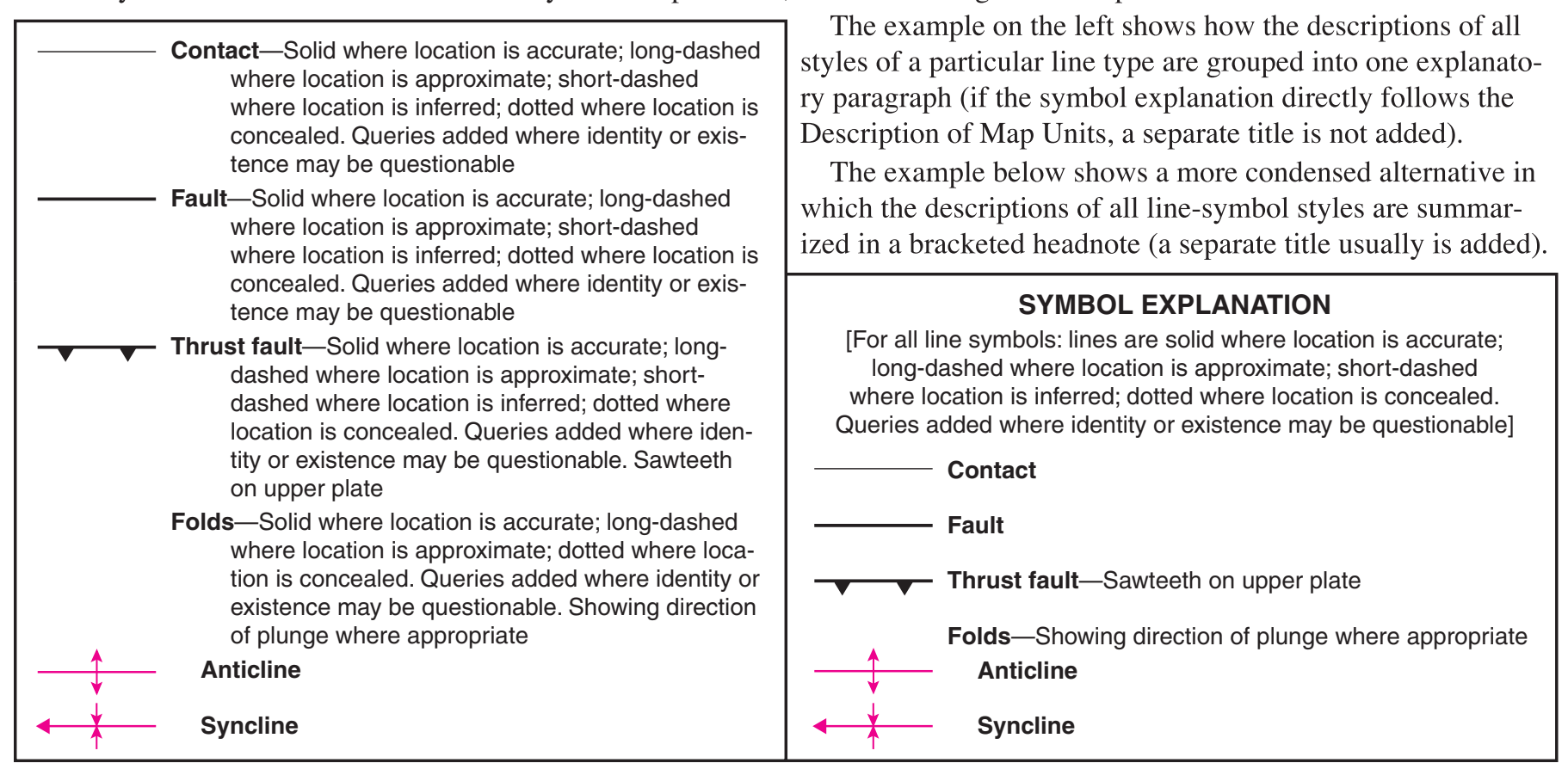


Table 1. Chart showing conversion values from inches (in) to points (pts) to millimeters (mm).

\begin{tabular}{|c|c|c|c|c|c|c|c|c|c|c|c|}
\hline in & pts & $\mathrm{nm}$ & in & pts & $\mathrm{mm}$ & in & pts & $\mathrm{mm}$ & in & pts & $\mathbf{m m}$ \\
\hline 0.001 & .072 & 025 & 0.051 & 3.672 & 1.295 & .101 & 7.272 & 2.565 & .151 & 0.872 & 3.835 \\
\hline 0.002 & .144 & .051 & 0.052 & .744 & 1.321 & .102 & 7.344 & 2.591 & 152 & & 3.861 \\
\hline 0.003 & 216 & & 0.053 & 16 & 346 & 103 & 416 & 616 & 53 & & .886 \\
\hline 0.004 & 0.288 & & 0.054 & 3.888 & 1.372 & 0.104 & 7.488 & 2.642 & .154 & 1.088 & 3.912 \\
\hline 0.005 & 0.360 & 127 & 0.055 & 3.960 & 1.397 & .105 & 7.560 & 2.667 & 155 & .160 & .937 \\
\hline 0.006 & 0.432 & & 0.056 & 4.032 & .422 & 106 & 7.632 & 2.692 & 156 & .232 & 3.962 \\
\hline 0.007 & 0.504 & 0.178 & 0.057 & 4.104 & 1.448 & 0.107 & 7.704 & 2.718 & & & 3.988 \\
\hline 0.008 & 0.576 & & 0.058 & 4.176 & 73 & 108 & .776 & 2.743 & & .376 & 4.013 \\
\hline 0.009 & 0.648 & 0.229 & 0.059 & 4.248 & 1.499 & 0.109 & 7.848 & 2.769 & 0.159 & 11.448 & 4.039 \\
\hline 0.010 & 0.720 & 254 & 0.060 & 4.320 & & 0.110 & 7.920 & 2.794 & & 11.520 & 4.064 \\
\hline 0.011 & 0.792 & & 0.061 & 4.392 & & 111 & 992 & 2.819 & & 592 & 4.089 \\
\hline 0.012 & 0.864 & 305 & 0.062 & 4.464 & 1.575 & 0.112 & 8.064 & 2.845 & 0.162 & .664 & 4.115 \\
\hline 0.013 & 0.936 & 330 & 0.063 & 4.536 & 1.600 & 0.113 & 8.136 & 2.870 & 163 & .736 & 4.140 \\
\hline 0.014 & 1.008 & & 0.064 & 4.608 & 1.6 & .114 & 8.208 & 2.896 & & & 4.166 \\
\hline 0.015 & 1.080 & 0.381 & 0.065 & 4.680 & 1.651 & 0.115 & 8.280 & 2.921 & 0.165 & 1.880 & 4.191 \\
\hline 0.016 & 1.152 & & 0.066 & 4.7 & & & .352 & & & & .216 \\
\hline 0.017 & 1.224 & 432 & 0.067 & 4.824 & 1.702 & 0.117 & 8.424 & 2.972 & 0.167 & .024 & 4.242 \\
\hline 0.018 & 1.296 & 457 & 0.068 & 4.896 & 1.727 & 0.118 & 8.496 & 2.997 & 168 & .096 & 4.267 \\
\hline 0.019 & 1.368 & & 0.069 & 4.9 & & & 8.568 & & & & 4.293 \\
\hline 0.020 & 1.440 & 508 & 0.070 & 5.040 & 1.778 & 0.120 & 8.640 & 3.048 & 0.170 & .240 & 4.318 \\
\hline 0.021 & 1.512 & & 0.071 & 5.112 & & 21 & 8.712 & 3.073 & & & .343 \\
\hline 0.022 & 1.584 & & 0.072 & 5.184 & 1.829 & 0.122 & 8.784 & 3.099 & & & 4.369 \\
\hline 0.023 & 1.656 & 84 & 0.073 & 5.256 & 1.8 & 0.123 & 8.856 & 3.124 & 0.173 & .456 & 4.394 \\
\hline 0.024 & 1.728 & & 0.074 & 5.328 & 1.8 & & .928 & & & & .420 \\
\hline 0.025 & 1.800 & 35 & 0.075 & 5.400 & 1.905 & 0.125 & 9.000 & 3.175 & & 600 & 4.445 \\
\hline 0.026 & 1.872 & 660 & 0.076 & 5.472 & & 0.126 & 9.072 & 3.200 & & 672 & 4.470 \\
\hline 0.027 & 1.944 & & 0.077 & 5.5 & & 0.127 & 9.144 & 3.226 & & 744 & 4.496 \\
\hline 0.028 & 2.016 & & 0.078 & 5.616 & & 0.128 & 9.216 & 3.251 & & & 4.521 \\
\hline 0.029 & 2.088 & & 079 & 5.688 & & & 9.288 & 3.277 & & & 547 \\
\hline 0.030 & 2.160 & 762 & 0.080 & 5.760 & 2.032 & 0.130 & 9.360 & 3.302 & & 960 & 4.572 \\
\hline 0.031 & 2.232 & 0.787 & 0.081 & 5.832 & & & 9.432 & & & & 4.597 \\
\hline 0.032 & 2.304 & & 0.082 & 5.904 & 2.083 & 0.1 & 9.504 & 3.353 & & & 4.623 \\
\hline 0.033 & 2.376 & 0.838 & 0.083 & 5.976 & 2.108 & 0.133 & 9.576 & 3.378 & & 176 & 4.648 \\
\hline 0.034 & & & 0.084 & & & & 9.648 & & & & .674 \\
\hline 0.035 & 2.520 & 389 & 0.085 & 6.120 & 2.159 & 0.135 & 9.720 & 3.429 & 0.185 & 320 & 4.699 \\
\hline 0.036 & & & 0.086 & & & & & & & & 4.724 \\
\hline 0.037 & 2.664 & & 0.087 & 6.264 & 2.210 & 0.137 & 9.864 & 3.480 & & 464 & 4.750 \\
\hline 0.038 & 2.736 & 0.965 & 0.088 & 6.336 & 2.235 & 0.138 & 9.936 & 3.505 & 0.188 & 13.536 & 4.775 \\
\hline 0.039 & 2.808 & & 0.089 & & & & & & & & 4.801 \\
\hline 0.040 & 2.880 & 016 & 0.090 & 6.480 & 2.286 & 0.140 & 10.080 & 3.556 & 0.190 & 680 & 4.826 \\
\hline 0.041 & 2.952 & & 0.091 & 6.552 & 2.311 & 0.141 & 10.152 & 3.581 & & & 4.851 \\
\hline 0.042 & 3.024 & & 0.092 & 6.624 & 2.337 & 0.142 & 10.224 & 3.607 & & 824 & 4.877 \\
\hline 0.043 & 3.096 & 1.092 & 0.093 & 6.696 & 2.362 & 0.143 & 10.296 & 3.632 & 0.193 & .896 & 4.902 \\
\hline 0.044 & & & 0.094 & & & 0.144 & & 3.658 & & 968 & 4.928 \\
\hline 0.045 & 3.240 & & 0.095 & & & & & & & & 4.953 \\
\hline 0.046 & 3.312 & & 0.096 & & 2.438 & 0.146 & & 3.708 & 0.196 & 14.112 & 4.978 \\
\hline 0.047 & 3.384 & & 0.097 & 984 & 2.464 & 0.147 & 10.584 & 3.734 & 97 & 184 & 5.004 \\
\hline 0.048 & 3.456 & & 0.098 & 7.056 & 2.489 & 0.148 & 10.656 & 3.759 & & 14.256 & 5.029 \\
\hline 0.049 & 3.528 & & 0.099 & 7.128 & 2.515 & 0.149 & 10.728 & 3.785 & 0.199 & 14.328 & 5.055 \\
\hline 0.050 & 3.600 & 1.270 & 0.100 & 7.200 & 2.540 & 0.150 & 10.800 & 3.810 & 0.200 & 14.400 & 5.080 \\
\hline
\end{tabular}


Table 2. Abbreviations used in this standard.

\begin{tabular}{|c|c|c|}
\hline Abbreviation & Meaning & Example of usage \\
\hline B & brown [ink] & 422-B (pattern) \\
\hline C & cyan [ink] & 132-C (pattern) \\
\hline CMYK & cyan/magenta/yellow/black & CMYK color model \\
\hline DO & dropout [pattern] & 204-DO (pattern) \\
\hline FG-8 & FGDCGeoAge [font], 8 pt type & kg (unit label containing geologic age character) \\
\hline H-8 ${ }^{1}$ & Helvetica [font], 8 pt type & GOLDEN FAULT (name of fault) \\
\hline HB-8 ${ }^{1}$ & Helvetica Bold [font], 8 pt type & ? (query indicating "identity or existence questionable" fault) \\
\hline HI-6 ${ }^{1}$ & Helvetica Italic [font], 6 pt type & 40 (dip value) \\
\hline HSV & hue/saturation/value & HSV color model \\
\hline K & black [ink] & 134-K (pattern) \\
\hline M & magenta [ink] & 313-M (pattern) \\
\hline R & red [ink] & 405-R (pattern) \\
\hline RGB & red/green/blue & RGB color model \\
\hline T-9 ${ }^{2}$ & Times [font], 9 pt type & UNITED STATES (label on national boundary) \\
\hline TBI-12 & Times Bold Italic [font], 12 pt type & $\boldsymbol{A}^{\prime}$ (cross section labels) \\
\hline TI-8 ${ }^{2}$ & Times Italic [font], 8 pt type & Bass Lake (name of lake) \\
\hline Y & yellow [ink] & CMYK color model \\
\hline
\end{tabular}

${ }^{1}$ Although Helvetica has been specified, any sans-serif font (such as Univers or Arial) may be used. Note, however, that if other fonts are used, their appearance will not match that of FGDCGeoAge, whose character size and spacing is based on Helvetica.

${ }^{2}$ Although Times has been specified, any serif font (such as Times New Roman or Souvenir) may be used.

Table 3. Spot color specifications used in this standard, and their equivalent colors in other color models.

[Abbreviations: C, cyan; M, magenta; $\mathrm{Y}$, yellow; $\mathrm{K}$, black (standard process-color inks combined during offset printing). CMYK, cyan/magenta/yellow/black color model. R, red; G, green; B, blue (primary colors transmitted by computer monitors and televisions). RGB, red/green/blue color model.]

\begin{tabular}{|c|c|c|c|c|c|}
\hline Spot color 1 & Pantone color ${ }^{2}$ & $\begin{array}{c}\text { Directly converted } \\
\text { CMYK } \text { color }^{3}\end{array}$ & $\begin{array}{c}\text { Color on CMYK } \\
\text { Color Chart }{ }^{4} \\
\end{array}$ & $\begin{array}{c}\text { Directly converted } \\
\text { RGB color }{ }^{5}\end{array}$ & $\begin{array}{l}\text { "Web-safe" } \\
\text { RGB color } 6\end{array}$ \\
\hline red & $485 \mathrm{U}$ & 0/100/91/0 & $0 / 100 / 100$ & $254 / 0 / 12$ & $255 / 0 / 0$ \\
\hline $50 \%$ red & $\begin{array}{c}485 \mathrm{U} \\
\text { (screened 50\%) }\end{array}$ & 0/50/45.5/0 & $0 / 50 / 40$ & $251 / 128 / 104$ & $255 / 102 / 102$ \\
\hline green & $354 \mathrm{U}$ & $91 / 0 / 83 / 0$ & $100 / 0 / 100$ & $24 / 150 / 76$ & $51 / 153 / 102$ \\
\hline $50 \%$ green & $\begin{array}{c}354 \mathrm{U} \\
\text { (screened 50\%) }\end{array}$ & $45.5 / 0 / 41.5 / 0$ & $40 / 0 / 40$ & $139 / 207 / 144$ & $153 / 204 / 153$ \\
\hline violet & $253 \mathrm{U}$ & $47 / 91 / 0 / 0$ & $50 / 100 / 0$ & $136 / 22 / 135$ & $153 / 0 / 153$ \\
\hline purple & $2735 \mathrm{U}$ & $100 / 94 / 0 / 0$ & $100 / 100 / 0$ & $18 / 12 / 128$ & 0/0/153 \\
\hline brown & $470 \cup$ & 0/56/94/34 & $30 / 70 / 100$ & $168 / 74 / 9$ & $153 / 51 / 0$ \\
\hline orange & $1585 \mathrm{U}$ & $0 / 56 / 87 / 0$ & $0 / 60 / 100$ & $254 / 112 / 24$ & $255 / 102 / 0$ \\
\hline
\end{tabular}

${ }^{1}$ Generic name of spot color, as specified in this standard (note that cyan, magenta, yellow, and black are process-color inks, not spot colors, and so they have not been included in this table).

${ }^{2}$ Suggested Pantone color for offset printing on uncoated paper.

${ }^{3}$ Color value after direct conversion of suggested Pantone color to CMYK (C/M/Y/K) by Adobe Illustrator 8.0.1.

${ }^{4}$ Closest color on CMYK Color Chart (in pocket) to directly converted CMYK color value.

${ }^{5}$ Color value after direct conversion of suggested Pantone color to RGB (R/G/B) by Adobe Illustrator 8.0.1.

${ }^{6}$ Closest "web-safe" color (see discussion in Section 7.3, entitled "Color Specifications for Line and Point Symbols," in the introductory text) to directly converted RGB color value. 
Federal Geographic Data Committee (Doc. No. FGDC-STD-013-2006)

U.S. Geological Survey Techniques and Methods 11-A2 FGDC Digital Cartographic Standard for Geologic Map Symbolization (PostScript Implementation)

DOWNLOAD this Illustrator EPS file: Al8 / $\underline{\text { CS2 }}$

\section{1-CONTACTS, KEY BEDS, AND DIKES}

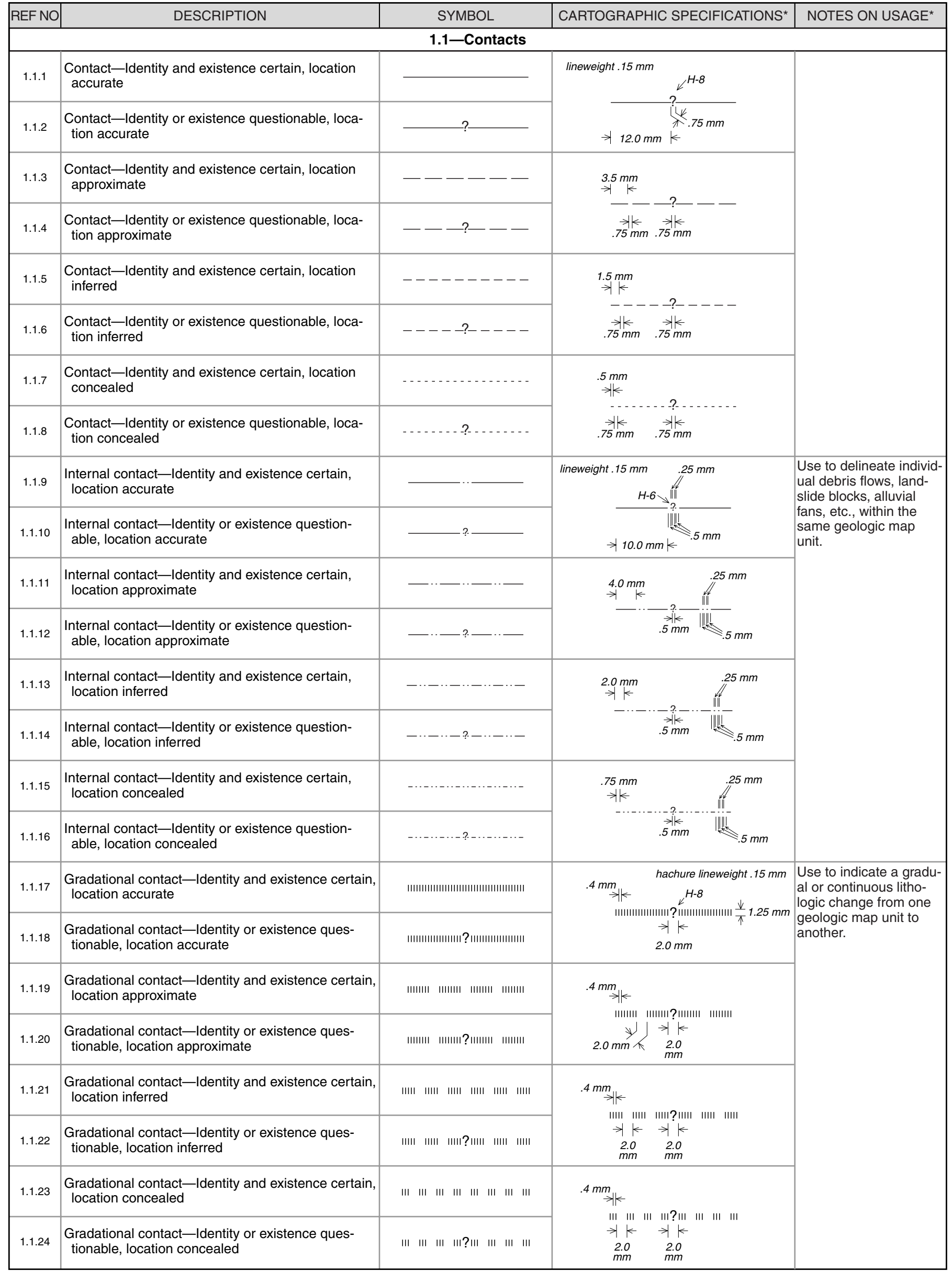


Federal Geographic Data Committee (Doc. No. FGDC-STD-013-2006)

U.S. Geological Survey Techniques and Methods 11-A2 FGDC Digital Cartographic Standard for Geologic Map Symbolization (PostScript Implementation) DOWNLOAD this Illustrator EPS file: Al8 / CS2

\section{1-CONTACTS, KEY BEDS, AND DIKES (continued)}

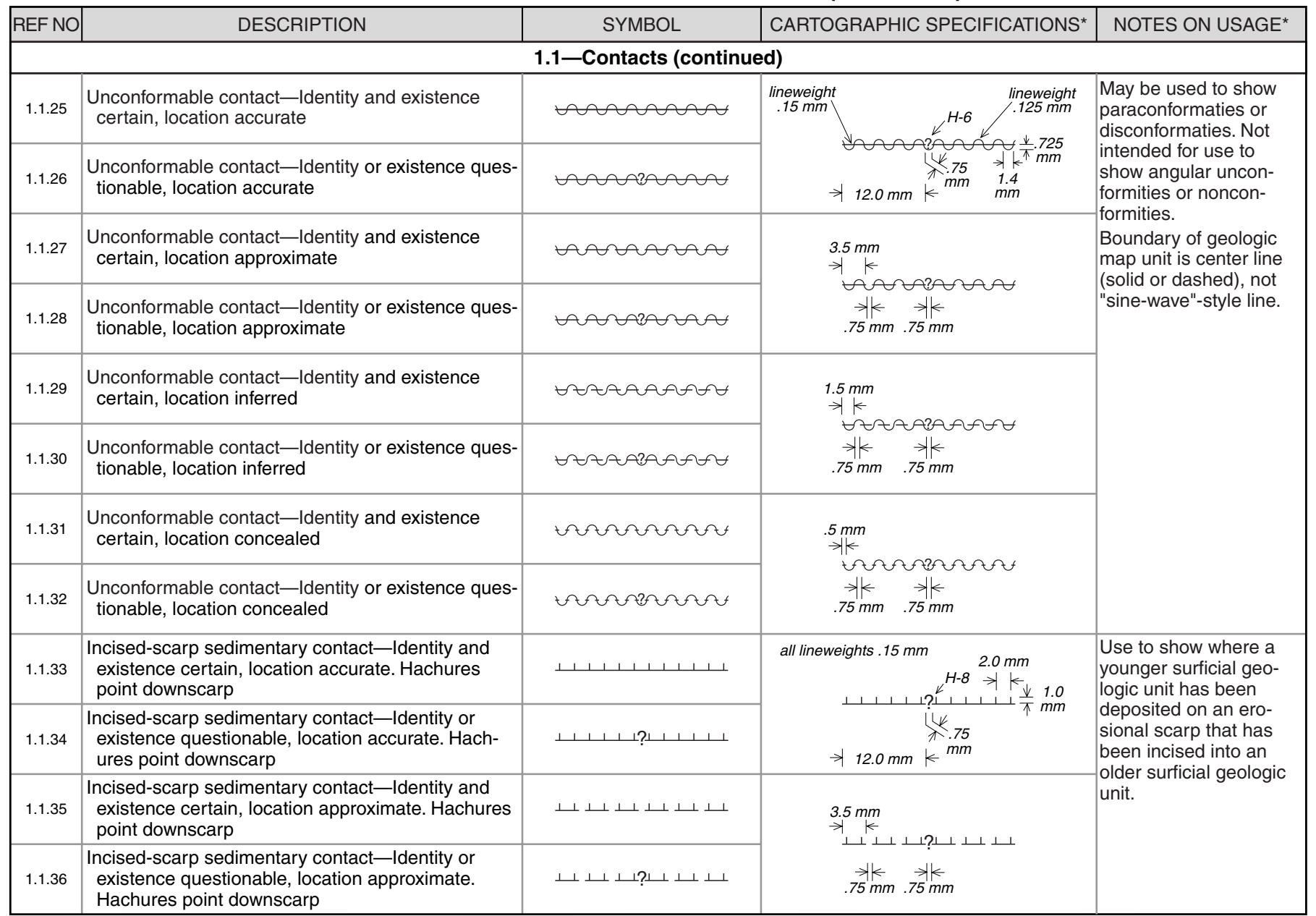

${ }^{*}$ For more information, see general guidelines on pages $A-i$ to $A-v$. 
Federal Geographic Data Committee (Doc. No. FGDC-STD-013-2006)

U.S. Geological Survey Techniques and Methods 11-A2 FGDC Digital Cartographic Standard for Geologic Map Symbolization (PostScript Implementation)

DOWNLOAD this Illustrator EPS file: Al8 / CS2

1-CONTACTS, KEY BEDS, AND DIKES (continued)

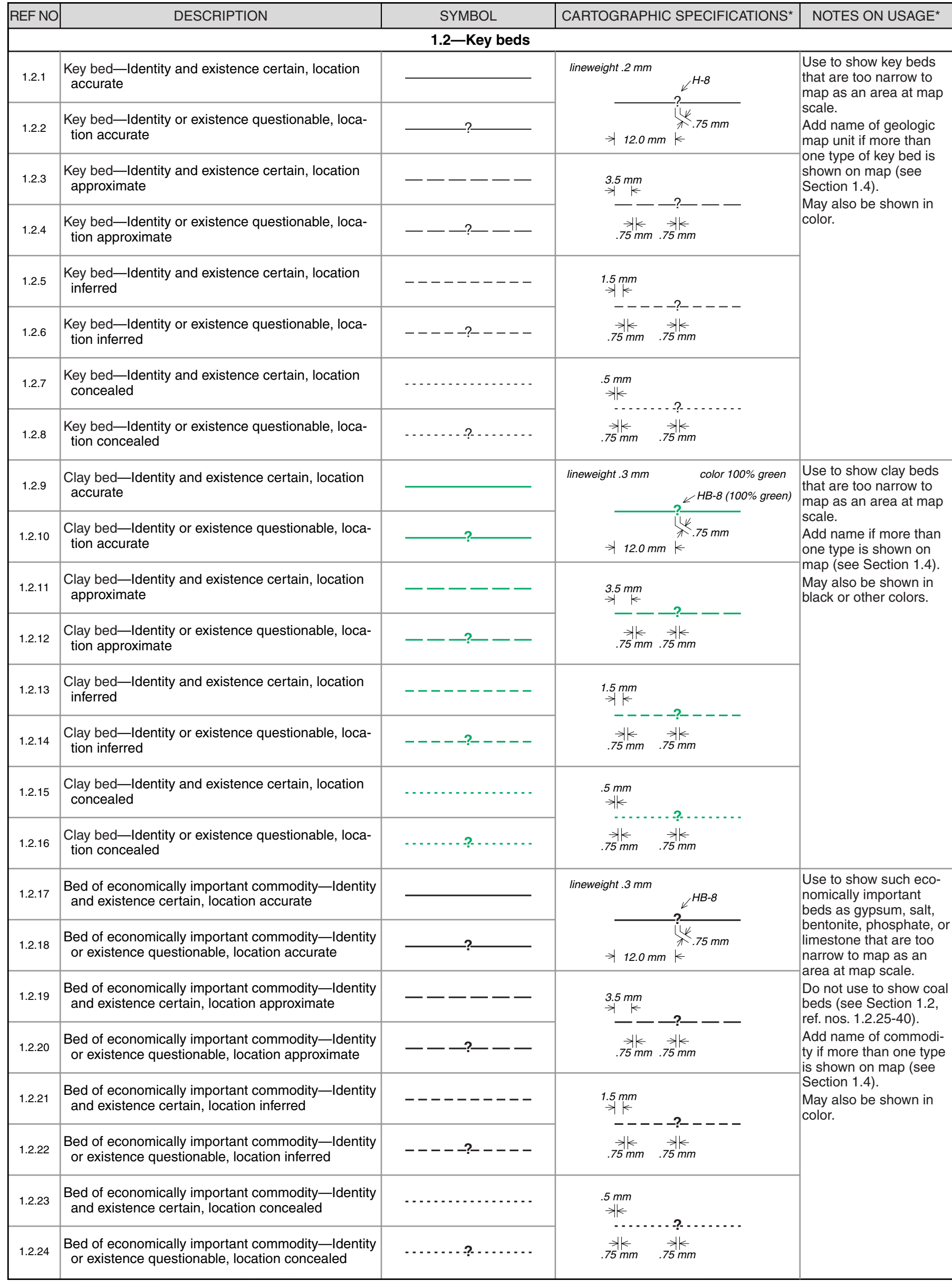


Federal Geographic Data Committee (Doc. No. FGDC-STD-013-2006)

U.S. Geological Survey Techniques and Methods 11-A2 FGDC Digital Cartographic Standard for Geologic Map Symbolization (PostScript Implementation)

DOWNLOAD this Illustrator EPS file: Al8 / CS2

1-CONTACTS, KEY BEDS, AND DIKES (continued)

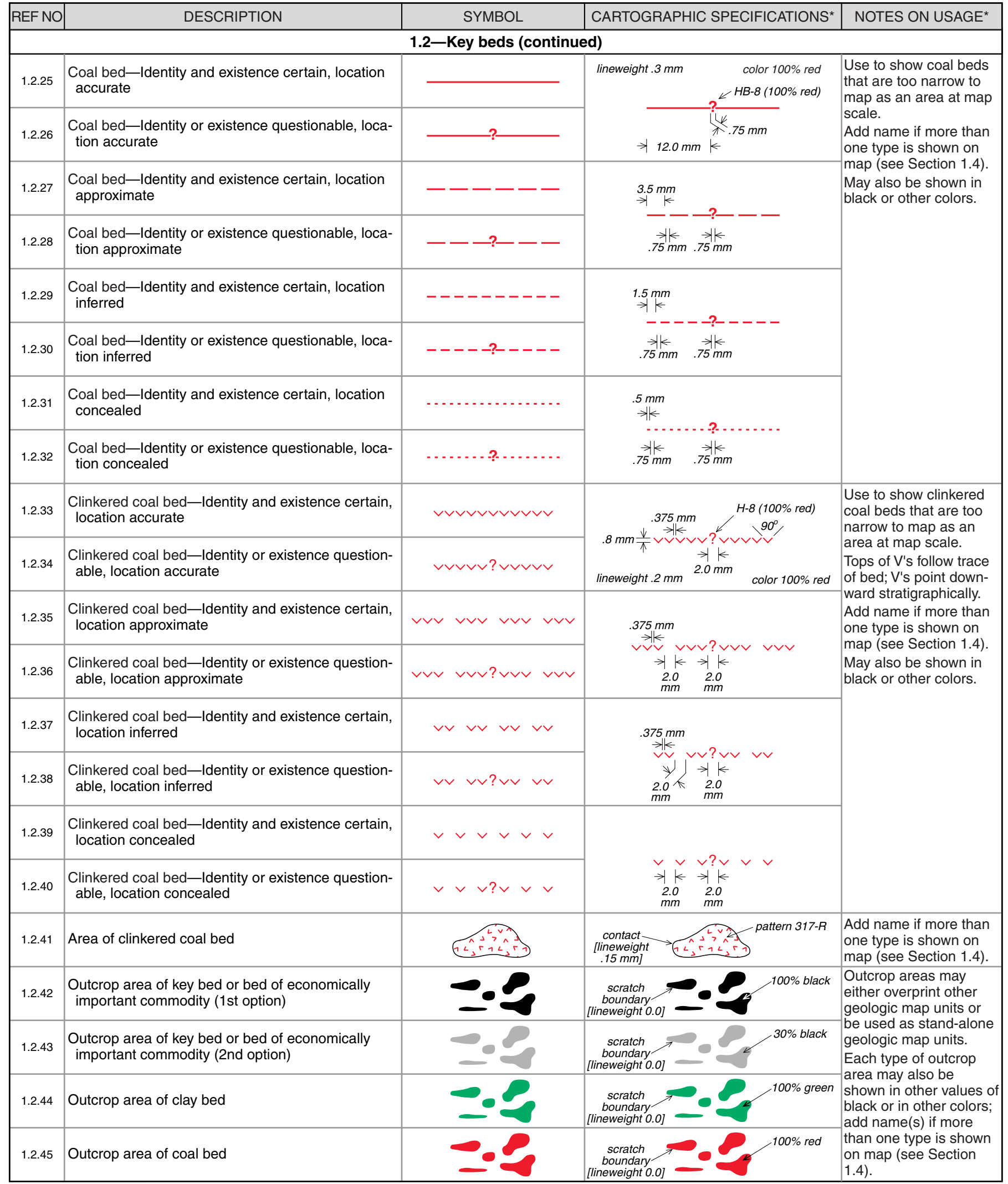

${ }^{*}$ For more information, see general guidelines on pages $A-i$ to $A-v$. 
Federal Geographic Data Committee (Doc. No. FGDC-STD-013-2006)

U.S. Geological Survey Techniques and Methods 11-A2 FGDC Digital Cartographic Standard for Geologic Map Symbolization (PostScript Implementation)

DOWNLOAD this Illustrator EPS file: Al8 / CS2

\section{1-CONTACTS, KEY BEDS, AND DIKES (continued)}

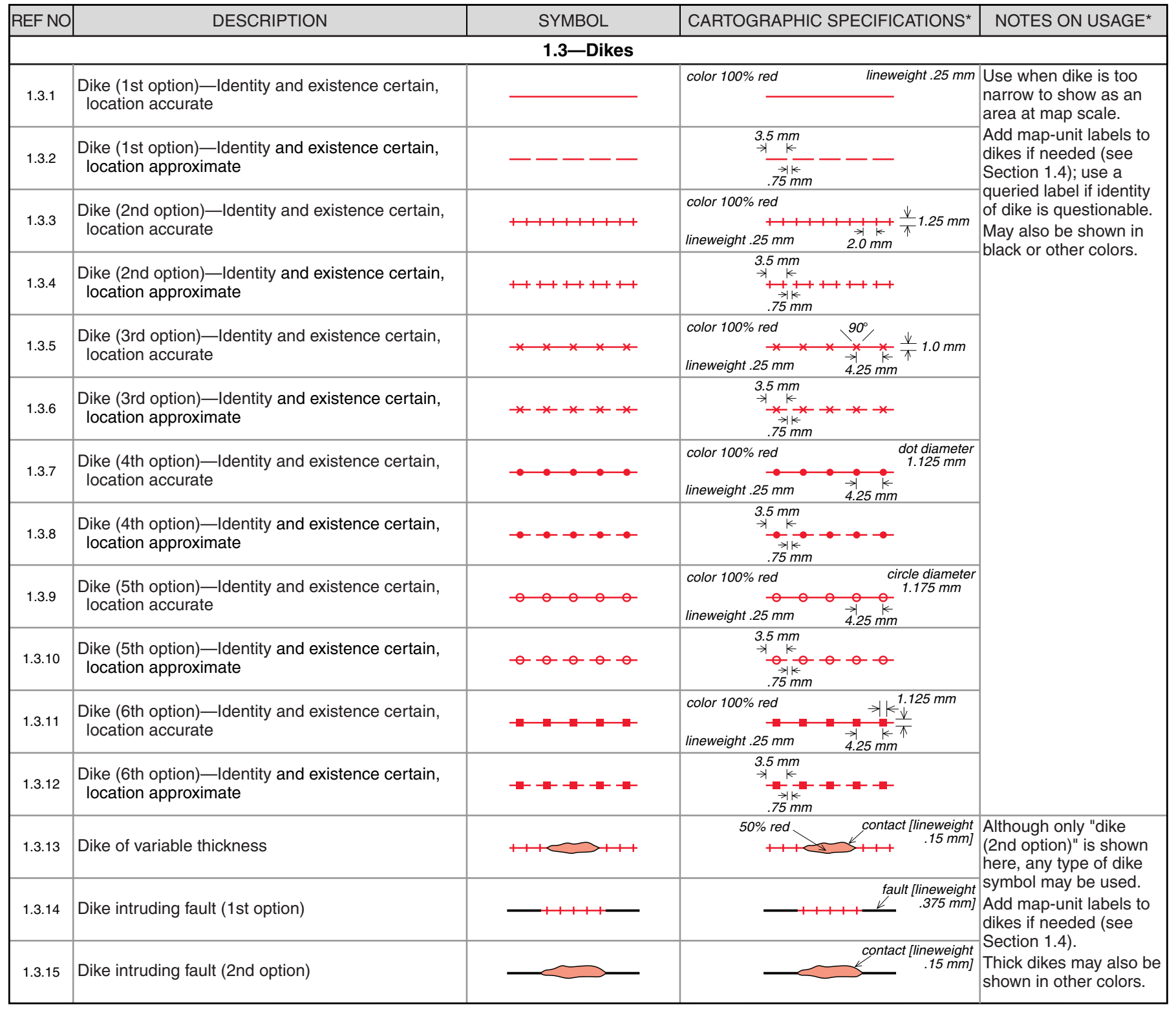

${ }^{*}$ For more information, see general guidelines on pages $A-i$ to $A-v$. 
Federal Geographic Data Committee (Doc. No. FGDC-STD-013-2006)

U.S. Geological Survey Techniques and Methods 11-A2 FGDC Digital Cartographic Standard for Geologic Map Symbolization (PostScript Implementation)

DOWNLOAD this Illustrator EPS file: Al8 / CS2

\section{1-CONTACTS, KEY BEDS, AND DIKES (continued)}

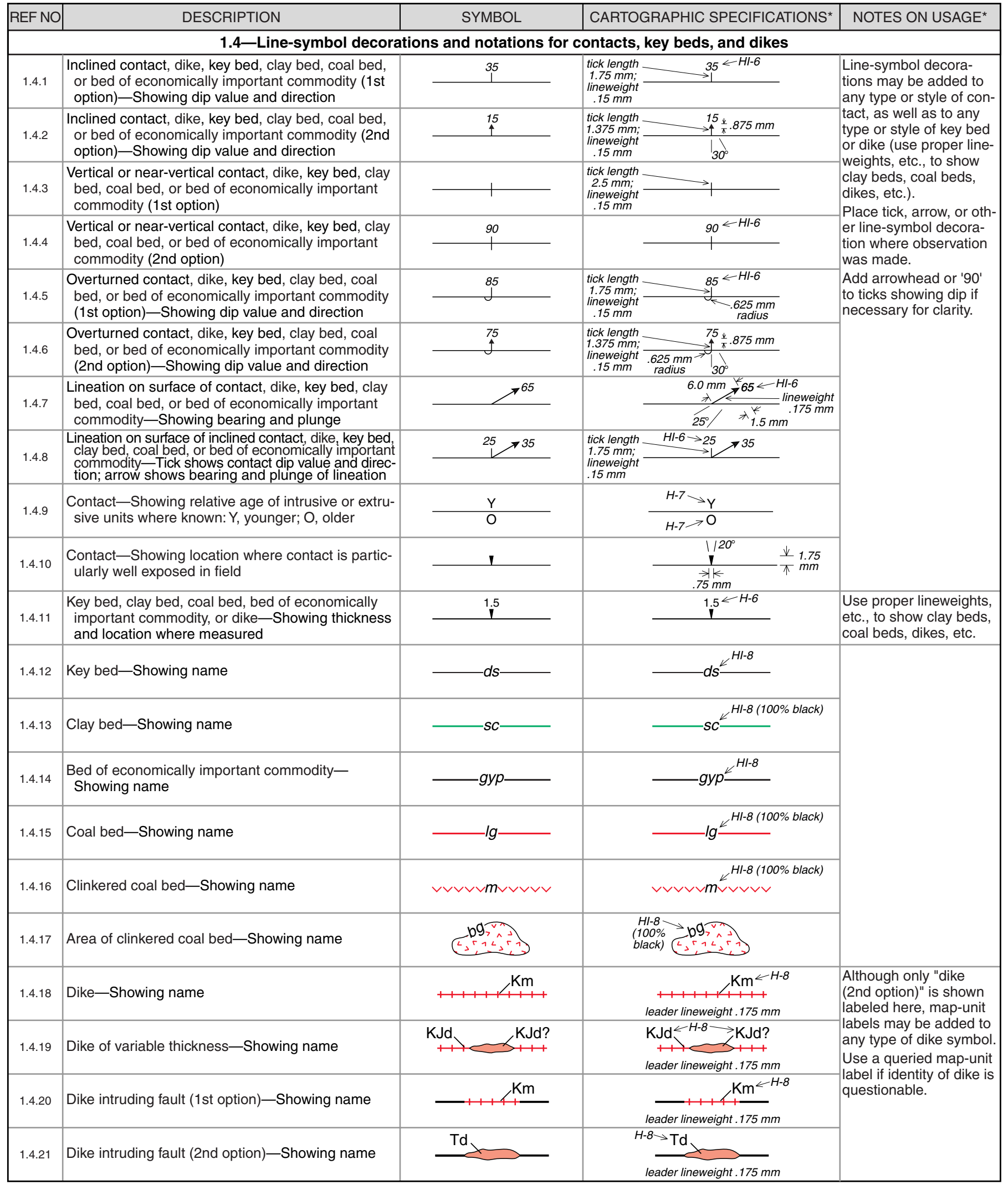

${ }^{*}$ For more information, see general guidelines on pages $A-i$ to $A-v$. 


\begin{tabular}{|c|c|c|c|c|}
\hline \multicolumn{5}{|c|}{ 2-FAULTS } \\
\hline REF NO & DESCRIPTION & SYMBOL & CARTOGRAPHIC SPECIFICATIONS* & NOTES ON USAGE* \\
\hline \multicolumn{5}{|c|}{ 2.1-Faults (generic; vertical, subvertical, or high-angle; or unknown or unspecified orientation or sense of slip) } \\
\hline 2.1.1 & $\begin{array}{l}\text { Fault (generic; vertical, subvertical, or high-angle; or } \\
\text { unknown or unspecified orientation or sense of slip) } \\
\text { - Identity and existence certain, location accurate }\end{array}$ & & lineweight $.375 \mathrm{~mm}$ & \multirow{8}{*}{$\begin{array}{l}\text { Use generic, nonspecif- } \\
\text { ic (non-ornamented) } \\
\text { fault symbols when ori- } \\
\text { entation or sense of slip } \\
\text { is not known or not } \\
\text { specified; use also on } \\
\text { small-scale maps to } \\
\text { show regional fault pat- } \\
\text { terns. } \\
\text { If orientation or sense of } \\
\text { slip is known and if } \\
\text { scale allows, use more } \\
\text { specific types of orna- } \\
\text { mented fault symbols to } \\
\text { indicate fault geometry } \\
\text { and (or) relative motion. }\end{array}$} \\
\hline 2.1 .2 & $\begin{array}{l}\text { Fault (generic; vertical, subvertical, or high-angle; or } \\
\text { unknown or unspecified orientation or sense of slip)- } \\
\text { Identity or existence questionable, location accurate }\end{array}$ & ? & $\rightarrow 12.0 \mathrm{~mm} \stackrel{k}{k} .75 \mathrm{~mm}$ & \\
\hline 2.1.3 & $\begin{array}{l}\text { Fault (generic; vertical, subvertical, or high-angle; or } \\
\text { unknown or unspecified orientation or sense of slip)- } \\
\text { Identity and existence certain, location approximate }\end{array}$ & ------ & \multirow{2}{*}{ 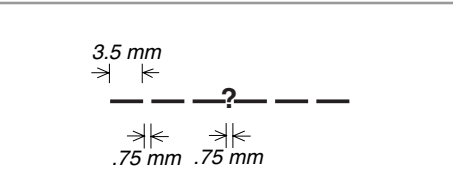 } & \\
\hline 2.1 .4 & $\begin{array}{l}\text { Fault (generic; vertical, subvertical, or high-angle; or } \\
\text { unknown or unspecified orientation or sense of slip)- } \\
\text { Identity or existence questionable, location approximate }\end{array}$ & $-?--$ & & \\
\hline 2.1 .5 & $\begin{array}{l}\text { Fault (generic; vertical, subvertical, or high-angle; or } \\
\text { unknown or unspecified orientation or sense of slip) } \\
\text { - Identity and existence certain, location inferred }\end{array}$ & - - - - - - - & \multirow{2}{*}{ 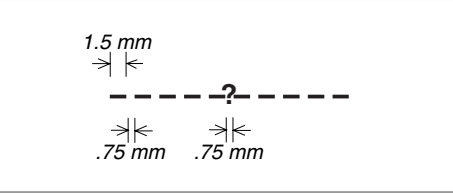 } & \\
\hline 2.1.6 & $\begin{array}{l}\text { Fault (generic; vertical, subvertical, or high-angle; or } \\
\text { unknown or unspecified orientation or sense of slip)- } \\
\text { Identity or existence questionable, location inferred }\end{array}$ & - - - - ?- - - - & & \\
\hline 2.1 .7 & $\begin{array}{l}\text { Fault (generic; vertical, subvertical, or high-angle; or } \\
\text { unknown or unspecified orientation or sense of slip) } \\
\text { - Identity and existence certain, location concealed }\end{array}$ & - . & \multirow{2}{*}{ 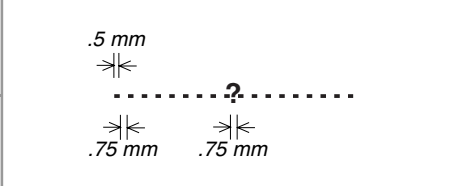 } & \\
\hline 2.1 .8 & $\begin{array}{l}\text { Fault (generic; vertical, subvertical, or high-angle; or } \\
\text { unknown or unspecified orientation or sense of slip)- } \\
\text { Identity or existence questionable, location concealed }\end{array}$ & $\cdots$ & & \\
\hline
\end{tabular}


2-FAULTS (continued)

\begin{tabular}{|c|c|c|c|c|}
\hline REF NO & DESCRIPTION & SYMBOL & CARTOGRAPHIC SPECIFICATIONS* & NOTES ON USAGE* \\
\hline \multicolumn{5}{|c|}{ 2.2-Normal faults } \\
\hline 2.2.1 & $\begin{array}{l}\text { Normal fault-Identity and existence certain, loca- } \\
\text { tion accurate. Ball and bar on downthrown block }\end{array}$ & $\stackrel{1}{i}$ & $\begin{array}{l}\text { tick length } 1.0 \mathrm{~mm} ; \\
\text { lineweight } .175 \mathrm{~mm}\end{array}$ & \multirow{6}{*}{$\begin{array}{l}\text { Ball and bar symbols } \\
\text { are placed along a fault } \\
\text { to indicate its overall } \\
\text { fault type (normal fault). } \\
\text { Ball and bar symbols } \\
\text { may also be placed } \\
\text { along other types of } \\
\text { faults at specific locali- } \\
\text { ties where observations } \\
\text { of normal (or apparent } \\
\text { normal) offset have } \\
\text { been made (see Sec- } \\
\text { tion } 2.11 \text { ). } \\
\text { Ball and bar symbols } \\
\text { may be combined with } \\
\text { paired arrows to show } \\
\text { oblique offset (see Sec- } \\
\text { tions } 2.7,2.11 \text { ). } \\
\text { In cross section, use } \\
\text { paired arrows to show } \\
\text { relative motion of nor- } \\
\text { mal faults (see Section } \\
2.11 \text { ). }\end{array}$} \\
\hline 2.2.2 & $\begin{array}{l}\text { Normal fault-Identity or existence questionable, } \\
\text { location accurate. Ball and bar on downthrown } \\
\text { block }\end{array}$ & $? \quad ?$ & $\begin{array}{c}\text { lineweight } .375 \mathrm{~mm} \\
\Rightarrow 12.0 \mathrm{~mm} \\
k\end{array}$ & \\
\hline 2.2.3 & $\begin{array}{l}\text { Normal fault-Identity and existence certain, loca- } \\
\text { tion approximate. Ball and bar on downthrown } \\
\text { block }\end{array}$ & ーー・ーー & $\underset{\rightarrow \rightarrow}{3.5 \mathrm{~mm}}$ & \\
\hline 2.2 .4 & $\begin{array}{l}\text { Normal fault-Identity or existence questionable, } \\
\text { location approximate. Ball and bar on downthrown } \\
\text { block }\end{array}$ & - - & 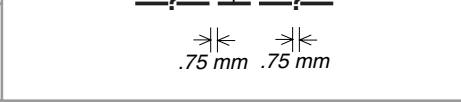 & \\
\hline 2.2.5 & $\begin{array}{l}\text { Normal fault-Identity and existence certain, loca- } \\
\text { tion inferred. Ball and bar on downthrown block }\end{array}$ & -ーーーPーーーー & 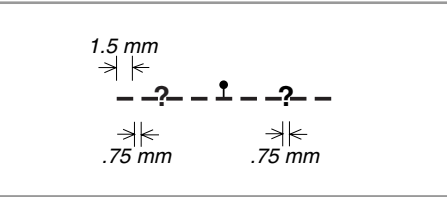 & \\
\hline 2.2.8 & $\begin{array}{l}\text { Normal fault-Identity or existence questionable, } \\
\text { location concealed. Ball and bar on downthrown } \\
\text { block }\end{array}$ & . . . . . . . . . . . . . & 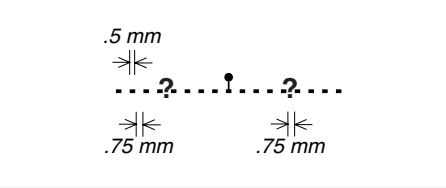 & \\
\hline 2.2 .9 & $\begin{array}{l}\text { Low-angle normal fault-Identity and existence cer- } \\
\text { tain, location accurate. Half-circles on downthrown } \\
\text { block }\end{array}$ & & \multirow{2}{*}{ 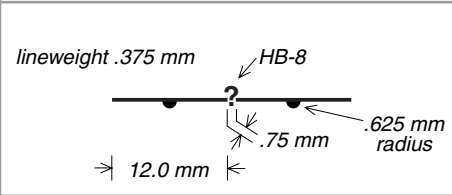 } & \multirow{5}{*}{$\begin{array}{l}\text { Half-circles indicate } \\
\text { overall fault type (low- } \\
\text { angle normal fault); they } \\
\text { are not placed at spec } \\
\text { ific localities where } \\
\text { observations have been } \\
\text { made. } \\
\text { In cross section, use } \\
\text { paired arrows to show } \\
\text { relative motion of low- } \\
\text { angle normal faults (see } \\
\text { Section 2.11). }\end{array}$} \\
\hline 2.2.10 & $\begin{array}{l}\text { Low-angle normal fault-Identity or existence ques- } \\
\text { tionable, location accurate. Half-circles on down- } \\
\text { thrown block }\end{array}$ & & & \\
\hline 2.2.11 & $\begin{array}{l}\text { Low-angle normal fault-Identity and existence cer- } \\
\text { tain, location approximate. Half-circles on down- } \\
\text { thrown block }\end{array}$ & & 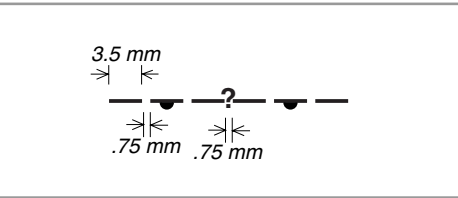 & \\
\hline 2.2.15 & $\begin{array}{l}\text { Low-angle normal fault-Identity and existence cer- } \\
\text { tain, location concealed. Half-circles on down- } \\
\text { thrown block }\end{array}$ & & \multirow{2}{*}{ 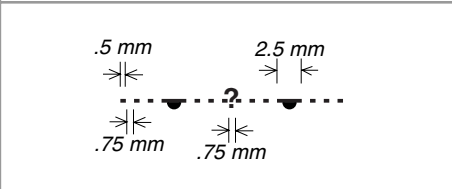 } & \\
\hline 2.2.16 & $\begin{array}{l}\text { Low-angle normal fault-Identity or existence ques- } \\
\text { tionable, location concealed. Half-circles on down- } \\
\text { thrown block }\end{array}$ & & & \\
\hline
\end{tabular}




\section{2-FAULTS (continued)}

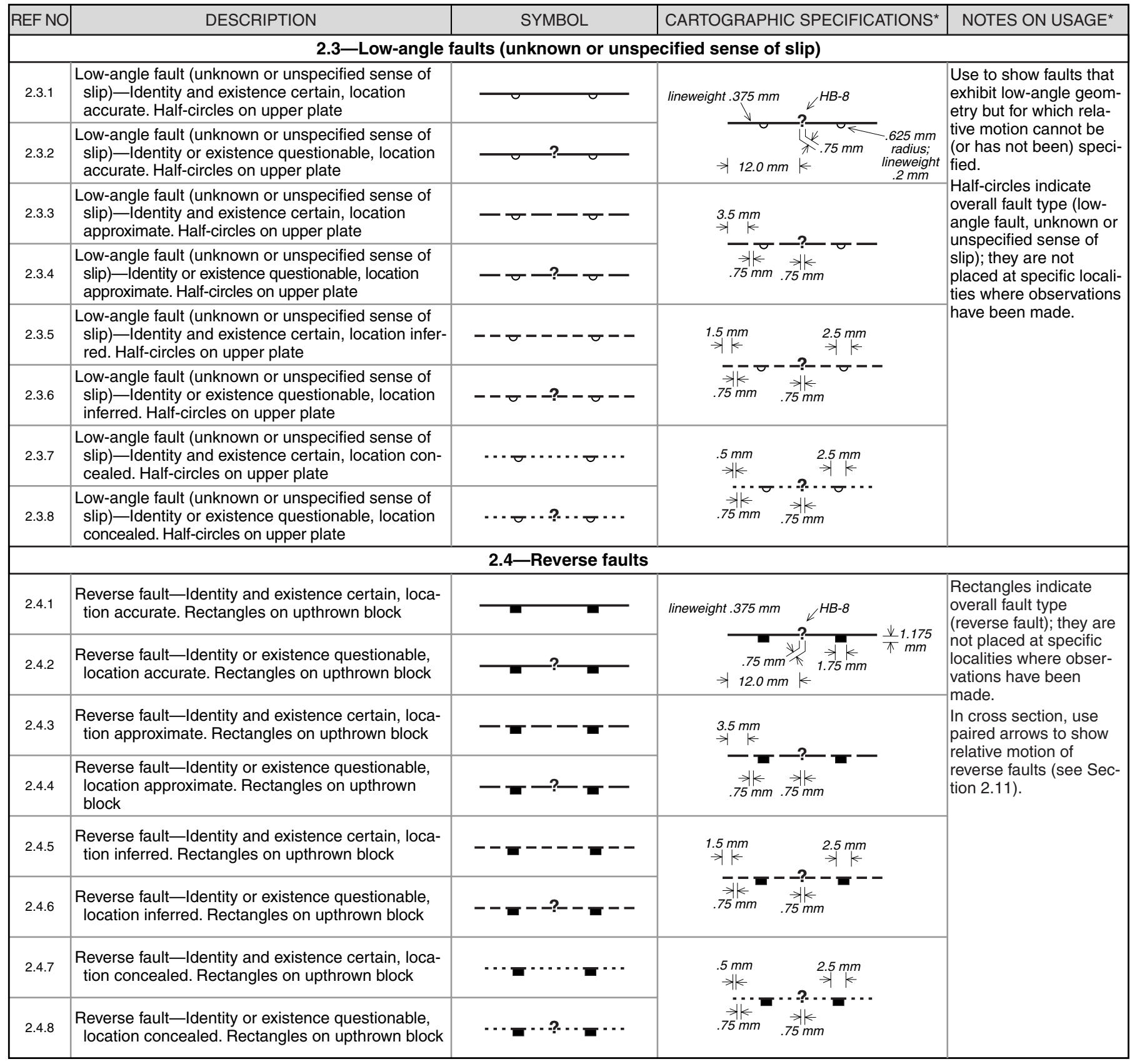




\section{2-FAULTS (continued)}

\begin{tabular}{|c|c|c|c|c|}
\hline REF NO & DESCRIPTION & SYMBOL & CARTOGRAPHIC SPECIFICATIONS* & NOTES ON USAGE* \\
\hline \multicolumn{5}{|c|}{ 2.5-Rotational or scissor faults } \\
\hline 2.5 .1 & $\begin{array}{l}\text { Rotational or scissor fault, reverse-slip offset- } \\
\text { Identity and existence certain, location accurate. } \\
\text { Rectangles on upthrown block }\end{array}$ & & \multirow{2}{*}{ lineweight $.375 \mathrm{~mm}$} & \multirow{5}{*}{$\begin{array}{l}\text { Rectangles indicate } \\
\text { overall fault type (rota- } \\
\text { tional or scissor fault, } \\
\text { reverse-slip offset); they } \\
\text { are not placed at specif- } \\
\text { ic localities where } \\
\text { observations have been } \\
\text { made. } \\
\text { In cross section, use } \\
\text { paired arrows to show } \\
\text { relative motion of rota- } \\
\text { tional or scissor faults } \\
\text { (see Section 2.11). }\end{array}$} \\
\hline 2.5 .2 & $\begin{array}{l}\text { Rotational or scissor fault, reverse-slip offset- } \\
\text { Identity or existence questionable, location accu- } \\
\text { rate. Rectangles on upthrown block }\end{array}$ & & & \\
\hline 2.5 .4 & $\begin{array}{l}\text { Rotational or scissor fault, reverse-slip offset- } \\
\text { Identity or existence questionable, location } \\
\text { approximate. Rectangles on upthrown block }\end{array}$ & - - ?- - & 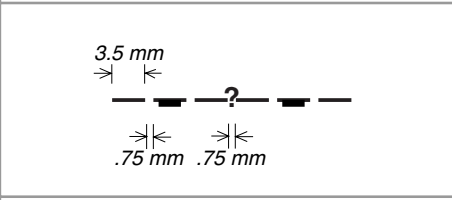 & \\
\hline 2.5 .5 & $\begin{array}{l}\text { Rotational or scissor fault, reverse-slip offset- } \\
\text { Identity and existence certain, location inferred. } \\
\text { Rectangles on upthrown block }\end{array}$ & -ーーーー-ー・ー・ & 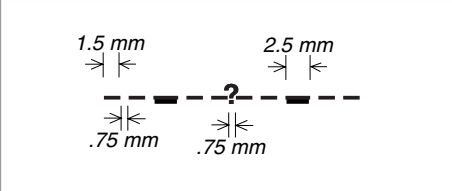 & \\
\hline 2.5 .8 & $\begin{array}{l}\text { Rotational or scissor fault, reverse-slip offset- } \\
\text { Identity or existence questionable, location con- } \\
\text { cealed. Rectangles on upthrown block }\end{array}$ & $\cdots-\cdots+\cdots$ & 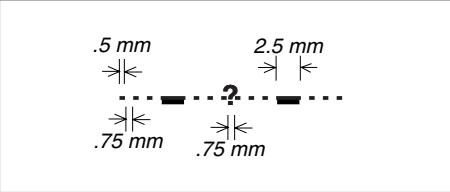 & \\
\hline 2.5 .9 & $\begin{array}{l}\text { Rotational or scissor fault, normal-slip offset- } \\
\text { Identity and existence certain, location accurate. } \\
\text { Rectangles on downthrown block }\end{array}$ & & \multirow{2}{*}{ 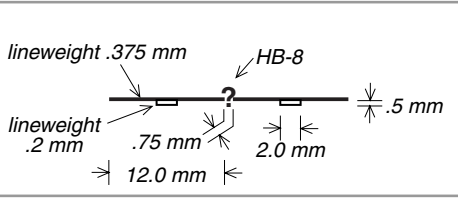 } & \multirow{5}{*}{$\begin{array}{l}\text { Rectangles indicate } \\
\text { overall fault type (rota- } \\
\text { tional or scissor fault, } \\
\text { normal-slip offset); they } \\
\text { are not placed at spe- } \\
\text { cific localities where } \\
\text { observations have been } \\
\text { made. } \\
\text { In cross section, use } \\
\text { paired arrows to show } \\
\text { relative motion of rota- } \\
\text { tional or scissor faults } \\
\text { (see Section 2.11). }\end{array}$} \\
\hline 2.5.10 & $\begin{array}{l}\text { Rotational or scissor fault, normal-slip offset- } \\
\text { Identity or existence questionable, location accu- } \\
\text { rate. Rectangles on downthrown block }\end{array}$ & $-?$ & & \\
\hline 2.5.11 & $\begin{array}{l}\text { Rotational or scissor fault, normal-slip offset- } \\
\text { Identity and existence certain, location approxi- } \\
\text { mate. Rectangles on downthrown block }\end{array}$ & ーேーーーー & 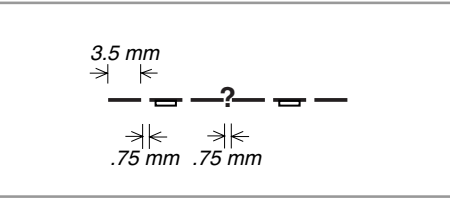 & \\
\hline 2.5.15 & $\begin{array}{l}\text { Rotational or scissor fault, normal-slip offset- } \\
\text { Identity and existence certain, location concealed. } \\
\text { Rectangles on downthrown block }\end{array}$ & $\cdots$ & \multirow{2}{*}{ 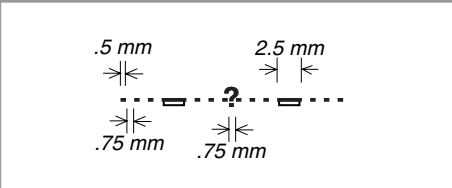 } & \\
\hline 2.5.16 & $\begin{array}{l}\text { Rotational or scissor fault, normal-slip offset- } \\
\text { Identity or existence questionable, location con- } \\
\text { cealed. Rectangles on downthrown block }\end{array}$ & $\cdots \square \cdots ?$ & & \\
\hline
\end{tabular}


Federal Geographic Data Committee (Doc. No. FGDC-STD-013-2006)

U.S. Geological Survey Techniques and Methods 11-A2 FGDC Digital Cartographic Standard for Geologic Map Symbolization (PostScript Implementation)

DOWNLOAD this Illustrator EPS file: Al8 / CS2

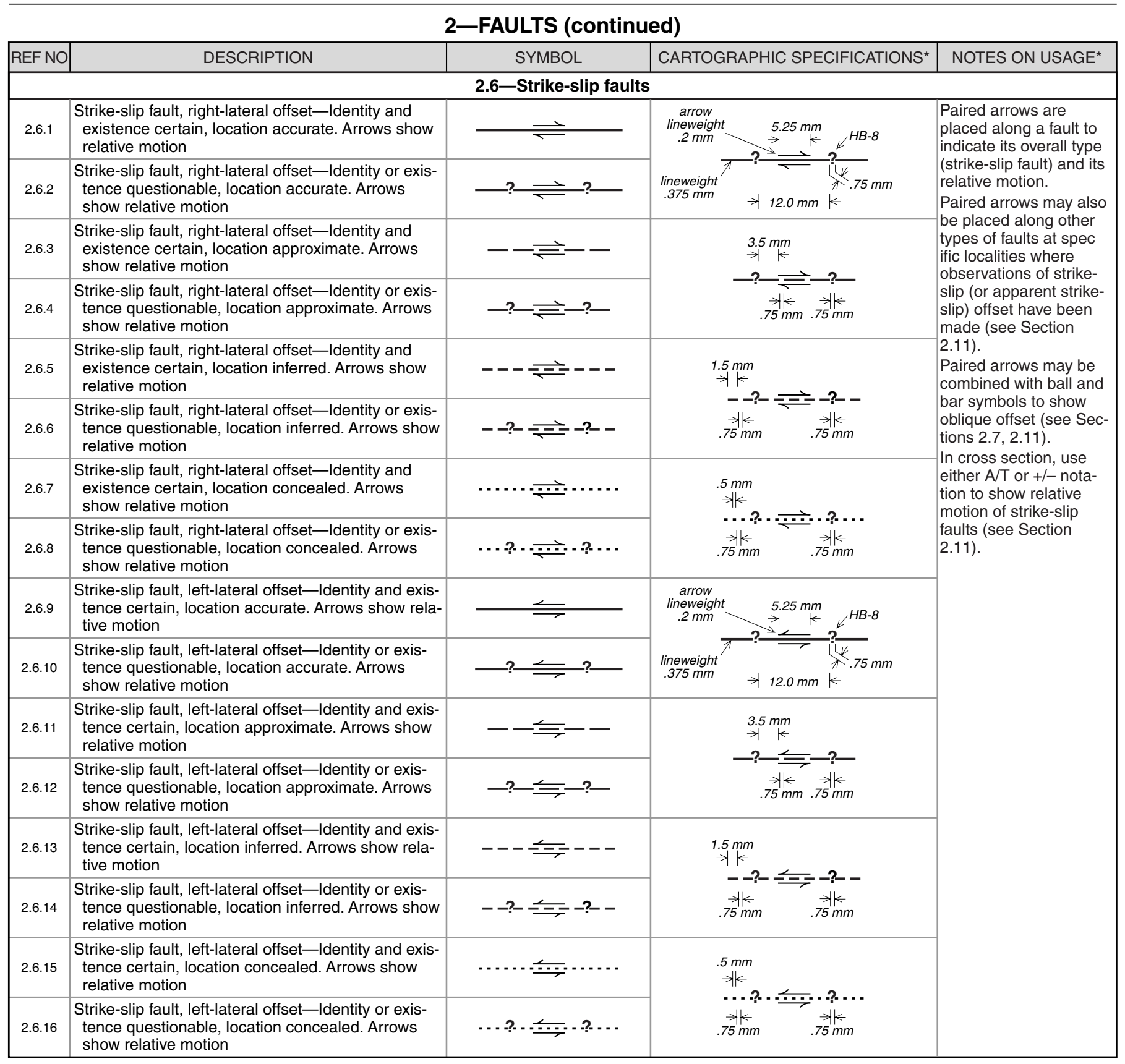

${ }^{*}$ For more information, see general guidelines on pages $A-i$ to $A-v$. 
2-FAULTS (continued)

\begin{tabular}{|c|c|c|c|c|}
\hline REF NO & DESCRIPTION & SYMBOL & CARTOGRAPHIC SPECIFICATIONS* & NOTES ON USAGE* \\
\hline \multicolumn{5}{|c|}{ 2.7-Oblique-slip faults } \\
\hline 2.7 .1 & $\begin{array}{l}\text { Oblique-slip fault, right-lateral offset-Identity and } \\
\text { existence certain, location accurate. Arrows show } \\
\text { relative motion; ball and bar on downthrown block }\end{array}$ & $\rightleftharpoons$ & $\begin{array}{lll}\text { arrow } \\
\text { lineweight } \\
.2 \mathrm{~mm}\end{array}$ & \multirow{2}{*}{$\begin{array}{l}\text { Sets of paired arrows } \\
\text { and ball and bar sym- } \\
\text { bols are placed along a } \\
\text { fault to indicate its over- } \\
\text { all type (oblique-slip } \\
\text { fault) and its relative } \\
\text { motion }\end{array}$} \\
\hline 2.7.2 & $\begin{array}{l}\text { Oblique-slip fault, right-lateral offset-Identity or exis- } \\
\text { tence questionable, location accurate. Arrows show } \\
\text { relative motion; ball and bar on downthrown block }\end{array}$ & $\longrightarrow ? \rightleftharpoons ?$ & $\begin{array}{l}\text { lineweight } \\
.375 \mathrm{~mm}\end{array} \rightarrow 12.0 \mathrm{~mm} \stackrel{\mathbb{k} .75 \mathrm{~mm}}{\mathrm{~K}}$ & \\
\hline 2.7.4 & $\begin{array}{l}\text { Oblique-slip fault, right-lateral offset-Identity or exis- } \\
\text { tence questionable, location approximate. Arrows show } \\
\text { relative motion; ball and bar on downthrown block }\end{array}$ & 一一三??- & 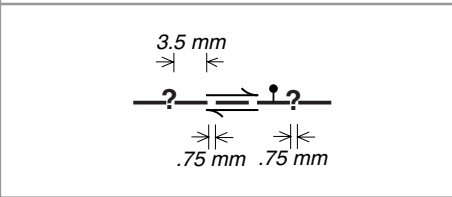 & \multirow{8}{*}{$\begin{array}{l}\text { Sets of paired arrows } \\
\text { and ball and bar sym- } \\
\text { bols may also be placed } \\
\text { along other types of } \\
\text { faults at specific locali- } \\
\text { ties where observations } \\
\text { of oblique-slip (or appa- } \\
\text { rent oblique-slip) offset } \\
\text { have been made (see } \\
\text { Section } 2.11 \text { ). } \\
\text { In cross section, use } \\
\text { paired arrows with } \\
\text { either A/T or +/- nota- } \\
\text { tion to show relative } \\
\text { motion of oblique-slip } \\
\text { faults (see Section } \\
2.11 \text { ). }\end{array}$} \\
\hline 2.7.5 & $\begin{array}{l}\text { Oblique-slip fault, right-lateral offset-Identity and } \\
\text { existence certain, location inferred. Arrows show } \\
\text { relative motion; ball and bar on downthrown block }\end{array}$ & $---\rightleftharpoons$ & 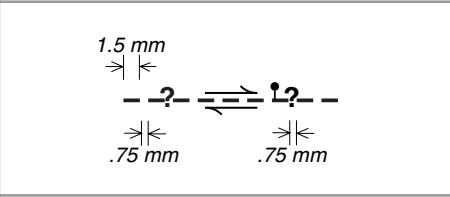 & \\
\hline 2.7.8 & $\begin{array}{l}\text { Oblique-slip fault, right-lateral offset-Identity or exis- } \\
\text { tence questionable, location concealed. Arrows show } \\
\text { relative motion; ball and bar on downthrown block }\end{array}$ & 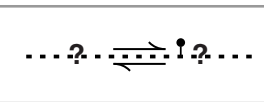 & 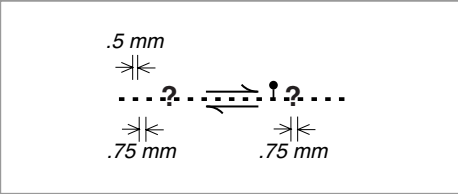 & \\
\hline 2.7.9 & $\begin{array}{l}\text { Oblique-slip fault, left-lateral offset-Identity and ex- } \\
\text { istence certain, location accurate. Arrows show } \\
\text { relative motion; ball and bar on downthrown block }\end{array}$ & $i \leftrightharpoons$ & \multirow{2}{*}{$\begin{array}{l}.875 \mathrm{~mm} \text { diameter } \\
\text { tick length } \\
1.0 \mathrm{~mm} \\
\text { lineweight } \\
.175 \mathrm{~mm} \\
\text { lineweight } \\
.375 \mathrm{~mm}\end{array} \rightarrow \begin{array}{c}\text { arrow } \\
5.25 \mathrm{~mm}\end{array}$} & \\
\hline 2.7.10 & $\begin{array}{l}\text { Oblique-slip fault, left-lateral offset-Identity or exis- } \\
\text { tence questionable, location accurate. Arrows show } \\
\text { relative motion; ball and bar on downthrown block }\end{array}$ & 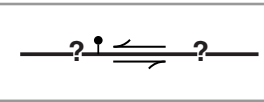 & & \\
\hline 2.7.11 & $\begin{array}{l}\text { Oblique-slip fault, left-lateral offset-Identity and } \\
\text { existence certain, location approximate. Arrows show } \\
\text { relative motion; ball and bar on downthrown block }\end{array}$ & - $\leftrightharpoons-$ & 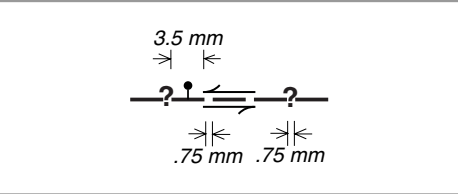 & \\
\hline 2.7.15 & $\begin{array}{l}\text { Oblique-slip fault, left-lateral offset-Identity and } \\
\text { existence certain, location concealed. Arrows show } \\
\text { relative motion; ball and bar on downthrown block }\end{array}$ & 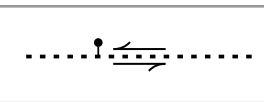 & \multirow{2}{*}{ 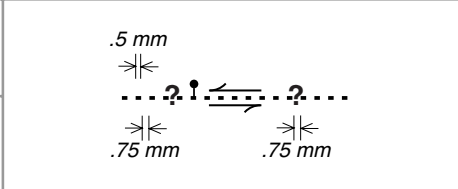 } & \\
\hline 2.7.16 & $\begin{array}{l}\text { Oblique-slip fault, left-lateral offset-Identity or exis- } \\
\text { tence questionable, location concealed. Arrows show } \\
\text { relative motion; ball and bar on downthrown block }\end{array}$ & - . - ? & & \\
\hline
\end{tabular}


2-FAULTS (continued)

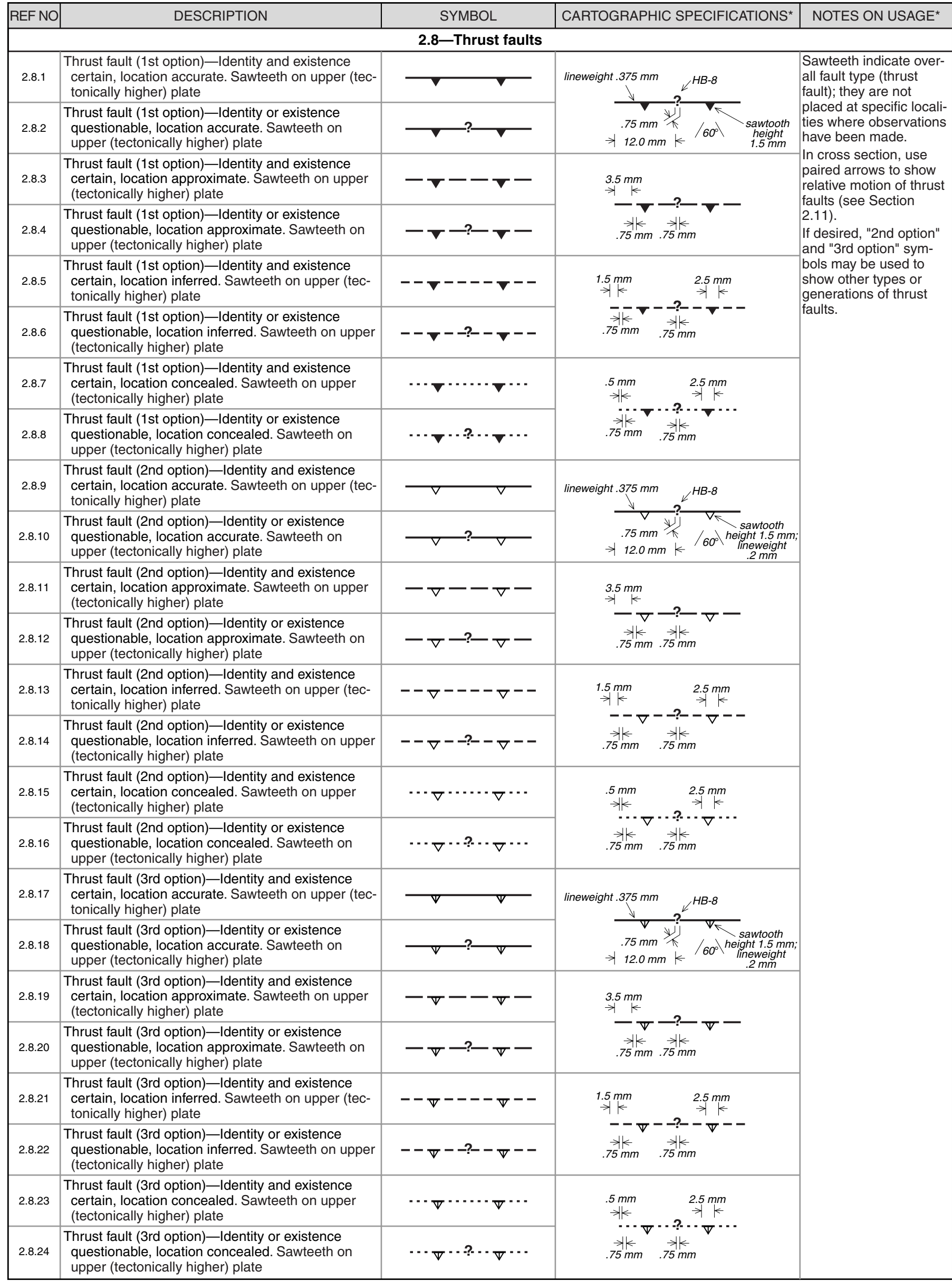




\section{2-FAULTS (continued)}

\begin{tabular}{|c|c|c|c|c|}
\hline REF NO & DESCRIPTION & SYMBOL & CARTOGRAPHIC SPECIFICATIONS* & NOTES ON USAGE* \\
\hline \multicolumn{5}{|c|}{ 2.9-Overturned thrust faults } \\
\hline 2.9.1 & $\begin{array}{l}\text { Overturned thrust fault (1st option)—Identity and exis- } \\
\text { tence certain, location accurate. Bars on tectonically } \\
\text { higher plate (footwall); sawteeth in direction of dip }\end{array}$ & & \multirow{2}{*}{ 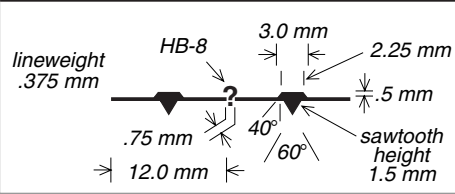 } & \multirow{18}{*}{$\begin{array}{l}\text { Bars and sawteeth indi- } \\
\text { cate overall fault type } \\
\text { (overturned thrust fault) } \\
\text { they are not placed at } \\
\text { specific localities where } \\
\text { observations have been } \\
\text { made. } \\
\text { In cross section, use } \\
\text { paired arrows to show } \\
\text { relative motion of over- } \\
\text { turned thrust faults (see } \\
\text { Section 2.11). } \\
\text { If desired, "2nd option" } \\
\text { and "3rd option" sym- } \\
\text { bols may be used to } \\
\text { show other types or } \\
\text { generations of over- } \\
\text { turned thrust faults. }\end{array}$} \\
\hline 2.9.2 & $\begin{array}{l}\text { Overturned thrust fault (1st option)—-Identity or exis- } \\
\text { tence questionable, location accurate. Bars on tectoni- } \\
\text { cally higher plate (footwall); sawteeth in direction of dip }\end{array}$ & & & \\
\hline 2.9 .3 & $\begin{array}{l}\text { Overturned thrust fault (1st option)-Identity and exis- } \\
\text { tence certain, location approximate. Bars on tectoni- } \\
\text { cally higher plate (footwall); sawteeth in direction of dip }\end{array}$ & & \multirow{2}{*}{ 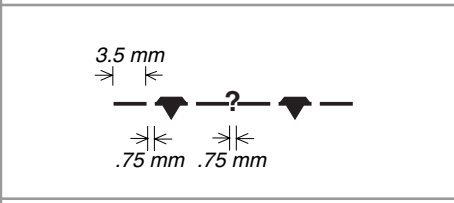 } & \\
\hline 2.9 .4 & $\begin{array}{l}\text { Overturned thrust fault (1st option)—Identity or existence } \\
\text { questionable, location approximate. Bars on tectonical- } \\
\text { ly higher plate (footwall); sawteeth in direction of dip }\end{array}$ & & & \\
\hline 2.9 .5 & $\begin{array}{l}\text { Overturned thrust fault (1st option)-Identity and ex- } \\
\text { istence certain, location inferred. Bars on tectonical- } \\
\text { ly higher plate (footwall); sawteeth in direction of dip }\end{array}$ & -- & 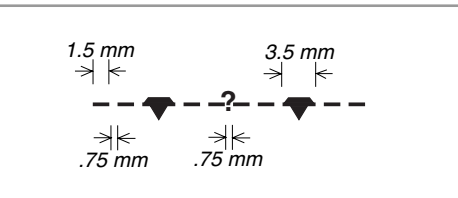 & \\
\hline 2.9 .8 & $\begin{array}{l}\text { Overturned thrust fault (1st option)—Identity or existence } \\
\text { questionable, location concealed. Bars on tectonically } \\
\text { higher plate (footwall); sawteeth in direction of dip }\end{array}$ & & 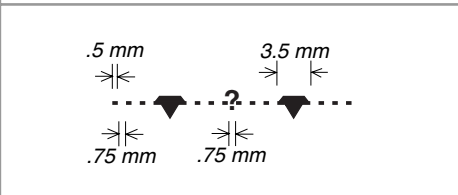 & \\
\hline 2.9 .9 & $\begin{array}{l}\text { Overturned thrust fault (2nd option)—Identity and ex- } \\
\text { istence certain, location accurate. Bars on tectonical- } \\
\text { ly higher plate (footwall); sawteeth in direction of dip }\end{array}$ & & \multirow{2}{*}{ 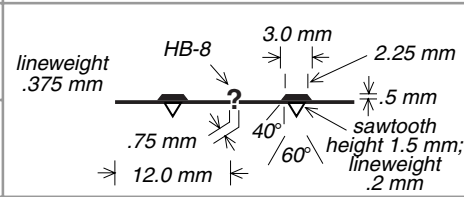 } & \\
\hline 2.9 .10 & $\begin{array}{l}\text { Overturned thrust fault (2nd option)—Identity or exis- } \\
\text { tence questionable, location accurate. Bars on tectoni- } \\
\text { cally higher plate (footwall); sawteeth in direction of dip }\end{array}$ & & & \\
\hline 2.9.11 & $\begin{array}{l}\text { Overturned thrust fault (2nd option)-Identity and exis- } \\
\text { tence certain, location approximate. Bars on tectoni- } \\
\text { cally higher plate (footwall); sawteeth in direction of dip }\end{array}$ & 一 & 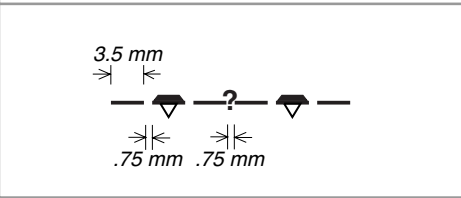 & \\
\hline 2.9.15 & $\begin{array}{l}\text { Overturned thrust fault (2nd option)_-Identity and exis- } \\
\text { tence certain, location concealed. Bars on tectonical- } \\
\text { ly higher plate (footwall); sawteeth in direction of dip }\end{array}$ & & \multirow{2}{*}{ 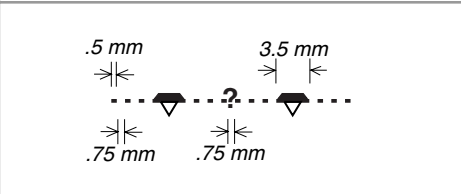 } & \\
\hline 2.9.16 & $\begin{array}{l}\text { Overturned thrust fault (2nd option)—Identity or exis- } \\
\text { tence questionable, location concealed. Bars on tecton- } \\
\text { ically higher plate (footwall); sawteeth in direction of dip }\end{array}$ & & & \\
\hline 2.9.17 & $\begin{array}{l}\text { Overturned thrust fault (3rd option)-Identity and exis- } \\
\text { tence certain, location accurate. Bars on tectonically } \\
\text { higher plate (footwall); sawteeth in direction of dip }\end{array}$ & & \multirow{2}{*}{ 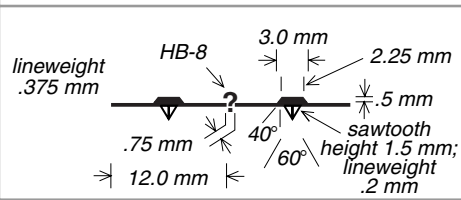 } & \\
\hline 2.9.18 & $\begin{array}{l}\text { Overturned thrust fault (3rd option)-Identity or exis- } \\
\text { tence questionable, location accurate. Bars on tectoni- } \\
\text { cally higher plate (footwall); sawteeth in direction of dip }\end{array}$ & & & \\
\hline 2.9.19 & $\begin{array}{l}\text { Overturned thrust fault (3rd option)—Identity and exis- } \\
\text { tence certain, location approximate. Bars on tectoni- } \\
\text { cally higher plate (footwall); sawteeth in direction of dip }\end{array}$ & $-\nabla-$ & \multirow{2}{*}{ 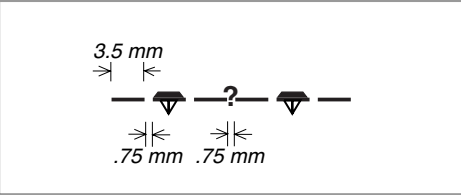 } & \\
\hline 2.9.20 & $\begin{array}{l}\text { Overturned thrust fault (3rd option)—Identity or existence } \\
\text { questionable, location approximate. Bars on tectonically } \\
\text { higher plate (footwall); sawteeth in direction of dip }\end{array}$ & $-\nabla-?-\nabla-$ & & \\
\hline 2.9.21 & $\begin{array}{l}\text { Overturned thrust fault (3rd option)-Identity and ex- } \\
\text { istence certain, location inferred. Bars on tectonical- } \\
\text { ly higher plate (footwall); sawteeth in direction of dip }\end{array}$ & $--\nabla----\nabla--$ & \multirow{2}{*}{ 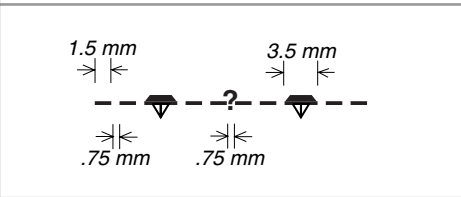 } & \\
\hline 2.9.22 & $\begin{array}{l}\text { Overturned thrust fault (3rd option)-Identity or exis- } \\
\text { tence questionable, location inferred. Bars on tectoni- } \\
\text { cally higher plate (footwall); sawteeth in direction of dip }\end{array}$ & $--\nabla--?--\nabla--$ & & \\
\hline 2.9.23 & $\begin{array}{l}\text { Overturned thrust fault (3rd option)_-Identity and exis- } \\
\text { tence certain, location concealed. Bars on tectonical- } \\
\text { ly higher plate (footwall); sawteeth in direction of dip }\end{array}$ & $\cdots$ & 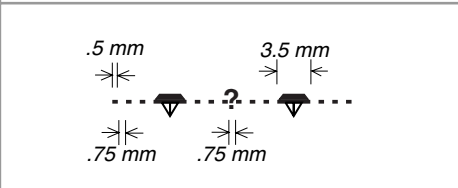 & \\
\hline
\end{tabular}




\section{2-FAULTS (continued)}

\begin{tabular}{|c|c|c|c|c|}
\hline REF NO & DESCRIPTION & SYMBOL & CARTOGRAPHIC SPECIFICATIONS* & NOTES ON USAGE* \\
\hline \multicolumn{5}{|c|}{ 2.10-Detachment faults (sense of slip unspecified) } \\
\hline 2.10.1 & $\begin{array}{l}\text { Detachment fault (sense of slip unspecified) (1st } \\
\text { option)-Identity and existence certain, location } \\
\text { accurate. Hachures on upper plate }\end{array}$ & 71 & lineweight .375 mm HB-8 & \multirow{6}{*}{$\begin{array}{l}\text { May be used to show } \\
\text { either normal (exten- } \\
\text { sional) or thrust (com- } \\
\text { pressional) offset. } \\
\text { Hachures indicate over- } \\
\text { all fault type (detach- } \\
\text { ment fault); they are not } \\
\text { placed at specific locali- } \\
\text { ties where observations } \\
\text { have been made. } \\
\text { In cross section, use } \\
\text { paired arrows to show } \\
\text { relative motion of } \\
\text { detachment faults (see } \\
\text { Section 2.11). } \\
\text { If desired, "2nd option" } \\
\text { and "3rd option" sym- } \\
\text { bols may be used to } \\
\text { show other types or } \\
\text { generations of detach- } \\
\text { ment faults. }\end{array}$} \\
\hline 2.10.2 & $\begin{array}{l}\text { Detachment fault (sense of slip unspecified) (1st } \\
\text { option)—Identity or existence questionable, loca- } \\
\text { tion accurate. Hachures on upper plate }\end{array}$ & 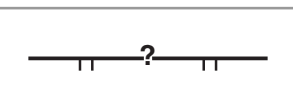 & 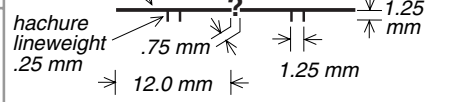 & \\
\hline 2.10 .3 & $\begin{array}{l}\text { Detachment fault (sense of slip unspecified) (1st } \\
\text { option)—Identity and existence certain, location } \\
\text { approximate. Hachures on upper plate }\end{array}$ & $-\pi--\pi-$ & \multirow{2}{*}{ 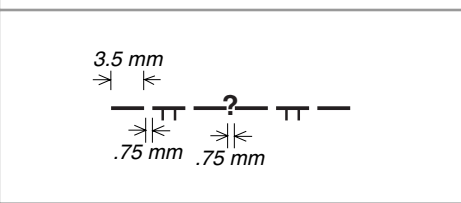 } & \\
\hline 2.10 .4 & $\begin{array}{l}\text { Detachment fault (sense of slip unspecified) (1st } \\
\text { option)—Identity or existence questionable, loca- } \\
\text { tion approximate. Hachures on upper plate }\end{array}$ & $-\pi-?-\pi$ & & \\
\hline 2.10 .5 & $\begin{array}{l}\text { Detachment fault (sense of slip unspecified) (1st } \\
\text { option)-Identity and existence certain, location } \\
\text { inferred. Hachures on upper plate }\end{array}$ & $--\pi----\pi--$ & 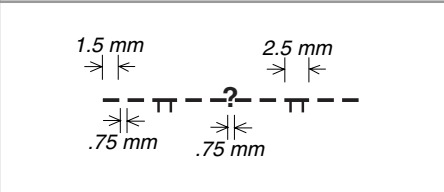 & \\
\hline 2.10 .8 & $\begin{array}{l}\text { Detachment fault (sense of slip unspecified) (1st } \\
\text { option)—Identity or existence questionable, loca- } \\
\text { tion concealed. Hachures on upper plate }\end{array}$ & $\cdots \pi \cdots ?+\cdots$ & 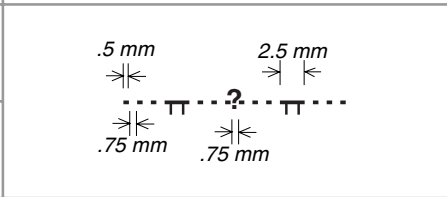 & \\
\hline 2.10 .9 & $\begin{array}{l}\text { Detachment fault (sense of slip unspecified) (2nd } \\
\text { option)—Identity and existence certain, location } \\
\text { accurate. Boxes on upper plate }\end{array}$ & प & \multirow{2}{*}{ 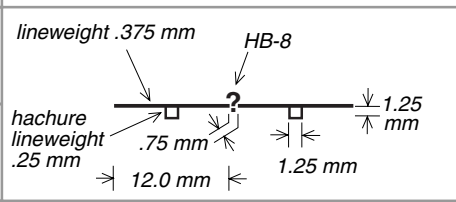 } & \multirow{12}{*}{$\begin{array}{l}\text { May be used to show } \\
\text { either normal (exten- } \\
\text { sional) or thrust (com- } \\
\text { pressional) offset. } \\
\text { Boxes indicate overall } \\
\text { fault type (detachment } \\
\text { fault); they are not } \\
\text { placed at specific locali- } \\
\text { ties where observations } \\
\text { have been made. } \\
\text { In cross section, use } \\
\text { paired arrows to show } \\
\text { relative motion of } \\
\text { detachment faults (see } \\
\text { Section 2.11). } \\
\text { If desired, "2nd option" } \\
\text { and "3rd option" sym- } \\
\text { bols may be used to } \\
\text { show other types or } \\
\text { generations of detach- } \\
\text { ment faults. }\end{array}$} \\
\hline 2.10.10 & $\begin{array}{l}\text { Detachment fault (sense of slip unspecified) (2nd } \\
\text { option) - Identity or existence questionable, loca- } \\
\text { tion accurate. Boxes on upper plate }\end{array}$ & $?$ & & \\
\hline 2.10.11 & $\begin{array}{l}\text { Detachment fault (sense of slip unspecified) (2nd } \\
\text { option)—Identity and existence certain, location } \\
\text { approximate. Boxes on upper plate }\end{array}$ & 一ேーーчー & 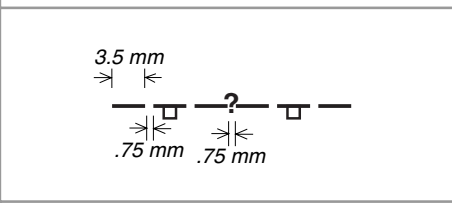 & \\
\hline 2.10 .15 & $\begin{array}{l}\text { Detachment fault (sense of slip unspecified) (2nd } \\
\text { option) - Identity and existence certain, location } \\
\text { concealed. Boxes on upper plate }\end{array}$ & $\cdots \square \cdot$ & \multirow{2}{*}{ 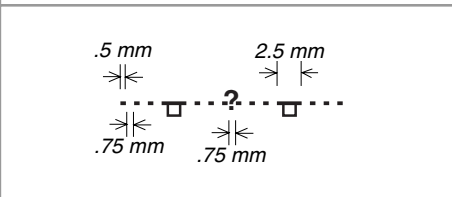 } & \\
\hline 2.10 .16 & $\begin{array}{l}\text { Detachment fault (sense of slip unspecified) (2nd } \\
\text { option)-Identity or existence questionable, loca- } \\
\text { tion concealed. Boxes on upper plate }\end{array}$ & 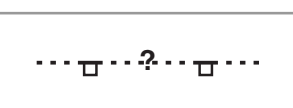 & & \\
\hline 2.10 .17 & $\begin{array}{l}\text { Detachment fault (sense of slip unspecified) (3rd } \\
\text { option) - Identity and existence certain, location } \\
\text { accurate. Boxes on upper plate }\end{array}$ & ए & \multirow{2}{*}{ 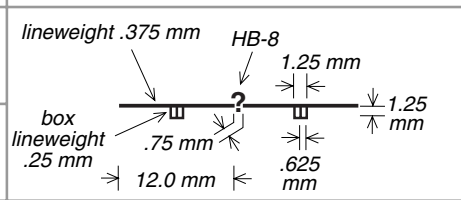 } & \\
\hline 2.10 .18 & $\begin{array}{l}\text { Detachment fault (sense of slip unspecified) (3rd } \\
\text { option)—Identity or existence questionable, loca- } \\
\text { tion accurate. Boxes on upper plate }\end{array}$ & ए? & & \\
\hline 2.10 .19 & $\begin{array}{l}\text { Detachment fault (sense of slip unspecified) (3rd } \\
\text { option)-Identity and existence certain, location } \\
\text { approximate. Boxes on upper plate }\end{array}$ & 一шーーшー & \multirow{2}{*}{ 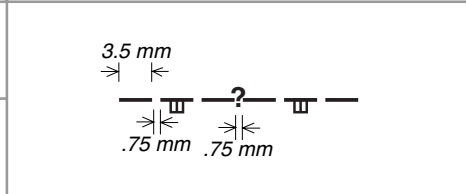 } & \\
\hline 2.10 .20 & $\begin{array}{l}\text { Detachment fault (sense of slip unspecified) (3rd } \\
\text { option)—Identity or existence questionable, loca- } \\
\text { tion approximate. Boxes on upper plate }\end{array}$ & 一ш-?-ш- & & \\
\hline 2.10 .21 & $\begin{array}{l}\text { Detachment fault (sense of slip unspecified) (3rd } \\
\text { option)—Identity and existence certain, location } \\
\text { inferred. Boxes on upper plate }\end{array}$ & -ーயー-ーーயーー & \multirow{2}{*}{ 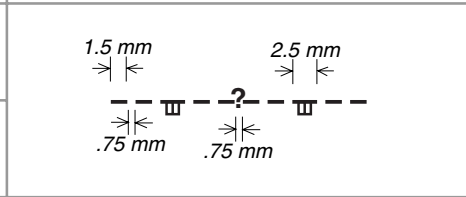 } & \\
\hline 2.10 .22 & $\begin{array}{l}\text { Detachment fault (sense of slip unspecified) (3rd } \\
\text { option)-Identity or existence questionable, loca- } \\
\text { tion inferred. Boxes on upper plate }\end{array}$ & $--\square--?--\square--$ & & \\
\hline 2.10 .23 & $\begin{array}{l}\text { Detachment fault (sense of slip unspecified) (3rd } \\
\text { option) - Identity and existence certain, location } \\
\text { concealed. Boxes on upper plate }\end{array}$ & $\cdots$ т $\cdots \cdots$ & 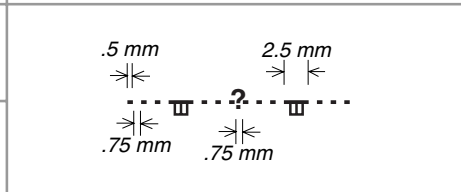 & \\
\hline
\end{tabular}




\section{2-FAULTS (continued)}

\begin{tabular}{|c|c|c|c|c|}
\hline REF NO & DESCRIPTION & SYMBOL & CARTOGRAPHIC SPECIFICATIONS* & NOTES ON USAGE* \\
\hline \multicolumn{5}{|c|}{ 2.10-Detachment faults (sense of slip unspecified) (continued) } \\
\hline 2.10 .25 & $\begin{array}{l}\text { Master detachment fault (sense of slip unspecified) } \\
\text { - Identity and existence certain, location accurate. } \\
\text { Hachures on upper plate }\end{array}$ & TIII & 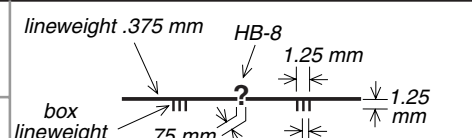 & \multirow{6}{*}{$\begin{array}{l}\text { May be used to show } \\
\text { either normal (exten- } \\
\text { sional) or thrust (com- } \\
\text { pressional) offset. } \\
\text { Hachures indicate over- } \\
\text { all fault type (master } \\
\text { detachment fault); they } \\
\text { are not placed at spec } \\
\text { ific localities where } \\
\text { observations have been } \\
\text { made. } \\
\text { In cross section, use } \\
\text { paired arrows to show } \\
\text { relative motion of mas- } \\
\text { ter detachment faults } \\
\text { (see Section 2.11). }\end{array}$} \\
\hline 2.10 .26 & $\begin{array}{l}\text { Master detachment fault (sense of slip unspecified) } \\
\text { - Identity or existence questionable, location } \\
\text { accurate. Hachures on upper plate }\end{array}$ & III ? - & 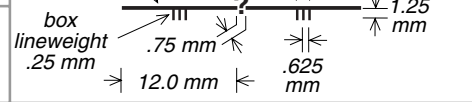 & \\
\hline 2.10 .27 & $\begin{array}{l}\text { Master detachment fault (sense of slip unspecified) } \\
\text { - Identity and existence certain, location approxi- } \\
\text { mate. Hachures on upper plate }\end{array}$ & - $\pi \frac{\pi}{-\pi}-$ & \multirow{2}{*}{ 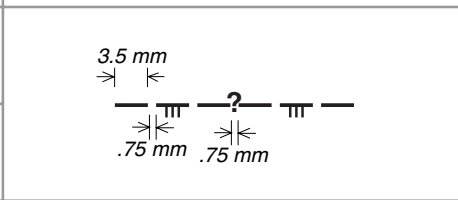 } & \\
\hline 2.10 .28 & $\begin{array}{l}\text { Master detachment fault (sense of slip unspecified) } \\
\text { - Identity or existence questionable, location } \\
\text { approximate. Hachures on upper plate }\end{array}$ & 一 $\pi$ ? & & \\
\hline 2.10 .29 & $\begin{array}{l}\text { Master detachment fault (sense of slip unspecified) } \\
\text { - Identity and existence certain, location inferred. } \\
\text { Hachures on upper plate }\end{array}$ & $--\pi+---m--$ & 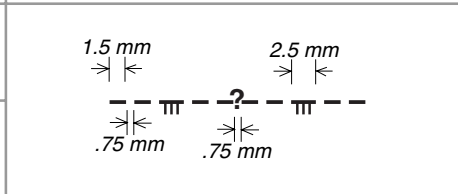 & \\
\hline 2.10 .32 & $\begin{array}{l}\text { Master detachment fault (sense of slip unspecified) } \\
\text { - Identity or existence questionable, location con- } \\
\text { cealed. Hachures on upper plate }\end{array}$ & $\cdots \pi+\cdots$ & 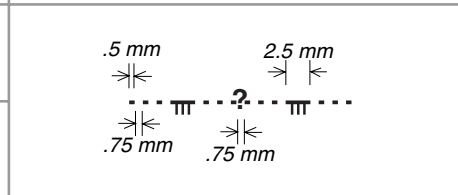 & \\
\hline 2.10 .33 & $\begin{array}{l}\text { Listric fault at head of detachment fault (sense of } \\
\text { slip unspecified)-Identity and existence certain, } \\
\text { location accurate. Ticks on upper plate }\end{array}$ & & \multirow{2}{*}{$\begin{array}{l}\text { lineweight } .375 \mathrm{~mm} \\
\text { tick } \\
\text { lineweight }\end{array}$} & \multirow{5}{*}{$\begin{array}{l}\text { May be used to show } \\
\text { either normal (exten- } \\
\text { sional) or thrust (com- } \\
\text { pressional) offset. } \\
\text { Ticks indicate overall } \\
\text { fault type (listric fault at } \\
\text { head of detachment } \\
\text { fault); they are not } \\
\text { placed at specific locali- } \\
\text { ties where observations } \\
\text { have been made. } \\
\text { In cross section, use } \\
\text { paired arrows to show } \\
\text { relative motion of listric } \\
\text { faults at head of detach- } \\
\text { ment faults (see Section } \\
\text { 2.11). }\end{array}$} \\
\hline 2.10 .34 & $\begin{array}{l}\text { Listric fault at head of detachment fault (sense of } \\
\text { slip unspecified)-Identity or existence question- } \\
\text { able, location accurate. Ticks on upper plate }\end{array}$ & - ? & & \\
\hline 2.10 .35 & $\begin{array}{l}\text { Listric fault at head of detachment fault (sense of } \\
\text { slip unspecified)-Identity and existence certain, } \\
\text { location approximate. Ticks on upper plate }\end{array}$ & - & 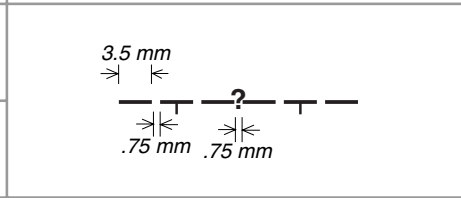 & \\
\hline 2.10 .39 & $\begin{array}{l}\text { Listric fault at head of detachment fault (sense of } \\
\text { slip unspecified)—Identity and existence certain, } \\
\text { location concealed. Ticks on upper plate }\end{array}$ & & \multirow{2}{*}{ 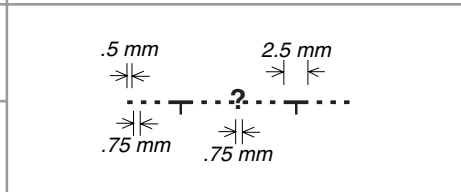 } & \\
\hline 2.10 .40 & $\begin{array}{l}\text { Listric fault at head of detachment fault (sense of } \\
\text { slip unspecified)-Identity or existence question- } \\
\text { able, location concealed. Ticks on upper plate }\end{array}$ & " & & \\
\hline
\end{tabular}


2-FAULTS (continued)

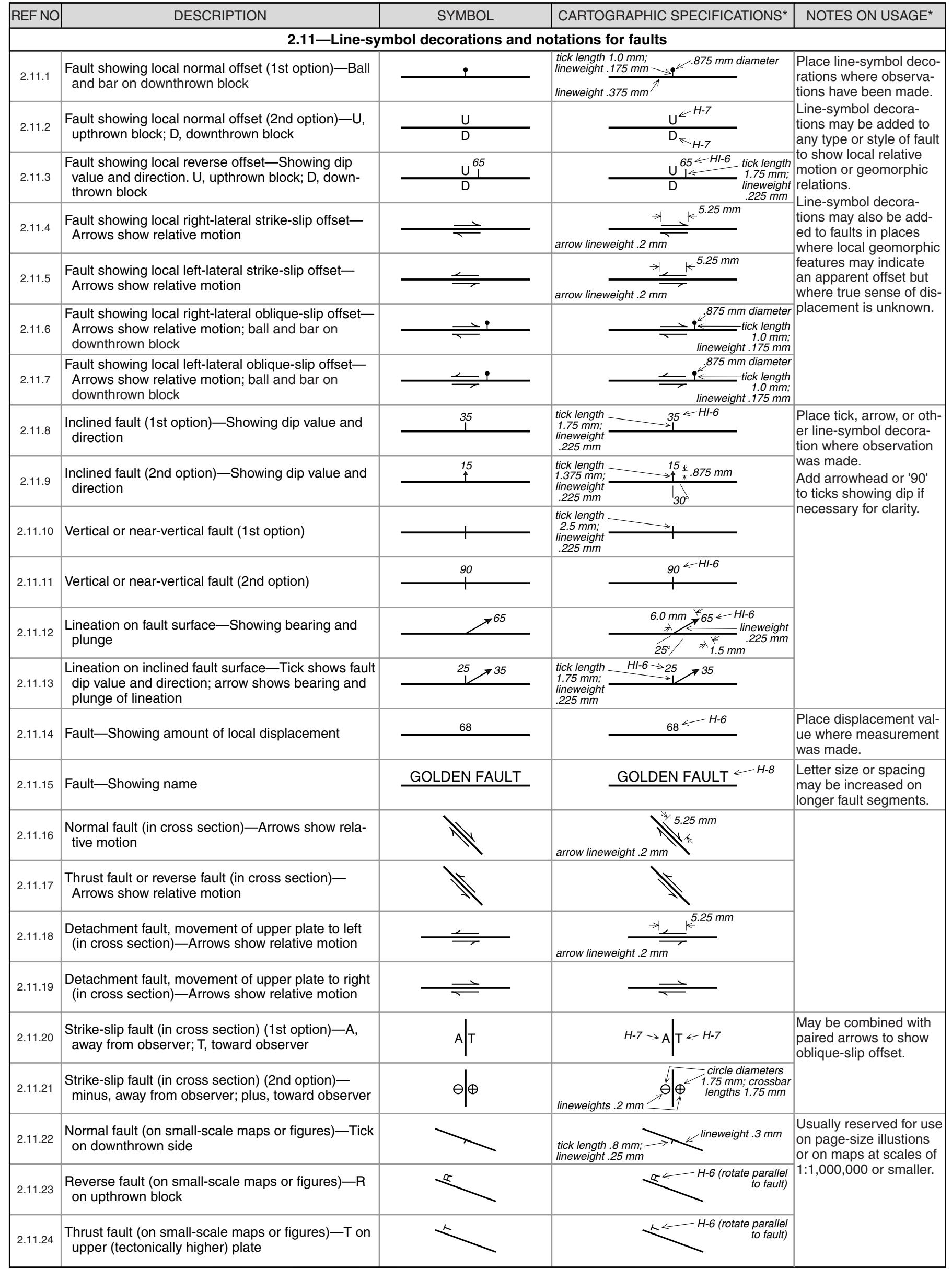




\section{2-FAULTS (continued)}

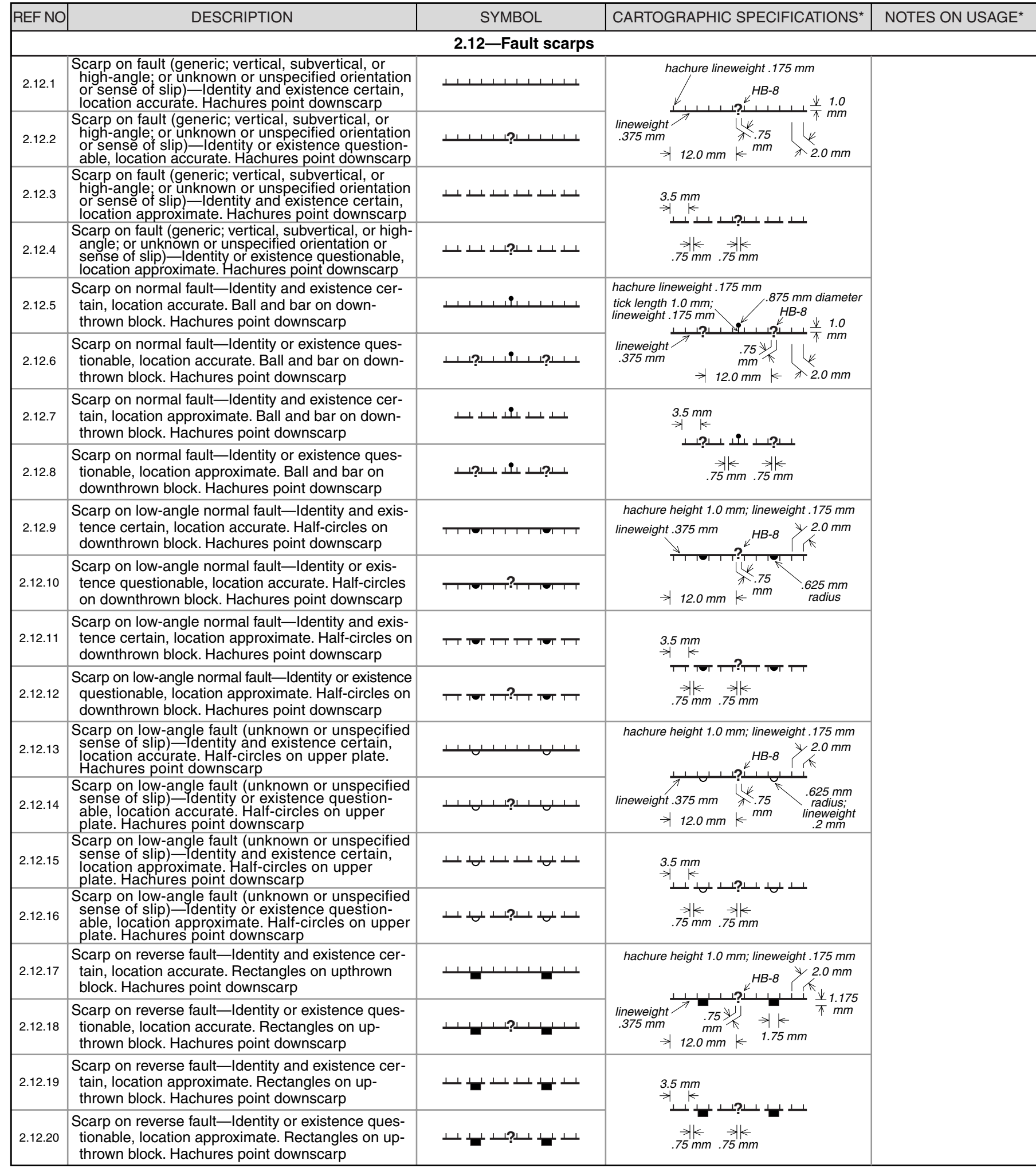




\section{2-FAULTS (continued)}

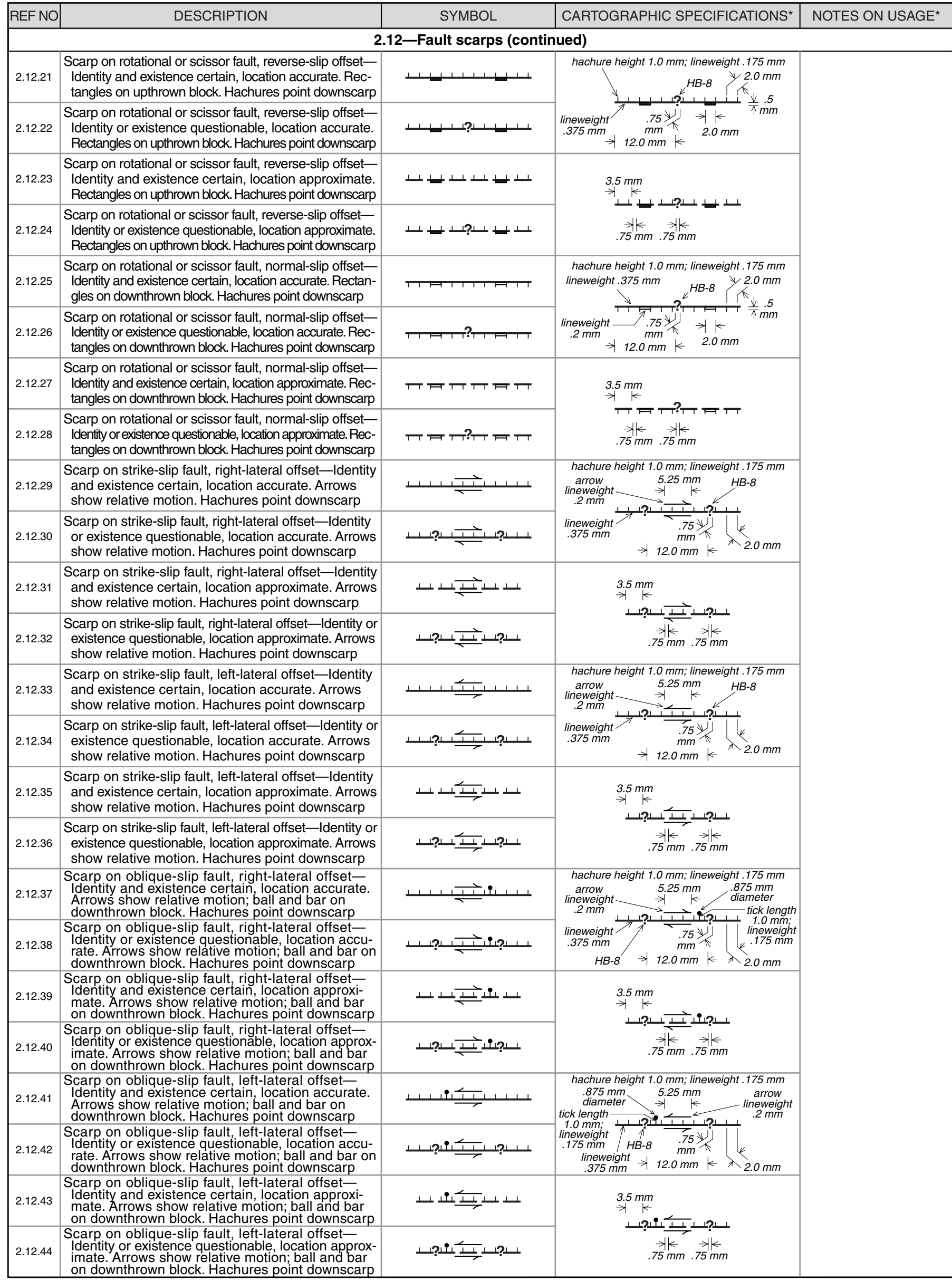




\section{2-FAULTS (continued)}

\begin{tabular}{|c|c|c|c|c|}
\hline REF NO & DESCRIPTION & SYMBOL & CARTOGRAPHIC SPECIFICATIONS* & NOTES ON USAGE* \\
\hline \multicolumn{5}{|c|}{ 2.12-Fault scarps (continued) } \\
\hline 2.12 .45 & $\begin{array}{l}\text { Scarp on thrust fault (1st option)-Identity and exis- } \\
\text { tence certain, location accurate. Sawteeth on upper } \\
\text { (tectonically higher) plate. Hachures point downscarp }\end{array}$ & Lس & hachure lineweight $175 \mathrm{~mm}$; height $1.0 \mathrm{~mm}$ & \\
\hline 2.12 .46 & $\begin{array}{l}\text { Scarp on thrust fault (1st option)-Identity or existence } \\
\text { questionable, location accurate. Sawteeth on upper } \\
\text { (tectonically higher) plate. Hachures point downscarp }\end{array}$ & ـ & 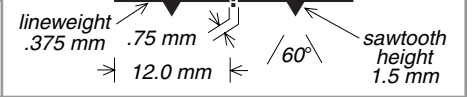 & \\
\hline 2.12 .48 & $\begin{array}{l}\text { Scarp on thrust fault (1st option)-Identity or existence } \\
\text { questionable, location approximate. Sawteeth on upper } \\
\text { (tectonically higher) plate. Hachures point downscarp }\end{array}$ & $+\perp-\perp \perp$ & 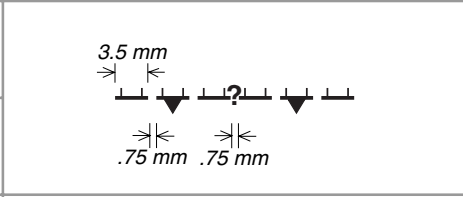 & \\
\hline 2.12 .49 & $\begin{array}{l}\text { Scarp on thrust fault (2nd option)-Identity and exis- } \\
\text { tence certain, location accurate. Sawteeth on upper } \\
\text { (tectonically higher) plate. Hachures point downscarp }\end{array}$ & 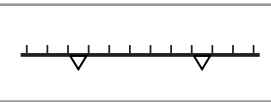 & 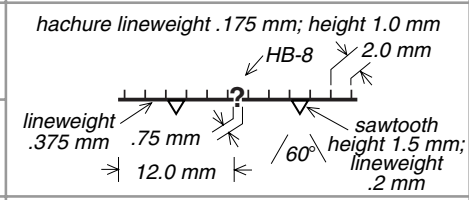 & \\
\hline 2.12 .52 & $\begin{array}{l}\text { Scarp on thrust fault (2nd option)-Identity or existence } \\
\text { questionable, location approximate. Sawteeth on upper } \\
\text { (tectonically higher) plate. Hachures point downscarp }\end{array}$ & $\perp \forall-\nabla^{\perp} \nabla^{\perp}$ & 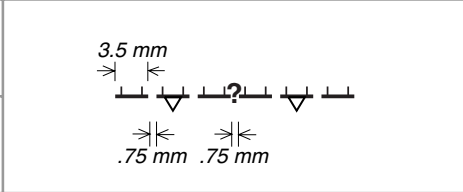 & \\
\hline 2.12 .53 & $\begin{array}{l}\text { Scarp on thrust fault (3rd option)-Identity and exis- } \\
\text { tence certain, location accurate. Sawteeth on upper } \\
\text { (tectonically higher) plate. Hachures point downscarp }\end{array}$ & 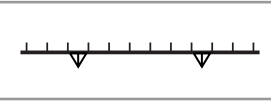 & \multirow{2}{*}{ 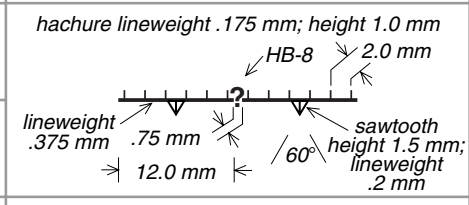 } & \\
\hline 2.12 .54 & $\begin{array}{l}\text { Scarp on thrust fault (3rd option)-Identity or existence } \\
\text { questionable, location accurate. Sawteeth on upper } \\
\text { (tectonically higher) plate. Hachures point downscarp }\end{array}$ & 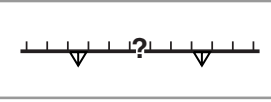 & & \\
\hline 2.12 .55 & $\begin{array}{l}\text { Scarp on thrust fault (3rd option)—Identity and existence } \\
\text { certain, location approximate. Sawteeth on upper } \\
\text { (tectonically higher) plate. Hachures point downscarp }\end{array}$ & $\perp \nabla \perp \perp \nabla \perp$ & 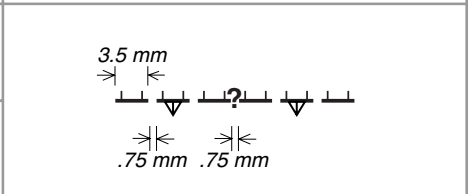 & \\
\hline 2.12 .59 & $\begin{array}{l}\text { Scarp on overturned thrust fault (1st option)- } \\
\text { ldentity and existence certain, location approximate. } \\
\text { Bars on tectonically higher plate (footwall); saw- } \\
\text { teeth in direction of dip. Hachures point downscarp }\end{array}$ & & \multirow{2}{*}{ 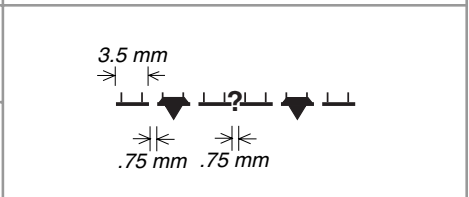 } & \\
\hline 2.12 .60 & $\begin{array}{l}\text { Scarp on overturned thrust fault (1st option)- } \\
\text { ldentity or existence questionable, location approxi- } \\
\text { mate. Bars on tectonically higher plate (footwall); saw- } \\
\text { teeth in direction of dip. Hachures point downscarp }\end{array}$ & $\perp$ & & \\
\hline 2.12 .61 & $\begin{array}{l}\text { Scarp on overturned thrust fault (2nd option)- } \\
\text { ldentity and existence certain, location accurate. } \\
\text { Bars on tectonically higher plate (footwall); saw- } \\
\text { teeth in direction of dip. Hachures point downscarp }\end{array}$ & & \multirow{2}{*}{$\begin{array}{c}\text { hachure lineweight } .175 \mathrm{~mm} \text {; height } 1.0 \mathrm{~mm} \\
2.0 \mathrm{~mm}\end{array}$} & \\
\hline 2.12 .62 & $\begin{array}{l}\text { Scarp on overturned thrust fault (2nd option)- } \\
\text { Identity or existence questionable, location accurate. } \\
\text { Bars on tectonically, higher plate (footwall); sawteeth } \\
\text { in direction of dip. Hachures point downscarp }\end{array}$ & 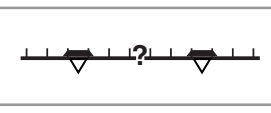 & & \\
\hline 2.12 .63 & $\begin{array}{l}\text { Scarp on overturned thrust fault (2nd option)- } \\
\text { ldentity and existence certain, location approximate. } \\
\text { Bars on teetonically higher plate (footwall); saw- } \\
\text { teeth in direction of dip. Hachures point downscarp }\end{array}$ & $-\nabla \perp \perp \nabla \perp$ & \multirow{2}{*}{ 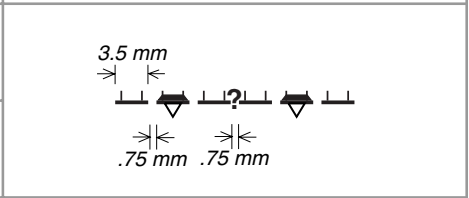 } & \\
\hline 2.12 .64 & $\begin{array}{l}\text { Scarp on overturned thrust fault (2nd option)- } \\
\text { Identity or existence questionable, location approxi- } \\
\text { mate. Bars on tectonically higher plate (footwall); saw- } \\
\text { teeth in direction of dip. Hachures point downscarp }\end{array}$ & $\perp \nabla \perp ?-\nabla \nu$ & & \\
\hline 2.12 .65 & $\begin{array}{l}\text { Scarp on overturned thrust fault (3rd option)- } \\
\text { ldentity and existence certain, location accurate. } \\
\text { Bars on tectonically higher plate (footwall); saw- } \\
\text { teeth in direction of dip. Hachures point downscarp }\end{array}$ & & \multirow{2}{*}{$\begin{array}{c}\text { hachure lineweight. } 175 \mathrm{~mm} \text {; height } 1.0 \mathrm{~mm} \\
2.0 \mathrm{~mm}\end{array}$} & \\
\hline 2.12 .66 & $\begin{array}{l}\text { Scarp on overturned thrust fault (3rd option)- } \\
\text { Identity or existence questionable, location accurate. } \\
\text { Bars on tectonically higher plate (footwall); sawteeth } \\
\text { in direction of dip. Hachures point downscarp }\end{array}$ & 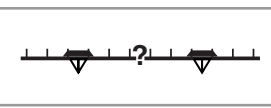 & & \\
\hline 2.12 .67 & $\begin{array}{l}\text { Scarp on overturned thrust fault (3rd option)- } \\
\text { ldentity and existence certain, location approximate. } \\
\text { Bars on tectonically higher plate (footwall); saw- } \\
\text { teeth in direction of dip. Hachures point downscarp }\end{array}$ & $\perp \nabla \perp-\nabla \perp$ & 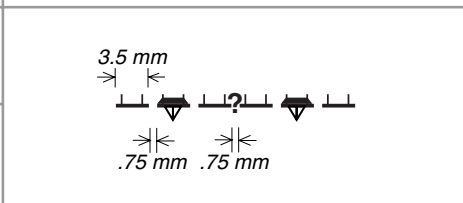 & \\
\hline
\end{tabular}




\section{2-FAULTS (continued)}

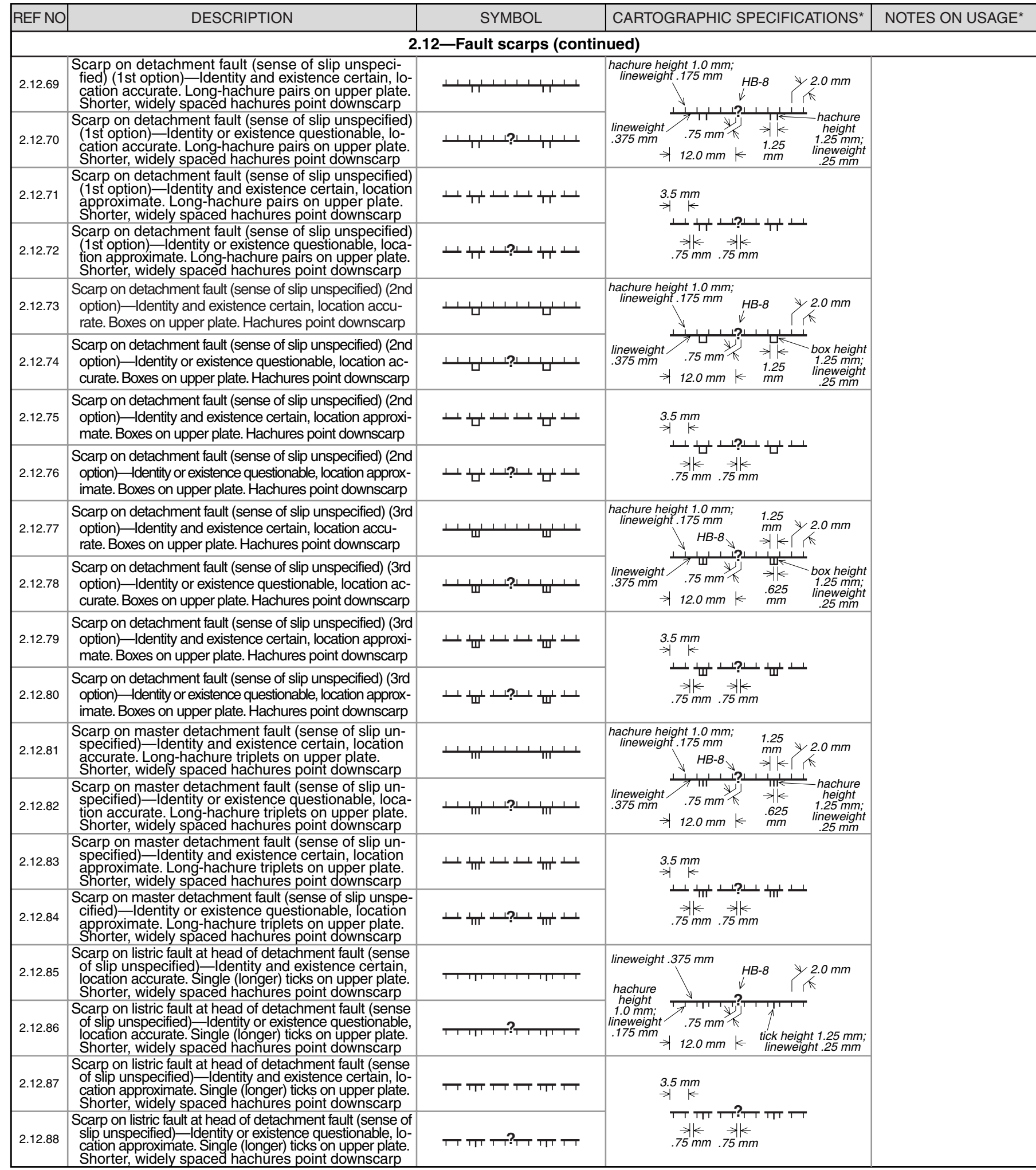

${ }^{*}$ For more information, see general guidelines on pages $A-i$ to $A-v$. 
Federal Geographic Data Committee (Doc. No. FGDC-STD-013-2006)

U.S. Geological Survey Techniques and Methods 11-A2 FGDC Digital Cartographic Standard for Geologic Map Symbolization (PostScript Implementation) DOWNLOAD this Illustrator EPS file: Al8 / $\underline{\mathrm{CS} 2}$

\begin{tabular}{|c|c|c|c|c|}
\hline \multicolumn{5}{|c|}{ 2-FAULTS (continued) } \\
\hline REF NO & DESCRIPTION & SYMBOL & CARTOGRAPHIC SPECIFICATIONS* & NOTES ON USAGE* \\
\hline \multicolumn{5}{|c|}{ 2.13-Quaternary faulting } \\
\hline 2.13 .1 & $\begin{array}{l}\text { Fault showing displacement during historic time } \\
\text { (includes areas of known fault creep) }\end{array}$ & & lineweight $1.25 \mathrm{~mm}$; color $100 \%$ red & \multirow{4}{*}{$\begin{array}{l}\text { Although only shown } \\
\text { here on "identity and } \\
\text { existence certain, loca- } \\
\text { tion accurate," generic } \\
\text { faults, color may be } \\
\text { added to any type or } \\
\text { style of fault to highlight } \\
\text { where geomorphic evi- } \\
\text { dence indicates dis- } \\
\text { placement during Qua- } \\
\text { ternary time. }\end{array}$} \\
\hline 2.13 .2 & Fault showing displacement during Holocene time & & lineweight $1.25 \mathrm{~mm}$; color $100 \%$ orange & \\
\hline 2.13 .3 & $\begin{array}{l}\text { Fault showing displacement during late Quaternary } \\
\text { time }\end{array}$ & & lineweight $1.25 \mathrm{~mm}$; color $100 \%$ green & \\
\hline 2.13 .4 & $\begin{array}{l}\text { Fault showing displacement during Quaternary time } \\
\text { (undifferentiated) }\end{array}$ & & lineweight $1.25 \mathrm{~mm}$; color $100 \%$ violet & \\
\hline \multicolumn{5}{|c|}{ 2.14-Shear zones; mylonite zones; fault-breccia zones } \\
\hline 2.14 .1 & $\begin{array}{l}\text { Ductile shear zone or mylonite zone-May or may } \\
\text { not be associated with mappable faults }\end{array}$ & & $\overbrace{\text { all lineweights } .2}^{\sim}$ & \multirow{4}{*}{$\begin{array}{l}\text { Orient S-shaped sym- } \\
\text { bols to indicate linear } \\
\text { trend of zone; spacing } \\
\text { may be varied to show } \\
\text { intensity of shear. } \\
\text { Width of zones may } \\
\text { vary. } \\
\text { Patterns may either } \\
\text { overprint other map } \\
\text { units or be used as } \\
\text { stand-alone map units } \\
\text { (if zones have well- } \\
\text { defined boundaries). }\end{array}$} \\
\hline 2.14 .2 & Zone of sheared rock within fault & & 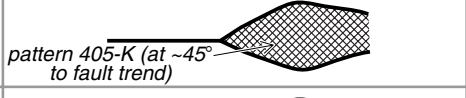 & \\
\hline 2.14 .3 & $\begin{array}{l}\text { Fault-breccia zone or zone of broken rock within } \\
\text { fault }\end{array}$ & & & \\
\hline 2.14 .4 & $\begin{array}{l}\text { Fault-breccia zone or zone of broken rock around } \\
\text { fault }\end{array}$ & 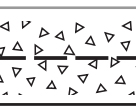 & 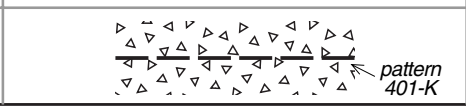 & \\
\hline \multicolumn{5}{|c|}{ 2.15-Small, minor faults } \\
\hline 2.15 .1 & Small, minor inclined fault-Showing strike and dip & 35 & 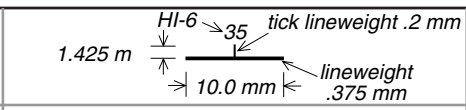 & \multirow{3}{*}{$\begin{array}{l}\text { Use to show small, } \\
\text { minor faults that are } \\
\text { observed in outcrop but } \\
\text { that cannot be traced } \\
\text { away from that outcrop. }\end{array}$} \\
\hline 2.15 .2 & $\begin{array}{l}\text { Small, minor vertical or near-vertical fault- } \\
\text { Showing strike }\end{array}$ & & $2.5 \mathrm{~mm} \frac{\Downarrow}{\uparrow}$ & \\
\hline 2.15 .3 & $\begin{array}{l}\text { Small, minor shear fault-Showing dip. Arrow } \\
\text { shows direction of relative horizontal displacement }\end{array}$ & $\stackrel{85}{\perp}$ & $\underset{\text { arrow lineweight } .2 \mathrm{~mm}}{\stackrel{85}{\longrightarrow}}$ & \\
\hline
\end{tabular}

${ }^{\star}$ For more information, see general guidelines on pages $A-i$ to $A-v$. 
Federal Geographic Data Committee (Doc. No. FGDC-STD-013-2006)

U.S. Geological Survey Techniques and Methods 11-A2

FGDC Digital Cartographic Standard for Geologic Map Symbolization (PostScript Implementation)

DOWNLOAD this Illustrator EPS file: Al8 / CS2

\section{3-BOUNDARIES LOCATED BY GEOPHYSICAL SURVEYS}

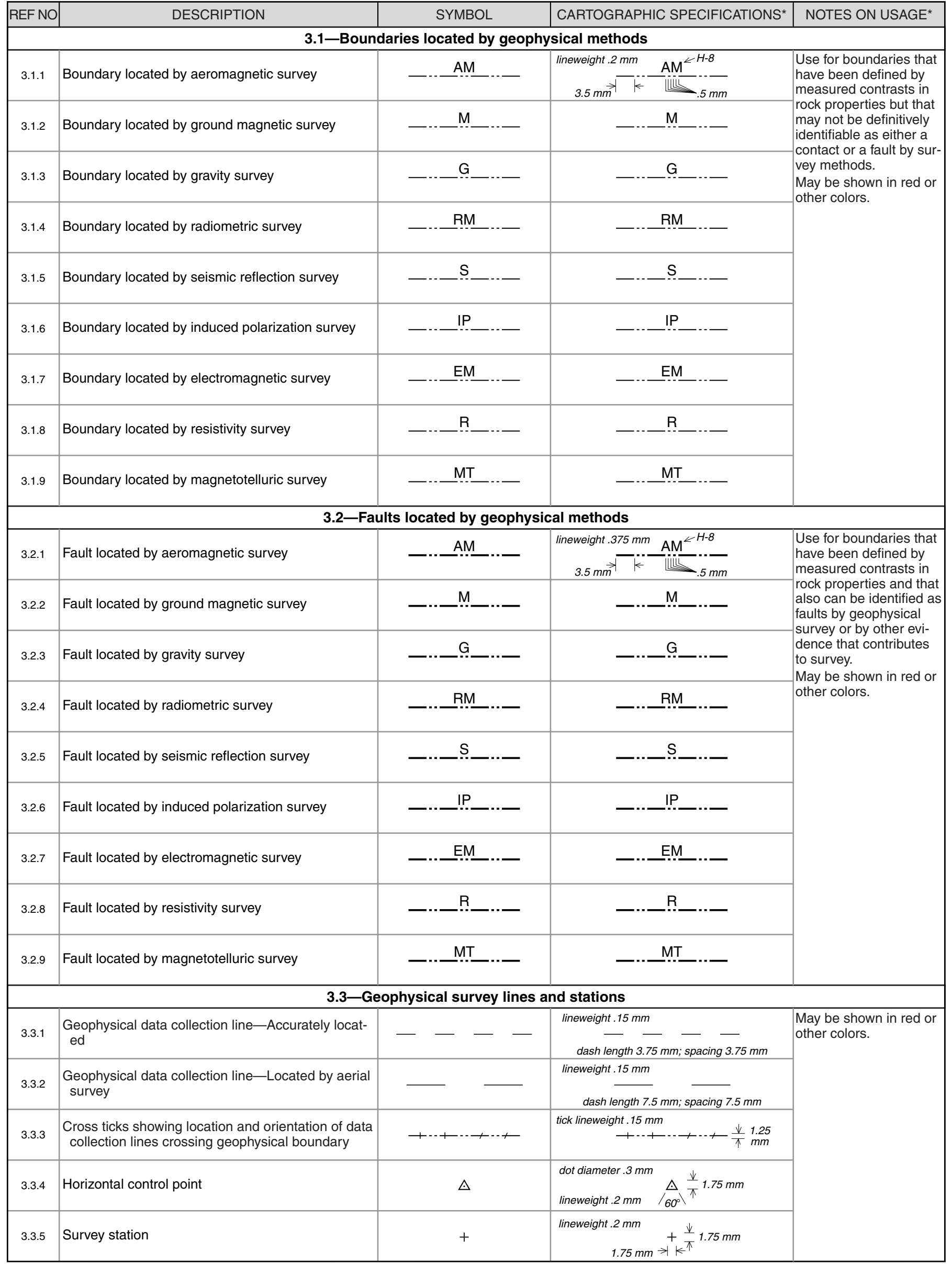


Federal Geographic Data Committee (Doc. No. FGDC-STD-013-2006)

U.S. Geological Survey Techniques and Methods 11-A2

FGDC Digital Cartographic Standard for Geologic Map Symbolization (PostScript Implementation)

DOWNLOAD this Illustrator EPS file: Al8 / $\underline{\mathrm{CS} 2}$

\section{4-LINEAMENTS AND JOINTS}

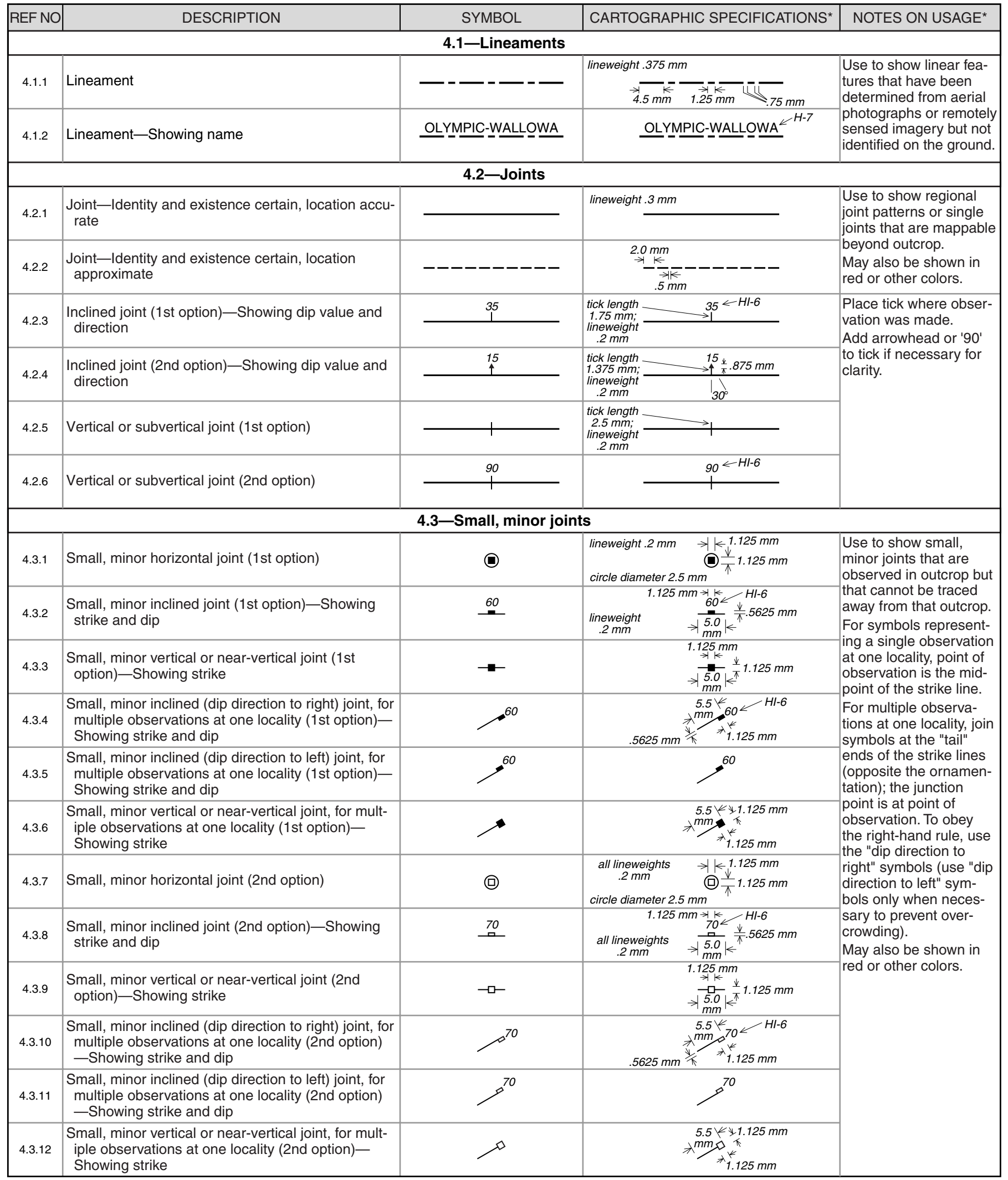

${ }^{*}$ For more information, see general guidelines on pages $A-i$ to $A-v$. 
Federal Geographic Data Committee (Doc. No. FGDC-STD-013-2006)

U.S. Geological Survey Techniques and Methods 11-A2 FGDC Digital Cartographic Standard for Geologic Map Symbolization (PostScript Implementation)

DOWNLOAD this Illustrator EPS file: Al8 / CS2

\section{5-FOLDS}

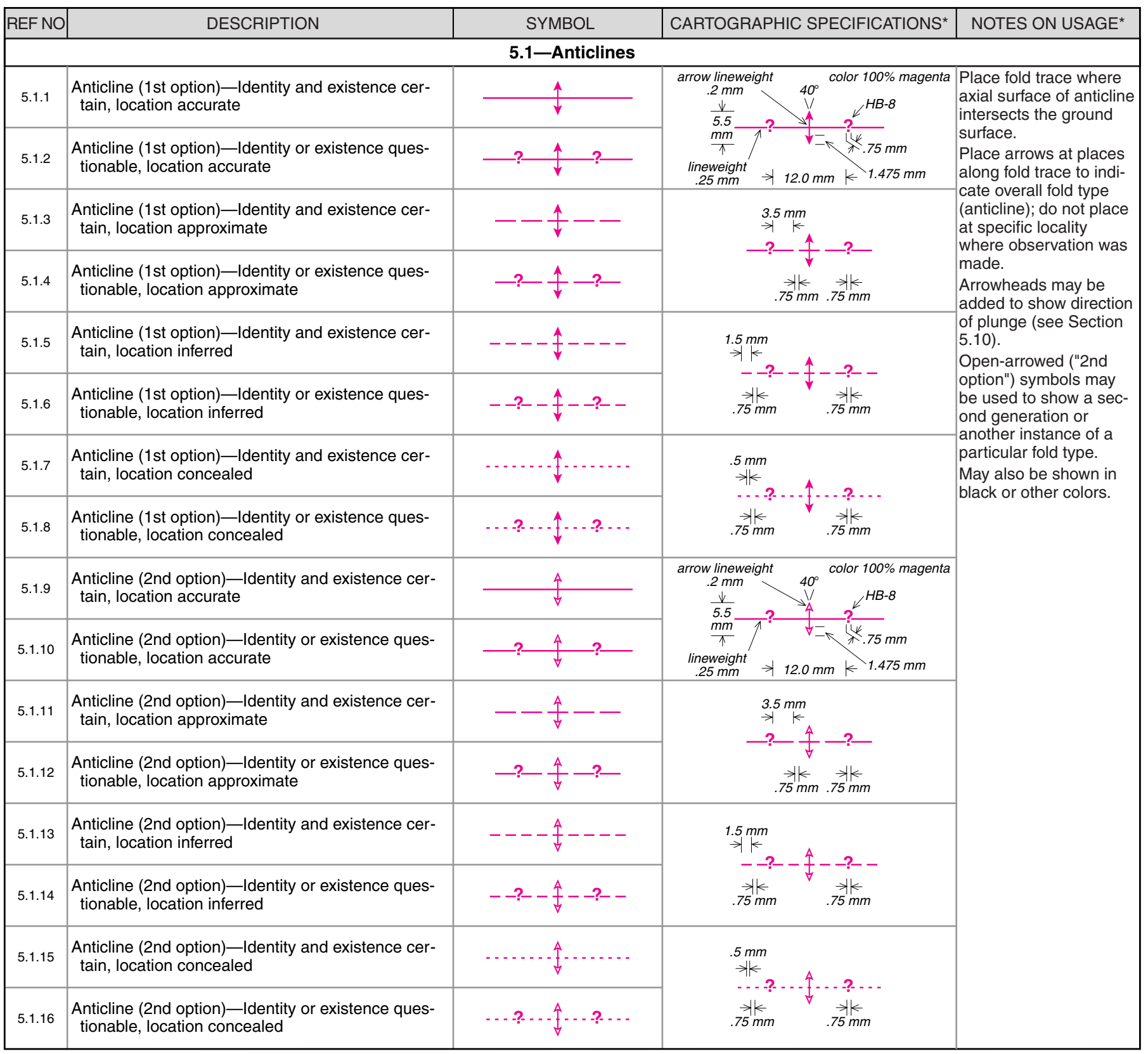

${ }^{*}$ For more information, see general guidelines on pages $A-i$ to $A-v$. 
Federal Geographic Data Committee (Doc. No. FGDC-STD-013-2006)

U.S. Geological Survey Techniques and Methods 11-A2 FGDC Digital Cartographic Standard for Geologic Map Symbolization (PostScript Implementation)

DOWNLOAD this Illustrator EPS file: Al8 / $\underline{\text { CS2 }}$

\section{5-FOLDS (continued)}

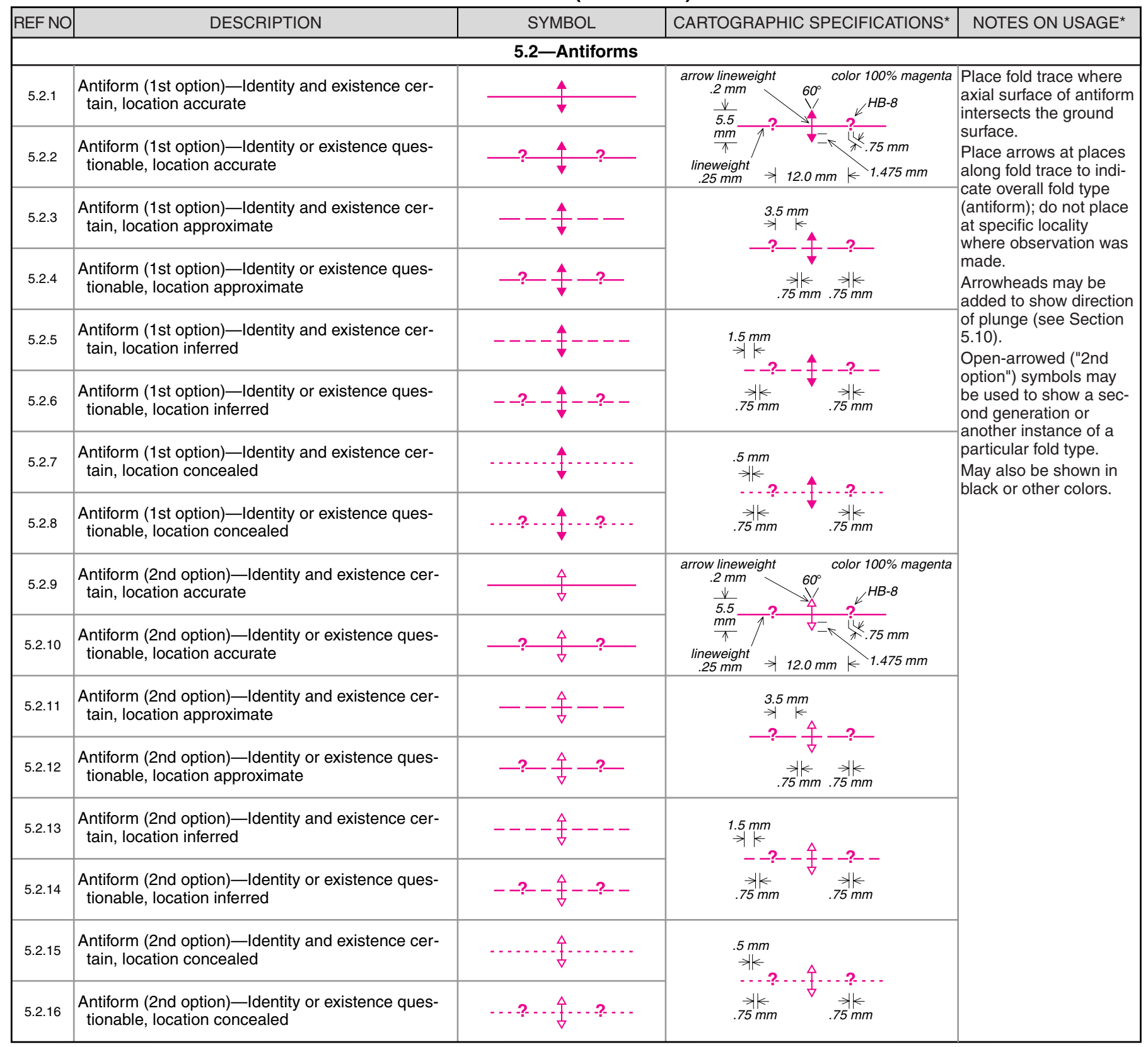

${ }^{*}$ For more information, see general guidelines on pages $A-i$ to $A-v$. 


\section{5-FOLDS (continued)}

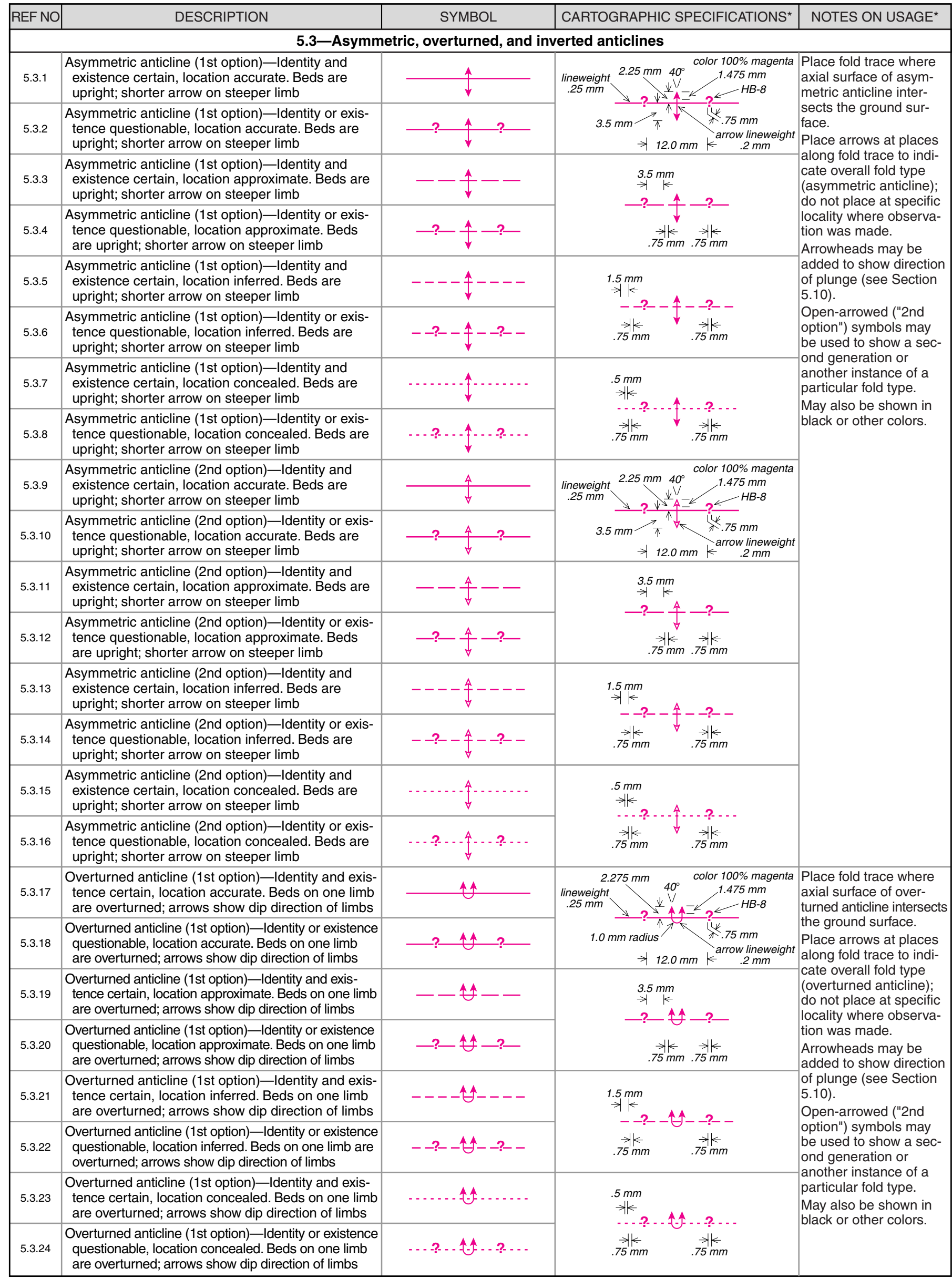




\section{5-FOLDS (continued)}

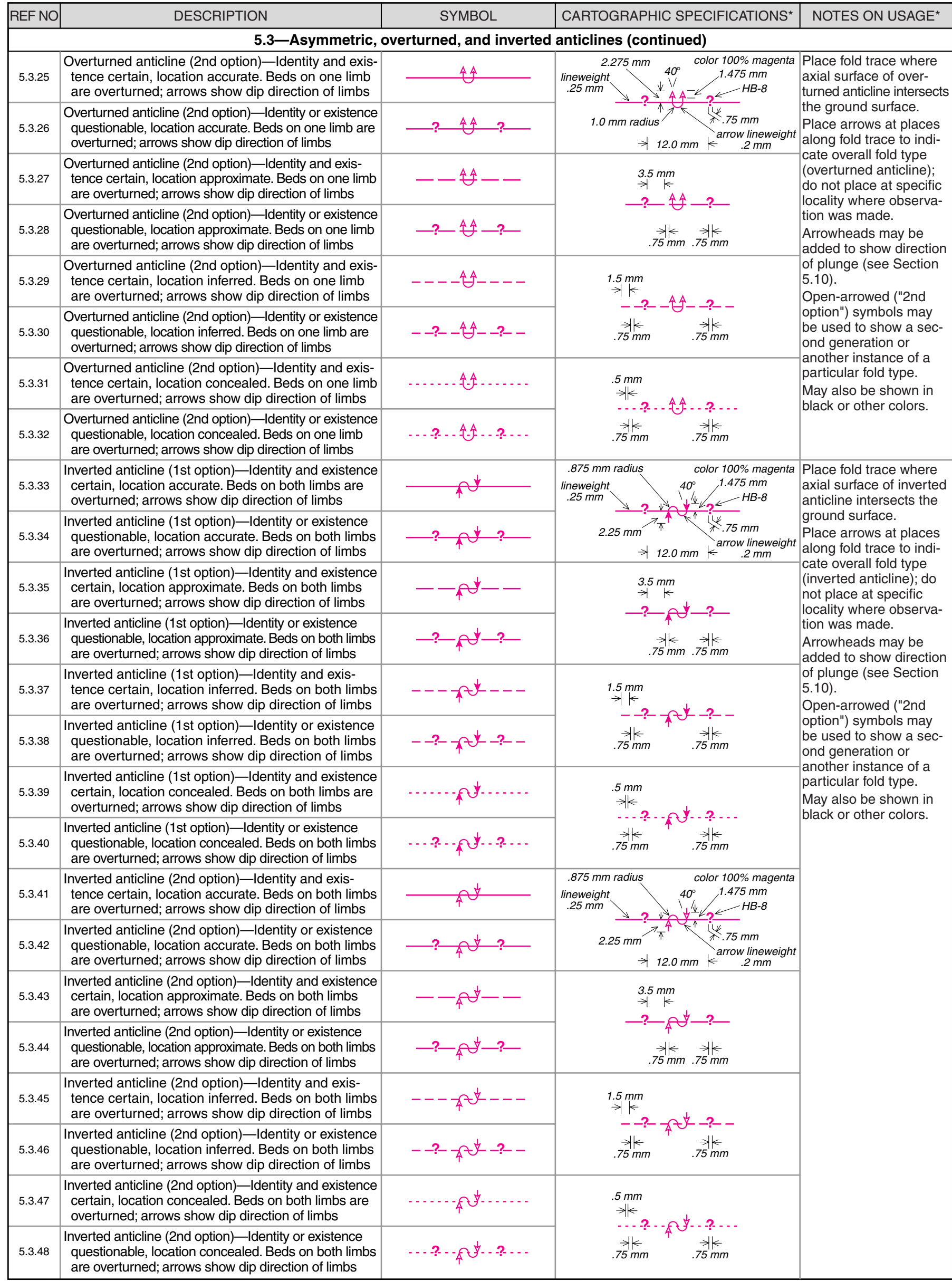


Federal Geographic Data Committee (Doc. No. FGDC-STD-013-2006)

U.S. Geological Survey Techniques and Methods 11-A2 FGDC Digital Cartographic Standard for Geologic Map Symbolization (PostScript Implementation)

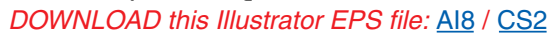

\section{5-FOLDS (continued)}

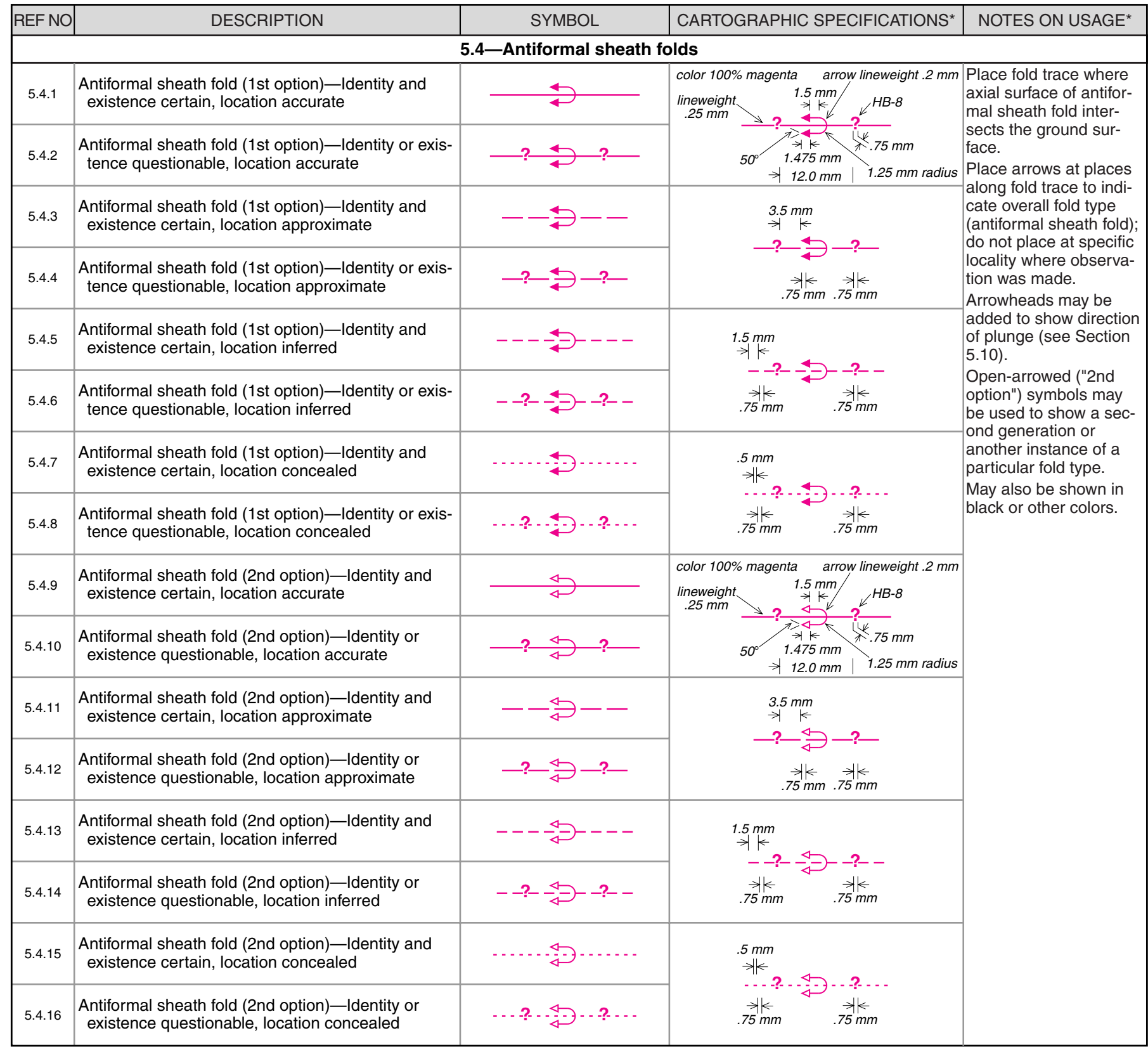

${ }^{*}$ For more information, see general guidelines on pages $A-i$ to $A-v$. 
Federal Geographic Data Committee (Doc. No. FGDC-STD-013-2006)

U.S. Geological Survey Techniques and Methods 11-A2 FGDC Digital Cartographic Standard for Geologic Map Symbolization (PostScript Implementation)

DOWNLOAD this Illustrator EPS file: Al8 / $\underline{\text { CS2 }}$

\section{5-FOLDS (continued)}

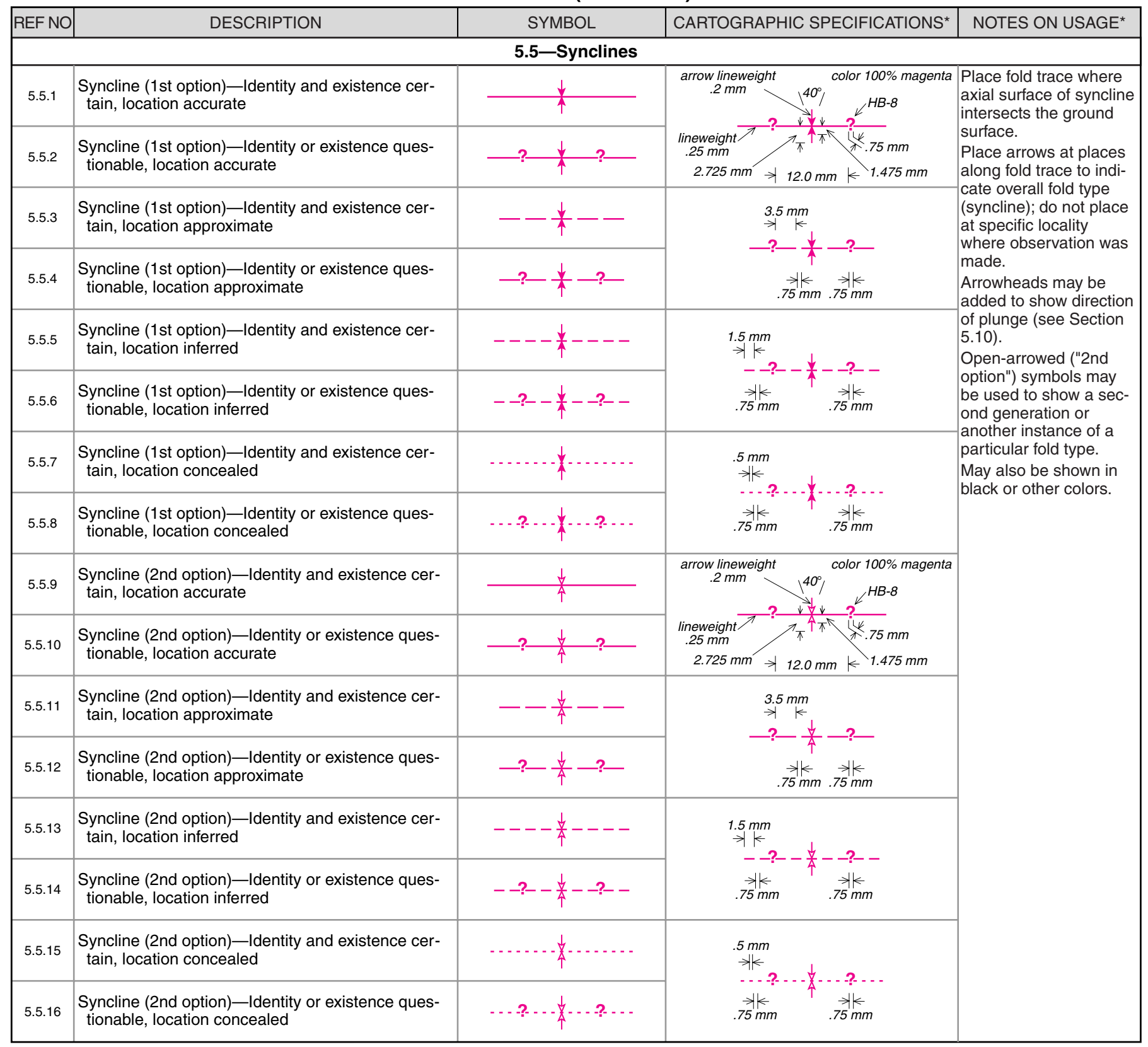

${ }^{*}$ For more information, see general guidelines on pages $A-i$ to $A-v$. 
Federal Geographic Data Committee (Doc. No. FGDC-STD-013-2006)

U.S. Geological Survey Techniques and Methods 11-A2 FGDC Digital Cartographic Standard for Geologic Map Symbolization (PostScript Implementation)

DOWNLOAD this Illustrator EPS file: Al8 / CS2

\section{5-FOLDS (continued)}

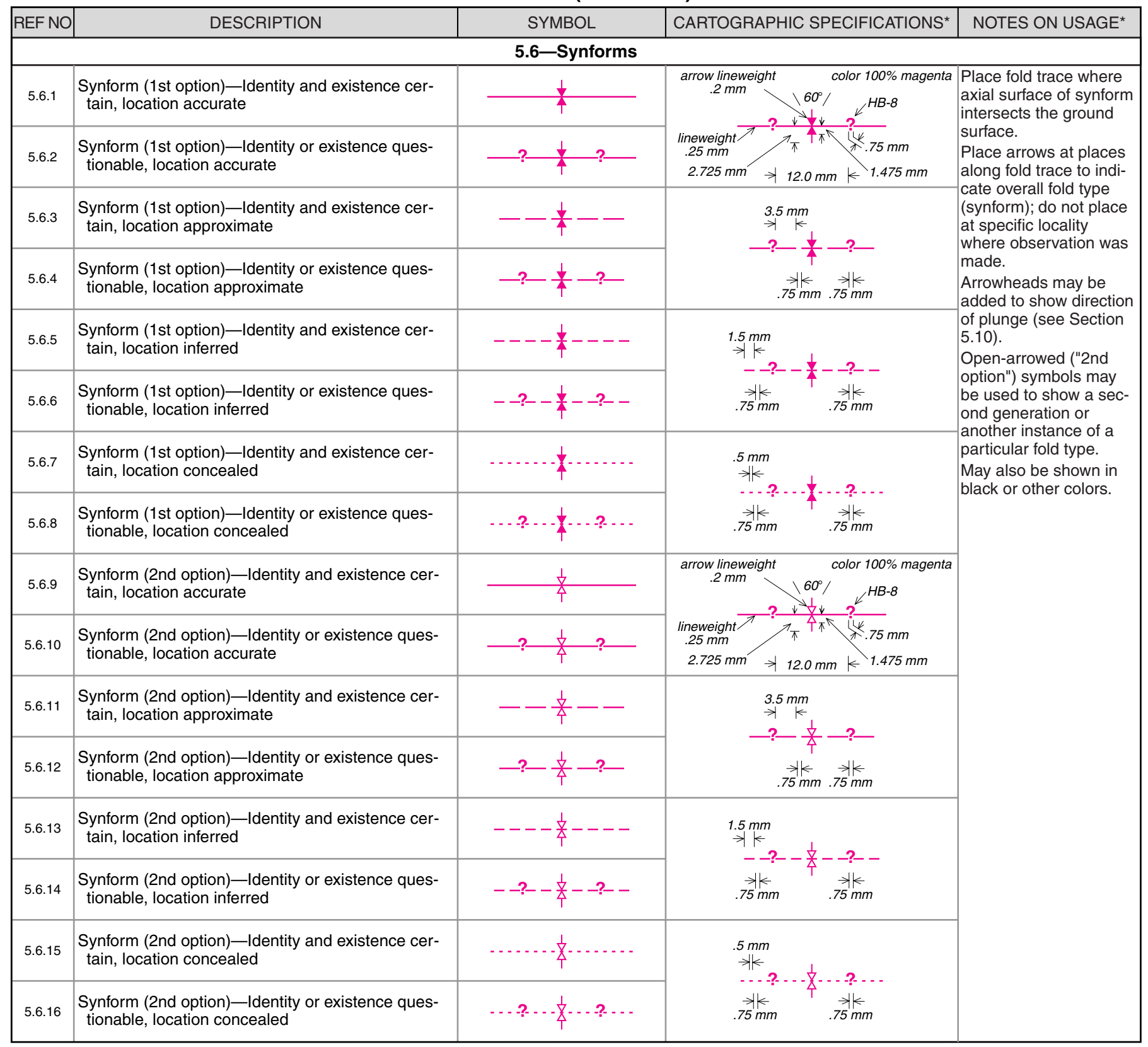

${ }^{*}$ For more information, see general guidelines on pages $A-i$ to $A-v$. 


\section{5-FOLDS (continued)}

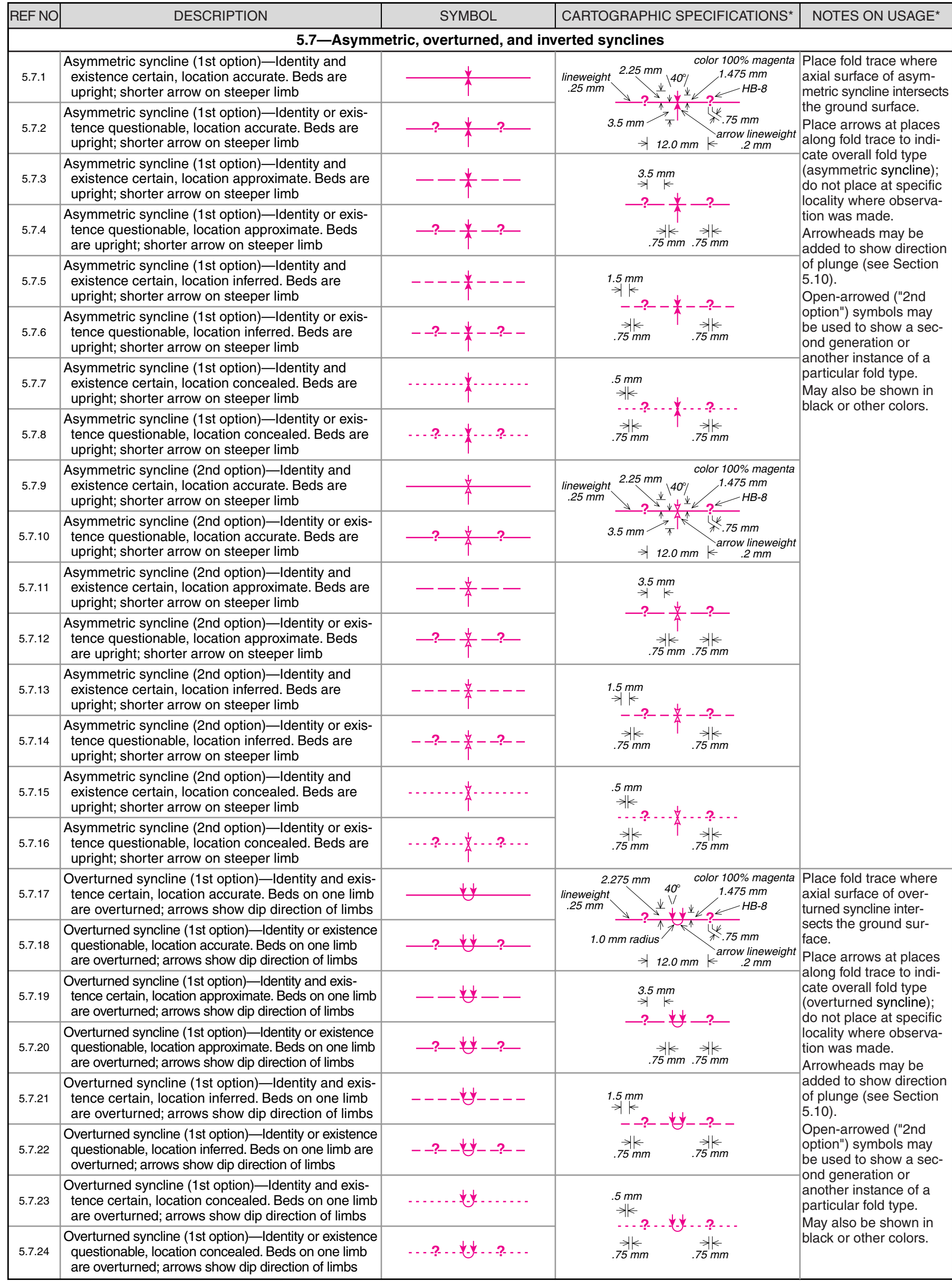




\section{5-FOLDS (continued)}

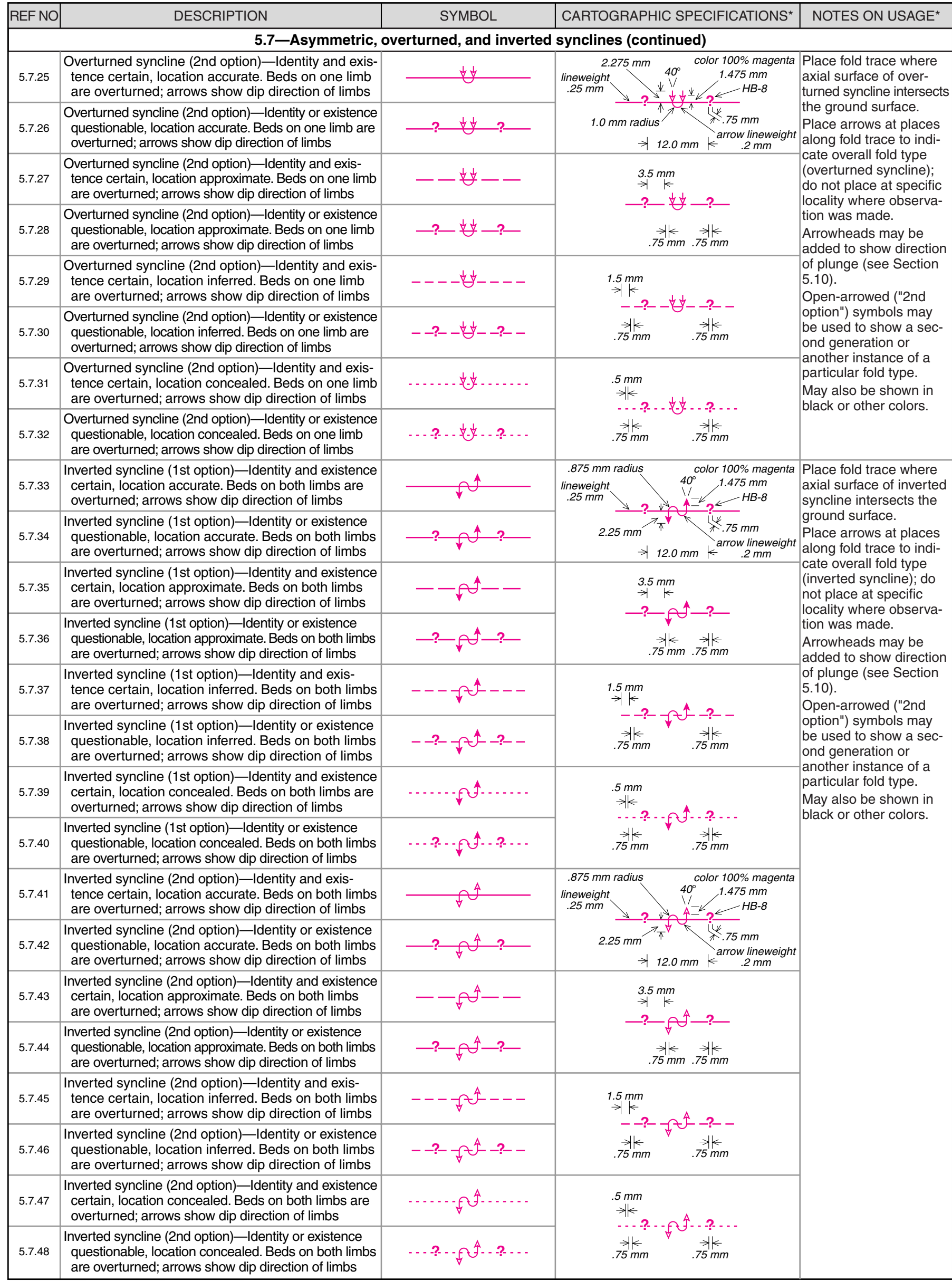


Federal Geographic Data Committee (Doc. No. FGDC-STD-013-2006)

U.S. Geological Survey Techniques and Methods 11-A2 FGDC Digital Cartographic Standard for Geologic Map Symbolization (PostScript Implementation)

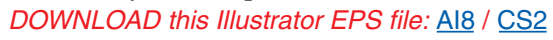

\section{5-FOLDS (continued)}

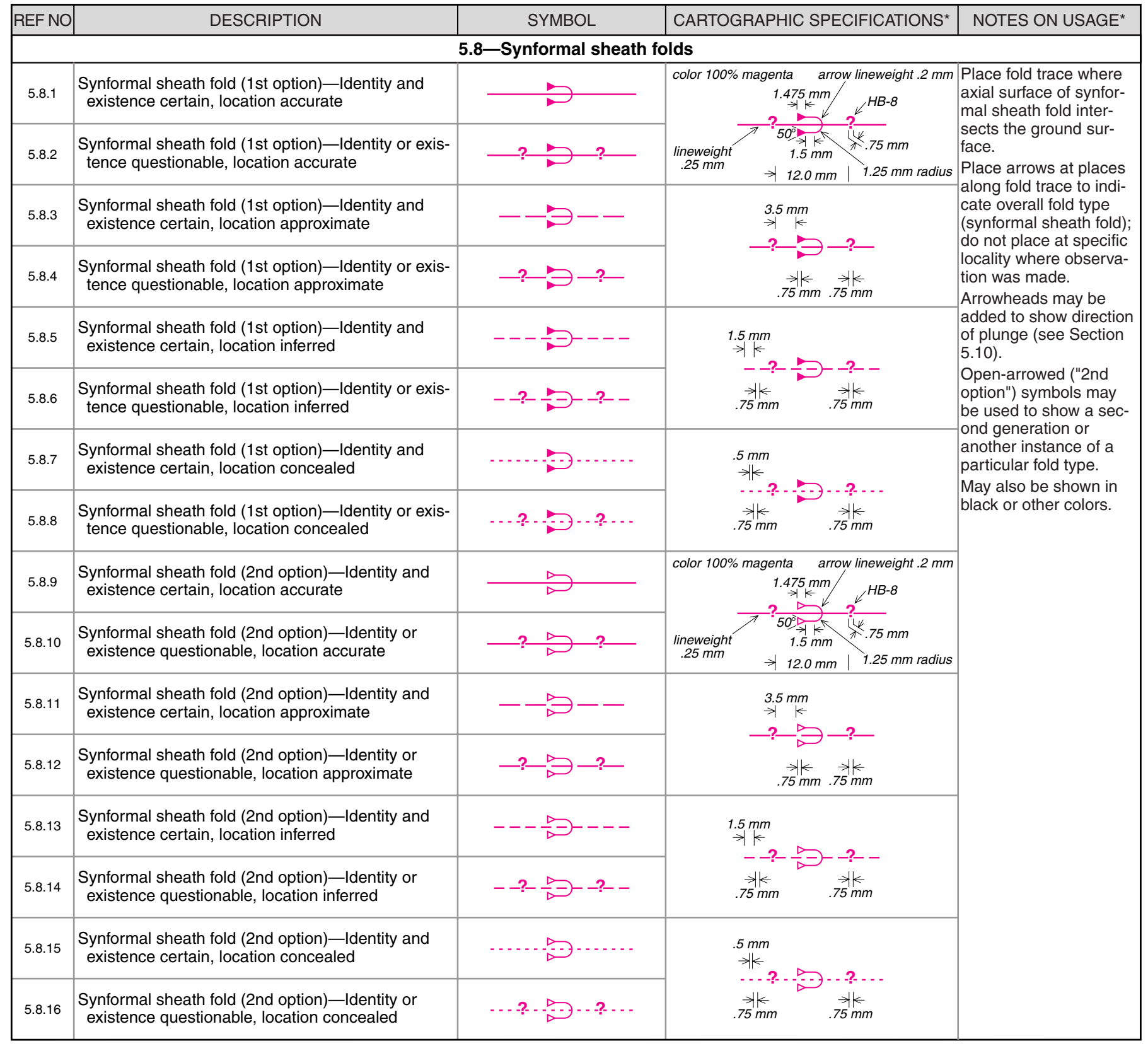

${ }^{*}$ For more information, see general guidelines on pages $A-i$ to $A-v$. 


\section{5-FOLDS (continued)}

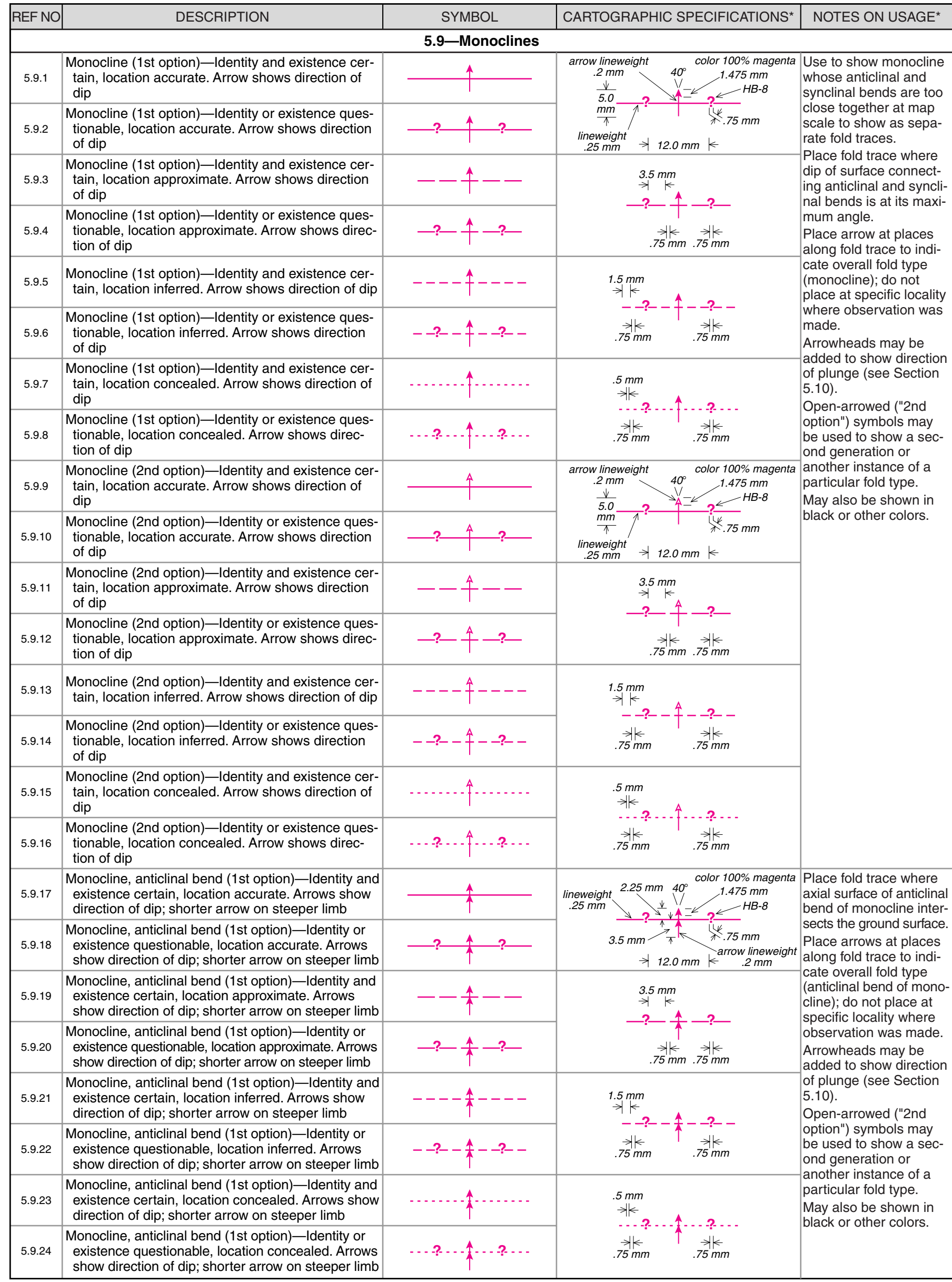




\section{5-FOLDS (continued)}

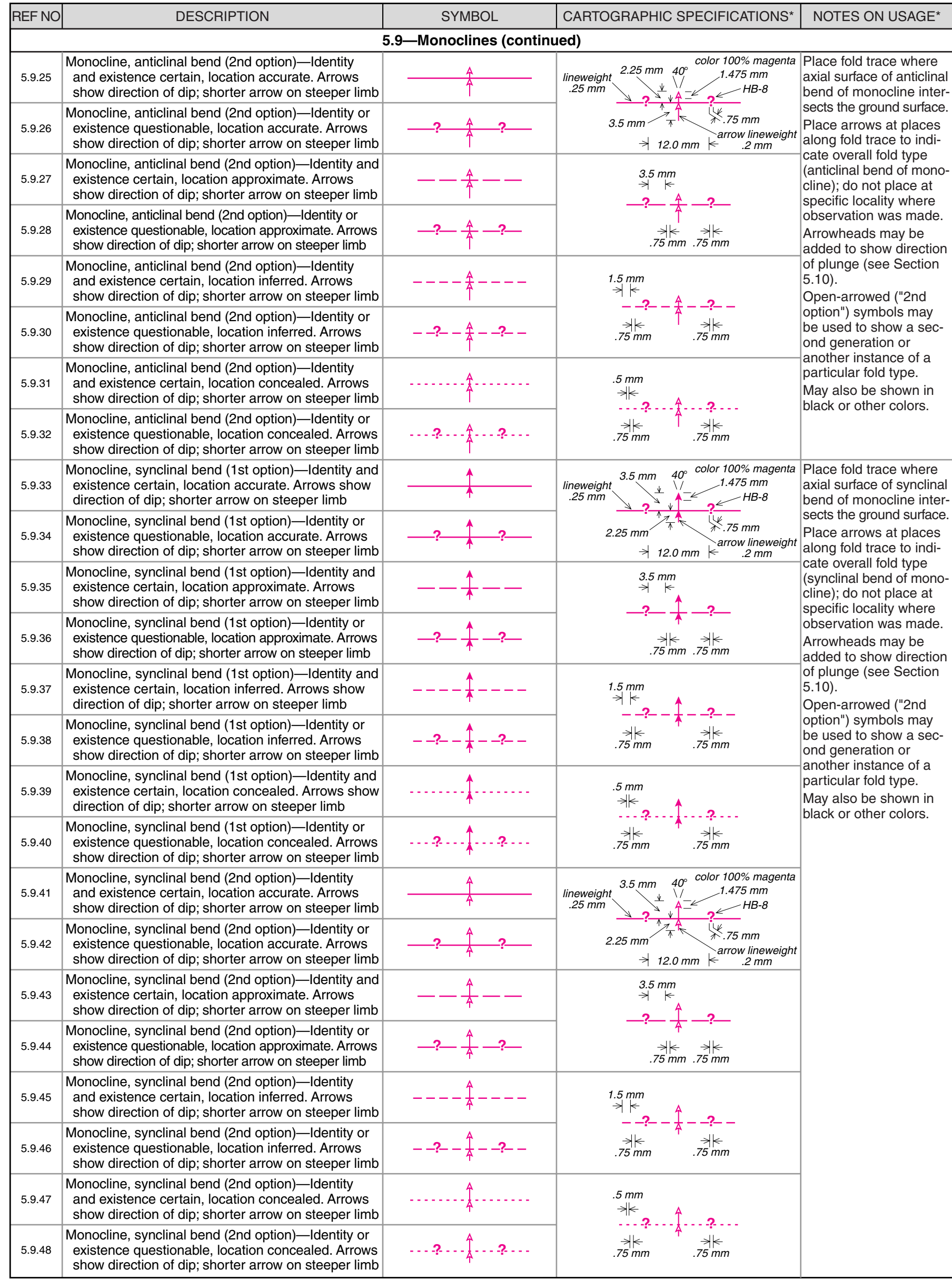


Federal Geographic Data Committee (Doc. No. FGDC-STD-013-2006)

U.S. Geological Survey Techniques and Methods 11-A2 FGDC Digital Cartographic Standard for Geologic Map Symbolization (PostScript Implementation)

\section{5-FOLDS (continued)}

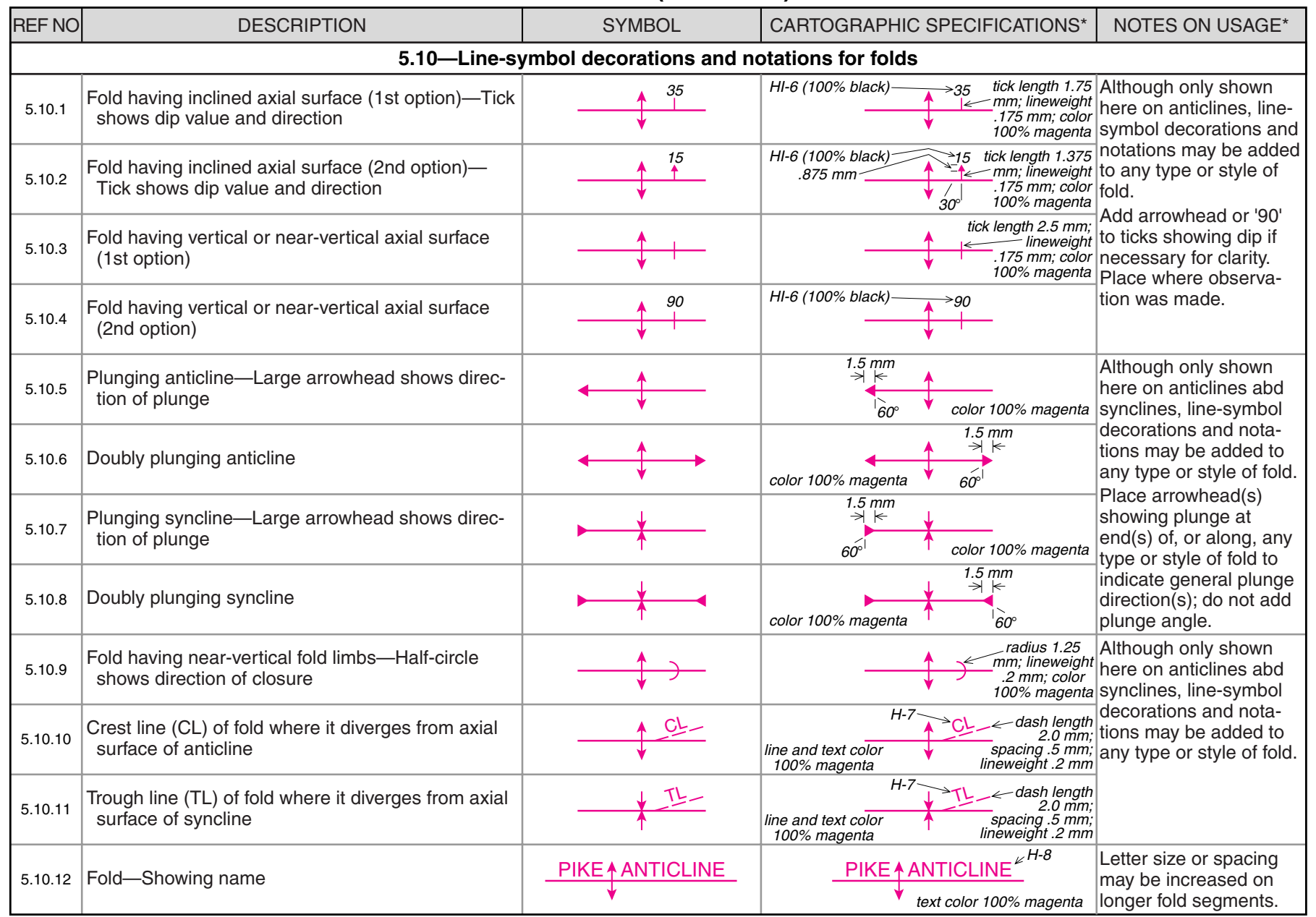

${ }^{*}$ For more information, see general guidelines on pages $A-i$ to $A-v$. 
Federal Geographic Data Committee (Doc. No. FGDC-STD-013-2006)

U.S. Geological Survey Techniques and Methods 11-A2

FGDC Digital Cartographic Standard for Geologic Map Symbolization (PostScript Implementation)

DOWNLOAD this Illustrator EPS file: Al8 / CS2

\section{5-FOLDS (continued)}

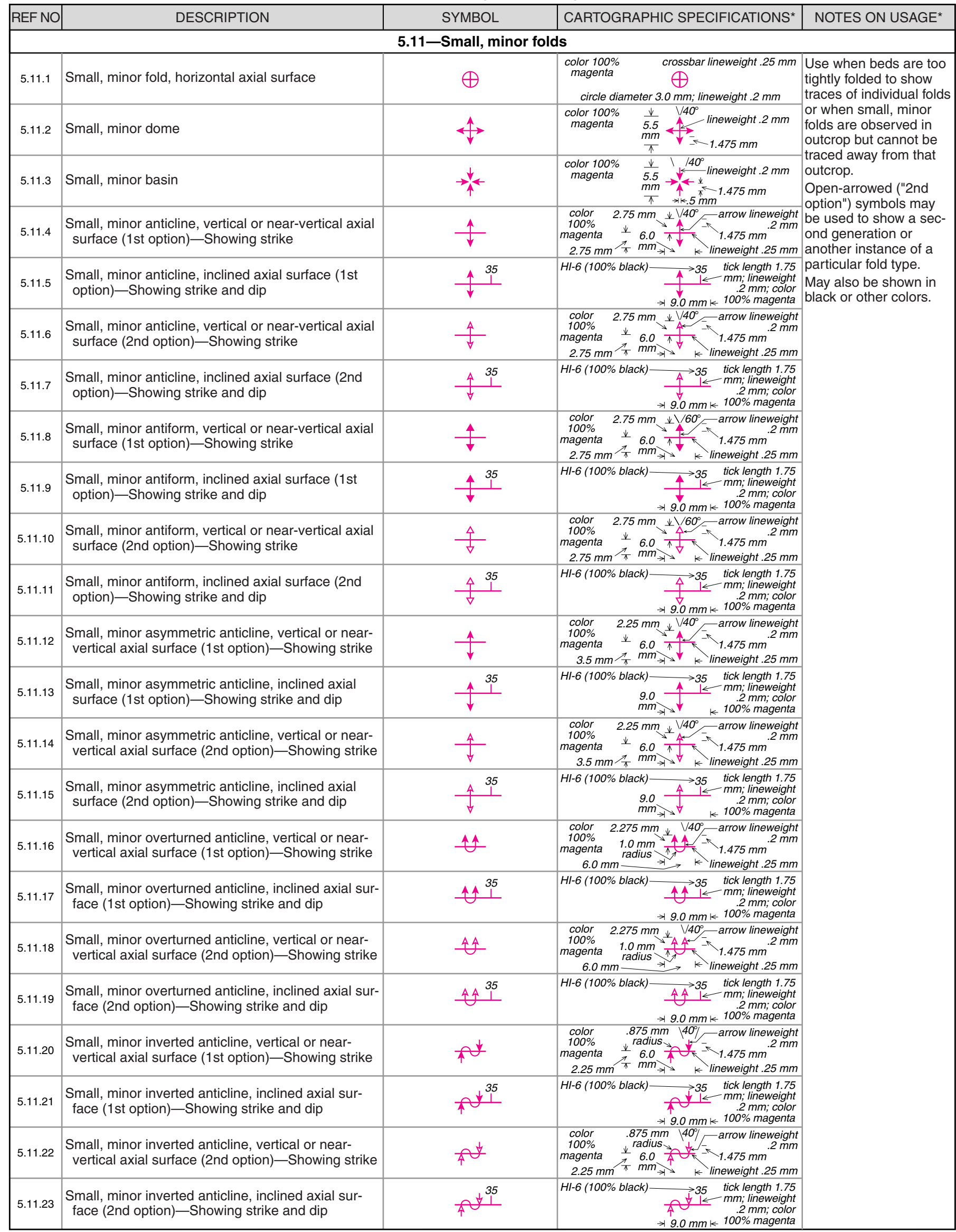

${ }^{*}$ For more information, see general guidelines on pages $A-i$ to $A-v$ 
Federal Geographic Data Committee (Doc. No. FGDC-STD-013-2006)

U.S. Geological Survey Techniques and Methods 11-A2

FGDC Digital Cartographic Standard for Geologic Map Symbolization (PostScript Implementation)

DOWNLOAD this Illustrator EPS file: Al8 / CS2

\section{5-FOLDS (continued)}

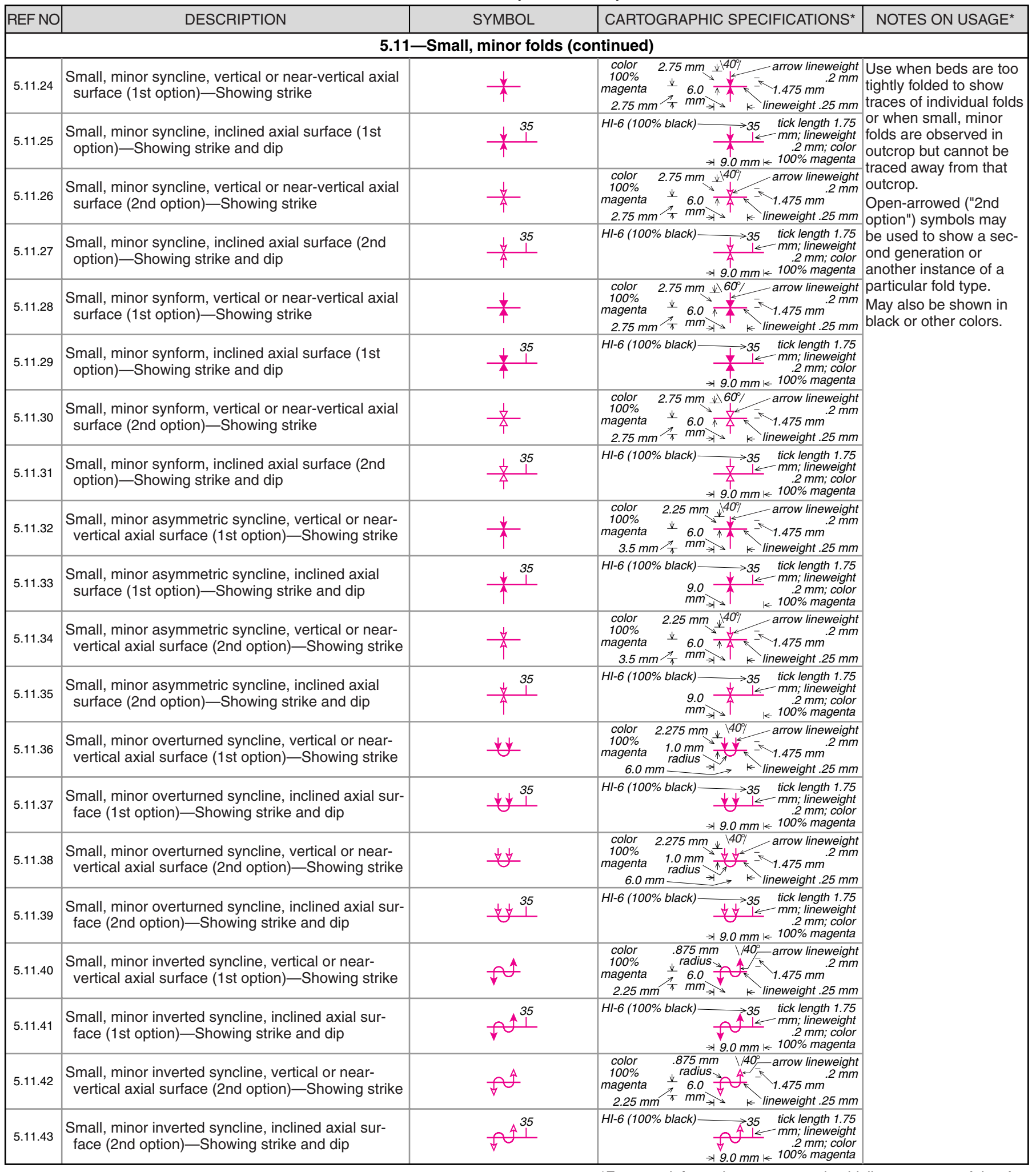

${ }^{*}$ For more information, see general guidelines on pages $A-i$ to $A-V$. 
Federal Geographic Data Committee (Doc. No. FGDC-STD-013-2006)

U.S. Geological Survey Techniques and Methods 11-A2 FGDC Digital Cartographic Standard for Geologic Map Symbolization (PostScript Implementation)

DOWNLOAD this Illustrator EPS file: Al8 / CS2

\section{6-BEDDING}

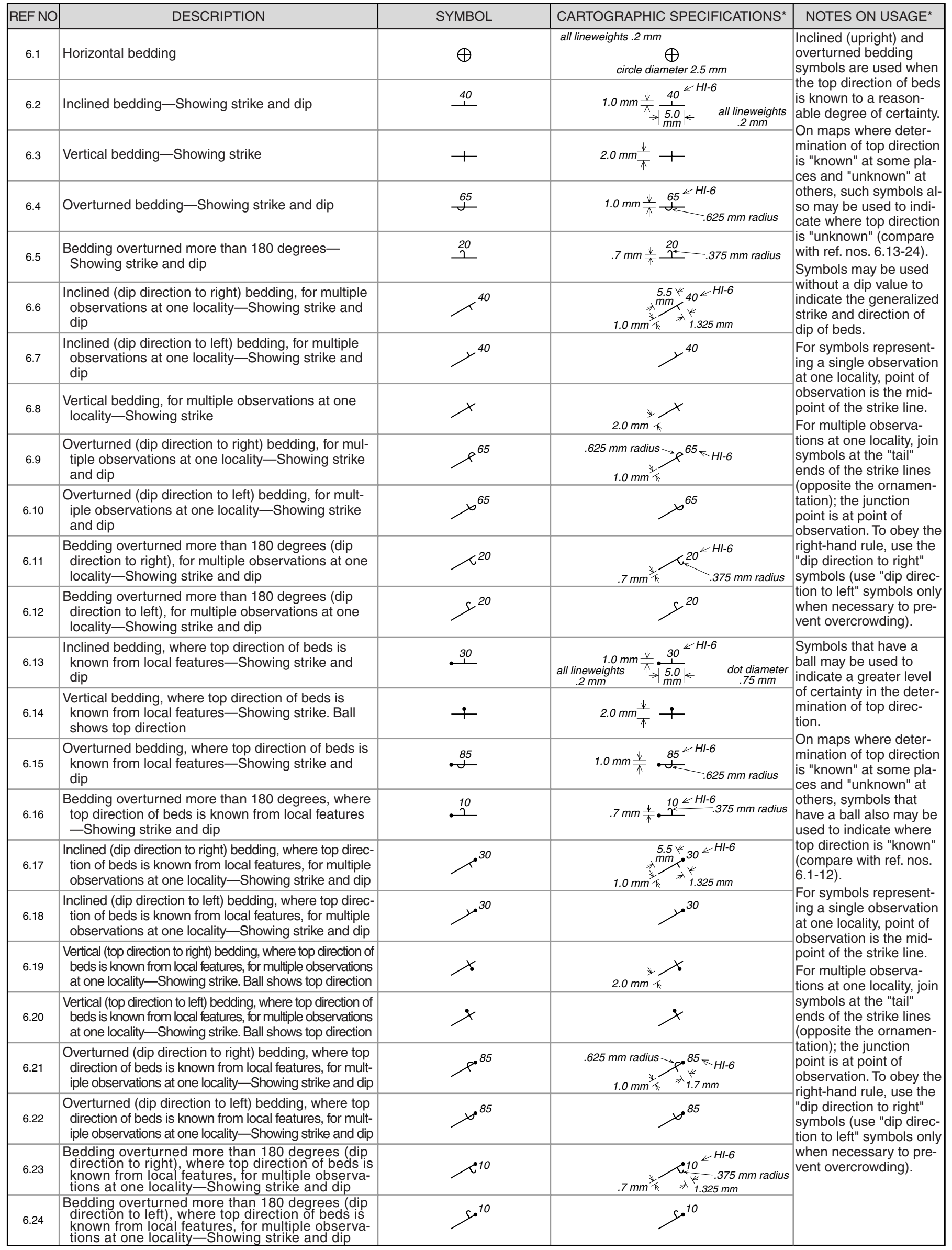


Federal Geographic Data Committee (Doc. No. FGDC-STD-013-2006)

U.S. Geological Survey Techniques and Methods 11-A2 FGDC Digital Cartographic Standard for Geologic Map Symbolization (PostScript Implementation)

DOWNLOAD this Illustrator EPS file: Al8 / CS2

\section{6-BEDDING (continued)}

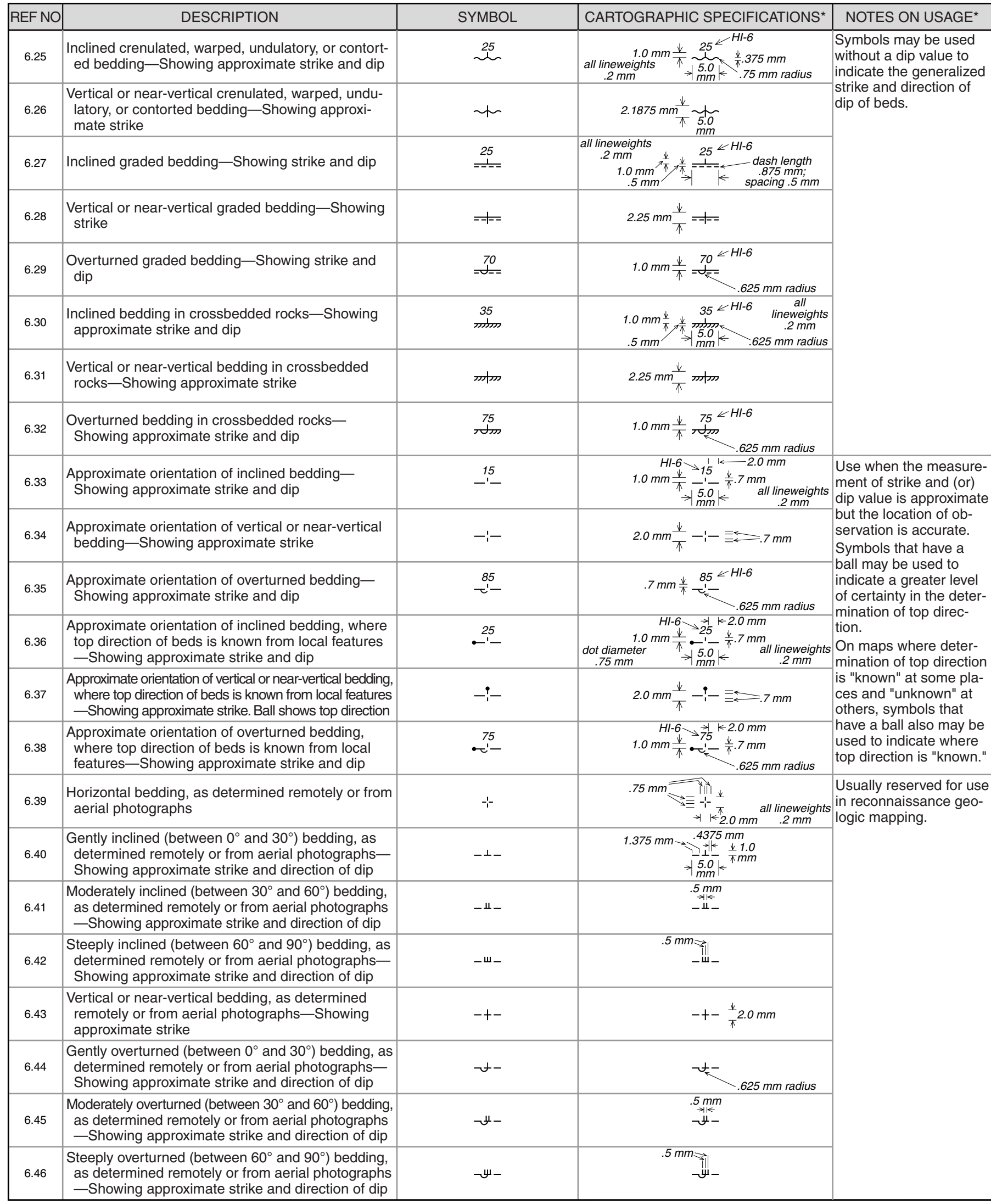

${ }^{*}$ For more information, see general guidelines on pages $A-i$ to $A-v$. 
Federal Geographic Data Committee (Doc. No. FGDC-STD-013-2006)

U.S. Geological Survey Techniques and Methods 11-A2 FGDC Digital Cartographic Standard for Geologic Map Symbolization (PostScript Implementation)

DOWNLOAD this Illustrator EPS file: Al8 / CS2

7-CLEAVAGE

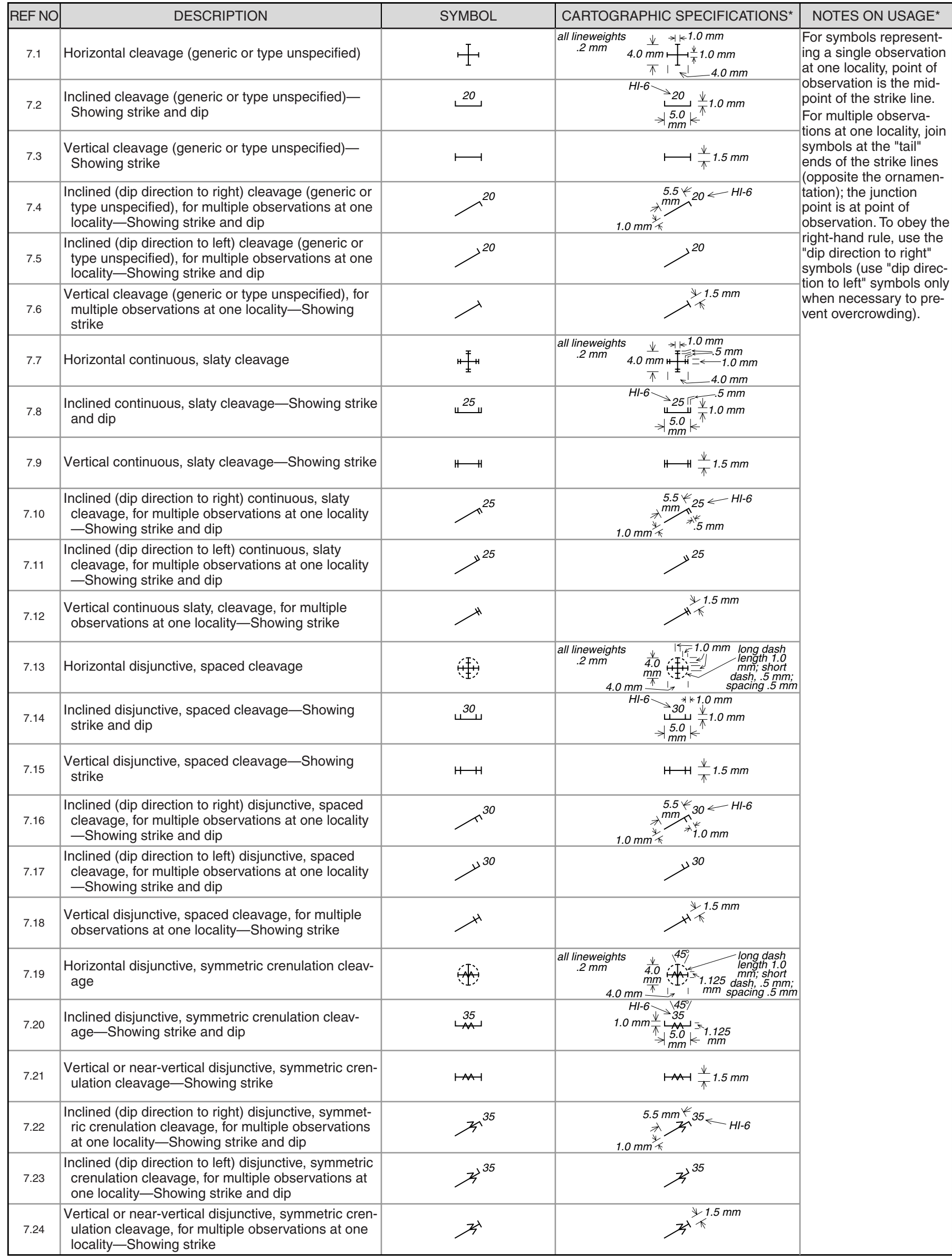


Federal Geographic Data Committee (Doc. No. FGDC-STD-013-2006)

U.S. Geological Survey Techniques and Methods 11-A2 FGDC Digital Cartographic Standard for Geologic Map Symbolization (PostScript Implementation)

DOWNLOAD this Illustrator EPS file: $\underline{\mathrm{Al} 8}$ / $\underline{\mathrm{CS} 2}$

\section{7-CLEAVAGE (continued)}

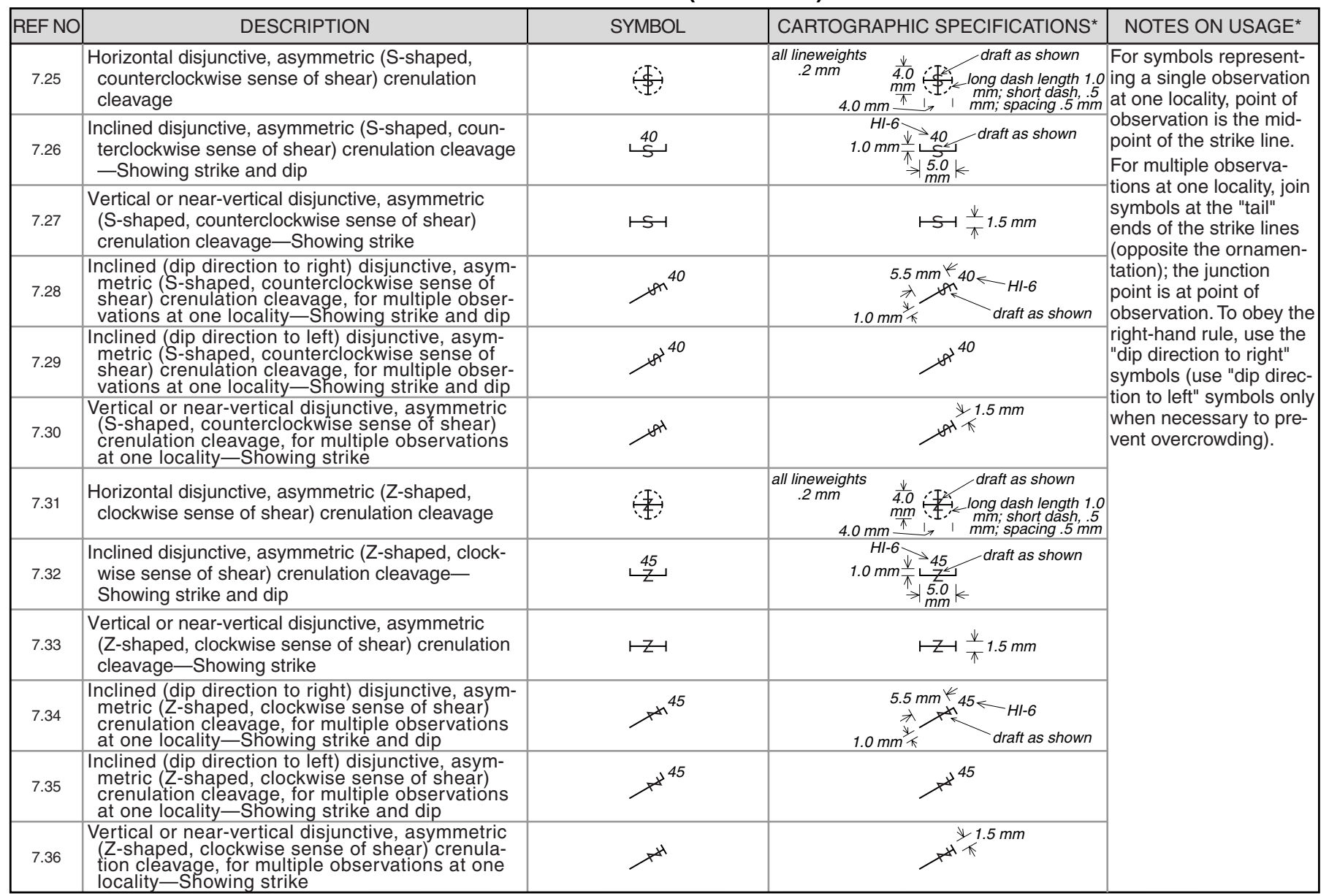

${ }^{*}$ For more information, see general guidelines on pages $A-i$ to $A-v$. 


\section{8-FOLIATION}

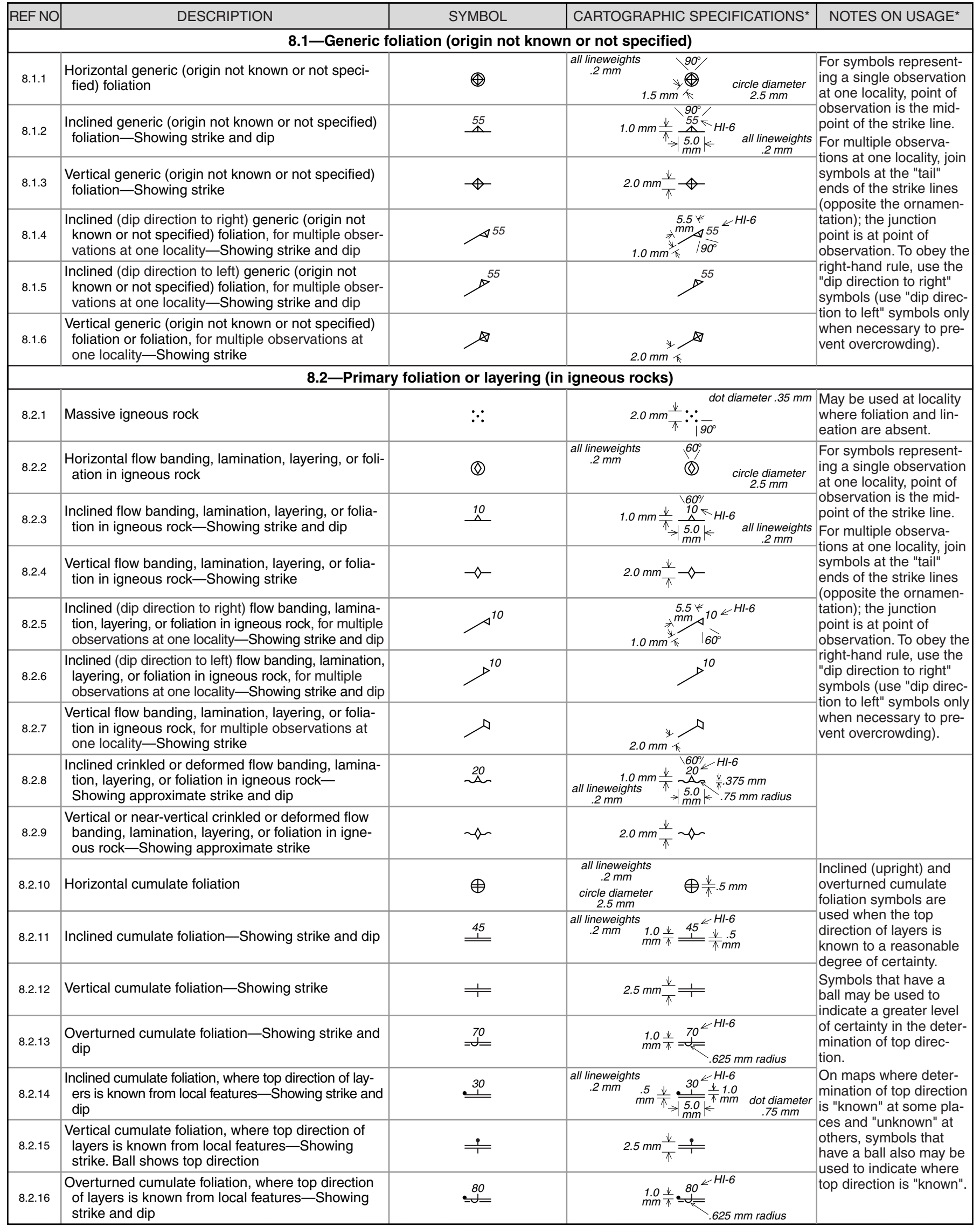


Federal Geographic Data Committee (Doc. No. FGDC-STD-013-2006)

U.S. Geological Survey Techniques and Methods 11-A2 FGDC Digital Cartographic Standard for Geologic Map Symbolization (PostScript Implementation)

\section{8-FOLIATION (continued)}

\begin{tabular}{|c|c|c|c|c|}
\hline REF NO & DESCRIPTION & SYMBOL & CARTOGRAPHIC SPECIFICATIONS* & NOTES ON USAGE* \\
\hline \multicolumn{5}{|c|}{ 8.2-Primary foliation or layering (in igneous rocks) (continued) } \\
\hline 8.2.17 & $\begin{array}{l}\text { Inclined crinkled or deformed cumulate foliation- } \\
\text { Showing approximate strike and dip }\end{array}$ & $\stackrel{25}{\sim}$ & 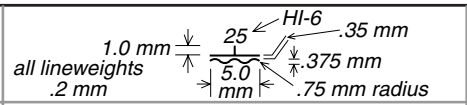 & \multirow{10}{*}{$\begin{array}{l}\text { For symbols represent- } \\
\text { ing a single observation } \\
\text { at one locality, point of } \\
\text { observation is the mid- } \\
\text { point of the strike line. } \\
\text { For multiple observa- } \\
\text { tions at one locality, join } \\
\text { symbols at the "tail" } \\
\text { ends of the strike lines } \\
\text { (opposite the ornamen- } \\
\text { tation); the junction } \\
\text { point is at point of } \\
\text { observation. To obey the } \\
\text { right-hand rule, use the } \\
\text { "dip direction to right" } \\
\text { symbols (use "dip direc- } \\
\text { tion to left" symbols only } \\
\text { when necessary to pre- } \\
\text { vent overcrowding). }\end{array}$} \\
\hline 8.2 .18 & $\begin{array}{l}\text { Vertical or near-vertical crinkled or deformed cumu- } \\
\text { late foliation-Showing approximate strike }\end{array}$ & $\approx$ & 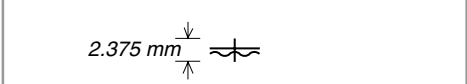 & \\
\hline 8.2.19 & Horizontal eutaxitic foliation & $\theta$ & $\begin{array}{cc}.75 \mathrm{~mm} \frac{\downarrow}{\uparrow} \Theta & \begin{array}{c}\text { all lineweights } \\
.2 \mathrm{~mm} \\
\text { circle diameter } \\
2.5 \mathrm{~mm}\end{array} \\
\end{array}$ & \\
\hline 8.2 .20 & Inclined eutaxitic foliation-Showing strike and dip & 5 & 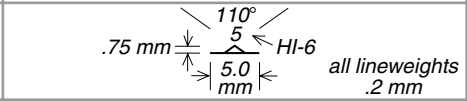 & \\
\hline 8.2 .21 & $\begin{array}{l}\text { Vertical or near-vertical eutaxitic foliation-Showing } \\
\text { strike }\end{array}$ & & $1.5 \mathrm{~mm} \frac{\downarrow}{\pi}$ & \\
\hline 8.2.22 & $\begin{array}{l}\text { Inclined (dip direction to right) eutaxitic foliation, for } \\
\text { multiple observations at one locality-Showing } \\
\text { strike and dip }\end{array}$ & & 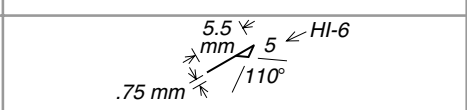 & \\
\hline 8.2 .23 & $\begin{array}{l}\text { Inclined (dip direction to left) eutaxitic foliation, for } \\
\text { multiple observations at one locality-Showing } \\
\text { strike and dip }\end{array}$ & & & \\
\hline 8.2 .24 & $\begin{array}{l}\text { Vertical or near-vertical eutaxitic foliation, for mult- } \\
\text { iple observations at one locality-Showing strike }\end{array}$ & & $1.5 \mathrm{~mm} \pi$ & \\
\hline 8.2 .25 & $\begin{array}{l}\text { Inclined crinkled or deformed eutaxitic foliation- } \\
\text { Showing approximate strike and dip }\end{array}$ & $\stackrel{15}{\approx}$ & 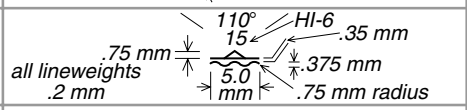 & \\
\hline 8.2.26 & $\begin{array}{l}\text { Vertical or near-vertical crinkled or deformed eutax- } \\
\text { itic foliation-Showing approximate strike }\end{array}$ & $\widehat{\approx a}$ & $1.5 \mathrm{~mm} \frac{\downarrow}{\pi} \precsim \widehat{\diamond}$ & \\
\hline
\end{tabular}

${ }^{\star}$ For more information, see general guidelines on pages A-i to A-v. 
Federal Geographic Data Committee (Doc. No. FGDC-STD-013-2006)

U.S. Geological Survey Techniques and Methods 11-A2 FGDC Digital Cartographic Standard for Geologic Map Symbolization (PostScript Implementation)

DOWNLOAD this Illustrator EPS file: Al8 / CS2

\section{8-FOLIATION (continued)}

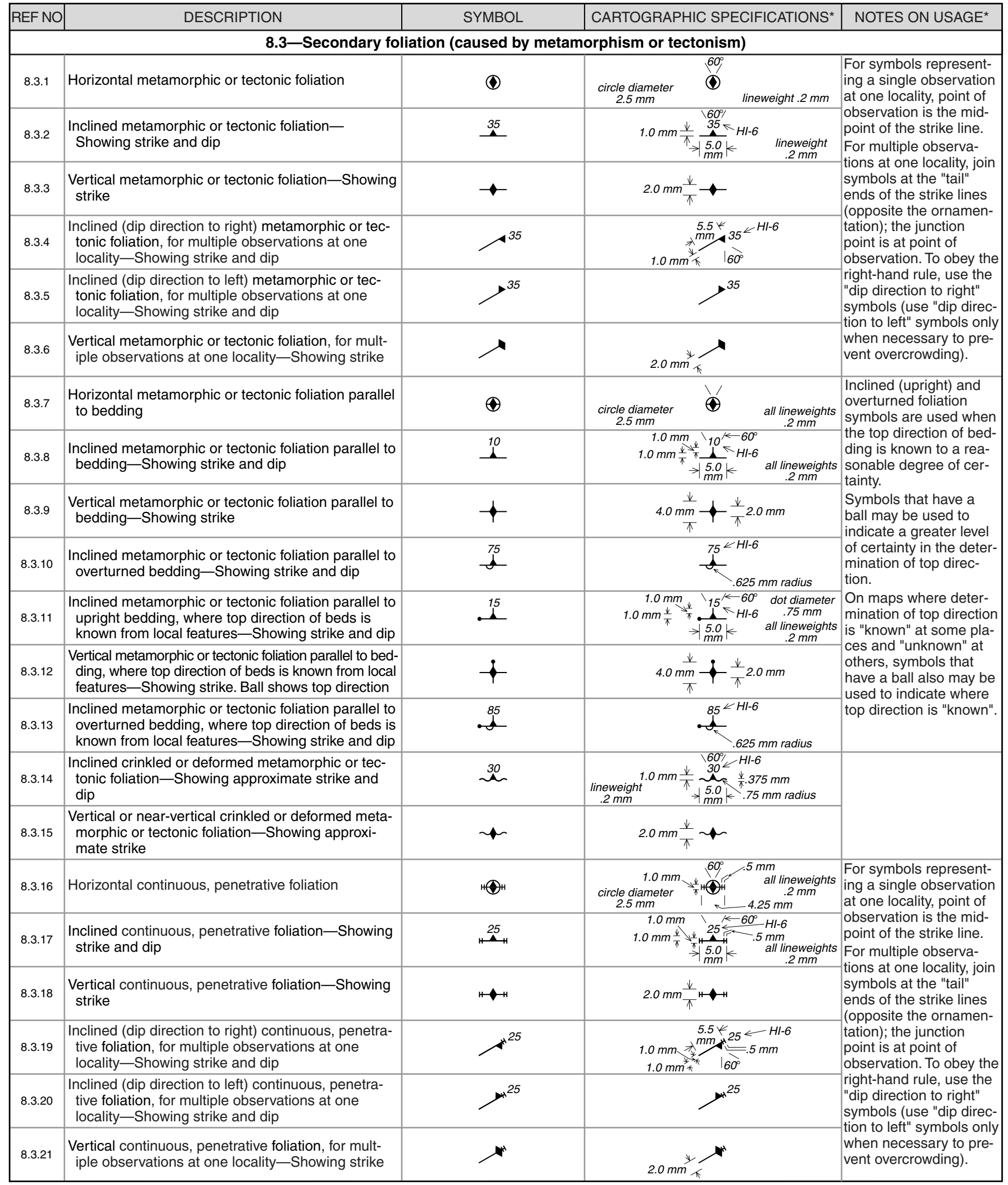

${ }^{*}$ For more information, see general guidelines on pages $A-i$ to $A-v$. 
Federal Geographic Data Committee (Doc. No. FGDC-STD-013-2006)

U.S. Geological Survey Techniques and Methods 11-A2 FGDC Digital Cartographic Standard for Geologic Map Symbolization (PostScript Implementation)

DOWNLOAD this Illustrator EPS file: Al8 / CS2

\section{8-FOLIATION (continued)}

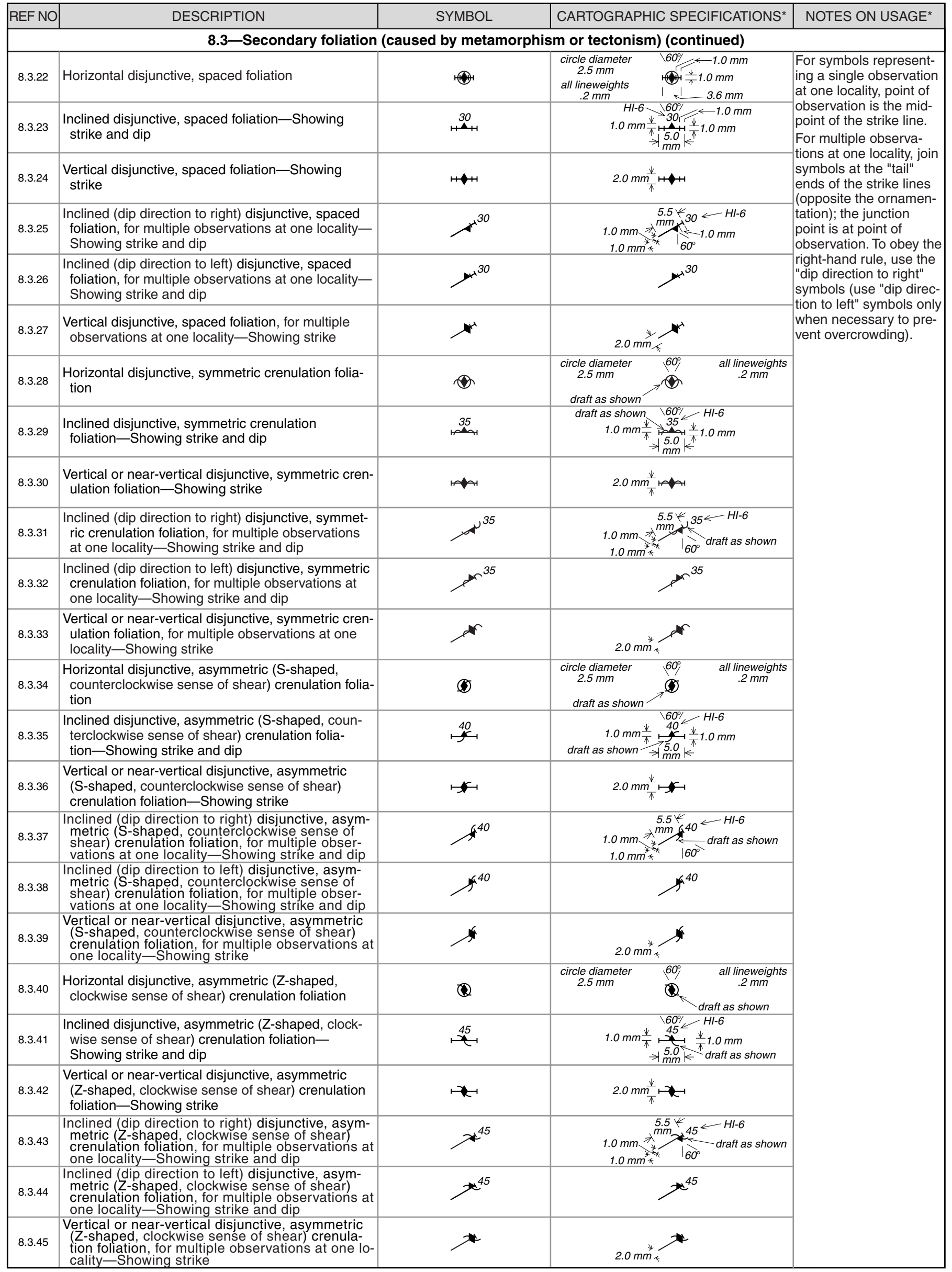


Federal Geographic Data Committee (Doc. No. FGDC-STD-013-2006)

U.S. Geological Survey Techniques and Methods 11-A2 FGDC Digital Cartographic Standard for Geologic Map Symbolization (PostScript Implementation)

DOWNLOAD this Illustrator EPS file: Al8 / $\underline{\text { CS2 }}$

\section{8-FOLIATION (continued)}

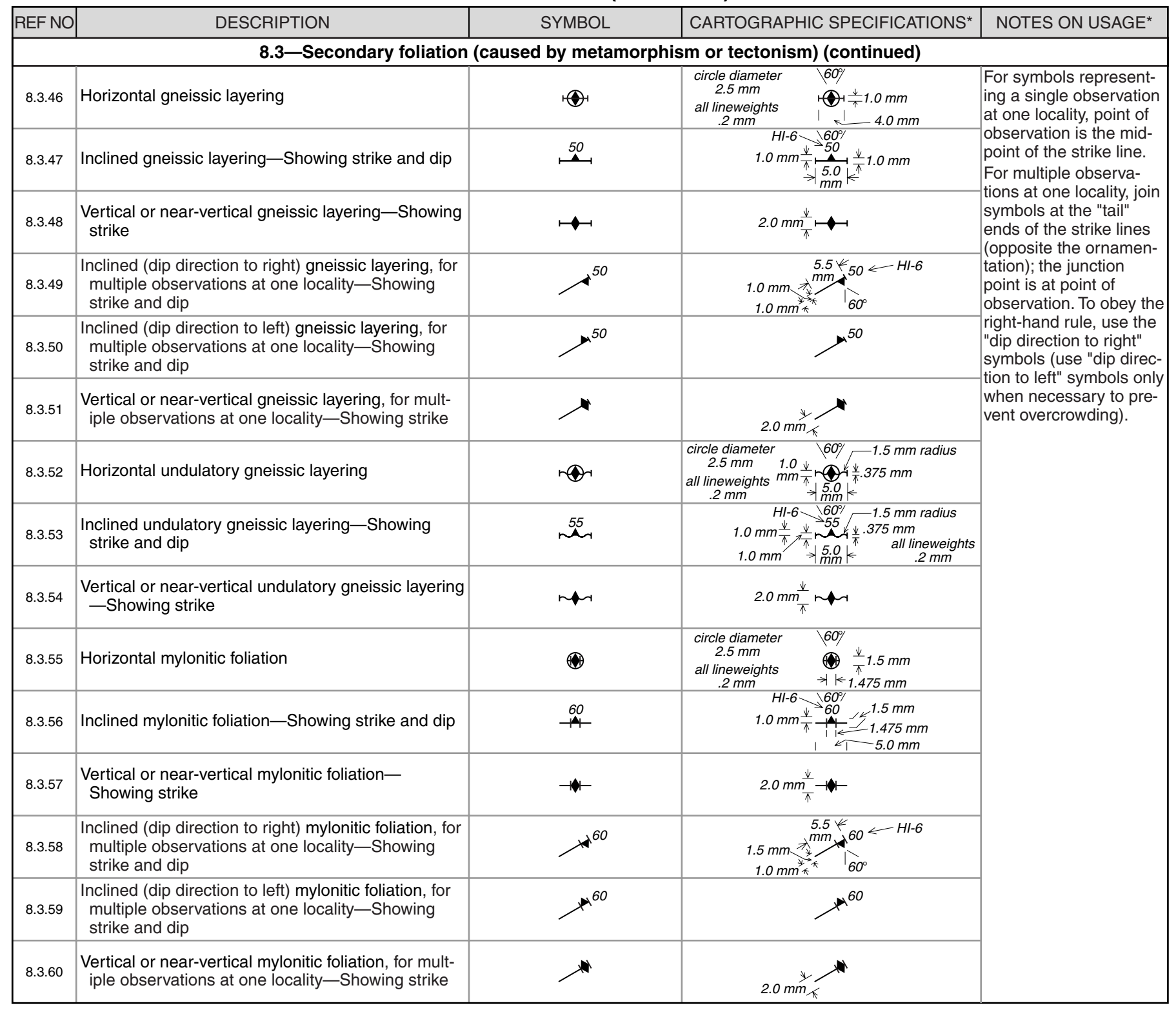

${ }^{*}$ For more information, see general guidelines on pages $A-i$ to $A-v$. 
Federal Geographic Data Committee (Doc. No. FGDC-STD-013-2006)

U.S. Geological Survey Techniques and Methods 11-A2

FGDC Digital Cartographic Standard for Geologic Map Symbolization (PostScript Implementation)

DOWNLOAD this Illustrator EPS file: Al8 / CS2

\section{9-LINEATION}

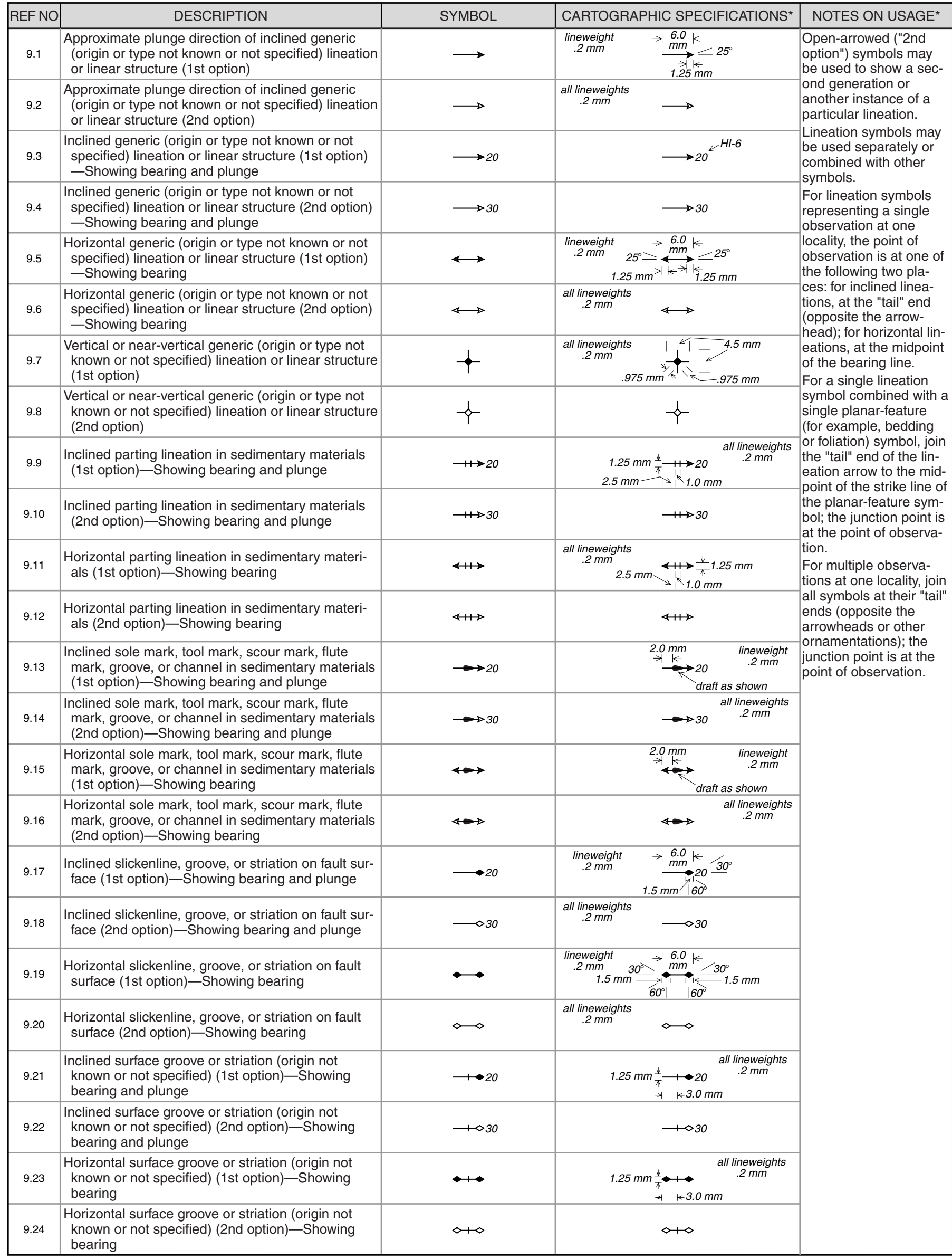


Federal Geographic Data Committee (Doc. No. FGDC-STD-013-2006)

U.S. Geological Survey Techniques and Methods 11-A2

FGDC Digital Cartographic Standard for Geologic Map Symbolization (PostScript Implementation)

DOWNLOAD this Illustrator EPS file: Al8 / CS2

\section{9-LINEATION (continued)}

\begin{tabular}{|c|c|c|c|c|}
\hline REF NO & DESCRIPTION & SYMBOL & CARTOGRAPHIC SPECIFICATIONS* & NOTES ON USAGE* \\
\hline 9.25 & $\begin{array}{l}\text { Inclined aligned-object lineation (1st option)- } \\
\text { Showing bearing and plunge }\end{array}$ & $\rightarrow 20$ & 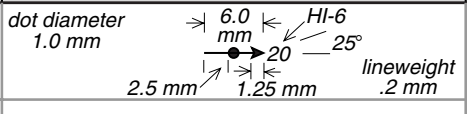 & \multirow{24}{*}{$\begin{array}{l}\text { Open-arrowed ("2nd } \\
\text { option") symbols may } \\
\text { be used to show a sec- } \\
\text { ond generation or } \\
\text { another instance of a } \\
\text { particular lineation. } \\
\text { Lineation symbols may } \\
\text { be used separately or } \\
\text { combined with other } \\
\text { symbols. } \\
\text { For lineation symbols } \\
\text { representing a single } \\
\text { observation at one } \\
\text { locality, the point of } \\
\text { observation is at one of } \\
\text { the following two pla- } \\
\text { ces: for inclined linea- } \\
\text { tions, at the "tail" end } \\
\text { (opposite the arrow- } \\
\text { head); for horizontal lin- } \\
\text { eations, at the midpoint } \\
\text { of the bearing line. } \\
\text { For a single lineation } \\
\text { symbol combined with a } \\
\text { single planar-feature } \\
\text { (for example, bedding } \\
\text { or foliation) symbol, join } \\
\text { the "tail" end of the lin- } \\
\text { eation arrow to the mid- } \\
\text { point of the strike line of } \\
\text { the planar-feature sym- } \\
\text { bol; the junction point is } \\
\text { at the point of observa- } \\
\text { tion. } \\
\text { For multiple observa- } \\
\text { tions at one locality, join } \\
\text { all symbols at their "tail" } \\
\text { ends (opposite the } \\
\text { arrowheads or other } \\
\text { ornamentations); the } \\
\text { junction point is at the } \\
\text { point of observation. } \\
\end{array}$} \\
\hline 9.26 & $\begin{array}{l}\text { Inclined aligned-object lineation (2nd option)- } \\
\text { Showing bearing and plunge }\end{array}$ & $\rightarrow 30$ & $\longrightarrow 30 \quad \begin{array}{c}\text { all lineweights } \\
.2 \mathrm{~mm}\end{array}$ & \\
\hline 9.27 & $\begin{array}{l}\text { Horizontal aligned-object lineation (1st option)—- } \\
\text { Showing bearing }\end{array}$ & $\leftrightarrow$ & 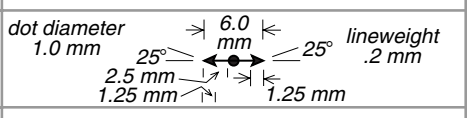 & \\
\hline 9.28 & $\begin{array}{l}\text { Horizontal aligned-object lineation (2nd option)- } \\
\text { Showing bearing }\end{array}$ & $\leftrightarrow$ & $\begin{array}{c}\text { all lineweights } \\
.2 \mathrm{~mm}\end{array}$ & \\
\hline 9.29 & $\begin{array}{l}\text { Inclined aligned-clast or aligned-grain lineation (in } \\
\text { sedimentary materials) (1st option)-Showing } \\
\text { bearing and plunge }\end{array}$ & $\rightarrow 20$ & 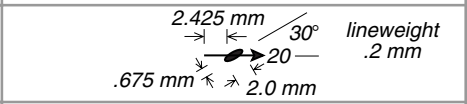 & \\
\hline 9.30 & $\begin{array}{l}\text { Inclined aligned-clast or aligned-grain lineation (in } \\
\text { sedimentary materials) (2nd option)—Showing } \\
\text { bearing and plunge }\end{array}$ & $\hookrightarrow 30$ & $\begin{array}{lc}\text { all lineweights } \\
.2 \mathrm{~mm}\end{array}$ & \\
\hline 9.31 & $\begin{array}{l}\text { Horizontal aligned-clast or aligned-grain lineation } \\
\text { (in sedimentary materials) (1st option)-Showing } \\
\text { bearing }\end{array}$ & $\leftrightarrow$ & 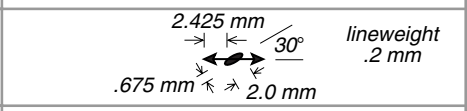 & \\
\hline 9.32 & $\begin{array}{l}\text { Horizontal aligned-clast or aligned-grain lineation } \\
\text { (in sedimentary materials) (2nd option)—Showing } \\
\text { bearing }\end{array}$ & $\leftrightarrow$ & $\leftrightarrow \quad \begin{array}{c}\text { all lineweights } \\
.2 \mathrm{~mm}\end{array}$ & \\
\hline 9.33 & $\begin{array}{l}\text { Inclined aligned-inclusion lineation (in igneous } \\
\text { rocks) (1st option)-Showing bearing and plunge }\end{array}$ & $\longrightarrow 20$ & 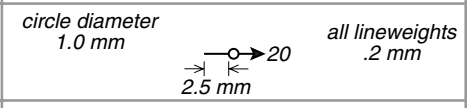 & \\
\hline 9.34 & $\begin{array}{l}\text { Inclined aligned-inclusion lineation (in igneous } \\
\text { rocks) (2nd option)-Showing bearing and plunge }\end{array}$ & $\longrightarrow 30$ & $\longrightarrow 30$ & \\
\hline 9.35 & $\begin{array}{l}\text { Horizontal aligned-inclusion lineation (in igneous } \\
\text { rocks) (1st option)-Showing bearing }\end{array}$ & $\leftrightarrow$ & 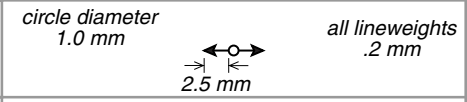 & \\
\hline 9.36 & $\begin{array}{l}\text { Horizontal aligned-inclusion lineation (in igneous } \\
\text { rocks) ( } 2 \text { nd option)-Showing bearing }\end{array}$ & $\leftrightarrow \circ$ & & \\
\hline 9.37 & $\begin{array}{l}\text { Inclined aligned-mineral lineation (1st option)- } \\
\text { Showing bearing and plunge }\end{array}$ & $\longrightarrow 20$ & 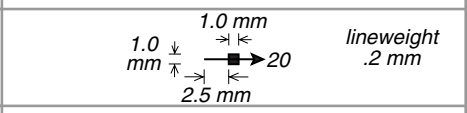 & \\
\hline 9.38 & $\begin{array}{l}\text { Inclined aligned-mineral lineation (2nd option)- } \\
\text { Showing bearing and plunge }\end{array}$ & $\rightarrow 30$ & $\begin{array}{l}\text { all lineweights } \\
.2 \mathrm{~mm}\end{array}$ & \\
\hline 9.39 & $\begin{array}{l}\text { Horizontal aligned-mineral lineation (1st option)- } \\
\text { Showing bearing }\end{array}$ & $\leftrightarrow$ & 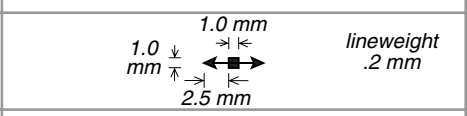 & \\
\hline 9.40 & $\begin{array}{l}\text { Horizontal aligned-mineral lineation (2nd option)- } \\
\text { Showing bearing }\end{array}$ & $\leftrightarrow$ & $\begin{array}{l}\text { all lineweights } \\
.2 \mathrm{~mm}\end{array}$ & \\
\hline 9.41 & $\begin{array}{l}\text { Inclined aligned mineral-aggregate lineation (1st } \\
\text { option)—Showing bearing and plunge }\end{array}$ & $\rightarrow 20$ & 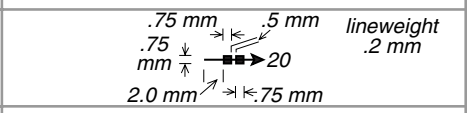 & \\
\hline 9.42 & $\begin{array}{l}\text { Inclined aligned mineral-aggregate lineation (2nd } \\
\text { option)—Showing bearing and plunge }\end{array}$ & $\rightarrow 30$ & $\Longrightarrow 30 \quad \begin{array}{c}\text { all lineweights } \\
.2 \mathrm{~mm}\end{array}$ & \\
\hline 9.43 & $\begin{array}{l}\text { Horizontal aligned mineral-aggregate lineation (1st } \\
\text { option)-Showing bearing }\end{array}$ & $\leftrightarrow$ & 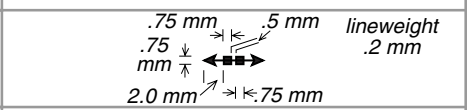 & \\
\hline 9.44 & $\begin{array}{l}\text { Horizontal aligned mineral-aggregate lineation ( } 2 \text { nd } \\
\text { option)-Showing bearing }\end{array}$ & $\leftrightarrow$ & $\leftrightarrow \quad \begin{array}{cc}\text { all lineweights } \\
.2 \mathrm{~mm}\end{array}$ & \\
\hline 9.45 & $\begin{array}{l}\text { Inclined aligned deformed-mineral lineation (1st } \\
\text { option)-Showing bearing and plunge }\end{array}$ & $\rightarrow 20$ & 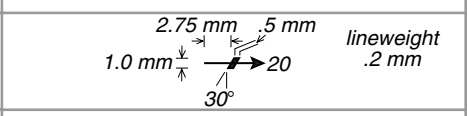 & \\
\hline 9.46 & $\begin{array}{l}\text { Inclined aligned deformed-mineral lineation ( } 2 \text { nd } \\
\text { option)-Showing bearing and plunge }\end{array}$ & $\leftrightarrow 30$ & $\begin{array}{l}\text { all lineweights } \\
.2 \mathrm{~mm}\end{array}$ & \\
\hline 9.47 & $\begin{array}{l}\text { Horizontal aligned deformed-mineral lineation (1st } \\
\text { option)-Showing bearing }\end{array}$ & $\leftrightarrow$ & 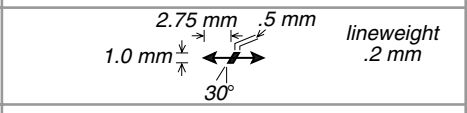 & \\
\hline 9.48 & $\begin{array}{l}\text { Horizontal aligned deformed-mineral lineation (2nd } \\
\text { option)—Showing bearing }\end{array}$ & $\leftrightarrow \leftrightarrow$ & $\leftrightarrow \leftrightarrow \quad \begin{array}{c}\text { all lineweights } \\
.2 \mathrm{~mm}\end{array}$ & \\
\hline
\end{tabular}


Federal Geographic Data Committee (Doc. No. FGDC-STD-013-2006)

U.S. Geological Survey Techniques and Methods 11-A2 FGDC Digital Cartographic Standard for Geologic Map Symbolization (PostScript Implementation)

DOWNLOAD this Illustrator EPS file: Al8 / CS2

\section{9-LINEATION (continued)}

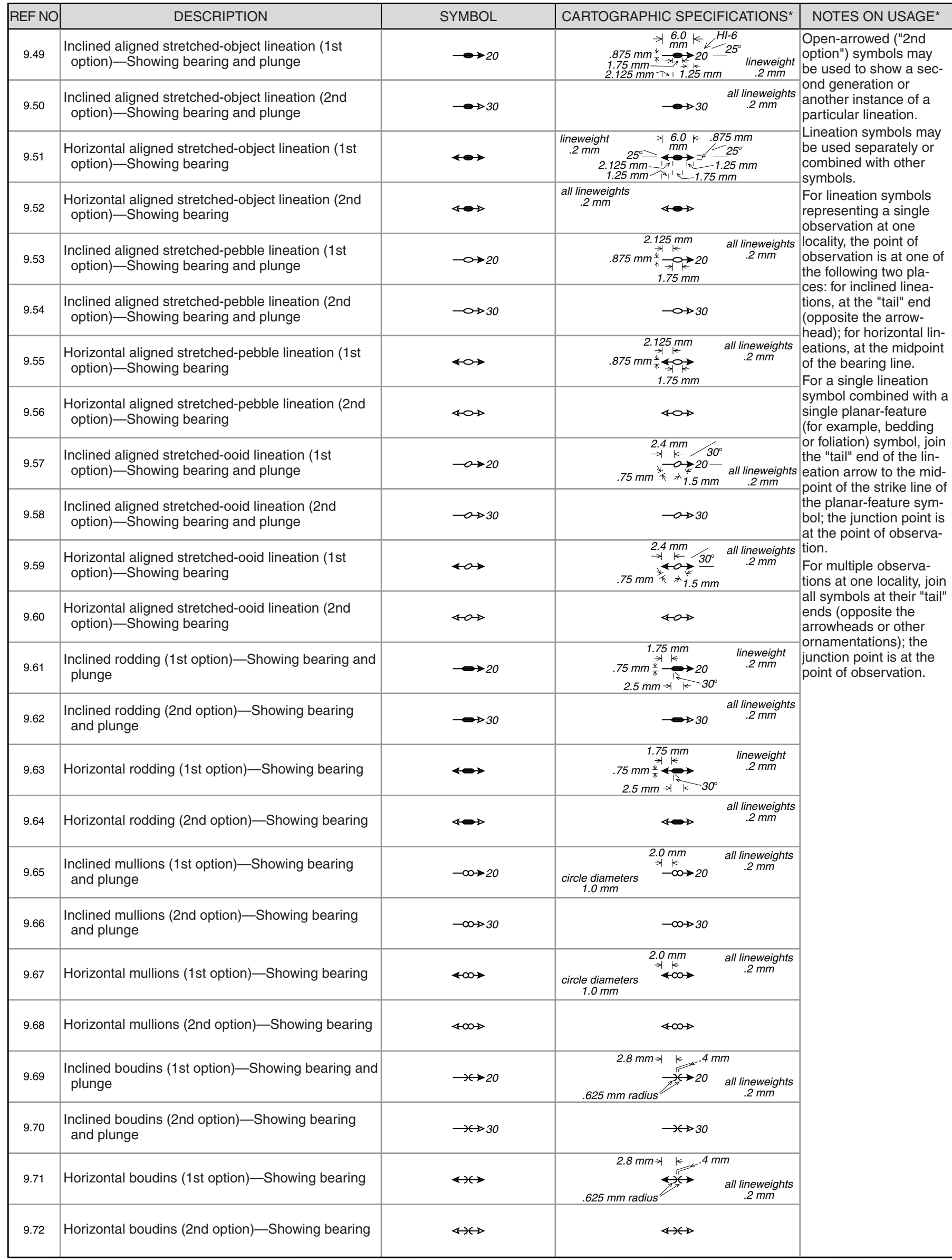


Federal Geographic Data Committee (Doc. No. FGDC-STD-013-2006)

U.S. Geological Survey Techniques and Methods 11-A2

FGDC Digital Cartographic Standard for Geologic Map Symbolization (PostScript Implementation)

DOWNLOAD this Illustrator EPS file: Al8 / CS2

\section{9-LINEATION (continued)}

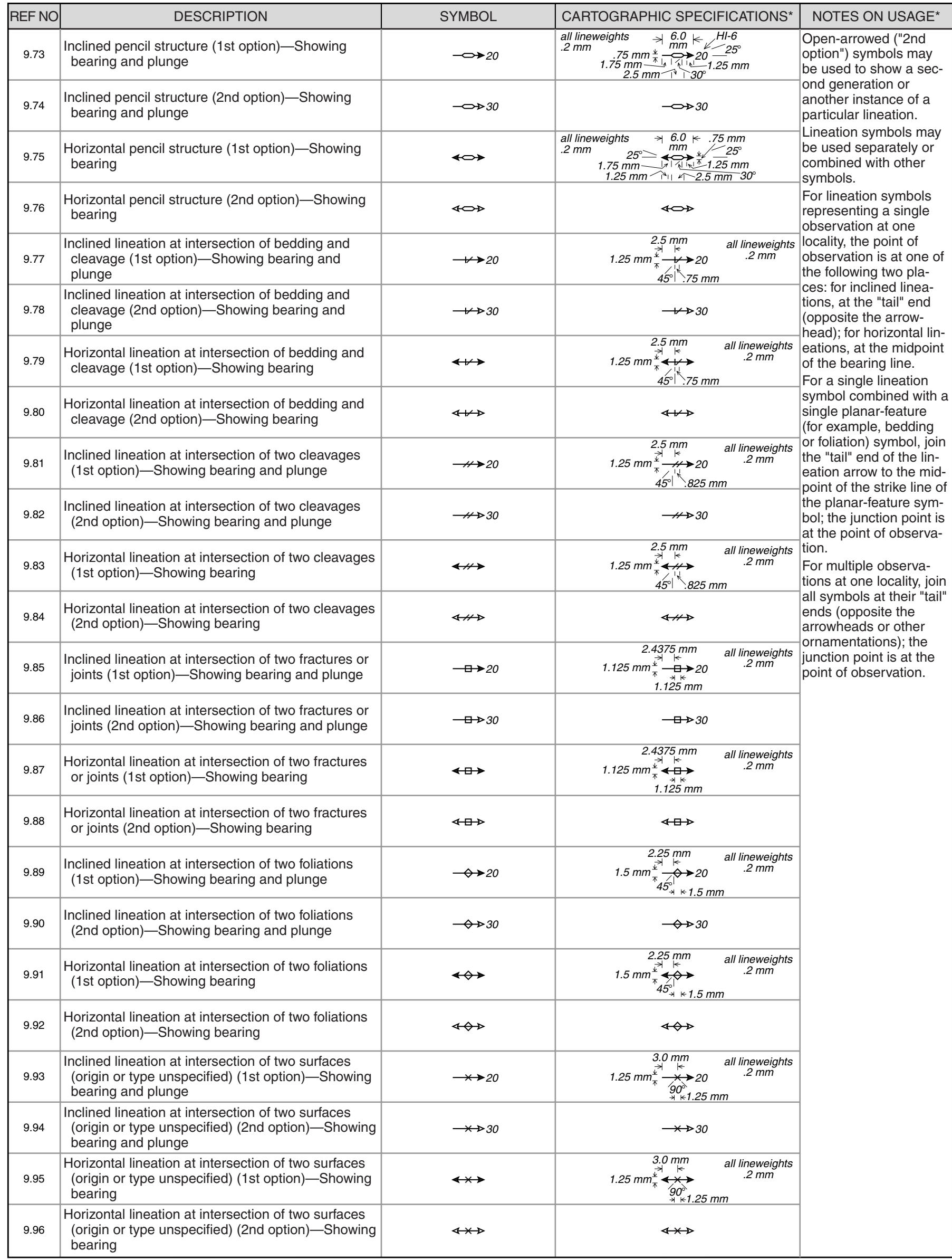


Federal Geographic Data Committee (Doc. No. FGDC-STD-013-2006)

U.S. Geological Survey Techniques and Methods 11-A2

FGDC Digital Cartographic Standard for Geologic Map Symbolization (PostScript Implementation)

DOWNLOAD this Illustrator EPS file: Al8 / CS2

\section{9-LINEATION (continued)}

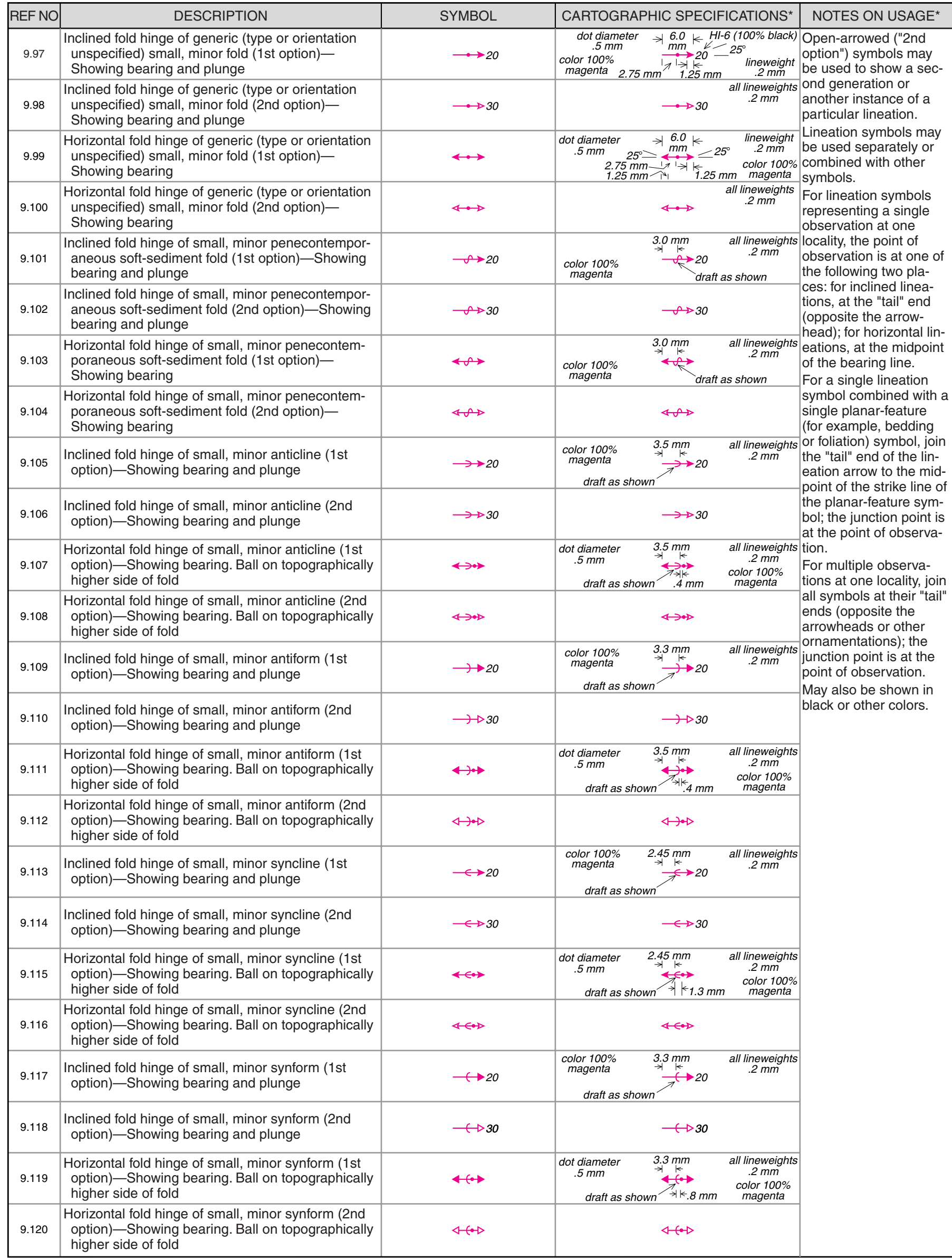


Federal Geographic Data Committee (Doc. No. FGDC-STD-013-2006)

U.S. Geological Survey Techniques and Methods 11-A2 FGDC Digital Cartographic Standard for Geologic Map Symbolization (PostScript Implementation)

DOWNLOAD this Illustrator EPS file: Al8 / CS2

\section{9-LINEATION (continued)}

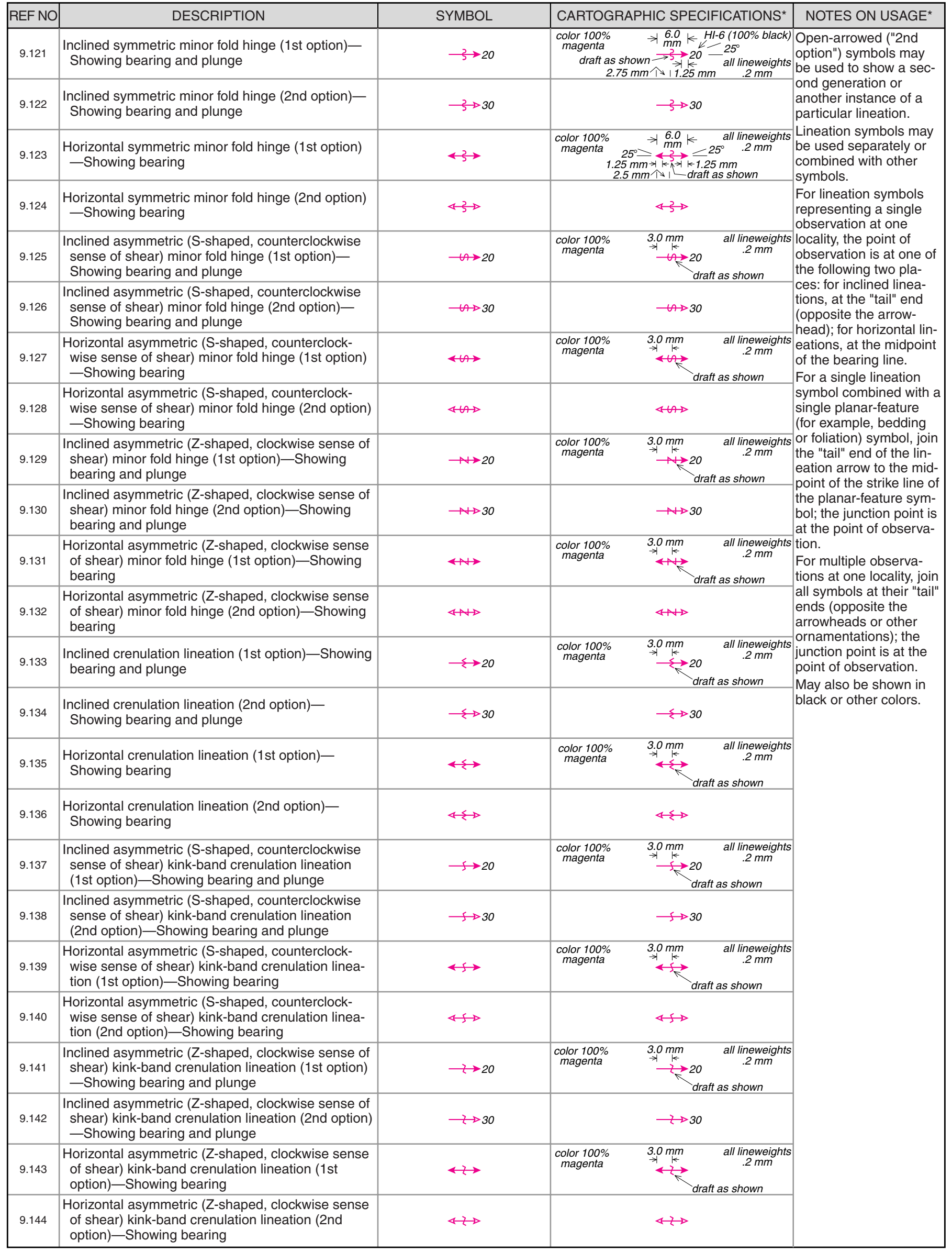


Federal Geographic Data Committee (Doc. No. FGDC-STD-013-2006)

U.S. Geological Survey Techniques and Methods 11-A2

FGDC Digital Cartographic Standard for Geologic Map Symbolization (PostScript Implementation)

DOWNLOAD this Illustrator EPS file: Al8 / $\underline{\mathrm{CS} 2}$

10-PALEONTOLOGICAL FEATURES

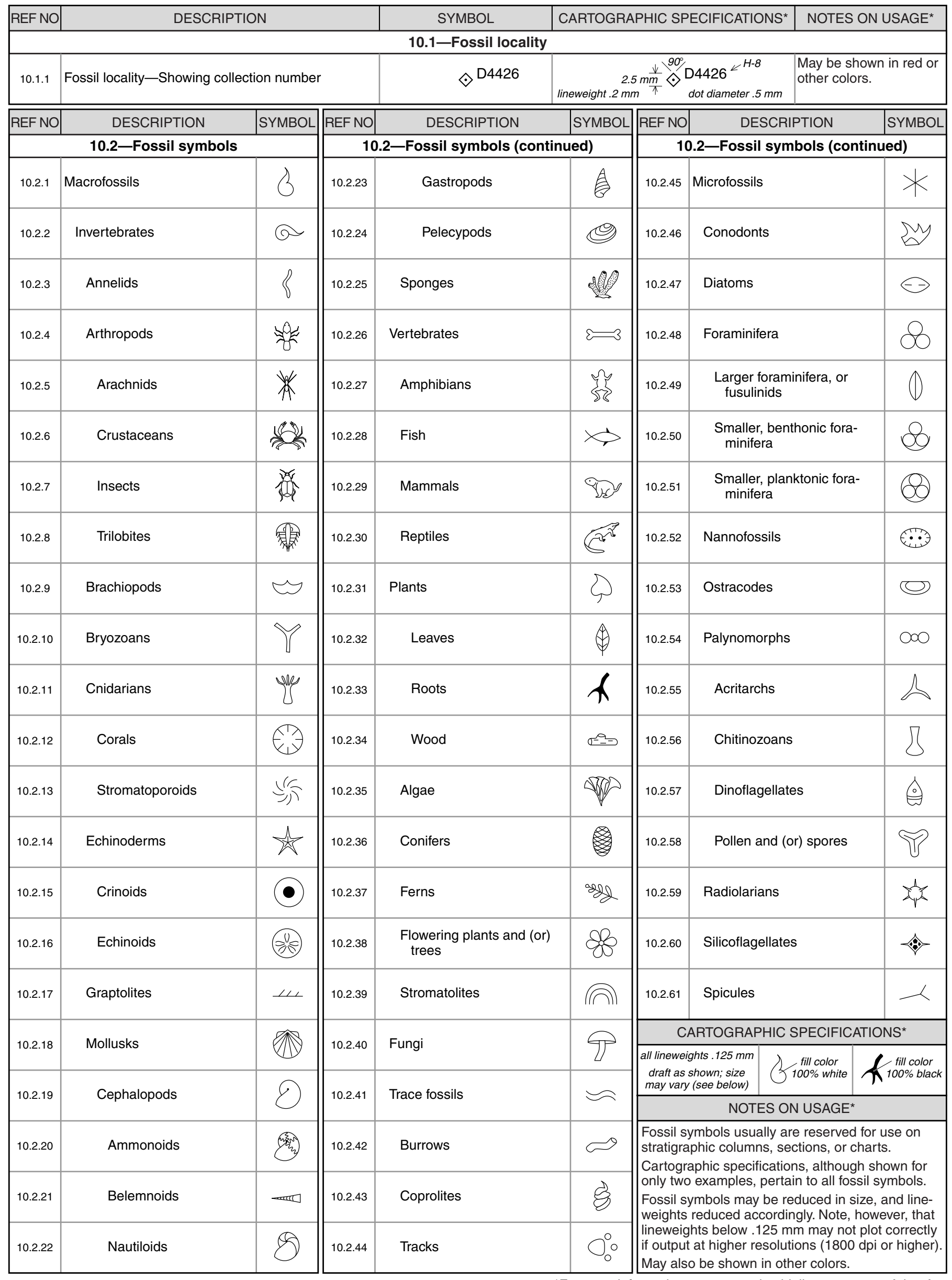


Federal Geographic Data Committee (Doc. No. FGDC-STD-013-2006)

U.S. Geological Survey Techniques and Methods 11-A2 FGDC Digital Cartographic Standard for Geologic Map Symbolization (PostScript Implementation)

DOWNLOAD this Illustrator EPS file: Al8 / $\underline{\mathrm{CS} 2}$

\section{1-GEOPHYSICAL AND STRUCTURE CONTOURS}

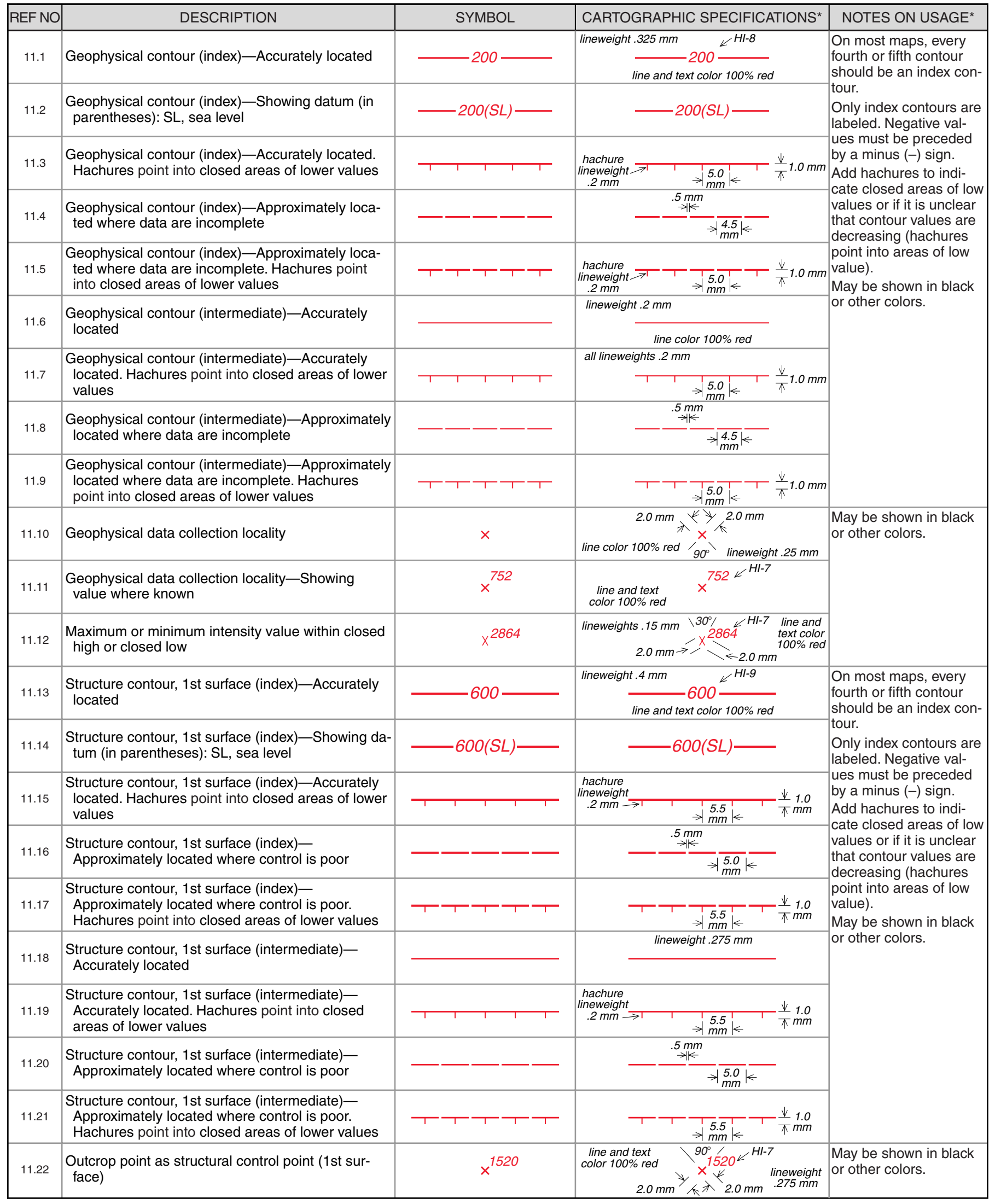

${ }^{\star}$ For more information, see general guidelines on pages $A-i$ to $A-v$. 
Federal Geographic Data Committee (Doc. No. FGDC-STD-013-2006)

U.S. Geological Survey Techniques and Methods 11-A2

FGDC Digital Cartographic Standard for Geologic Map Symbolization (PostScript Implementation)

DOWNLOAD this Illustrator EPS file: Al8 / CS2

\section{1-GEOPHYSICAL AND STRUCTURE CONTOURS (continued)}

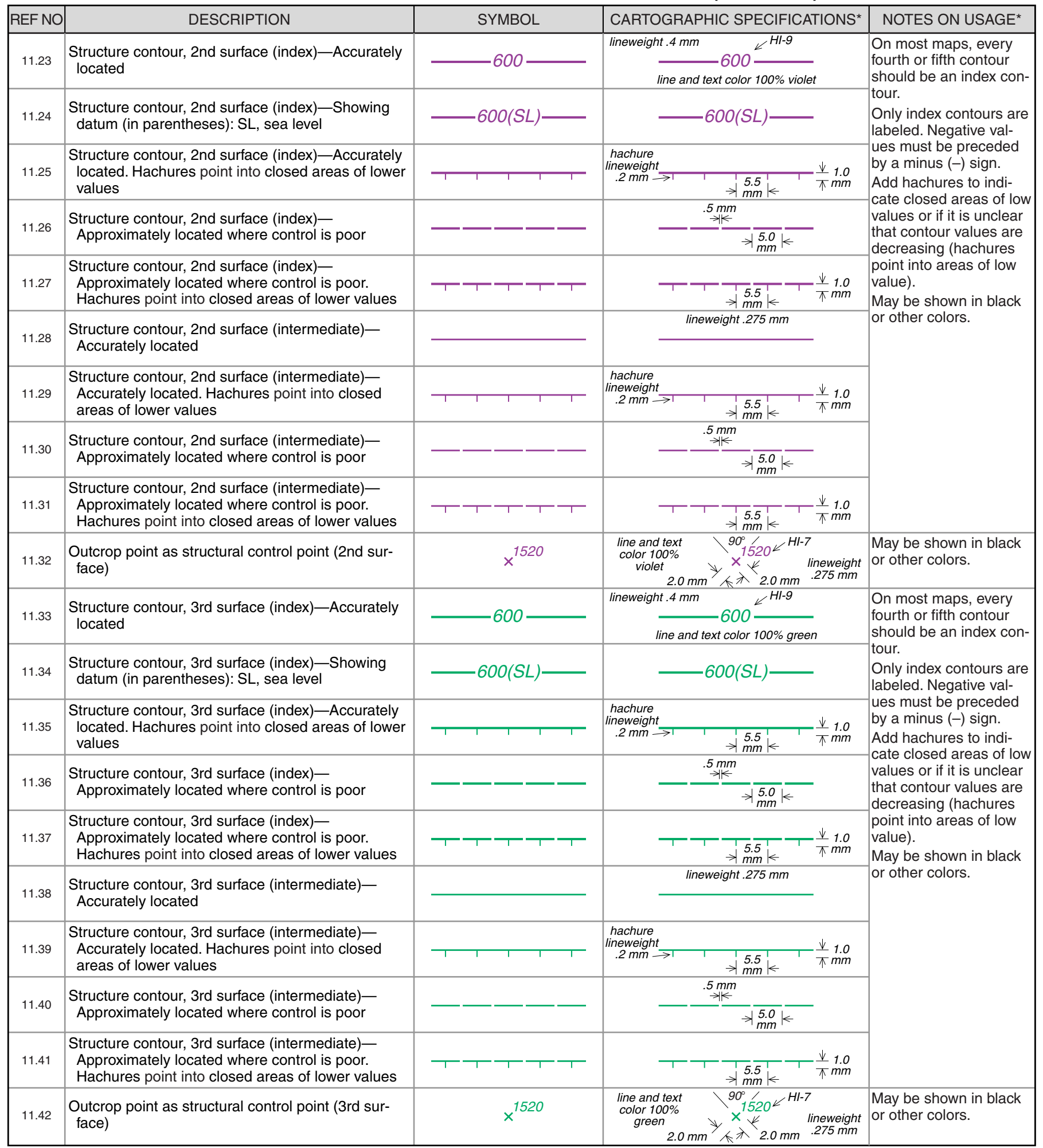

${ }^{\star}$ For more information, see general guidelines on pages $A-i$ to $A-v$. 
Federal Geographic Data Committee (Doc. No. FGDC-STD-013-2006)

U.S. Geological Survey Techniques and Methods 11-A2 FGDC Digital Cartographic Standard for Geologic Map Symbolization (PostScript Implementation) DOWNLOAD this Illustrator EPS file: $\underline{\mathrm{Al} 8}$ / $\underline{\mathrm{CS} 2}$

\section{2-FLUVIAL AND ALLUVIAL FEATURES}

\begin{tabular}{|c|c|c|c|c|}
\hline REF NO & DESCRIPTION & SYMBOL & CARTOGRAPHIC SPECIFICATIONS* & NOTES ON USAGE* \\
\hline 12.1 & $\begin{array}{l}\text { Fluvial terrace scarp-Identity and existence cer- } \\
\text { tain, location accurate. Hachures point downscarp }\end{array}$ & 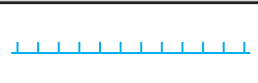 & \multirow{2}{*}{ 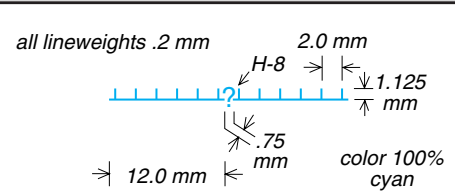 } & \multirow[t]{8}{*}{$\begin{array}{l}\text { May also be shown in } \\
\text { black or other colors. }\end{array}$} \\
\hline 12.2 & $\begin{array}{l}\text { Fluvial terrace scarp-Identity or existence ques- } \\
\text { tionable, location accurate. Hachures point down- } \\
\text { scarp }\end{array}$ & 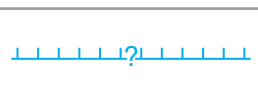 & & \\
\hline 12.3 & $\begin{array}{l}\text { Fluvial terrace scarp-Identity and existence cer- } \\
\text { tain, location approximate. Hachures point down- } \\
\text { scarp }\end{array}$ & $\perp \perp \perp \perp \perp \perp \perp \perp$ & \multirow{2}{*}{ 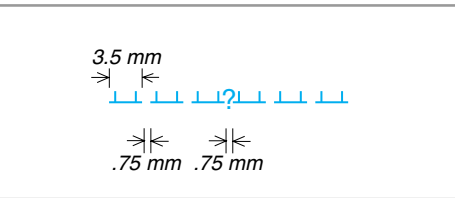 } & \\
\hline 12.4 & $\begin{array}{l}\text { Fluvial terrace scarp-Identity or existence ques- } \\
\text { tionable, location approximate. Hachures point } \\
\text { downscarp }\end{array}$ & $\perp \perp \perp \perp \perp \perp \perp \perp \perp \perp \perp \perp \perp \perp \perp$ & & \\
\hline 12.5 & Fluvial transport direction & $\longrightarrow$ & 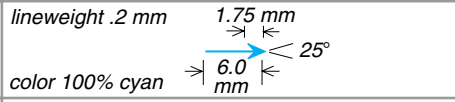 & \\
\hline 12.6 & $\begin{array}{l}\text { Sediment transport direction determined from im- } \\
\text { brication }\end{array}$ & $\infty \longrightarrow$ & circle diameters $.75 \mathrm{~mm}$ & \\
\hline 12.7 & $\begin{array}{l}\text { Sediment transport direction determined from } \\
\text { crossbeds }\end{array}$ & $\longleftrightarrow$ & 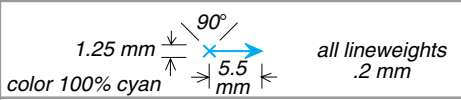 & \\
\hline 12.8 & $\begin{array}{l}\text { Sediment transport direction determined from flute } \\
\text { casts }\end{array}$ & $\longleftrightarrow$ & 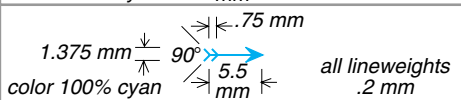 & \\
\hline
\end{tabular}

${ }^{*}$ For more information, see general guidelines on pages $A-i$ to $A-v$. 
Federal Geographic Data Committee (Doc. No. FGDC-STD-013-2006)

U.S. Geological Survey Techniques and Methods 11-A2 FGDC Digital Cartographic Standard for Geologic Map Symbolization (PostScript Implementation)

DOWNLOAD this Illustrator EPS file: Al8 / CS2

\section{3-GLACIAL AND GLACIOFLUVIAL FEATURES}

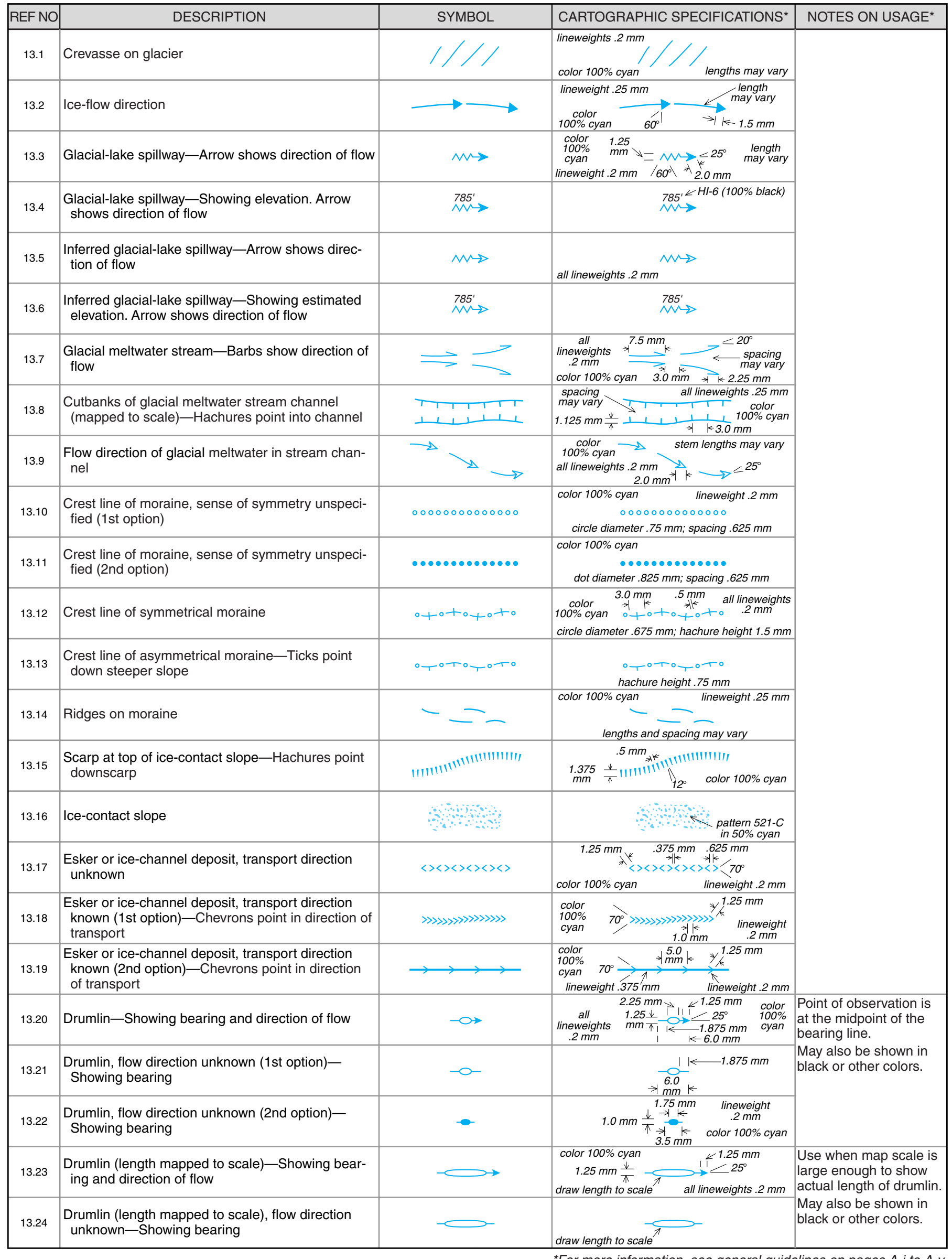

${ }^{*}$ For more information, see general guidelines on pages $A-i$ to $A-v$. 
Federal Geographic Data Committee (Doc. No. FGDC-STD-013-2006)

U.S. Geological Survey Techniques and Methods 11-A2 FGDC Digital Cartographic Standard for Geologic Map Symbolization (PostScript Implementation)

DOWNLOAD this Illustrator EPS file: Al8 / CS2

\section{3-GLACIAL AND GLACIOFLUVIAL FEATURES (continued)}

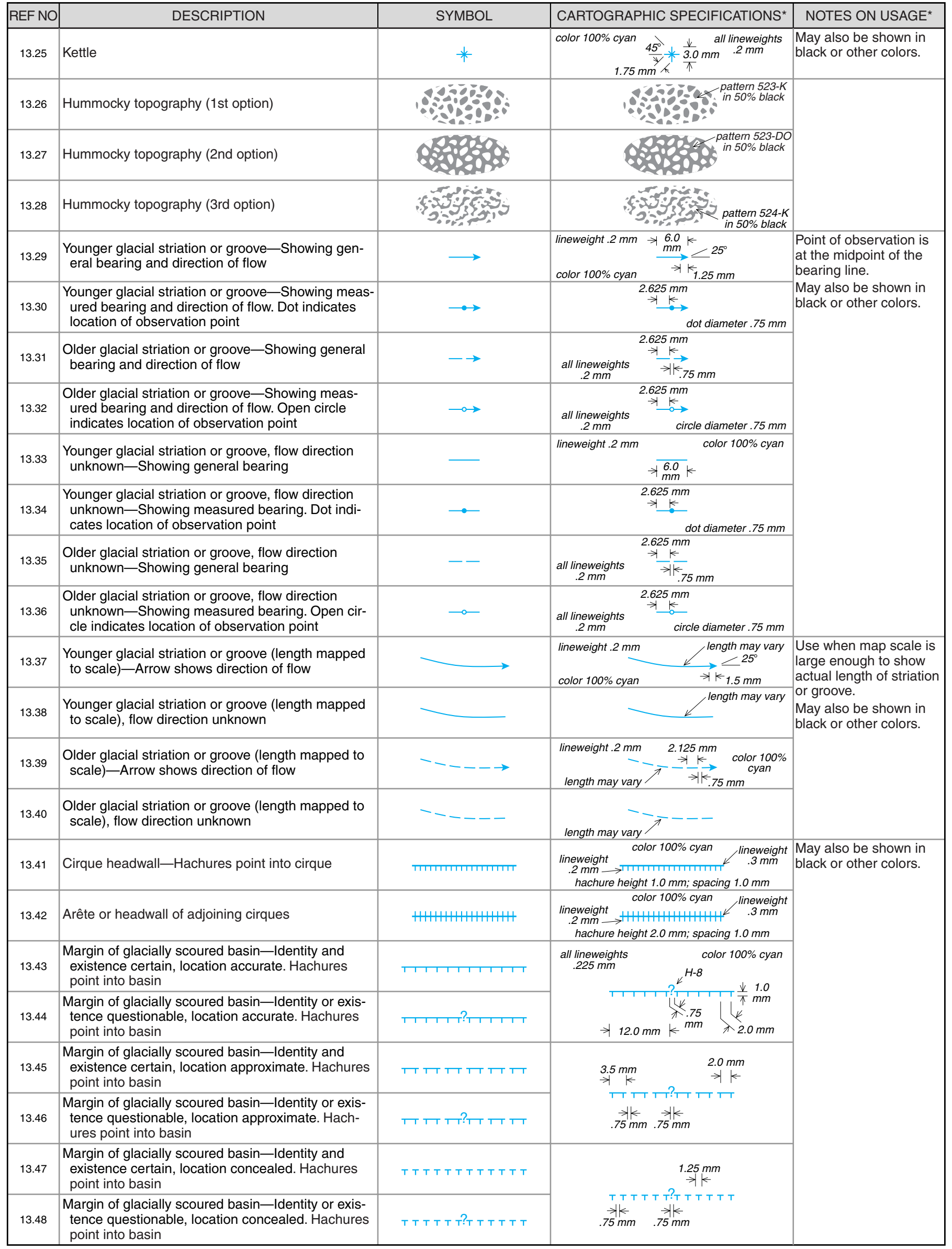

${ }^{*}$ For more information, see general guidelines on pages $A-i$ to $A-v$. 
Federal Geographic Data Committee (Doc. No. FGDC-STD-013-2006)

U.S. Geological Survey Techniques and Methods 11-A2 FGDC Digital Cartographic Standard for Geologic Map Symbolization (PostScript Implementation)

DOWNLOAD this Illustrator EPS file: Al8 / CS2

\section{3-GLACIAL AND GLACIOFLUVIAL FEATURES (continued)}

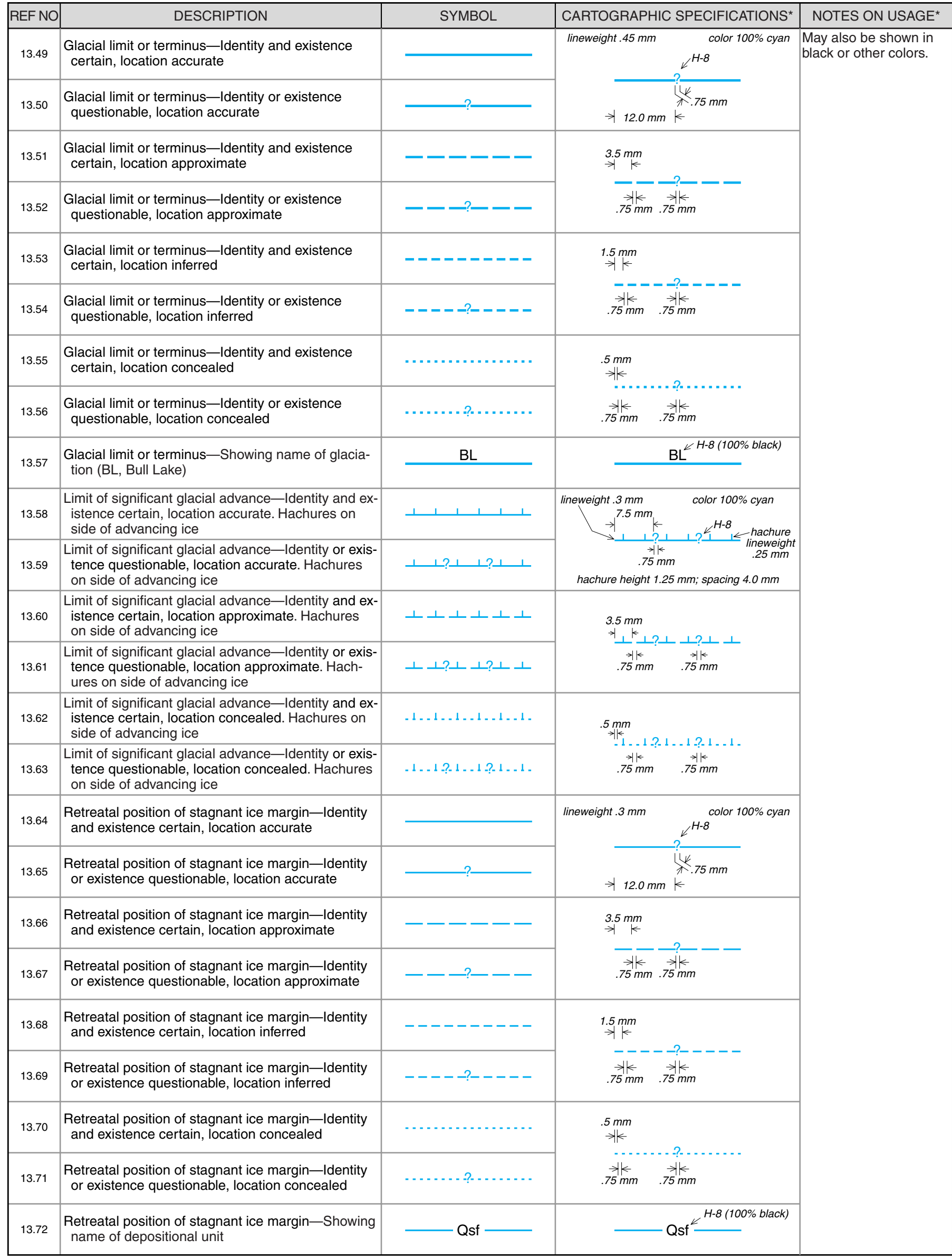


Federal Geographic Data Committee (Doc. No. FGDC-STD-013-2006)

U.S. Geological Survey Techniques and Methods 11-A2 FGDC Digital Cartographic Standard for Geologic Map Symbolization (PostScript Implementation)

14-PERIGLACIAL FEATURES

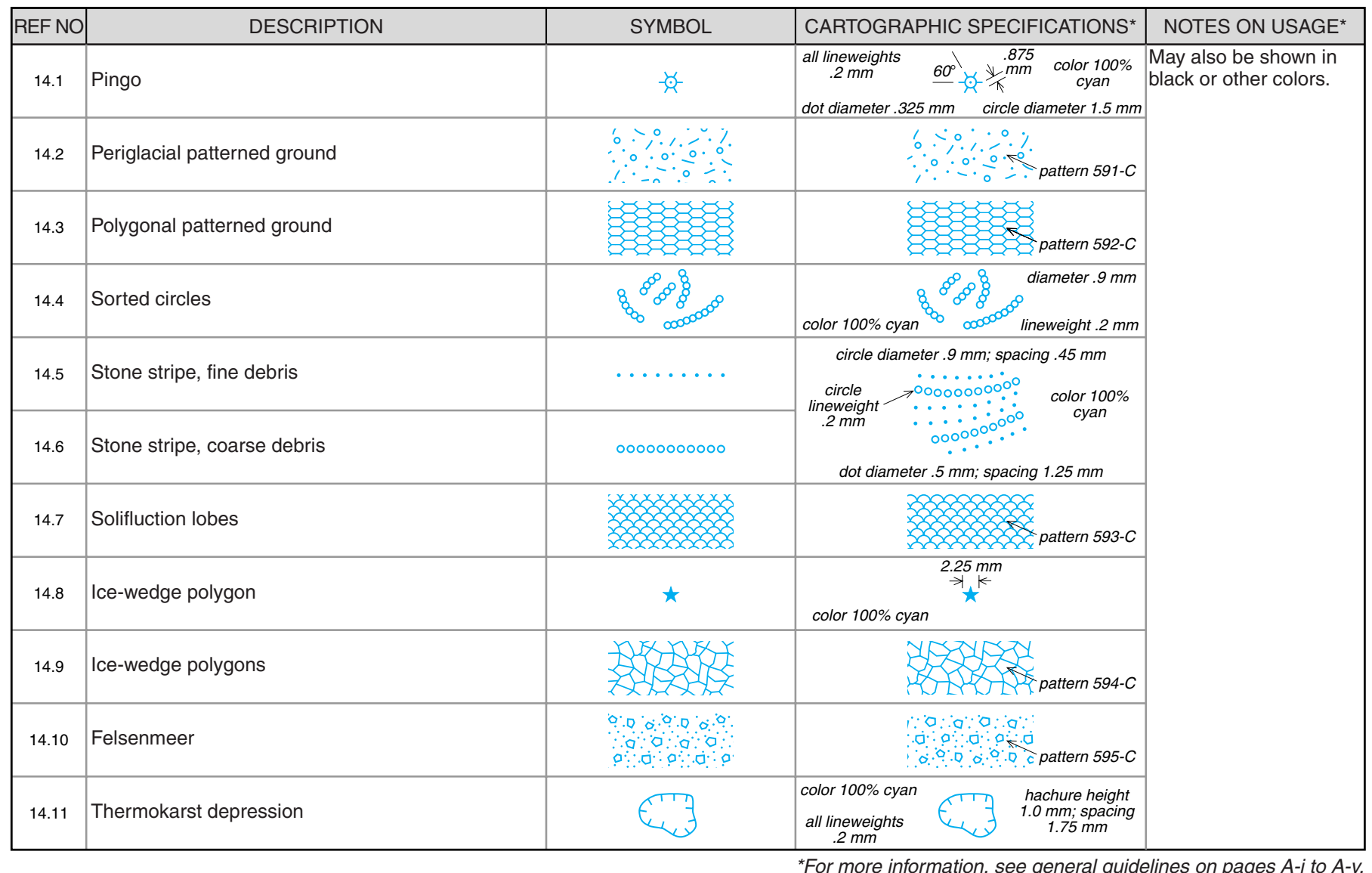


Federal Geographic Data Committee (Doc. No. FGDC-STD-013-2006)

U.S. Geological Survey Techniques and Methods 11-A2 FGDC Digital Cartographic Standard for Geologic Map Symbolization (PostScript Implementation) DOWNLOAD this Illustrator EPS file: Al8 / CS2

\section{5-LACUSTRINE AND MARINE FEATURES}

\begin{tabular}{|c|c|c|c|c|}
\hline REF NO & DESCRIPTION & SYMBOL & CARTOGRAPHIC SPECIFICATIONS* & NOTES ON USAGE* \\
\hline 15.1 & Beach & •......... & $\begin{array}{c}\text { color } 100 \% \text { cyan } \\
\qquad \ldots \ldots \ldots \ldots \\
\text { dot diameter } .75 \mathrm{~mm} \text {; spacing } .75 \mathrm{~mm}\end{array}$ & \multirow[t]{3}{*}{$\begin{array}{l}\text { May also be shown in } \\
\text { black or other colors. }\end{array}$} \\
\hline 15.2 & Beach ridges & & color $100 \%$ cyan lineweight $.2 \mathrm{~mm}$ & \\
\hline 15.3 & Marine-abrasion platform (1st option) & WIIIIIIIIIIIIIIIIII, & 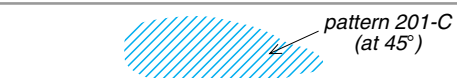 & \\
\hline 15.4 & Marine-abrasion platform (2nd option) & 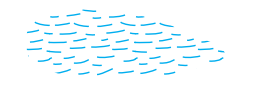 & & \\
\hline 15.5 & $\begin{array}{l}\text { Aggradational shoreline-Identity and existence } \\
\text { certain, location accurate. Triangles point offshore }\end{array}$ & $\overline{1111111}$ & \multirow{2}{*}{ 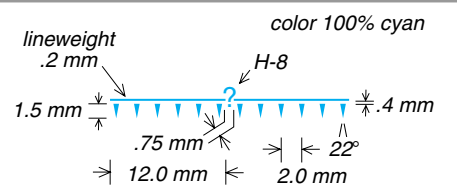 } & \\
\hline 15.6 & $\begin{array}{l}\text { Aggradational shoreline-Identity or existence } \\
\text { questionable, location accurate. Triangles point off- } \\
\text { shore }\end{array}$ & 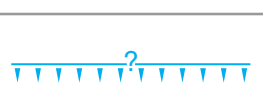 & & \\
\hline 15.7 & $\begin{array}{l}\text { Aggradational shoreline-Identity and existence } \\
\text { certain, location approximate. Triangles point off- } \\
\text { shore }\end{array}$ & ॠ ॠ ॠ ॠ & \multirow{2}{*}{ 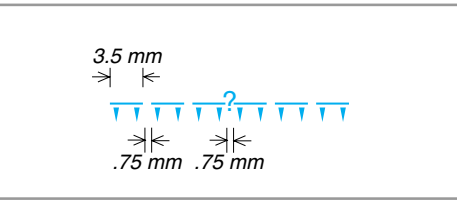 } & \\
\hline 15.8 & $\begin{array}{l}\text { Aggradational shoreline-Identity or existence } \\
\text { questionable, location approximate. Triangles point } \\
\text { offshore }\end{array}$ & $\overline{ा प ~ क ् ष ा प ~}$ & & \\
\hline 15.9 & $\begin{array}{l}\text { Erosional shoreline-Identity and existence certain, } \\
\text { location accurate. Triangles point onshore }\end{array}$ & I111111111 & \multirow{2}{*}{ 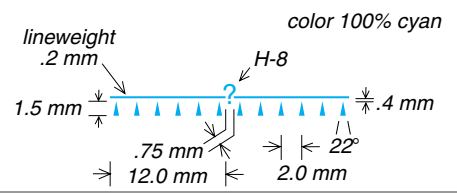 } & \\
\hline 15.10 & $\begin{array}{l}\text { Erosional shoreline-Identity or existence question- } \\
\text { able, location accurate. Triangles point onshore }\end{array}$ & $\overline{11111} ? \overline{11111}$ & & \\
\hline 15.11 & $\begin{array}{l}\text { Erosional shoreline-Identity and existence certain, } \\
\text { location approximate. Triangles point onshore }\end{array}$ & 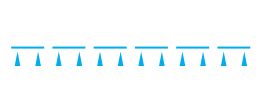 & \multirow{2}{*}{ 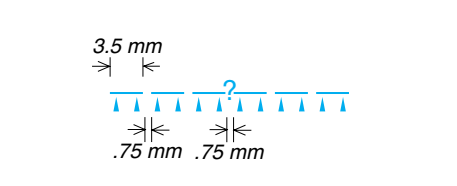 } & \\
\hline 15.12 & $\begin{array}{l}\text { Erosional shoreline-Identity or existence question- } \\
\text { able, location approximate. Triangles point on- } \\
\text { shore }\end{array}$ & $\overline{11} \overline{11} T \overline{11}$ & & \\
\hline 15.13 & $\begin{array}{l}\text { Former shoreline or marine limit-Identity and exis- } \\
\text { tence certain, location accurate }\end{array}$ & & \multirow[t]{2}{*}{ lineweight $.25 \mathrm{~mm} \quad \swarrow^{\mathrm{H}-8}$} & \\
\hline 15.14 & $\begin{array}{l}\text { Former shoreline or marine limit-Identity or exis- } \\
\text { tence questionable, location accurate }\end{array}$ & $?$ & & \\
\hline 15.15 & $\begin{array}{l}\text { Former shoreline or marine limit-Identity and exis- } \\
\text { tence certain, location approximate }\end{array}$ & ----- & \multirow{2}{*}{ 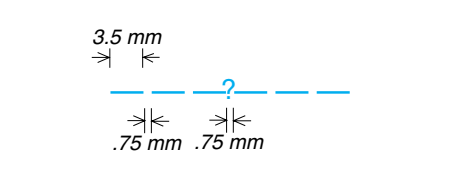 } & \\
\hline 15.16 & $\begin{array}{l}\text { Former shoreline or marine limit-Identity or exis- } \\
\text { tence questionable, location approximate }\end{array}$ & $---?--$ & & \\
\hline 15.17 & $\begin{array}{l}\text { Former shoreline or marine limit-Identity and exis- } \\
\text { tence certain, location inferred }\end{array}$ & ---------- & \multirow{2}{*}{ 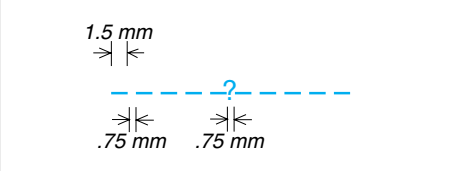 } & \\
\hline 15.18 & $\begin{array}{l}\text { Former shoreline or marine limit-Identity or exis- } \\
\text { tence questionable, location inferred }\end{array}$ & $-----?-----$ & & \\
\hline 15.19 & $\begin{array}{l}\text { Former shoreline or marine limit-Identity and exis- } \\
\text { tence certain, location concealed }\end{array}$ & $\cdots$ & \multirow{2}{*}{ 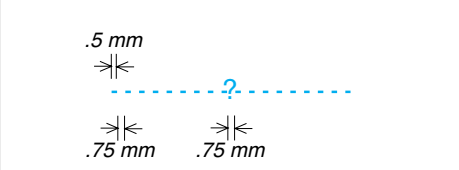 } & \\
\hline 15.20 & $\begin{array}{l}\text { Former shoreline or marine limit-Identity or exis- } \\
\text { tence questionable, location concealed }\end{array}$ & . & & \\
\hline 15.21 & $\begin{array}{l}\text { Former shoreline or marine limit-Showing name } \\
\text { (B, Bonneville) }\end{array}$ & $-\mathrm{B}-$ & $-\mathrm{B}^{\mathrm{K-8(100 \% \text {black } )}}$ & \\
\hline
\end{tabular}

${ }^{\star}$ For more information, see general guidelines on pages $A-i$ to $A-v$. 
Federal Geographic Data Committee (Doc. No. FGDC-STD-013-2006)

U.S. Geological Survey Techniques and Methods 11-A2 FGDC Digital Cartographic Standard for Geologic Map Symbolization (PostScript Implementation) DOWNLOAD this Illustrator EPS file: Al8 / CS2

\section{5-LACUSTRINE AND MARINE FEATURES (continued)}

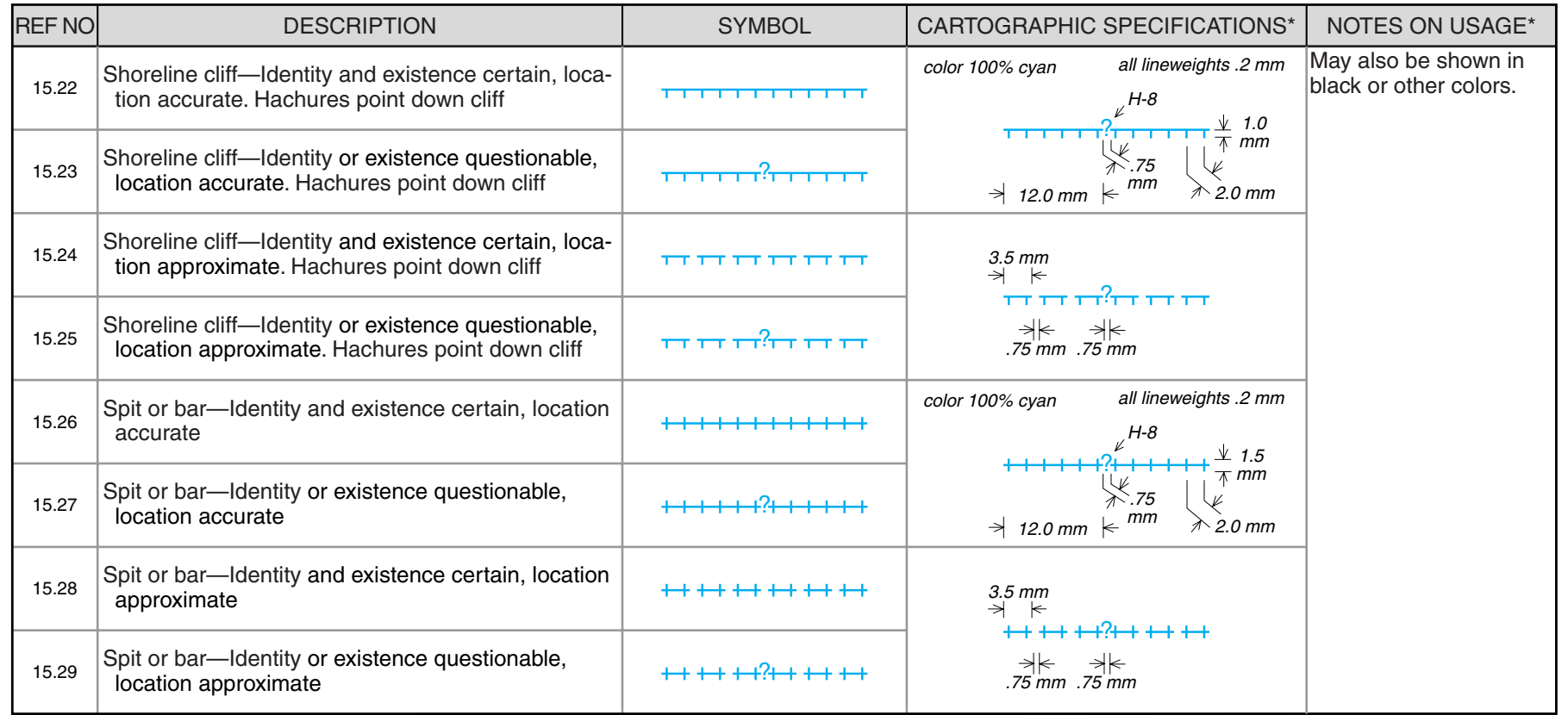

${ }^{*}$ For more information, see general guidelines on pages $A-i$ to $A-v$. 
Federal Geographic Data Committee (Doc. No. FGDC-STD-013-2006)

U.S. Geological Survey Techniques and Methods 11-A2 FGDC Digital Cartographic Standard for Geologic Map Symbolization (PostScript Implementation)

\section{6-EOLIAN FEATURES}

\begin{tabular}{|c|c|c|c|c|}
\hline REF NO & DESCRIPTION & SYMBOL & CARTOGRAPHIC SPECIFICATIONS* & NOTES ON USAGE* \\
\hline 16.1 & Dune crest & & lineweight $.25 \mathrm{~mm} \quad \begin{array}{c}375 \mathrm{~mm} \text {; } \\
\text { space } .3 \mathrm{~mm}\end{array}$ & \multirow[t]{2}{*}{$\begin{array}{l}\text { Dune forms shown by } \\
\text { traces of dune crests. }\end{array}$} \\
\hline 16.2 & $\begin{array}{l}\text { Scarp on dune crest, caused by slip-Hachures } \\
\text { point down slip face of dune }\end{array}$ & & $\begin{array}{l}\text { hachure lineweight } .2 \mathrm{~mm} \text {; } \\
\text { height } 1.0 \mathrm{~mm} \text {; spacing } \\
4.75 \mathrm{~mm}\end{array}$ & \\
\hline 16.3 & $\begin{array}{l}\text { Blowout rim around closed depression of eolian } \\
\text { origin in dune field-Hachures point into closed } \\
\text { depression }\end{array}$ & & $\begin{array}{l}\text { all lineweights.15 mm } 15 \text {. long dash } 1.4 \mathrm{~mm} \text {; } \\
\text { hachure } \\
\text { height } 875 \mathrm{~mm} ; \\
\text { spacing } 3.5 \mathrm{~mm}\end{array}$ & \\
\hline 16.4 & $\begin{array}{l}\text { Blowout rim around closed depression of eolian } \\
\text { origin in bedrock-Accurately located. Hachures } \\
\text { point into closed depression }\end{array}$ & & $\begin{array}{l}\text { all lineweights } \\
.2 \mathrm{~mm}\end{array}$ & \\
\hline 16.5 & $\begin{array}{l}\text { Blowout rim around closed depression of eolian } \\
\text { origin in bedrock-Approximately located. Hach- } \\
\text { ures point into closed depression }\end{array}$ & & $2.5 \underset{\pi}{\underline{w}}$ & \\
\hline 16.6 & $\begin{array}{l}\text { Edge of dry lakebed within closed depression of } \\
\text { eolian origin in bedrock }\end{array}$ & & $\begin{array}{l}\text { lineweight } .15 \mathrm{~mm} \\
\text { dash length } 1.5 \mathrm{~mm} \text {; } \\
\text { space. } 375 \mathrm{~mm}\end{array}$ & \\
\hline 16.7 & $\begin{array}{l}\text { Sediment transport direction determined from dune } \\
\text { forms }\end{array}$ & $\leftrightarrow$ & 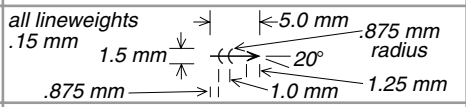 & $\begin{array}{l}\text { Point of observation is } \\
\text { at the midpoint of the } \\
\text { bearing line. }\end{array}$ \\
\hline 16.8 & $\begin{array}{l}\text { Sediment transport direction determined from dune } \\
\text { bedding in horizontal section }\end{array}$ & 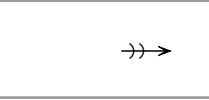 & 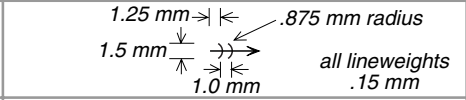 & \\
\hline 16.9 & $\begin{array}{l}\text { Sediment transport direction determined from } \\
\text { eolian crossbedding in vertical or near-vertical } \\
\text { section }\end{array}$ & $\stackrel{\leftrightarrow}{\longrightarrow}$ & 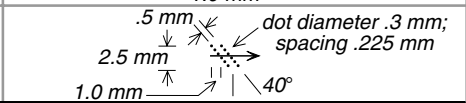 & \\
\hline
\end{tabular}


Federal Geographic Data Committee (Doc. No. FGDC-STD-013-2006)

U.S. Geological Survey Techniques and Methods 11-A2 FGDC Digital Cartographic Standard for Geologic Map Symbolization (PostScript Implementation)

DOWNLOAD this Illustrator EPS file: Al8 / $\underline{\mathrm{CS}}$

\section{7-LANDSLIDE AND MASS-WASTING FEATURES}

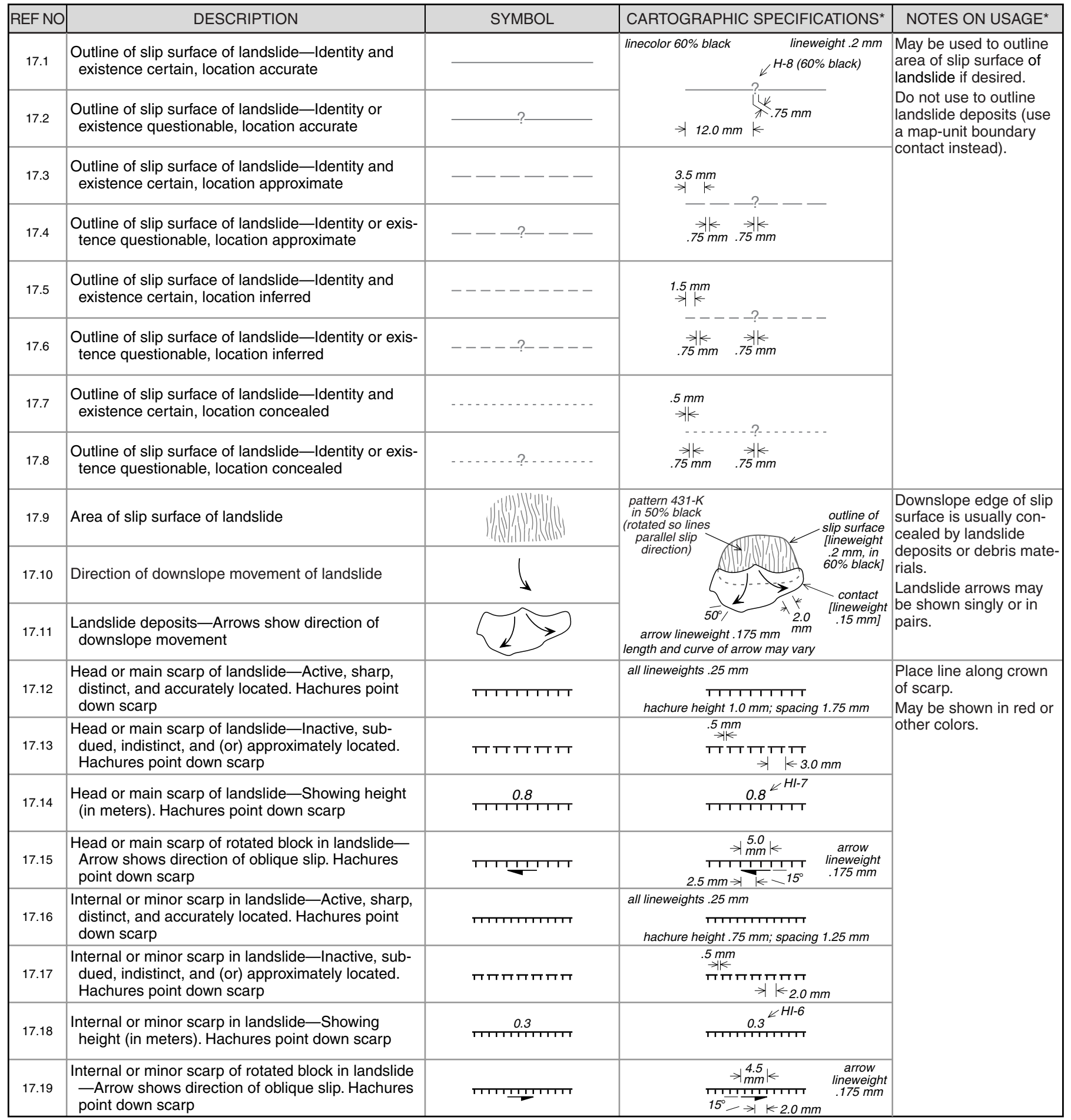

${ }^{*}$ For more information, see general guidelines on pages $A-i$ to $A-v$. 


\section{7-LANDSLIDE AND MASS-WASTING FEATURES (continued)}

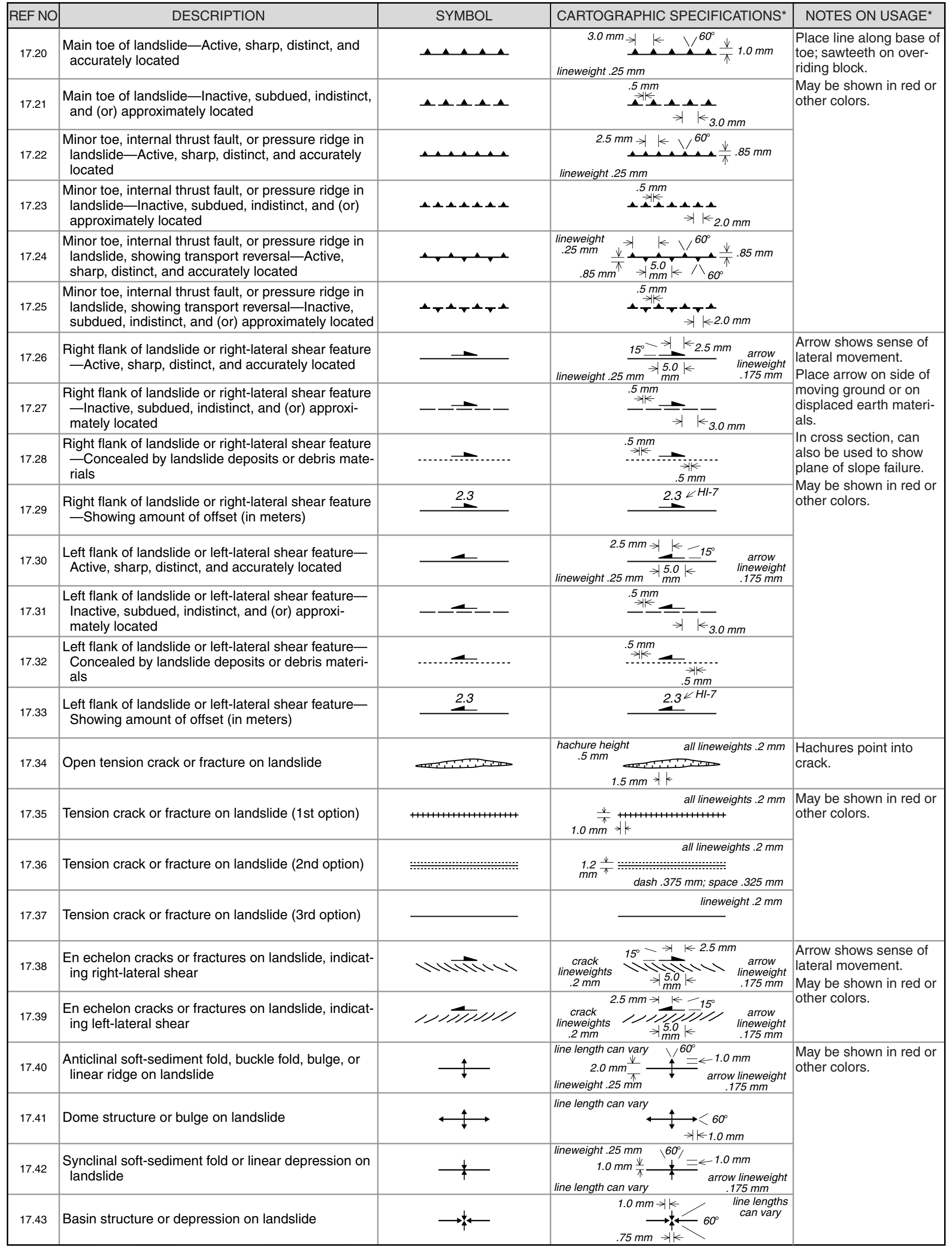


Federal Geographic Data Committee (Doc. No. FGDC-STD-013-2006)

U.S. Geological Survey Techniques and Methods 11-A2

FGDC Digital Cartographic Standard for Geologic Map Symbolization (PostScript Implementation)

DOWNLOAD this Illustrator EPS file: Al8 / CS2

\section{7-LANDSLIDE AND MASS-WASTING FEATURES (continued)}

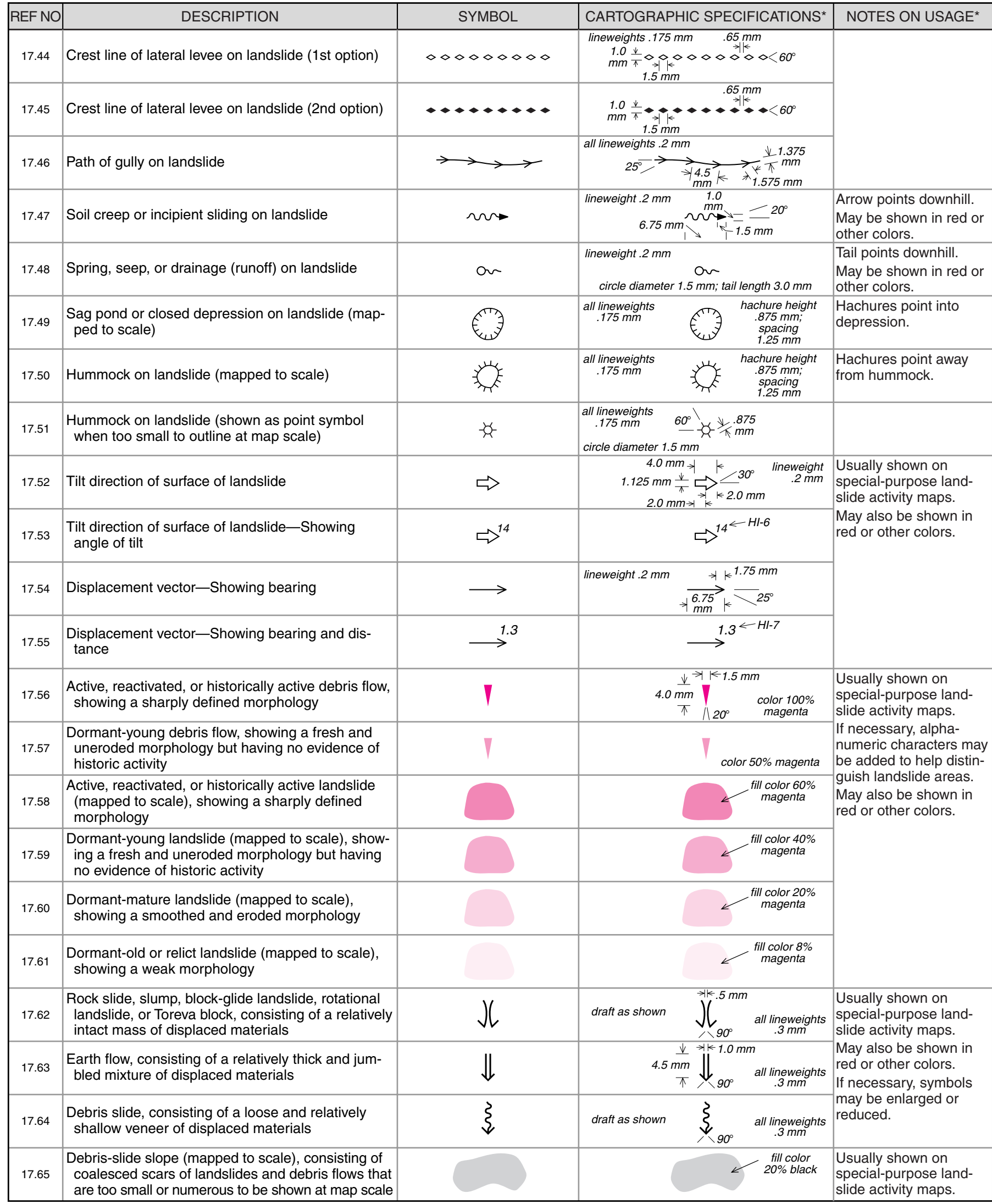

${ }^{*}$ For more information, see general guidelines on pages $A-i$ to $A-v$. 
Federal Geographic Data Committee (Doc. No. FGDC-STD-013-2006)

U.S. Geological Survey Techniques and Methods 11-A2

FGDC Digital Cartographic Standard for Geologic Map Symbolization (PostScript Implementation)

DOWNLOAD this Illustrator EPS file: Al8 / CS2

\section{8-VOLCANIC FEATURES}

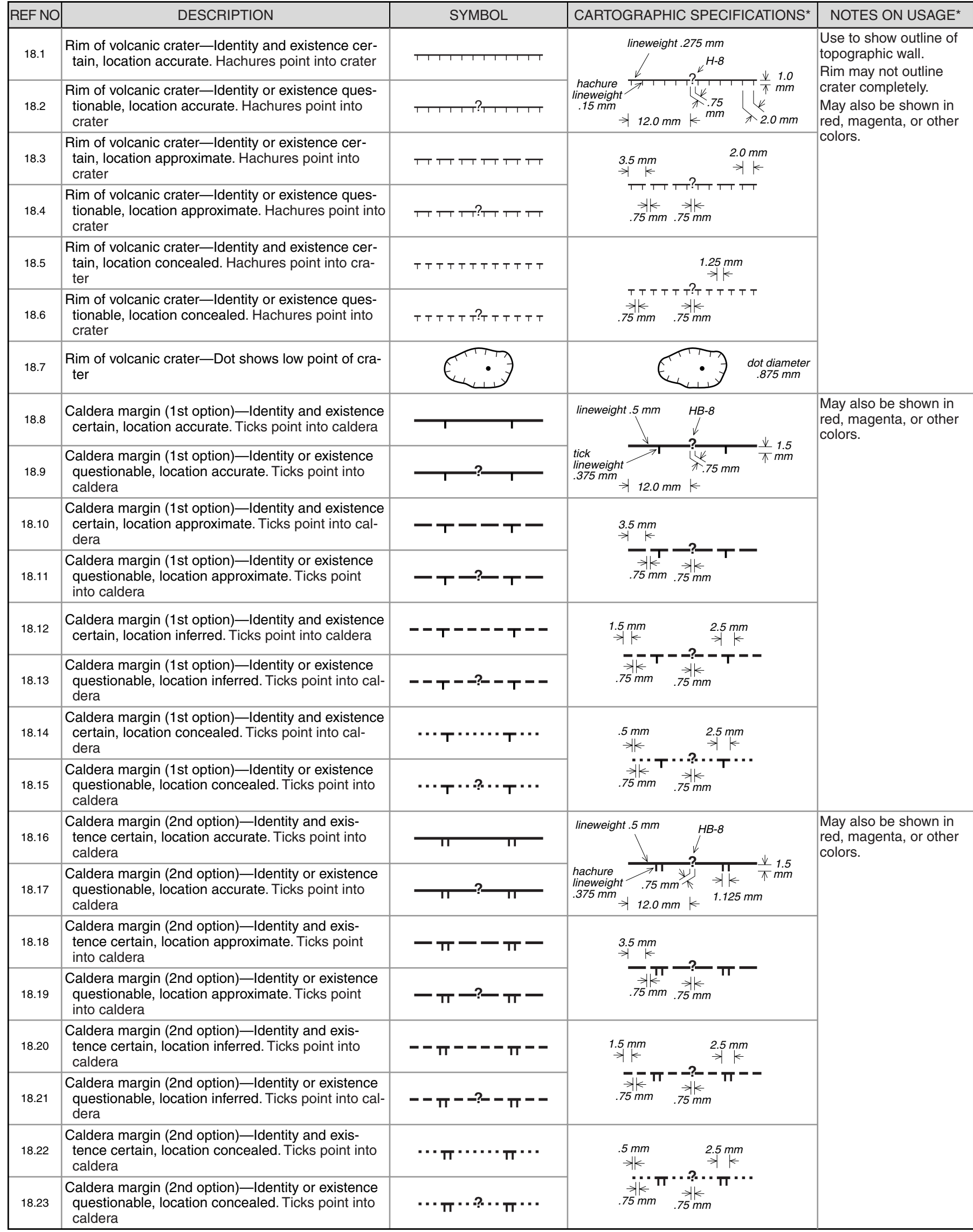

${ }^{*}$ For more information, see general guidelines on pages $A-i$ to $A-v$. 
Federal Geographic Data Committee (Doc. No. FGDC-STD-013-2006)

U.S. Geological Survey Techniques and Methods 11-A2

FGDC Digital Cartographic Standard for Geologic Map Symbolization (PostScript Implementation)

DOWNLOAD this Illustrator EPS file: Al8 / $\underline{\text { CS2 }}$

\section{8-VOLCANIC FEATURES (continued)}

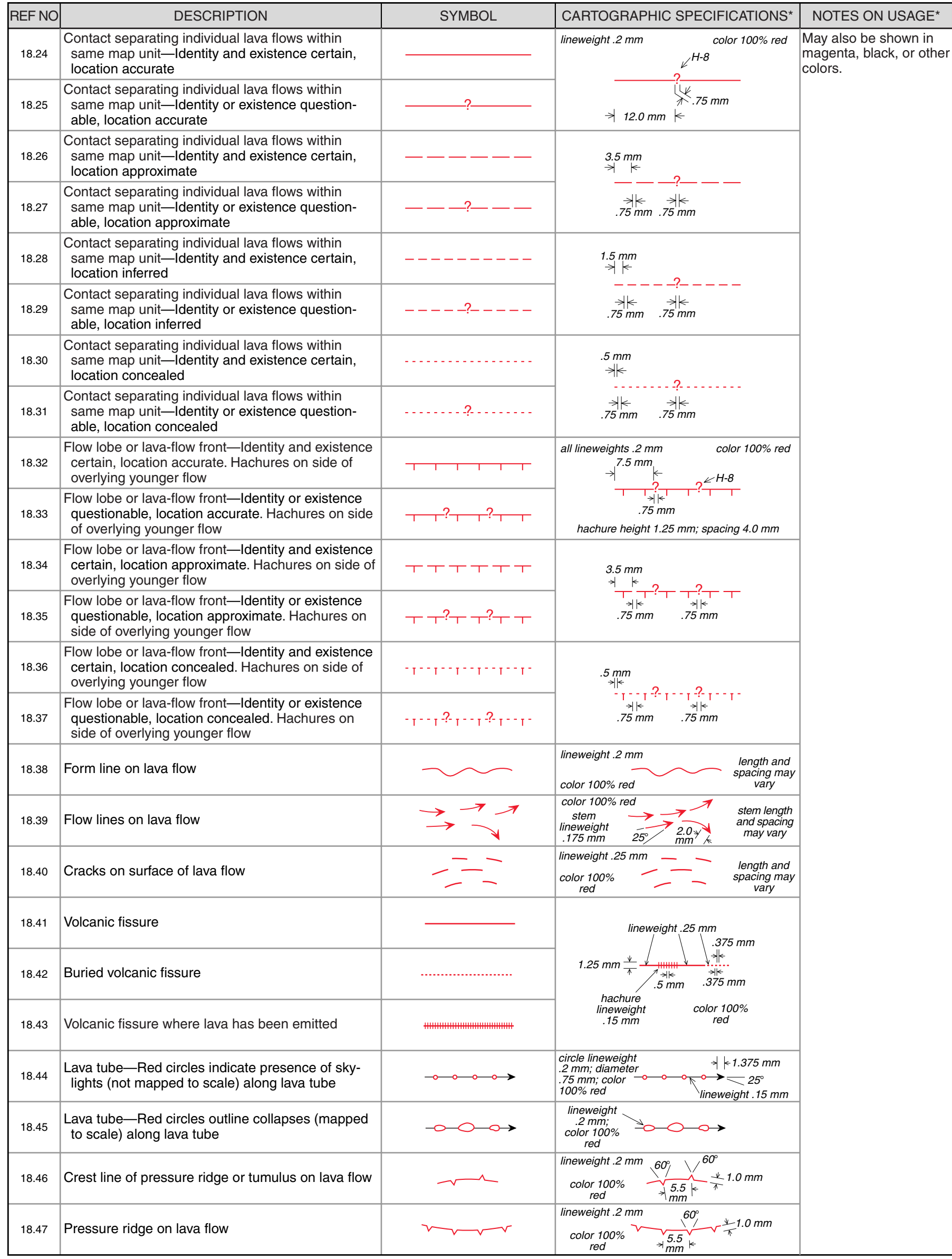

${ }^{*}$ For more information, see general guidelines on pages $A-i$ to $A-v$. 
Federal Geographic Data Committee (Doc. No. FGDC-STD-013-2006)

U.S. Geological Survey Techniques and Methods 11-A2

FGDC Digital Cartographic Standard for Geologic Map Symbolization (PostScript Implementation)

DOWNLOAD this Illustrator EPS file: Al8 / $\underline{\mathrm{CS}}$

\section{8-VOLCANIC FEATURES (continued)}

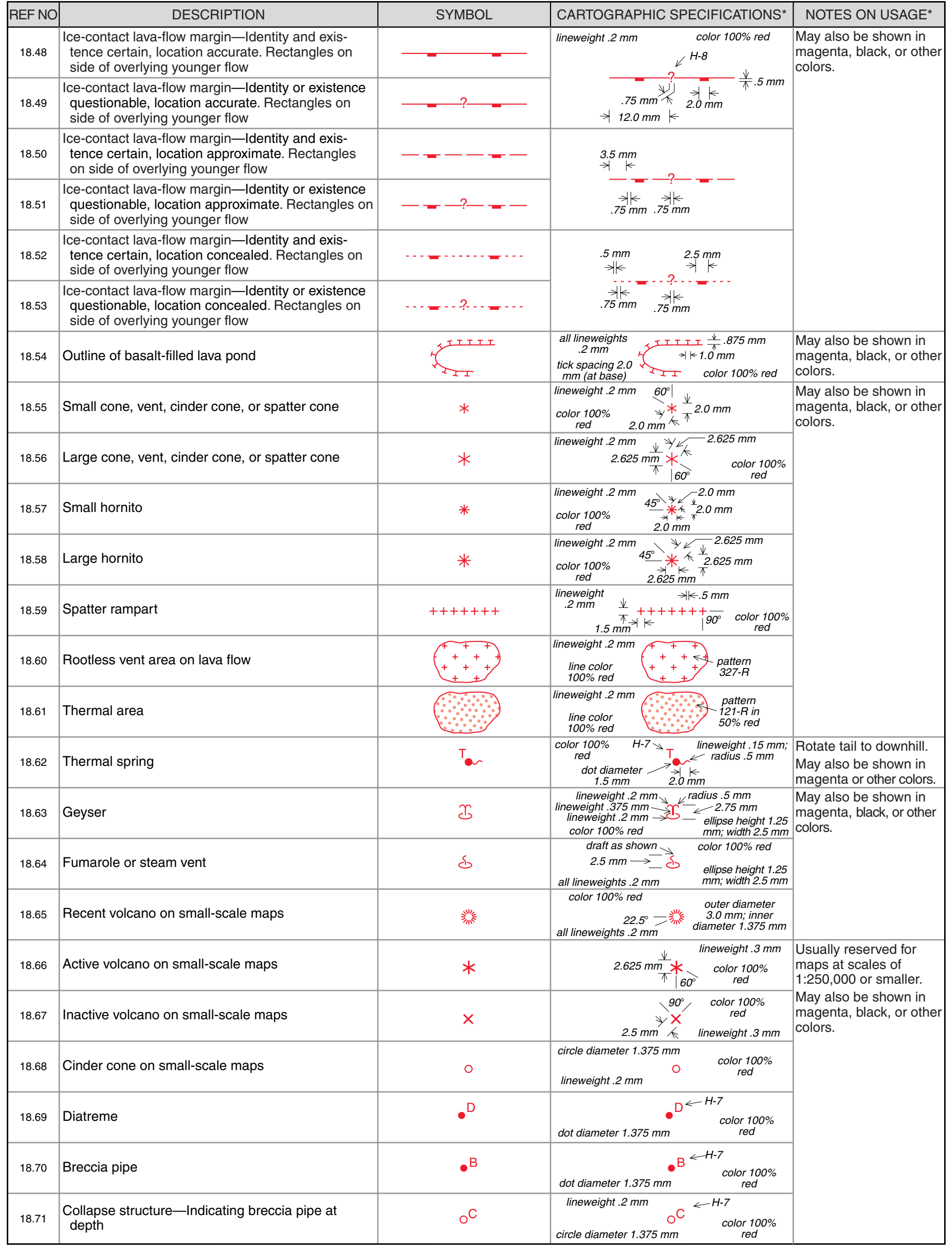

${ }^{*}$ For more information, see general guidelines on pages $A-i$ to $A-v$. 
Federal Geographic Data Committee (Doc. No. FGDC-STD-013-2006)

U.S. Geological Survey Techniques and Methods 11-A2

FGDC Digital Cartographic Standard for Geologic Map Symbolization (PostScript Implementation)

DOWNLOAD this Illustrator EPS file: Al8 / $\underline{\mathrm{CS} 2}$

\section{9-NATURAL RESOURCES}

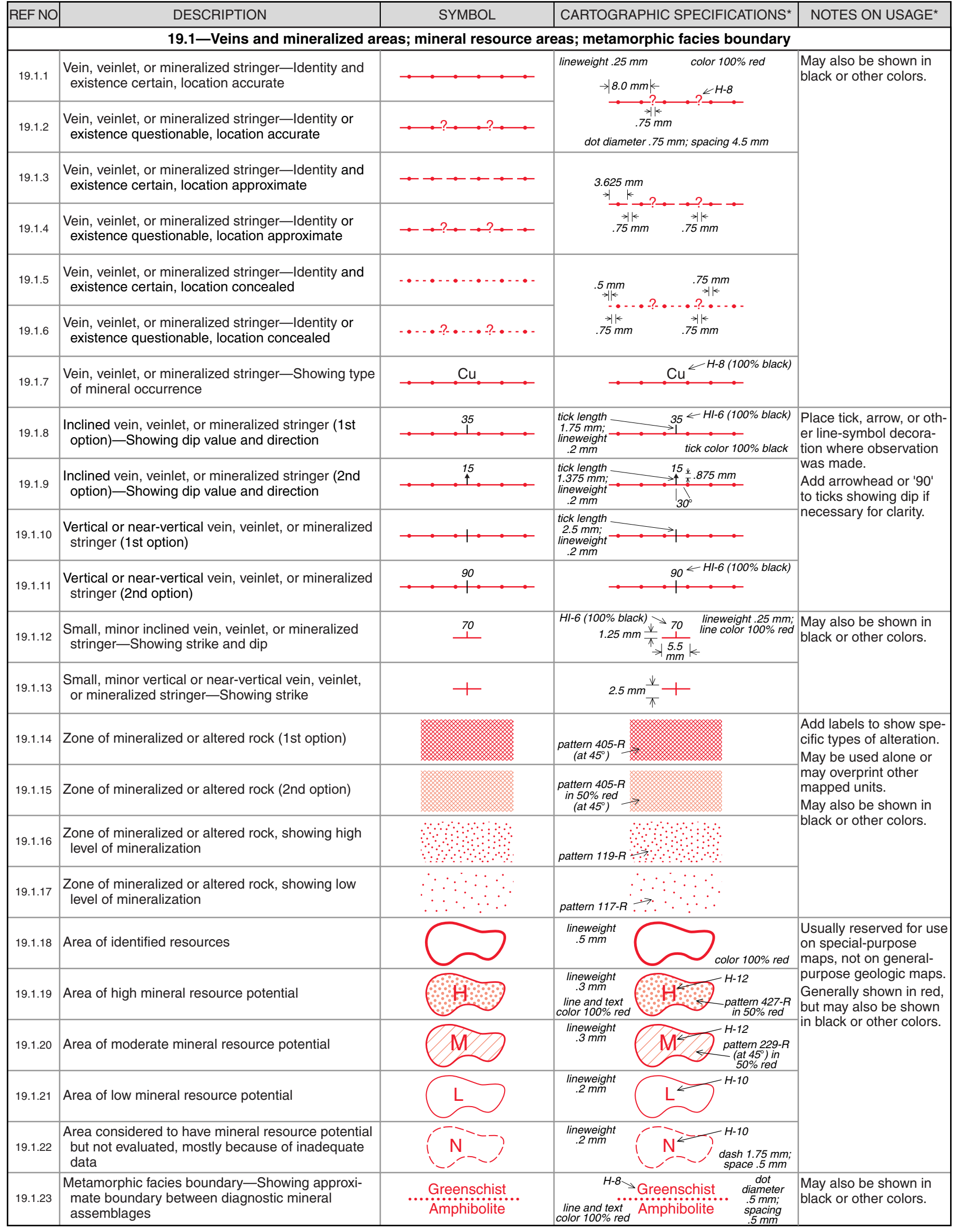

${ }^{*}$ For more information, see general guidelines on pages $A-i$ to $A-v$. 
Federal Geographic Data Committee (Doc. No. FGDC-STD-013-2006)

U.S. Geological Survey Techniques and Methods 11-A2 FGDC Digital Cartographic Standard for Geologic Map Symbolization (PostScript Implementation)

\section{9-NATURAL RESOURCES (continued)}

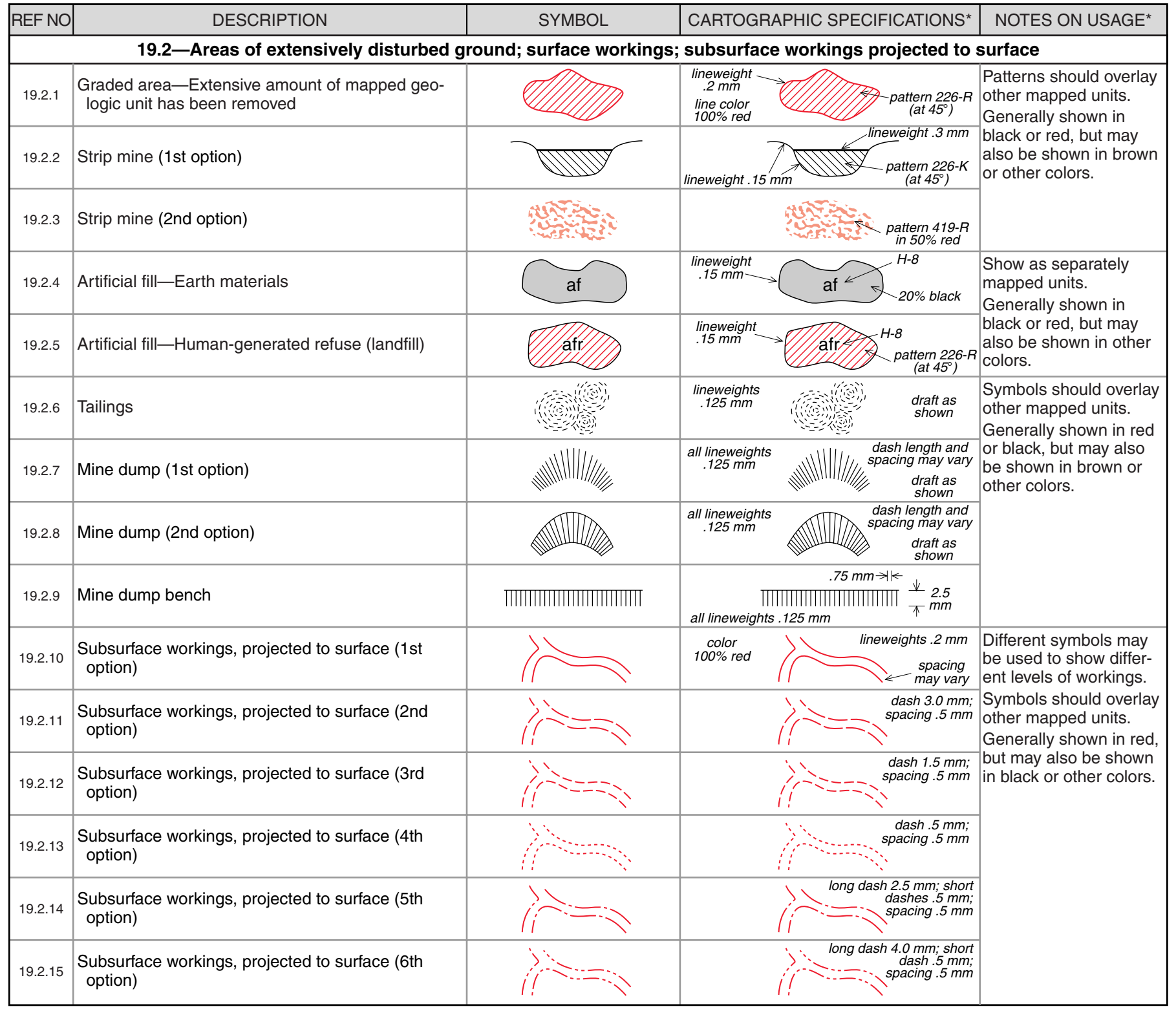

${ }^{*}$ For more information, see general guidelines on pages $A-i$ to $A-v$. 
Federal Geographic Data Committee (Doc. No. FGDC-STD-013-2006)

U.S. Geological Survey Techniques and Methods 11-A2

FGDC Digital Cartographic Standard for Geologic Map Symbolization (PostScript Implementation)

DOWNLOAD this Illustrator EPS file: Al8 / $\underline{\text { CS2 }}$

\section{9-NATURAL RESOURCES (continued)}

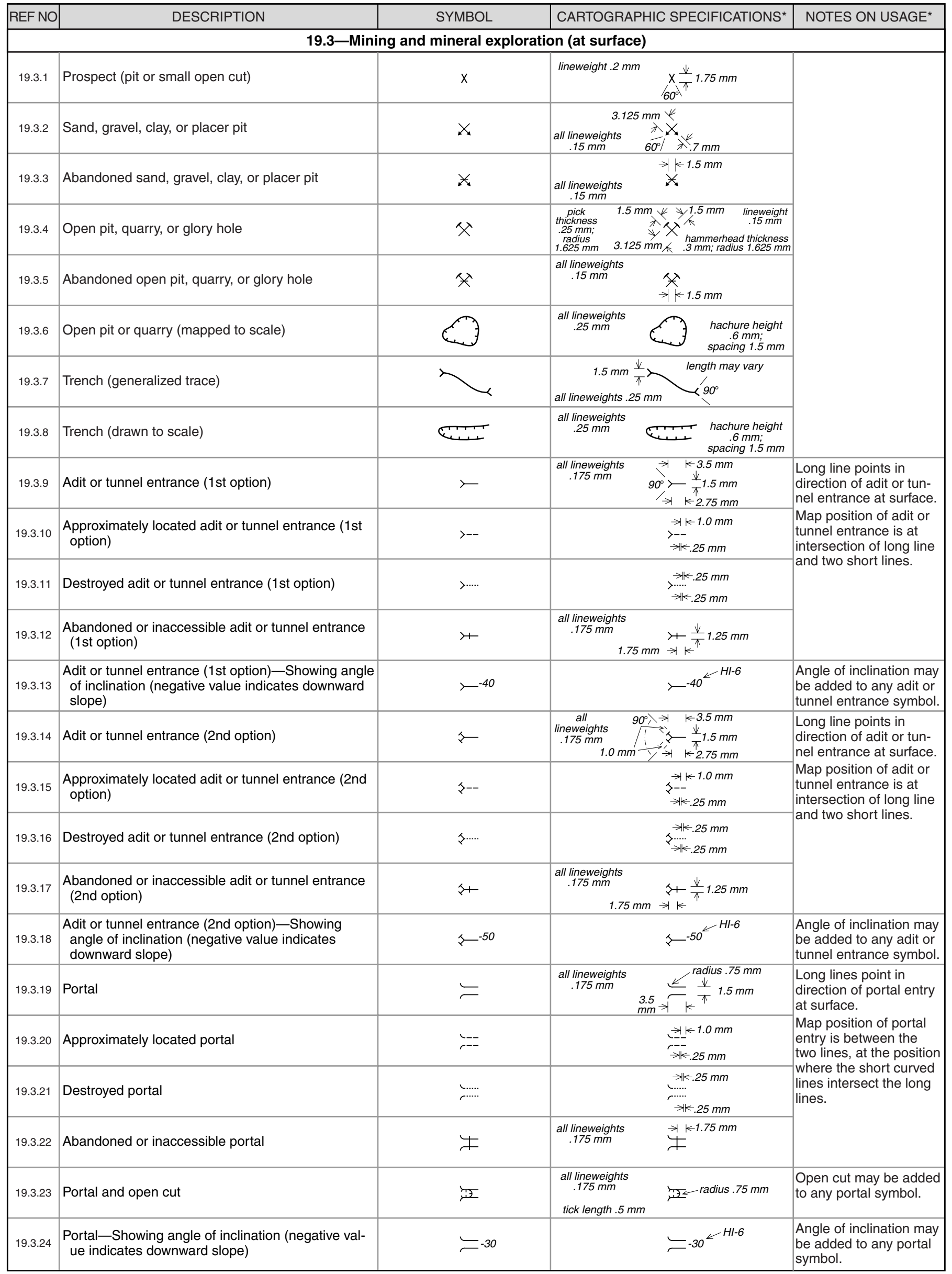


Federal Geographic Data Committee (Doc. No. FGDC-STD-013-2006)

U.S. Geological Survey Techniques and Methods 11-A2 FGDC Digital Cartographic Standard for Geologic Map Symbolization (PostScript Implementation)

\section{9-NATURAL RESOURCES (continued)}

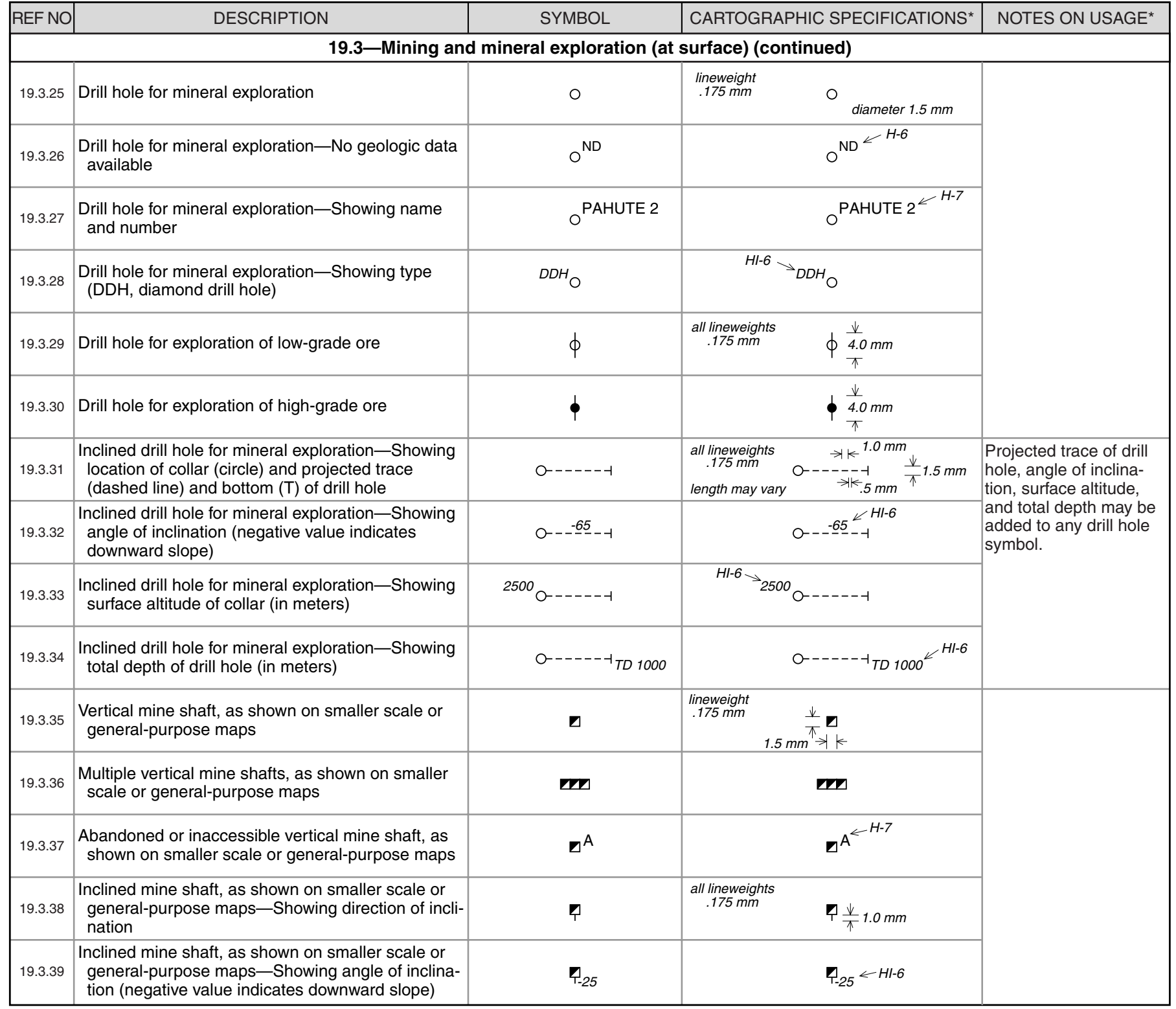

${ }^{*}$ For more information, see general guidelines on pages $A-i$ to $A-v$. 
Federal Geographic Data Committee (Doc. No. FGDC-STD-013-2006)

U.S. Geological Survey Techniques and Methods 11-A2 FGDC Digital Cartographic Standard for Geologic Map Symbolization (PostScript Implementation)

DOWNLOAD this Illustrator EPS file: $\underline{\mathrm{Al} 8}$ / $\underline{\mathrm{CS} 2}$

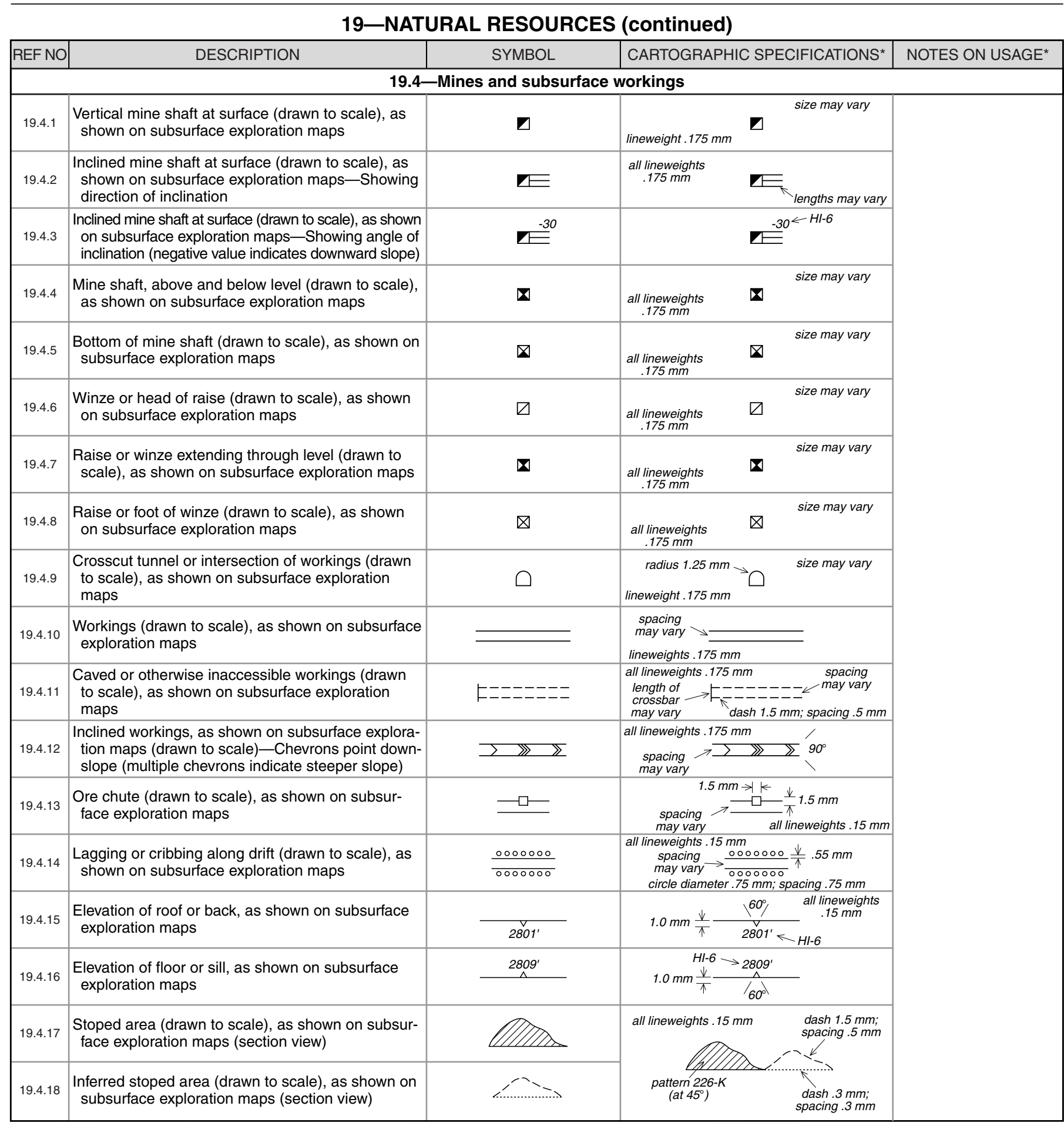

${ }^{*}$ For more information, see general guidelines on pages $A-i$ to $A-v$. 
Federal Geographic Data Committee (Doc. No. FGDC-STD-013-2006)

U.S. Geological Survey Techniques and Methods 11-A2 FGDC Digital Cartographic Standard for Geologic Map Symbolization (PostScript Implementation)

\section{9-NATURAL RESOURCES (continued)}

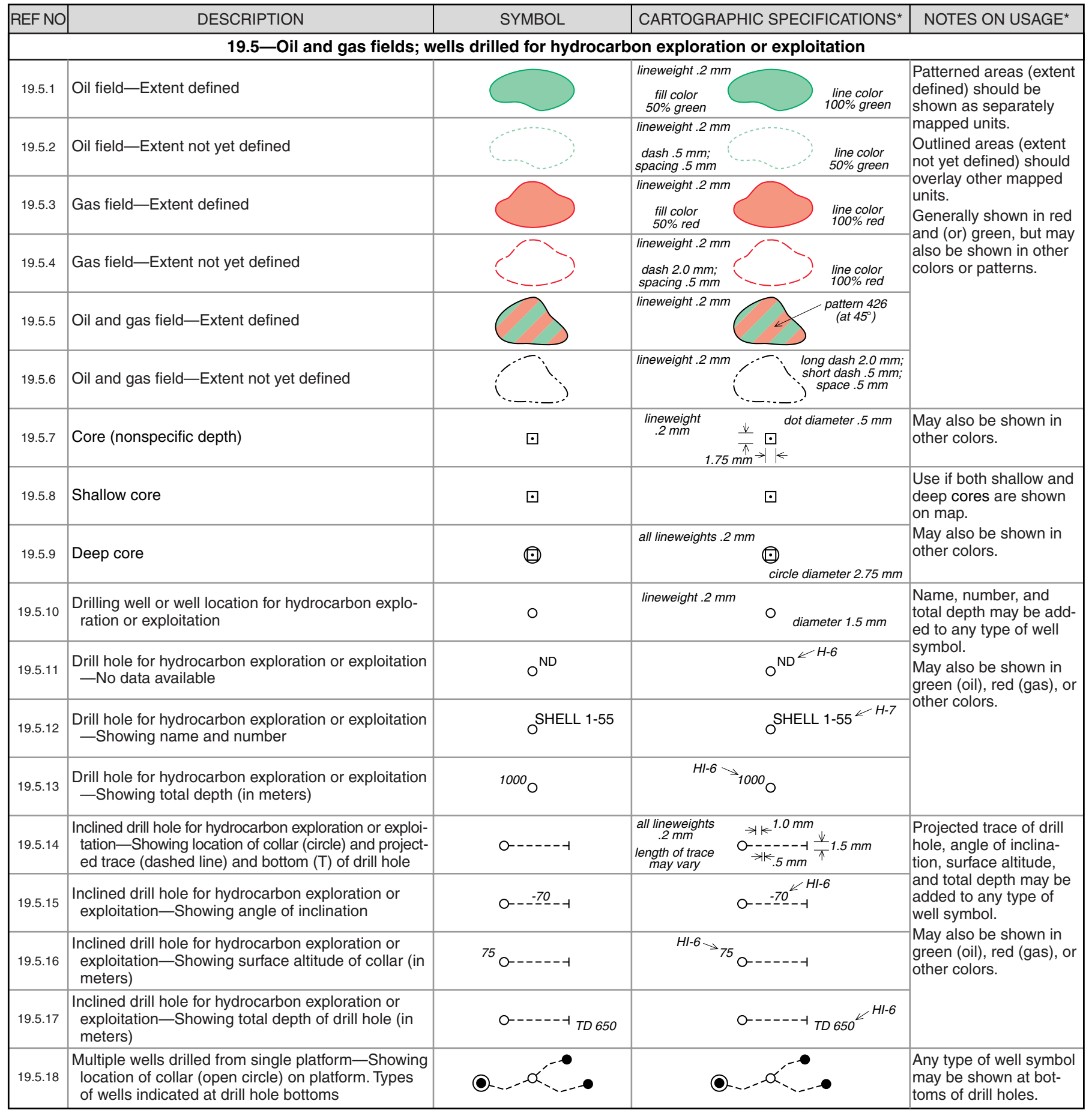


Federal Geographic Data Committee (Doc. No. FGDC-STD-013-2006)

U.S. Geological Survey Techniques and Methods 11-A2 FGDC Digital Cartographic Standard for Geologic Map Symbolization (PostScript Implementation)

DOWNLOAD this Illustrator EPS file: $\underline{\mathrm{Al} 8}$ / $\underline{\mathrm{CS} 2}$

\section{9-NATURAL RESOURCES (continued)}

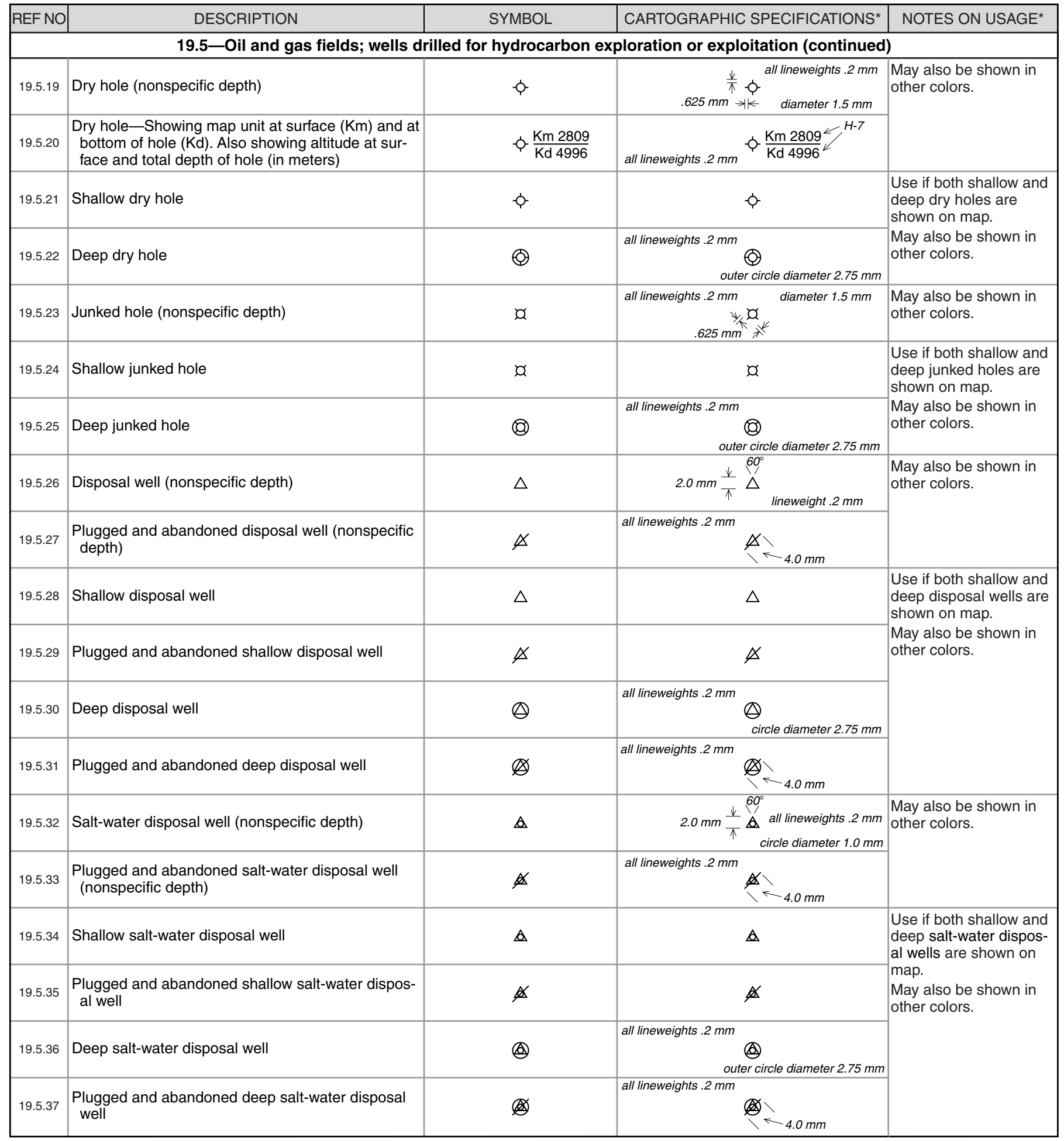

${ }^{*}$ For more information, see general guidelines on pages $A-i$ to $A-v$. 
Federal Geographic Data Committee (Doc. No. FGDC-STD-013-2006)

U.S. Geological Survey Techniques and Methods 11-A2 FGDC Digital Cartographic Standard for Geologic Map Symbolization (PostScript Implementation)

DOWNLOAD this Illustrator EPS file: Al8 / $\underline{\mathrm{CS} 2}$

\section{9-NATURAL RESOURCES (continued)}

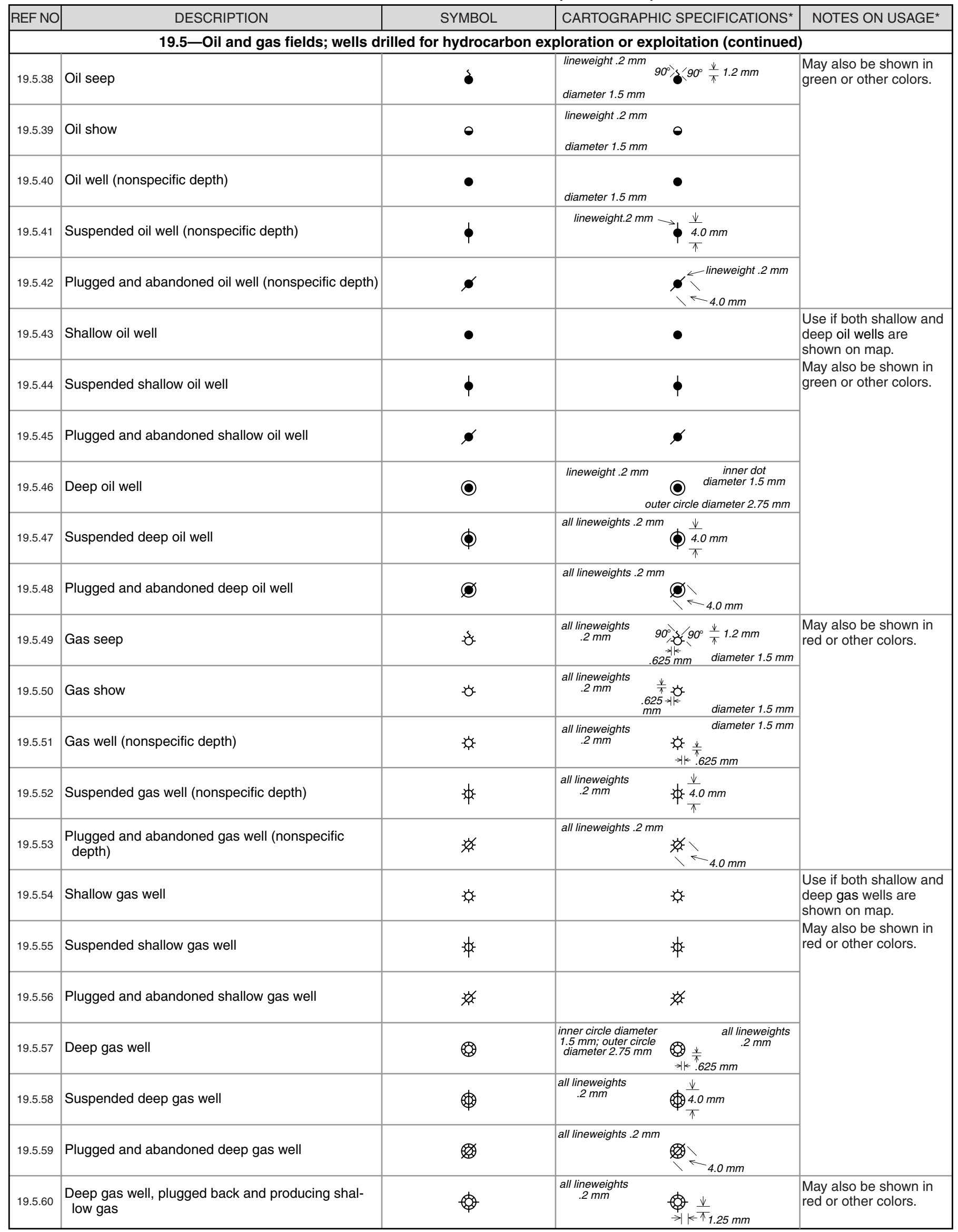


Federal Geographic Data Committee (Doc. No. FGDC-STD-013-2006)

U.S. Geological Survey Techniques and Methods 11-A2 FGDC Digital Cartographic Standard for Geologic Map Symbolization (PostScript Implementation)

DOWNLOAD this Illustrator EPS file: Al8 / $\underline{\mathrm{CS} 2}$

\section{9-NATURAL RESOURCES (continued)}

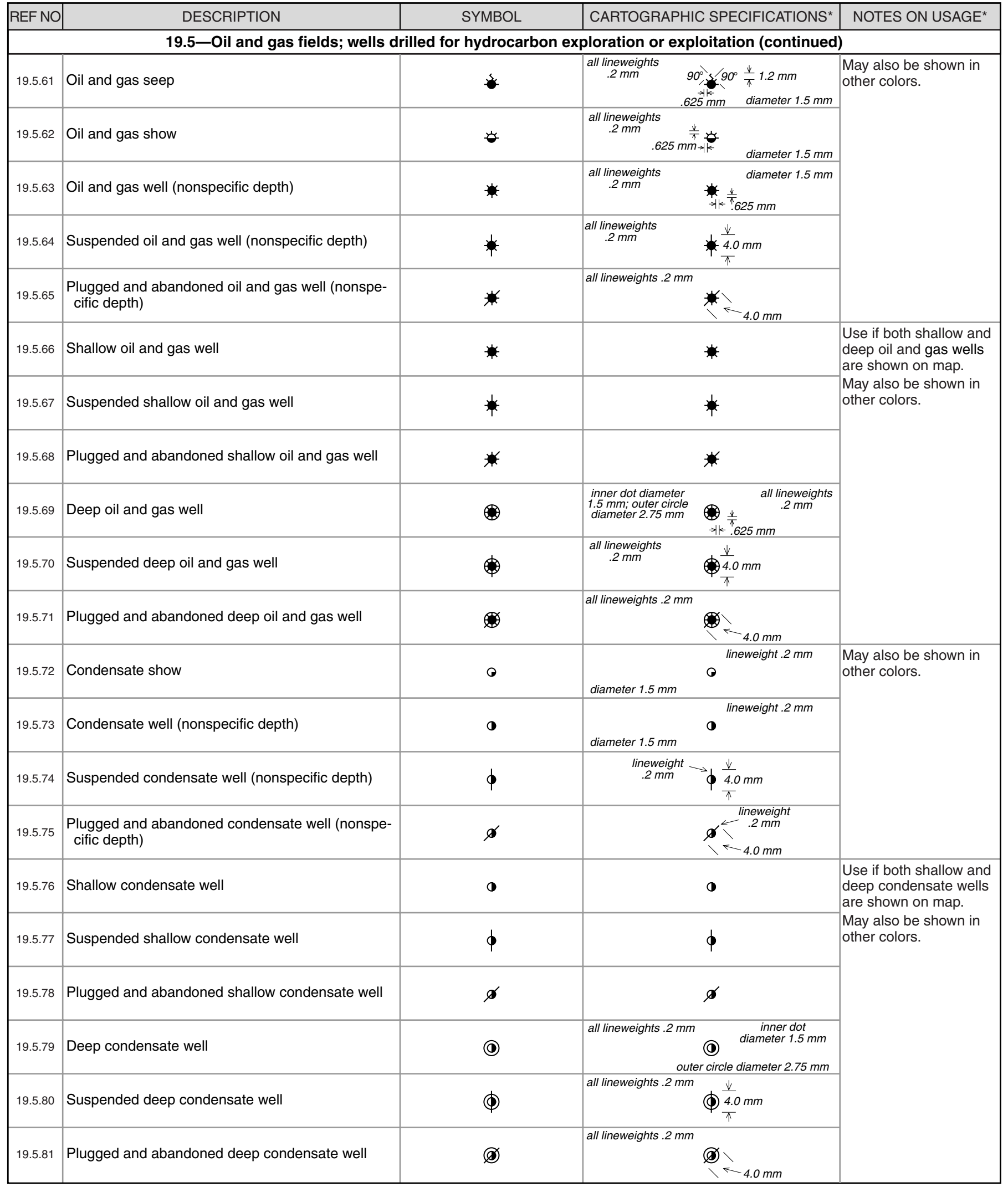

${ }^{*}$ For more information, see general guidelines on pages $A-i$ to $A-v$. 
Federal Geographic Data Committee (Doc. No. FGDC-STD-013-2006)

U.S. Geological Survey Techniques and Methods 11-A2 FGDC Digital Cartographic Standard for Geologic Map Symbolization (PostScript Implementation)

DOWNLOAD this Illustrator EPS file: Al8 / $\underline{\mathrm{CS} 2}$

\section{9-NATURAL RESOURCES (continued)}

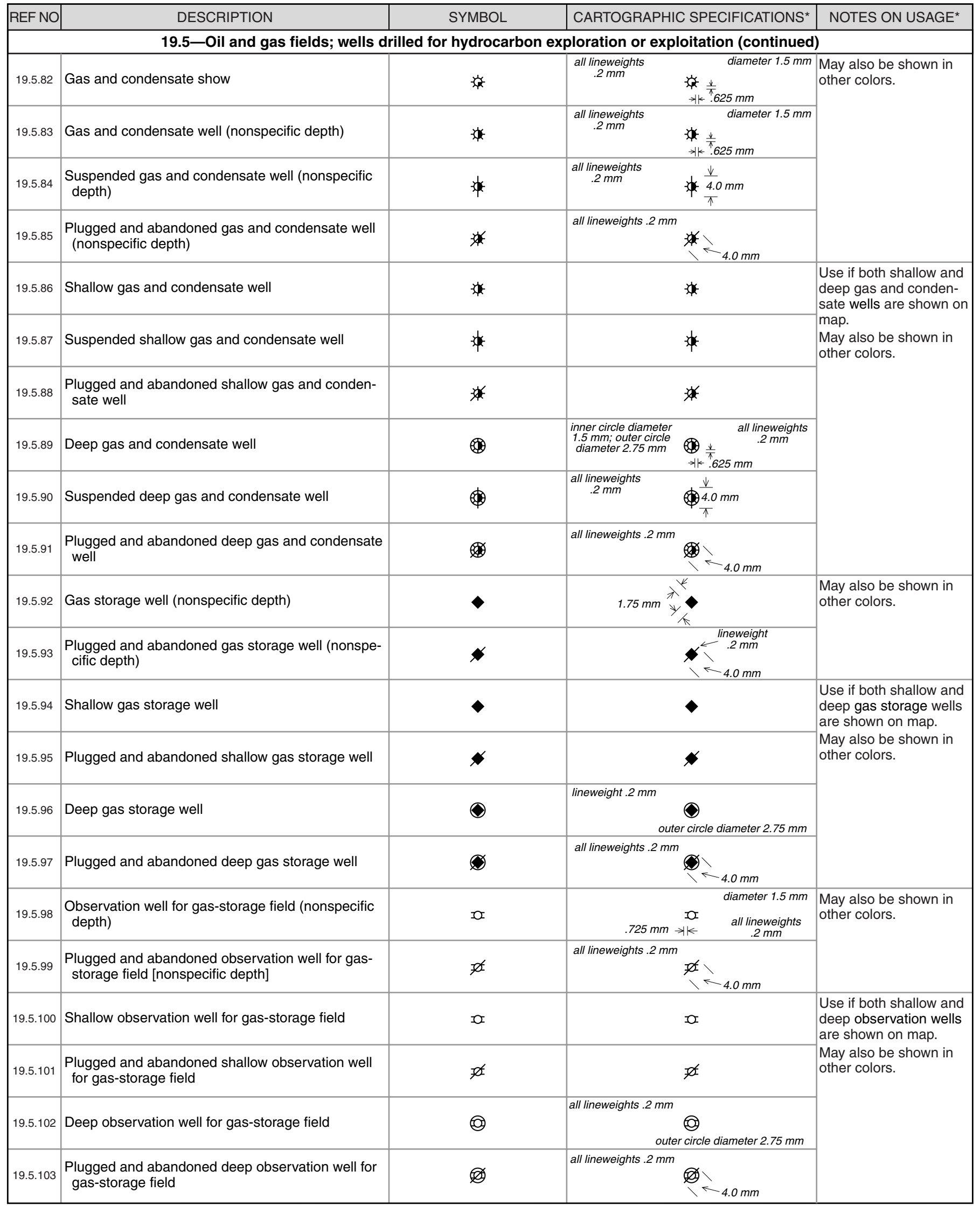

${ }^{*}$ For more information, see general guidelines on pages $A-i$ to $A-v$. 
Federal Geographic Data Committee (Doc. No. FGDC-STD-013-2006)

U.S. Geological Survey Techniques and Methods 11-A2 FGDC Digital Cartographic Standard for Geologic Map Symbolization (PostScript Implementation)

\section{0-HAZARDOUS WASTE SITES}

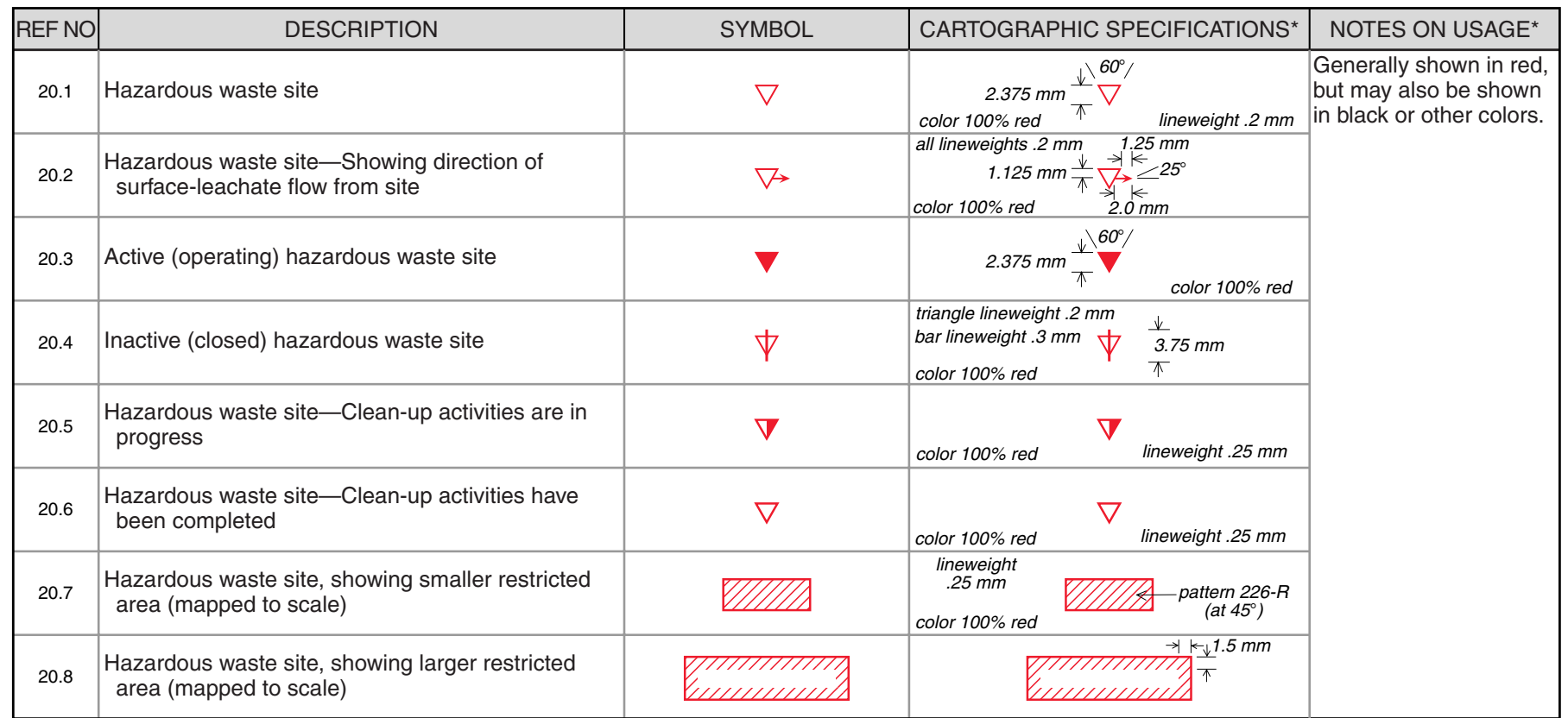

${ }^{*}$ For more information, see general guidelines on pages $A-i$ to $A-v$. 
Federal Geographic Data Committee (Doc. No. FGDC-STD-013-2006)

U.S. Geological Survey Techniques and Methods 11-A2

FGDC Digital Cartographic Standard for Geologic Map Symbolization (PostScript Implementation)

DOWNLOAD this Illustrator EPS file: Al8 / CS2

\section{1-NEOTECTONIC AND EARTHQUAKE-HAZARD FEATURES}

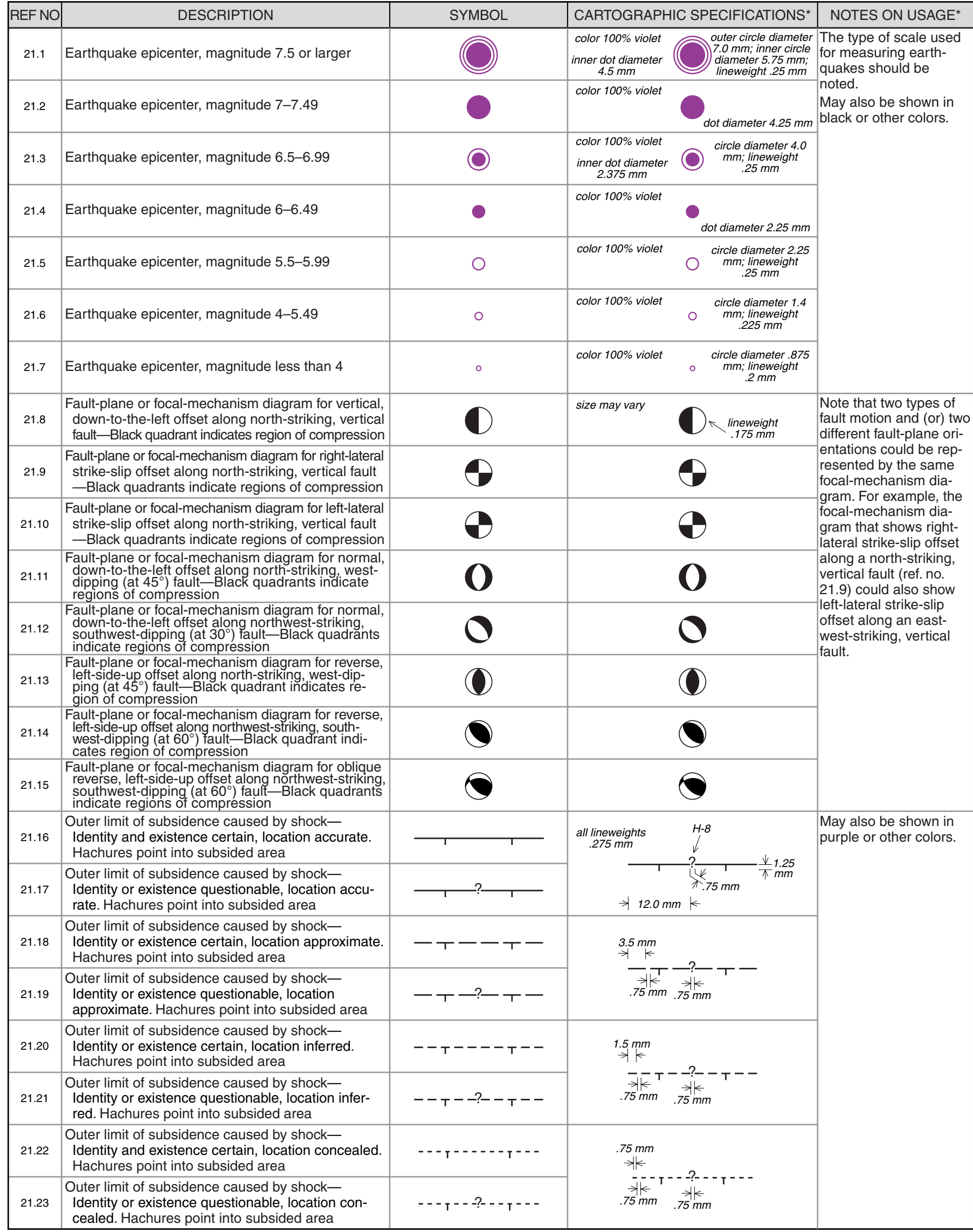

${ }^{*}$ For more information, see general guidelines on pages $A-i$ to $A-v$. 
Federal Geographic Data Committee (Doc. No. FGDC-STD-013-2006)

U.S. Geological Survey Techniques and Methods 11-A2 FGDC Digital Cartographic Standard for Geologic Map Symbolization (PostScript Implementation)

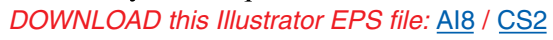

\section{1-NEOTECTONIC AND EARTHQUAKE-HAZARD FEATURES (continued)}

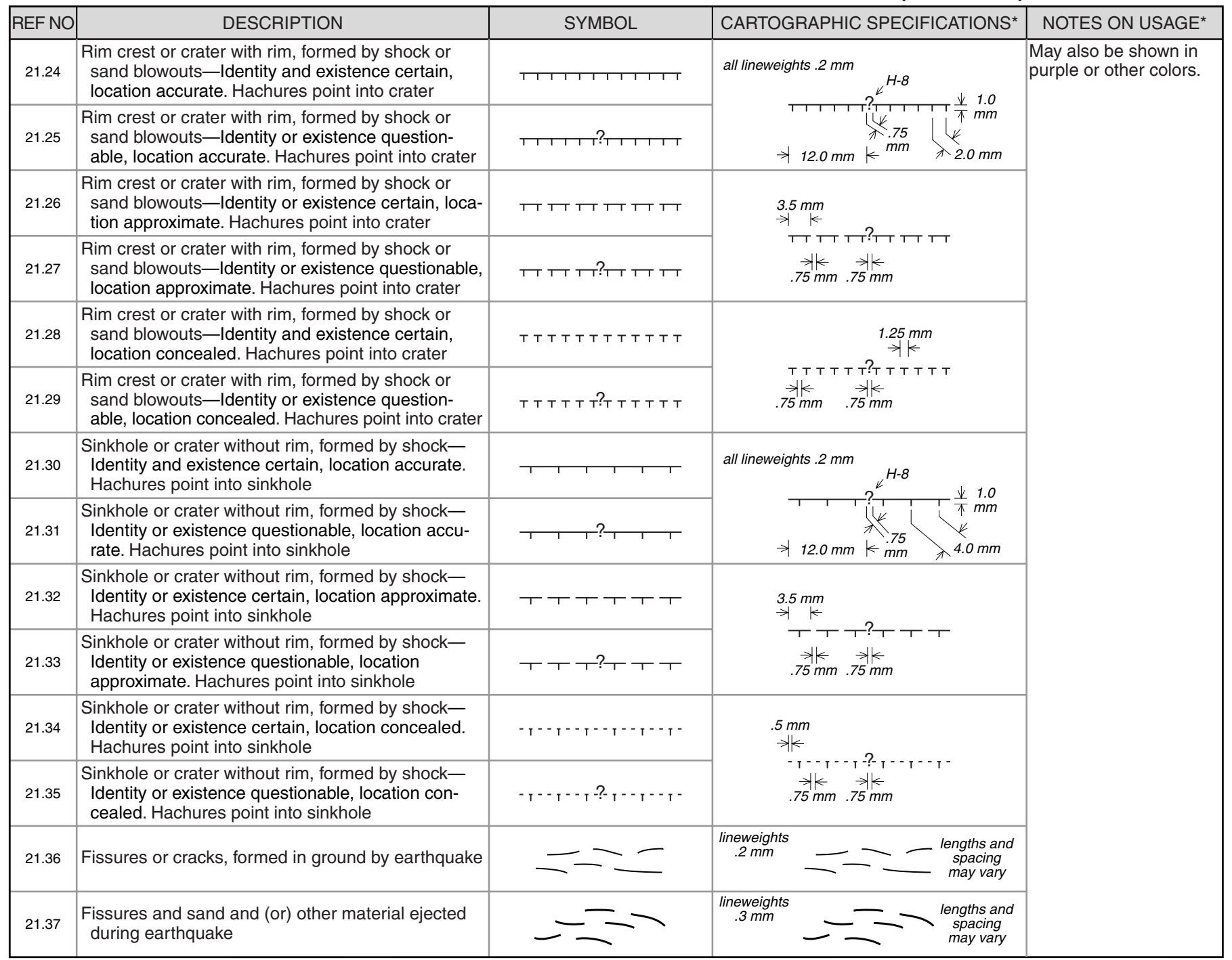

${ }^{*}$ For more information, see general guidelines on pages $A-i$ to $A-v$. 
Federal Geographic Data Committee (Doc. No. FGDC-STD-013-2006)

U.S. Geological Survey Techniques and Methods 11-A2

FGDC Digital Cartographic Standard for Geologic Map Symbolization (PostScript Implementation)

DOWNLOAD this Illustrator EPS file: Al8 / CS2

\section{2-PLATE-TECTONIC FEATURES}

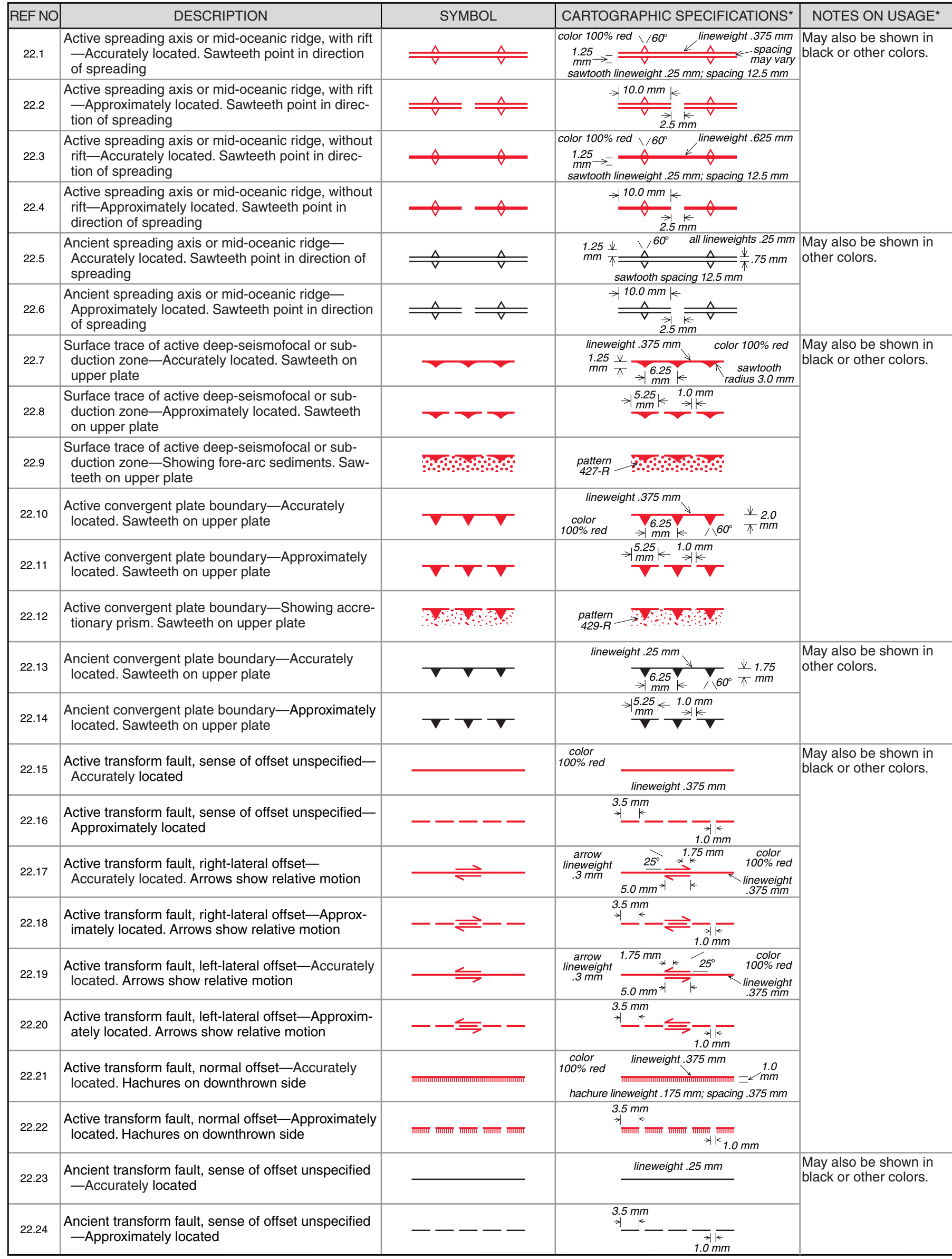

${ }^{*}$ For more information, see general guidelines on pages $A-i$ to $A-v$. 
Federal Geographic Data Committee (Doc. No. FGDC-STD-013-2006)

U.S. Geological Survey Techniques and Methods 11-A2 FGDC Digital Cartographic Standard for Geologic Map Symbolization (PostScript Implementation) DOWNLOAD this Illustrator EPS file: Al8 / $\underline{\mathrm{CS} 2}$

22-PLATE-TECTONIC FEATURES (continued)

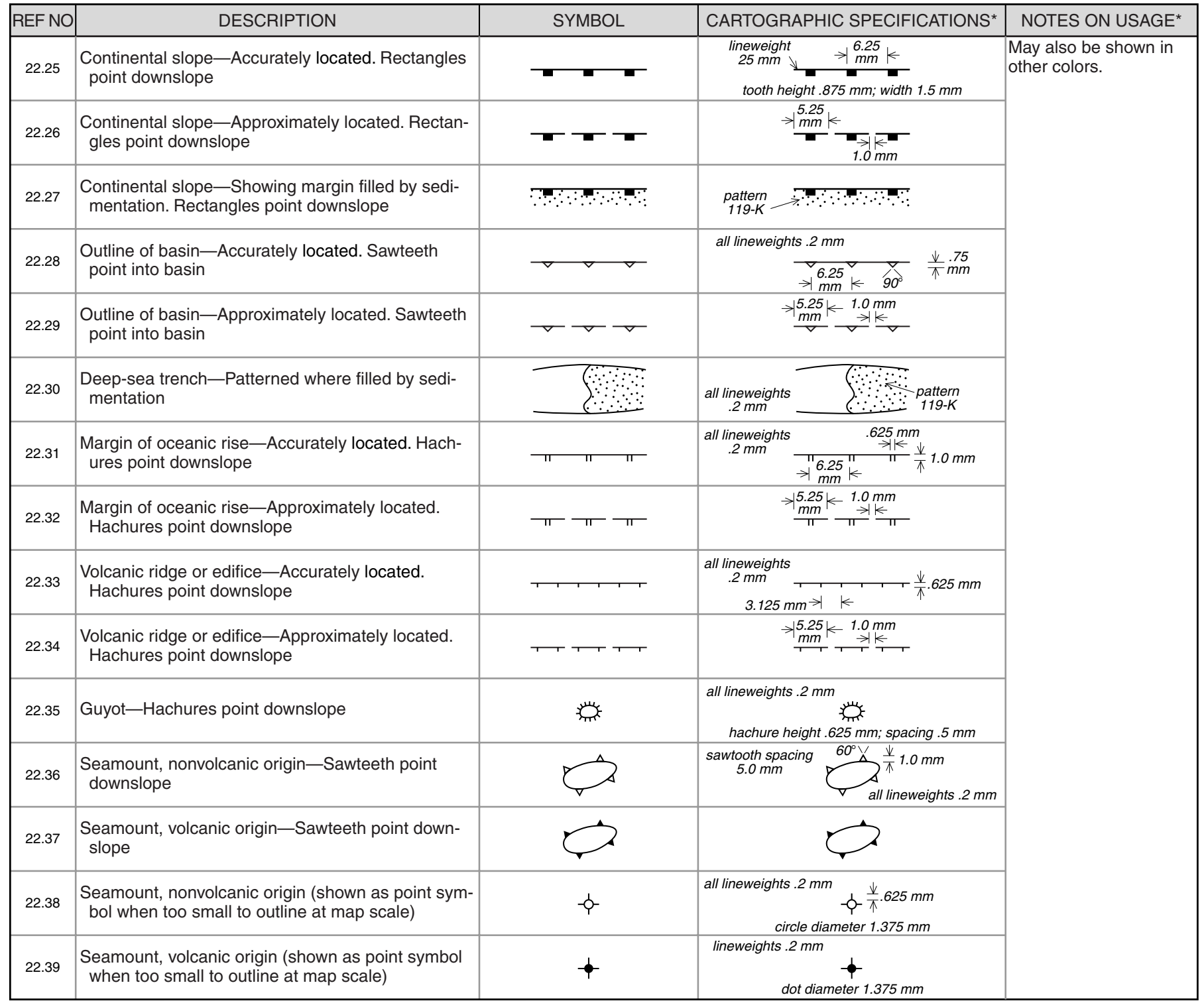

${ }^{*}$ For more information, see general guidelines on pages $A-i$ to $A-v$. 
Federal Geographic Data Committee (Doc. No. FGDC-STD-013-2006)

U.S. Geological Survey Techniques and Methods 11-A2

FGDC Digital Cartographic Standard for Geologic Map Symbolization (PostScript Implementation)

DOWNLOAD this Illustrator EPS file: Al8 / CS2

\section{3-MISCELLANEOUS UPLIFT AND COLLAPSE FEATURES}

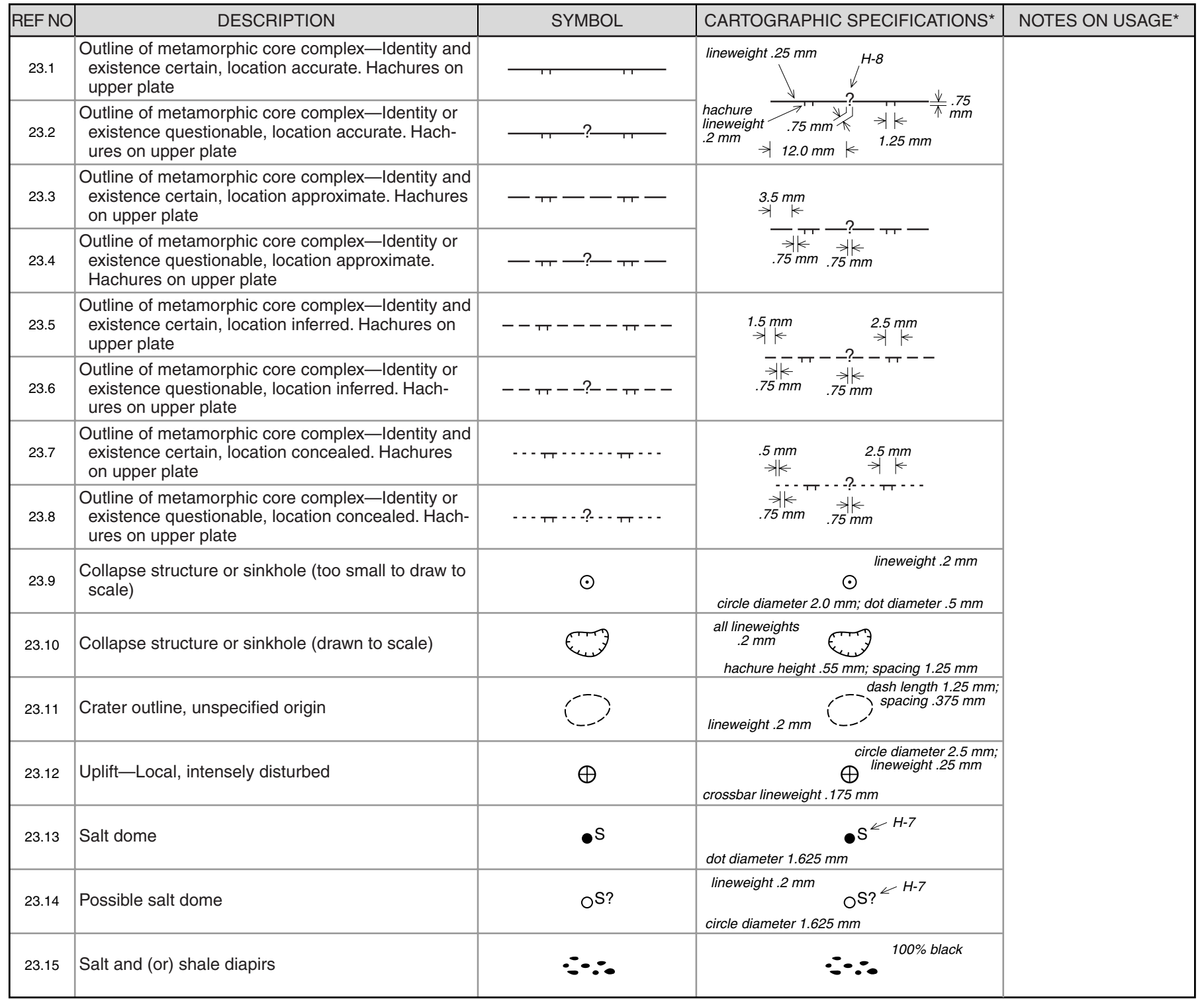

${ }^{*}$ For more information, see general guidelines on pages $A-i$ to $A-v$. 
Federal Geographic Data Committee (Doc. No. FGDC-STD-013-2006)

U.S. Geological Survey Techniques and Methods 11-A2

FGDC Digital Cartographic Standard for Geologic Map Symbolization (PostScript Implementation)

DOWNLOAD this Illustrator EPS file: $\underline{\mathrm{Al} 8}$ / $\underline{\mathrm{CS} 2}$

\section{4-TERRESTRIAL IMPACT FEATURES}

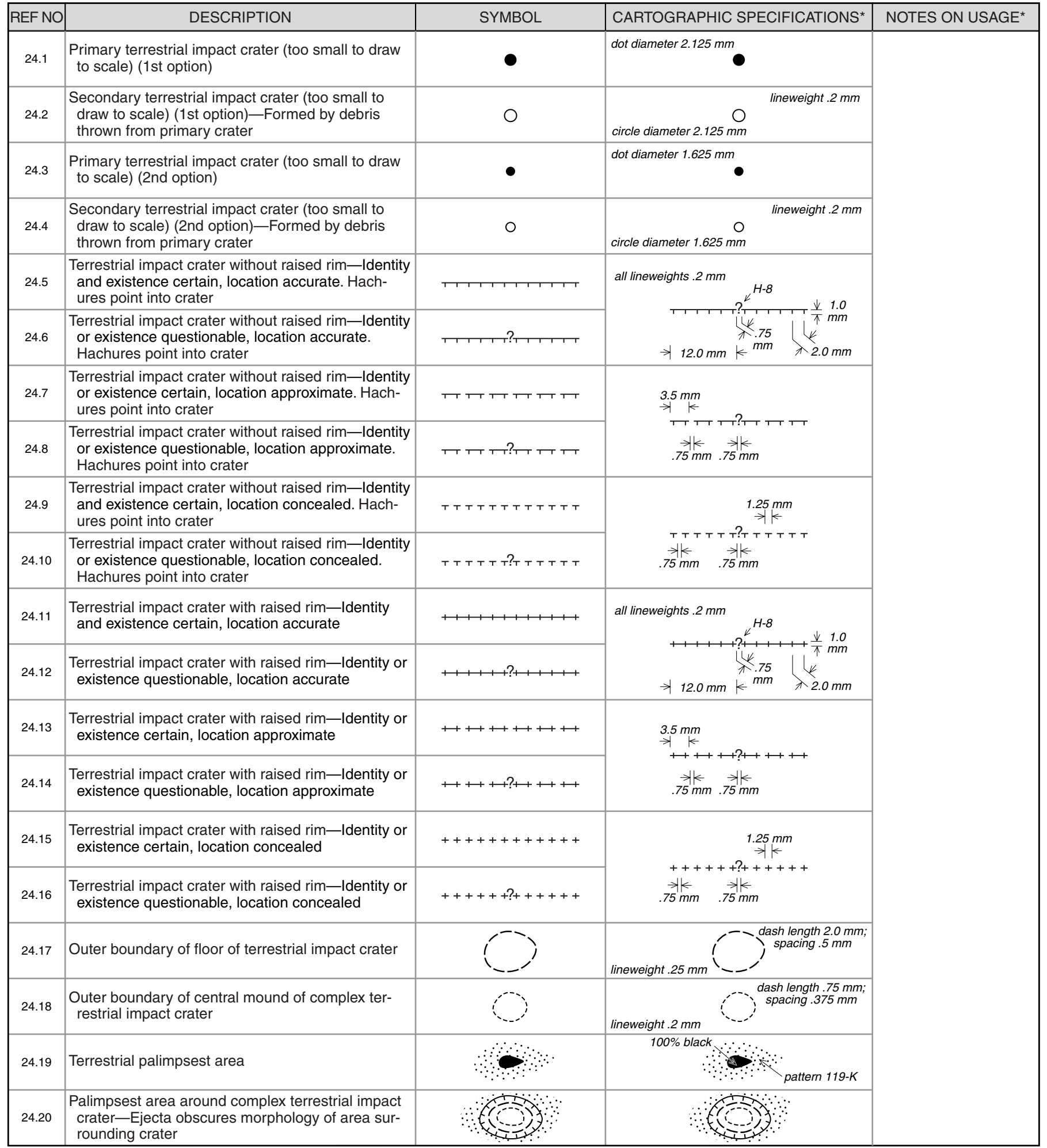

${ }^{*}$ For more information, see general guidelines on pages $A-i$ to $A-v$. 
Federal Geographic Data Committee (Doc. No. FGDC-STD-013-2006)

U.S. Geological Survey Techniques and Methods 11-A2

FGDC Digital Cartographic Standard for Geologic Map Symbolization (PostScript Implementation)

DOWNLOAD this Illustrator EPS file: Al8 / $\underline{\mathrm{CS} 2}$

\section{5-PLANETARY GEOLOGY FEATURES}

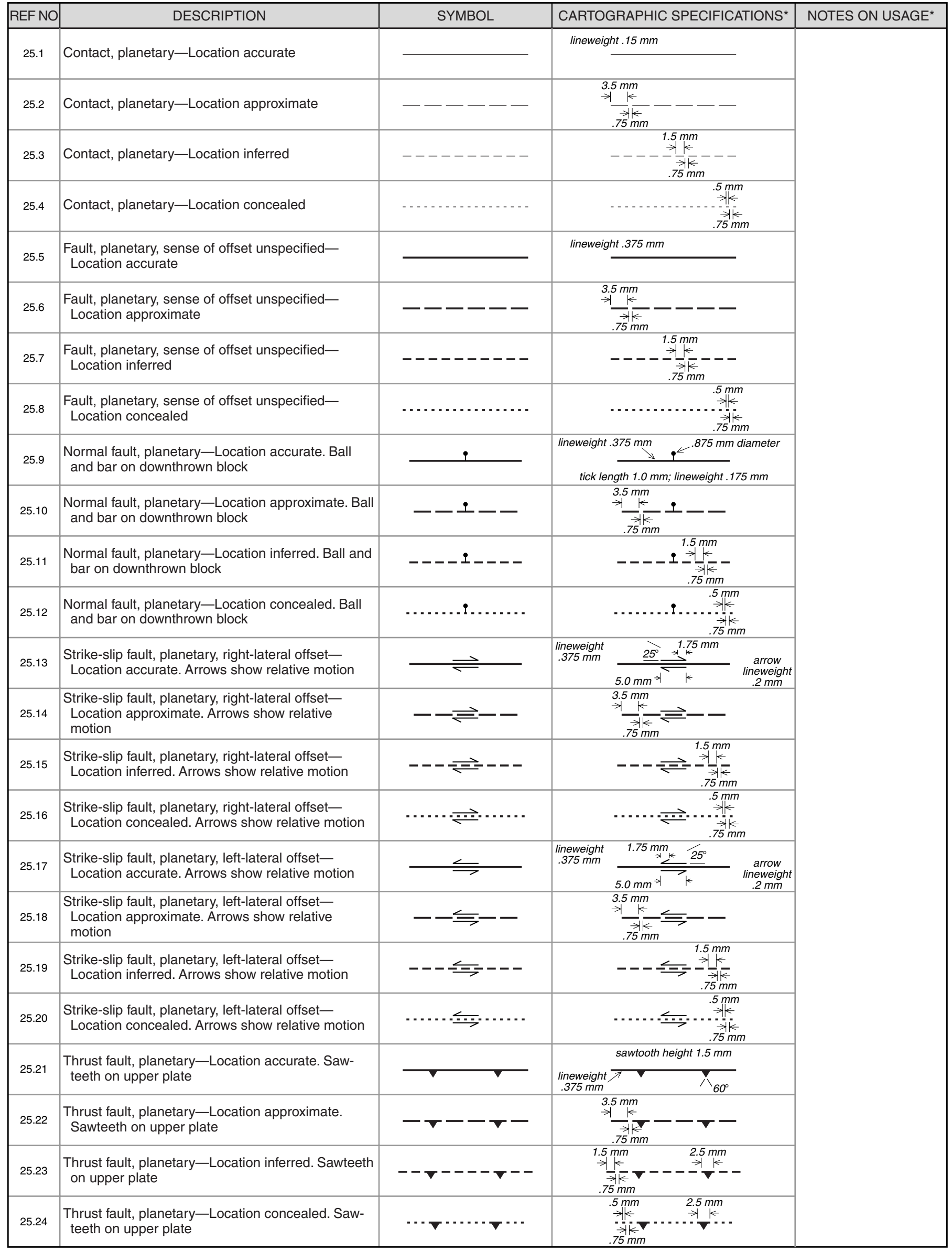

${ }^{*}$ For more information, see general guidelines on pages $A-i$ to $A-v$. 
Federal Geographic Data Committee (Doc. No. FGDC-STD-013-2006)

U.S. Geological Survey Techniques and Methods 11-A2

FGDC Digital Cartographic Standard for Geologic Map Symbolization (PostScript Implementation)

DOWNLOAD this Illustrator EPS file: Al8 / CS2

\section{5-PLANETARY GEOLOGY FEATURES (continued)}

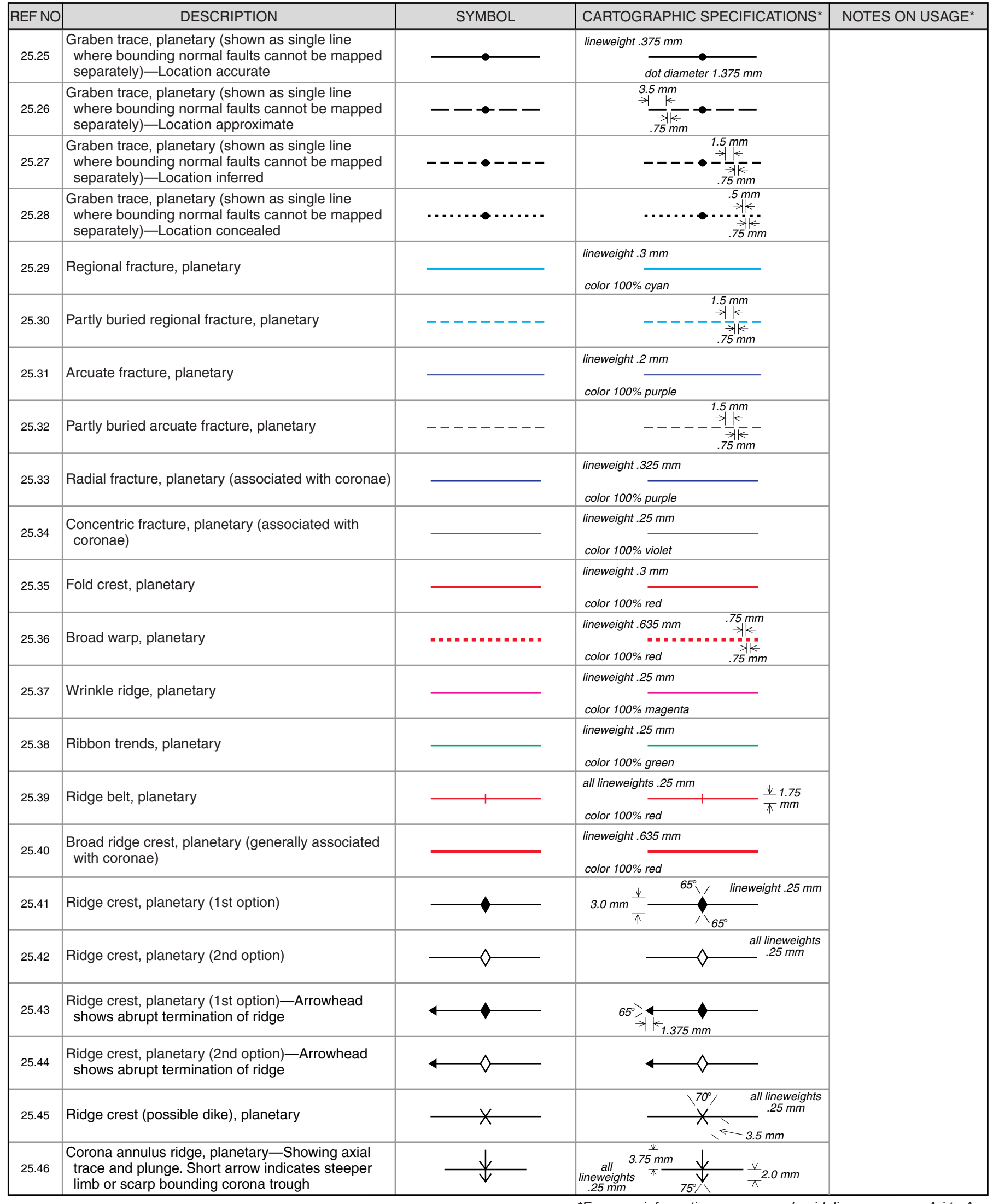

${ }^{*}$ For more information, see general guidelines on pages $A-i$ to $A-v$. 
Federal Geographic Data Committee (Doc. No. FGDC-STD-013-2006)

U.S. Geological Survey Techniques and Methods 11-A2 FGDC Digital Cartographic Standard for Geologic Map Symbolization (PostScript Implementation)

DOWNLOAD this Illustrator EPS file: Al8 / CS2

\section{5-PLANETARY GEOLOGY FEATURES (continued)}

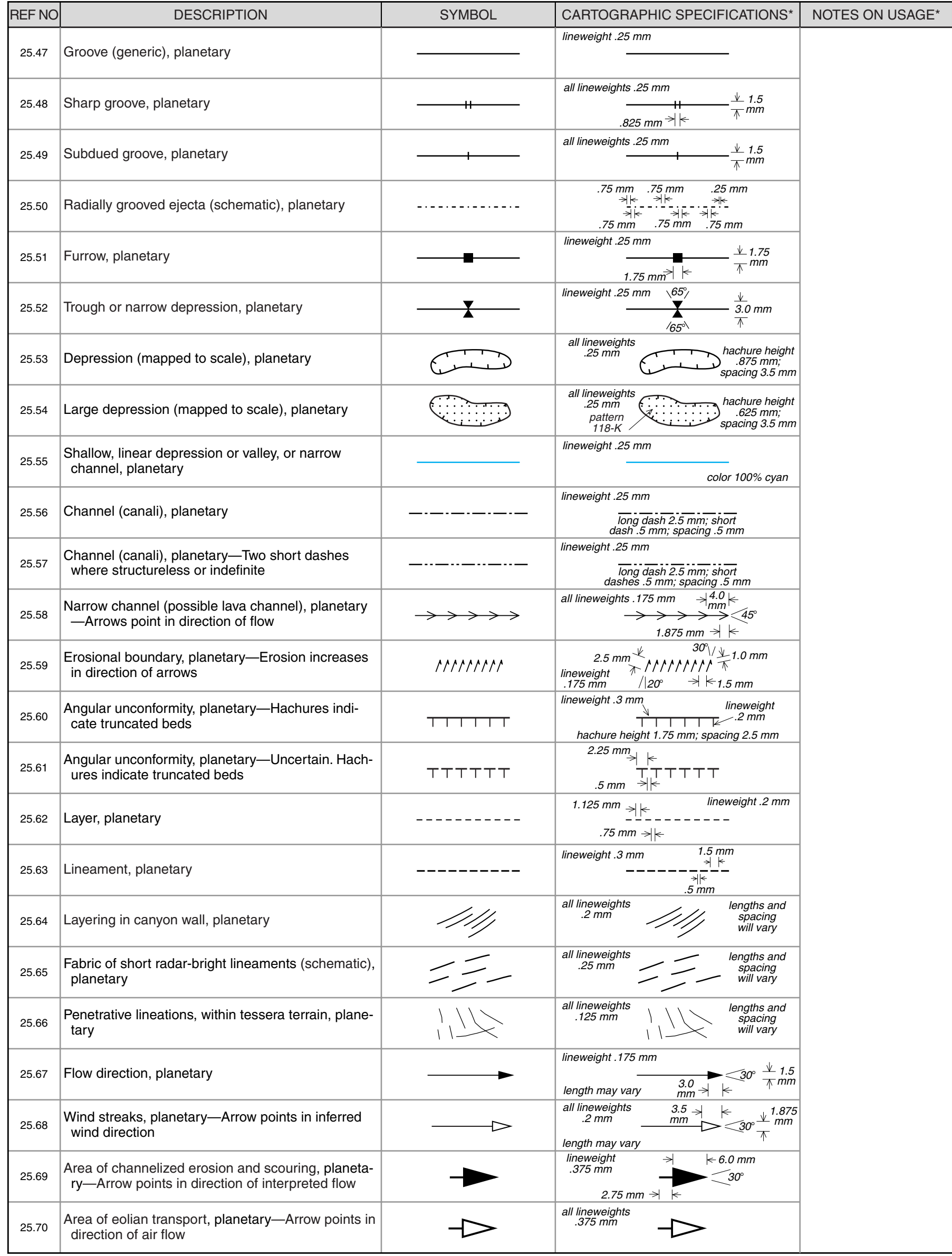

${ }^{\star}$ For more information, see general guidelines on pages $A-i$ to $A-v$. 
Federal Geographic Data Committee (Doc. No. FGDC-STD-013-2006)

U.S. Geological Survey Techniques and Methods 11-A2 FGDC Digital Cartographic Standard for Geologic Map Symbolization (PostScript Implementation)

DOWNLOAD this Illustrator EPS file: Al8 / CS2

\section{5-PLANETARY GEOLOGY FEATURES (continued)}

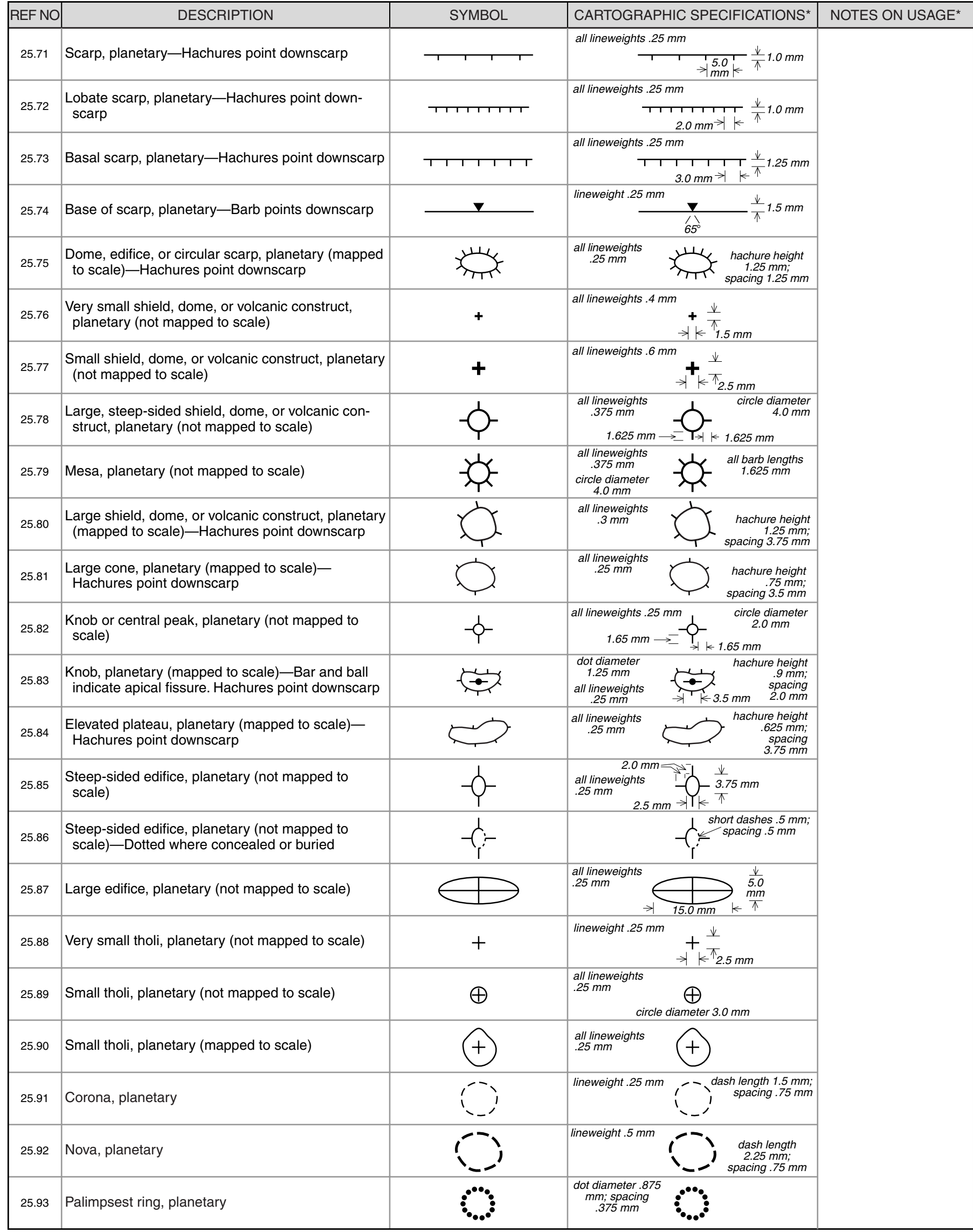

${ }^{*}$ For more information, see general guidelines on pages $A-i$ to $A-v$. 
Federal Geographic Data Committee (Doc. No. FGDC-STD-013-2006)

U.S. Geological Survey Techniques and Methods 11-A2

FGDC Digital Cartographic Standard for Geologic Map Symbolization (PostScript Implementation)

DOWNLOAD this Illustrator EPS file: Al8 / CS2

\section{5-PLANETARY GEOLOGY FEATURES (continued)}

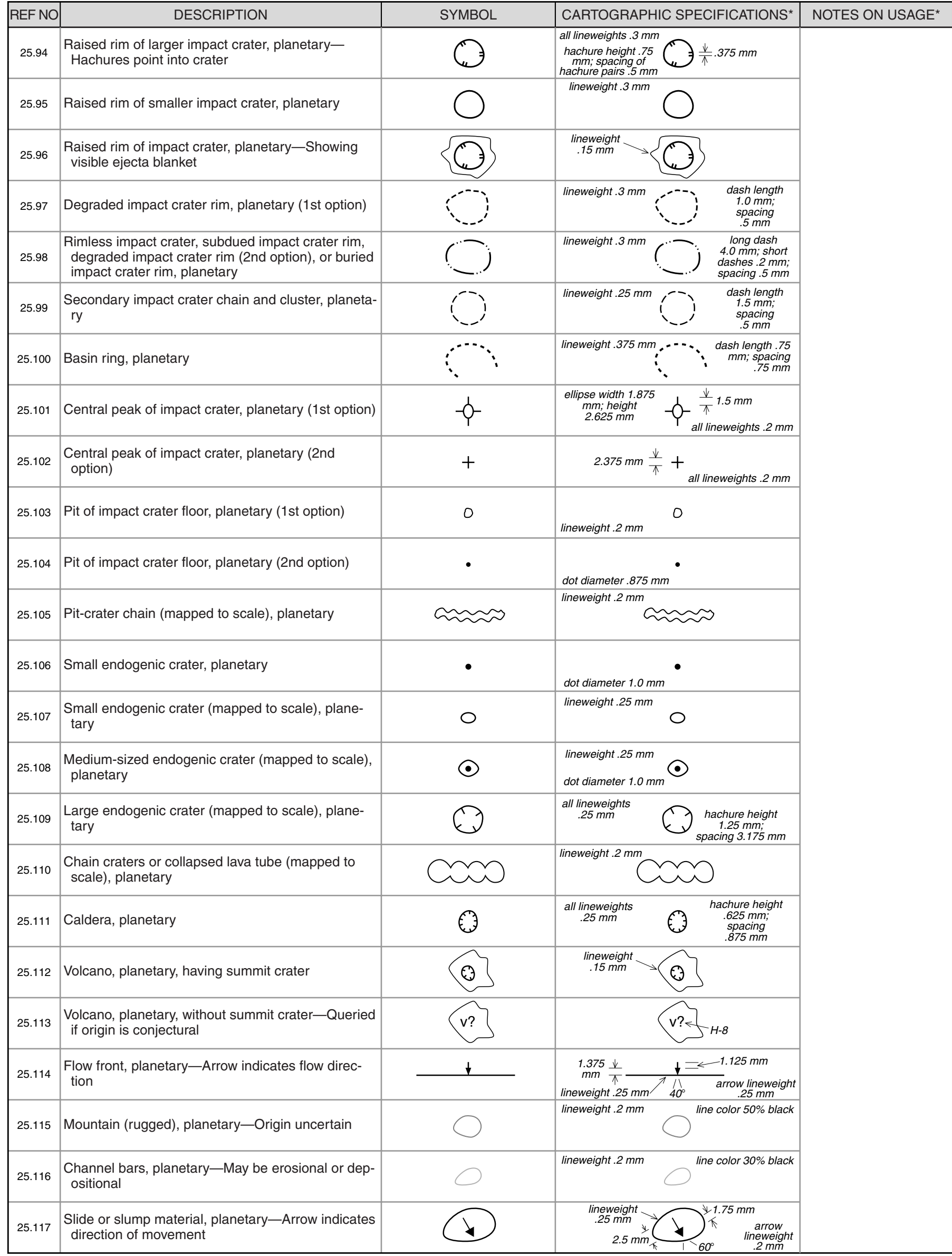

${ }^{*}$ For more information, see general guidelines on pages $A-i$ to $A-v$. 
Federal Geographic Data Committee (Doc. No. FGDC-STD-013-2006)

U.S. Geological Survey Techniques and Methods 11-A2 FGDC Digital Cartographic Standard for Geologic Map Symbolization (PostScript Implementation) DOWNLOAD this Illustrator EPS file: Al8 / $\underline{\text { CS2 }}$

\section{5-PLANETARY GEOLOGY FEATURES (continued)}

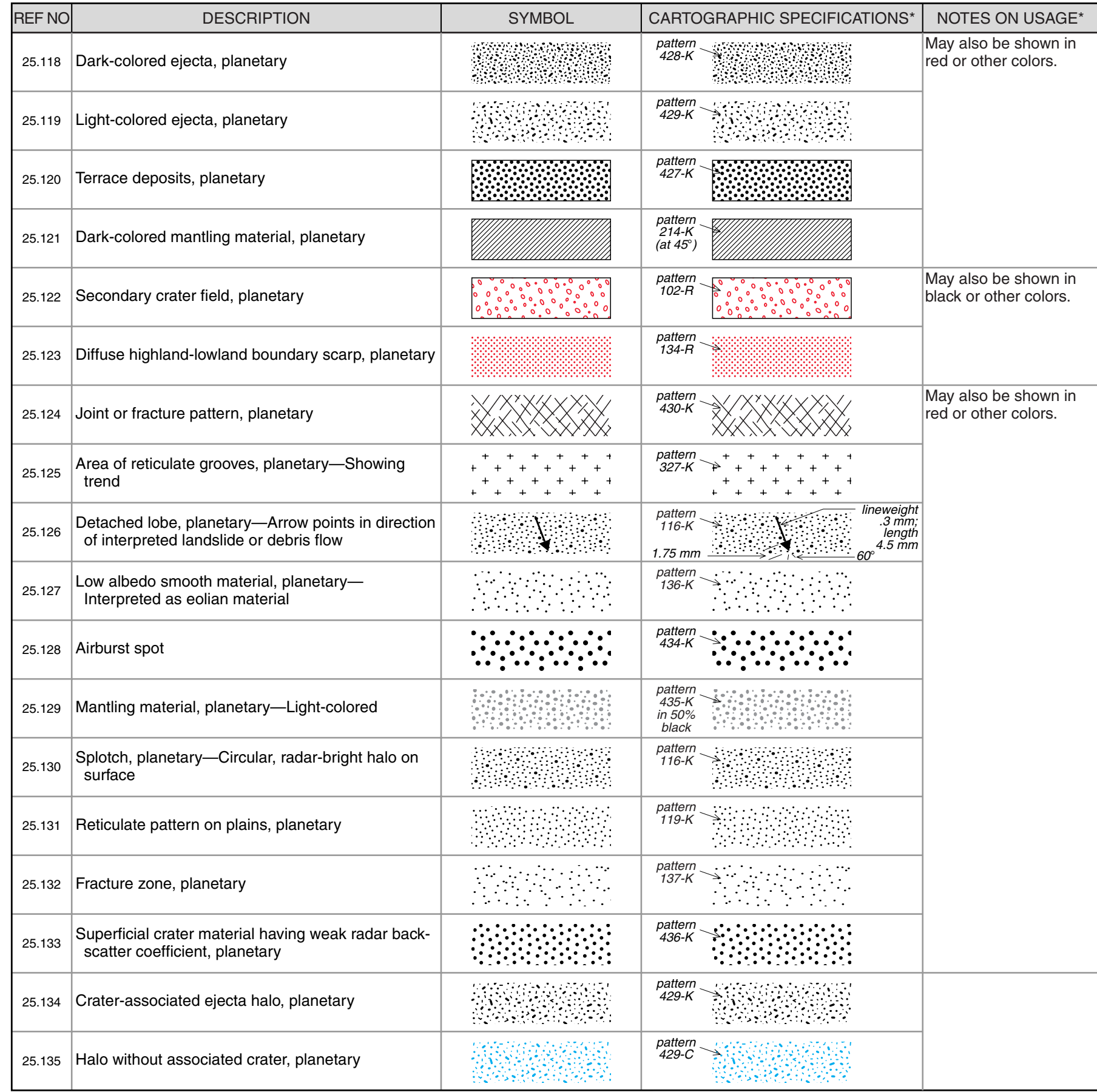

${ }^{*}$ For more information, see general guidelines on pages $A-i$ to $A-v$. 
Federal Geographic Data Committee (Doc. No. FGDC-STD-013-2006)

U.S. Geological Survey Techniques and Methods 11-A2

FGDC Digital Cartographic Standard for Geologic Map Symbolization (PostScript Implementation)

DOWNLOAD this Illustrator EPS file: Al8 / $\underline{\text { CS2 }}$

\section{6-GEOHYDROLOGIC FEATURES}

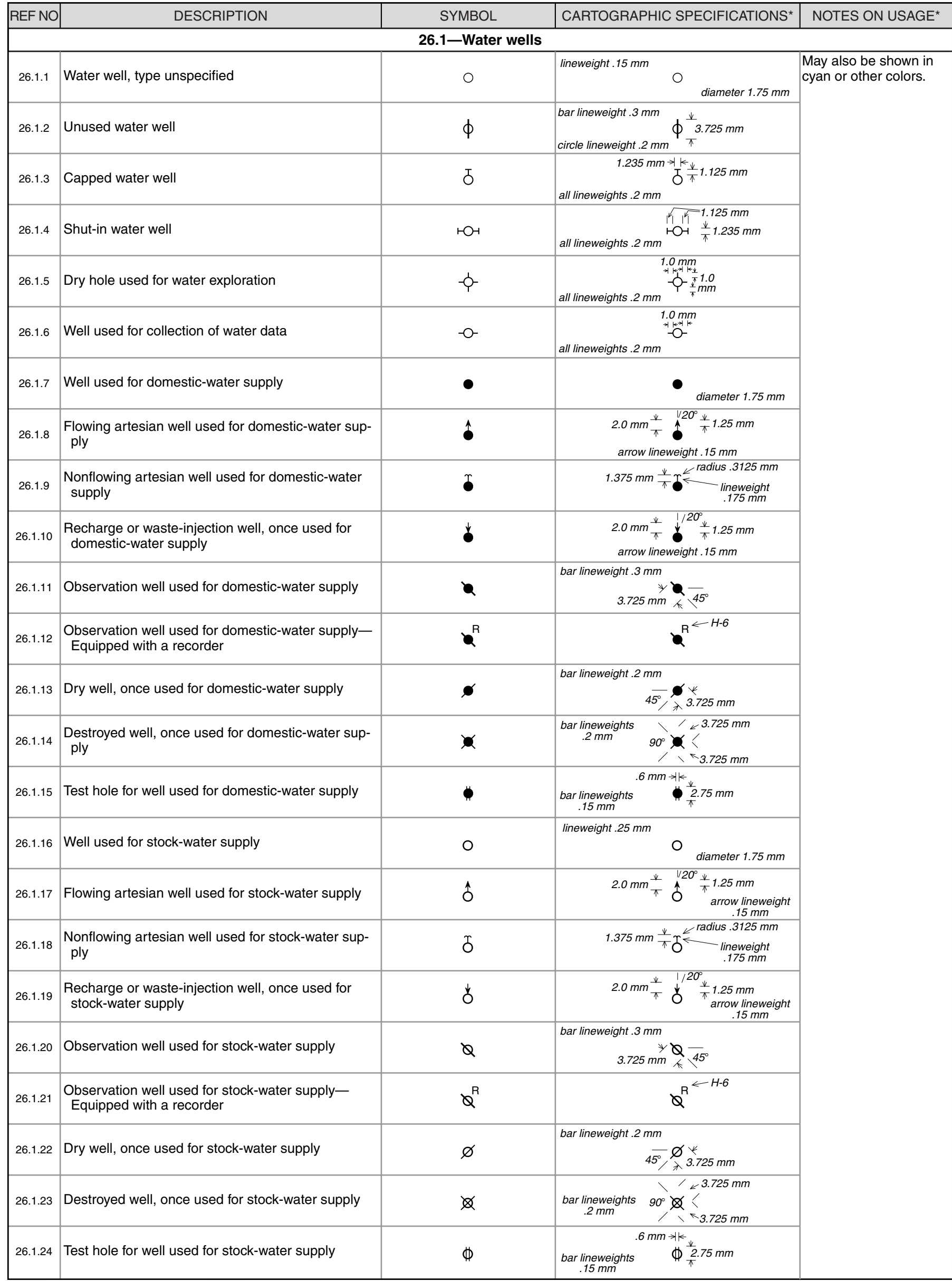


Federal Geographic Data Committee (Doc. No. FGDC-STD-013-2006)

U.S. Geological Survey Techniques and Methods 11-A2

FGDC Digital Cartographic Standard for Geologic Map Symbolization (PostScript Implementation)

DOWNLOAD this Illustrator EPS file: Al8 / CS2

\section{6-GEOHYDROLOGIC FEATURES (continued)}

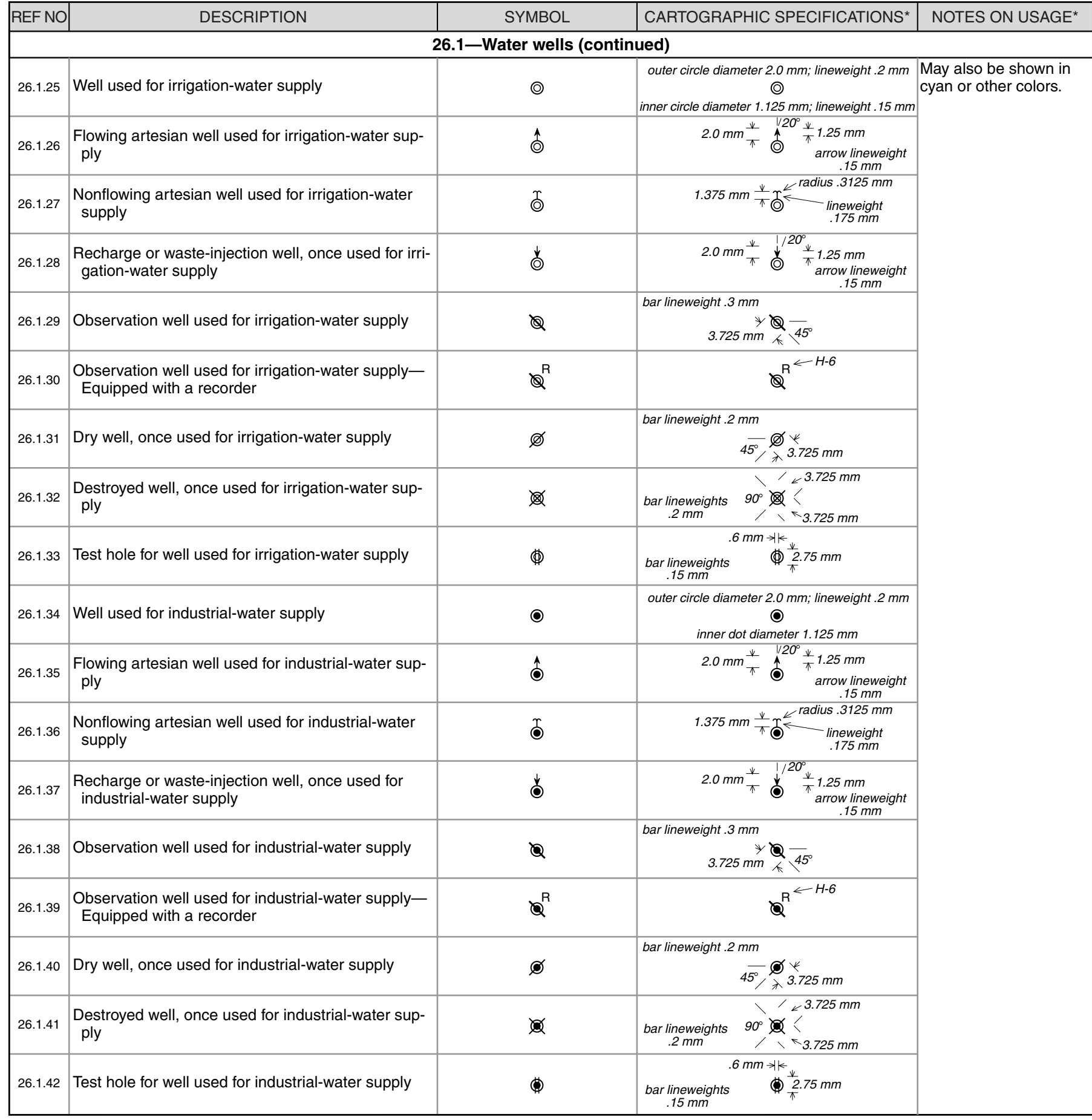

${ }^{*}$ For more information, see general guidelines on pages $A-i$ to $A-v$. 
Federal Geographic Data Committee (Doc. No. FGDC-STD-013-2006)

U.S. Geological Survey Techniques and Methods 11-A2 FGDC Digital Cartographic Standard for Geologic Map Symbolization (PostScript Implementation) DOWNLOAD this Illustrator EPS file: Al8 / CS2

\section{6-GEOHYDROLOGIC FEATURES (continued)}

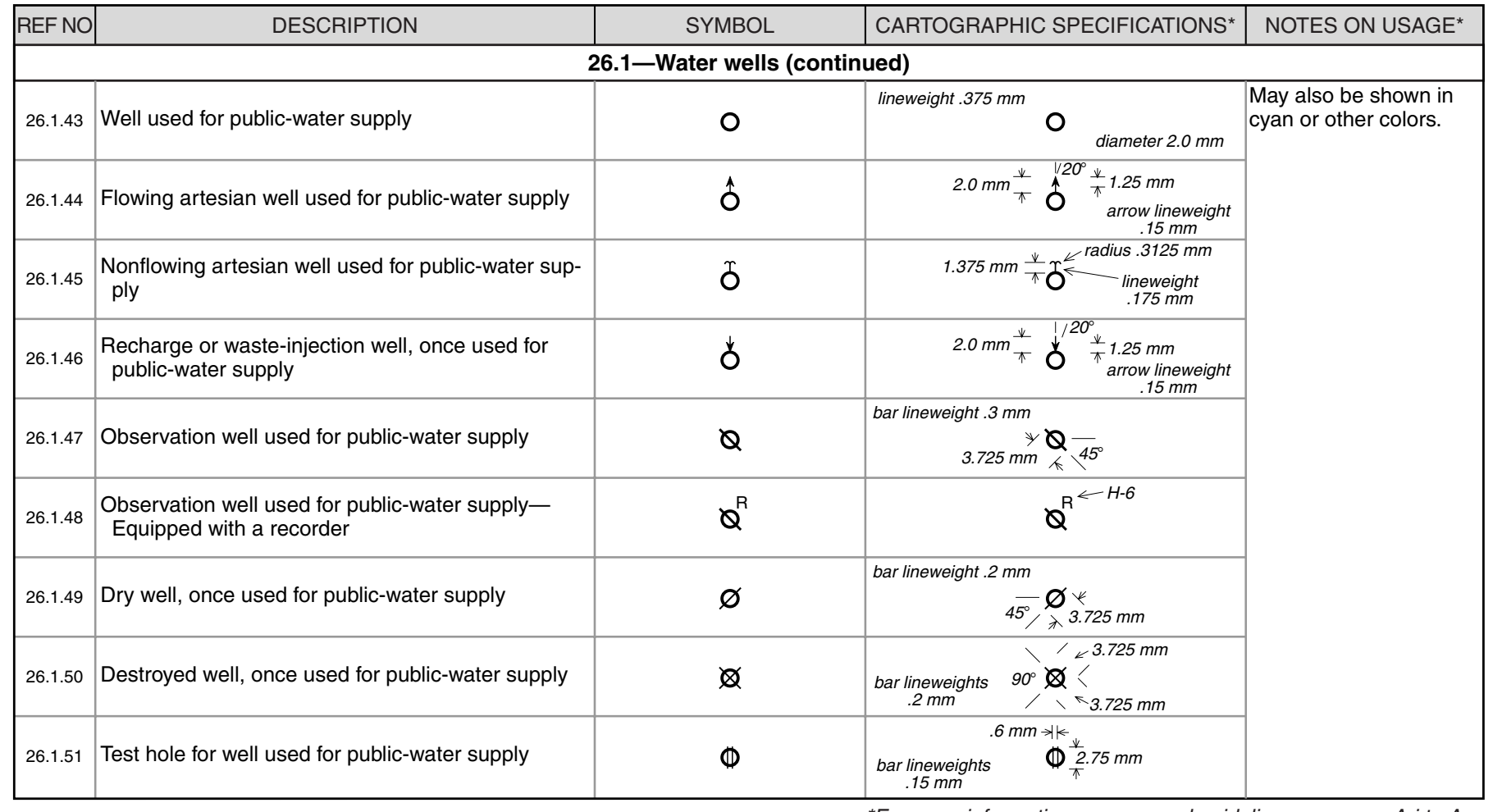

${ }^{*}$ For more information, see general guidelines on pages $A-i$ to $A-v$. 
Federal Geographic Data Committee (Doc. No. FGDC-STD-013-2006)

U.S. Geological Survey Techniques and Methods 11-A2

FGDC Digital Cartographic Standard for Geologic Map Symbolization (PostScript Implementation)

DOWNLOAD this Illustrator EPS file: Al8 / CS2

\section{6-GEOHYDROLOGIC FEATURES (continued)}

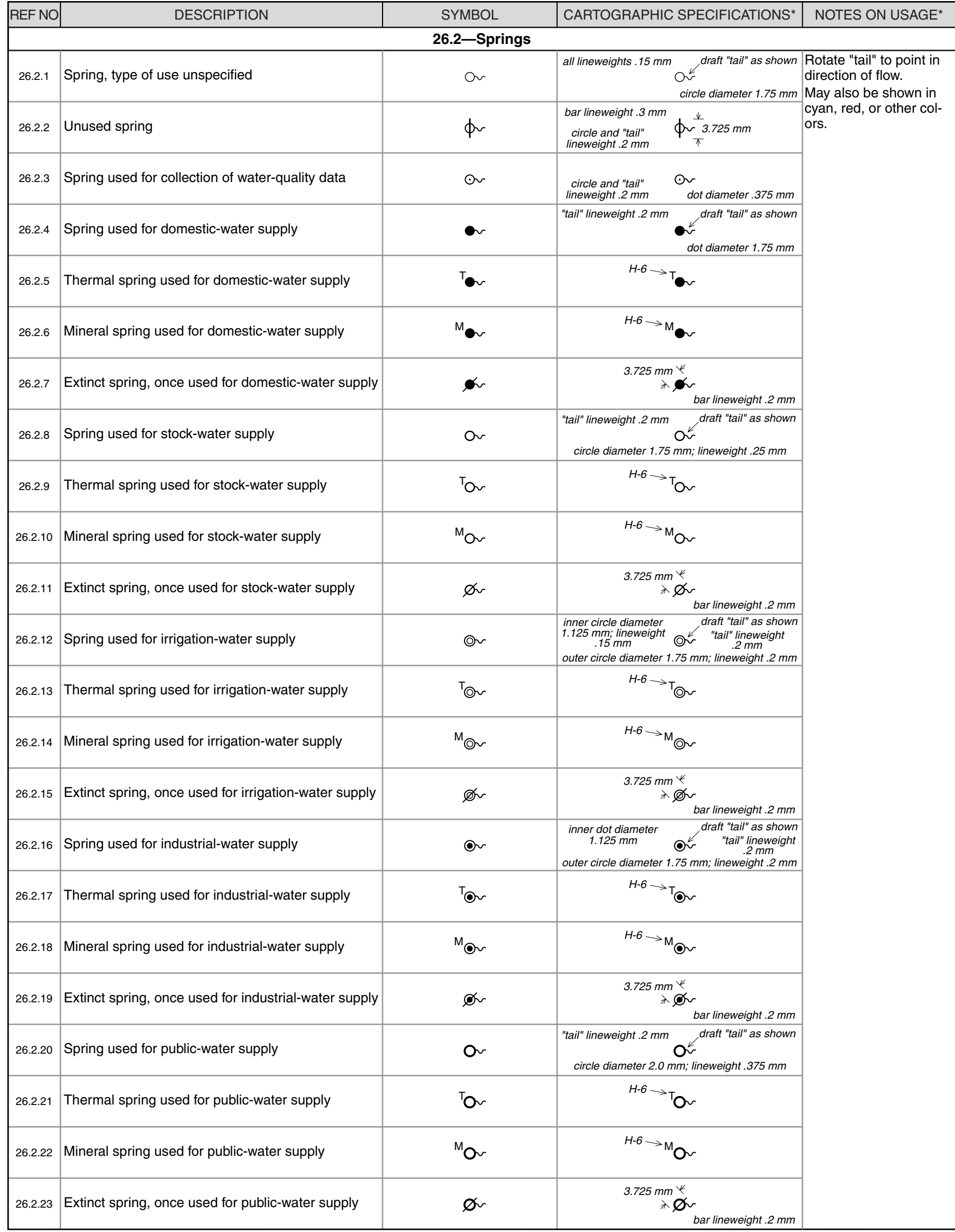

${ }^{*}$ For more information, see general guidelines on pages $A-i$ to $A-v$. 
Federal Geographic Data Committee (Doc. No. FGDC-STD-013-2006)

U.S. Geological Survey Techniques and Methods 11-A2 FGDC Digital Cartographic Standard for Geologic Map Symbolization (PostScript Implementation)

DOWNLOAD this Illustrator EPS file: Al8 / CS2

\section{6-GEOHYDROLOGIC FEATURES (continued)}

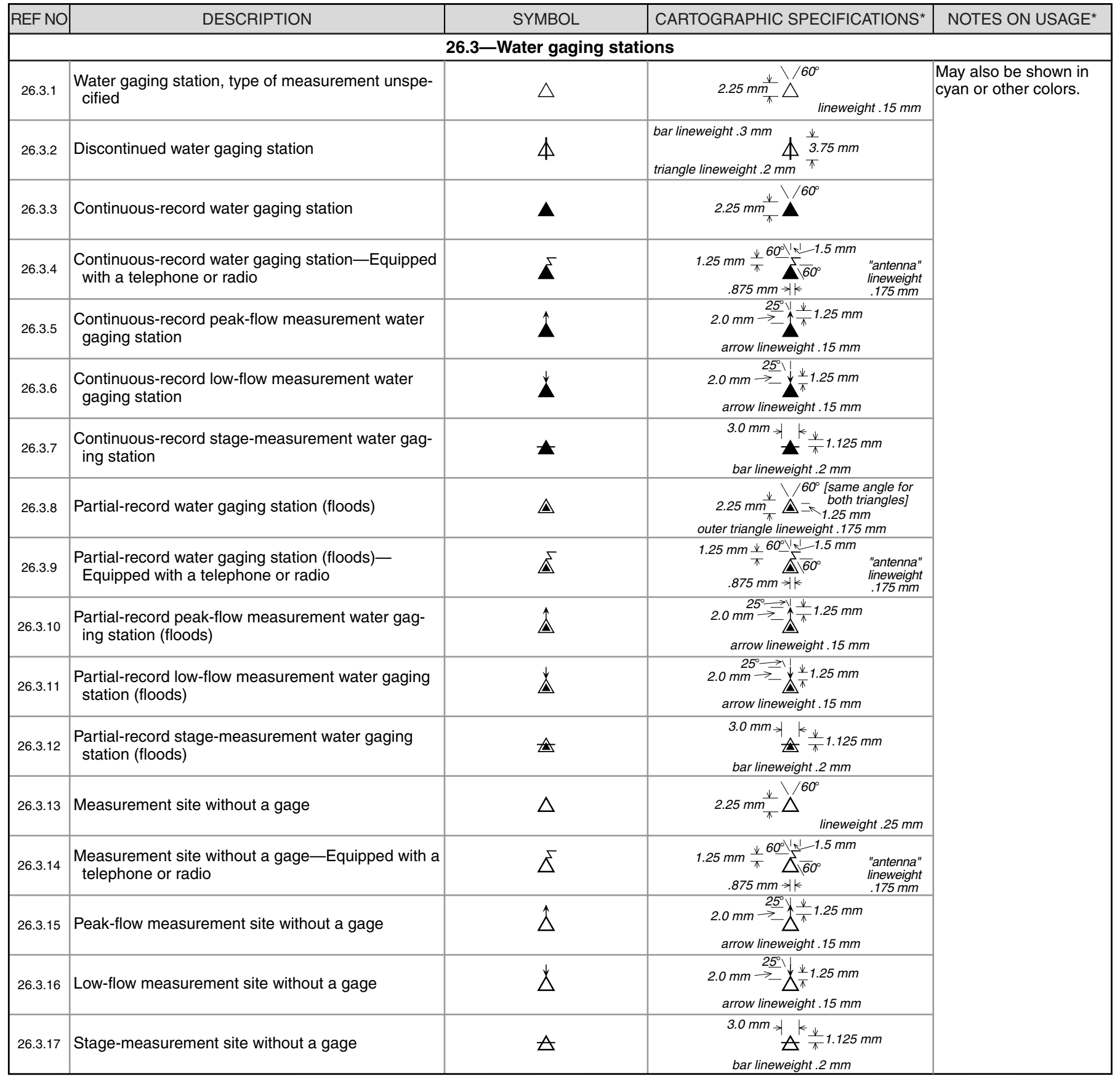

${ }^{*}$ For more information, see general guidelines on pages $A-i$ to $A-v$. 
Federal Geographic Data Committee (Doc. No. FGDC-STD-013-2006)

U.S. Geological Survey Techniques and Methods 11-A2 FGDC Digital Cartographic Standard for Geologic Map Symbolization (PostScript Implementation) DOWNLOAD this Illustrator EPS file: Al8 / $\underline{\mathrm{CS} 2}$

\section{6-GEOHYDROLOGIC FEATURES (continued)}

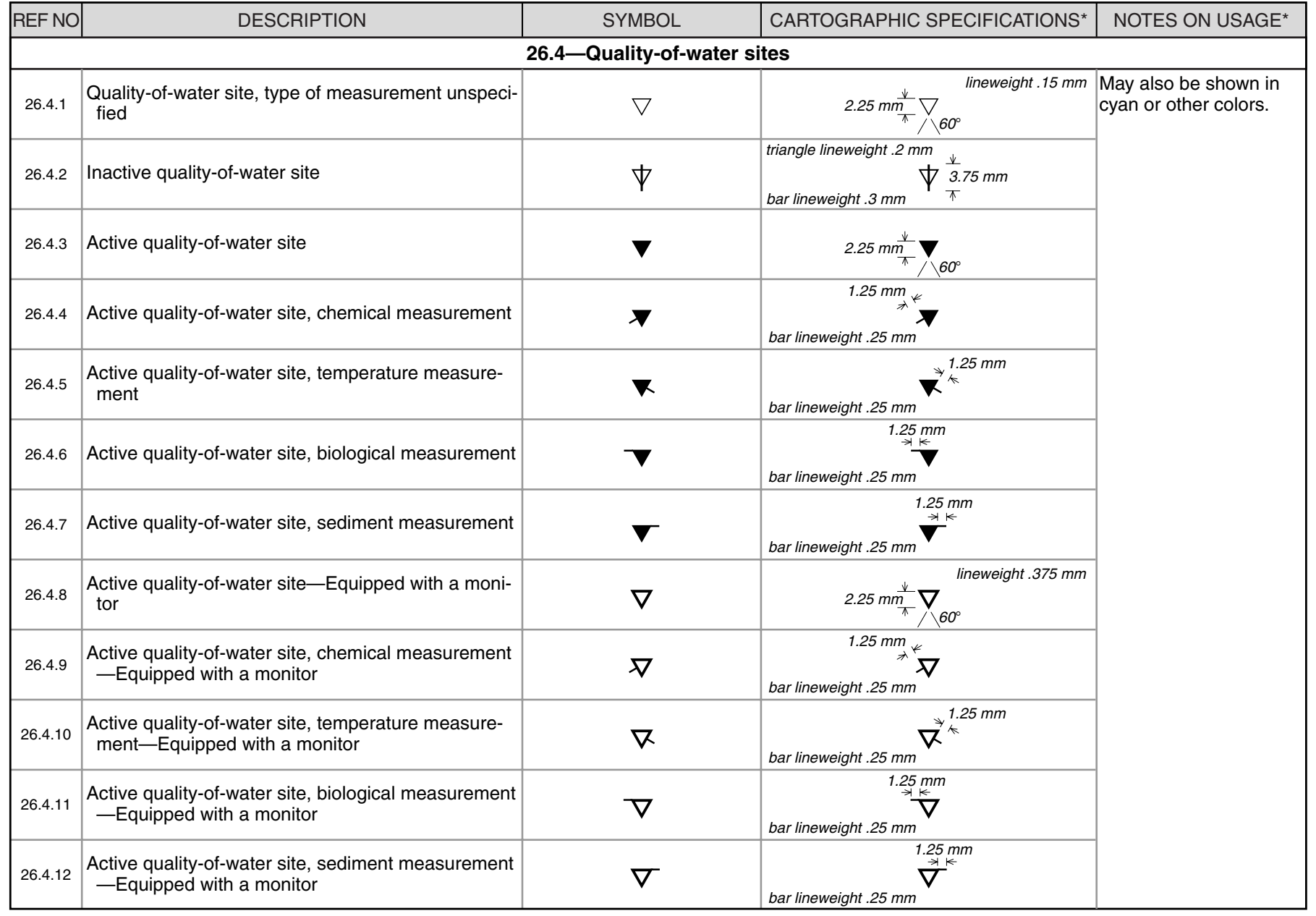

${ }^{*}$ For more information, see general guidelines on pages $A-i$ to $A-v$. 


\section{6-GEOHYDROLOGIC FEATURES (continued)}

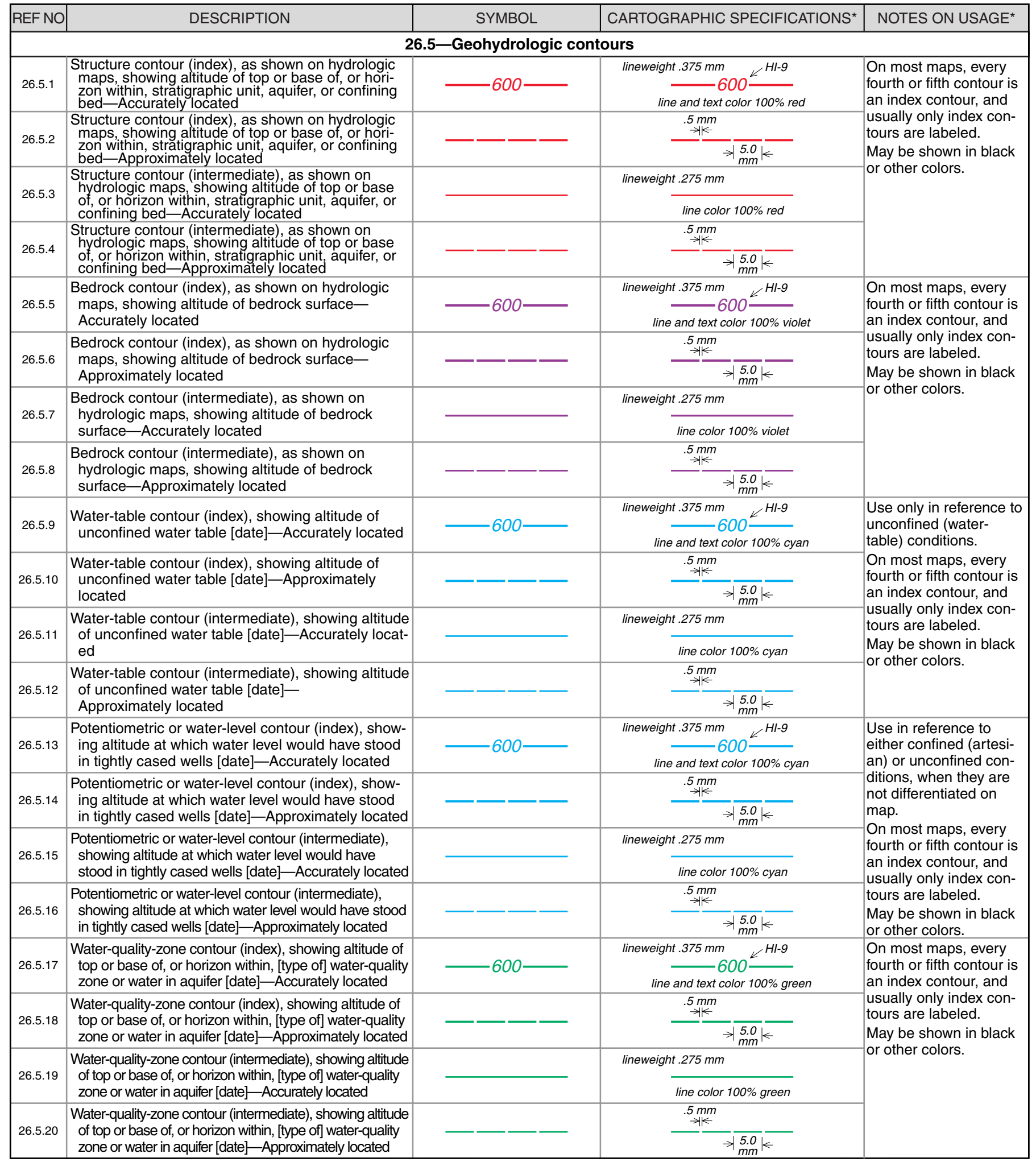


Federal Geographic Data Committee (Doc. No. FGDC-STD-013-2006)

U.S. Geological Survey Techniques and Methods 11-A2

FGDC Digital Cartographic Standard for Geologic Map Symbolization (PostScript Implementation)

DOWNLOAD this Illustrator EPS file: Al8 / CS2

\section{6-GEOHYDROLOGIC FEATURES (continued)}

\begin{tabular}{|c|c|c|c|c|}
\hline REF NO & DESCRIPTION & SYMBOL & CARTOGRAPHIC SPECIFICATIONS* & NOTES ON USAGE* \\
\hline \multicolumn{5}{|c|}{ 26.6-Geohydrologic lines } \\
\hline 26.6.1 & $\begin{array}{l}\text { Line of equal, average, mean, or median (etc.) } \\
\text { annual, monthly, or daily (etc.) precipitation [date] } \\
\text { —Accurately located }\end{array}$ & $-24-$ & 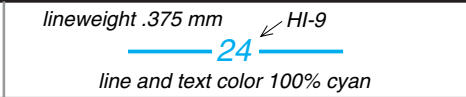 & \multirow{18}{*}{$\begin{array}{l}\text { Negative values must } \\
\text { be preceded by a minus } \\
(-) \text { sign. } \\
\text { Date needed only for } \\
\text { parameters that vary } \\
\text { with time. } \\
\text { May be shown in black } \\
\text { or other colors. }\end{array}$} \\
\hline \multirow{2}{*}{26.6 .2} & \multirow{2}{*}{$\begin{array}{l}\text { Line of equal, average, mean, or median (etc.) } \\
\text { annual, monthly, or daily (etc.) precipitation [date] } \\
\text { —Approximately located }\end{array}$} & \multirow{2}{*}{$\longrightarrow-\longrightarrow$} & \multirow{2}{*}{$\stackrel{.5 \mathrm{~mm}}{\rightarrow} \frac{\| k}{\rightarrow} \underset{\substack{5.0 \\
\mathrm{~mm}}}{K} \bar{K}$} & \\
\hline & & & & \\
\hline \multirow{2}{*}{26.6 .4} & \multirow{2}{*}{$\begin{array}{l}\text { Line of equal depth to geologic formation, bedrock, } \\
\text { aquifer, or water (etc.) [date]_Approximately } \\
\text { located }\end{array}$} & \multirow[t]{2}{*}{$-\square-\longrightarrow$} & 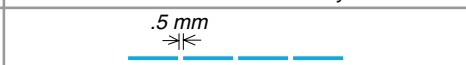 & \\
\hline & & & $\rightarrow \mid \begin{array}{c}5.0 \\
\mathrm{~mm}\end{array}$ & \\
\hline 26.6 .5 & $\begin{array}{l}\text { Line of equal thickness of geologic formation, aqui- } \\
\text { fer, confining bed, or saturated material (etc.) } \\
\text { [date]-Accurately located }\end{array}$ & $-50 \longrightarrow$ & 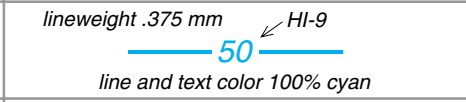 & \\
\hline \multirow[t]{2}{*}{26.6 .8} & \multirow{2}{*}{$\begin{array}{l}\text { Line of equal water temperature [date]- } \\
\text { Approximately located }\end{array}$} & \multirow[t]{2}{*}{$-\square-$} & $\underset{\substack{.5 \mathrm{~mm} \\
\rightarrow \mathbb{K}}}{-}$ & \\
\hline & & & $\rightarrow \mid \begin{array}{c}5.0 \\
\mathrm{~mm}\end{array}$ & \\
\hline 26.6 .9 & $\begin{array}{l}\text { Line of equal specific conductance [date]- } \\
\text { Accurately located }\end{array}$ & $-2000-$ & $\begin{array}{l}\text { lineweight } .375 \mathrm{~mm} \\
\quad 2000 \frac{\swarrow^{H I}-9}{\text { line and text color } 100 \% \text { cyan }}\end{array}$ & \\
\hline \multirow{2}{*}{26.6 .10} & Line of equal specific conductance [date]- & \multirow{2}{*}{$-\square-\longrightarrow$} & $\underset{\substack{.5 \mathrm{~mm} \\
\rightarrow \mathbb{K}}}{\longrightarrow}$ & \\
\hline & Approximately located & & 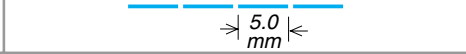 & \\
\hline 26.6.11 & $\begin{array}{l}\text { Line of equal dissolved-solids concentration, hard- } \\
\text { ness, or chemical-constituent concentration [date] } \\
\text {-Accurately located }\end{array}$ & $-500-$ & $\begin{array}{l}\text { lineweight } .375 \mathrm{~mm} \\
\quad \text { line and text color } 100 \% \text { cyan }\end{array}$ & \\
\hline 26.6.15 & Line of equal runoff [date]—Accurately located & $-6 \longrightarrow$ & 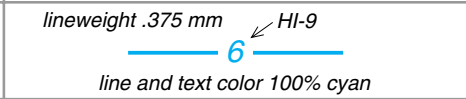 & \\
\hline 26.6 .16 & Line of equal runoff [date]_Approximately located & & $\underset{\rightarrow \mathbb{k}}{.5 \mathrm{~mm}}$ & \\
\hline & 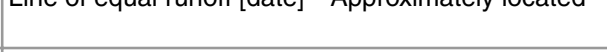 & & $\rightarrow \rightarrow \begin{array}{ll}5.0 \\
\mathrm{~mm}\end{array}$ & \\
\hline 26.6.17 & $\begin{array}{l}\text { Line of equal transmissivity, hydraulic conductivity, } \\
\text { or porosity (etc.) - Accurately located }\end{array}$ & $-10,000-$ & 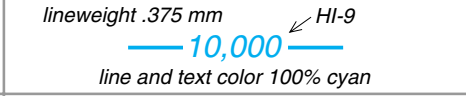 & \\
\hline 26.6.18 & Line of equal transmissivity, hydraulic conductivity, & & $\underset{\rightarrow \mathbb{K}}{.5 \mathrm{~mm}}$ & \\
\hline & or porosity (etc.)—Approximately located & & $\rightarrow\left|\begin{array}{c}5.0 \\
\mathrm{~mm}\end{array}\right|<$ & \\
\hline
\end{tabular}

${ }^{*}$ For more information, see general guidelines on pages $A-i$ to $A-v$. 
Federal Geographic Data Committee (Doc. No. FGDC-STD-013-2006)

U.S. Geological Survey Techniques and Methods 11-A2 FGDC Digital Cartographic Standard for Geologic Map Symbolization (PostScript Implementation)

DOWNLOAD this Illustrator EPS file: Al8 / $\underline{\mathrm{CS} 2}$

\section{6-GEOHYDROLOGIC FEATURES (continued)}

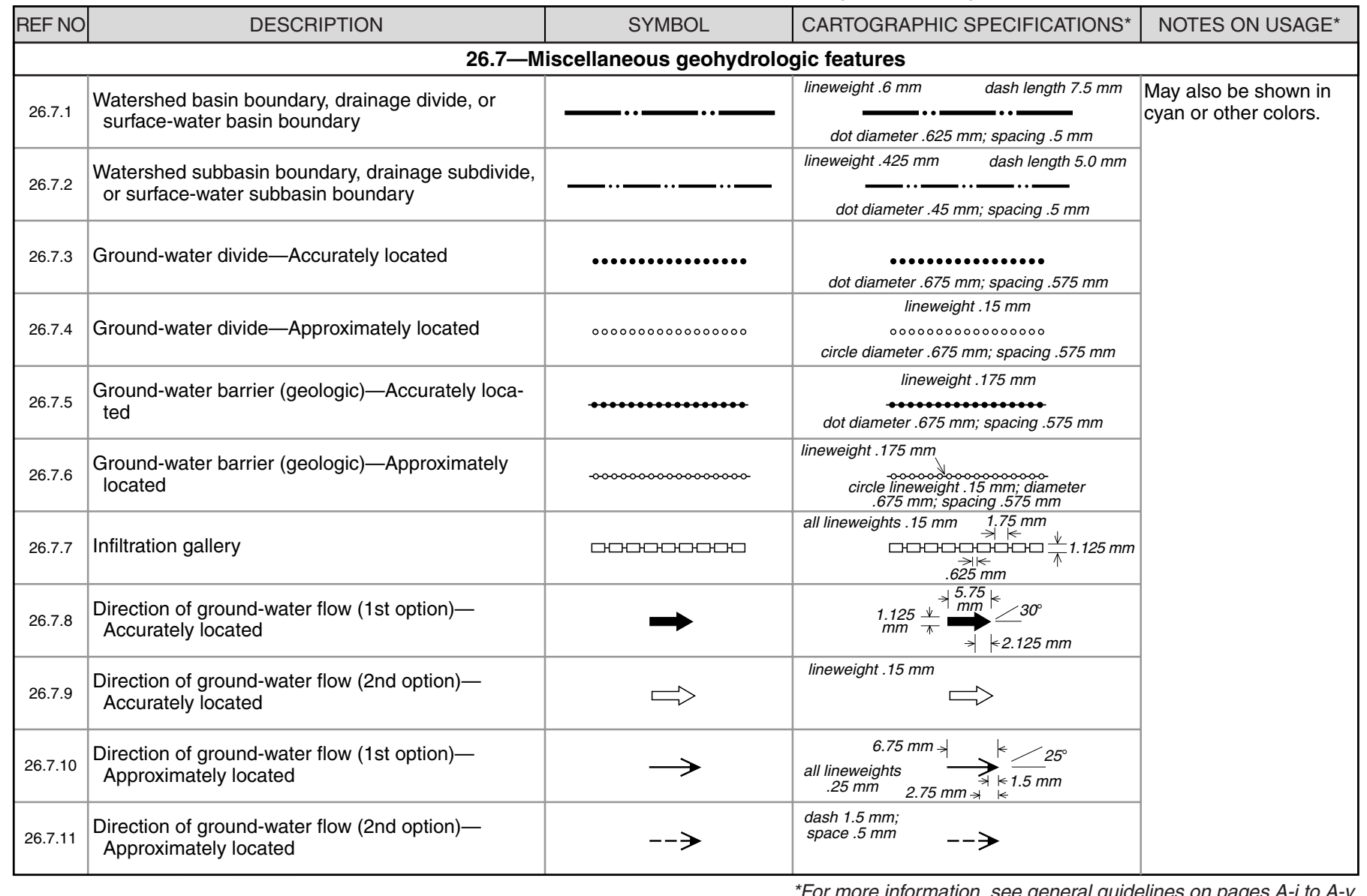


Federal Geographic Data Committee (Doc. No. FGDC-STD-013-2006)

U.S. Geological Survey Techniques and Methods 11-A2 FGDC Digital Cartographic Standard for Geologic Map Symbolization (PostScript Implementation)

\section{7-WEATHER STATIONS}

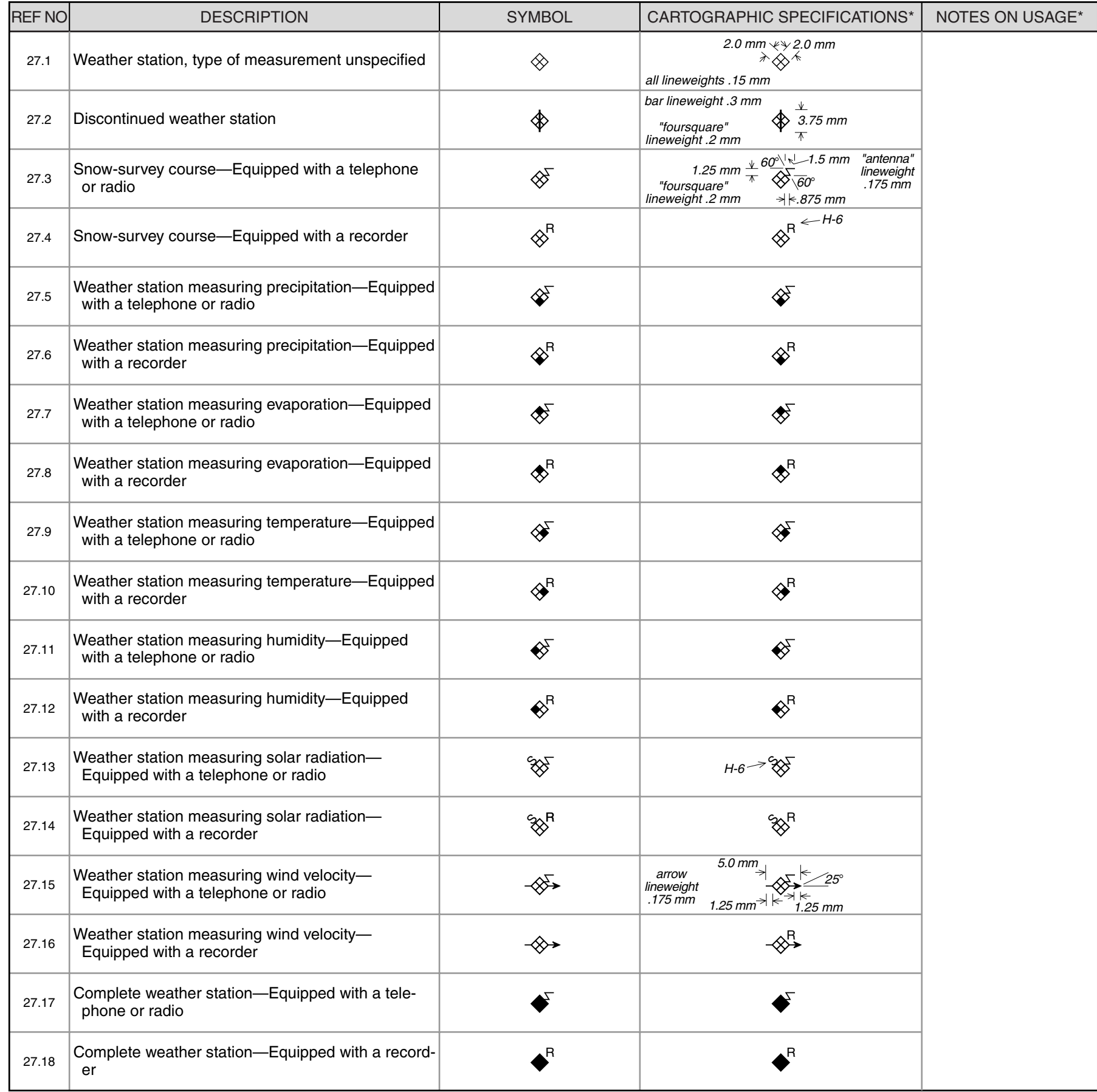

${ }^{\star}$ For more information, see general guidelines on pages $A-i$ to $A-v$. 
Federal Geographic Data Committee (Doc. No. FGDC-STD-013-2006)

U.S. Geological Survey Techniques and Methods 11-A2

FGDC Digital Cartographic Standard for Geologic Map Symbolization (PostScript Implementation) DOWNLOAD this Illustrator EPS file: Al8 / $\underline{\mathrm{CS}}$

\section{8-TRANSPORTATION FEATURES}

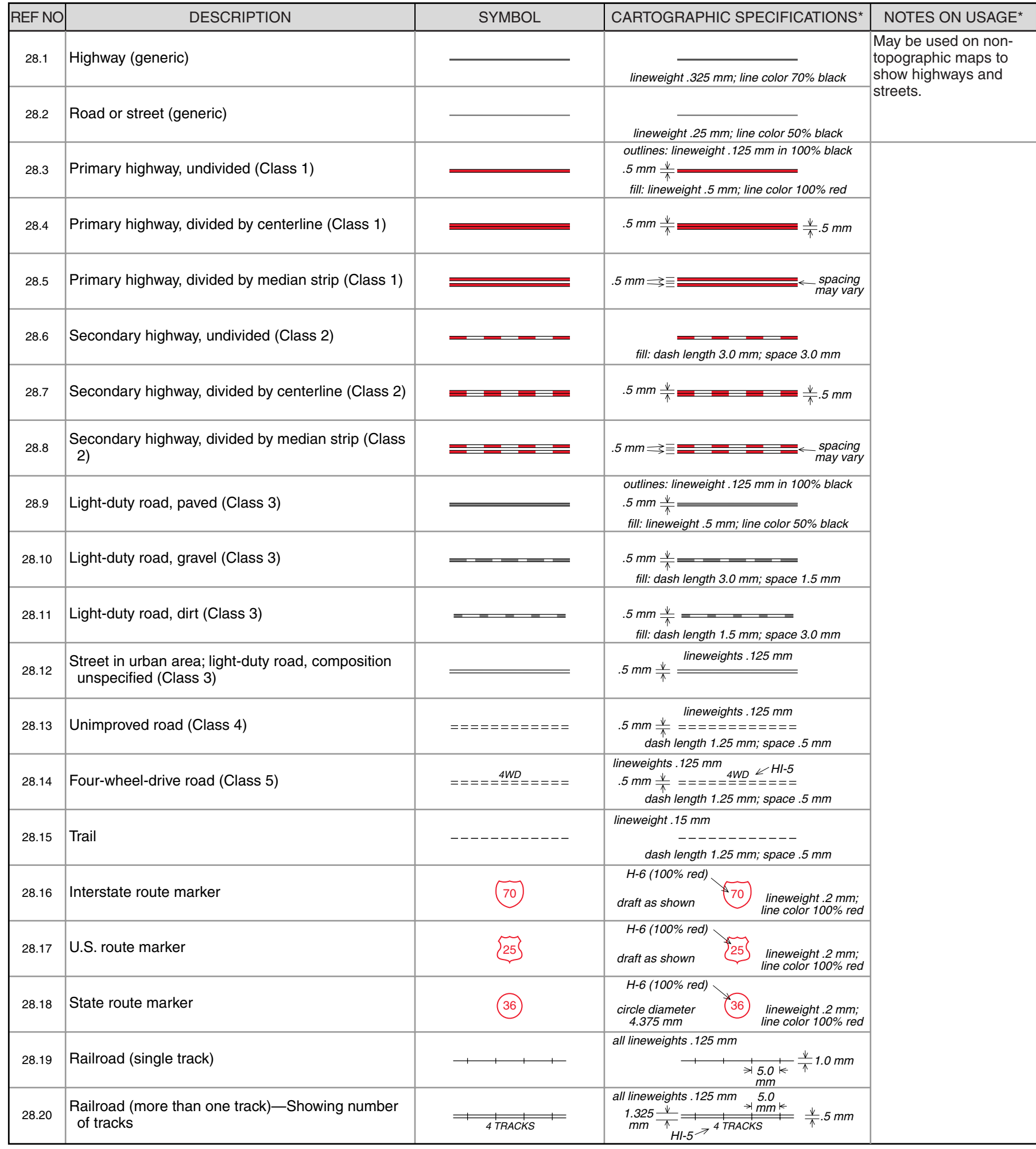

${ }^{*}$ For more information, see general guidelines on pages $A-i$ to $A-\mathrm{V}$. 
Federal Geographic Data Committee (Doc. No. FGDC-STD-013-2006)

U.S. Geological Survey Techniques and Methods 11-A2 FGDC Digital Cartographic Standard for Geologic Map Symbolization (PostScript Implementation) DOWNLOAD this Illustrator EPS file: Al8 / CS2

\section{9-BOUNDARIES}

\begin{tabular}{|c|c|c|c|c|}
\hline REF NO & DESCRIPTION & SYMBOL & CARTOGRAPHIC SPECIFICATIONS* & NOTES ON USAGE* \\
\hline 29.1 & Boundary—National & UNITED STATES & $\begin{array}{l}\begin{array}{l}\text { long dash } \\
6.35 \mathrm{~mm} ; \\
\text { short dashes } 1.75 \\
\text { mm; space } 835 \mathrm{~mm}\end{array} \\
\text { MEXICO STATES }\end{array}$ & \\
\hline 29.3 & $\begin{array}{l}\text { Boundary—County, parish, Alaska borough, muni- } \\
\text { cipio, judicial division }\end{array}$ & $-\frac{\mathrm{HUMBOLDT}}{\mathrm{MENDOC}} \overline{\mathrm{N}}$ & 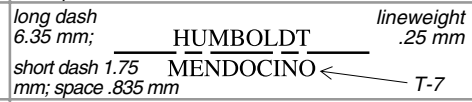 & \\
\hline 29.5 & $\begin{array}{l}\text { Boundary-Incorporated city, village, town, bor- } \\
\text { ough, or hamlet }\end{array}$ & & $\begin{array}{l}\text { lineweight } .175 \mathrm{~mm} \\
\text { long dash } 2.0 \mathrm{~mm} ; \overline{\mathrm{mh}}-\overline{\mathrm{sht}} \text { dash } \overline{1.0} \mathrm{~mm} \\
\text { space } \overline{\mathrm{mm}}\end{array}$ & \\
\hline 29.6 & $\begin{array}{l}\text { Boundary-National or state park, monument, res- } \\
\text { ervation, forest, grassland, wilderness area, or } \\
\text { wildlife refuge; Hawaii Homestead, Forest Reserve }\end{array}$ & - & $\begin{array}{l}\text { lineweight } .175 \mathrm{~mm} \\
\text { dash length } 6.35 \mathrm{~mm} ; \text { space } 2.5 \mathrm{~mm}\end{array}$ & \\
\hline 29.8 & Continental Divide & $\begin{array}{c}\text { CONTINENTAL } \\
\text { DIVIDE }\end{array}$ & $\begin{array}{c}\text { lineweight } .3 \mathrm{~mm} \text { CONTINENTAL } \leftarrow \\
\frac{\text { DIVIDE }}{\text { dash } 10.0 \mathrm{~mm} \text {; space } 2.5 \mathrm{~mm}}\end{array}$ & \\
\hline
\end{tabular}

${ }^{*}$ For more information, see general guidelines on pages $A-i$ to $A-v$. 
Federal Geographic Data Committee (Doc. No. FGDC-STD-013-2006)

U.S. Geological Survey Techniques and Methods 11-A2 FGDC Digital Cartographic Standard for Geologic Map Symbolization (PostScript Implementation)

DOWNLOAD this Illustrator EPS file: Al8 / $\underline{\mathrm{CS} 2}$

\section{0-TOPOGRAPHIC AND HYDROGRAPHIC FEATURES}

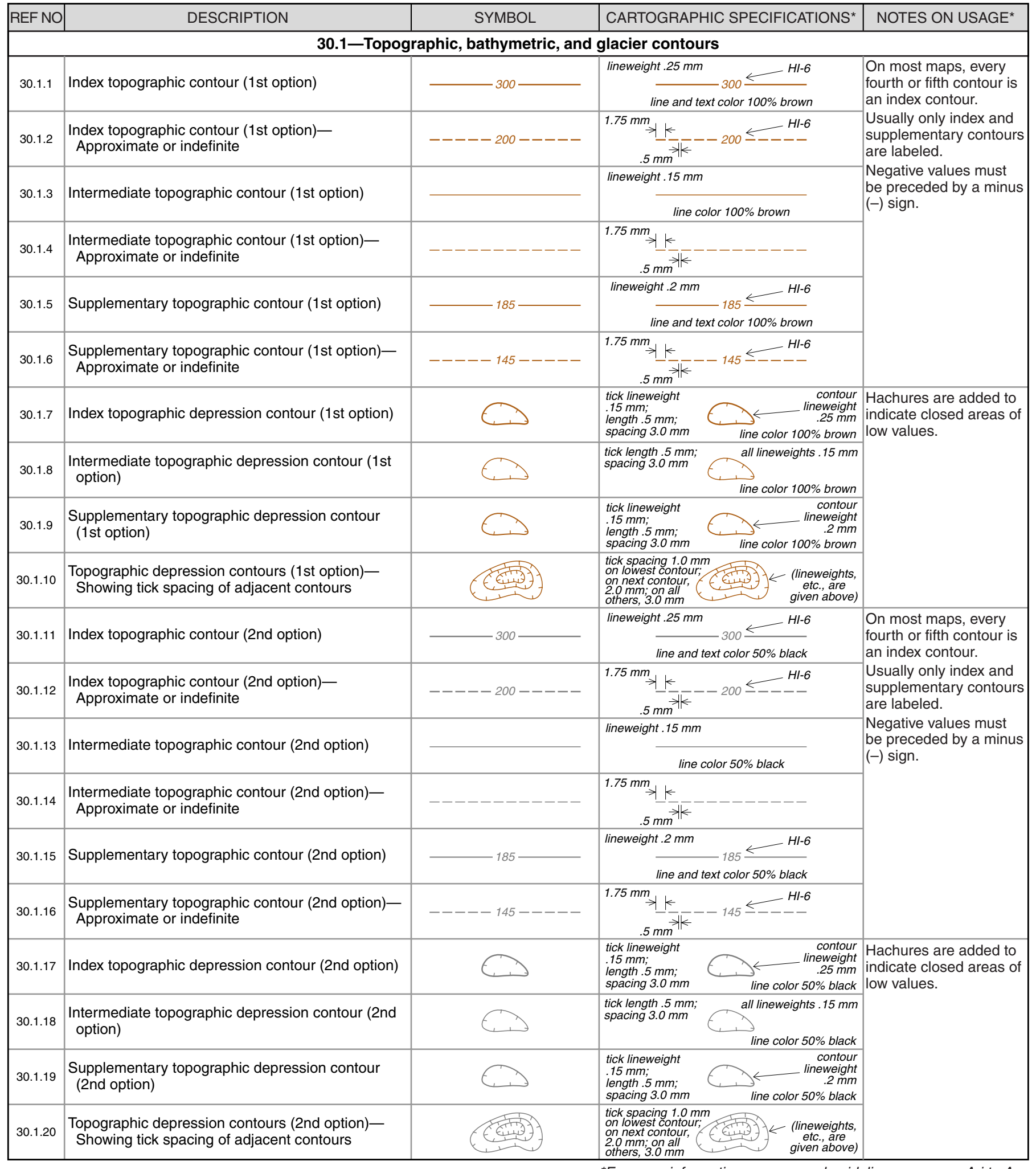

${ }^{*}$ For more information, see general guidelines on pages $A-i$ to $A-v$. 
Federal Geographic Data Committee (Doc. No. FGDC-STD-013-2006)

U.S. Geological Survey Techniques and Methods 11-A2

FGDC Digital Cartographic Standard for Geologic Map Symbolization (PostScript Implementation)

DOWNLOAD this Illustrator EPS file: Al8 / $\underline{\mathrm{CS} 2}$

\section{0-TOPOGRAPHIC AND HYDROGRAPHIC FEATURES (continued)}

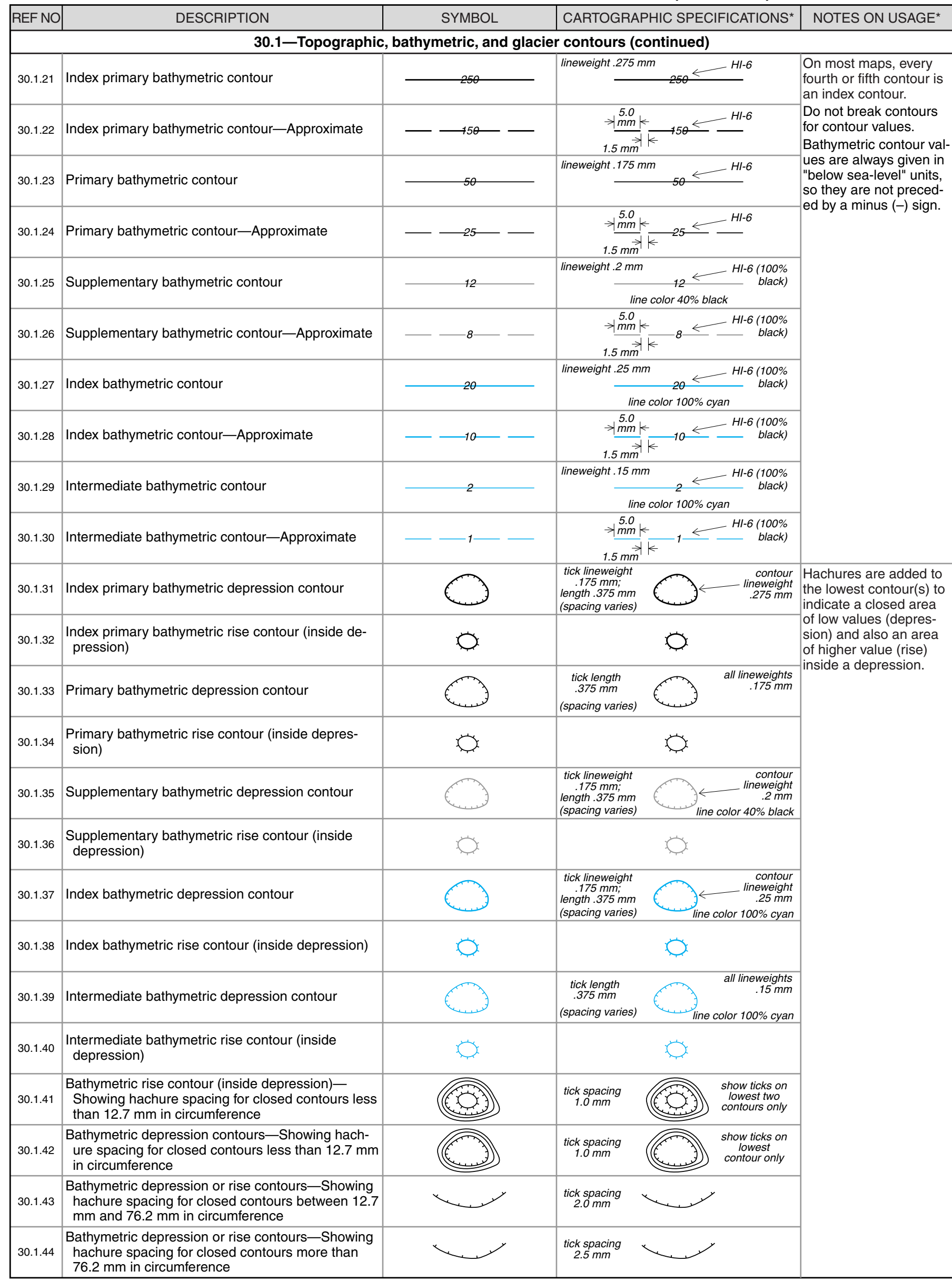


Federal Geographic Data Committee (Doc. No. FGDC-STD-013-2006)

U.S. Geological Survey Techniques and Methods 11-A2 FGDC Digital Cartographic Standard for Geologic Map Symbolization (PostScript Implementation)

30-TOPOGRAPHIC AND HYDROGRAPHIC FEATURES (continued)

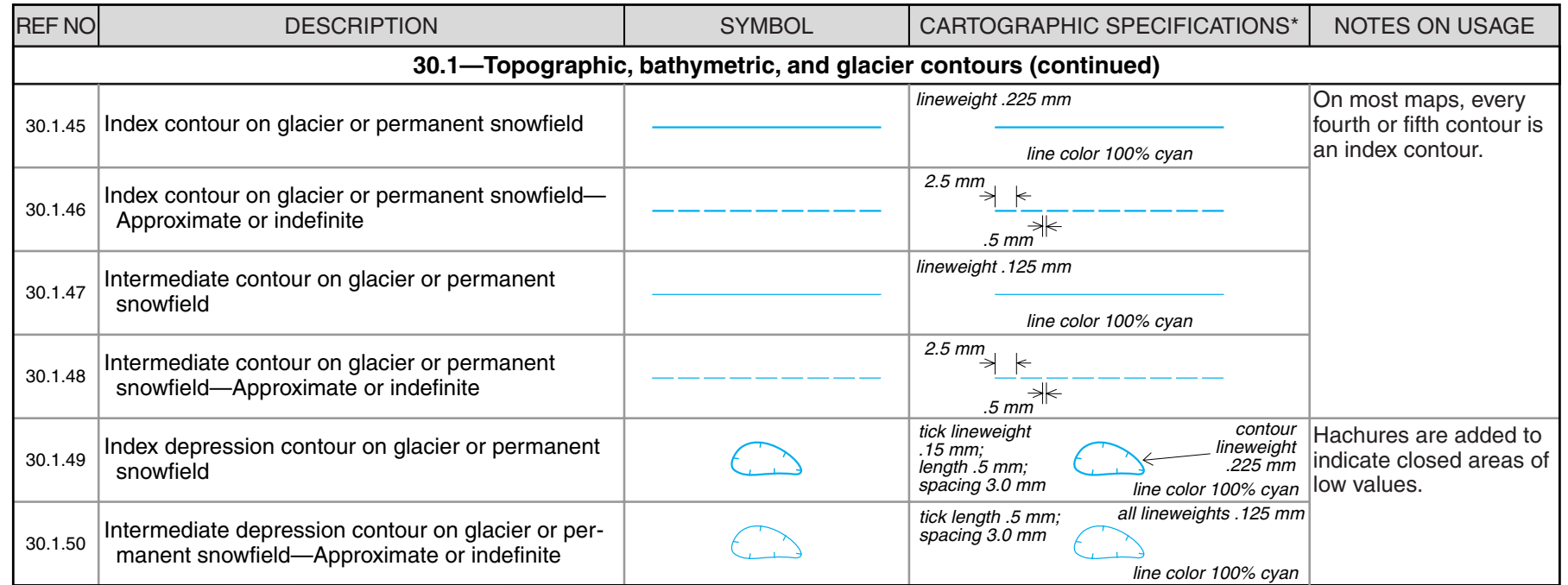

${ }^{*}$ For more information, see general guidelines on pages $A-i$ to $A-v$. 
Federal Geographic Data Committee (Doc. No. FGDC-STD-013-2006)

U.S. Geological Survey Techniques and Methods 11-A2

FGDC Digital Cartographic Standard for Geologic Map Symbolization (PostScript Implementation)

DOWNLOAD this Illustrator EPS file: Al8 / $\underline{\mathrm{CS} 2}$

\section{0-TOPOGRAPHIC AND HYDROGRAPHIC FEATURES (continued)}

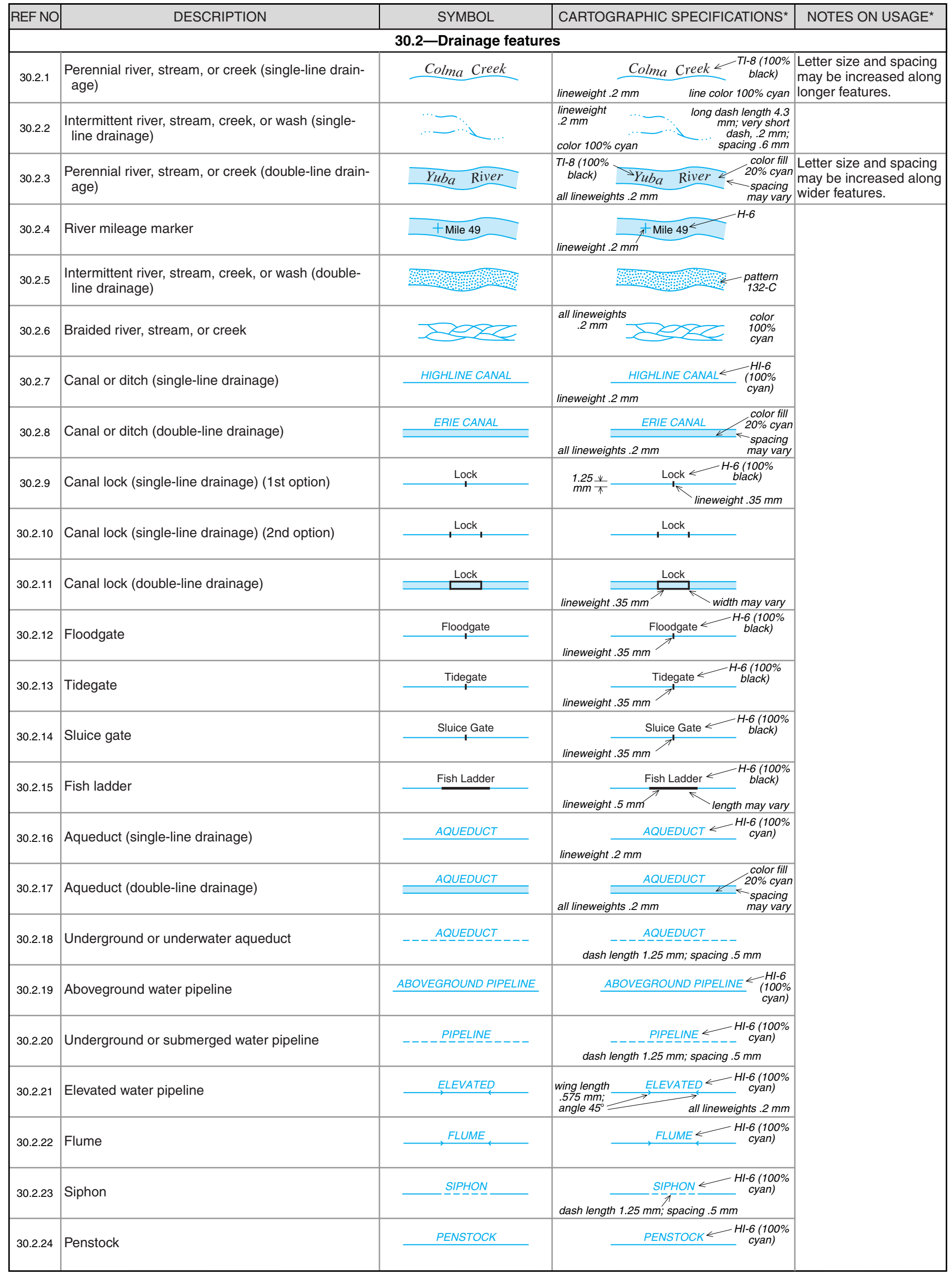


Federal Geographic Data Committee (Doc. No. FGDC-STD-013-2006)

U.S. Geological Survey Techniques and Methods 11-A2

FGDC Digital Cartographic Standard for Geologic Map Symbolization (PostScript Implementation)

DOWNLOAD this Illustrator EPS file: Al8 / $\underline{\mathrm{CS}}$

\section{0-TOPOGRAPHIC AND HYDROGRAPHIC FEATURES (continued)}

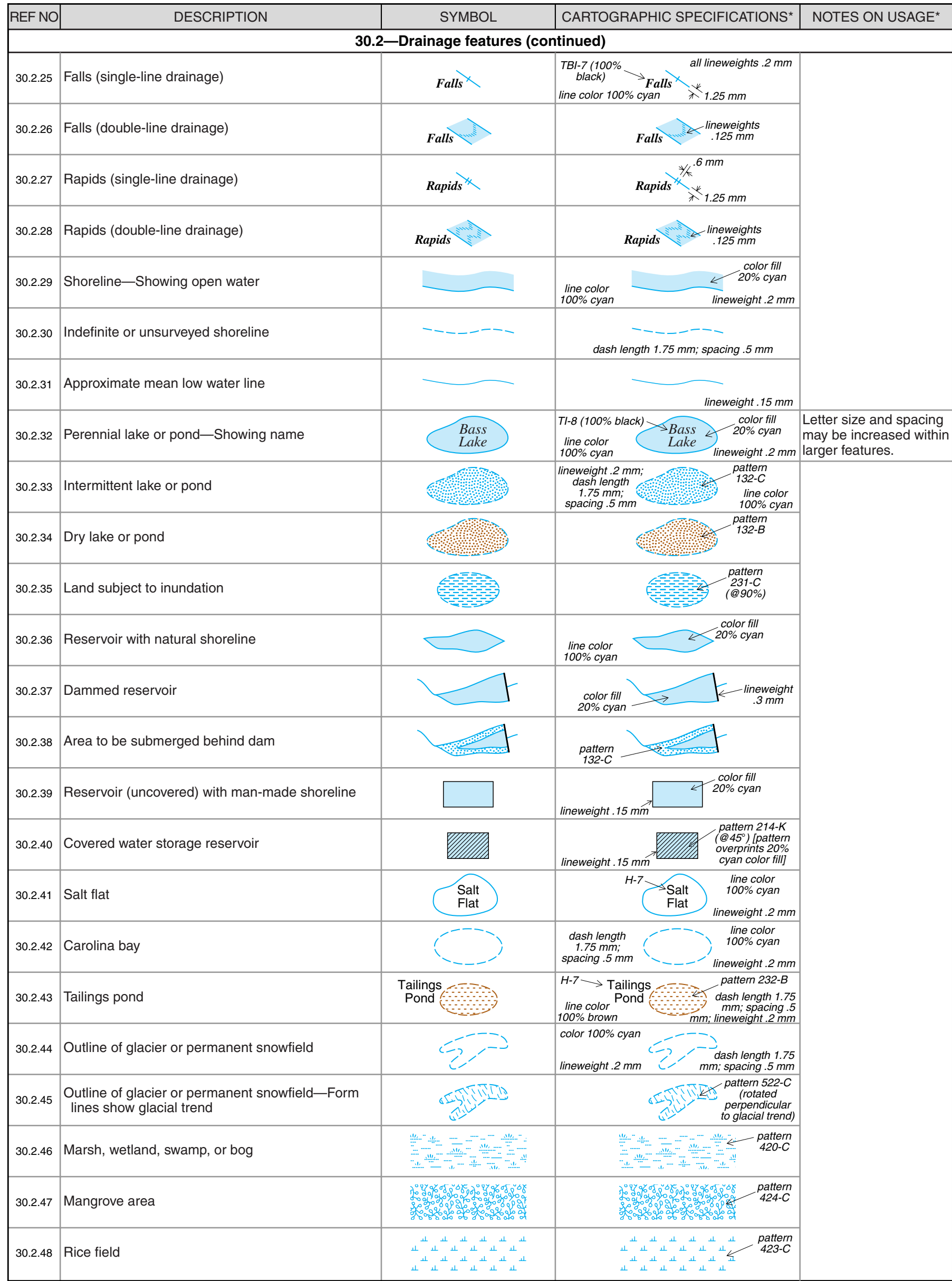


Federal Geographic Data Committee (Doc. No. FGDC-STD-013-2006)

U.S. Geological Survey Techniques and Methods 11-A2

FGDC Digital Cartographic Standard for Geologic Map Symbolization (PostScript Implementation)

DOWNLOAD this Illustrator EPS file: Al8 / CS2

\section{0-TOPOGRAPHIC AND HYDROGRAPHIC FEATURES (continued)}

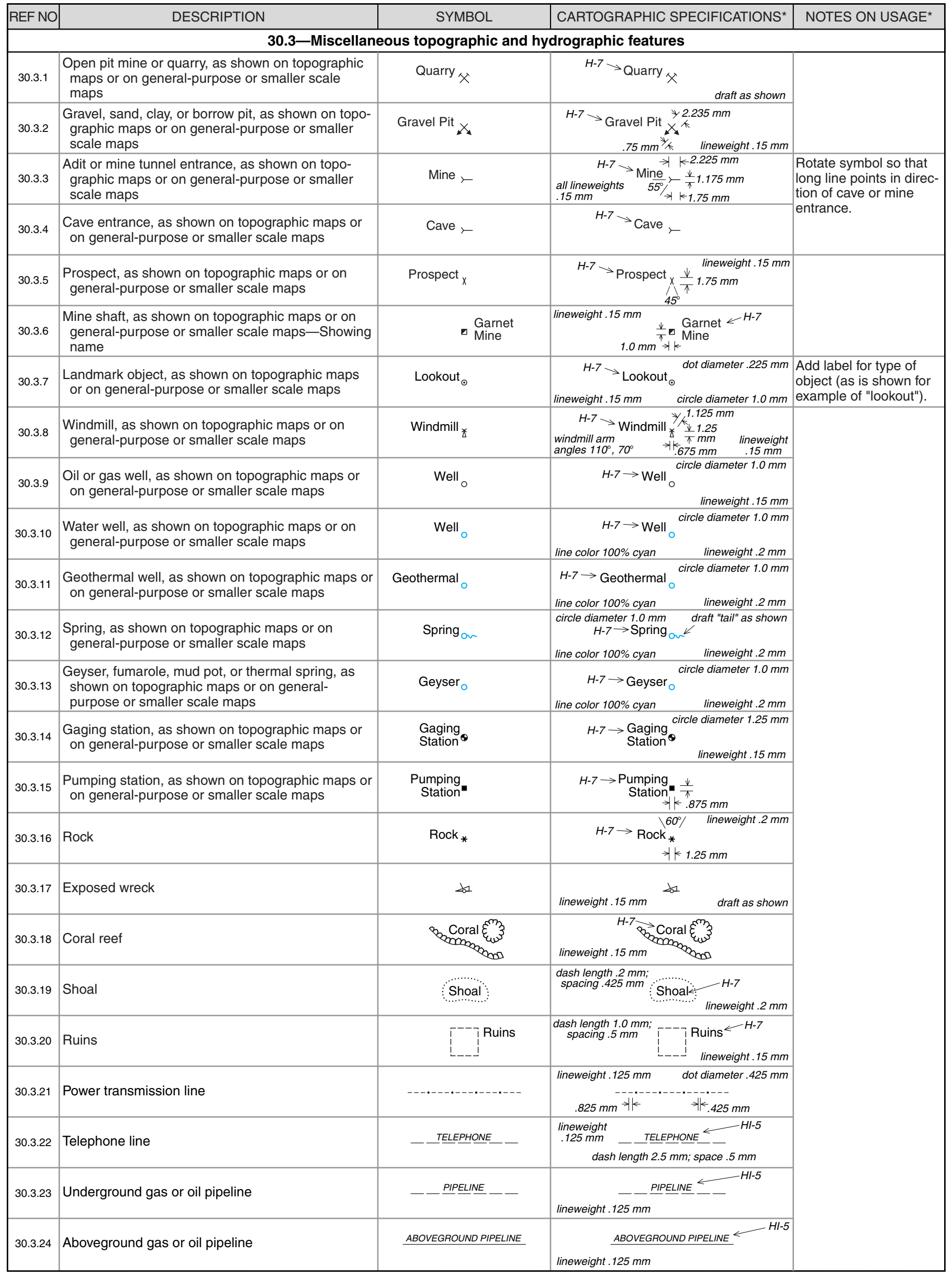


Federal Geographic Data Committee (Doc. No. FGDC-STD-013-2006)

U.S. Geological Survey Techniques and Methods 11-A2

FGDC Digital Cartographic Standard for Geologic Map Symbolization (PostScript Implementation)

DOWNLOAD this Illustrator EPS file: Al8 / $\underline{\text { CS2 }}$

\section{1-MISCELLANEOUS MAP ELEMENTS}

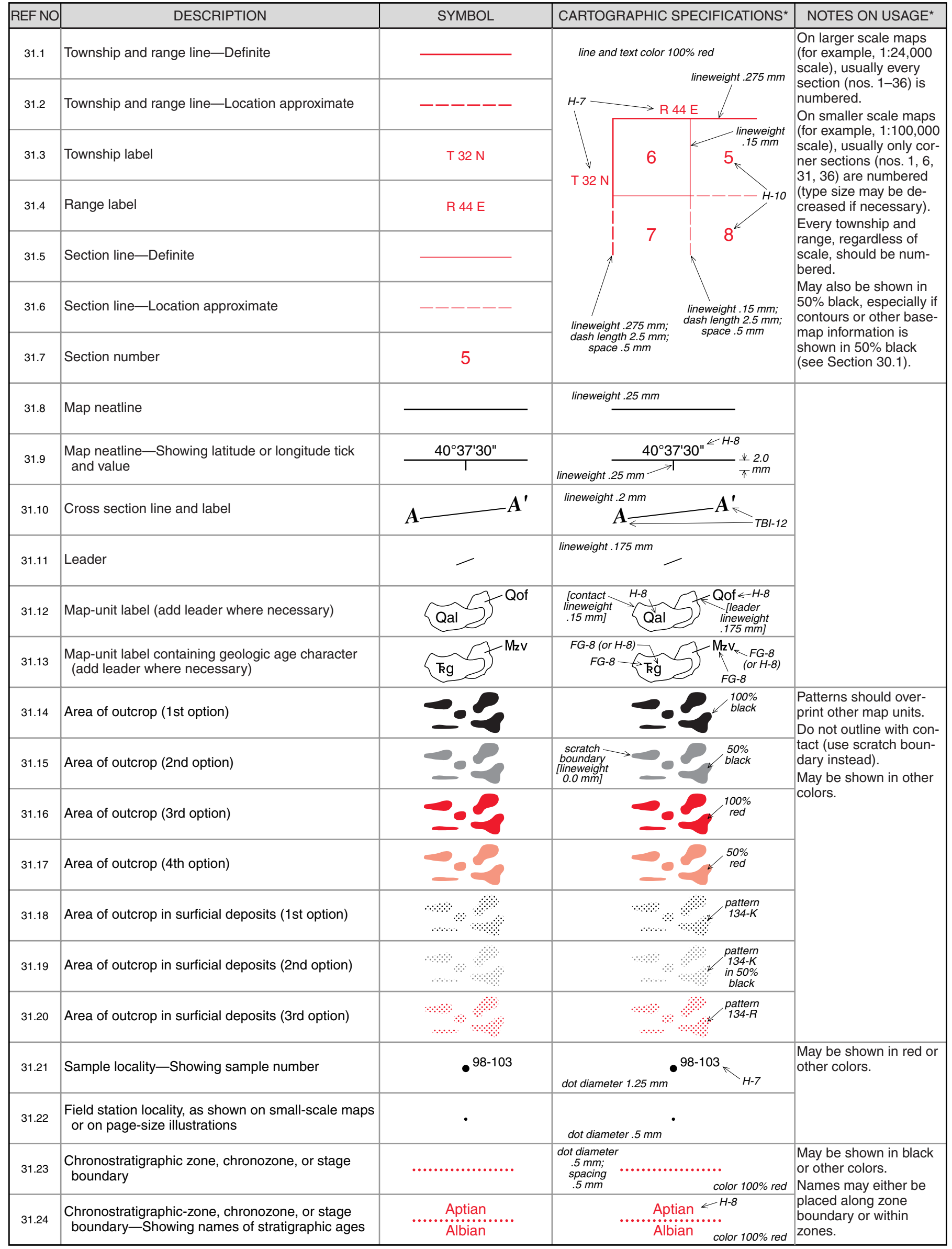

${ }^{*}$ For more information, see general guidelines on pages $A-i$ to $A-v$. 
Federal Geographic Data Committee (Doc. No. FGDC-STD-013-2006)

U.S. Geological Survey Techniques and Methods 11-A2

FGDC Digital Cartographic Standard for Geologic Map Symbolization (PostScript Implementation)

DOWNLOAD this Illustrator EPS file: Al8 / CS2

\section{2-GEOLOGIC AGE SYMBOL FONT ("FGDCGeoAge")}

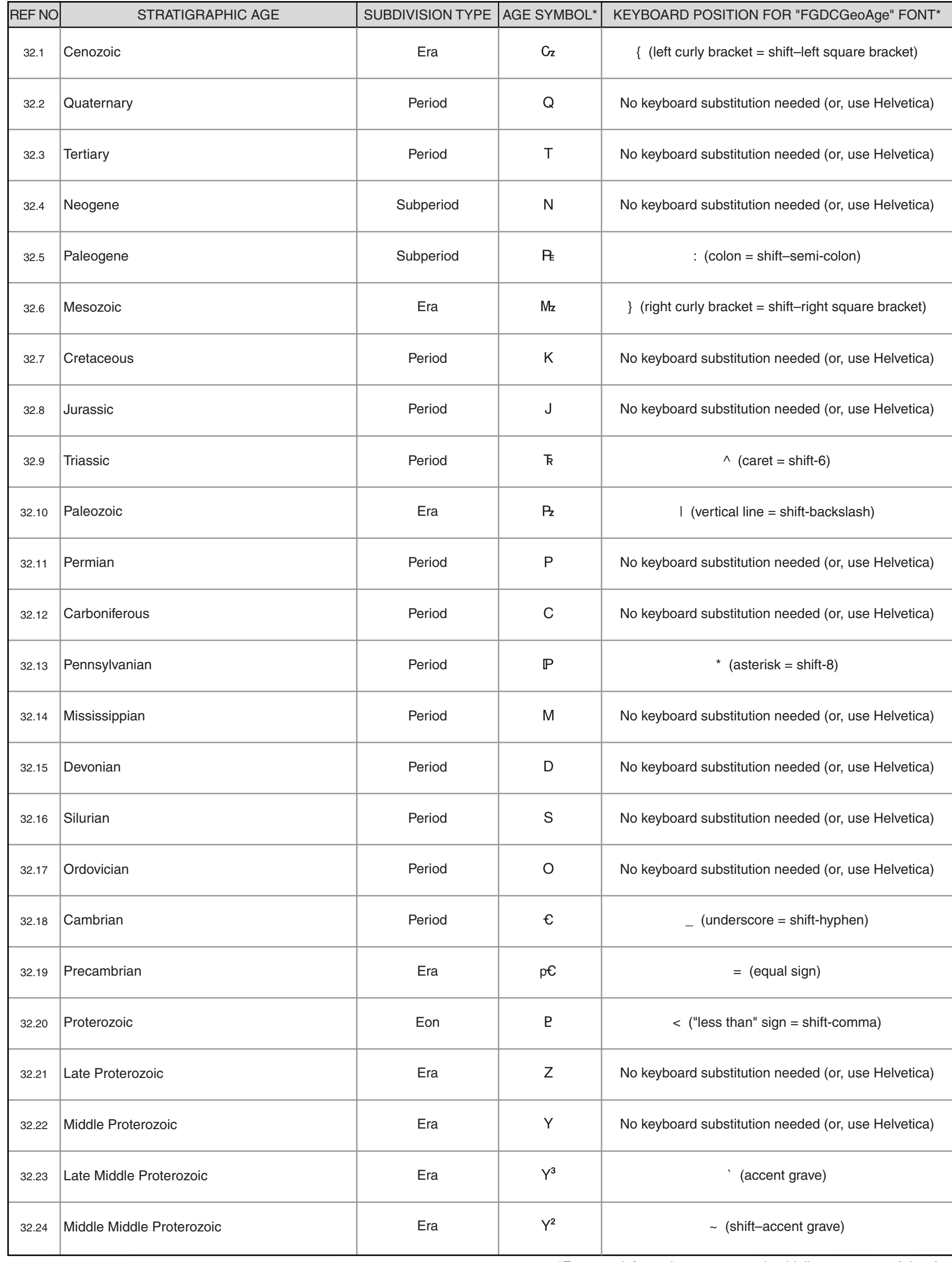

${ }^{\star}$ For more information, see general guidelines on pages $A-i$ to $A-v$. 
Federal Geographic Data Committee (Doc. No. FGDC-STD-013-2006)

U.S. Geological Survey Techniques and Methods 11-A2 FGDC Digital Cartographic Standard for Geologic Map Symbolization (PostScript Implementation) DOWNLOAD this Illustrator EPS file: Al8 / CS2

\section{2-GEOLOGIC AGE SYMBOL FONT ("FGDCGeoAge") (continued)}

\begin{tabular}{|c|c|c|c|c|}
\hline REF NO & STRATIGRAPHIC AGE & SUBDIVISION TYPE & AGE SYMBOL* & KEYBOARD POSITION FOR "FGDCGeoAge" FONT* \\
\hline 32.25 & Early Middle Proterozoic & Era & $Y^{1}$ & ! $($ exclamation point $=$ shift- $1[$ one $])$ \\
\hline 32.27 & Late Early Proterozoic & Era & $X^{3}$ & @ ("at" sign = shift-2) \\
\hline 32.28 & Middle Early Proterozoic & Era & $\mathrm{X}^{2}$ & $\#($ pound sign $=$ shift- 3$)$ \\
\hline 32.29 & Early Early Proterozoic & Era & $X^{1}$ & $\$($ dollar sign $=$ shift- 4$)$ \\
\hline 32.30 & Archean & Eon & A & No keyboard substitution needed (or, use Helvetica) \\
\hline 32.32 & Middle Archean & Era & $\mathrm{V}$ & No keyboard substitution needed (or, use Helvetica) \\
\hline 32.33 & Early Archean & Era & U & No keyboard substitution needed (or, use Helvetica) \\
\hline 32.34 & pre-Archean & Eon & $\mathrm{pA}$ & > ("greater than" sign = shift-period $)$ \\
\hline
\end{tabular}

${ }^{*}$ For more information, see general guidelines on pages $A-i$ to $A-v$. 


\section{3-SUGGESTED RANGES OF MAP-UNIT COLORS FOR VOLCANIC AND PLUTONIC ROCKS AND FOR STRATIGRAPHIC AGES OF SEDIMENTARY AND METAMORPHIC ROCKS}

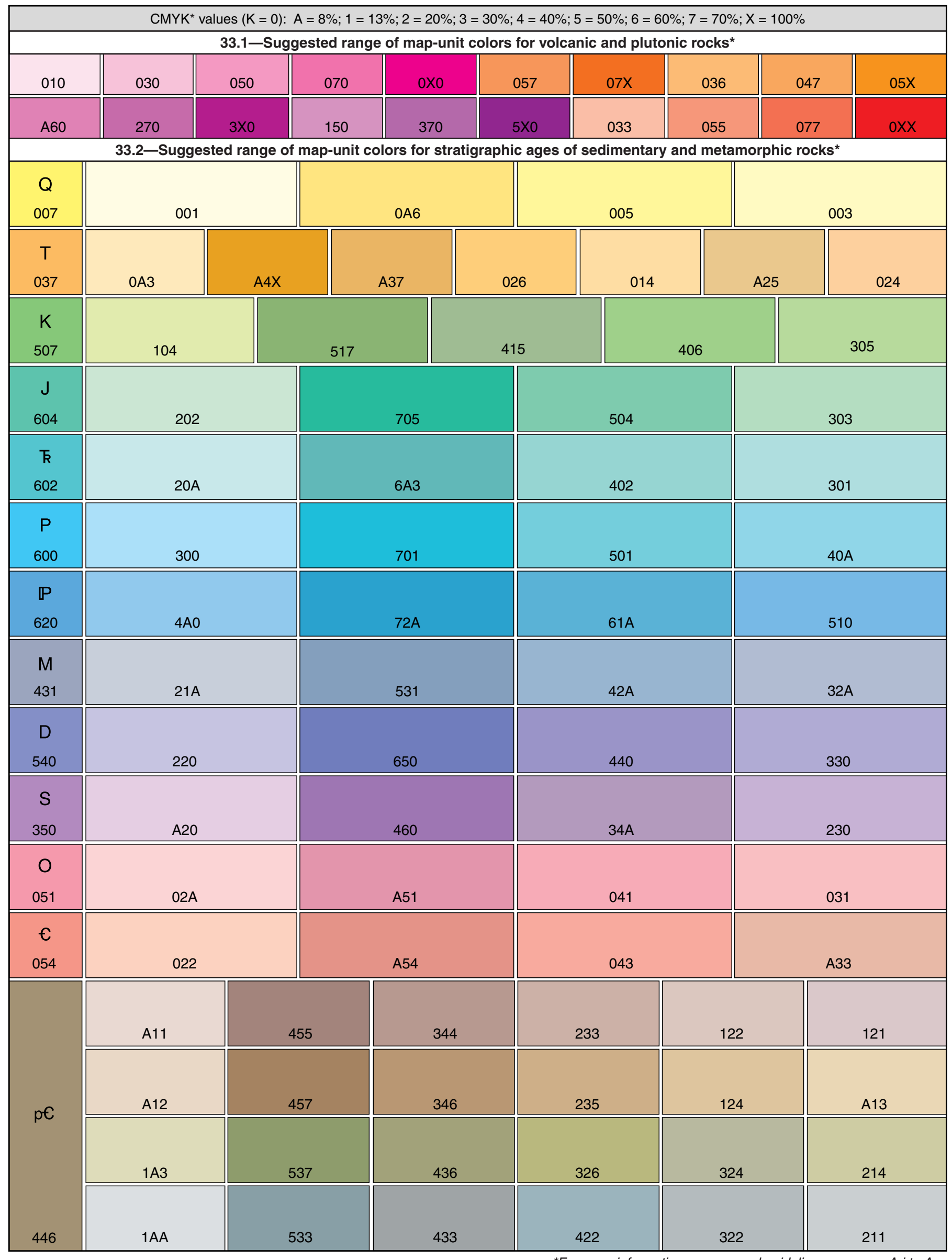




\section{4-STATE LOCATION MAPS}

\begin{tabular}{|c|c|}
\hline CARTOGRAPHIC SPECIFICATIONS & NOTES ON USAGE \\
\hline MAP LOCATION & $\begin{array}{l}\text { State location maps are at various scales; projection is Albers Equal-Area, } \\
\text { based on parallels } 291 / 2^{\circ} \text { and } 451 / 2^{\circ} \text {. } \\
\text { Maps are modified from the United States Base Map (U.S. Geological Survey, } \\
\text { 1965, scale } 1: 3,168,000 \text { ) and the Digital Shaded-Relief Image of Alaska (J.R. } \\
\text { Riehle and others, 1997, U.S. Geological Survey Miscellaneous Investigations } \\
\text { Map I-2585, scale 1:2,500,000; see fig. } 2 \text {, approximate scale } 1: 8,000,000) \text {. } \\
\text { To show a quadrangle or map-area location, place a small black-filled rectangle } \\
\text { ( } \text { ) or polygon ( } \text { ) that shows the approximate location within state (adjust } \\
\text { size and shape accordingly). Reposition state name if necessary. } \\
\text { If a quadrangle or map-area location is within two or more adjoining states, cre- } \\
\text { ate one new location map that contains each state by extracting states from } \\
\text { location map of } 48 \text { conterminous states (see Section } 34.2 \text { ). Rotate new location } \\
\text { map so that it is approximately horizontal, and resize it so that it is about } 2-2.5 \\
\text { cm high. Add rectangle or polygon showing location of mapped area, then add } \\
\text { names to each state. Center "MAP LOCATION" below new location map. }\end{array}$ \\
\hline
\end{tabular}

34.1-Individual states; District of Columbia; Guam; Puerto Rico; U.S. Virgin Islands

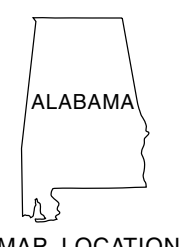

MAP LOCATION

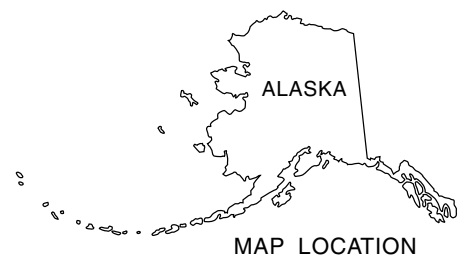

MAP LOCATION

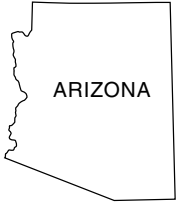

MAP LOCATION

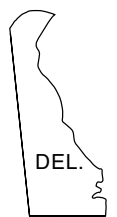

MAP LOCATION

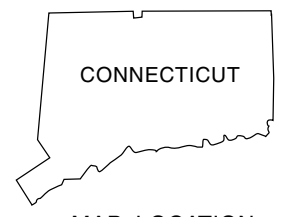

MAP LOCATION

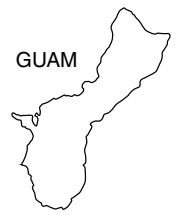

MAP LOCATION

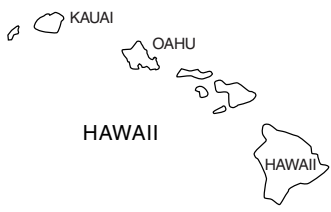

MAP LOCATION

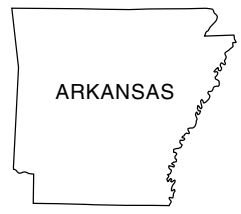

MAP LOCATION

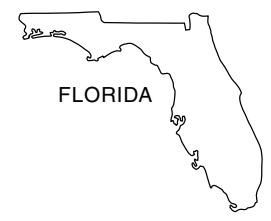

MAP LOCATION

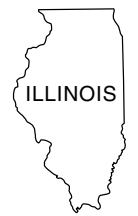

MAP LOCATION

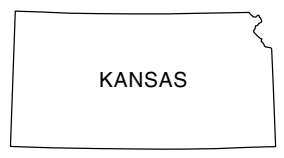

MAP LOCATION

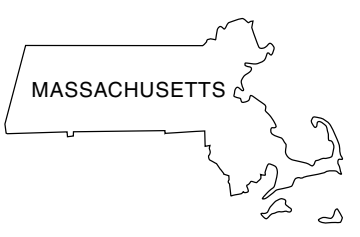

MAP LOCATION

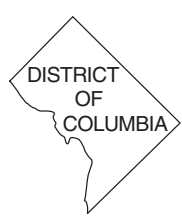

MAP LOCATION

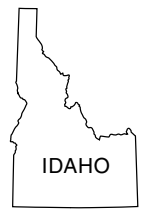

MAP LOCATION

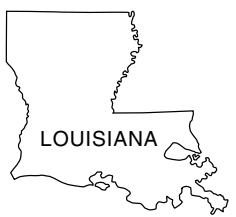

MAP LOCATION

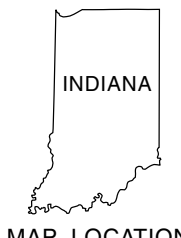

MAP LOCATION

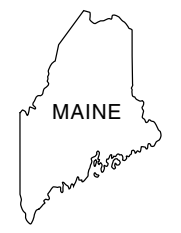

MAP LOCATION

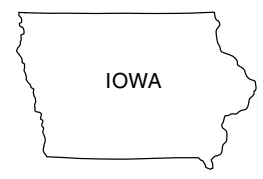

MAP LOCATION

MAP LOCATION
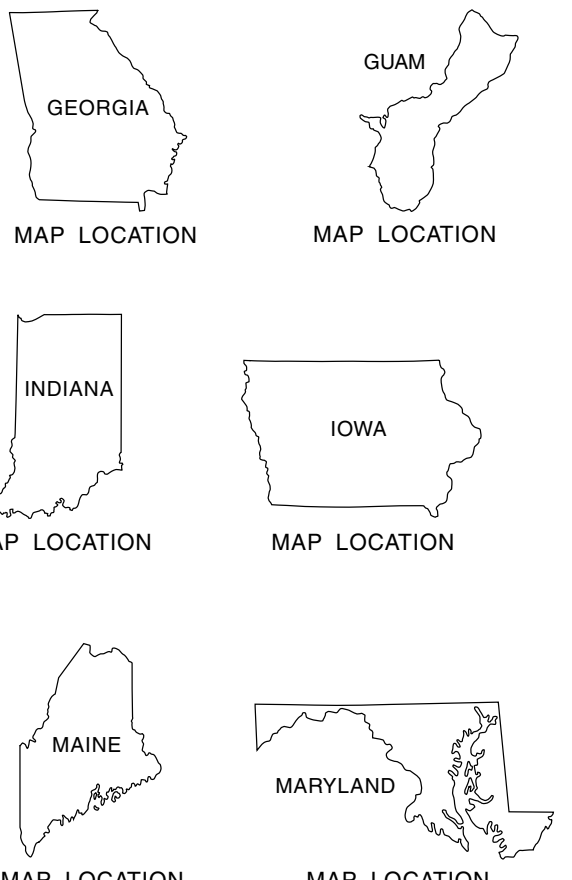

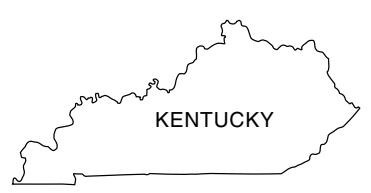

MAP LOCATION 


\section{4-STATE LOCATION MAPS (continued)}

34.1-Individual states; District of Columbia; Guam; Puerto Rico; U.S. Virgin Islands (continued)

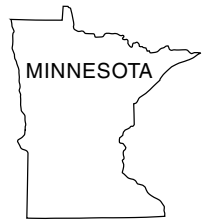

MAP LOCATION

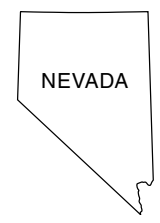

MAP LOCATION

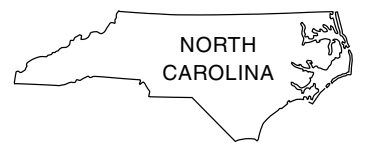

MAP LOCATION

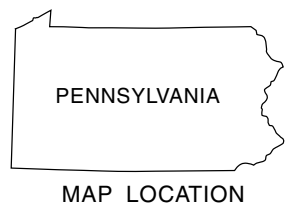

MAP LOCATION

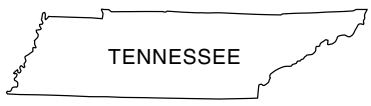

MAP LOCATION

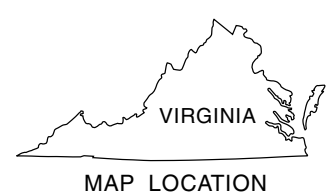

MAP LOCATION

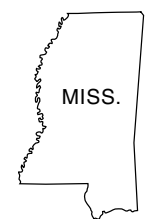

MAP LOCATION

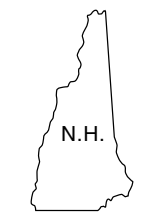

MAP LOCATION

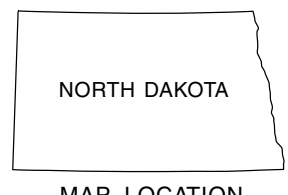

MAP LOCATION
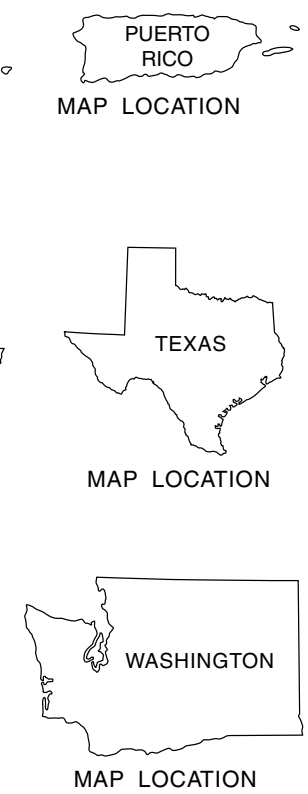

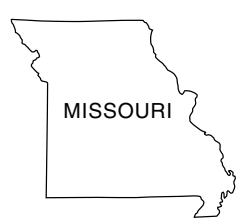

MAP LOCATION

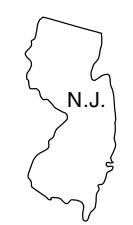

MAP LOCATION
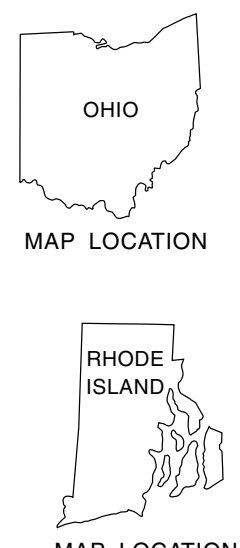

MAP LOCATION
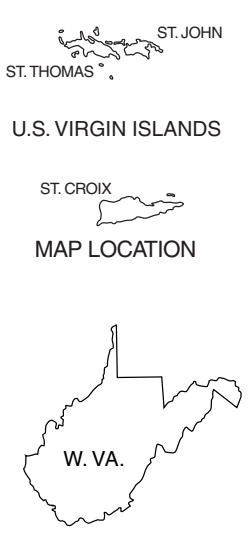

MAP LOCATION

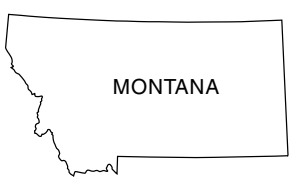

MAP LOCATION

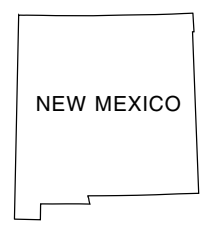

MAP LOCATION

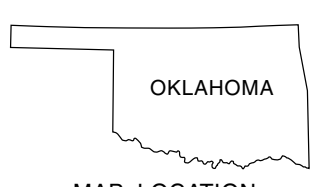

MAP LOCATION

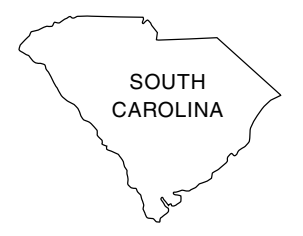

MAP LOCATION

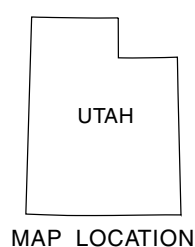

MAP LOCATION

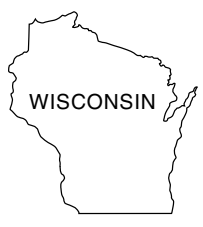

MAP LOCATION

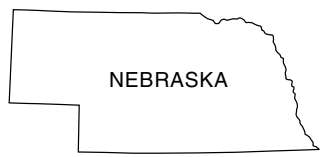

MAP LOCATION

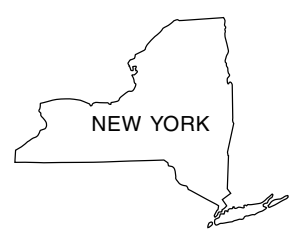

MAP LOCATION

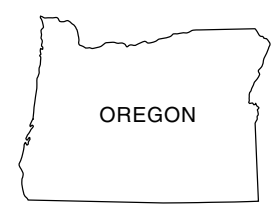

MAP LOCATION

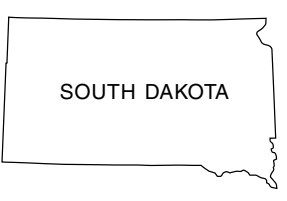

MAP LOCATION

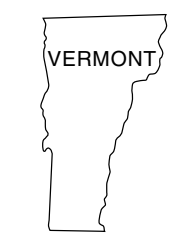

MAP LOCATION

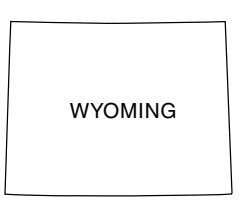

MAP LOCATION 


\section{4-STATE LOCATION MAPS (continued)}

34.2-Conterminous states

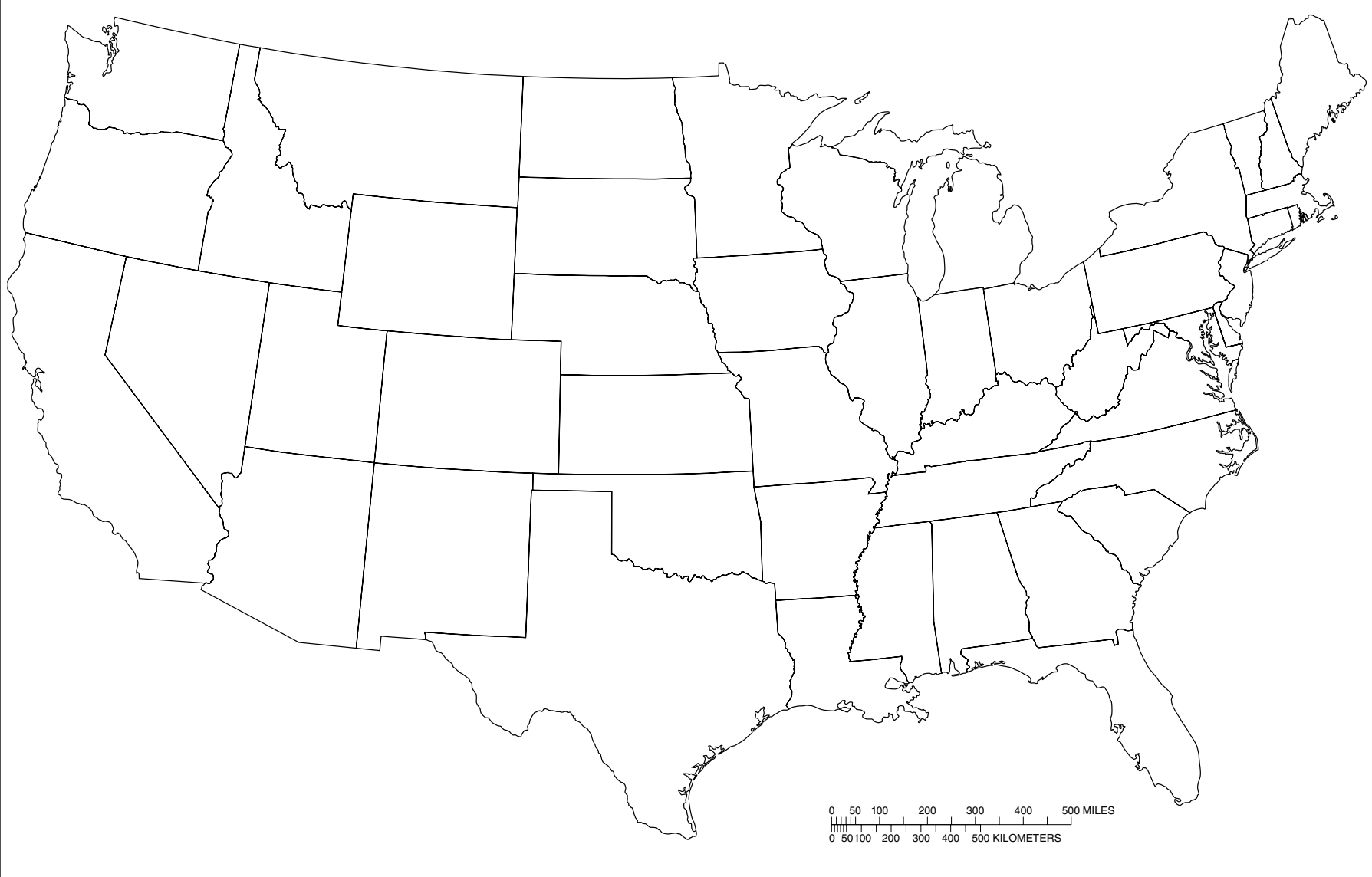




\section{5-BAR SCALES}

CARTOGRAPHIC SPECIFICATIONS

2 UNITS OF MEASUREMENT:

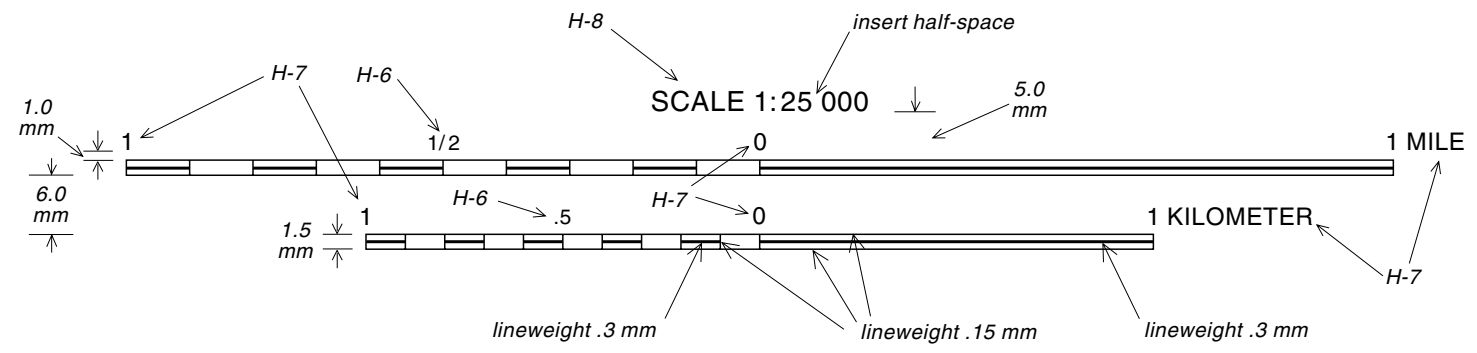

3 UNITS OF MEASUREMENT:

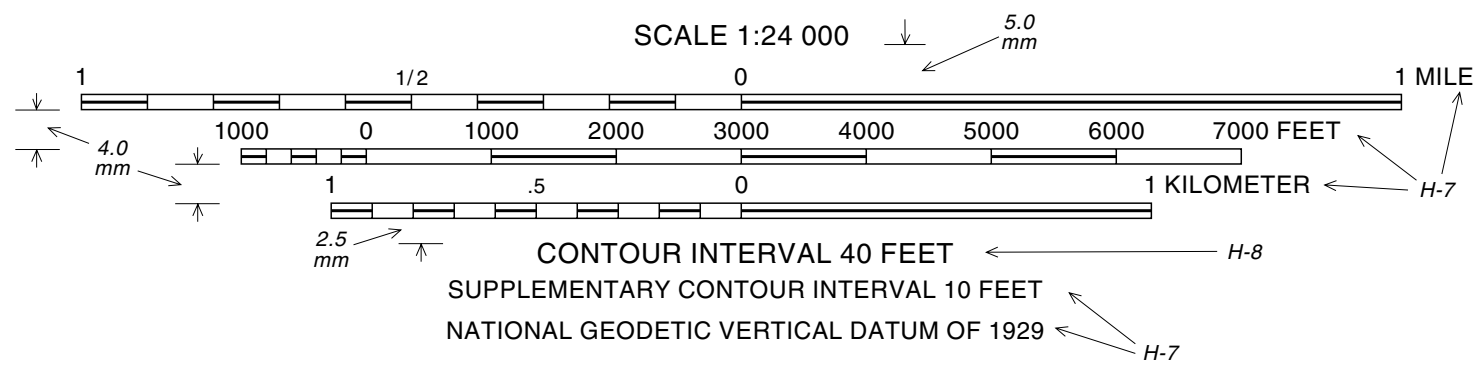

\begin{tabular}{|c|c|c|c|c|}
\hline \multicolumn{2}{|c|}{ DISTANCE MEASURES } & \multicolumn{3}{|c|}{ MEASUREMENT EQUIVALENTS } \\
\hline \multirow[b]{2}{*}{1 mile $(\mathrm{mi})$} & \multirow[b]{2}{*}{$\begin{array}{l}=63,360 \text { inches }(\mathrm{in}) \\
=5,280 \text { feet }(\mathrm{ft})\end{array}$} & \multicolumn{2}{|c|}{ Metric } & \multirow{2}{*}{$\begin{array}{l}\text { English } \\
=0.039 \mathrm{in}\end{array}$} \\
\hline & & 1 millimeter $(\mathrm{mm})$ & $\begin{array}{l}=1 / 10 \mathrm{~cm} \\
=1 / 1000 \mathrm{~m}\end{array}$ & \\
\hline \multirow[t]{3}{*}{1 kilometer $(\mathrm{km})$} & $\begin{array}{l}=3,280.833 \mathrm{ft} \\
=0.62137 \mathrm{mi}\end{array}$ & 1 centimeter $(\mathrm{cm})$ & $\begin{array}{l}=10 \mathrm{~mm} \\
=1 / 100 \mathrm{~m}\end{array}$ & $=0.393 \mathrm{in}$ \\
\hline & & 1 meter $(m)$ & $\begin{array}{l}=100 \mathrm{~cm} \\
=1,000 \mathrm{~mm} \\
=1 / 1,000 \mathrm{~km}\end{array}$ & $\begin{aligned}= & 39.37 \mathrm{in} \\
& \text { or } 3.28 \mathrm{ft} \\
& \text { or } 0.00062 \mathrm{mi}\end{aligned}$ \\
\hline & & 1 kilometer $(\mathrm{km})$ & $\begin{array}{l}=1,000 \mathrm{~m} \\
=100,000 \mathrm{~cm} \\
=1,000,000 \mathrm{~mm}\end{array}$ & $\begin{array}{l}=3,280.833 \mathrm{ft} \\
\\
\text { or } 0.62137 \mathrm{mi}\end{array}$ \\
\hline
\end{tabular}


Federal Geographic Data Committee (Doc. No. FGDC-STD-013-2006)

U.S. Geological Survey Techniques and Methods 11-A2

FGDC Digital Cartographic Standard for Geologic Map Symbolization (PostScript Implementation)

\section{5-BAR SCALES (continued)}

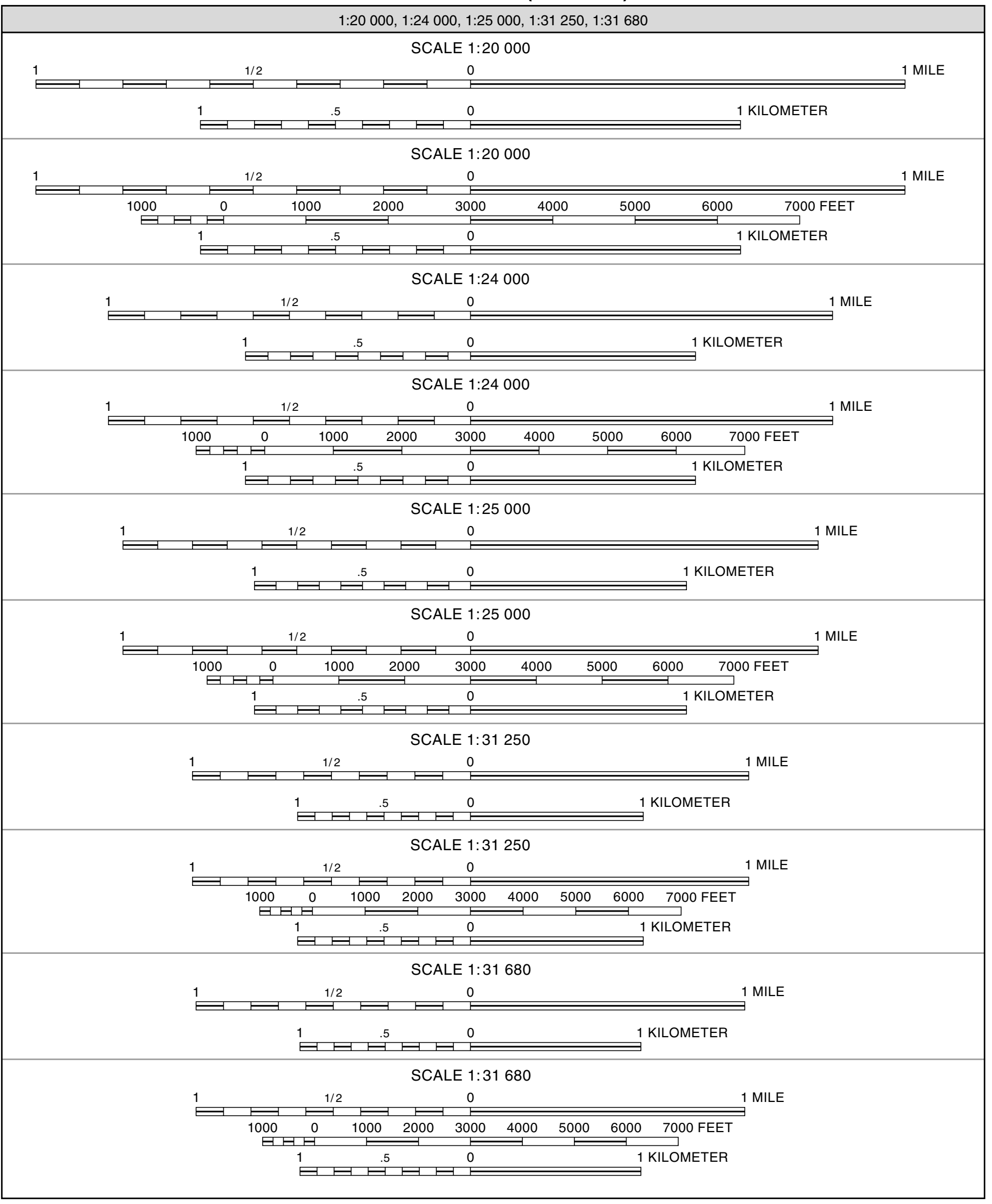




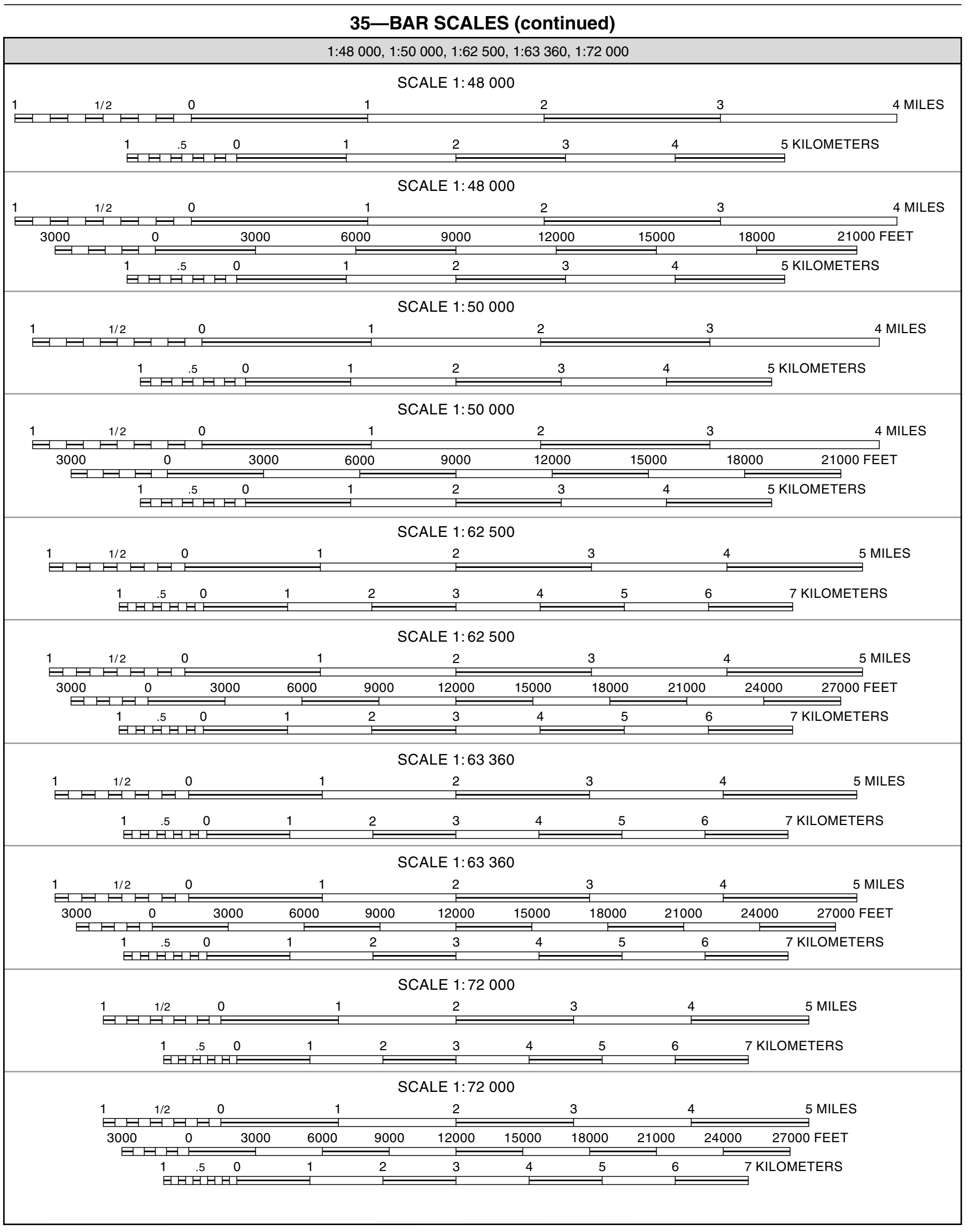




\section{5-BAR SCALES (continued)}

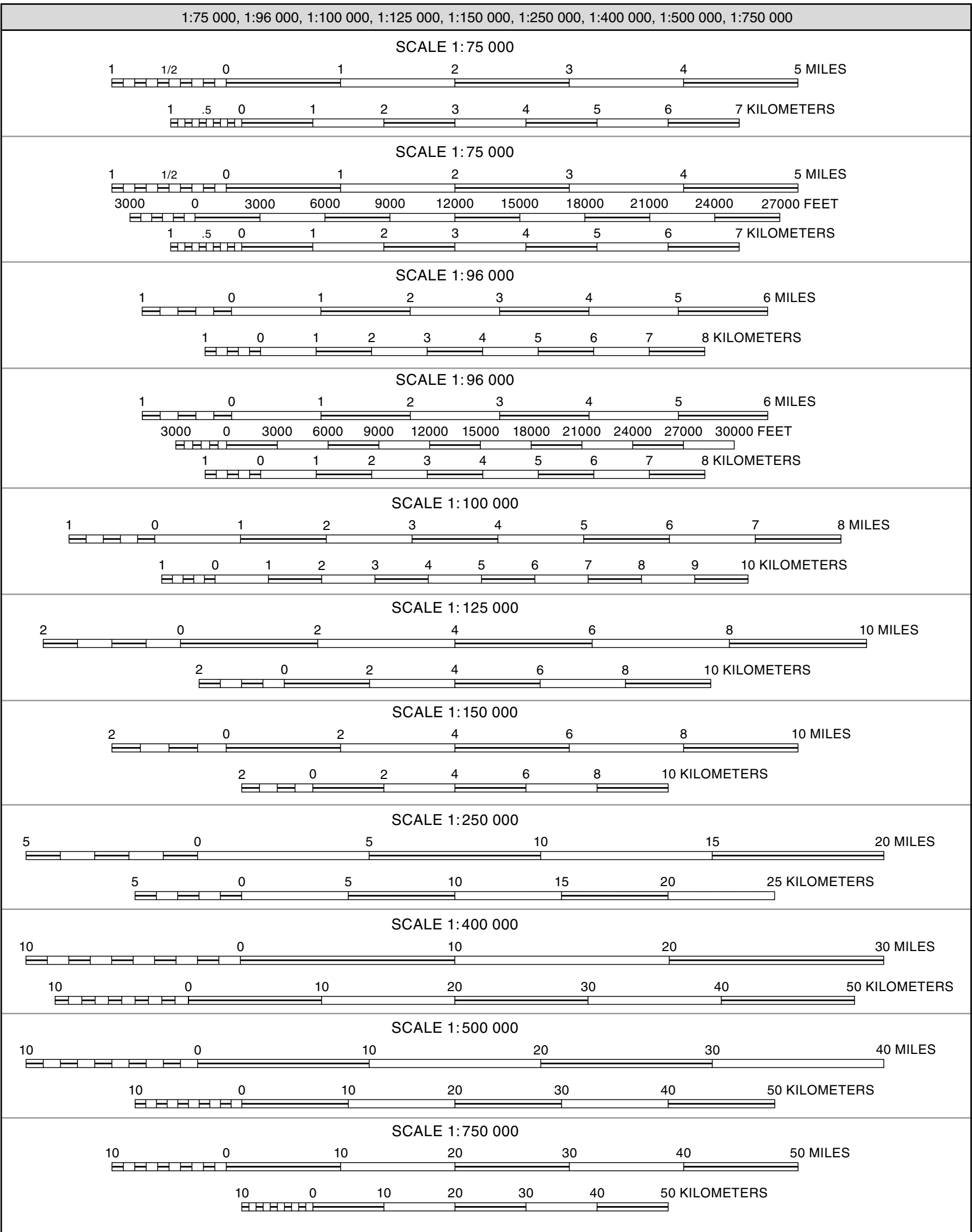


35-BAR SCALES (continued)

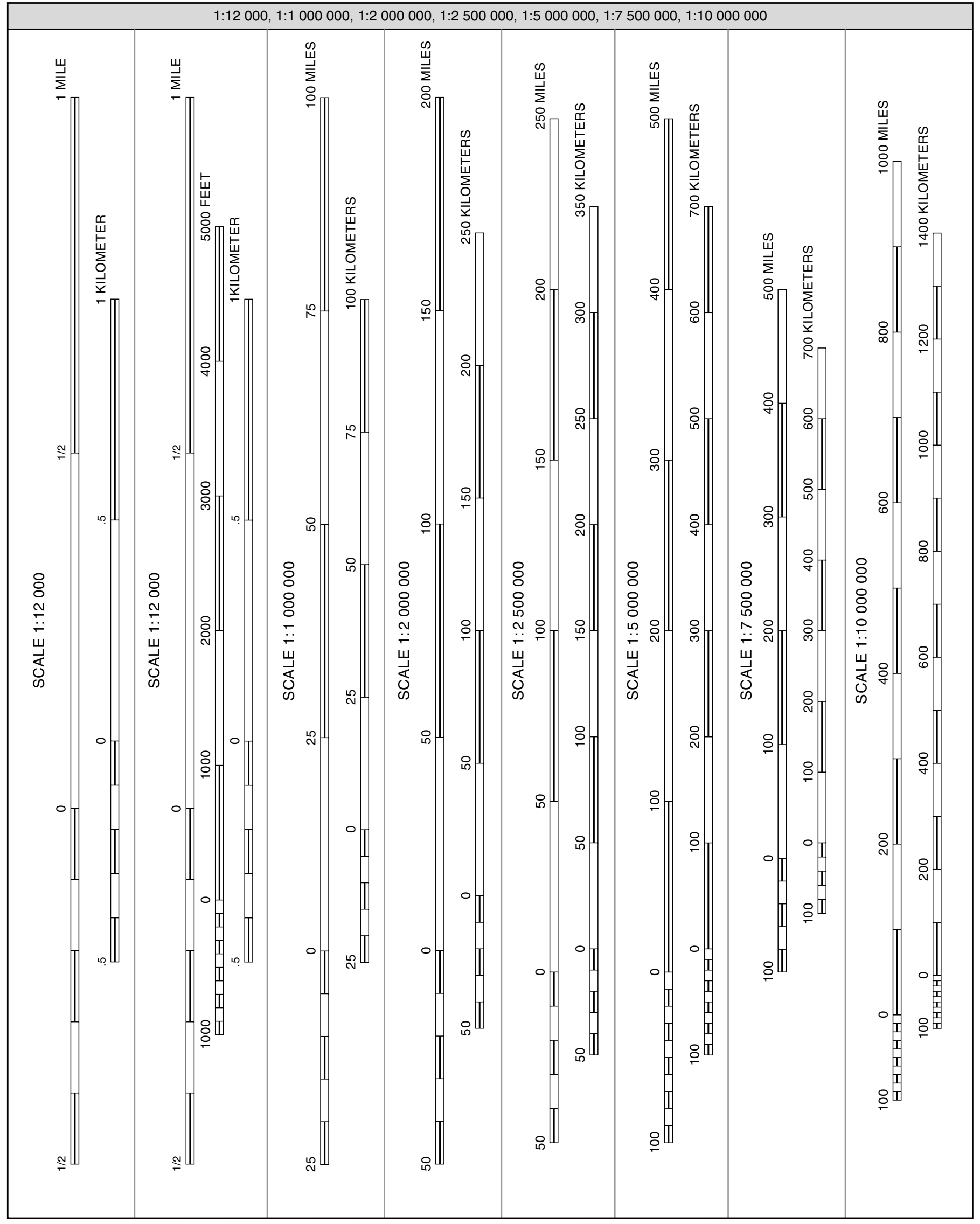




\section{5-BAR SCALES (continued)}

\begin{tabular}{|c|c|c|c|c|c|c|c|}
\hline \multicolumn{8}{|c|}{ BAR SCALE CALCULATIONS - MILES ( 1 mile $=63,360$ inches) } \\
\hline \multirow{2}{*}{$\begin{array}{l}\text { FRACTIONAL } \\
\text { SCALE }\end{array}$} & \multicolumn{2}{|c|}{$\begin{array}{l}\text { SCALE TO MAP } \\
\text { REPRESENTATION }\end{array}$} & \multirow{2}{*}{$\begin{array}{c}\text { TO FIND } \\
\text { MILES PER INCH } \\
\text { ( } x \text { in ratio) } \\
\text { Use ratio below or } \frac{\text { SCALE }}{63360}\end{array}$} & \multirow{2}{*}{$\begin{array}{c}\text { MILES } \\
\text { PER } \\
\text { INCH }\end{array}$} & \multirow{2}{*}{$\begin{array}{l}\text { TOTAL } \\
\text { MILES } \\
\text { ON } \\
\text { SCALE }\end{array}$} & \multirow{2}{*}{$\begin{array}{c}\text { TO FIND TOTAL } \\
\text { SCALE LENGTH IN } \\
\text { INCHES ( } y \text { in ratio) } \\
\text { Use ratio below or } \frac{\text { Miles on scale }}{\text { Miles per inch }}\end{array}$} & \multirow{2}{*}{$\begin{array}{l}\text { TOTAL } \\
\text { SCALE } \\
\text { LENGTH } \\
\text { (INCHES) }\end{array}$} \\
\hline & Scale & represents & & & & & \\
\hline \multirow{2}{*}{$1: 12000$} & \multirow[t]{2}{*}{1 inch } & \multirow{2}{*}{12000 in } & $63360=12000$ & \multirow{2}{*}{0.1893939} & \multirow{2}{*}{1.5} & $0.1893939=$ & \multirow{2}{*}{7.920} \\
\hline & & & 1 & & & 1 & \\
\hline \multirow{2}{*}{$1: 20000$} & \multirow{2}{*}{1 inch } & \multirow{2}{*}{20000 in } & $63360=20000$ & \multirow{2}{*}{0.3156565} & \multirow{2}{*}{2} & $0.3156565=$ & \multirow{2}{*}{6.336} \\
\hline & & & $\mathbf{x}$ & & & 1 & \\
\hline \multirow{2}{*}{$1: 24000$} & \multirow{2}{*}{1 inch } & 24000 in & $63360=24000$ & 0.3787878 & 2 & $0.3787878=$ & 5280 \\
\hline & & & $11 \quad x$ & | & 5 & 1 & $0 .<00$ \\
\hline 1.25000 & 1 inch & 25000 in & $63360=25000$ & 0.3945707 & 2 & $0.3945707=$ & 5068 \\
\hline & & & $x$ & 0.054010 & $<$ & $y$ & 0.000 \\
\hline $1 \cdot 31250$ & 1 inch & $31250 \mathrm{in}$ & $63360=31250$ & 04932133 & 2 & $0.4932133=$ & 4055 \\
\hline & & & $\mathbf{x}$ & & & $\mathbf{y}$ & \\
\hline 1.31680 & 1 inch & 31680 in & $63360=31680$ & 0500 & 2 & 0.500 & 1000 \\
\hline 1.01000 & 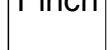 & III & $\begin{array}{ll}1 & x\end{array}$ & 0.500 & 2 & $\overline{1}$ & 4.000 \\
\hline $1: 48000$ & 1 inch & 48000 in & $63360=48000$ & 0.7575757 & 5 & $0.7575757=$ & 6.600 \\
\hline & & & $\mathbf{x}$ & & & $\mathbf{y}$ & \\
\hline 1.50000 & 1 inch & 50000 in & $63360=50000$ & 07891414 & 5 & $0.7891414=$ & 6336 \\
\hline 1.00000 & 1 & II & $11 \quad x$ & $0.1001414]$ & & 1 & \\
\hline 1.62500 & 1 inch & 62500 in & $63360=62500$ & 0 9864267 & 6 & $0.9864267=$ & 6082 \\
\hline $1.0<-600$ & 1 пाँा & oc & $\mathbf{x}$ & $0.0004<01$ & 0 & 1 & 0.002 \\
\hline 1.63360 & 1 inch & 63360 in & $63360=63360$ & 1000 & 6 & 1.000 & 6000 \\
\hline & & & $\mathbf{x}$ & & & $\mathbf{y}$ & \\
\hline 1.72000 & 1 inch & 72000 in & $63360=72000$ & 11363636 & 6 & $1.1363636=$ & 5280 \\
\hline & & & $\mathbf{x}$ & & & 1 & \\
\hline 1.75000 & 1 inch & 75000 in & $63360=75000$ & 11837121 & 6 & $1.1837121=$ & 5068 \\
\hline & & & 1 & & & $\mathbf{y}$ & \\
\hline 1.06 $\cap \cap 0$ & 1 inch & o6 000 in & $63360=96000$ & 15151515 & 7 & $1.5151515=$ & 1600 \\
\hline & & & $\mathbf{x}$ & & & 1 & $4.0<0$ \\
\hline 1.100 & 1 inch & 100000 in & $63360=100000$ & 15780000 & 0 & $1.5782828=$ & $570 ?$ \\
\hline & & & $\mathbf{x}$ & & & 1 & \\
\hline $1 \cdot 125000$ & 1 inch & 125000 in & $63360=125000$ & 19728535 & 12 & $1.9728535=$ & 6082 \\
\hline & & & $\mathbf{x}$ & & & 11 & 0.002 \\
\hline $1: 150000$ & 1 inch & 150000 in & $63360=150000$ & 2.3674242 & 12 & $2.3674242=$ & 5068 \\
\hline & & & $\mathbf{x}$ & & & $y$ & \\
\hline To find miles & per incl & $\mathrm{h}$ on $1: 12000 \mathrm{r}$ & nap... & & Soluti & $63360 \cdot x=$ & $12000 \cdot 1$ \\
\hline 63,360 inches & $s=1$ mile & Let SCA & LE $(12000)$ be in inches & & 63360 & $=12000$ & 12000 \\
\hline Show in ratio & as $\ldots$ & Fraction & al scale says 1 inch repre & esents $12,000 \mathrm{i}$ & 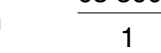 & $\overline{63360}$ & $\overline{63360}$ \\
\hline 633 & inch & Let $\mathbf{x}$ be & $\begin{array}{l}\text { miles that } 1 \text { inch represe } \\
\text { ratio as .... }\end{array}$ & ents on map & & & 12000 (SCALE) \\
\hline 1 & mile & & 12000 & hes & & & 63360 \\
\hline & & & $\mathbf{x}$ & les & & & 0.1893939 \\
\hline
\end{tabular}




\section{5-BAR SCALES (continued)}

\begin{tabular}{|c|c|c|c|c|c|c|c|}
\hline \multicolumn{8}{|c|}{ BAR SCALE CALCULATIONS - MILES ( 1 mile $=63,360$ inches) - continued } \\
\hline \multirow{2}{*}{$\begin{array}{c}\text { FRACTIONAL } \\
\text { SCALE }\end{array}$} & \multicolumn{2}{|c|}{$\begin{array}{c}\text { SCALE TO MAP } \\
\text { REPRESENTATION }\end{array}$} & \multirow{2}{*}{\begin{tabular}{|c|} 
TO FIND \\
MILES PER INCH \\
$(\mathbf{x}$ in ratio) \\
Use ratio below or $\frac{\text { SCALE }}{63360}$ \\
\end{tabular}} & \multirow{2}{*}{$\begin{array}{c}\text { MILES } \\
\text { PER } \\
\text { INCH }\end{array}$} & \multirow{2}{*}{$\begin{array}{l}\text { TOTAL } \\
\text { MILES } \\
\text { ON } \\
\text { SCALE }\end{array}$} & \multirow{2}{*}{\begin{tabular}{|c|} 
TO FIND TOTAL \\
SCALE LENGTH IN \\
INCHES ( $y$ in ratio) \\
Use ratio below or Miles on scale \\
Miles per inch
\end{tabular}} & \multirow{2}{*}{$\begin{array}{l}\text { TOTAL } \\
\text { SCALE } \\
\text { LENGTH } \\
\text { (INCHES) }\end{array}$} \\
\hline & Scale & $\begin{array}{c}\text { represents } \\
\text { Map Unit }\end{array}$ & & & & & \\
\hline \multirow[t]{2}{*}{$1: 250000$} & \multirow[t]{2}{*}{1 inch } & \multirow[t]{2}{*}{250000 in } & $63360=250000$ & \multirow[t]{2}{*}{3.945707} & \multirow[t]{2}{*}{25} & $3.945707=25$ & \multirow{2}{*}{6.336} \\
\hline & & & 1 & & & 1 & \\
\hline \multirow{2}{*}{$1: 400000$} & \multirow{2}{*}{1 inch } & \multirow{2}{*}{$400000 \mathrm{in}$} & $63360=400000$ & \multirow{2}{*}{6.3131313} & \multirow{2}{*}{40} & $6.3131313=40$ & \multirow{2}{*}{6.336} \\
\hline & & & $1 \quad \frac{x}{x}$ & & & 1 & \\
\hline \multirow{2}{*}{$1: 500000$} & \multirow{2}{*}{1 inch } & \multirow{2}{*}{500000 in } & $63360=500000$ & \multirow{2}{*}{7.8914141} & \multirow{2}{*}{50} & $7.8914141=50$ & \multirow{2}{*}{6.336} \\
\hline & & & 1 & & & $y$ & \\
\hline \multirow{2}{*}{$1: 750000$} & \multirow{2}{*}{1 inch } & \multirow{2}{*}{750000 in } & $63360=750000$ & 11837121 & 60 & $11.837121=60$ & 5068 \\
\hline & & & 1 & & & $\mathbf{y}$ & \\
\hline 1.1000000 & 1 inch & 1000000 in & $63360=1000000$ & 15700000 & 125 & $15.782828=125$ & 7000 \\
\hline 1.1000000 & I IIICn & $1000000 \mathrm{im}$ & 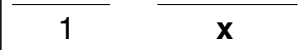 & $15 . / 82828$ & 125 & $1 \quad-\frac{y}{y}$ & 7.920 \\
\hline $1: 2000000$ & 1 inch & 2000000 in & $63360=2000000$ & 31.565656 & 250 & $31.565656=250$ & 7920 \\
\hline & & & 1 & & & $\mathbf{y}$ & \\
\hline $1: 2500000$ & 1 inch & 2500000 in & $63360=2500000$ & 39.45707 & 300 & $39.45707=300$ & 7.603 \\
\hline & & & $1 \quad x$ & & & 1 & \\
\hline $1: 5000000$ & 1 inch & 5000000 in & $63360=5000000$ & 78.914141 & 600 & $78.914141=600$ & 7.603 \\
\hline & & & $1 \quad x$ & & & $1 \quad y$ & \\
\hline $1: 7500000$ & 1 inch & 7500000 in & $63360=7500000$ & 118.37121 & 600 & $118.37121=600$ & 5.068 \\
\hline & & & $\begin{array}{l}1 \\
\end{array}$ & & & 1 & \\
\hline $1: 10000000$ & 1 inch & 10000000 in & $63360=10000000$ & 157.82828 & 1100 & $157.82828=1100$ & 6.969 \\
\hline & & & 1 & & & 1 & \\
\hline To find mile & per inc & ch on 1: 250000 & map... & & Solutic & $63360 \cdot x$ & $250000 \cdot 1$ \\
\hline 63,360 inche & $\mathrm{s}=1 \mathrm{mil}$ & Let SCA & ALE (250 000) be in inche & & 63360 & $=250000$ & 250000 \\
\hline Show in ratio & as ... & Fraction & al scale says 1 inch repre & esents 250,00 & 1 & 63360 & $\overline{63360}$ \\
\hline 633 & & Show in & ratio as ... & & & & 250000 (SCALE) \\
\hline 1 & & & 250000 & ches & & & 63360 \\
\hline & & & $\mathbf{x} \quad$ mi & iles & & & 3.945707 \\
\hline
\end{tabular}




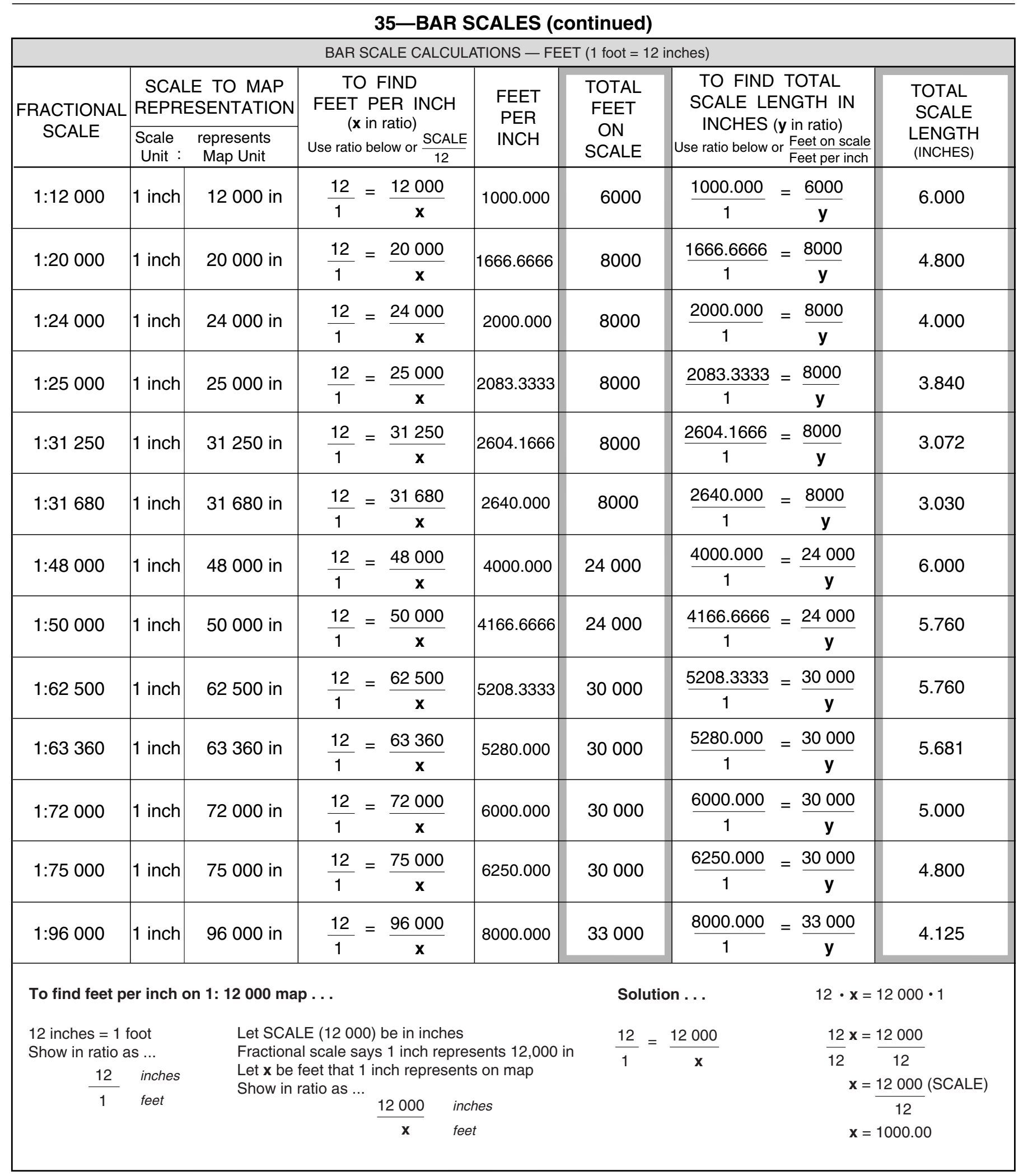




\section{5-BAR SCALES (continued)}

\begin{tabular}{|c|c|c|c|c|c|c|c|c|c|}
\hline \multicolumn{10}{|c|}{ BAR SCALE CALCULATIONS - KILOMETERS ( 1 kilometer $=100,000$ centimeters $)$} \\
\hline \multirow{2}{*}{$\begin{array}{l}\text { FRACTIONAL } \\
\text { SCALE }\end{array}$} & \multicolumn{2}{|c|}{$\begin{array}{c}\text { SCALE TO MAP } \\
\text { REPRESENTATION }\end{array}$} & \multirow{2}{*}{$\begin{array}{l}\text { TO FIND } \\
\text { KILOMETERS PER } \\
\text { CENTIMETER (CM) } \\
(\mathbf{x} \text { in ratio }) \\
\text { Use ratio below or } \frac{\text { SCALE }}{100000}\end{array}$} & \multirow{2}{*}{\begin{tabular}{|c|} 
KILO- \\
METERS \\
PER \\
CM \\
\end{tabular}} & \multirow{2}{*}{$\begin{array}{c}\text { TOTAL } \\
\text { KILOMETERS } \\
\text { ON } \\
\text { SCALE }\end{array}$} & \multirow{2}{*}{\multicolumn{2}{|c|}{$\begin{array}{c}\text { TO FIND TOTAL } \\
\text { SCALE LENGTH IN } \\
\text { CENTIMETERS }(y \text { in ratio) } \\
\begin{array}{c}\text { Use ratio or Kilometers on scale } \\
\text { below }\end{array} \text { Kilometers per cm }\end{array}$}} & \multicolumn{2}{|c|}{$\begin{array}{l}\text { TOTAL SCALE } \\
\text { LENGTH IN }\end{array}$} \\
\hline & $\begin{array}{l}\text { Scale } \\
\text { Unit : }\end{array}$ & $\begin{array}{c}\text { represents } \\
\text { Map Unit }\end{array}$ & & & & & & \begin{tabular}{|l} 
CENTI- \\
METERS
\end{tabular} & \begin{tabular}{|c|} 
MILLI- \\
METERS \\
\end{tabular} \\
\hline \multirow{2}{*}{$1: 12000$} & \multirow{2}{*}{$1 \mathrm{~cm}$} & \multirow{2}{*}{$12000 \mathrm{~cm}$} & $100000=12000$ & \multirow{2}{*}{0.120} & \multirow{2}{*}{1.5} & \multirow{2}{*}{\multicolumn{2}{|c|}{$\frac{0.120}{1}=\frac{1.5}{y}$}} & \multirow{2}{*}{12.500} & \multirow{2}{*}{125.00} \\
\hline & & & 1 & & & & & & \\
\hline \multirow{2}{*}{$1: 20000$} & \multirow{2}{*}{$1 \mathrm{~cm}$} & \multirow{2}{*}{$20000 \mathrm{~cm}$} & $100000=20000$ & \multirow{2}{*}{0.200} & \multirow[t]{2}{*}{2} & \multirow{2}{*}{\multicolumn{2}{|c|}{$\frac{0.200}{1}=\frac{2}{y}$}} & \multirow{2}{*}{10.000} & 100.00 \\
\hline & & & $\begin{array}{l}1 \\
x\end{array}$ & & & & & & \\
\hline $1: 24000$ & $1 \mathrm{~cm}$ & $24000 \mathrm{~cm}$ & $100000=24000$ & 0.240 & 2 & $0.240=$ & & 8.333 & 83.33 \\
\hline & & & $1 \quad \frac{x}{1}$ & & & & $\mathbf{y}$ & & \\
\hline $1 \cdot 25000$ & $1 \mathrm{~cm}$ & $25000 \mathrm{~cm}$ & $100000=25000$ & 0250 & 2 & $0.250=$ & & 8000 & 8000 \\
\hline $1 .<000$ & 1 cin & $25000 \mathrm{cmi}$ & $\begin{array}{ll}1 & x\end{array}$ & 0.250 & 2 & $\overline{1}$ & $y$ & 8.000 & 80.00 \\
\hline $1: 31250$ & $1 \mathrm{~cm}$ & $31250 \mathrm{~cm}$ & $100000=31250$ & 0.3125 & 2 & $\underline{0.3125}=$ & & 6.400 & 64.00 \\
\hline & & & $1 \quad \frac{x}{x}$ & & & 1 & $y$ & & \\
\hline $1: 31680$ & $1 \mathrm{~cm}$ & $31680 \mathrm{~cm}$ & $100000=31680$ & 0.3168 & 2 & $0.3168=$ & & 6.313 & 63.13 \\
\hline & & & $\mathbf{x}$ & & & 1 & $\mathbf{y}$ & & \\
\hline $1: 48000$ & $1 \mathrm{~cm}$ & $48000 \mathrm{~cm}$ & $100000=48000$ & 0.480 & 6 & $0.480=$ & & 12.500 & 125.00 \\
\hline & - & & $1 \quad x$ & & & 1 & $\mathbf{y}$ & & \\
\hline $1: 50000$ & $1 \mathrm{~cm}$ & $50000 \mathrm{~cm}$ & $100000=50000$ & 0.500 & 6 & $\underline{0.500}=$ & & 12.000 & 120.00 \\
\hline & & & $\mathbf{x}$ & & & 1 & $y$ & & \\
\hline $1: 62500$ & $1 \mathrm{~cm}$ & $62500 \mathrm{~cm}$ & $100000=62500$ & 0.625 & 8 & $\underline{0.625}=$ & & 12.800 & 128.00 \\
\hline & & & 1 & & & 1 & $\mathbf{y}$ & & \\
\hline 1.63360 & $1 \mathrm{~cm}$ & $63360 \mathrm{~cm}$ & $100000=63360$ & 06336 & 8 & $0.6336=$ & & 12626 & 12626 \\
\hline 1.00000 & ( vim & (20) & $\begin{array}{ll}1 & x\end{array}$ & 0.0000 & 0 & 1 & $y$ & $1<.0<0$ & $1<0 .<0$ \\
\hline 1.72 & $1 \mathrm{~cm}$ & $72000 \mathrm{~cm}$ & $100000=72000$ & 0720 & 8 & $\underline{0.720}=$ & 8 & 11111 & 11111 \\
\hline $1.1<000$ & $10 \pi 1$ & $1<0000111$ & $\begin{array}{ll}1 & x\end{array}$ & $0.1<0$ & 0 & 1 & $y$ & 11.171 & 1111.11 \\
\hline $1 \cdot 75000$ & $1 \mathrm{~cm}$ & $75000 \mathrm{~cm}$ & $100000=75000$ & 0750 & 8 & $\underline{0.750}=$ & & 10666 & 10666 \\
\hline סט & ( & 18000 cin & 1 & 0.160 & 0 & 1 & $\mathbf{y}$ & 10.000 & 100.00 \\
\hline $1: 96000$ & $1 \mathrm{~cm}$ & $96000 \mathrm{~cm}$ & $100000=96000$ & 0.960 & 9 & $\underline{0.960}=$ & & 9.375 & 93.75 \\
\hline & & & $1 \quad \frac{x}{x}$ & & & 1 & $y$ & & \\
\hline $1: 100000$ & $1 \mathrm{~cm}$ & $100000 \mathrm{~cm}$ & $100000=100000$ & 1.000 & 11 & $1.000=$ & 11 & 11.000 & 110.00 \\
\hline & & & $1 \quad x$ & & & 1 & y & & \\
\hline $1: 125000$ & $1 \mathrm{~cm}$ & $125000 \mathrm{~cm}$ & $100000=125000$ & 1.250 & 12 & $\frac{1.250}{1}=$ & & 9.600 & 96.00 \\
\hline & & & 1 & & & 1 & $\mathbf{y}$ & & \\
\hline $1: 150000$ & $1 \mathrm{~cm}$ & $150000 \mathrm{~cm}$ & $100000=150000$ & 1.500 & 12 & $1.500=$ & 12 & 8.000 & 80.00 \\
\hline & & & $1 \quad x$ & & & 1 & $\mathbf{y}$ & & \\
\hline To find kilome & eters pe & er centimeter on & 1: 12000 map ... & & & ution... & $100000 \cdot x$ & $=12000$ & \\
\hline 100000 centim & neters $=$ & $=1$ kilometer & et SCALE (12 000) be in ce & entimeters & & $0000=12000$ & $100000 x$ & $=12000$ & \\
\hline Show in ratio as & & $\mathrm{Fr}$ & actional scale says 1 cent & timeter repres & sents & $1 \quad x$ & $\overline{100000}$ & 100000 & \\
\hline & 0000 & centimeters & $\begin{array}{l}12,000 \text { centimeters } \\
\text { tet } \mathbf{x} \text { be kilometers that } 1 \mathrm{cn}\end{array}$ & $\mathrm{m}$ represents & on map & & & $=12000$ & (SCALE) \\
\hline & 1 & kilometers & how in ratio as ... 12000 & centimeter & & & & 10000 & \\
\hline & & & $x$ & & & & & $=0.120$ & \\
\hline
\end{tabular}




\section{5-BAR SCALES (continued)}

\begin{tabular}{|c|c|c|c|c|c|c|c|c|c|}
\hline \multicolumn{10}{|c|}{ BAR SCALE CALCULATIONS - KILOMETERS ( 1 kilometer $=100,000$ centimeters) - continued } \\
\hline \multirow{2}{*}{$\begin{array}{l}\text { FRACTIONAL } \\
\text { SCALE }\end{array}$} & \multicolumn{2}{|c|}{$\begin{array}{l}\text { SCALE TO MAP } \\
\text { REPRESENTATION }\end{array}$} & \multirow{2}{*}{\begin{tabular}{|c|} 
TO FIND \\
KILOMETERS PER \\
CENTIMETER (CM) \\
$(\mathbf{x}$ in ratio) \\
Use ratio below or $\frac{\text { SCALE }}{100000}$ \\
\end{tabular}} & \multirow{2}{*}{\begin{tabular}{|c|} 
KILO- \\
METERS \\
PER \\
CM \\
\end{tabular}} & \multirow{2}{*}{\begin{tabular}{|c|} 
TOTAL \\
KILOMETERS \\
ON \\
SCALE \\
\end{tabular}} & \multirow{2}{*}{\multicolumn{2}{|c|}{\begin{tabular}{|c|} 
TO FIND TOTAL \\
SCALE LENGTH IN \\
CENTIMETERS $(y$ in ratio) \\
$\begin{array}{c}\text { Use ratio or Kilometers on scale } \\
\text { below }\end{array}$ \\
\end{tabular}}} & \multicolumn{2}{|c|}{$\begin{array}{l}\text { TOTAL SCALE } \\
\text { LENGTH IN }\end{array}$} \\
\hline & $\begin{array}{l}\text { Scale } \\
\text { Unit : }\end{array}$ & $\begin{array}{c}\text { represents } \\
: \quad \text { Map Unit }\end{array}$ & & & & & & $\begin{array}{c}\text { CENTI- } \\
\text { METERS }\end{array}$ & $\begin{array}{c}\text { MILLI- } \\
\text { METERS }\end{array}$ \\
\hline $1: 250000$ & $1 \mathrm{~cm}$ & $250000 \mathrm{~cm}$ & $\frac{100000}{1}=\frac{250000}{x}$ & 2.500 & 30 & $\frac{2.500}{1}=$ & & 12.000 & 120.00 \\
\hline 1:400 000 & $1 \mathrm{~cm}$ & $400000 \mathrm{~cm}$ & $\frac{100000}{1}=\frac{400000}{x}$ & 4.000 & 60 & $\frac{4.000}{1}=$ & & 15.000 & 150.00 \\
\hline $1: 500000$ & $1 \mathrm{~cm}$ & $500000 \mathrm{~cm}$ & $\frac{100000}{1}=\frac{500000}{x}$ & 5.000 & 60 & $\frac{5.000}{1}=$ & & 12.000 & 120.00 \\
\hline 1:750 000 & $1 \mathrm{~cm}$ & $750000 \mathrm{~cm}$ & $\frac{100000}{1}=\frac{750000}{x}$ & 7.500 & 60 & $\frac{7.500}{1}=$ & & 8.000 & 80.00 \\
\hline 1:1000 000 & $1 \mathrm{~cm}$ & $1000000 \mathrm{~cm}$ & $\frac{100000}{1}=\frac{1000000}{x}$ & 10.000 & 125 & \multicolumn{2}{|c|}{$\frac{10.000}{1}=\frac{125}{y}$} & 12.500 & 125.00 \\
\hline $1: 2000000$ & $1 \mathrm{~cm}$ & $2000000 \mathrm{~cm}$ & $\frac{100000}{1}=\frac{2000000}{x}$ & 20.000 & 300 & $\frac{20.000}{1}=3$ & $\frac{300}{y}$ & 15.000 & 150.00 \\
\hline $1: 2500000$ & $1 \mathrm{~cm}$ & $2500000 \mathrm{~cm}$ & $\frac{100000}{1}=\frac{2500000}{x}$ & 25.000 & 400 & $\frac{25.000}{1}=$ & $\frac{400}{y}$ & 16.000 & 160.00 \\
\hline $1: 5000000$ & $1 \mathrm{~cm}$ & $5000000 \mathrm{~cm}$ & $\frac{100000}{1}=\frac{5000000}{x}$ & 50.000 & 800 & $\frac{50.000}{1}=$ & $\frac{800}{y}$ & 16.000 & 160.00 \\
\hline $1: 7500000$ & $1 \mathrm{~cm}$ & $7500000 \mathrm{~cm}$ & $\frac{100000}{1}=\frac{7500000}{x}$ & 75.000 & 800 & $\frac{75.000}{1}=$ & $\frac{800}{y}$ & 10.666 & 106.66 \\
\hline $1: 10000000$ & $1 \mathrm{~cm}$ & $10000000 \mathrm{~cm}$ & $\frac{100000}{1}=\frac{10000000}{x}$ & 100.000 & 1500 & $\frac{100.000}{1}=1$ & $\frac{1500}{y}$ & 15.000 & 150.00 \\
\hline $\begin{array}{l}\text { To find kilomet } \\
100000 \text { centime } \\
\text { Show in ratio as } \\
100\end{array}$ & $\begin{array}{l}\text { ters pe } \\
\text { neters }= \\
\text { s... } \\
\frac{0000}{1}\end{array}$ & $\begin{array}{lr}\text { er centimeter on } 1 \\
& \begin{array}{lr}1 \text { kilometer } & \text { Let } \\
& \text { Fra } \\
\text { centimeters } & 2 \\
\text { kilometers } & \text { Let } \\
& \text { Sh }\end{array}\end{array}$ & $\begin{array}{l}\text { 1: } 250000 \text { map ... } \\
\text { t SCALE (250 000) be in o } \\
\text { actional scale says } 1 \text { centi } \\
250,000 \text { centimeters } \\
\text { t } \mathbf{x} \text { be kilometers that } 1 \mathrm{~cm} \\
\text { ow in ratio as ... } \\
\qquad \frac{250000}{\mathbf{x}}\end{array}$ & $\begin{array}{l}\text { centimeters } \\
\text { timeter repre } \\
\text { m represents } \\
0 \quad \text { centimete } \\
\text { kilometer }\end{array}$ & $\begin{array}{l}\text { sents } \\
\text { on map } \\
\text { ss }\end{array}$ & $\begin{array}{l}\text { ution ... } \\
\frac{0000}{1}=\frac{250000}{x}\end{array}$ & $\begin{array}{r}\frac{100000 \cdot x}{100000} \times 00 x \\
x \\
x\end{array}$ & $\begin{array}{l}x=250000 \\
x=\frac{250000}{100000} \\
x=\frac{250000}{100000} \\
x=2.5\end{array}$ & $\frac{1}{5}(\mathrm{SCALE})$ \\
\hline
\end{tabular}




\section{6-MEAN DECLINATION ARROWS}

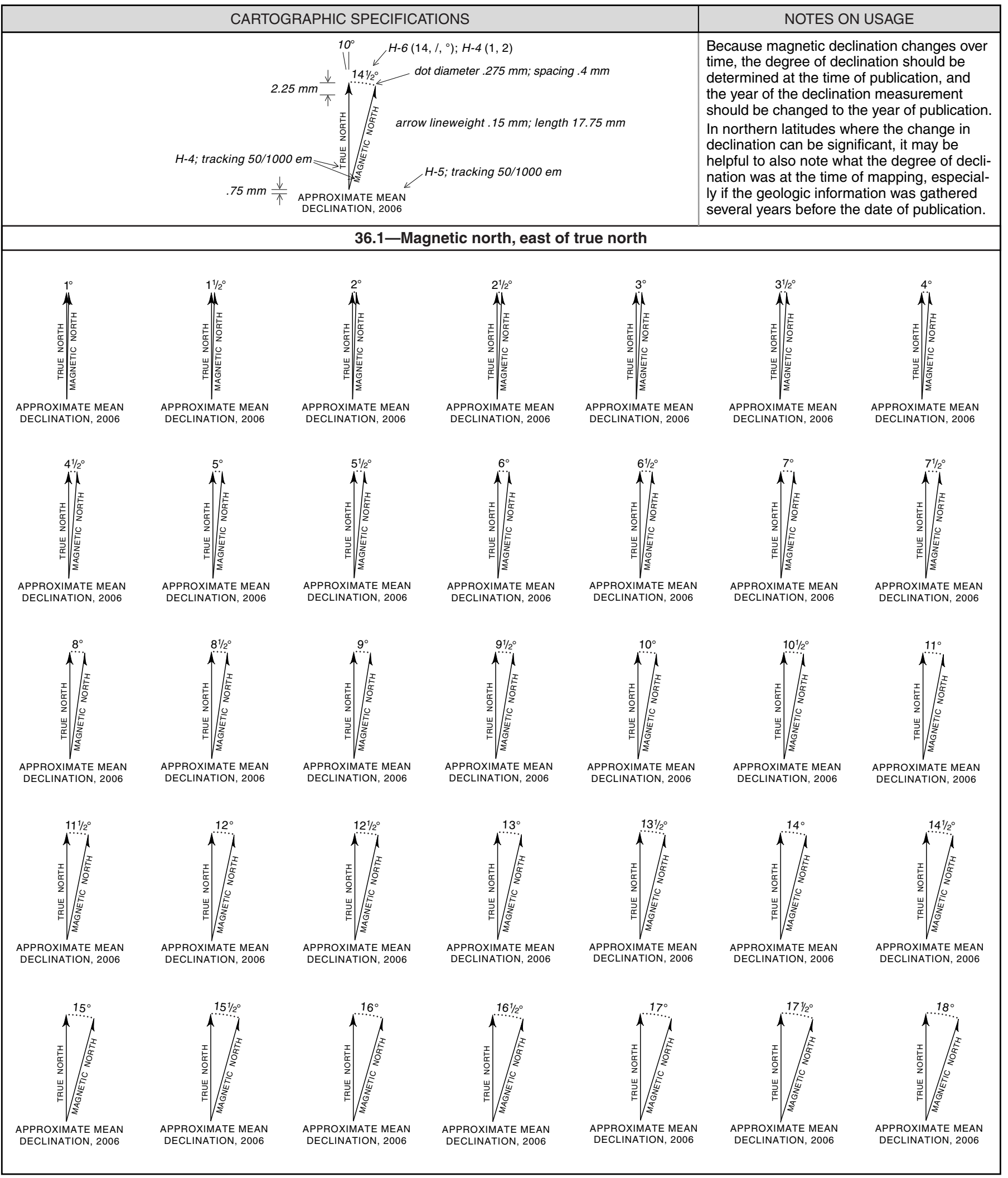




\section{6-MEAN DECLINATION ARROWS (continued)}

36.1-Magnetic north, east of true north (continued)

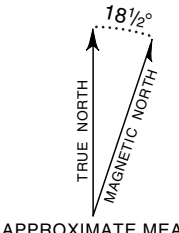

APPROXIMATE MEAN

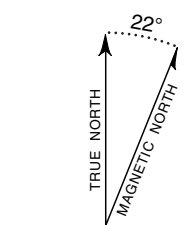

APPROXIMATE MEAN DECLINATION, 2006

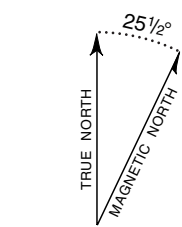

APPROXIMATE MEAN DECLINATION, 2006

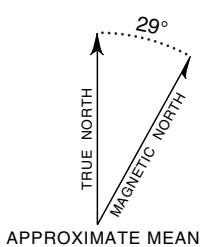

APPROXIMATE MEAN

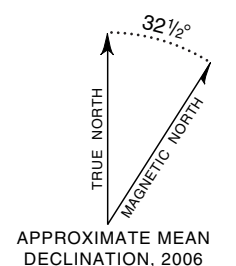

DECLINATION, 2006

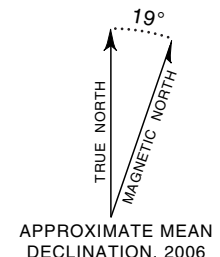

DECLINATION, 2006
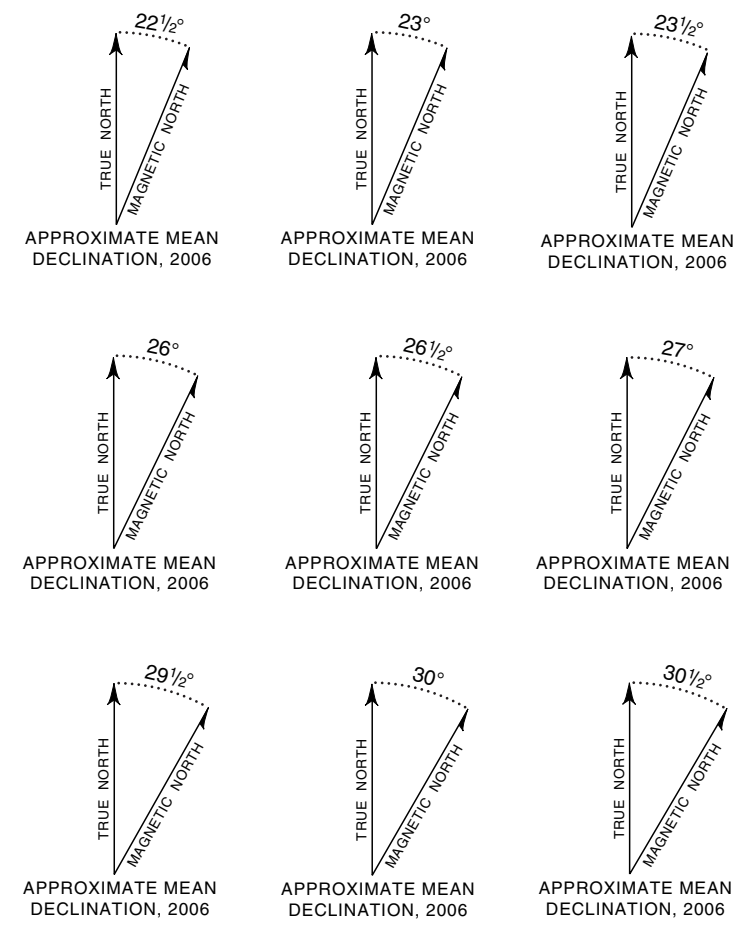

APPROXIMATE MEAN DECLINATION, 2006
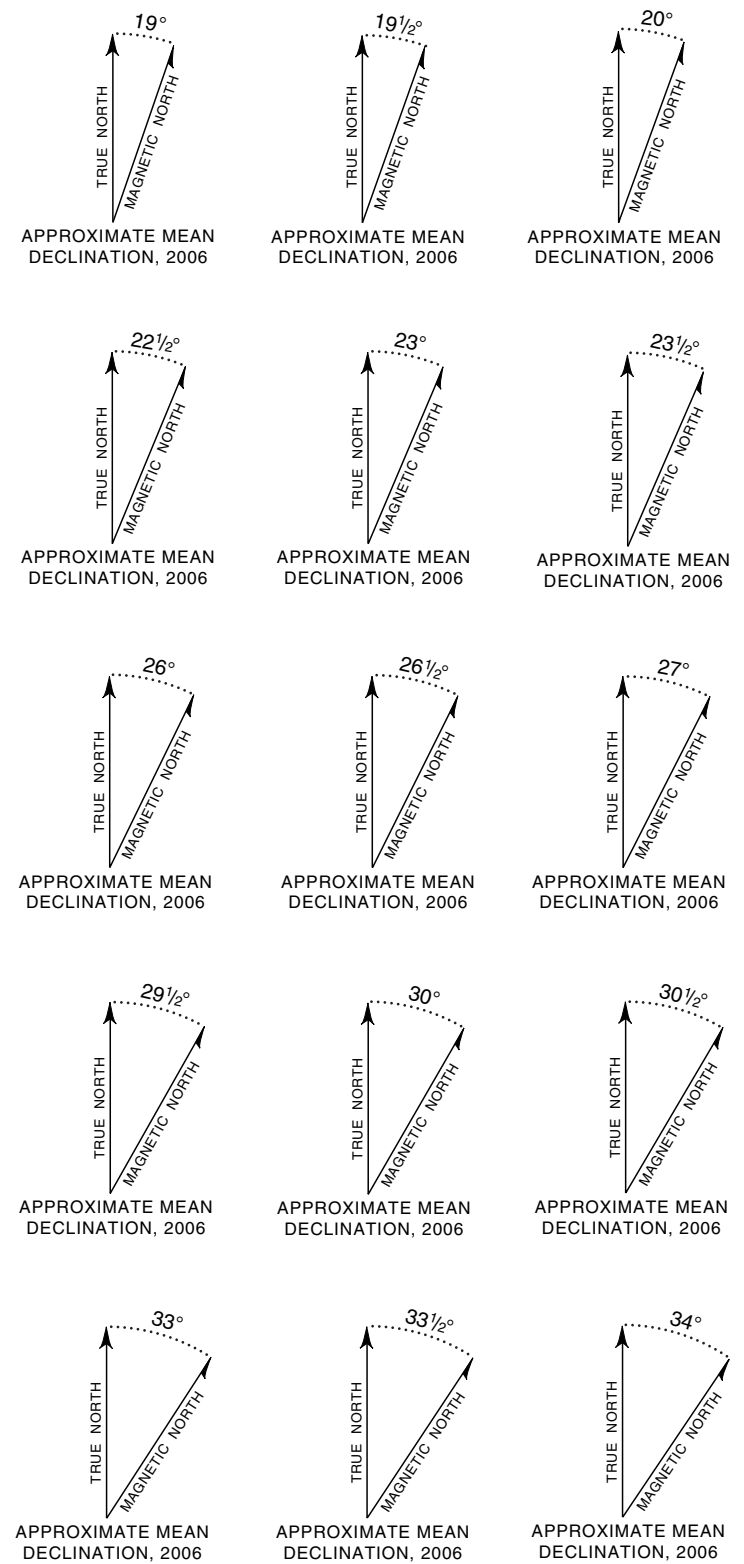
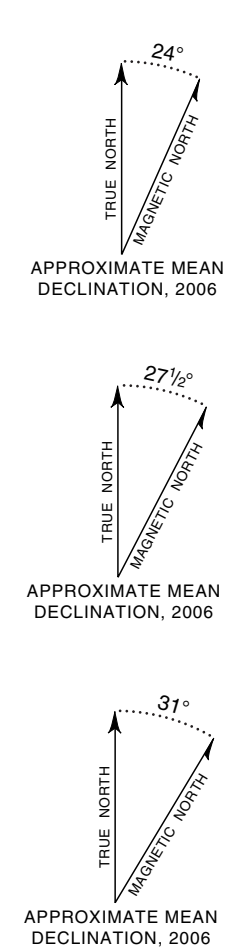

DECLINATION, 2006

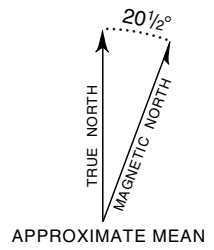

APPROXIMATE MEAN

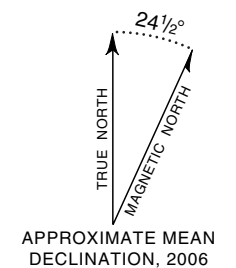

DECLINATION, 2006
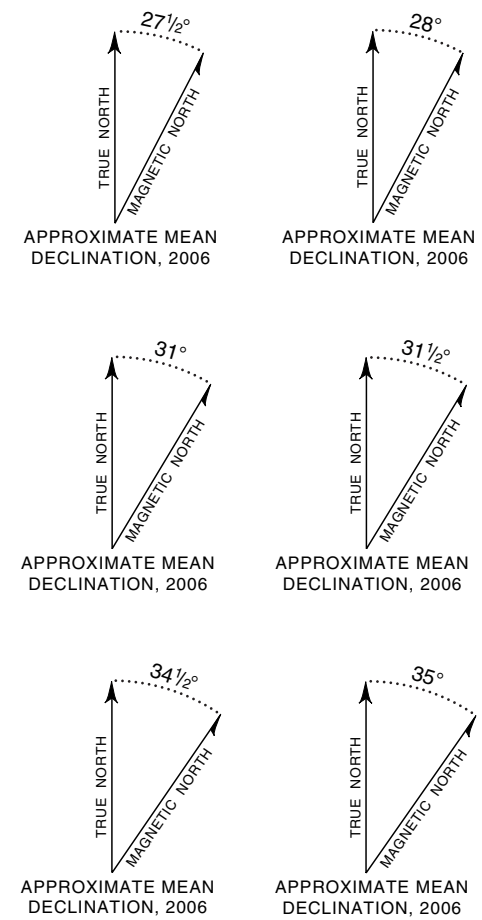
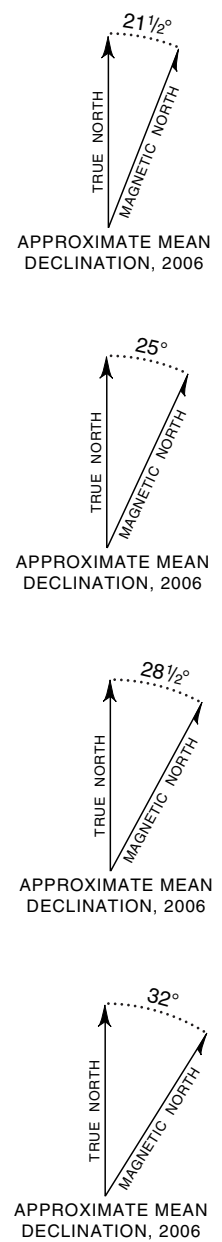

DECLINATION 2006
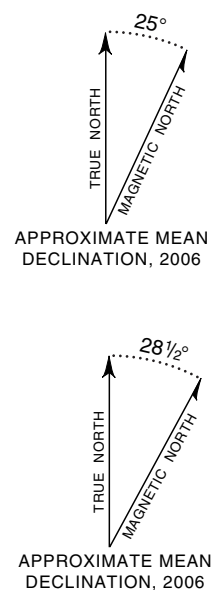

DECLINATION, 2006 


\section{6-MEAN DECLINATION ARROWS (continued)}

36.2-Magnetic north, west of true north

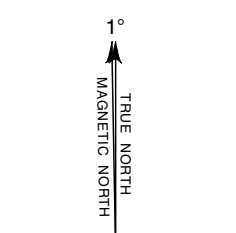

APPROXIMATE MEAN
DECLINATION, 2006

$41 / 20$

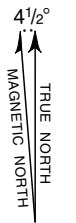

APPROXIMATE MEAN DECLINATION, 2006

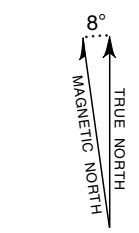

APPROXIMATE MEAN DECLINATION, 2006

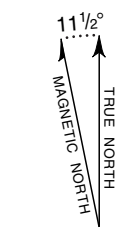

APPROXIMATE MEAN DECLINATION, 2006

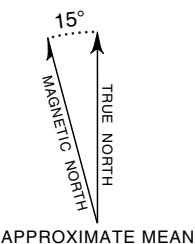

APPROXIMATE MEAN
DECLINATION, 2006
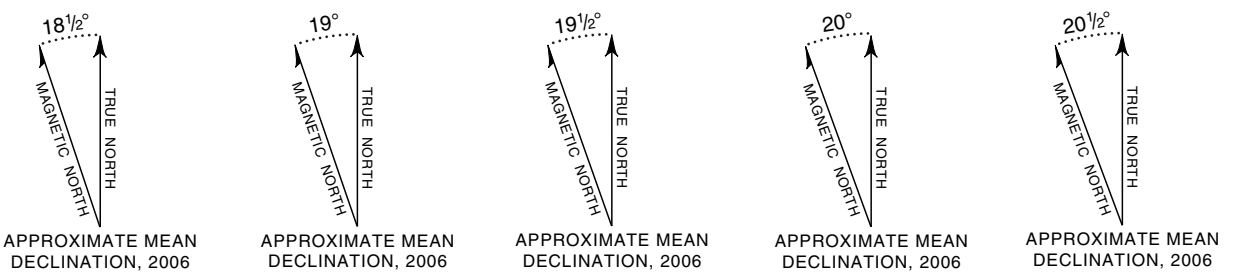

APPROXIMATE MEAN
DECLINATION, 2006

DECLINATION, 2006
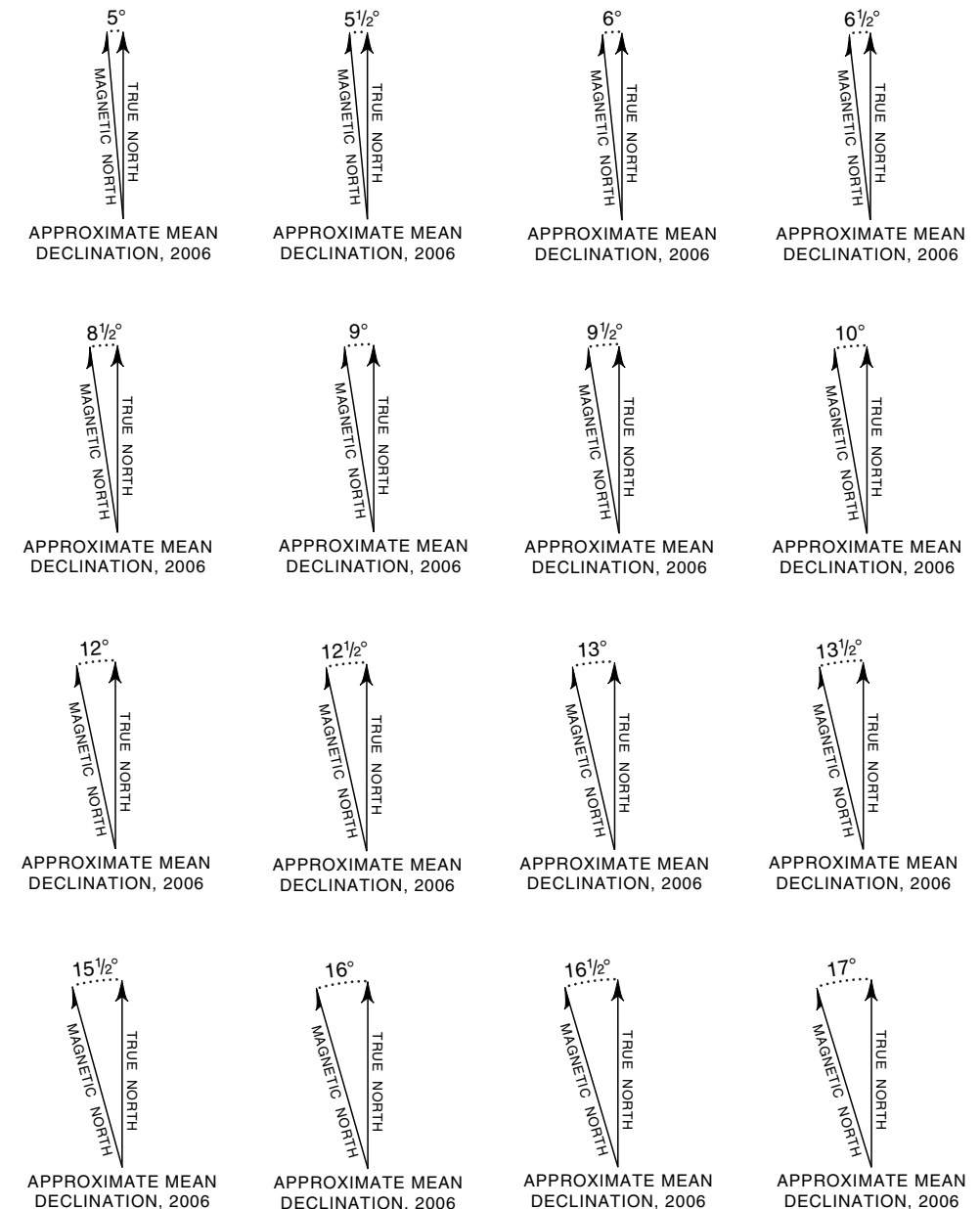
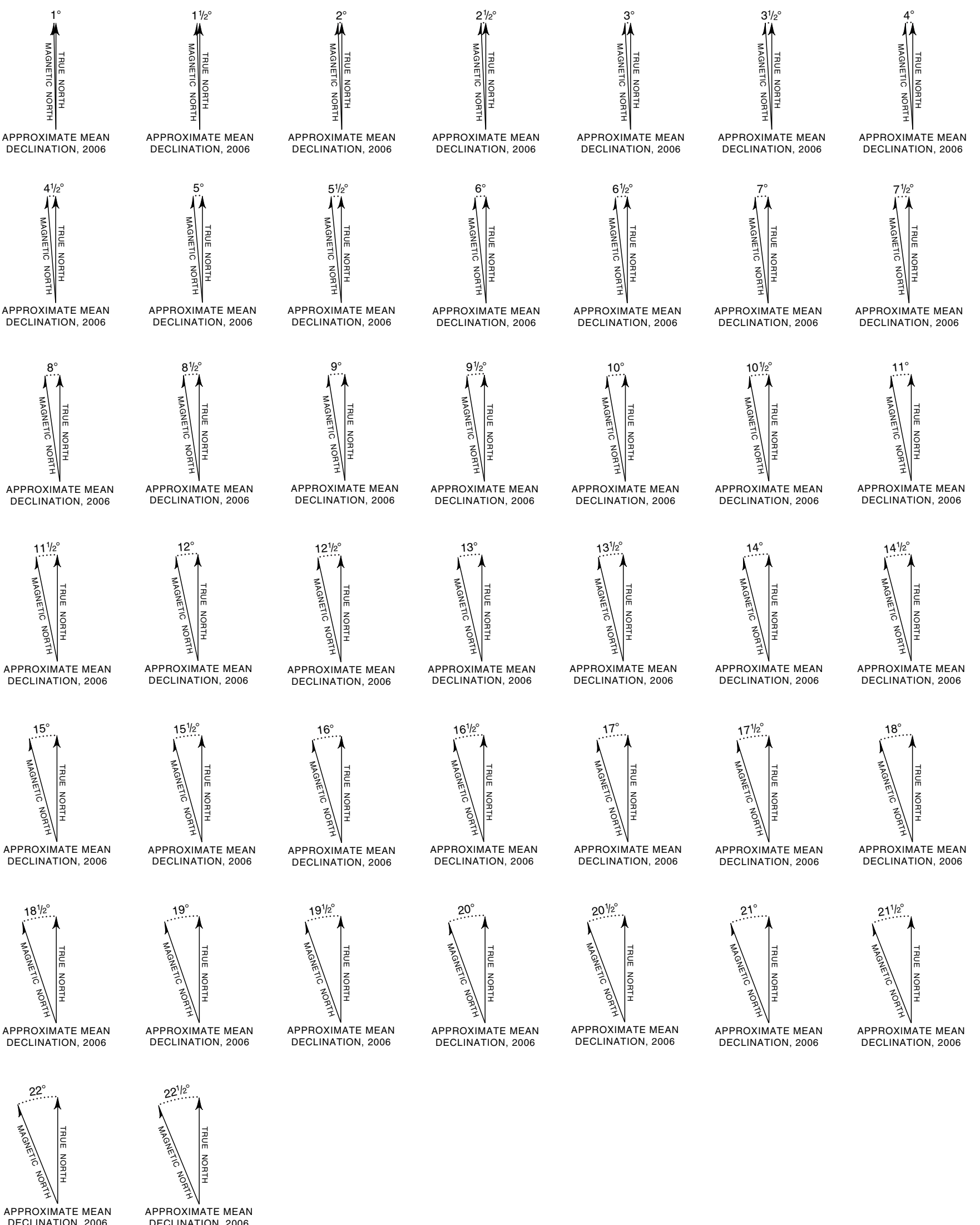
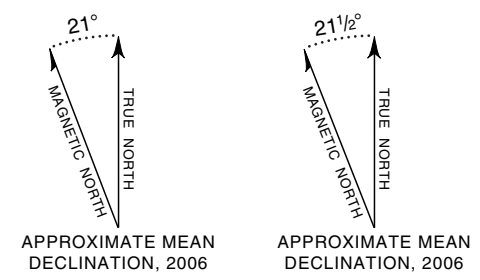


\section{7-LITHOLOGIC PATTERNS}

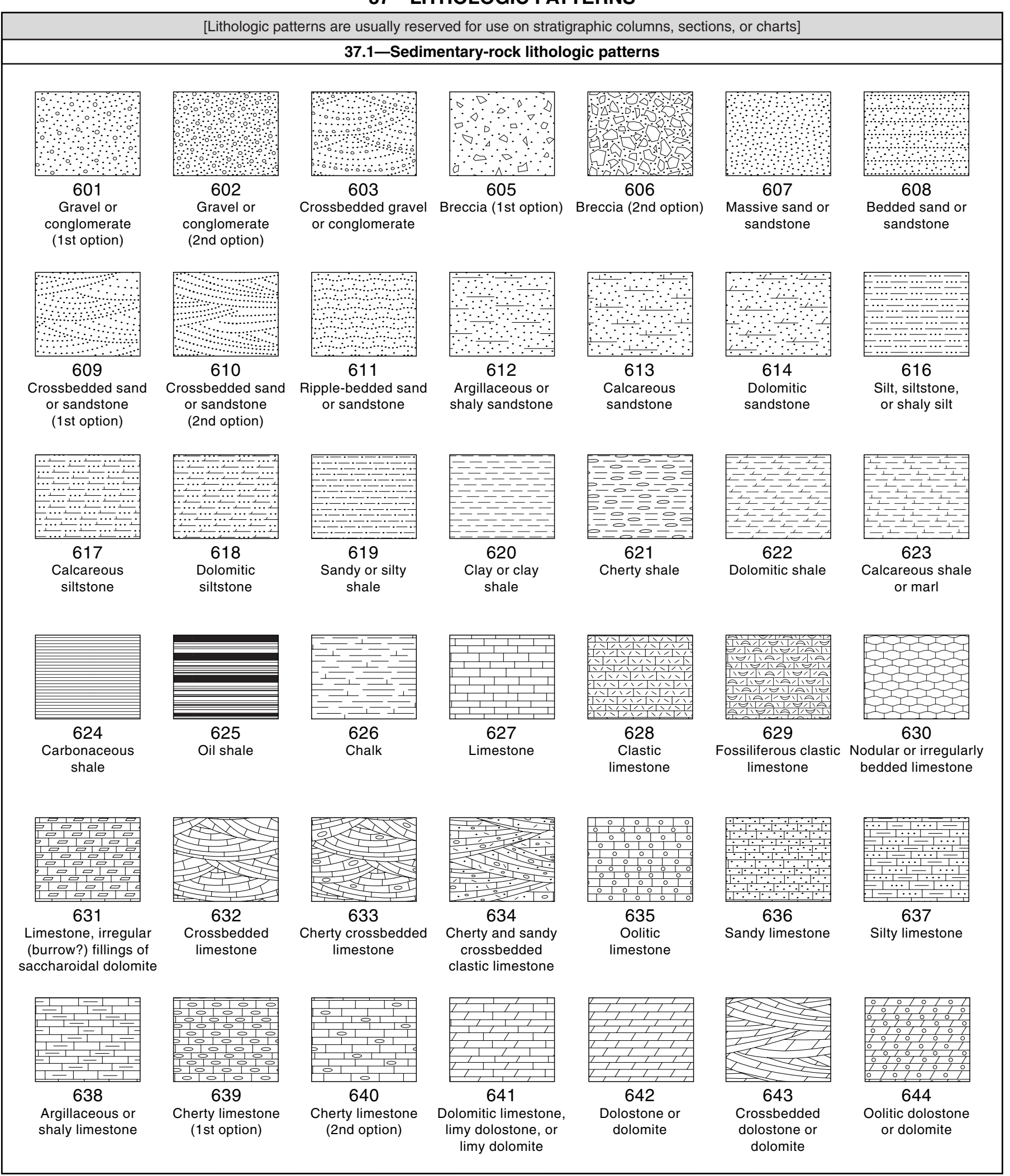

${ }^{*}$ For more information, see general guidelines on pages $A$-i to A-v. 


\section{7-LITHOLOGIC PATTERNS (continued)}

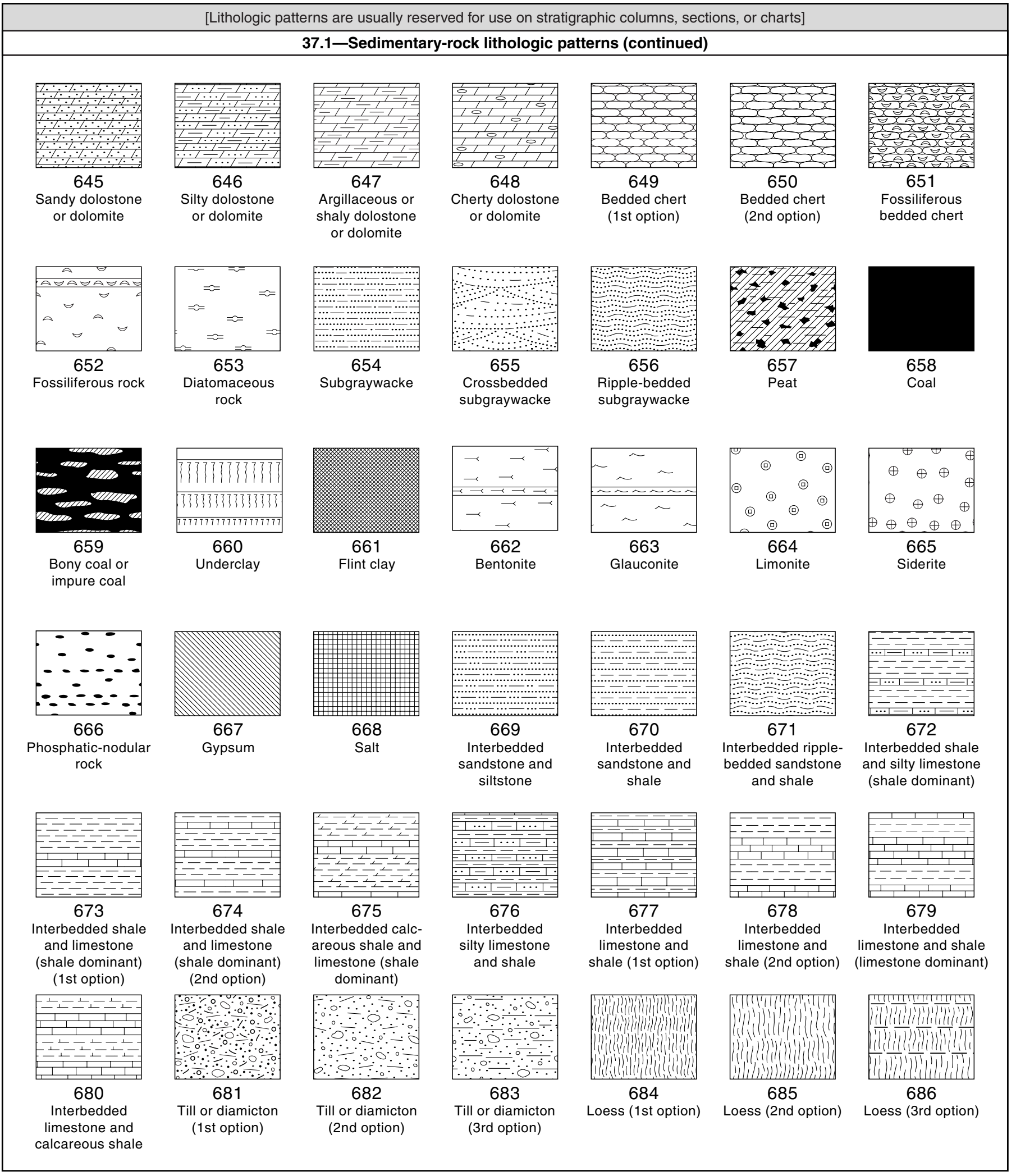

${ }^{*}$ For more information, see general guidelines on pages $A-i$ to $A-v$. 


\section{7-LITHOLOGIC PATTERNS (continued)}

[Lithologic patterns are usually reserved for use on stratigraphic columns, sections, or charts]

37.2-Metamorphic-rock, igneous-rock, and vein-matter lithologic patterns

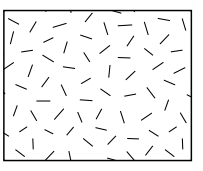

701 Metamorphism
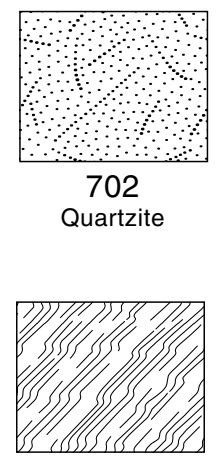

Schist and gneiss

Granite (1st option)

Basaltic flows

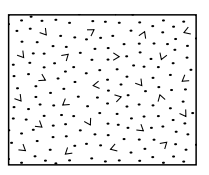

723

Igneous rock (3rd option)
702

Quartzite

707
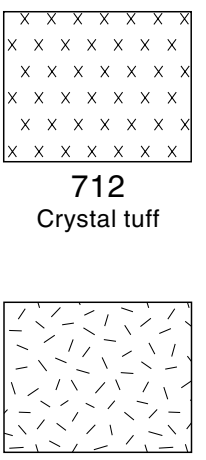

718

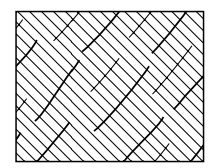

703

Slate

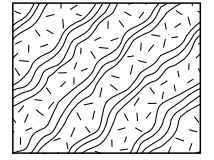

708

Gneiss

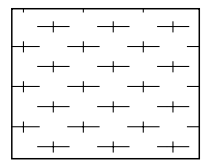

713

Devitrified

tuff

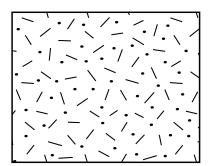

719

Granite (2nd option)

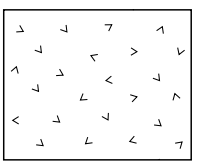

724

Igneous rock

(4th option)

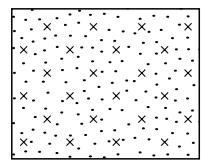

725

Igneous rock (5th option)

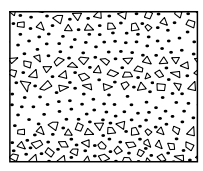

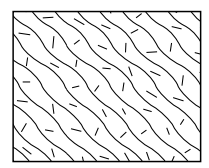

704

Schistose or gneissoid granite

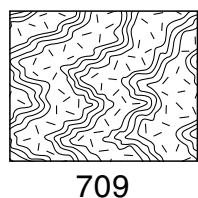

Contorted gneiss

714

Volcanic breccia and tuff

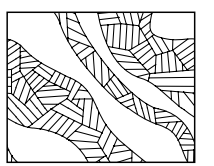

720

Banded igneous rock

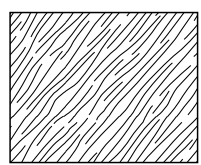

705

Schist

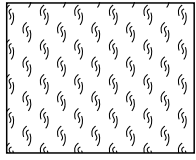

710

Soapstone, talc, or serpentinite

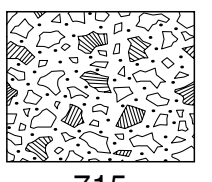

715

Volcanic breccia or agglomerate

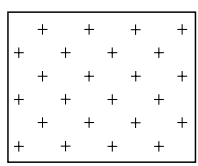

721

Igneous rock

(1st option)

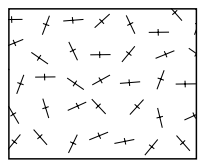

726

Igneous rock

(6th option)

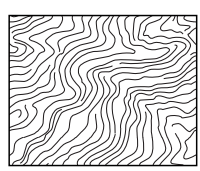

706

Contorted schist

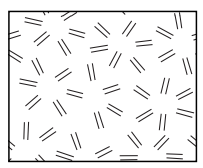

729

Porphyritic rock (1st option)

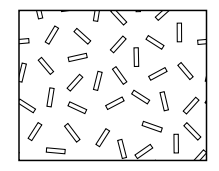

730

Porphyritic rock (2nd option)

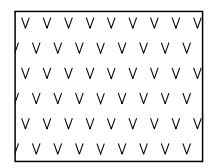

731

Vitrophyre

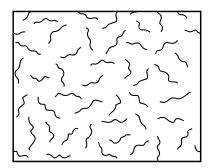

732

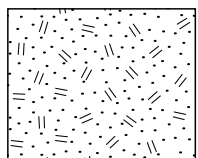

727

Igneous rock

(7th option)
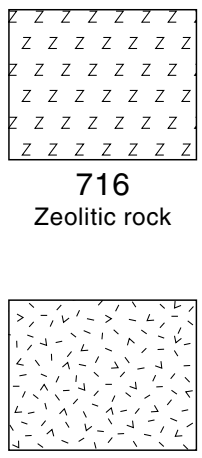

722

Igneous rock

(2nd option)

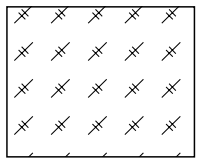

728

Igneous rock

(8th option)

${ }^{*}$ For more information, see general guidelines on pages $A-i$ to $A-v$. 


\section{8-EXPLANATION FOR PATTERN CHART}

DISCUSSION*

This diagram provides some basic information on how to use the new Pattern Chart, which is enclosed in the sleeve on the inside back cover of this standard volume. For more specific information on the use of patterns (and color) on geologic maps, see Section 5, entitled "Guidelines for Map Color and Pattern Selection," in the accompanying introductory text.

Most patterns on this new chart were designed (in Adobe Illustrator 8.0.1) to closely replicate patterns in the informal "Technical Cartographic Standards" volume (U.S. Geological Survey, ca. 1975). In some cases, however, lineweights of pattern elements had to be increased to facilitate higher resolution (1800 dpi) digital output; therefore, some patterns may not plot or print correctly if output at lower resolutions.

Each pattern has been assigned a new pattern number (see below each box). In addition, each pattern now has associated with it a generic look-up table number that can be used to access a pattern if it has been incorporated into a patternset.

\section{DESCRIPTION}

\begin{tabular}{|l|l|}
\hline Abbreviations used in pattern numbers: & $\bullet \mathrm{K}$, black; C, cyan; M, magenta; DO, dropout; R, red; B, brown \\
\hline Overprint patterns have white background & $\begin{array}{l}\cdot \text { Pattern is in front. One bounding box (having Fill and Stroke set to 'None') is in back } \\
\text { - White background is transparent (underlying map-unit color will be visible) }\end{array}$ \\
\hline Dropout patterns have black background & $\begin{array}{l}\cdot \text { Pattern is in front. Two bounding boxes are in back: box directly beneath pattern has Fill set } \\
\text { to } 100 \% \text { black and Stroke set to 'None'; box to rear has both Fill and Stroke set to 'None' } \\
\text { - Black background represents underlying map-unit color. If white pattern is used "as is," it will } \\
\text { knock out the underlying map-unit color; if pattern is changed to one of the CMYK values in } \\
\text { the underlying map-unit color, it will knock out the other CMYK value(s) in map-unit color }\end{array}$ \\
\hline
\end{tabular}

Pattern number shown below box

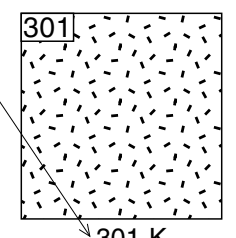

$\sqrt{301-\mathrm{K}}$

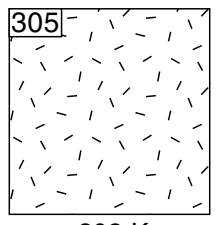

$302-\mathrm{K}$

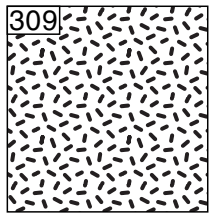

$303-\mathrm{K}$

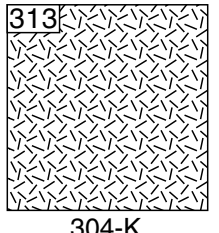

304-K

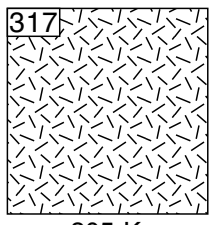

305-K

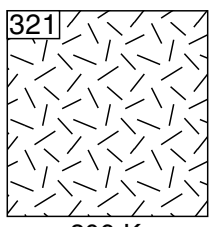

306-K
IGNEOUS PATTERNS (Series 300)

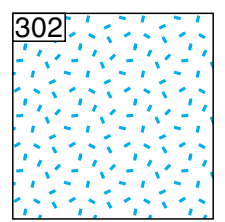

$301-\mathrm{C}$

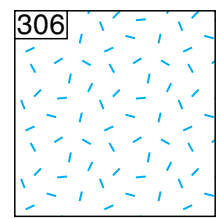

$302-\mathrm{C}$

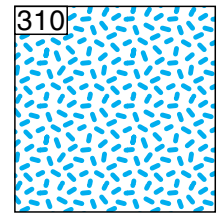

$303-\mathrm{C}$

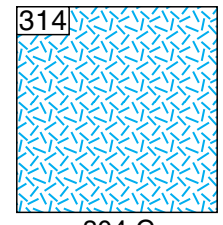

304-C

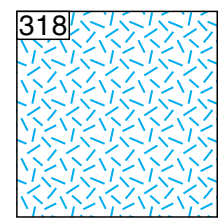

305-C

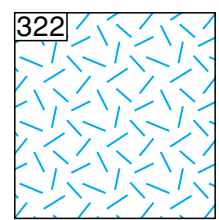

306-C

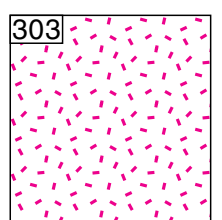

301-M

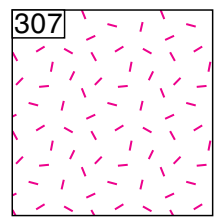

302-M

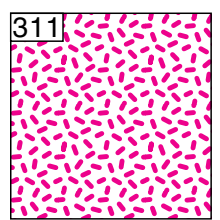

303-M

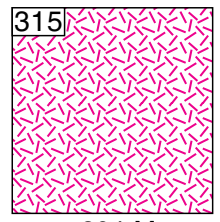

304-M

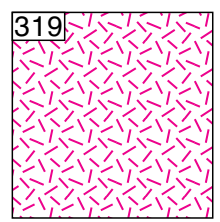

305-M

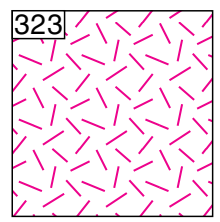

306-M
Generic lookup-table number shown in upper left-hand corner of box (can be used to access a particular pattern from a patternset) 


\section{9-EXPLANATION FOR CMYK COLOR CHART}

\begin{tabular}{|l|}
\hline \multicolumn{1}{|c|}{ DISCUSSION*} \\
\hline This diagram explains how to use the new CMYK Color Chart, which is enclosed in the sleeve on the inside back cover of this standard volume. \\
For more specific information on the use of color (and patterns) on geologic maps, see Section 5, entitled "Guidelines for Map Color and Pattern \\
Selection," in the accompanying introductory text. \\
This new CMYK Color Chart was designed in Adobe Illustrator 8.0.1 (Macintosh) to closely replicate the colors on the U.S. Geological Survey's \\
original offset-printed process-ink color chart, entitled "Printing Colors and Screens in Use by the U.S. Geological Survey for Geologic and \\
Hydrologic Maps" [yellow/magenta/cyan version], which has long been used at the USGS for choosing colors on geologic maps. The new color \\
chart contains the same colors that were in the original offset-printed USGS color chart; however, the old color codes indicating the YMC \\
(yellow/magenta/cyan) values have been updated to show CMYK (cyan/magenta/yellow, with K $=0$ ) values, to conform to industry standards. \\
In addition, each color now has associated with it a generic look-up table number that can be used to access a particular color if it has been \\
incorporated into a shadeset.
\end{tabular}

CMYK $(K=0)$ value shown below box (see left side of diagram for explanation of abbreviations used)

$$
\text { o }
$$

Abbreviations used to specify CMY values

8\% CYAN WITH MAGENTA AND YELLOW

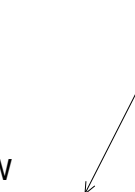

Generic lookup-table number shown in upper left-hand corner of box (can be used to access a particular color from a shadeset)
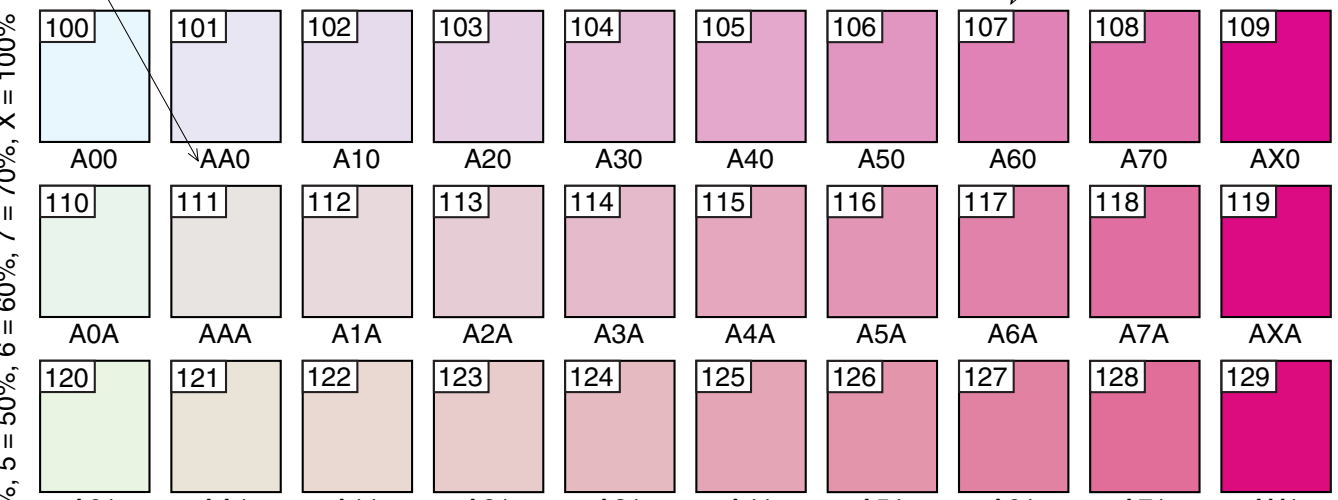

129
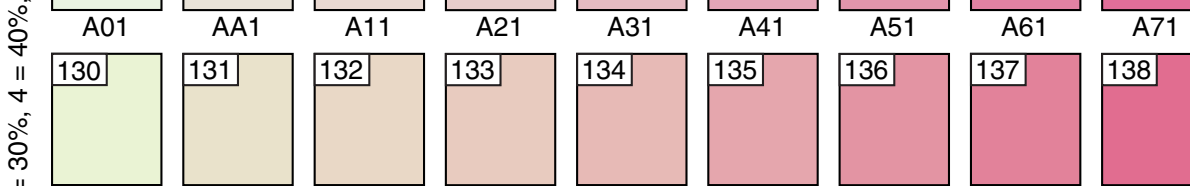

AX1
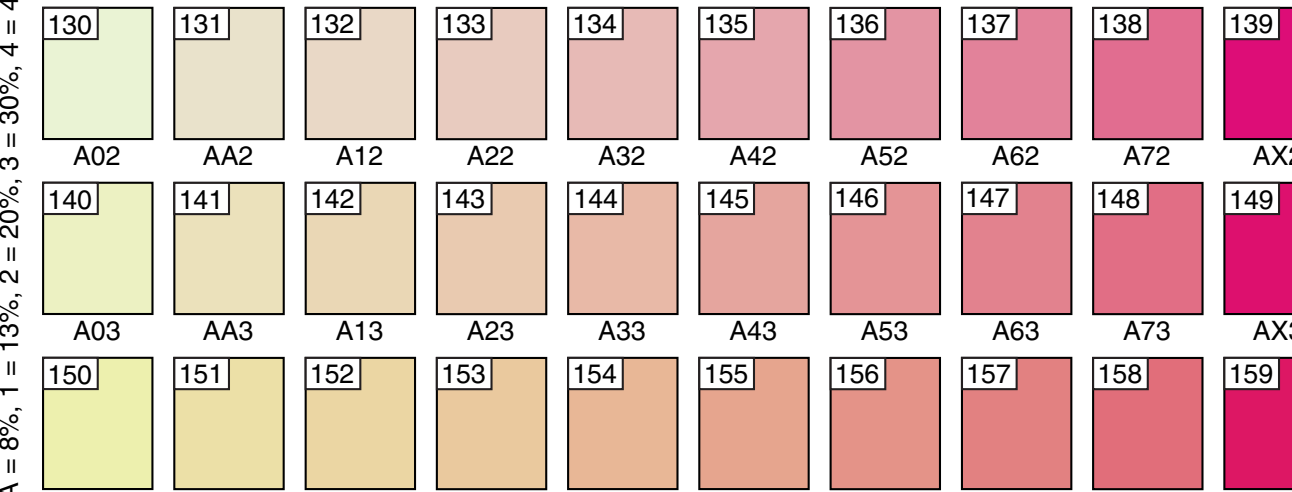

AX2
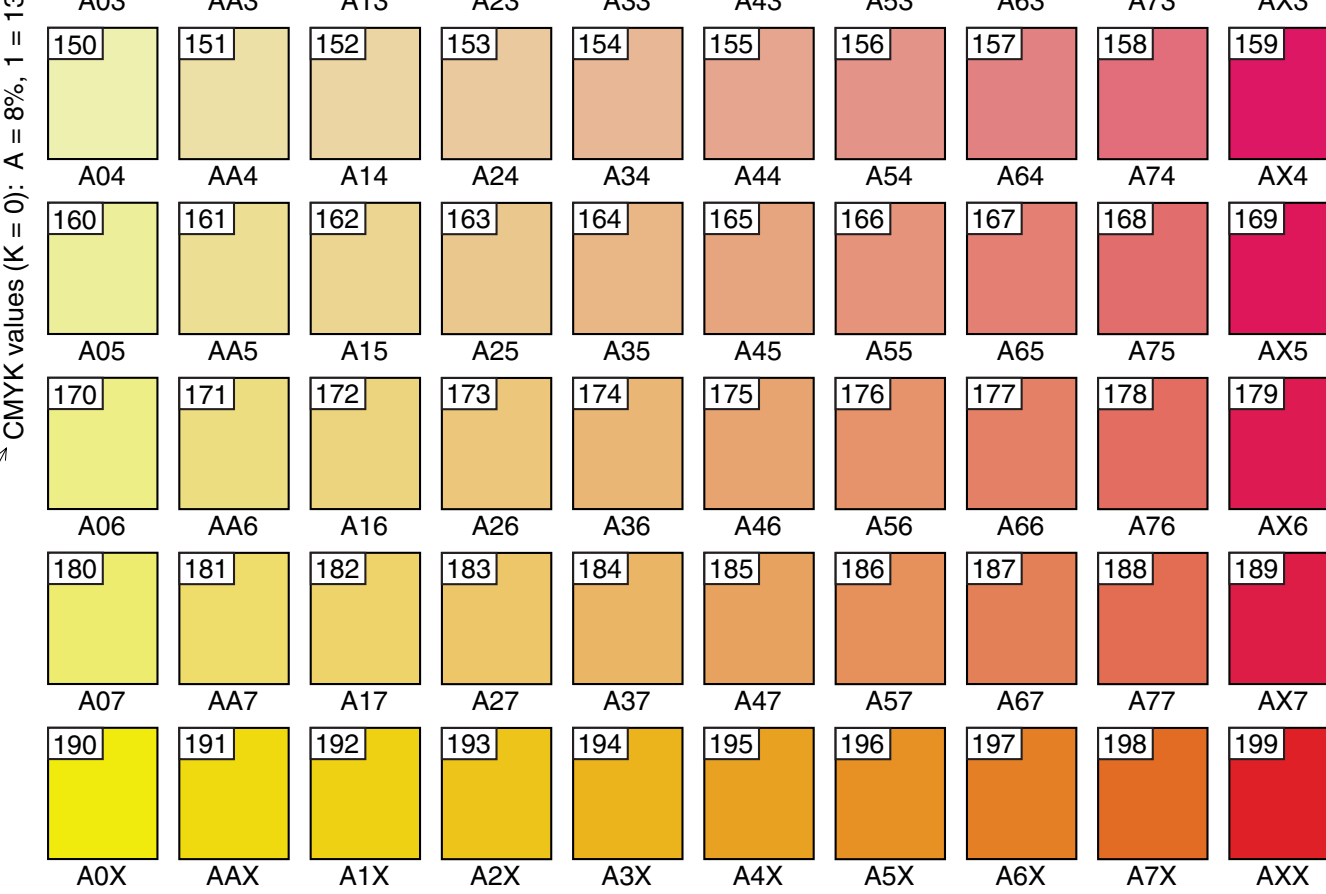


\title{
INDEX TO
}

\section{INTRODUCTORY TEXT}

\author{
AND APPENDIX A
}




\section{INDEX TO INTRODUCTORY TEXT AND APPENDIX A}

\begin{tabular}{|c|c|}
\hline Page & Ref. No. \\
\hline bandoned adit (1st option).. & 19.3.12 \\
\hline Abandoned adit (2nd option).. & 19.3.17 \\
\hline Abandoned clay pit.............. & 19.3.3 \\
\hline Abandoned glory hole.. & 19.3.5 \\
\hline Abandoned gravel pit .. & 19.3.3 \\
\hline Abandoned open pit... & 19.3.5 \\
\hline Abandoned placer pit.. & 19.3.3 \\
\hline Abandoned portal... & 19.3.22 \\
\hline Abandoned quarry ... & 19.3.5 \\
\hline Abandoned sand pit ....... & 19.3.3 \\
\hline Abandoned tunnel entrance (1st option).. & 19.3.12 \\
\hline Abandoned tunnel entrance (2nd option).. & 19.3.17 \\
\hline Abandoned vertical mine shaft, as shown on smaller scale or general-purpose maps . & 19.3.37 \\
\hline Abbreviations used in this $x_{1}$ & Table 2 \\
\hline About this version of the standard... & $\mathrm{n} / \mathrm{a}$ \\
\hline Aboveground gas pipeline............ & 30.3 .24 \\
\hline Aboveground oil pipeline...... & 30.3.24 \\
\hline Aboveground water pipeline ........... & 30.2 .19 \\
\hline Accommodating different values of the zone of confidence [concepts and definitions]. & $\mathrm{n} / \mathrm{a}$ \\
\hline Acknowledgments & $\mathrm{n} / \mathrm{a}$ \\
\hline Acritarchs............. & 10.2 .55 \\
\hline Active convergent plate boundary-Accurately located.. & 22.10 \\
\hline Active convergent plate boundary-Approximately located ... & 22.11 \\
\hline Active convergent plate boundary, showing accretionary prism. & 22.12 \\
\hline Active debris flow, showing a sharply defined morphology....... & 17.56 \\
\hline Active landslide (mapped to scale), showing a sharply defined morphology ...... & 17.58 \\
\hline 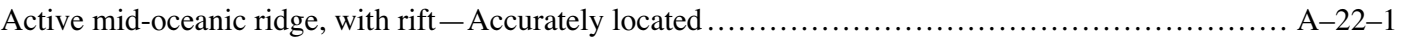 & 22.1 \\
\hline Active mid-oceanic ridge, with rift-Approximately located ............. & 22.2 \\
\hline 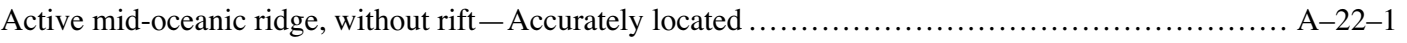 & 22.3 \\
\hline Active mid-oceanic ridge, without rift-Approximately located .................... & 22.4 \\
\hline 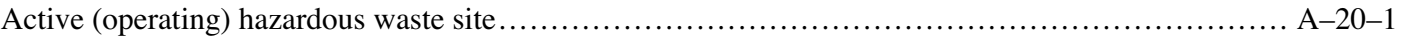 & 20.3 \\
\hline Active quality-of-water site & 26.4.3 \\
\hline Active quality-of-water site, biological measurement $\ldots \ldots \ldots \ldots \ldots \ldots \ldots \ldots \ldots \ldots \ldots \ldots \ldots \ldots$ & 26.4.6 \\
\hline Active quality-of-water site, biological measurement, equipped with a monitor..... & 26.4.11 \\
\hline 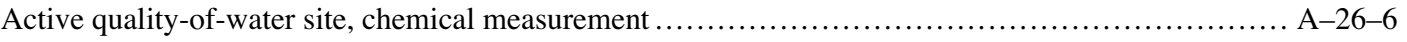 & 26.4 .4 \\
\hline Active quality-of-water site, chemical measurement, equipped with a monitor ........ & 26.4.9 \\
\hline Active quality-of-water site, equipped with a monitor $\ldots \ldots \ldots \ldots \ldots \ldots \ldots \ldots \ldots \ldots$ & 26.4.8 \\
\hline Active quality-of-water site, sediment measurement $\ldots \ldots \ldots \ldots \ldots \ldots \ldots \ldots \ldots \ldots \ldots$ & 26.4.7 \\
\hline Active quality-of-water site, sediment measurement, equipped with a monitor $\ldots \ldots \ldots \ldots \ldots \ldots \ldots \ldots \ldots$ A-26-6 & 26.4.12 \\
\hline 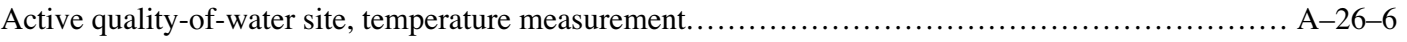 & 26.4 .5 \\
\hline Active quality-of-water site, temperature measurement, equipped with a monitor..................... A-26-6 & 26.4.10 \\
\hline 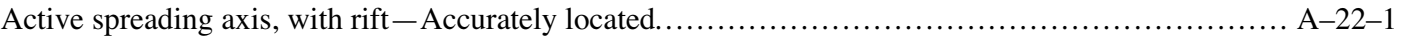 & 22.1 \\
\hline Active spreading axis, with rift-Approximately located..................... & 22.2 \\
\hline 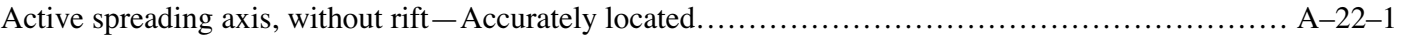 & 22.3 \\
\hline 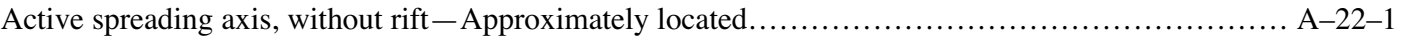 & 22.4 \\
\hline 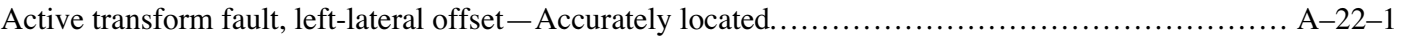 & 22.19 \\
\hline Active transform fault, left-lateral offset-Approximately located.................................... A-22-1 & 22.20 \\
\hline 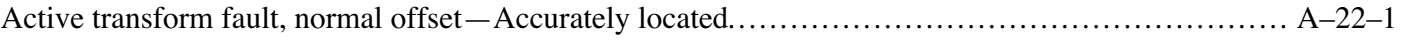 & 22.21 \\
\hline 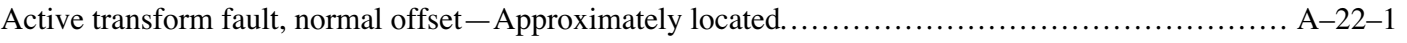 & 22.22 \\
\hline 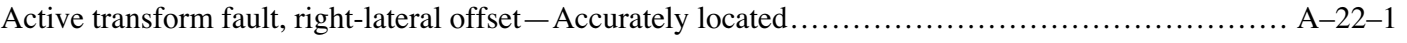 & 22.17 \\
\hline Active transform fault, right-lateral offset-Approximately located.................................... A-22-1 & 22.18 \\
\hline Active transform fault, sense of offset unspecified-Accurately located.............................. A-22-1 & 22.15 \\
\hline Active transform fault, sense of offset unspecified-Approximately locate & 22.16 \\
\hline
\end{tabular}




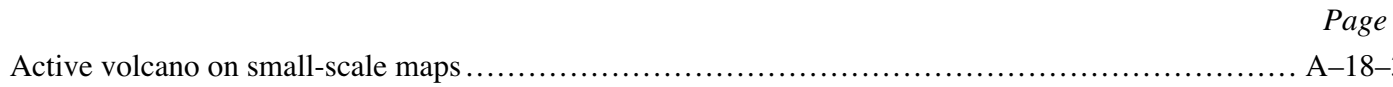

Ref. No.

Adit (1st option)

A-19-3

Adit (1st option), showing angle of inclination.

A-19-3

Adit (2nd option)

A-19-3

Adit (2nd option), showing angle of inclination....

A-19-3

Adit, as shown on topographic maps or on general-purpose or smaller scale maps.

A-30-6

Aeromagnetic survey....

A-3-1

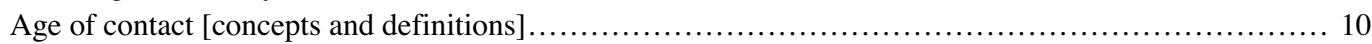

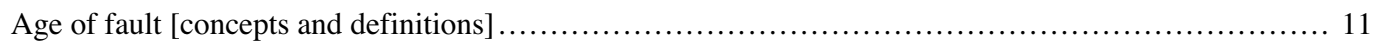

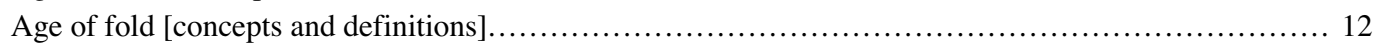

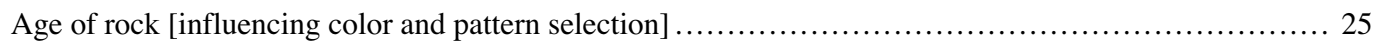

Age symbol font

A-32-1

Ages of rock units [concepts and definitions]

... 8

Aggradational shoreline - Identity and existence certain, location accurate

A-15-1

Aggradational shoreline-Identity and existence certain, location approximate

A-15-1

Aggradational shoreline-Identity or existence questionable, location accurate

A-15-1

Aggradational shoreline - Identity or existence questionable, location approximate .................. A-15-1

Airburst spot.

A-25-6

Alabama [state location map].

A-34-1

Alaska [state location map]

A-34-1

Alaska borough boundary

A-29-1

Algae [fossil symbol]

A-10-1

18.66

19.3.9

19.3.13

19.3.14

19.3 .18

30.3.3

Sec. 3

$\mathrm{n} / \mathrm{a}$

$\mathrm{n} / \mathrm{a}$

$\mathrm{n} / \mathrm{a}$

$\mathrm{n} / \mathrm{a}$

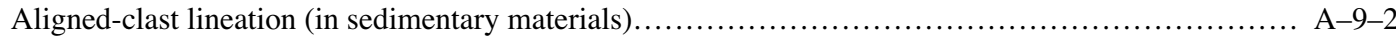

A-9-2

Aligned deformed-mineral lineation .

A-9-2

Aligned-grain lineation (in sedimentary materials)

A-9-2

Aligned-inclusion lineation (in igneous rocks)

A-9-2

Aligned mineral-aggregate lineation....

A-9-2

Aligned-mineral lineation

A-9-2

Aligned-object lineation

A-9-3

Aligned stretched-object lineation....

A-9-3

Aligned stretched-pebble lineation...

A-9-3

Alluvial features

A-12-1

Altered rock (1st option)...

A-19-1

Altered rock (2nd option).....

A-19-1

Altered rock, showing high level of mineralization.

A-19-1

Altered rock, showing low level of mineralization

A-19-1

Ammonoids.

A-10-1

Amphibians [fossil symbol].

A-10-1

Ancient convergent plate boundary-Accurately located.

A-22-1

Ancient convergent plate boundary-Approximately located.

A-22-1

Ancient mid-oceanic ridge-Accurately located

A-22-1

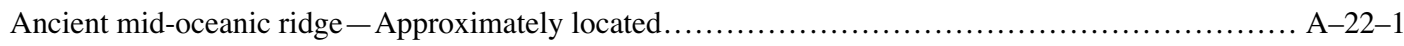

Ancient spreading axis - Accurately located ............................................... A $-22-1$

Ancient spreading axis - Approximately located .......................................... A $-22-1$

Ancient transform fault, sense of offset unspecified-Accurately located ........................... A-22-1

Ancient transform fault, sense of offset unspecified-Approximately located ...................... A-22-1

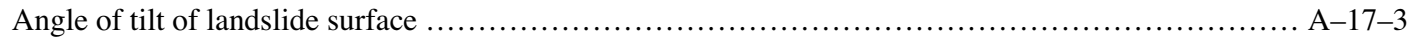

A-25-3

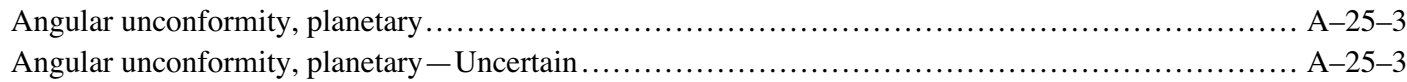

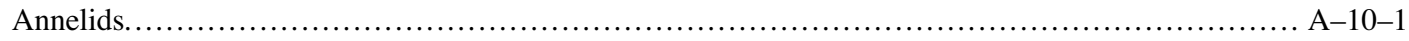

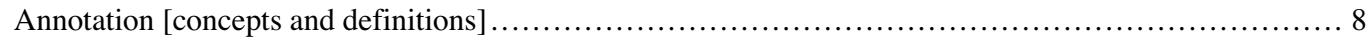

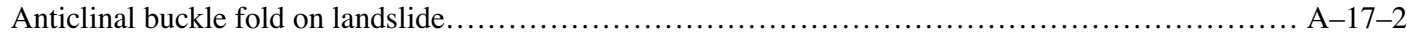

A-17-2

Sec. 32

$\mathrm{n} / \mathrm{a}$

15.5

15.7

15.6

15.8

25.128

Sec. 34.1

Sec. 34.1

29.3

10.2.35

9.29

9.45

9.29

9.33

9.41

9.37

9.25

9.49

9.57

9.53

Sec. 12

19.1.14

19.1 .15

19.1.16

19.1.17

10.2.20

10.2.27

22.13

22.14

22.5

22.6

22.5

22.6

22.23

22.24

17.53

25.60

25.61

10.2 .3

$\mathrm{n} / \mathrm{a}$

17.40

Anticlinal bulge on landslide.

17.40 
Page Ref. No.

Anticlinal linear ridge on landslide............................................................ A-17-2

17.40

Anticlinal soft-sediment fold on landslide .................................................... A-17-2

17.40

Anticline (1st option) - Identity and existence certain, location accurate …...................... A-5-1

5.1 .1

Anticline (1st option) - Identity and existence certain, location approximate ........................ A-5-1

5.1 .3

Anticline (1st option) - Identity and existence certain, location concealed ........................ A-5-1

5.1 .7

Anticline (1st option) - Identity and existence certain, location inferred......................... A-5-1

5.1 .5

Anticline (1st option) - Identity or existence questionable, location accurate ...................... A-5-1

5.1 .2

Anticline (1st option) - Identity or existence questionable, location approximate ..................... A-5-1

5.1 .4

Anticline (1st option) - Identity or existence questionable, location concealed ..................... A-5-1

5.1 .8

Anticline (1st option) - Identity or existence questionable, location inferred...................... A-5-1

5.1 .6

Anticline (2nd option) - Identity and existence certain, location accurate ........................... A-5-1

5.1 .9

Anticline (2nd option) - Identity and existence certain, location approximate ….................. A-5-1

5.1 .11

Anticline (2nd option) - Identity and existence certain, location concealed ........................ A-5-1

5.1 .15

Anticline (2nd option) - Identity and existence certain, location inferred ......................... A-5-1

5.1 .13

Anticline (2nd option) - Identity or existence questionable, location accurate ...................... A-5-1

5.1 .10

Anticline (2nd option) - Identity or existence questionable, location approximate ..................... A-5-1

5.1 .12

Anticline (2nd option) - Identity or existence questionable, location concealed .................... A-5-1

5.1 .16

Anticline (2nd option) - Identity or existence questionable, location inferred....................... A-5-1

5.1 .14

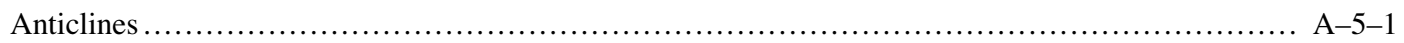

Antiform (1st option) - Identity and existence certain, location accurate ......................... A-5-2

Sec. 5.1

Antiform (1st option) - Identity and existence certain, location approximate ....................... A-5-2

5.2 .1

5.2 .3

Antiform (1st option) - Identity and existence certain, location concealed ....................... A-5-2

5.2 .7

Antiform (1st option) - Identity and existence certain, location inferred......................... A-5-2

5.2 .5

Antiform (1st option) - Identity or existence questionable, location accurate ...................... A-5-2

5.2 .2

Antiform (1st option) - Identity or existence questionable, location approximate .................... A-5-2

5.2 .4

Antiform (1st option) - Identity or existence questionable, location concealed ..................... A-5-2

5.2 .8

Antiform (1st option) - Identity or existence questionable, location inferred...................... A-5-2

5.2 .6

Antiform (2nd option) - Identity and existence certain, location accurate ….................... A-5-2

5.2 .9

Antiform (2nd option) - Identity and existence certain, location approximate ..................... A-5-2

5.2 .11

Antiform (2nd option) - Identity and existence certain, location concealed ....................... A-5-2

Antiform (2nd option) - Identity and existence certain, location inferred .......................... A-5-2

Antiform (2nd option) - Identity or existence questionable, location accurate ...................... A-5-2

5.2 .15

5.2 .13

5.2 .10

Antiform (2nd option) - Identity or existence questionable, location approximate ................... A-5-2

5.2 .12

Antiform (2nd option) - Identity or existence questionable, location concealed .................... A-5-2

5.2 .16

Antiform (2nd option) - Identity or existence questionable, location inferred....................... A-5-2

5.2 .14

Antiformal sheath fold (1st option) - Identity and existence certain, location accurate ................ A-5-5

5.4 .1

Antiformal sheath fold (1st option) - Identity and existence certain, location approximate .............. A-5-5

5.4 .3

Antiformal sheath fold (1st option) - Identity and existence certain, location concealed ................ A-5-5

5.4 .7

Antiformal sheath fold (1st option) - Identity and existence certain, location inferred................. A-5-5

5.4 .5

Antiformal sheath fold (1st option) - Identity or existence questionable, location accurate .............. A-5-5

5.4 .2

Antiformal sheath fold (1st option) - Identity or existence questionable, location approximate .......... A-5-5

5.4 .4

Antiformal sheath fold (1st option) - Identity or existence questionable, location concealed ............. A-5-5

5.4 .8

Antiformal sheath fold (1st option) - Identity or existence questionable, location inferred.............. A-5-5

5.4 .6

Antiformal sheath fold (2nd option) - Identity and existence certain, location accurate ................ A-5-5

5.4 .9

Antiformal sheath fold (2nd option) - Identity and existence certain, location approximate ............. A-5-5

5.4 .11

Antiformal sheath fold (2nd option) - Identity and existence certain, location concealed ............... A-5-5

5.4 .15

Antiformal sheath fold (2nd option) - Identity and existence certain, location inferred................ A-5-5

5.4 .13

Antiformal sheath fold (2nd option) - Identity or existence questionable, location accurate ............ A-5-5

5.4 .10

Antiformal sheath fold (2nd option) - Identity or existence questionable, location approximate ......... A-5-5

5.4 .12

Antiformal sheath fold (2nd option) - Identity or existence questionable, location concealed ............ A-5-5

5.4 .16

Antiformal sheath fold (2nd option) - Identity or existence questionable, location inferred.............. A-5-5

5.4 .14

Antiformal sheath folds ............................................................. A-5-5

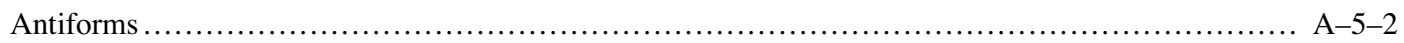

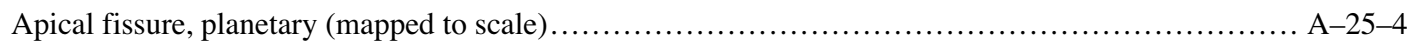

Sec. 5.4

Sec. 5.2

25.83 


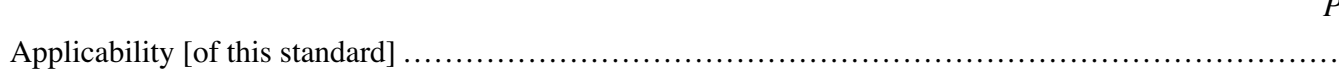

Approximate mean low water line.

Page

Approximate orientation of inclined bedding.

Ref. No.

Approximate orientation of inclined bedding, where top direction of beds is known from local features

A-6-2

30.231

pproximate orientation of overturned bedding

A-6-2

6.33

Approximate orientation of overturned bedding, where top direction of beds is known from local features

A-6-2

6.36

pproximate orientation of vertical or near-vertical bedding .

Approximate orientation of vertical or near-vertical bedding, where top direction of beds is known from local features....

Approximate plunge direction of inclined generic (origin or type not known or not specified) lineation or linear structure (1st option)

Approximate plunge direction of inclined generic (origin or type not known or not specified) lineation or linear structure (2nd option) .

Approximately located adit (1st option)

Approximately located adit (2nd option)

Approximately located portal

Approximately located tunnel entrance (1st option)

Approximately located tunnel entrance (2nd option)

Arcuate fracture, planetary

Area considered to have mineral resource potential but not evaluated.

Area of channelized erosion and scouring, planetary....

Area of clinkered coal bed.

A $-25-3$

A-1-6

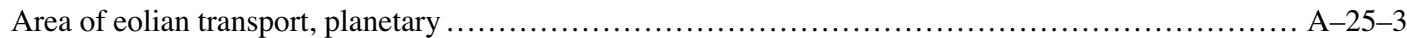

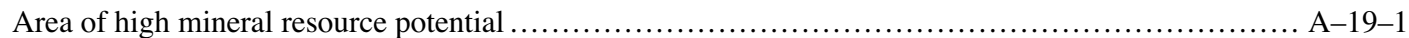

Area of identified resources

A-19-1

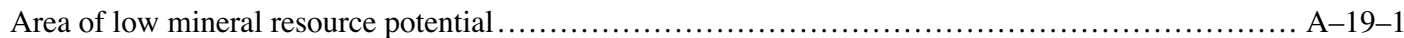

Area of moderate mineral resource potential ................................................ A $-19-1$

Area of outcrop (1st option).

A-31-1

Area of outcrop (2nd option).

A-31-1

Area of outcrop (3rd option)

A-31-1

Area of outcrop (4th option)

A-31-1

Area of outcrop in surficial deposits (1st option)

A-31-1

Area of outcrop in surficial deposits (2nd option)

A-31-1

Area of outcrop in surficial deposits (3rd option)

A-31-1

Area of reticulate grooves, planetary

Area of slip surface of landslide.....

A-17-1

Area to be submerged behind dam ....

A-30-5

Areas in geologic map databases [concepts and definitions]

.. 8

Areas of extensively disturbed ground.

A-19-2

Areas on geologic maps [concepts and definitions].

.. 7

19.3.20

19.3.10

19.3.15

30.2.17

30.2 .16

26.6 .3

26.6.5

10.2 .5

$\mathrm{n} / \mathrm{a}$

32.30

25.31

19.1.22

25.69

1.2.41

1.4.17

25.70

19.1.19

19.1.18

19.1.21

19.1.20

31.16

31.18

31.19

31.13

31.14

31.15

31.20

25.125

17.9

30.2 .38

$\mathrm{n} / \mathrm{a}$

Arête ....................................................................................... A-13-2

Sec. 19.2

$\mathrm{n} / \mathrm{a}$

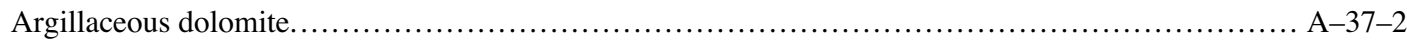

13.42

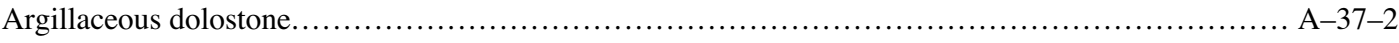

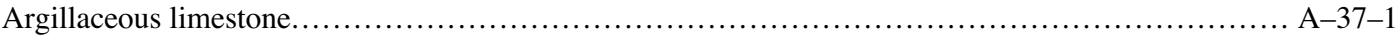

Argillaceous sandstone 
Page
Page

Arial [font].....

Arizona [state location map].....

Arkansas [state location map].

Arthropods....

Artificial fill consisting of earth materials

Artificial fill consisting of human-generated refuse (landfill).

Asymmetric anticline (1st option) - Identity and existence certain, location accurate........

Asymmetric anticline (1st option) - Identity and existence certain, location approximate

Asymmetric anticline (1st option) - Identity and existence certain, location concealed.

Asymmetric anticline (1st option) - Identity and existence certain, location inferred ..

Asymmetric anticline (1st option) - Identity or existence questionable, location accurate

Asymmetric anticline (1st option) - Identity or existence questionable, location approximate

Asymmetric anticline (1st option) - Identity or existence questionable, location concealed ............ A-5-3

Asymmetric anticline (1st option) - Identity or existence questionable, location inferred.............. A-5-3

Asymmetric anticline (2nd option) - Identity and existence certain, location accurate................ A-5-3

Asymmetric anticline (2nd option) - Identity and existence certain, location approximate............. A-5-3

Asymmetric anticline (2nd option) - Identity and existence certain, location concealed............... A-5-3

Asymmetric anticline (2nd option) - Identity and existence certain, location inferred ................. A-5-3

Asymmetric anticline (2nd option) - Identity or existence questionable, location accurate ............ A-5-3

Asymmetric anticline (2nd option) - Identity or existence questionable, location approximate .......... A-5-3

Asymmetric anticline (2nd option) - Identity or existence questionable, location concealed ............ A-5-3

Asymmetric anticline (2nd option) - Identity or existence questionable, location inferred............. A-5-3

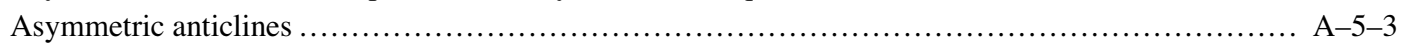

Asymmetric (S-shaped, counterclockwise sense of shear) crenulation cleavage, for multiple

observations at one locality

A-7-2

Asymmetric (S-shaped, counterclockwise sense of shear) crenulation foliation, for multiple

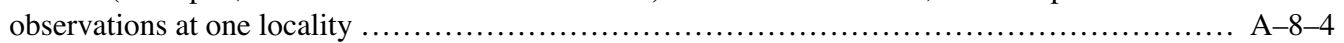

Asymmetric (S-shaped, counterclockwise sense of shear) kink-band crenulation lineation .............. A-9-6

Asymmetric (S-shaped, counterclockwise sense of shear) minor fold hinge........................ A-9-6

Asymmetric syncline (1st option) - Identity and existence certain, location accurate ................. A-5-8

Asymmetric syncline (1st option) - Identity and existence certain, location approximate .............. A-5-8

Asymmetric syncline (1st option) - Identity and existence certain, location concealed ................. A-5-8

Asymmetric syncline (1st option) - Identity and existence certain, location inferred ................. A-5-8

Asymmetric syncline (1st option) - Identity or existence questionable, location accurate............. A-5-8

Asymmetric syncline (1st option) - Identity or existence questionable, location approximate.......... A-5-8

Asymmetric syncline (1st option) - Identity or existence questionable, location concealed............. A-5-8

Asymmetric syncline (1st option) - Identity or existence questionable, location inferred .............. A-5-8

Asymmetric syncline (2nd option) - Identity and existence certain, location accurate................ A-5-8

Asymmetric syncline (2nd option) - Identity and existence certain, location approximate .............. A-5-8

Asymmetric syncline (2nd option) - Identity and existence certain, location concealed ............... A-5-8

Asymmetric syncline (2nd option) - Identity and existence certain, location inferred ................. A-5-8

Asymmetric syncline (2nd option) - Identity or existence questionable, location accurate............. A-5-8

Asymmetric syncline (2nd option) - Identity or existence questionable, location approximate.......... A-5-8

Asymmetric syncline (2nd option) - Identity or existence questionable, location concealed............. A-5-8

Asymmetric syncline (2nd option) - Identity or existence questionable, location inferred ............. A-5-8

Asymmetric synclines.............................................................. A-5-8

Asymmetric (Z-shaped, clockwise sense of shear) crenulation cleavage, for multiple observations at one locality ....

A-7-2

Asymmetric (Z-shaped, clockwise sense of shear) crenulation foliation, for multiple observations at

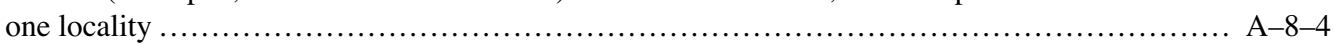

Asymmetric (Z-shaped, clockwise sense of shear) kink-band crenulation lineation .................... A-9-6

Asymmetric (Z-shaped, clockwise sense of shear) minor fold hinge $\ldots \ldots \ldots \ldots \ldots \ldots \ldots \ldots \ldots \ldots \ldots \ldots$ A-9 -6

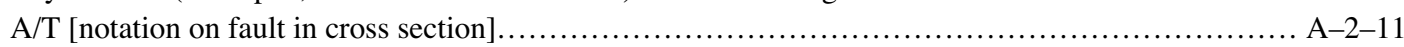

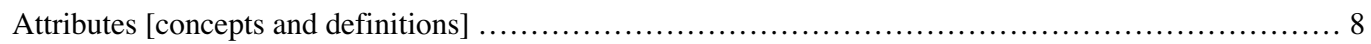

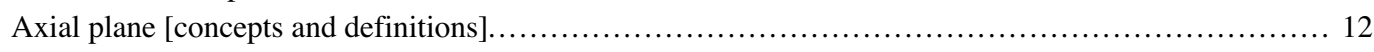

Ref. No. $\mathrm{n} / \mathrm{a}$

Sec. 34.1

Sec. 34.1

10.2.4

19.2.4

19.2.5

5.3 .1

5.3 .3

5.3 .7

5.3 .5

5.3 .2

5.3 .4

5.3 .8

5.3 .6

5.3 .9

5.3 .11

5.3.15

5.3 .13

5.3 .10

5.3 .12

5.3 .16

5.3.14

Sec. 5.3

7.28

8.3.37

9.137

9.125

5.7 .1

5.7 .3

5.7 .7

5.7 .5

5.7 .2

5.7 .4

5.7 .8

5.7 .6

5.7 .9

5.7 .11

5.7.15

5.7 .13

5.7 .10

5.7 .12

5.7 .16

5.7.14

Sec. 5.7

8.3 .43

9.141

9.129

2.11 .20

$\mathrm{n} / \mathrm{a}$

n/a 


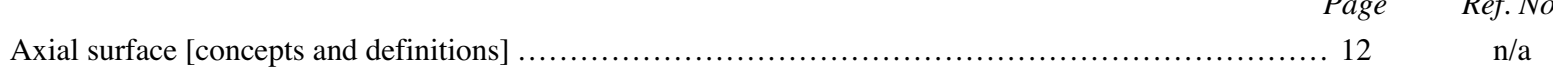

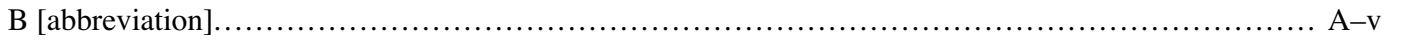

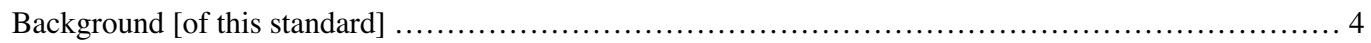

Ball and bar [notation on fault showing local normal offset] ................................. A $-2-11$

Ball and bar [notation on fault showing local oblique offset] .................................. A $-2-11$

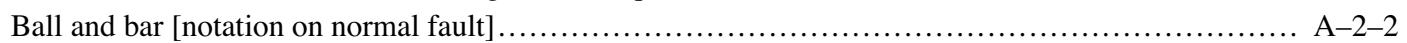

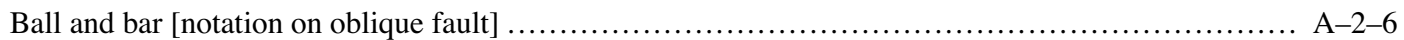

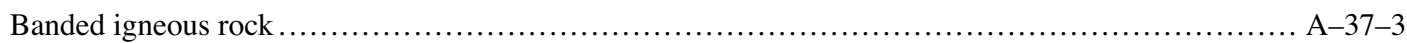

Bar-Identity and existence certain, location accurate ...................................... A-15-2

Bar-Identity and existence certain, location approximate ................................. A-15-2

Bar-Identity or existence questionable, location accurate................................. A-15-2

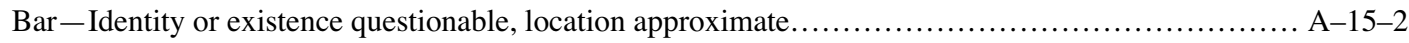

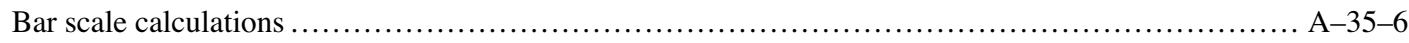

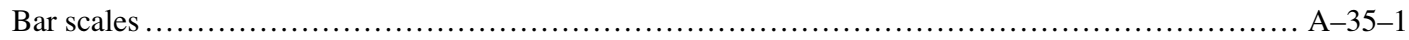

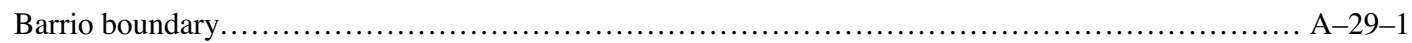

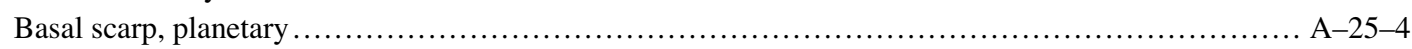

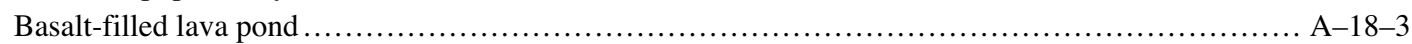

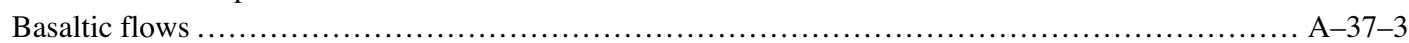

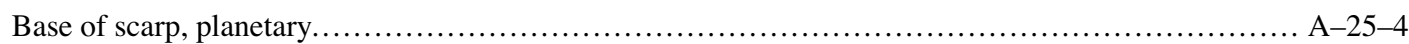

Basin ring, planetary ................................................................... A-25-5

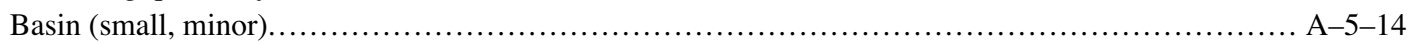

Basin structure on landslide ........................................................ A-17-2

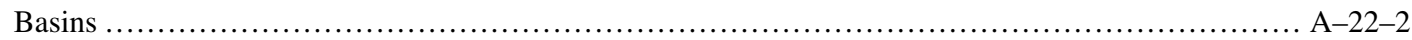

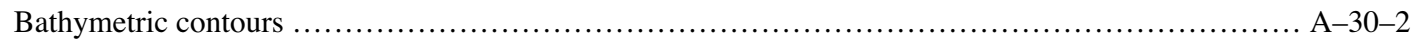

Bathymetric depression contours, showing hachure spacing for closed contours between $12.7 \mathrm{~mm}$

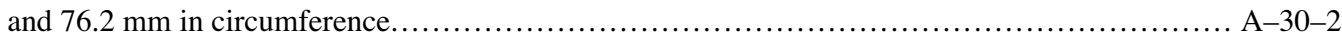

Bathymetric depression contours, showing hachure spacing for closed contours less than $12.7 \mathrm{~mm}$

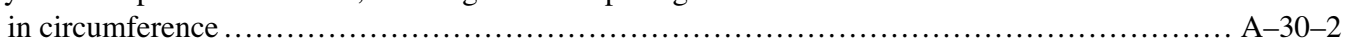

Bathymetric depression contours, showing hachure spacing for closed contours more than $76.2 \mathrm{~mm}$

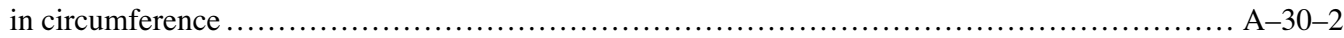

Bathymetric rise contour (inside depression), showing hachure spacing for closed contours between $12.7 \mathrm{~mm}$ and $76.2 \mathrm{~mm}$ in circumference ....................................................

Bathymetric rise contour (inside depression), showing hachure spacing for closed contours less than $12.7 \mathrm{~mm}$ in circumference.

Table 2 $\mathrm{n} / \mathrm{a}$

2.11 .1

2.11 .6

Sec. 2.2

Sec. 2.7

720

15.26

15.28

15.27

15.29

Sec. 35

Sec. 35

29.4

25.73

18.54

717

25.74

25.100

5.11 .3

17.43

Sec. 22

Sec. 30.1

30.1 .43

30.1 .42

30.1 .44

30.1 .43

30.1 .41

Bathymetric rise contour (inside depression), showing hachure spacing for closed contours more

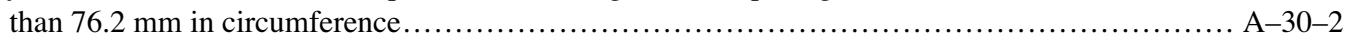

30.1 .44

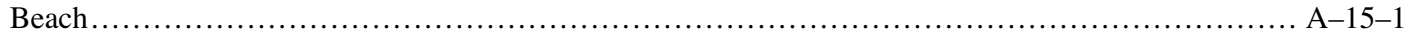

Bed of economically important commodity - Identity and existence certain, location accurate ......... A-1-3 $\mathrm{n} / \mathrm{a}$

1.2 .17

1.2 .19

1.2 .23

1.2 .21

1.2 .18

Bed of economically important commodity - Identity or existence questionable, location accurate ...... A-1-3

Bed of economically important commodity - Identity or existence questionable, location

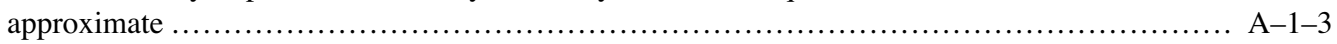

1.2 .20

1.2 .24

1.2 .22

1.2 .42

1.2 .43

1.4 .14

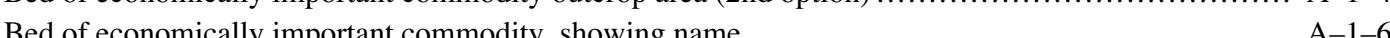

Bed of economically important commodity, showing thickness and location where thickness was measured 


\begin{tabular}{|c|c|}
\hline Page & Ref. No. \\
\hline Bedded chert (2nd option).. & 650 \\
\hline Bedded sand ................ & 608 \\
\hline Bedded sandstone.................. & 608 \\
\hline 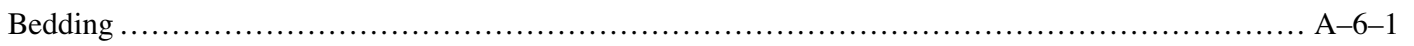 & Sec. 6 \\
\hline Bedding, as determined remotely or from aerial photographs.................................... A-6 -2 & 6.39 \\
\hline 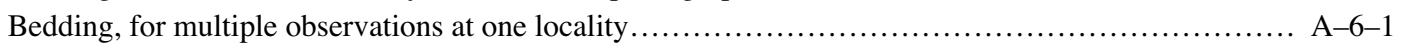 & 6.6 \\
\hline 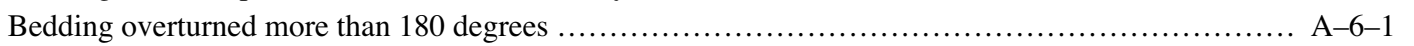 & 6.5 \\
\hline 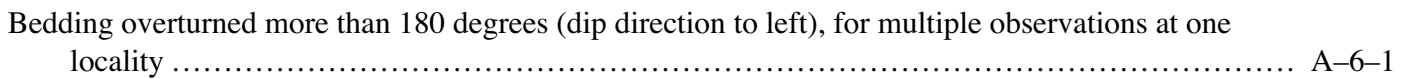 & 6.12 \\
\hline $\begin{array}{l}\text { Bedding overturned more than } 180 \text { degrees (dip direction to left), where top direction of beds is } \\
\quad \text { known from local features, for multiple observations at one locality } \ldots \ldots \ldots \ldots \ldots \ldots \ldots \ldots \ldots \ldots \ldots \text { A }-6-1\end{array}$ & 6.24 \\
\hline 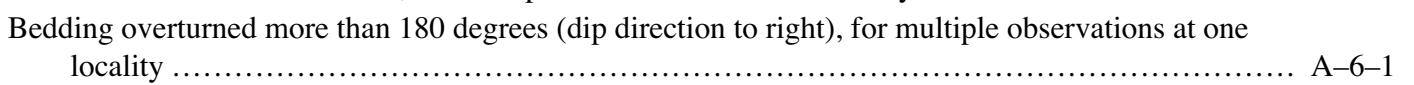 & 6.11 \\
\hline $\begin{array}{l}\text { Bedding overturned more than } 180 \text { degrees (dip direction to right), where top direction of beds is } \\
\quad \text { known from local features, for multiple observations at one locality } \ldots \ldots \ldots \ldots \ldots \ldots \ldots \ldots \ldots \ldots \ldots \ldots \ldots \ldots \\
\text {. }\end{array}$ & 6.23 \\
\hline Bedding overturned more than 180 degrees, where top direction of beds is known from local features.. A-6-1 & 6.16 \\
\hline Bedding where top direction of beds is known from local features.............................. A-6 -1 & 6.13 \\
\hline Bedding where top direction of beds is known from local features, for multiple observations at one & 6.17 \\
\hline $\begin{array}{l}\text { Bedrock contour (index), as shown on hydrologic maps, showing altitude of bedrock surface }- \\
\text { Accurately located } \ldots \ldots \ldots \ldots \ldots \ldots \ldots \ldots \ldots \ldots \ldots \ldots \ldots \ldots \ldots \ldots \ldots \ldots \ldots \ldots \ldots \ldots \ldots \ldots \ldots \ldots \ldots \ldots \ldots \ldots \ldots \ldots \ldots \ldots \ldots \\
\text { A }-26\end{array}$ & 26.5 .5 \\
\hline 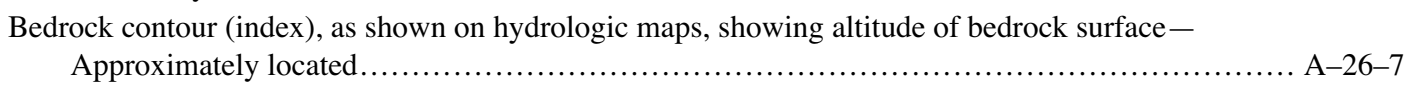 & 26.5 .6 \\
\hline 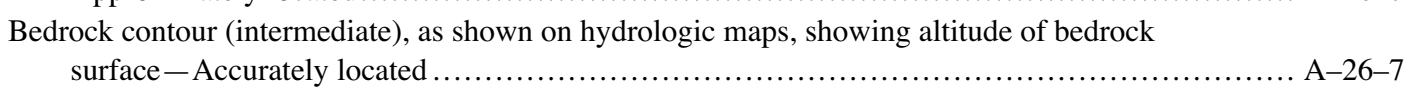 & 26.5 .7 \\
\hline $\begin{array}{l}\text { Bedrock contour (intermediate), as shown on hydrologic maps, showing altitude of bedrock } \\
\text { surface }- \text { Approximately located } \ldots \ldots \ldots \ldots \ldots \ldots \ldots \ldots \ldots \ldots \ldots \ldots \ldots \ldots \ldots \ldots \ldots \ldots \ldots \ldots \ldots \ldots \ldots \ldots \ldots \ldots \ldots\end{array}$ & 26.5 .8 \\
\hline 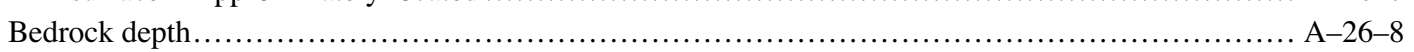 & 26.6 .3 \\
\hline 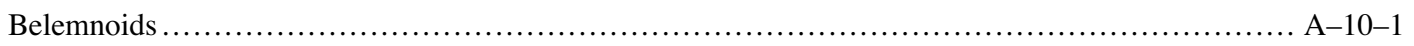 & 10.2 .21 \\
\hline 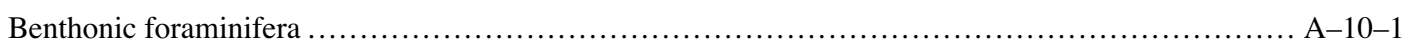 & 10.2 .50 \\
\hline Bentonite & 662 \\
\hline Block-glide landslide, consisting of a relatively intact mass of displaced materials . ................. A-17-3 & 17.62 \\
\hline Blowout rim around closed depression of eolian origin in bedrock - Accurately located............... A-16-1 & 16.4 \\
\hline Blowout rim around closed depression of eolian origin in bedrock - Approximately located............ A-16-1 & 16.5 \\
\hline 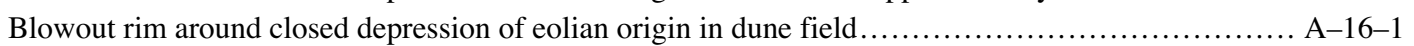 & 16.3 \\
\hline Bog & 30.2 .46 \\
\hline 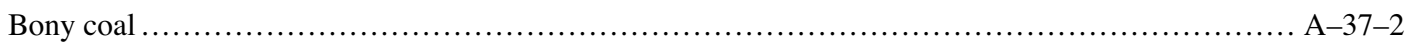 & 659 \\
\hline Borrow pit, as shown on topographic maps or on general-purpose or smaller scale maps .............. A-30-6 & 30.3 .2 \\
\hline Bottom of mine shaft (drawn to scale), as shown on subsurface exploration maps ................... A-19-5 & 19.4 .5 \\
\hline Boudins & 9.69 \\
\hline Boundaries.................................. & Sec. 29 \\
\hline Boundaries located by geophysical methods.... & Sec. 3.1 \\
\hline Boundary - Alaska borough .......................... & 29.3 \\
\hline Boundary - Barrio............................... & 29.4 \\
\hline 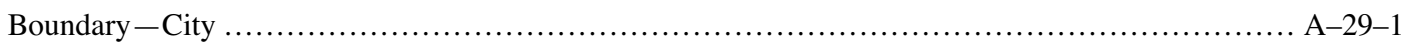 & 29.5 \\
\hline Boundary - Civil township............... & 29.4 \\
\hline 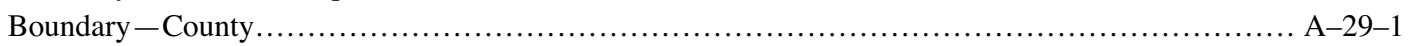 & 29.3 \\
\hline Boundary -District.......................................... & 29.4 \\
\hline Boundary -Forest Reserve............. & 29.6 \\
\hline 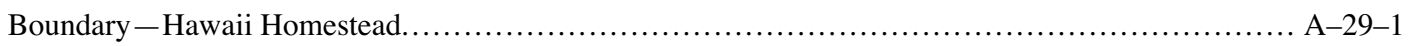 & 29.6 \\
\hline 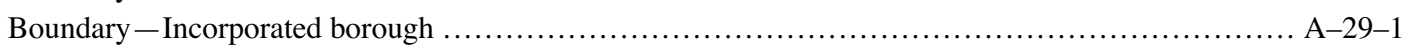 & 29.5 \\
\hline Boundary -Incorporated city ................ & 29.5 \\
\hline Boundary -Incorporated hamlet ..... & 29.5 \\
\hline Boundary - Incorporated town ....... & 29.5 \\
\hline Boundary - Incorporated village ..... & 29.5 \\
\hline
\end{tabular}




\begin{tabular}{|c|c|}
\hline Page & Ref. No. \\
\hline Boundary - Judicial division............................. & 29.3 \\
\hline 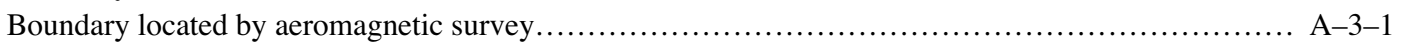 & 3.1 .1 \\
\hline Boundary located by electromagnetic survey................. & 3.1 .7 \\
\hline Boundary located by gravity survey ....................... & 3.1 .3 \\
\hline Boundary located by ground magnetic survey.............. & 3.1 .2 \\
\hline Boundary located by induced polarization survey ... & 3.1 .6 \\
\hline 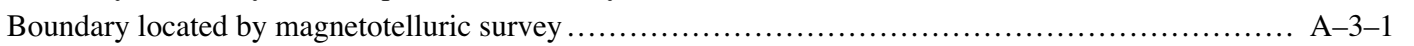 & 3.1 .9 \\
\hline 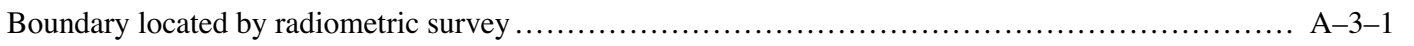 & 3.1 .4 \\
\hline 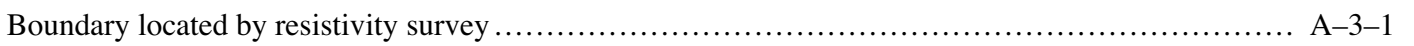 & 3.1 .8 \\
\hline Boundary located by seismic reflection survey............... & 3.1 .5 \\
\hline Boundary-Municipio ............................... & 29.3 \\
\hline Boundary - National $\ldots \ldots \ldots \ldots \ldots \ldots \ldots$ & 29.1 \\
\hline Boundary - National forest............... & 29.6 \\
\hline Boundary - National grassland ............ & 29.6 \\
\hline Boundary - National monument.......... & 29.6 \\
\hline Boundary - National park ............. & 29.6 \\
\hline Boundary - National reservation ........ & 29.6 \\
\hline Boundary -National wilderness area........... & 29.6 \\
\hline Boundary - National wildlife refuge............ & 29.6 \\
\hline Boundary - Parish ...................... & 29.3 \\
\hline Boundary -Park (small).......... & 29.7 \\
\hline Boundary - Precinct................. & 29.4 \\
\hline Boundary - Small park ...................... & 29.7 \\
\hline Boundary - State.................... & 29.2 \\
\hline Boundary - State forest................ & 29.6 \\
\hline Boundary - State grassland .............. & 29.6 \\
\hline Boundary - State monument.......... & 29.6 \\
\hline Boundary -State park ................. & 29.6 \\
\hline Boundary - State reservation ............. & 29.6 \\
\hline 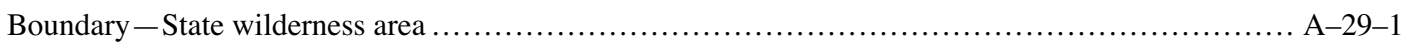 & 29.6 \\
\hline Boundary - State wildlife refuge ........ & 29.6 \\
\hline Boundary - Territory ...................... & 29.2 \\
\hline Boundary - Town......................... & 29.4 \\
\hline Boundary - Township.................. & 29.4 \\
\hline Brachiopods ........................ & 10.2 .9 \\
\hline Braided creek................. & 30.2 .6 \\
\hline Braided river ............... & 30.2 .6 \\
\hline Braided stream ............... & 30.2 .6 \\
\hline Breccia (1st option)..................... & 605 \\
\hline Breccia (2nd option)............................ & 606 \\
\hline 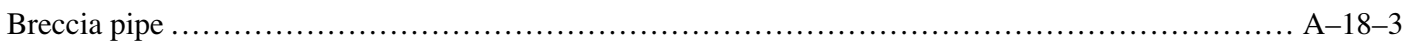 & 18.70 \\
\hline Broad ridge crest, planetary (generally associated with coronae)......... & 25.40 \\
\hline 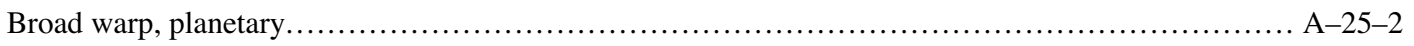 & 25.36 \\
\hline Broken rock around fault ............ & 2.14 .4 \\
\hline Broken rock within fault.............. & 2.14 .3 \\
\hline Brown [spot color] ..................... & Table 3 \\
\hline Bryozoans............................... & 10.2 .10 \\
\hline Bulge on landslide..................... & 17.41 \\
\hline 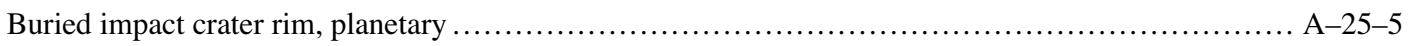 & 25.98 \\
\hline 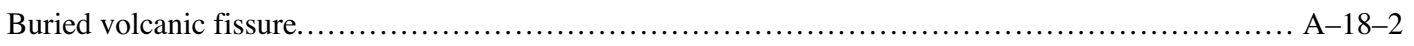 & 18.42 \\
\hline 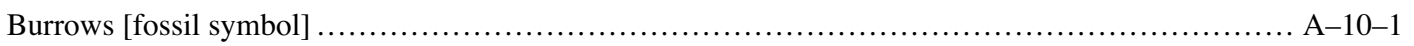 & 10.2 .42 \\
\hline $\mathrm{C}$ [abbreviation]........... & Table 2 \\
\hline Calcareous sandstone ...... & 613 \\
\hline
\end{tabular}




\begin{tabular}{|c|c|c|}
\hline Calcareous shale..... & $\begin{array}{c}\text { Page } \\
\text { A-37-1 }\end{array}$ & $\begin{array}{c}\text { Ref. No. } \\
\quad 623\end{array}$ \\
\hline Calcareous siltstone. & A-37-1 & 617 \\
\hline Caldera margin (1st option)-Identity and existence certain, location accurate. & A-18-1 & 18.8 \\
\hline Caldera margin (1st option)-Identity and existence certain, location approximate. & A-18-1 & 18.10 \\
\hline Caldera margin (1st option) - Identity and existence certain, location concealed.... & A-18-1 & 18.14 \\
\hline Caldera margin (1st option) - Identity and existence certain, location inferred ... & A-18-1 & 18.12 \\
\hline Caldera margin (1st option) - Identity or existence questionable, location accurate... & A-18-1 & 18.9 \\
\hline Caldera margin (1st option)-Identity or existence questionable, location approximate.. & A-18-1 & 18.11 \\
\hline Caldera margin (1st option)-Identity or existence questionable, location concealed.... & . A-18-1 & 18.15 \\
\hline Caldera margin (1st option) - Identity or existence questionable, location inferred ....... & . A-18-1 & 18.13 \\
\hline Caldera margin (2nd option) - Identity and existence certain, location accurate ...... & . A-18-1 & 18.16 \\
\hline Caldera margin (2nd option) - Identity and existence certain, location approximate ....... & . A-18-1 & 18.18 \\
\hline Caldera margin (2nd option) - Identity and existence certain, location concealed ......... & . A-18-1 & 18.22 \\
\hline Caldera margin (2nd option) - Identity and existence certain, location inferred .......... & A-18-1 & 18.20 \\
\hline Caldera margin (2nd option) - Identity or existence questionable, location accurate.......... & . A-18-1 & 18.17 \\
\hline Caldera margin (2nd option) - Identity or existence questionable, location approximate...... & . A-18-1 & 18.19 \\
\hline Caldera margin (2nd option) - Identity or existence questionable, location concealed...... & . A-18-1 & 18.23 \\
\hline Caldera margin (2nd option) - Identity or existence questionable, location inferred ........... & . A-18-1 & 18.21 \\
\hline Caldera, planetary & . A-25-5 & 25.111 \\
\hline California [state location map].............................. & . A-34-1 & Sec. 34.1 \\
\hline 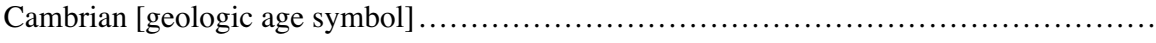 & . A-32-1 & 32.18 \\
\hline 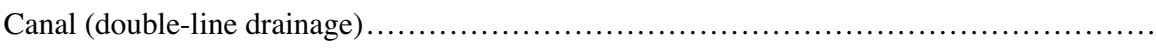 & . A-30-4 & 30.2 .8 \\
\hline Canal lock (double-line drainage) & . A-30-4 & 30.2 .11 \\
\hline 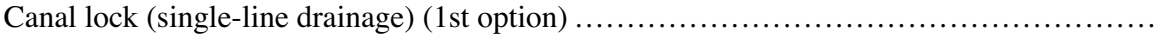 & . A-30-4 & 30.2 .9 \\
\hline Canal lock (single-line drainage) (2nd option) ...... & . A-30-4 & 30.2 .10 \\
\hline Canal (single-line drainage) ......................... & . A-30-4 & 30.2 .7 \\
\hline Capped water well..... & . A-26-1 & 26.1 .3 \\
\hline Carbonaceous shale . & . A-37-1 & 624 \\
\hline Carboniferous [geologic age symbol].. & A-32-1 & 32.12 \\
\hline 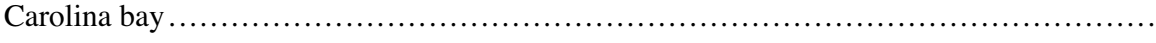 & . A-30-5 & 30.2 .42 \\
\hline Cartographic representation of locational accuracy [concepts and definitions]... & 23 & $\mathrm{n} / \mathrm{a}$ \\
\hline Cartographic representation of scientific confidence [concepts and definitions].. & .... 17 & $\mathrm{n} / \mathrm{a}$ \\
\hline Cave entrance, as shown on topographic maps or on general-purpose or smaller scale maps. & . A-30-6 & 30.3.4 \\
\hline 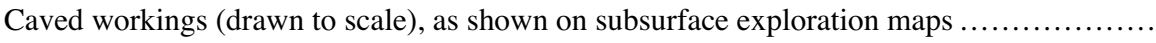 & . A-19-5 & 19.4.11 \\
\hline 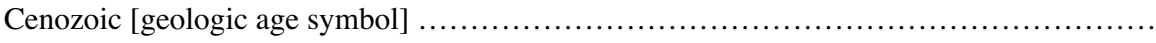 & . A-32-1 & 32.1 \\
\hline Central peak of impact crater, planetary (1st option).. & A-25-5 & 25.101 \\
\hline Central peak of impact crater, planetary (2nd option). & A-25-5 & 25.102 \\
\hline Central peak, planetary (not mapped to scale)........... & A-25-4 & 25.82 \\
\hline Cephalopods.................................... & A-10-1 & 10.2.19 \\
\hline "Certain" [concepts and definitions] ......... & $\ldots 16$ & $\mathrm{n} / \mathrm{a}$ \\
\hline Chain craters, planetary .................. & . A-25-5 & 25.110 \\
\hline Chalk ..................... & A-37-1 & 626 \\
\hline Changes [in this standard] from previous standards ................ & $\ldots .5$ & $\mathrm{n} / \mathrm{a}$ \\
\hline Channel bars, planetary, may be erosional or depositional ........ & . A-25-5 & 25.116 \\
\hline 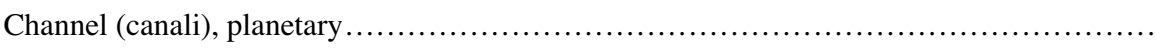 & $\mathrm{A}-25-3$ & 25.56 \\
\hline 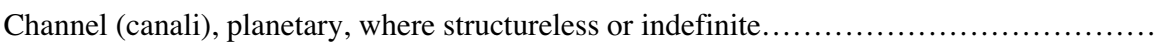 & A-25-3 & 25.57 \\
\hline 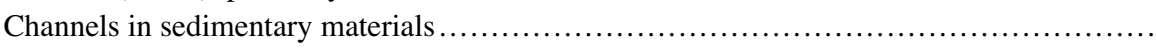 & A-9-1 & 9.13 \\
\hline Chart showing conversion values from inches (in) to points (pts) to millimeters (mm) ..... & .. A-iv & Table 1 \\
\hline 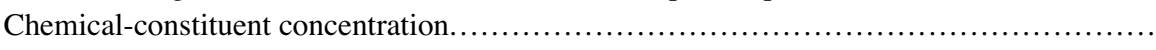 & A-26-8 & 26.6 .11 \\
\hline Cherty and sandy crossbedded clastic limestone & A-37-1 & 634 \\
\hline 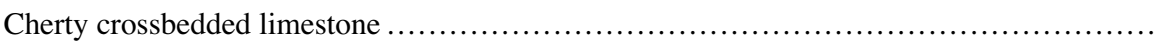 & A-37-1 & 633 \\
\hline Cherty dolomite.................. & A-37-2 & 648 \\
\hline Cherty dolostone................ & A-37-2 & 648 \\
\hline Cherty limestone (1st option). & A-37-1 & 639 \\
\hline
\end{tabular}




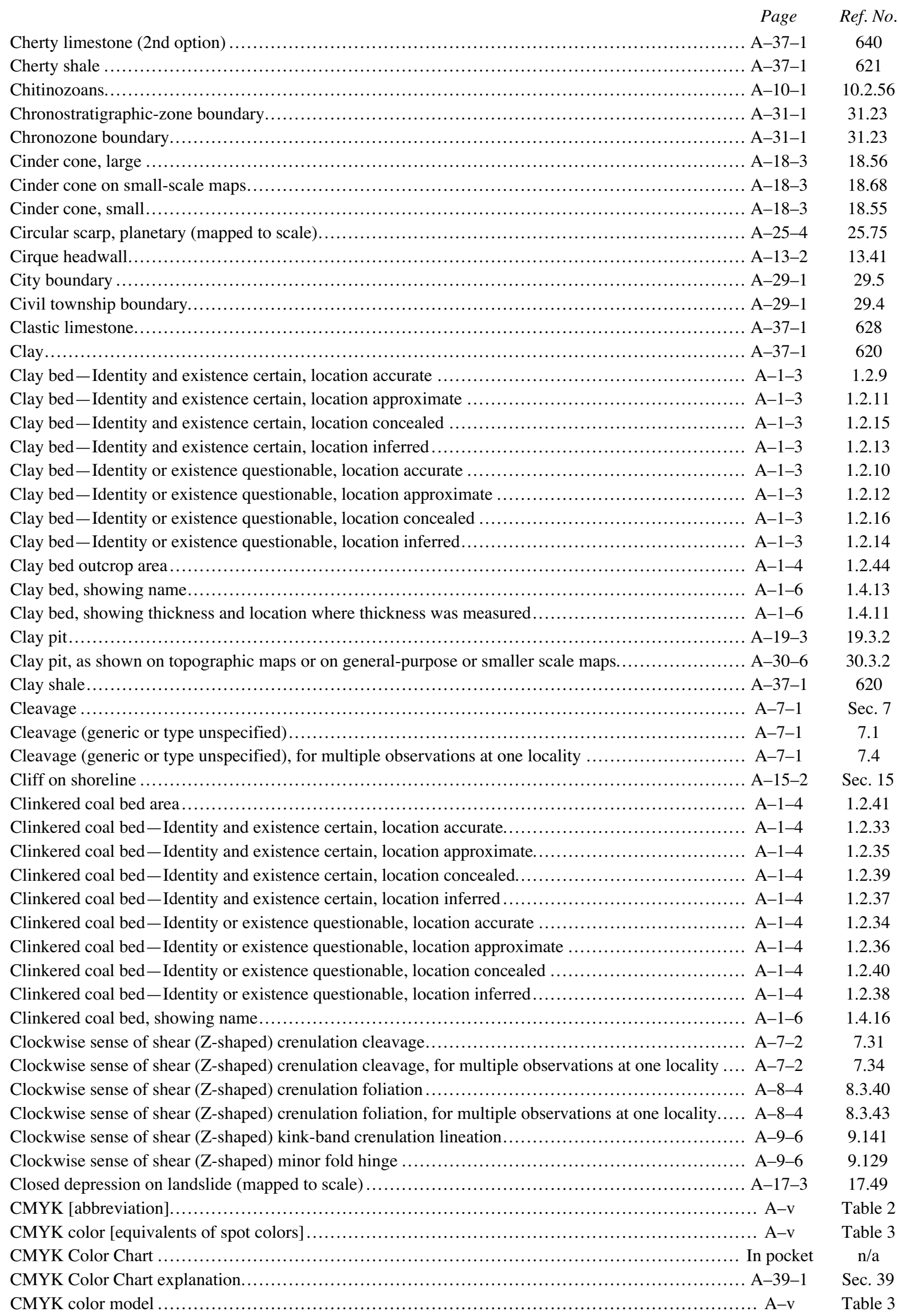




\begin{tabular}{|c|c|}
\hline 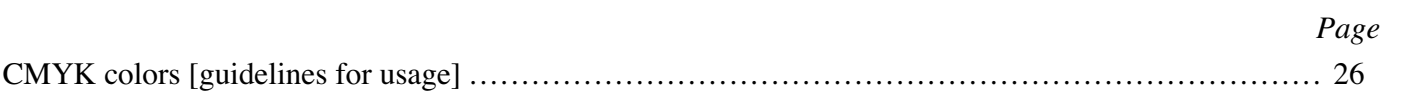 & $\begin{array}{l}\text { Ref. No. } \\
\mathrm{n} / \mathrm{a}\end{array}$ \\
\hline (2........................ A-10-1 & 10.2 .11 \\
\hline ...... A-37-2 & 658 \\
\hline Coal bed-Identity and existence certain, location accurate ..... & 1.2 .25 \\
\hline Coal bed-Identity and existence certain, location approximate & 1.2 .27 \\
\hline Coal bed-Identity and existence certain, location concealed ... & 1.2 .31 \\
\hline Coal bed-Identity and existence certain, location inferred... & 1.2 .29 \\
\hline Coal bed-Identity or existence questionable, location accurate . & 1.2 .26 \\
\hline Coal bed-Identity or existence questionable, location approximate & 1.2 .28 \\
\hline Coal bed-Identity or existence questionable, location concealed ... & 1.2 .32 \\
\hline Coal bed-Identity or existence questionable, location inferred... & 1.2 .30 \\
\hline Coal bed outcrop area & 1.2 .45 \\
\hline Coal bed, showing name.. & 1.4 .15 \\
\hline Coal bed, showing thickness and location where thickness was measured. & 1.4.11 \\
\hline Collapse structure (drawn to scale) & 23.10 \\
\hline Collapse structure indicating breccia pipe at depth . & 18.71 \\
\hline Collapse structure (too small to draw to scale) ...... & 23.9 \\
\hline Collapsed lava tube, planetary .................. & 25.110 \\
\hline Collapses (mapped to scale) along lava tube. & 18.45 \\
\hline Collection number of fossil .. & 10.1.1 \\
\hline Color Chart ....................... & $\mathrm{n} / \mathrm{a}$ \\
\hline Color conversion [during output]............. & $\mathrm{n} / \mathrm{a}$ \\
\hline 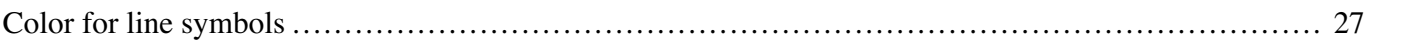 & $\mathrm{n} / \mathrm{a}$ \\
\hline Color for map-unit areas....................... & $\mathrm{n} / \mathrm{a}$ \\
\hline Color for point symbols & $\mathrm{n} / \mathrm{a}$ \\
\hline 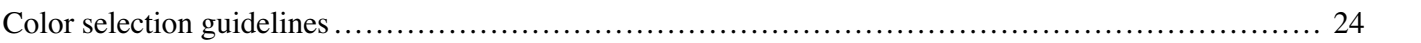 & $\mathrm{n} / \mathrm{a}$ \\
\hline Color specifications for line symbols [in this standard] ............... & $\mathrm{n} / \mathrm{a}$ \\
\hline Color specifications for map-unit areas [in this standard] . & $\mathrm{n} / \mathrm{a}$ \\
\hline Color specifications for point symbols [in this standard] .. & $\mathrm{n} / \mathrm{a}$ \\
\hline 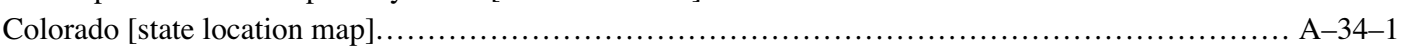 & Sec. 34.1 \\
\hline Complete weather station, equipped with a recorder ............ & 27.18 \\
\hline Complete weather station, equipped with a telephone or radio & 27.17 \\
\hline "Concealed" [concepts and definitions] $\ldots \ldots \ldots \ldots \ldots \ldots \ldots \ldots \ldots \ldots \ldots \ldots$ & $\mathrm{n} / \mathrm{a}$ \\
\hline Concentration of chemical constituent... & 26.6.11 \\
\hline Concentration of dissolved solids ............ & 26.6.11 \\
\hline Concentric fracture, planetary (associated with coronae). & 25.34 \\
\hline 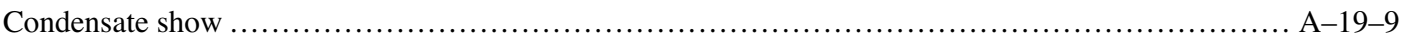 & 19.5.72 \\
\hline Condensate well (nonspecific depth) .. & 19.5.73 \\
\hline 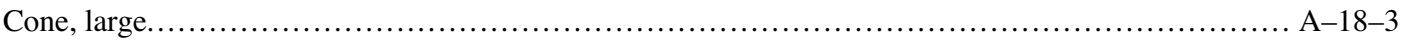 & 18.56 \\
\hline Cone, small ............... & 18.55 \\
\hline Confidence in a feature's existence [concepts and definitions]. & $\mathrm{n} / \mathrm{a}$ \\
\hline Confidence in a feature's identity [concepts and definitions]..... & $\mathrm{n} / \mathrm{a}$ \\
\hline Confidence in a feature's locatability [concepts and definitions] & $\mathrm{n} / \mathrm{a}$ \\
\hline Confidence in a feature's positioning [concepts and definitions] & $\mathrm{n} / \mathrm{a}$ \\
\hline Confining-bed thickness & 26.6.5 \\
\hline 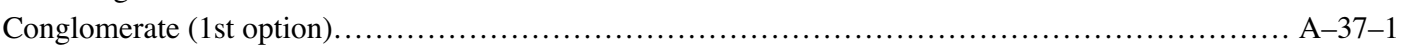 & 601 \\
\hline Conglomerate (2nd option)........ & 602 \\
\hline 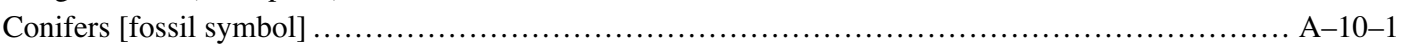 & 10.2 .36 \\
\hline 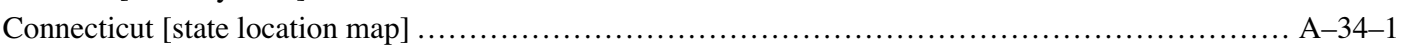 & Sec. 34.1 \\
\hline 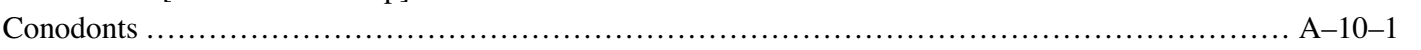 & 10.2 .46 \\
\hline 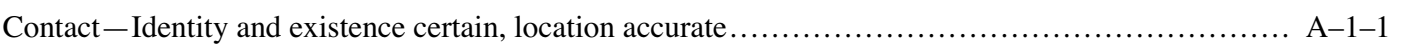 & 1.1 .1 \\
\hline 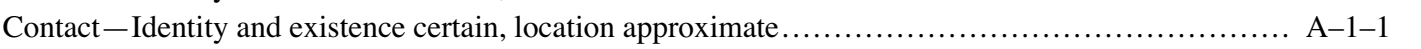 & 1.1 .3 \\
\hline 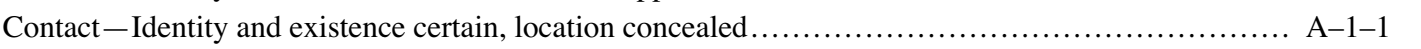 & 1.1 .7 \\
\hline Contact-Identity and existence certain, location inferred ... & 1.1.5 \\
\hline
\end{tabular}




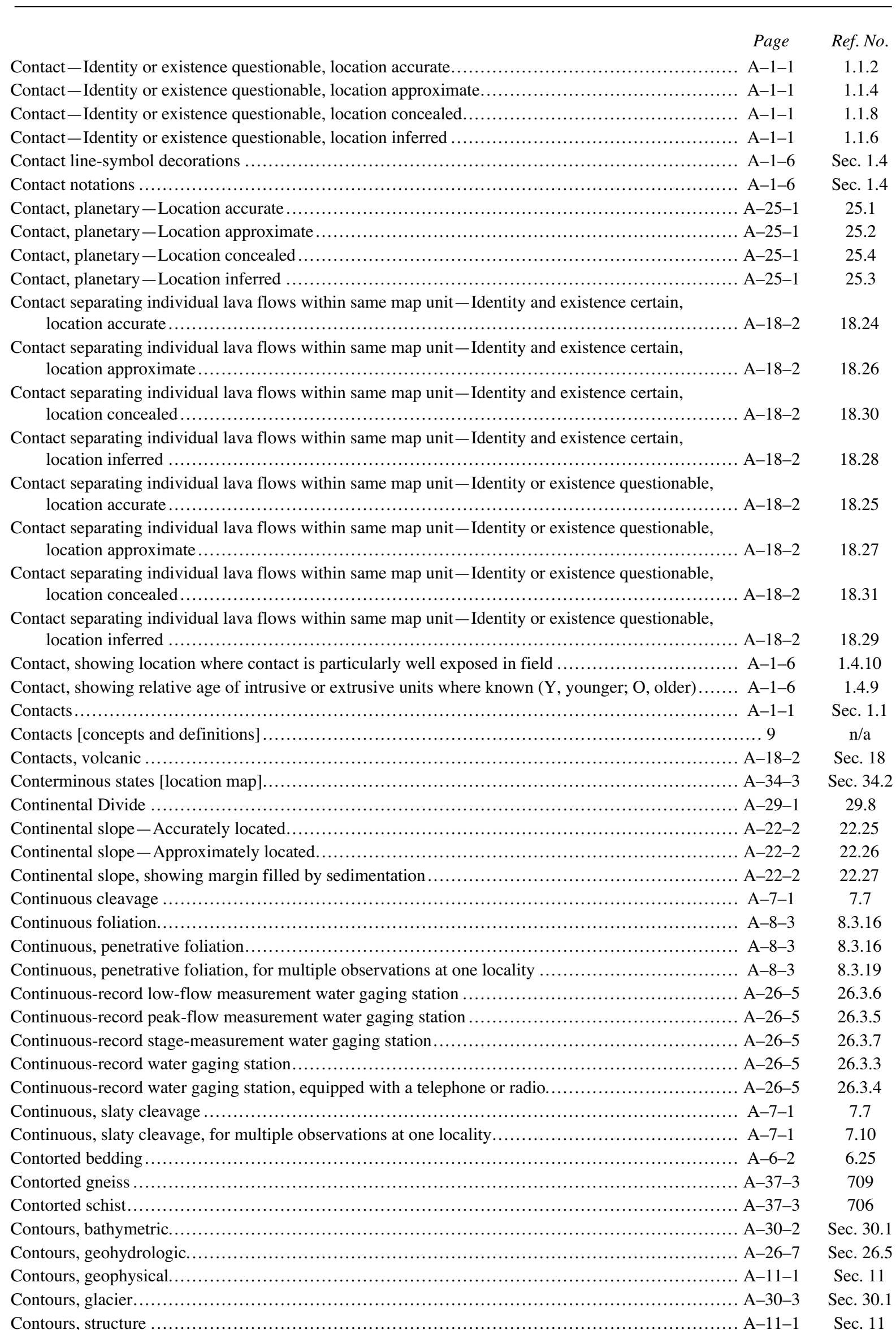




\begin{tabular}{|c|c|}
\hline ontours, topographic............................... & $\begin{array}{l}\text { Ref. No. } \\
\text { Sec. } 30.1\end{array}$ \\
\hline Contrast [influencing color and pattern selection] . & $\mathrm{n} / \mathrm{a}$ \\
\hline 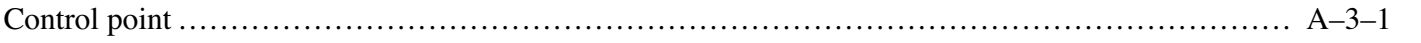 & 3.3 .4 \\
\hline Convergent plate boundaries.......... & Sec. 22 \\
\hline Conversion values from inches (in) to points (pts) to millimeters (mm)... & Table 1 \\
\hline 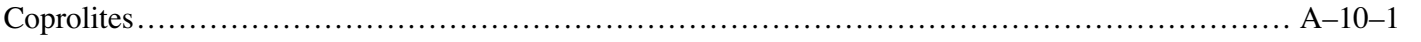 & 10.2 .43 \\
\hline Coral reef ..................... & 30.3 .18 \\
\hline 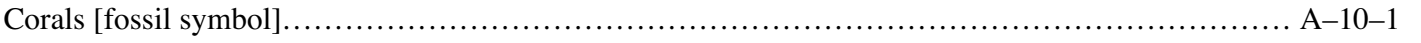 & 10.2.12 \\
\hline Core (nonspecific depth), & 19.5.7 \\
\hline 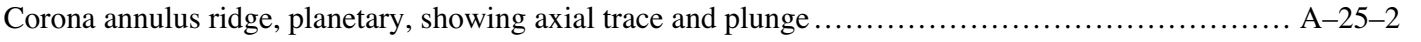 & 25.46 \\
\hline Corona, planetary & 25.91 \\
\hline 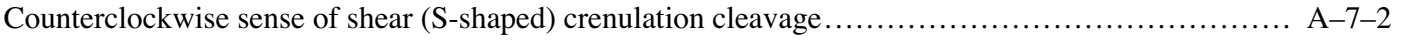 & 7.25 \\
\hline $\begin{array}{l}\text { Counterclockwise sense of shear (S-shaped) crenulation cleavage, for multiple observations at one } \\
\text { locality }\end{array}$ & 7.28 \\
\hline Counterclockwise sense of shear (S-shaped) crenulation foliation …..................................... A-8 & 8.3.34 \\
\hline $\begin{array}{l}\text { ounterclockwise sense of shear (S-shaped) crenulation foliation, for multiple observations at one } \\
\text { locality }\end{array}$ & 8.3.37 \\
\hline 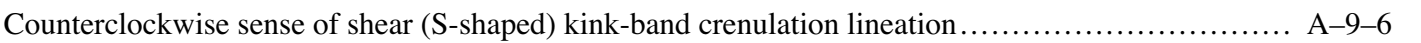 & 9.137 \\
\hline Counterclockwise sense of shear (S-shaped) minor fold hinge ................. & 9.125 \\
\hline 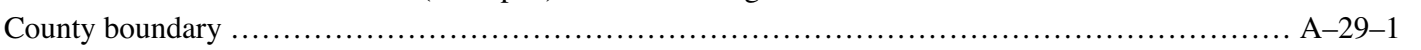 & 29.3 \\
\hline red water storage reservoir........... & 30.2 .40 \\
\hline Cracks formed in ground by earthquake. & 21.36 \\
\hline Cracks on landslides........................ & Sec. 17 \\
\hline Cracks on surface of lava flow............ & 18.40 \\
\hline Crater-associated ejecta halo, planetary . & 25.134 \\
\hline 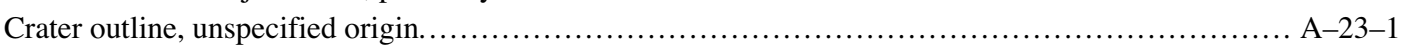 & 23.11 \\
\hline Crater, volcanic-Identity and existence certain, location accurate ..... & 18.1 \\
\hline Crater, volcanic-Identity and existence certain, location concealed ... & 18.5 \\
\hline Crater, volcanic-Identity or existence certain, location approximate.......... & 18.3 \\
\hline 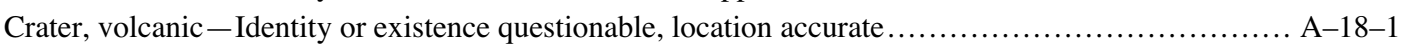 & 18.2 \\
\hline 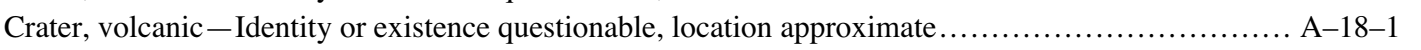 & 18.4 \\
\hline Crater, volcanic -Identity or existence questionable, location concealed............................. A-18-1 & 18.6 \\
\hline Crater with rim, formed by shock or sand blowouts - Identity and existence certain, location accurate.. A-21-2 & 21.24 \\
\hline rater with rim, formed by shock or sand blowouts - Identity and existence certain, location & 21.28 \\
\hline $\begin{array}{l}\text { Crater with rim, formed by shock or sand blowouts - Identity or existence certain, location } \\
\text { approximate }\end{array}$ & 21.26 \\
\hline rater with rim, formed by shock or sand blowouts - Identity or existence questionable, location & 21.25 \\
\hline 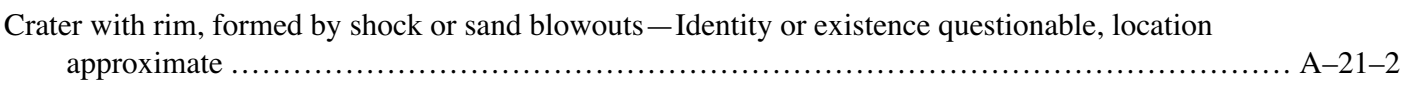 & 21.27 \\
\hline $\begin{array}{l}\text { Crater with rim, formed by shock or sand blowouts }- \text { Identity or existence questionable, location } \\
\text { concealed }\end{array}$ & 21.29 \\
\hline Crater without rim, formed by shock - Identity and existence certain, location accurate ............. & 21.30 \\
\hline Crater without rim, formed by shock - Identity or existence certain, location approximate .............. & 21.32 \\
\hline Crater without rim, formed by shock - Identity or existence certain, location concealed ............. & 21.34 \\
\hline Crater without rim, formed by shock - Identity or existence questionable, location accurate ....... & 21.31 \\
\hline Crater without rim, formed by shock - Identity or existence questionable, location approximate.. & 21.33 \\
\hline Crater without rim, formed by shock - Identity or existence questionable, location concealed... & 21.35 \\
\hline A-30-4 & Sec. 30.2 \\
\hline Crenulated bedding.. & 6.25 \\
\hline Crenulation cleavage. & 7.19 \\
\hline Crenulation cleavage, for mul & 7.22 \\
\hline A-8-4 & 8.3.28 \\
\hline Crenulation foli & 8.3 .31 \\
\hline
\end{tabular}




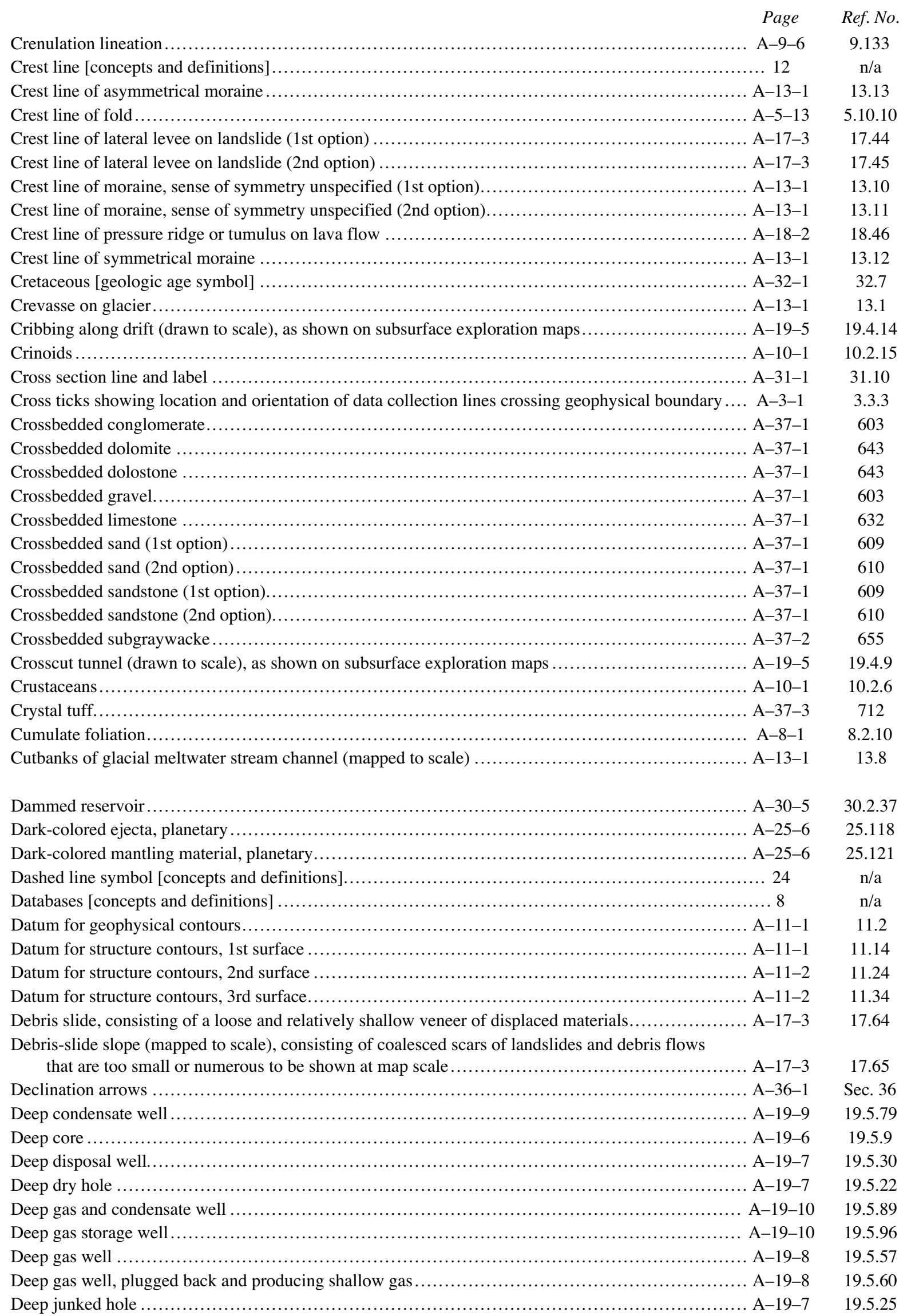




\begin{tabular}{|c|c|c|}
\hline & Page & Ref. No. \\
\hline Deep observation well for gas-storage field . & A-19-10 & 19.5.102 \\
\hline Deep oil and gas well .. & A-19-9 & 19.5.69 \\
\hline Deep oil well ............ & . A-19-8 & 19.5.46 \\
\hline Deep salt-water disposal well. & A-19-7 & 19.5.36 \\
\hline Deep-sea trench... & . A-22-2 & 22.30 \\
\hline Deep-seismofocal zones ........ & . A-22-1 & Sec. 22 \\
\hline Deformed-mineral lineation.............................. & . A-9-2 & 9.45 \\
\hline Degraded impact crater rim, planetary (1st option) & . A-25-5 & 25.97 \\
\hline Degraded impact crater rim, planetary (2nd option) & . A-25-5 & 25.98 \\
\hline Delaware $[$ state location map $]$ & . A-34-1 & Sec. 34.1 \\
\hline 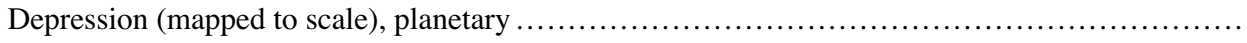 & . A-25-3 & 25.53 \\
\hline 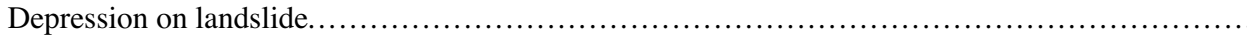 & . A-17-2 & 17.43 \\
\hline Depth to bedrock............ & . A-26-8 & 26.6 .3 \\
\hline Depth to formation .............. & . A-26-8 & 26.6 .3 \\
\hline Depth to geologic formation & . A-26-8 & 26.6 .3 \\
\hline Depth to water. & . A-26-8 & 26.6 .3 \\
\hline Destroyed adit (1st option) & . A-19-3 & 19.3.11 \\
\hline Destroyed adit (2nd option). & . A-19-3 & 19.3.16 \\
\hline Destroyed portal ............... & . A-19-3 & 19.3.21 \\
\hline 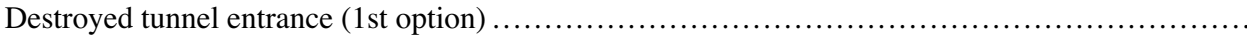 & . A-19-3 & 19.3.11 \\
\hline Destroyed tunnel entrance (2nd option). & . A-19-3 & 19.3.16 \\
\hline 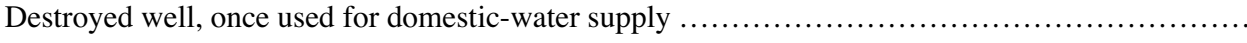 & . A-26-1 & 26.1.14 \\
\hline 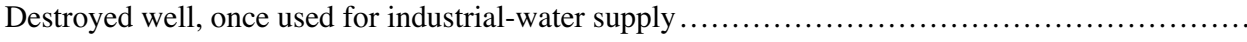 & . A-26-2 & 26.1 .41 \\
\hline 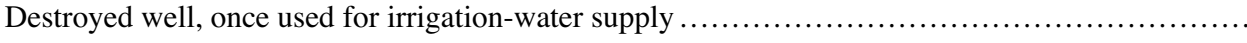 & . A-26-2 & 26.1.32 \\
\hline Destroyed well, once used for public-water supply..... & A-26-3 & 26.1 .50 \\
\hline Destroyed well, once used for stock-water supply. & A-26-1 & 26.1.23 \\
\hline Detached lobe, planetary ...... & A-25-6 & 25.126 \\
\hline 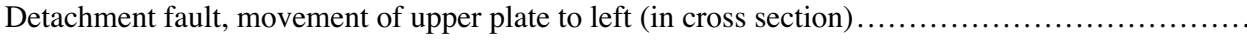 & A-2-11 & 2.11 .18 \\
\hline 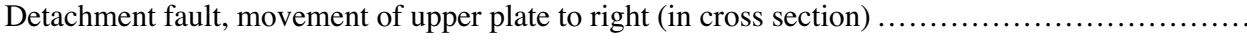 & A-2-11 & 2.11 .19 \\
\hline Detachment fault (sense of slip unspecified) (1st option) - Identity and existence certain, location & A-2-9 & 2.10 .1 \\
\hline Detachment fault (sense of slip unspecified) (1st option) - Identity and existence certain, location & A-2-9 & 2.10 .3 \\
\hline $\begin{array}{l}\text { Detachment fault (sense of slip unspecified) (1st option) - Identity and existence certain, location } \\
\text { concealed }\end{array}$ & A-2-9 & 2.10 .7 \\
\hline 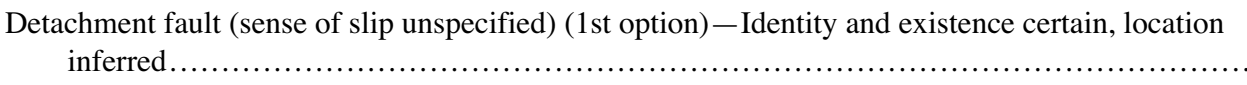 & A-2-9 & 2.10 .5 \\
\hline $\begin{array}{l}\text { Detachment fault (sense of slip unspecified) (1st option) - Identity or existence questionable } \\
\text { location accurate }\end{array}$ & A-2-9 & 2.10 .2 \\
\hline $\begin{array}{l}\text { Detachment fault (sense of slip unspecified) (1st option) - Identity or existence questionable, } \\
\text { location approximate }\end{array}$ & A $-2-9$ & 2.10 .4 \\
\hline $\begin{array}{l}\text { Detachment fault (sense of slip unspecified) (1st option) - Identity or existence questionable, } \\
\text { location concealed }\end{array}$ & A-2-9 & 2.10 .8 \\
\hline $\begin{array}{l}\text { Detachment fault (sense of slip unspecified) (1st option) - Identity or existence questionable, } \\
\text { location inferred }\end{array}$ & A-2-9 & 2.10 .6 \\
\hline Detachment fault (sense of slip unspecified) (2nd option) - Identity and existence certain, location & A-2-9 & 2.10 .9 \\
\hline $\begin{array}{l}\text { Detachment fault (sense of slip unspecified) (2nd option) - Identity and existence certain, location } \\
\text { approximate }\end{array}$ & A-2-9 & 2.10 .11 \\
\hline Detachment fault (sense of slip unspecified) (2nd option) - Identity and existence certain, location & A-2-9 & 2.10 .15 \\
\hline $\begin{array}{l}\text { Detachment fault (sense of slip unspecified) (2nd option) - Identity and existence certain, location } \\
\text { inferred }\end{array}$ & A-2-9 & 2.10 .13 \\
\hline $\begin{array}{l}\text { achment fault (sense of slip unspecified) (2nd option) - Identity or existence questionable, } \\
\text { location accurate }\end{array}$ & A-2-9 & 2.10 .10 \\
\hline
\end{tabular}


Detachment fault (sense of slip unspecified) (2nd option) - Identity or existence questionable, location approximate

Detachment fault (sense of slip unspecified) (2nd option) - Identity or existence questionable, location concealed

Detachment fault (sense of location inferred

Detachment fault (sense of slip unspecified) (3rd option) - Identity and existence certain, location accurate

Detachment fault (sense of slip unspecified) (3rd option) - Identity and existence certain, location approximate

Detachment fault (sense of slip unspecified) (3rd option) - Identity and existence certain, location concealed

Detachment fault (sense of slip unspecified) (3rd option) - Identity and existence certain, location inferred

Detachment fault (sense of slip unspecified) (3rd option) - Identity or existence questionable, location accurate...

Detachment fault (sense of slip unspecified) (3rd option) - Identity or existence questionable,

Detachment fault (sense of slip unspecified) (3rd option) - Identity or existence questionable, location concealed....

Detachment fault (sense of slip unspecified) (3rd option) - Identity or existence questionable, location inferred

Detachment faults (sense of slip unspecified)

Determining appropriate line symbol styles and associated terminology

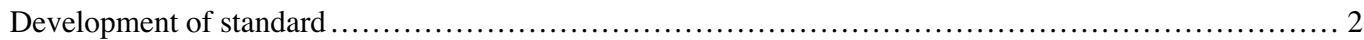

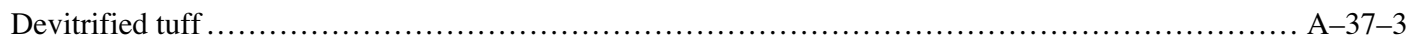

Fig. 2

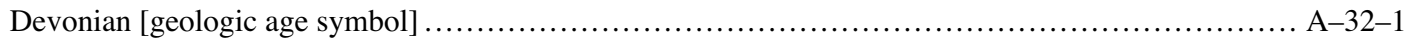

Diagram showing relation of new FGDC standard terminology to historically used terminology and

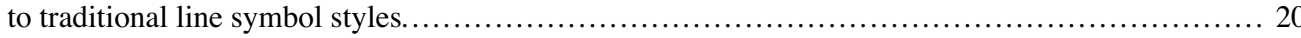

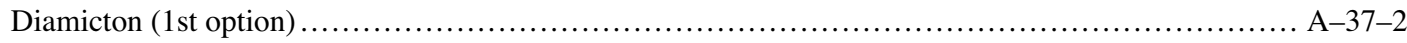

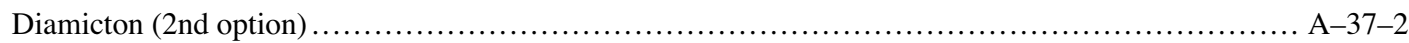

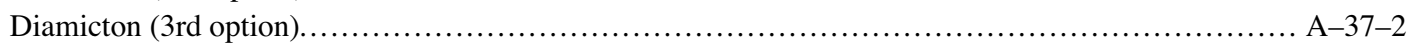

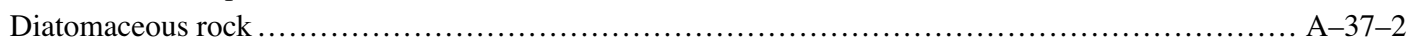

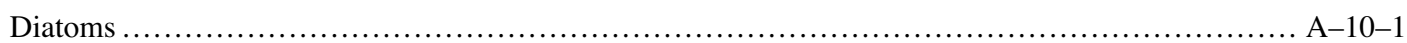

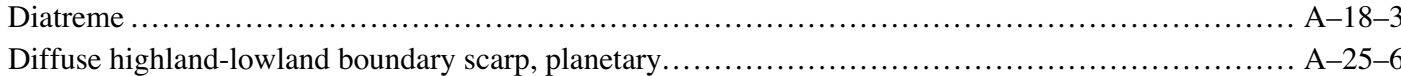

Fig. 1

Dike (1st option) - Identity and existence certain, location accurate ............................ A-1-5

Dike (1st option) - Identity and existence certain, location approximate........................ A-1-5

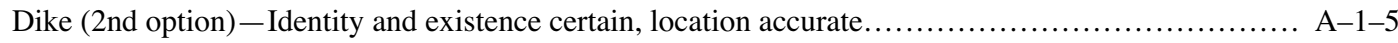

Dike (2nd option) - Identity and existence certain, location approximate....................... A-1-5

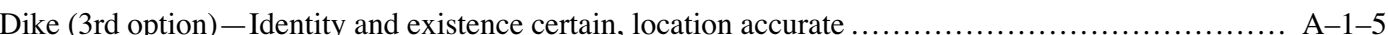

1.3 .4

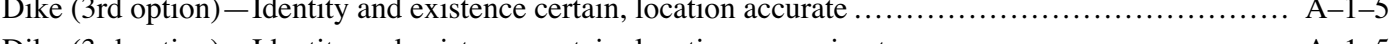

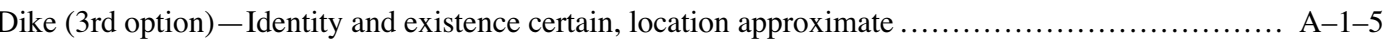

Dike (4th option) - Identity and existence certain, location accurate ........................... A-1-5

Dike (4th option) - Identity and existence certain, location approximate ......................... A-1-5

Dike (5th option) - Identity and existence certain, location accurate ........................... A-1-5

Dike (5th option) - Identity and existence certain, location approximate ........................... A-1-5

Dike (6th option) - Identity and existence certain, location accurate ............................ A-1-5

1.3 .11

Dike (6th option) - Identity and existence certain, location approximate ....................... A-1-5

1.3 .12

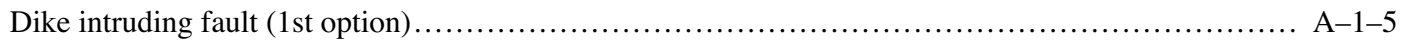

1.3 .14

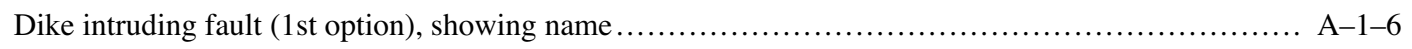

1.4 .20

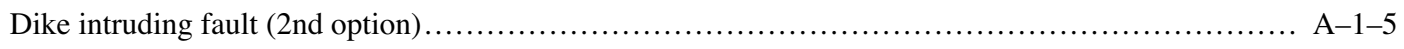

1.3 .15

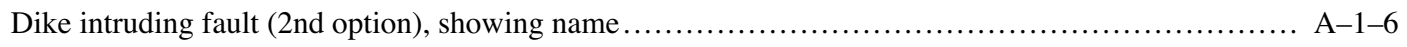

1.4 .21

Dike line-symbol decorations ......................................................... A-1-6

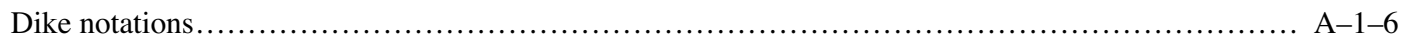

Sec. 1.4

Dike of variable thickness . 


\begin{tabular}{|c|c|c|}
\hline & Page & Ref. No. \\
\hline Dike of variable thickness, showing name. & A-1-6 & 1.4 .19 \\
\hline 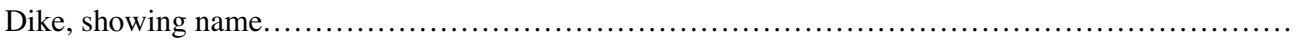 & A-1-6 & 1.4 .18 \\
\hline Dike, showing thickness and location where thickness was measured. & A-1-6 & 1.4 .11 \\
\hline Dikes & $\mathrm{A}-1-5$ & Sec. 1.3 \\
\hline Dinoflagellates. & A-10-1 & 10.2 .57 \\
\hline Dip [concepts and definitions]. & $\ldots 13$ & $\mathrm{n} / \mathrm{a}$ \\
\hline Direction of downslope movement of landslide. & A-17-1 & 17.10 \\
\hline Direction of flow of glacial meltwater in stream channel. & A-13-1 & 13.9 \\
\hline Direction of fluvial transport............................ & A-12-1 & 12.5 \\
\hline Direction of ground-water flow (1st option)-Accurately located . & A-26-9 & 26.7 .8 \\
\hline Direction of ground-water flow (1st option)-Approximately located & A-26-9 & 26.7 .9 \\
\hline Direction of ground-water flow (2nd option) - Accurately located ....... & A-26-9 & 26.7.10 \\
\hline Direction of ground-water flow (2nd option)-Approximately located . & A-26-9 & 26.7 .11 \\
\hline 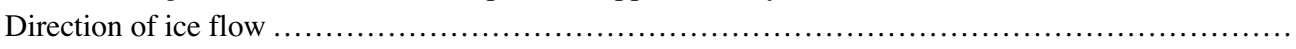 & A-13-1 & 13.2 \\
\hline 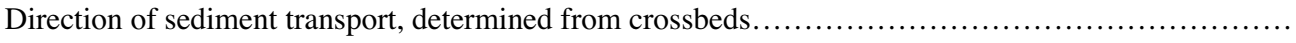 & A-12-1 & 12.7 \\
\hline Direction of sediment transport, determined from dune bedding in horizontal section ................. & A-16-1 & 16.8 \\
\hline 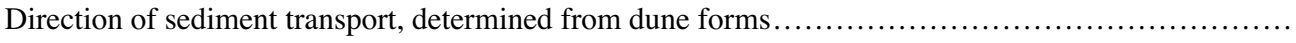 & A-16-1 & 16.7 \\
\hline $\begin{array}{l}\text { Direction of sediment transport, determined from eolian crossbedding in vertical or near-vertical } \\
\text { section }\end{array}$ & A-16-1 & 16.9 \\
\hline 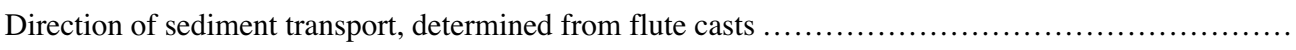 & A-12-1 & 12.8 \\
\hline Direction of sediment transport, determined from imbrication... & A-12-1 & 12.6 \\
\hline 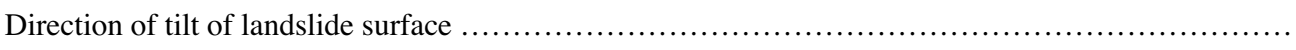 & A-17-3 & 17.52 \\
\hline 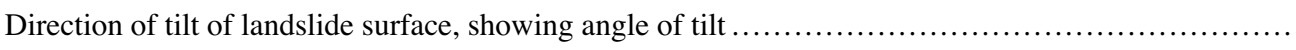 & A-17-3 & 17.53 \\
\hline Discontinued water gaging station. & A-26-5 & 26.3.2 \\
\hline Discontinued weather station ............... & A-27-1 & 27.2 \\
\hline Discrete faults [concepts and definitions] ........... & ... 11 & $\mathrm{n} / \mathrm{a}$ \\
\hline 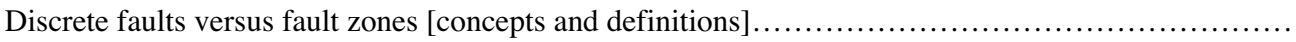 & $\ldots 11$ & $\mathrm{n} / \mathrm{a}$ \\
\hline Disjunctive, asymmetric (S-shaped, counterclockwise sense of shear) crenulation cleavage $\ldots \ldots \ldots \ldots \ldots$ & A-7-2 & 7.25 \\
\hline $\begin{array}{l}\text { Disjunctive, asymmetric (S-shaped, counterclockwise sense of shear) crenulation cleavage, for } \\
\text { multiple observations at one locality...................... }\end{array}$ & A-7-2 & 7.28 \\
\hline Disjunctive, asymmetric (S-shaped, counterclockwise sense of shear) crenulation foliation ............. & $\mathrm{A}-8-4$ & 8.3.34 \\
\hline 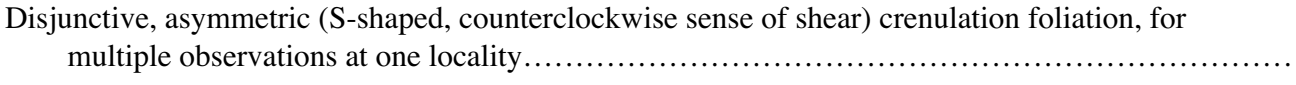 & A-8-4 & 8.3.37 \\
\hline Disjunctive, asymmetric (Z-shaped, clockwise sense of shear) crenulation cleavage................ & A-7-2 & 7.31 \\
\hline 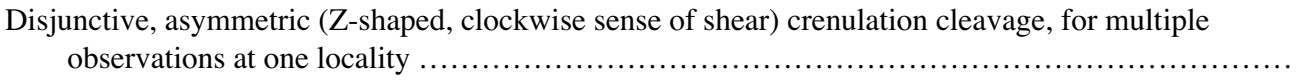 & A-7-2 & 7.34 \\
\hline Disjunctive, asymmetric (Z-shaped, clockwise sense of shear) crenulation foliation................. & A-8-4 & 8.3 .40 \\
\hline $\begin{array}{l}\text { Disjunctive, asymmetric (Z-shaped, clockwise sense of shear) crenulation foliation, for multiple } \\
\text { observations at one locality }\end{array}$ & A-8-4 & 8.3 .43 \\
\hline Disjunctive cleavage ............. & A-7-1 & 7.13 \\
\hline Disjunctive foliation... & A-8-4 & 8.3.22 \\
\hline Disjunctive, spaced cleavage.. & A-7-1 & 7.13 \\
\hline Disjunctive, spaced cleavage, for multiple observations at one locality & A-7-1 & 7.16 \\
\hline 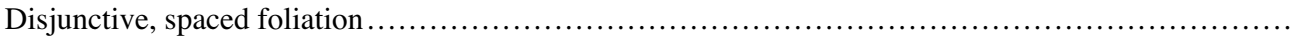 & A-8-4 & 8.3.22 \\
\hline Disjunctive, spaced foliation, for multiple observations at one locality.. & A-8-4 & 8.3.25 \\
\hline Disjunctive, symmetric crenulation cleavage ............ & A-7-1 & 7.19 \\
\hline Disjunctive, symmetric crenulation cleavage, for multiple observations at one locality . & A-7-1 & 7.22 \\
\hline Disjunctive, symmetric crenulation foliation . & A-8-4 & 8.3.28 \\
\hline Disjunctive, symmetric crenulation foliation, for multiple observations at one locality.. & A-8-4 & 8.3 .31 \\
\hline Displacement of fault during historic time (includes areas of known fault creep)..... & A-2-16 & 2.13 .1 \\
\hline Displacement of fault during Holocene time ..... & A-2-16 & 2.13.2 \\
\hline Displacement of fault during late Quaternary time ... & A-2-16 & 2.13 .3 \\
\hline Displacement of fault during Quaternary time (undifferentiated)... & A-2-16 & 2.13 .4 \\
\hline 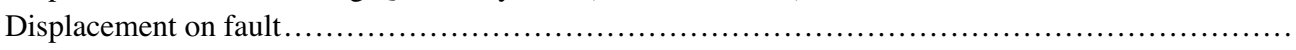 & A-2-11 & 2.11 .14 \\
\hline Displacement vector, showing bearing & A-17-3 & 17.54 \\
\hline
\end{tabular}




\begin{tabular}{|c|c|}
\hline Page & Ref. No. \\
\hline Displacement vector, showing bearing and distance....... & 17.55 \\
\hline Disposal well (nonspecific depth) ...................... & 19.5.26 \\
\hline Dissolved-solids concentration ............. & 26.6 .11 \\
\hline Distance specifications [in this standard]...... & $\mathrm{n} / \mathrm{a}$ \\
\hline District boundary ............................ & 29.4 \\
\hline District of Columbia [location map] ... & Sec. 34.1 \\
\hline Disturbed ground ...................... & Sec. 19.2 \\
\hline Ditch (double-line drainage) ..... & 30.2 .8 \\
\hline Ditch (single-line drainage) ... & 30.2 .7 \\
\hline DO [abbreviation] $\ldots \ldots \ldots \ldots$ & Table 2 \\
\hline Dolomite ............... & 642 \\
\hline Dolomitic limestone..... & 641 \\
\hline Dolomitic sandstone..... & 614 \\
\hline Dolomitic shale ............. & 622 \\
\hline Dolomitic siltstone ............... & 618 \\
\hline Dolostone ............................ & 642 \\
\hline Dome, planetary (mapped to scale) ... & 25.75 \\
\hline Dome (small, minor) ................. & 5.11 .2 \\
\hline 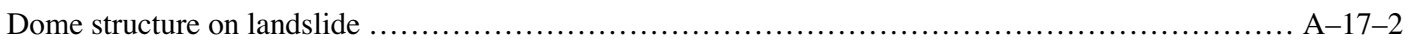 & 17.41 \\
\hline Dormant-mature landslide (mapped to scale), showing a smoothed and eroded morphology ............ A-17-3 & 17.60 \\
\hline Dormant-old landslide (mapped to scale), showing a weak morphology ........................... A-17-3 & 17.61 \\
\hline 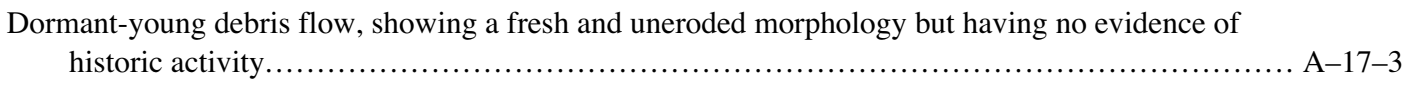 & 17.57 \\
\hline $\begin{array}{l}\text { Dormant-young landslide (mapped to scale), showing a fresh and uneroded morphology but having } \\
\text { no evidence of historic activity } \ldots \ldots \ldots \ldots \ldots \ldots \ldots \ldots \ldots \ldots \ldots \ldots \ldots \ldots \ldots \ldots \ldots \ldots \ldots \ldots \ldots \ldots \ldots \ldots \ldots \ldots \ldots \ldots \ldots \ldots \ldots \ldots \ldots \\
\text { A }\end{array}$ & 17.59 \\
\hline 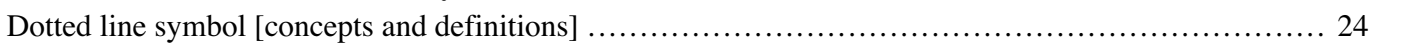 & $\mathrm{n} / \mathrm{a}$ \\
\hline Doubly plunging anticline......................... & 5.10 .6 \\
\hline Doubly plunging syncline $\ldots \ldots \ldots \ldots \ldots \ldots \ldots \ldots \ldots \ldots$ & 5.10 .8 \\
\hline Drainage divide ................ & 26.7.1 \\
\hline 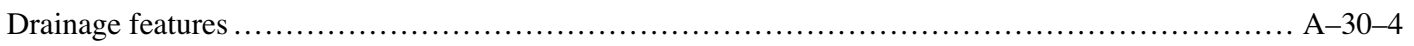 & Sec. 30.2 \\
\hline 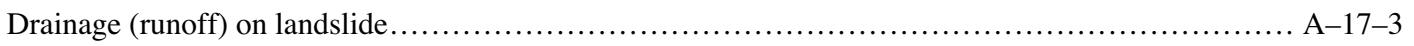 & 17.48 \\
\hline 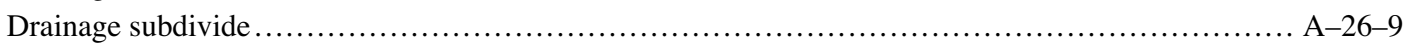 & 26.7 .2 \\
\hline Drill hole for exploration of high-grade ore....................................... & 19.3 .30 \\
\hline Drill hole for exploration of low-grade ore $\ldots \ldots \ldots \ldots \ldots \ldots \ldots \ldots \ldots \ldots \ldots \ldots \ldots \ldots \ldots \ldots \ldots \ldots \ldots \ldots \ldots \ldots \ldots \ldots \ldots \ldots \ldots, 19-4$ & 19.3.29 \\
\hline 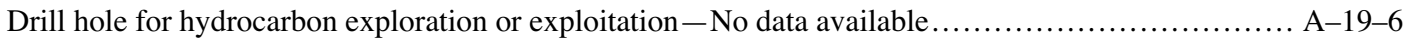 & 19.5 .11 \\
\hline Drill hole for hydrocarbon exploration or exploitation, showing name and number................... A-19-6 & 19.5 .12 \\
\hline Drill hole for hydrocarbon exploration or exploitation, showing total depth.......................... A-19-6 & 19.5 .13 \\
\hline 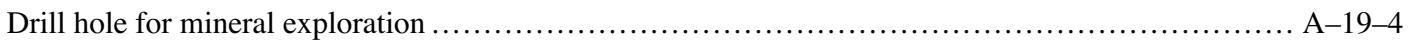 & 19.3 .25 \\
\hline 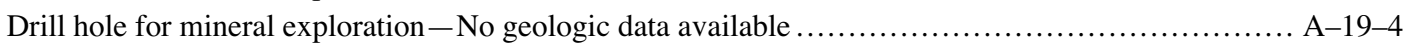 & 19.3.26 \\
\hline Drill hole for mineral exploration, showing name and number ............... & 19.3.27 \\
\hline Drill hole for mineral exploration, showing type.................................................. A-19-4 & 19.3.28 \\
\hline Drilling well for hydrocarbon exploration or exploitation $\ldots \ldots \ldots \ldots \ldots \ldots \ldots \ldots \ldots$ & 19.5.10 \\
\hline 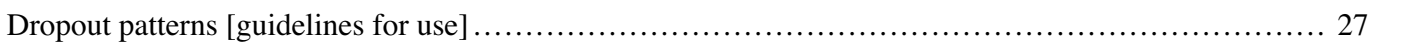 & $\mathrm{n} / \mathrm{a}$ \\
\hline 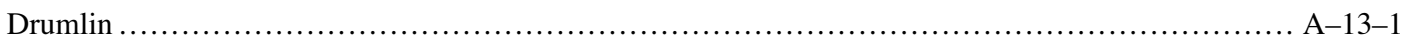 & 13.20 \\
\hline 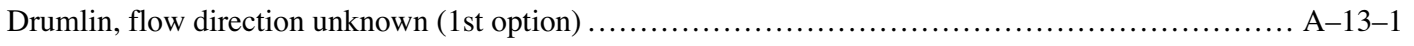 & 13.21 \\
\hline 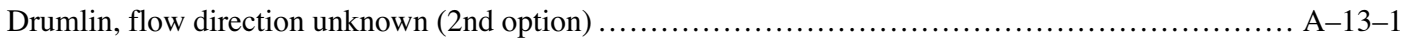 & 13.22 \\
\hline 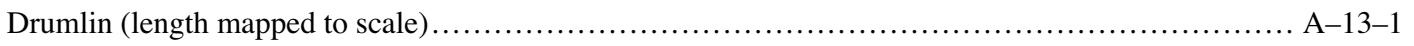 & 13.23 \\
\hline Drumlin (length mapped to scale), flow direction unknown.................................... A-13-1 & 13.24 \\
\hline 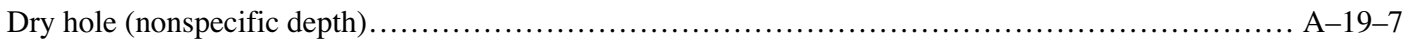 & 19.5.19 \\
\hline $\begin{array}{l}\text { Dry hole, showing map unit at surface and at bottom of hole, and showing altitude at surface and } \\
\text { total depth of hole }\end{array}$ & 19.5 .20 \\
\hline 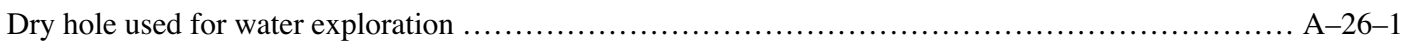 & 26.1 .5 \\
\hline 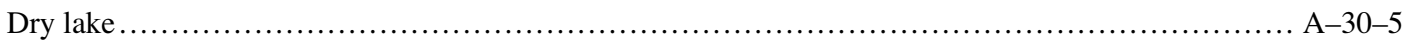 & 30.2 .34 \\
\hline Dry lakebed within closed depression of eolian origin in bedrock $\ldots \ldots \ldots \ldots \ldots \ldots \ldots \ldots$ & 16.6 \\
\hline 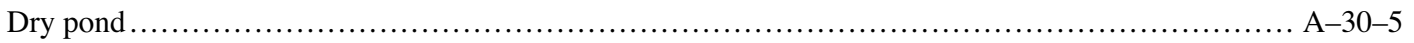 & 30.2 .34 \\
\hline
\end{tabular}




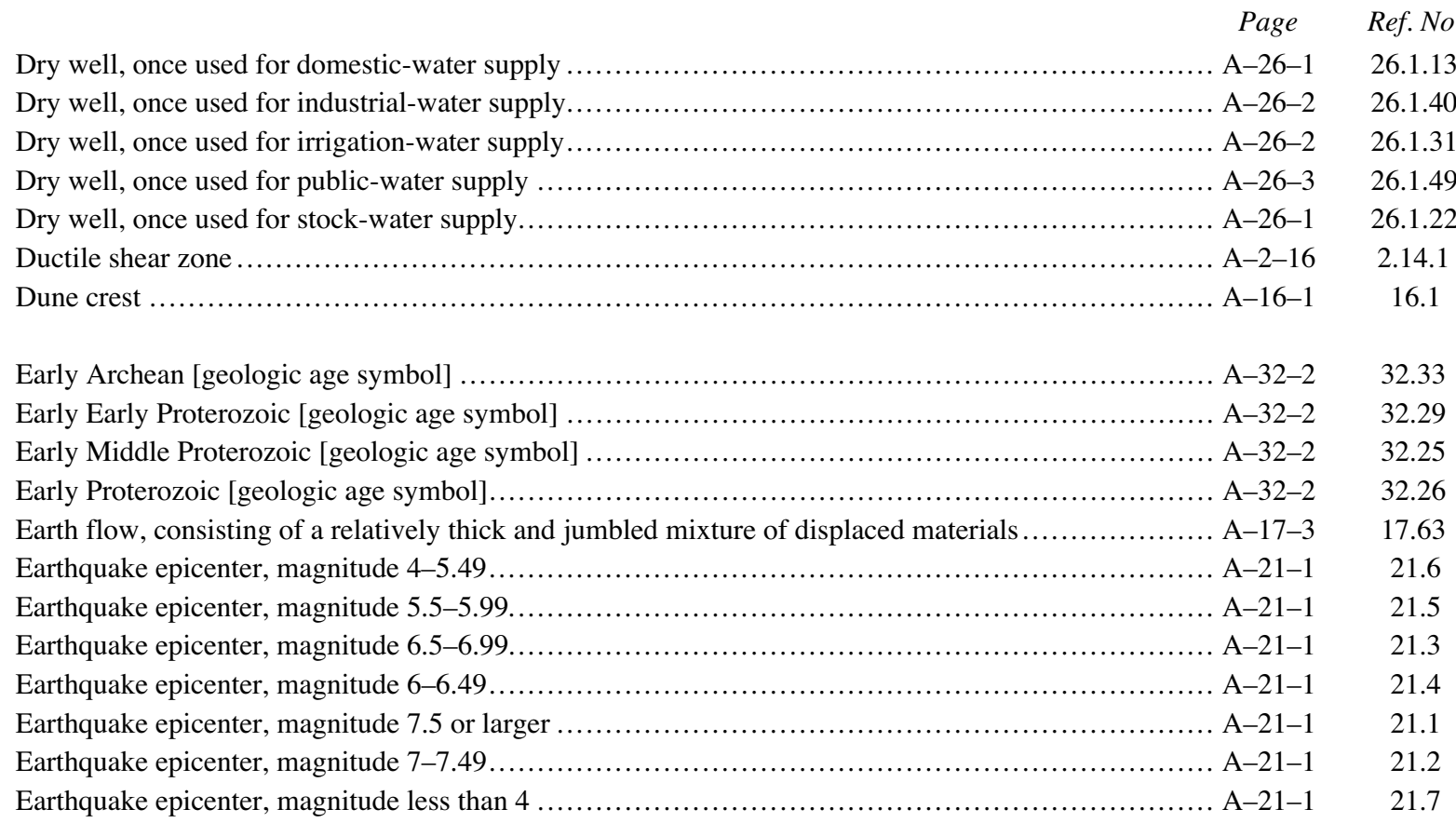

Earthquake-hazard features ...................................................................... A 21

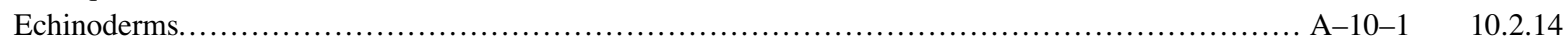

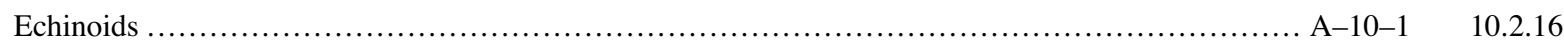

Edge of dry lakebed within closed depression of eolian origin in bedrock.......................... A-16-1 16.6

Edifice, planetary (mapped to scale) ............................................................ A-25-4 25.75

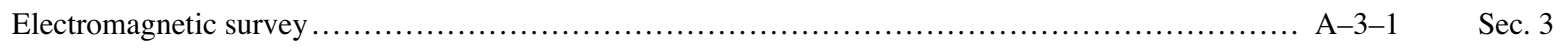

Elevated plateau, planetary (mapped to scale)....................................................... A-25-4 25.84

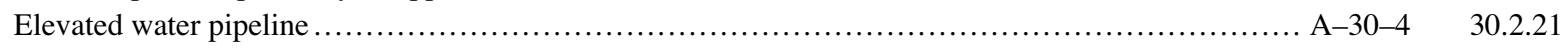

Elevation of back, as shown on subsurface exploration maps..................................... A-19-5 19.4.15

Elevation of floor, as shown on subsurface exploration maps..................................... A-19-5 19.4.16

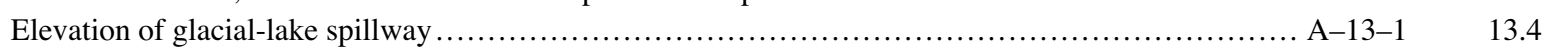

Elevation of roof, as shown on subsurface exploration maps .................................... A-19-5 19.4 .15

Elevation of sill, as shown on subsurface exploration maps........................................ A-19-5 19.4.16

En echelon cracks on landslide, indicating left-lateral shear .................................... A-17-2 17.39

En echelon cracks on landslide, indicating right-lateral shear...................................... A-17-2 17.38

En echelon fractures on landslide, indicating left-lateral shear..................................... A-17-2 17.39

En echelon fractures on landslide, indicating right-lateral shear.................................. A-17-2 17.38

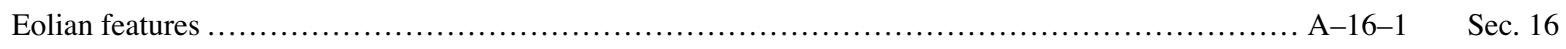

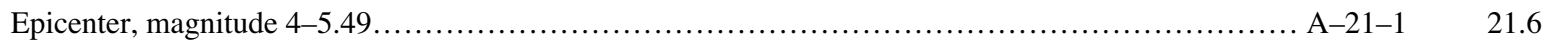

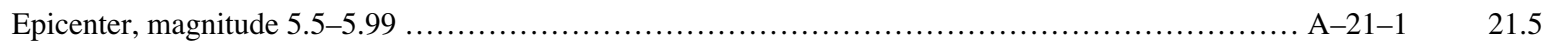

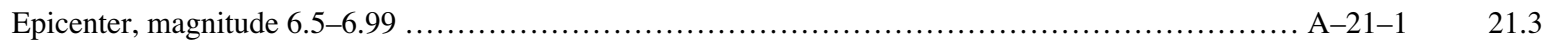

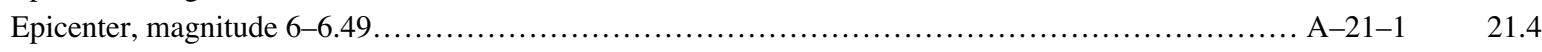

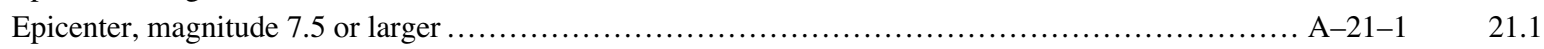

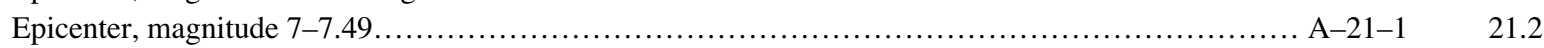

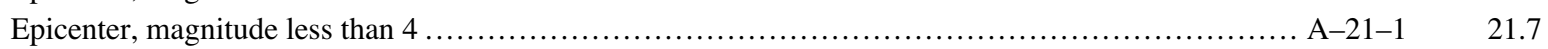

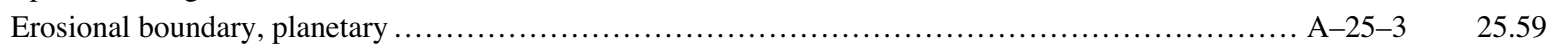

Erosional shoreline - Identity and existence certain, location accurate ............................ A-15-1 15.9

Erosional shoreline - Identity and existence certain, location approximate ........................... A-15-1 15.11

Erosional shoreline - Identity or existence questionable, location accurate ......................... A-15-1 15.10

Erosional shoreline -Identity or existence questionable, location approximate....................... A-15-1 15.12

Esker, transport direction known (1st option) ........................................................ A-1 13.18

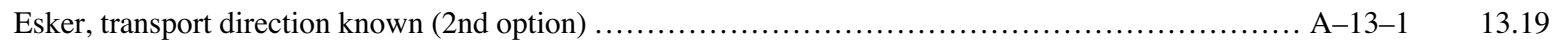

Esker, transport direction unknown ................................................................ A-13-1 13.17

Estimated elevation of inferred glacial-lake spillway .............................................. A-13-1 13.6 


\begin{tabular}{|c|c|}
\hline Page & Ref. No. \\
\hline 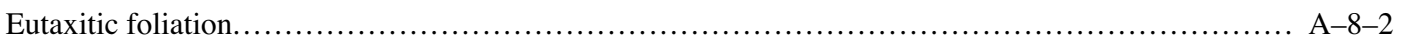 & 8.2 .19 \\
\hline Eutaxitic foliation, for multiple observations at one locality $\ldots \ldots \ldots \ldots \ldots \ldots \ldots \ldots \ldots \ldots \ldots \ldots \ldots \ldots \ldots \ldots \ldots \ldots \ldots \ldots \ldots \ldots$ & 8.2 .22 \\
\hline 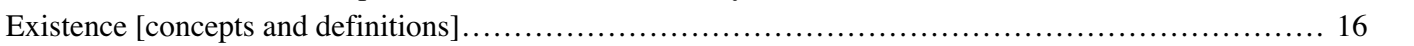 & $\mathrm{n} / \mathrm{a}$ \\
\hline "Existence certain" [concepts and definitions] ..................... & $\mathrm{n} / \mathrm{a}$ \\
\hline "Existence questionable" [concepts and definitions] .. & $\mathrm{n} / \mathrm{a}$ \\
\hline Explanation for CMYK Color Chart.................. & Sec. 39 \\
\hline Explanation for Pattern Chart ...................... & Sec. 38 \\
\hline Exposed wreck ................................... & 30.3 .17 \\
\hline 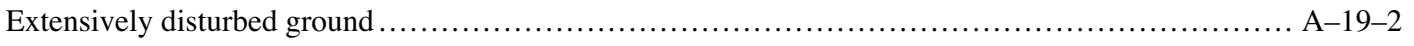 & Sec. 19.2 \\
\hline Extinct spring, once used for domestic-water supply............ & 26.2.7 \\
\hline Extinct spring, once used for industrial-water supply .............. & 26.2.19 \\
\hline Extinct spring, once used for irrigation-water supply ............. & 26.2 .15 \\
\hline Extinct spring, once used for public-water supply ................ & 26.2 .23 \\
\hline Extinct spring, once used for stock-water supply ............... & 26.2.11 \\
\hline 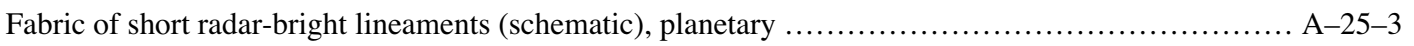 & 25.65 \\
\hline 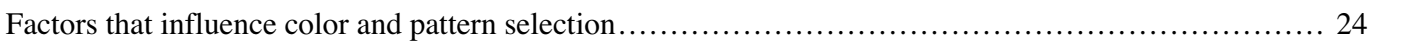 & $\mathrm{n} / \mathrm{a}$ \\
\hline Falls (double-line drainage) ................................... & 30.2 .26 \\
\hline Falls (single-line drainage) ................................ & 30.2 .25 \\
\hline Fault-breccia zone around fault............. & 2.14 .4 \\
\hline Fault-breccia zone within fault ........................... & 2.14 .3 \\
\hline 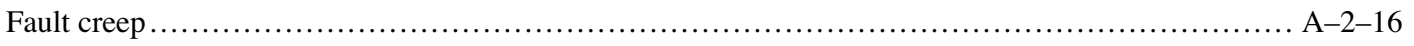 & 2.13 .1 \\
\hline Fault - Identity and existence certain, location accurate $\ldots \ldots \ldots \ldots \ldots \ldots \ldots \ldots \ldots \ldots \ldots \ldots \ldots \ldots \ldots \ldots \ldots \ldots \ldots \ldots \ldots \ldots \ldots$ & 2.1 .1 \\
\hline 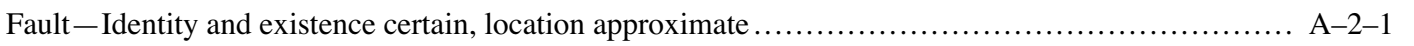 & 2.1 .3 \\
\hline Fault - Identity and existence certain, location concealed ............................ & 2.1 .7 \\
\hline Fault-Identity and existence certain, location inferred......... & 2.1 .5 \\
\hline Fault -Identity or existence questionable, location accurate............... & 2.1.2 \\
\hline 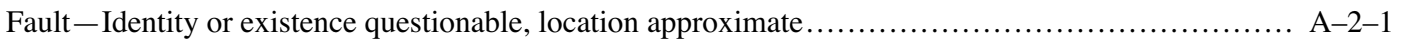 & 2.1 .4 \\
\hline Fault - Identity or existence questionable, location concealed $\ldots \ldots \ldots \ldots \ldots \ldots \ldots \ldots \ldots \ldots \ldots \ldots \ldots \ldots \ldots \ldots \ldots \ldots \ldots \ldots$ & 2.1.8 \\
\hline Fault - Identity or existence questionable, location inferred $\ldots \ldots \ldots \ldots \ldots \ldots \ldots \ldots \ldots \ldots \ldots \ldots \ldots \ldots \ldots \ldots \ldots \ldots \ldots \ldots \ldots \ldots \ldots \ldots$ & 2.1 .6 \\
\hline Fault line-symbol decorations................................ & Sec. 2.11 \\
\hline Fault located by aeromagnetic survey .......................... & 3.2 .1 \\
\hline Fault located by electromagnetic survey $\ldots \ldots \ldots \ldots \ldots \ldots \ldots \ldots . \ldots \ldots \ldots$ & 3.2 .7 \\
\hline Fault located by gravity survey ..................... & 3.2 .3 \\
\hline Fault located by ground magnetic survey $\ldots \ldots \ldots \ldots \ldots \ldots \ldots \ldots \ldots$ & 3.2 .2 \\
\hline 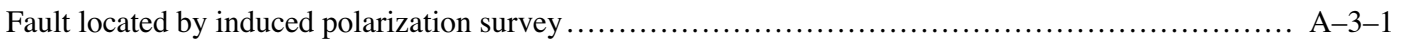 & 3.2 .6 \\
\hline 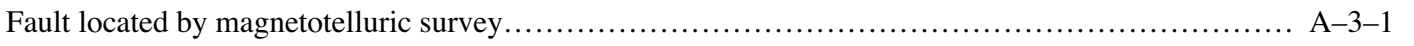 & 3.2 .9 \\
\hline Fault located by radiometric survey................................ & 3.2 .4 \\
\hline Fault located by resistivity survey........................... & 3.2 .8 \\
\hline Fault located by seismic reflection survey ...................... & 3.2 .5 \\
\hline 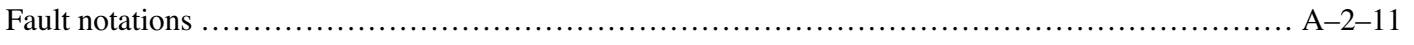 & Sec. 2.11 \\
\hline Fault-plane diagram for left-lateral strike-slip offset along north-striking, vertical fault.............. A-21-1 & 21.10 \\
\hline 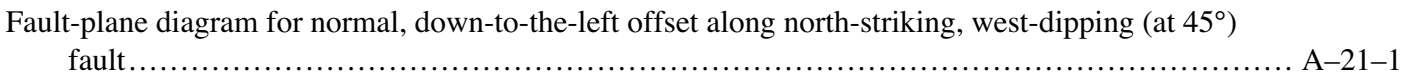 & 21.11 \\
\hline 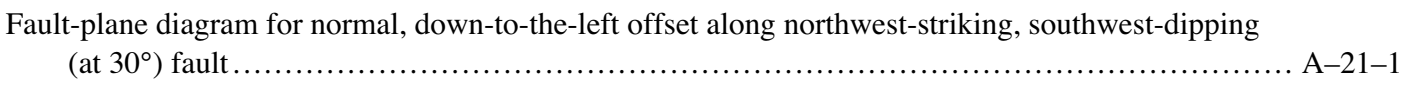 & 21.12 \\
\hline 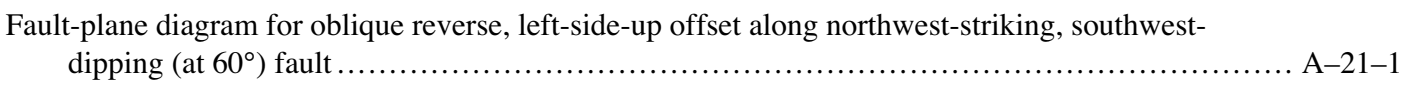 & 21.15 \\
\hline Fault-plane diagram for reverse, left-side-up offset along north-striking, west-dipping (at $45^{\circ}$ ) fault.... A-21-1 & 21.13 \\
\hline 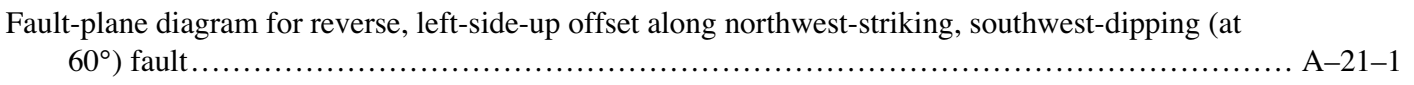 & 21.14 \\
\hline Fault-plane diagram for right-lateral strike-slip offset along north-striking, vertical fault .............. A-21-1 & 21.9 \\
\hline Fault-plane diagram for vertical, down-to-the-left offset along north-striking, vertical fault........... A-21-1 & 21.8 \\
\hline 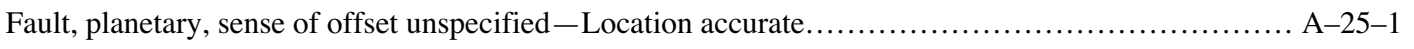 & 25.5 \\
\hline Fault, planetary, sense of offset unspecified-Location approximate..... & 25.6 \\
\hline
\end{tabular}




\begin{tabular}{|c|c|c|}
\hline & Page & Ref. No. \\
\hline ult, planetary, sense of offset unspecified-Location concealed........ & A-25-1 & 25.8 \\
\hline Fault, planetary, sense of offset unspecified-Location inferred ....... & A-25-1 & 25.7 \\
\hline Fault scarps & A-2-12 & Sec. 2.12 \\
\hline Fault, showing amount of local displacement . & A $-2-11$ & 2.11 .14 \\
\hline Fault showing displacement during historic time (includes areas of known fault creep) .... & A $-2-16$ & 2.13 .1 \\
\hline Fault showing displacement during Holocene time................... & A-2-16 & 2.13 .2 \\
\hline Fault showing displacement during late Quaternary time.. & A-2-16 & 2.13 .3 \\
\hline Fault showing displacement during Quaternary time (undifferentiated). & A-2-16 & 2.13 .4 \\
\hline Fault showing local left-lateral oblique-slip offset........................ & A-2-11 & 2.11 .7 \\
\hline Fault showing local left-lateral strike-slip offset.... & A-2-11 & 2.11 .5 \\
\hline Fault showing local normal offset (1st option: ball and bar on downthrown block). & A $-2-11$ & 2.11 .1 \\
\hline Fault showing local normal offset (2nd option: U, upthrown block; D, downthrown block). & A $-2-11$ & 2.11 .2 \\
\hline 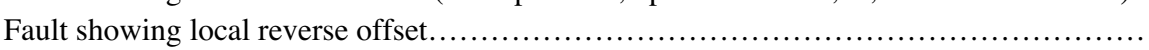 & A-2-11 & 2.11 .3 \\
\hline Fault showing local right-lateral oblique-slip offset. & A-2-11 & 2.11 .6 \\
\hline Fault showing local right-lateral strike-slip offset .... & A-2-11 & 2.11 .4 \\
\hline 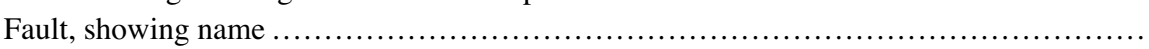 & A-2-11 & 2.11 .15 \\
\hline Fault symbols .................. & A-2-1 & Sec. 2 \\
\hline Fault zones [concepts and definitions]............... & $\ldots 11$ & $\mathrm{n} / \mathrm{a}$ \\
\hline Faults [concepts and definitions] .................. & $\ldots 11$ & $\mathrm{n} / \mathrm{a}$ \\
\hline Faults located by geophysical methods........ & A-3-1 & Sec. 3.2 \\
\hline 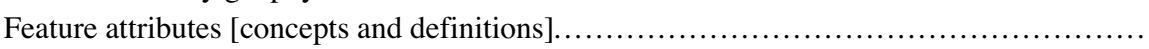 & $\ldots .8$ & $\mathrm{n} / \mathrm{a}$ \\
\hline 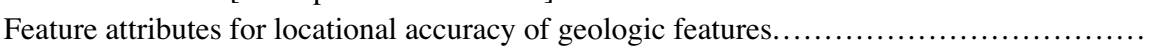 & .. A-ii & $\mathrm{n} / \mathrm{a}$ \\
\hline Feature attributes for scientific confidence of geologic features........ & .. A-ii & $\mathrm{n} / \mathrm{a}$ \\
\hline 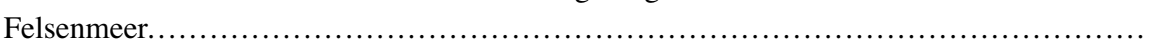 & A-14-1 & 14.10 \\
\hline 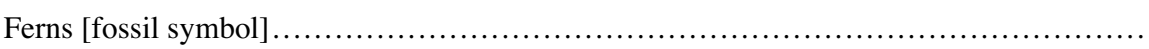 & A-10-1 & 10.2 .37 \\
\hline 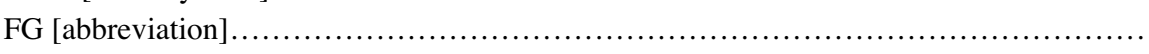 & $\ldots \mathrm{A}-\mathrm{v}$ & Table 2 \\
\hline FGDC-approved standard & $\ldots .3$ & $\mathrm{n} / \mathrm{a}$ \\
\hline "FGDCGeoAge" .......................... & A-32-1 & Sec. 32 \\
\hline "FGDCGeoAge" [technical specifications in this standard]. & $\ldots 31$ & $\mathrm{n} / \mathrm{a}$ \\
\hline FGDC standard terminology ................................... & $\ldots 20$ & Fig. 1 \\
\hline Field station locality, as shown on small-scale maps or on page-size illustrations ........... & A-31-1 & 31.22 \\
\hline Figure showing examples of the zone of confidence for planar, linear, and point features . & ... 22 & Fig. 3 \\
\hline 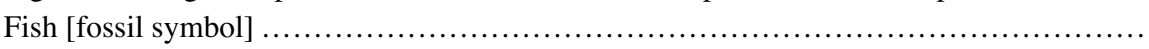 & A-10-1 & 10.2 .28 \\
\hline Fish ladder............. & A-30-4 & 30.2 .15 \\
\hline 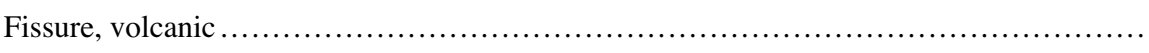 & A-18-2 & 18.41 \\
\hline Fissures and sand and (or) other material ejected during earthquake. & A-21-2 & 21.37 \\
\hline 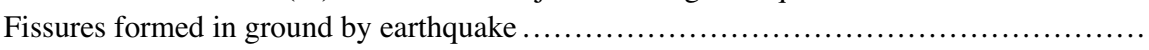 & A-21-2 & 21.36 \\
\hline 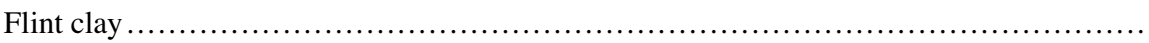 & A-37-2 & 661 \\
\hline Floodgate . & A-30-4 & 30.2 .12 \\
\hline Florida [state location map]. & A-34-1 & Sec. 34.1 \\
\hline Flow banding in $\mathrm{i}$ & A- $-8-1$ & 8.2.2 \\
\hline Flow banding in igneous rock, for multiple observations at one locality. & A-8-1 & 8.2 .5 \\
\hline Flow direction of glacial meltwater in stream channel ...................... & A-13-1 & 13.9 \\
\hline Flow direction, planetary ............................. & A-25-3 & 25.67 \\
\hline Flow front, planetary ....... & A-25-5 & 25.114 \\
\hline 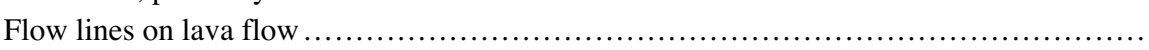 & A-18-2 & 18.39 \\
\hline Flow lobe-Identity and existence certain, location accurate ... & A-18-2 & 18.32 \\
\hline Flow lobe - Identity and existence certain, location approximate $\ldots \ldots \ldots \ldots \ldots \ldots \ldots \ldots \ldots \ldots \ldots \ldots \ldots$ & A-18-2 & 18.34 \\
\hline 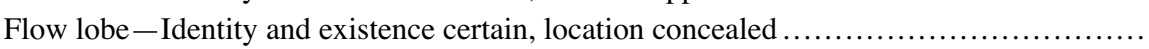 & A-18-2 & 18.36 \\
\hline 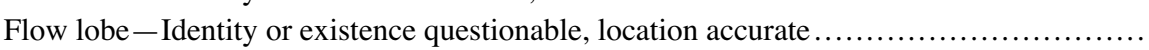 & A-18-2 & 18.33 \\
\hline 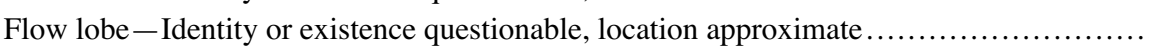 & A-18-2 & 18.35 \\
\hline Flow lobe - Identity or existence questionable, location concealed $\ldots \ldots \ldots \ldots \ldots \ldots \ldots \ldots \ldots \ldots$ & A-18-2 & 18.37 \\
\hline
\end{tabular}

Flowchart showing example of logical steps that might be used to determine appropriate line symbol 
Flowering plants [fossil symbol]

Page

Flowering trees [fossil symbol]...

Flowing artesian well used for domestic-water supply

Flowing artesian well used for industrial-water supply

Flowing artesian well used for irrigation-water supply....

Flowing artesian well used for public-water supply

Flowing artesian well used for stock-water supply

Flume.....

Flute mark in sedimentary materials

Fluvial features.

Fluvial terrace scarp-Identity and existence certain, location accurate.

Fluvial terrace scarp-Identity and existence certain, location approximate.

Fluvial terrace scarp-Identity or existence questionable, location accurate.

Fluvial terrace scarp-Identity or existence questionable, location approximate

Fluvial transport direction

Focal-mechanism diagram for left-lateral strike-slip offset along north-striking, vertical fault

Focal-mechanism diagram for normal, down-to-the-left offset along north-striking, west-dipping (at $\left.45^{\circ}\right)$ fault

Focal-mechanism diagram for normal, down-to-the-left offset along northwest-striking, southwestdipping (at $30^{\circ}$ ) fault

Focal-mechanism diagram for oblique reverse, left-side-up offset along northwest-striking, southwest-dipping (at $60^{\circ}$ ) fault

A-21-1

Focal-mechanism diagram for reverse, left-side-up offset along north-striking, west-dipping (at $45^{\circ}$ ) fault

Focal-mechanism diagram for reverse, left-side-up offset along northwest-striking, southwestdipping (at $60^{\circ}$ ) fault . . .

Focal-mechanism diagram for right-lateral strike-slip offset along north-striking, vertical fault.

Focal-mechanism diagram for vertical, down-to-the-left offset along north-striking, vertical fault ...... A-21-1

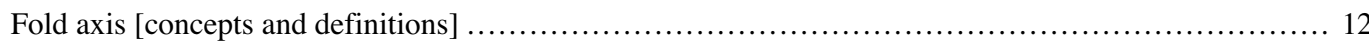

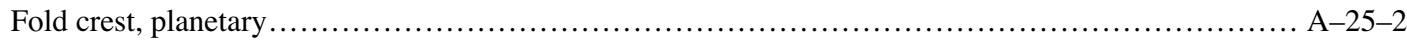

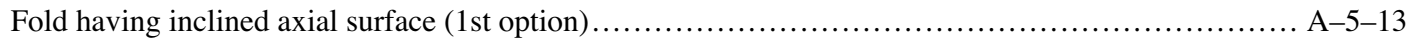

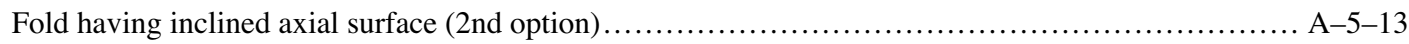

Fold having near-vertical fold limbs .................................................... A-5-13

Fold having vertical or near-vertical axial surface (1st option) ............................... A-5-13

Fold having vertical or near-vertical axial surface (2nd option) ................................ A-5-13

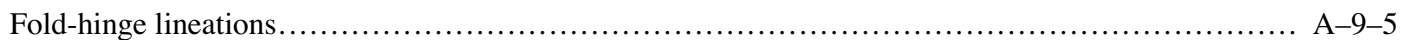

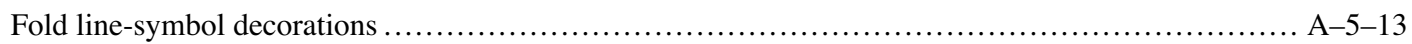

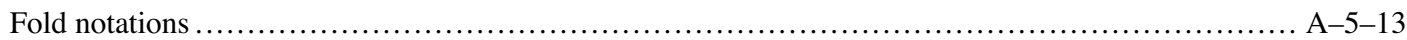

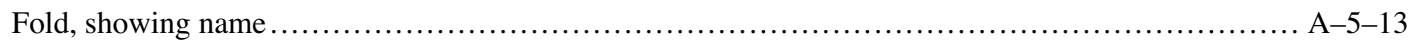

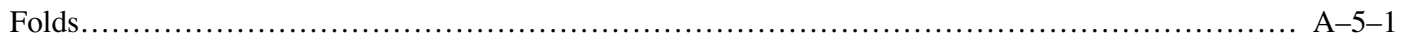

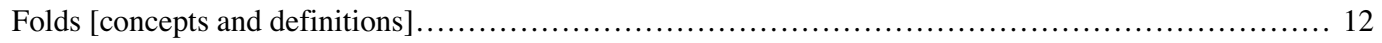

Folds on landslides $\ldots \ldots \ldots \ldots \ldots \ldots \ldots \ldots \ldots \ldots \ldots \ldots \ldots \ldots \ldots \ldots \ldots \ldots \ldots \ldots \ldots \ldots \ldots \ldots \ldots \ldots \ldots \ldots \ldots, 17-2$

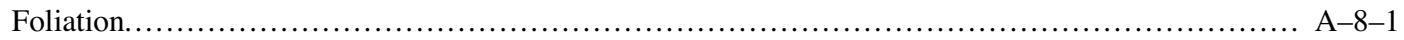

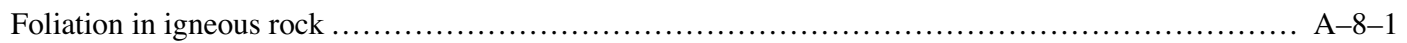

Foliation in igneous rock, for multiple observations at one locality ............................ A-8 -1

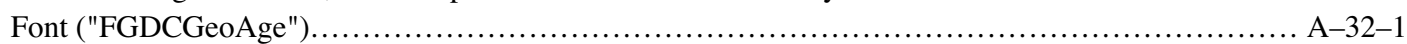

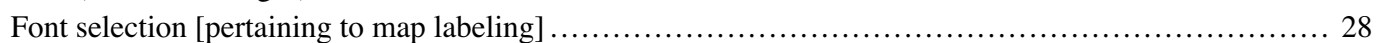

Foot of winze (drawn to scale), as shown on subsurface exploration maps .......................... A-19-5

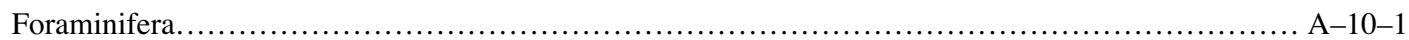

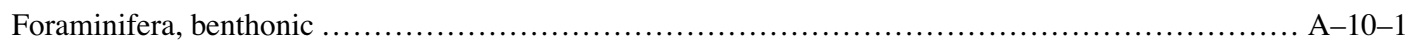

Foraminifera, planktonic........................................................... A-10

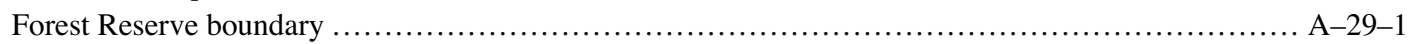

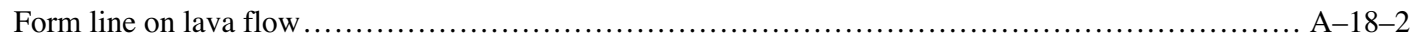

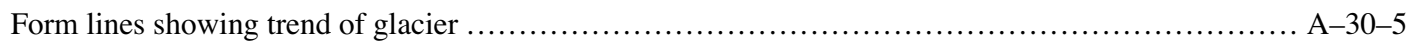

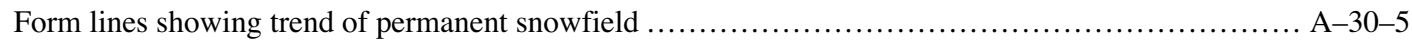

Ref. No.

10.2.38

10.2 .38

26.1 .8

26.1 .35

26.1 .26

26.1 .44

26.1.17

30.2 .22

9.13

Sec. 12

12.1

12.3

12.2

12.4

12.5

21.10

21.11

21.14

21.9

21.8

$\mathrm{n} / \mathrm{a}$

25.35

5.10 .1

5.10 .2

5.10 .9

5.10 .3

5.10 .4

9.97

Sec. 5.10

Sec. 5.10

5.10 .12

Sec. 5

n/a

Sec. 17

Sec. 8

8.2.2

8.2 .5

Sec. 32

$\mathrm{n} / \mathrm{a}$

19.4 .8

10.2 .48

10.2 .50

10.2 .51

29.6

18.38

30.2.45

30.2 .45 


\begin{tabular}{|c|c|}
\hline Formation [concepts and definitions] ... & $\begin{array}{c}\text { Ref. No. } \\
\text { n/a }\end{array}$ \\
\hline Formation depth ....................... & 26.6.3 \\
\hline Former marine limit-Identity and existence certain, location accurate...... & 15.13 \\
\hline Former marine limit-Identity and existence certain, location approximate. & 15.15 \\
\hline Former marine limit-Identity and existence certain, location concealed.... & 15.19 \\
\hline Former marine limit-Identity and existence certain, location inferred ....... & 15.17 \\
\hline Former marine limit-Identity or existence questionable, location accurate....... & 15.14 \\
\hline Former marine limit-Identity or existence questionable, location approximate.. & 15.16 \\
\hline Former marine limit-Identity or existence questionable, location concealed..... & 15.20 \\
\hline Former marine limit-Identity or existence questionable, location inferred .... & 15.18 \\
\hline Former shoreline-Identity and existence certain, location accurate........... & 15.13 \\
\hline Former shoreline - Identity and existence certain, location approximate........ & 15.15 \\
\hline Former shoreline-Identity and existence certain, location concealed........ & 15.19 \\
\hline Former shoreline-Identity and existence certain, location inferred ........ & 15.17 \\
\hline Former shoreline - Identity or existence questionable, location accurate ....... & 15.14 \\
\hline Former shoreline-Identity or existence questionable, location approximate .. & 15.16 \\
\hline Former shoreline - Identity or existence questionable, location concealed ..... & 15.20 \\
\hline Former shoreline-Identity or existence questionable, location inferred.... & 15.18 \\
\hline 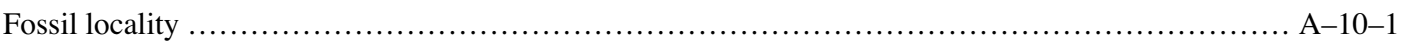 & Sec. 10.1 \\
\hline Fossil locality, showing collection number .... & 10.1.1 \\
\hline .... A-10-1 & Sec. 10.2 \\
\hline Fossiliferous bedded chert. & 651 \\
\hline Fossiliferous clastic limestone. & 629 \\
\hline Fossiliferous rock................... & 652 \\
\hline Four-wheel-drive road (Class 5). & 28.14 \\
\hline Fracture zone, planetary ........... & 25.132 \\
\hline Fractures on landslides... & Sec. 17 \\
\hline Fumarole................... & 18.64 \\
\hline Fumarole, as shown on topographic maps or on general-purpose or smaller scale maps.. & 30.3 .13 \\
\hline 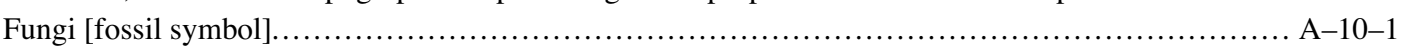 & 10.2 .40 \\
\hline Furrow, planetary ........ & 25.51 \\
\hline Fusulinids.............. & 10.2 .49 \\
\hline Gaging station, as shown on topographic maps or on general-purpose or smaller scale maps............ A-30-6 & 30.3.14 \\
\hline Gas and condensate show & 19.5.82 \\
\hline Gas and condensate well (nonspecific depth) $\ldots \ldots \ldots \ldots \ldots \ldots \ldots$ & 19.5.83 \\
\hline Gas field-Extent defined ...................... & 19.5.3 \\
\hline Gas field-Extent not yet defined.. & 19.5.4 \\
\hline Gas fields ........................... & Sec. 19.5 \\
\hline .... A-19-8 & 19.5.49 \\
\hline Gas show................................ & 19.5.50 \\
\hline Gas storage well (nonspecific depth) & 19.5.92 \\
\hline Gas well, as shown on topographic maps or on general-purpose or smaller scale maps................. A-30-6 & 30.3 .9 \\
\hline Gas well (nonspecific depth) & 19.5 .51 \\
\hline Gas wells & Sec. 19.5 \\
\hline 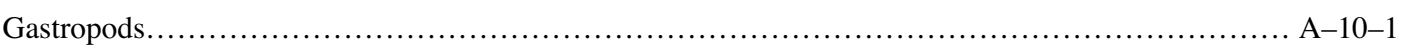 & 10.2 .23 \\
\hline 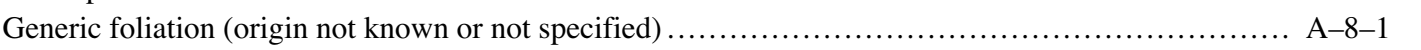 & Sec. 8 \\
\hline Generic foliation (origin not known or not specified), for multiple observations at one locality ......... A-8-1 & 8.1 .4 \\
\hline Gently inclined (between $0^{\circ}$ and $30^{\circ}$ ) bedding, as determined remotely or from aerial photographs..... A-6-2 & 6.40 \\
\hline Gently overturned (between $0^{\circ}$ and $30^{\circ}$ ) bedding, as determined remotely or from aerial photographs .. A-6-2 & 6.44 \\
\hline Geohydrologic contours & Sec. 26.5 \\
\hline Geohydrologic features ....... & Sec. 26 \\
\hline 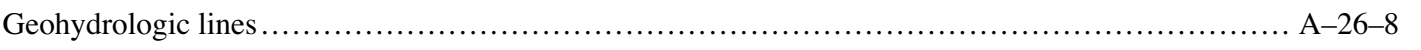 & Sec. 26.6 \\
\hline ...... 31 & $\mathrm{n} / \mathrm{a}$ \\
\hline
\end{tabular}




\begin{tabular}{|c|c|}
\hline Geologic age symbol font ("FGDCGeoAge").. & $\begin{array}{l}\text { Ref. No. } \\
\text { Sec. } 32\end{array}$ \\
\hline Geologic age symbols........................ & Sec. 32 \\
\hline Geologic age symbols [concepts and definitions]. & $\mathrm{n} / \mathrm{a}$ \\
\hline Geologic-formation depth ....................... & 26.6.3 \\
\hline Geologic-formation thickness.. & 26.6.5 \\
\hline Geologic map databases [concepts and definitions] . & $\mathrm{n} / \mathrm{a}$ \\
\hline Geologic map symbols [concepts and definitions]... & $\mathrm{n} / \mathrm{a}$ \\
\hline Geologic map units [concepts and definitions]..... & $\mathrm{n} / \mathrm{a}$ \\
\hline Geologic mapping concepts and definitions .... & $\mathrm{n} / \mathrm{a}$ \\
\hline Geologic maps [concepts and definitions] ... & $\mathrm{n} / \mathrm{a}$ \\
\hline Geologic point features [concepts and definitions] & $\mathrm{n} / \mathrm{a}$ \\
\hline Geologic time [concepts and definitions] ............ & $\mathrm{n} / \mathrm{a}$ \\
\hline Geophysical contour around closed area of lower values (index) - Accurately located ................ A-11-1 & 11.3 \\
\hline Geophysical contour around closed area of lower values (index) - Approximately located .............. A-11-1 & 11.5 \\
\hline Geophysical contour around closed area of lower values (intermediate) - Accurately located............ A-11-1 & 11.7 \\
\hline Geophysical contour around closed area of lower values (intermediate) - Approximately located........ A-11-1 & 11.9 \\
\hline Geophysical contour (index) - Accurately located $\ldots \ldots \ldots \ldots \ldots \ldots \ldots \ldots \ldots \ldots \ldots \ldots \ldots \ldots$ & 11.1 \\
\hline Geophysical contour (index)-Approximately located . & 11.4 \\
\hline Geophysical contour (index), showing datum.. & 11.2 \\
\hline Geophysical contour (intermediate)-Accurately located. & 11.6 \\
\hline Geophysical contour (intermediate) - Approximately located .. & 11.8 \\
\hline Geophysical contours .. & Sec. 11 \\
\hline Geophysical data collection line-Accurately located .. & 3.3 .1 \\
\hline Geophysical data collection line-Located by aerial survey .. & 3.3 .2 \\
\hline Geophysical data collection locality . & 11.10 \\
\hline Geophysical data collection locality - Showing value where known ....... & 11.11 \\
\hline Geophysical survey lines and stations.. & Sec. 3.3 \\
\hline Georgia [state location map] ............ & Sec. 34.1 \\
\hline Geothermal well, as shown on topographic maps or on general-purpose or smaller scale maps.. & 30.3 .11 \\
\hline .. A-18-3 & 18.63 \\
\hline Geyser, as shown on topographic maps or on general-purpose or smaller scale maps...... & 30.3 .13 \\
\hline Glacial advance... & Sec. 13 \\
\hline Glacial features & Sec. 13 \\
\hline Glacial grooves & Sec. 13 \\
\hline Glacial limit-Identity and existence certain, location accurate ..... & 13.49 \\
\hline Glacial limit-Identity and existence certain, location approximate. & 13.51 \\
\hline Glacial limit-Identity and existence certain, location concealed ... & 13.55 \\
\hline Glacial limit-Identity and existence certain, location inferred.... & 13.53 \\
\hline Glacial limit-Identity or existence questionable, location accurate...... & 13.50 \\
\hline Glacial limit-Identity or existence questionable, location approximate. & 13.52 \\
\hline Glacial limit-Identity or existence questionable, location concealed..... & 13.56 \\
\hline Glacial limit-Identity or existence questionable, location inferred .......... & 13.54 \\
\hline Glacial limit, showing name of glaciation... & 13.57 \\
\hline Glacial meltwater stream . & 13.7 \\
\hline Glacial meltwater stream channel (mapped to scale)... & 13.8 \\
\hline Glacial patterns (Series 500). & $\mathrm{n} / \mathrm{a}$ \\
\hline Glacial striations.. & Sec. 13 \\
\hline Glacial terminus - Identity and existence certain, location approximate .......... & 13.51 \\
\hline Glacial terminus - Identity and existence certain, location concealed & 13.55 \\
\hline 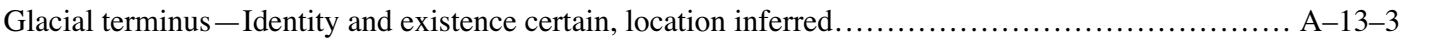 & 13.53 \\
\hline 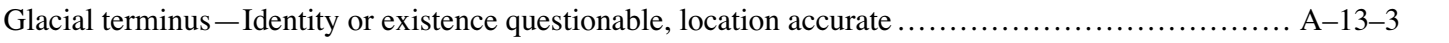 & 13.50 \\
\hline 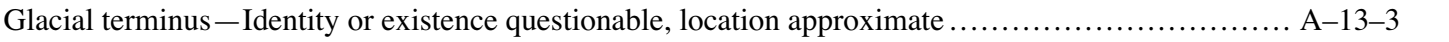 & 13.52 \\
\hline 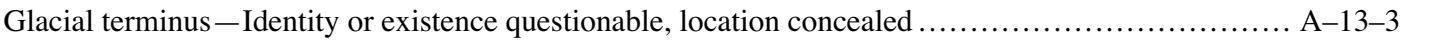 & 13.56 \\
\hline & 13.54 \\
\hline
\end{tabular}




\begin{tabular}{|c|c|}
\hline Page & Ref. No. \\
\hline Glacial terminus, showing name of glaciation.. & 13.57 \\
\hline Glacial-lake spillway........................... & 13.3 \\
\hline Glacial-lake spillway, showing elevation . & 13.4 \\
\hline Glacially scoured basin .................... & Sec. 13 \\
\hline Glacier........................ & 30.2 .44 \\
\hline Glacier contours ........ & Sec. 30.1 \\
\hline Glacier crevasse........ & 13.1 \\
\hline Glaciofluvial features ........ & Sec. 13 \\
\hline Glauconite ................ & 663 \\
\hline Glory hole .............. & 19.3.4 \\
\hline Gneiss ................. & 708 \\
\hline 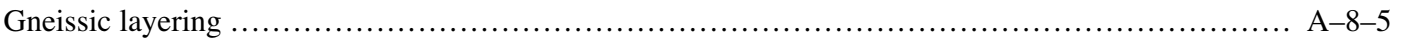 & Sec. 8 \\
\hline 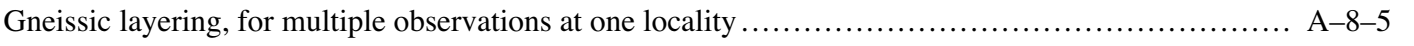 & 8.3 .49 \\
\hline 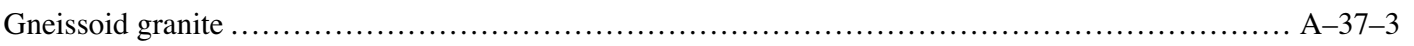 & 704 \\
\hline 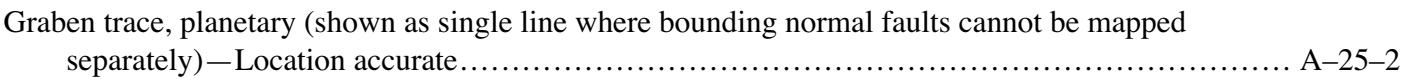 & 25.25 \\
\hline 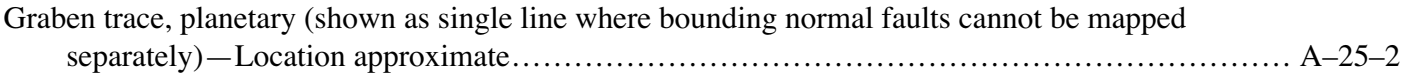 & 25.26 \\
\hline 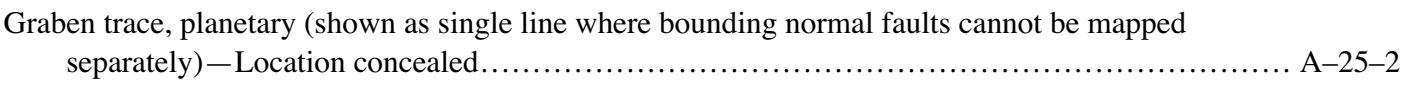 & 25.28 \\
\hline 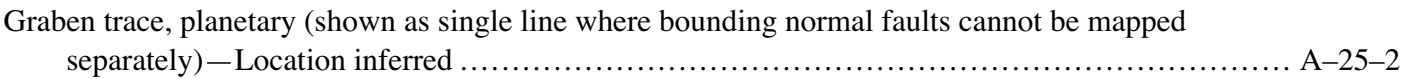 & 25.27 \\
\hline Gradational contact - Identity and existence certain, location accurate $\ldots \ldots \ldots \ldots \ldots \ldots \ldots \ldots \ldots \ldots \ldots \ldots \ldots \ldots$ & 1.1 .17 \\
\hline Gradational contact - Identity and existence certain, location approximate $\ldots \ldots \ldots \ldots \ldots \ldots \ldots$ & 1.1 .19 \\
\hline Gradational contact - Identity and existence certain, location concealed................... & 1.1 .23 \\
\hline Gradational contact - Identity and existence certain, location inferred $\ldots \ldots \ldots \ldots \ldots \ldots \ldots \ldots \ldots$ & 1.1 .21 \\
\hline Gradational contact-Identity or existence questionable, location accurate $\ldots \ldots \ldots \ldots \ldots \ldots$ & 1.1 .18 \\
\hline Gradational contact-Identity or existence questionable, location approximate $\ldots \ldots \ldots \ldots \ldots \ldots . . . .$. & 1.1 .20 \\
\hline Gradational contact - Identity or existence questionable, location concealed $\ldots \ldots \ldots \ldots \ldots \ldots \ldots \ldots \ldots \ldots \ldots$ & 1.1 .24 \\
\hline Gradational contact - Identity or existence questionable, location inferred $\ldots \ldots \ldots \ldots \ldots \ldots \ldots \ldots \ldots \ldots \ldots \ldots \ldots \ldots \ldots$ & 1.1 .22 \\
\hline 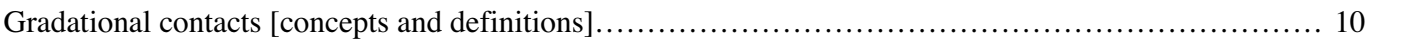 & $\mathrm{n} / \mathrm{a}$ \\
\hline Graded area where extensive amount of mapped geologic unit has been removed $\ldots \ldots \ldots \ldots \ldots \ldots$ & 19.2.1 \\
\hline 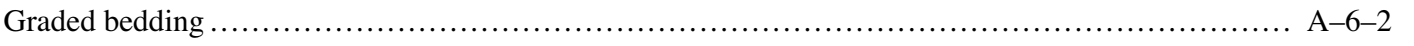 & 6.27 \\
\hline Granite (1st option)...... & 718 \\
\hline Granite (2nd option)................. & 719 \\
\hline Graptolites ........................... & 10.2 .17 \\
\hline Gravel (1st option)............ & 601 \\
\hline Gravel (2nd option).......... & 602 \\
\hline 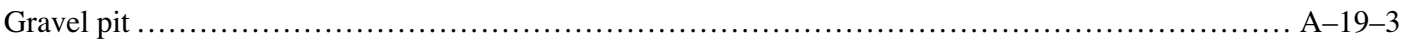 & 19.3.2 \\
\hline Gravel pit, as shown on topographic maps or on general-purpose or smaller scale maps ........... & 30.3 .2 \\
\hline 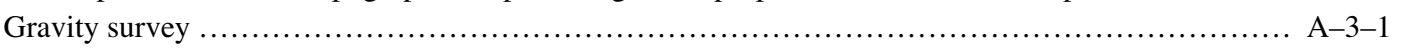 & Sec. 3 \\
\hline Green $[$ spot color $] \ldots \ldots \ldots \ldots \ldots \ldots \ldots \ldots$ & Table 3 \\
\hline Groove (generic), planetary ............... & 25.47 \\
\hline Groove, glacial.................... & Sec. 13 \\
\hline Groove on fault surface............. & 9.17 \\
\hline Grooves in sedimentary materials ....................... & 9.13 \\
\hline Ground magnetic survey $\ldots \ldots \ldots \ldots \ldots \ldots \ldots \ldots \ldots \ldots \ldots \ldots$ & Sec. 3 \\
\hline Ground-water barrier (geologic) - Accurately located ........ & 26.7 .5 \\
\hline Ground-water barrier (geologic) - Approximately located $\ldots \ldots \ldots \ldots \ldots \ldots \ldots \ldots \ldots$ & 26.7.6 \\
\hline 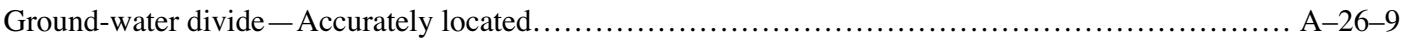 & 26.7 .3 \\
\hline Ground-water divide-Approximately located.......... & 26.7 .4 \\
\hline Guam [location map].......................... & Sec. 34.1 \\
\hline Guidelines for color selection................... & $\mathrm{n} / \mathrm{a}$ \\
\hline Guidelines for map labeling.............. & $\mathrm{n} / \mathrm{a}$ \\
\hline Guidelines for pattern selection....... & $\mathrm{n} / \mathrm{a}$ \\
\hline
\end{tabular}




\begin{tabular}{|c|c|}
\hline Page & Ref. No. \\
\hline Gully on landslide. & 17.46 \\
\hline$A-22-2$ & 22.35 \\
\hline Gypsum .... & 667 \\
\hline $\mathrm{H}[$ abbreviation] $\ldots \ldots \ldots \ldots \ldots \ldots \ldots \ldots \ldots \ldots$ & Table 2 \\
\hline Halo without associated crater, planetary ..... & 25.135 \\
\hline Hardness ...................................... & 26.6.11 \\
\hline Hawaii [state location map] ...... & Sec. 34.1 \\
\hline Hawaii Homestead boundary ... & 29.6 \\
\hline Hazardous waste site .................................... & 20.1 \\
\hline Hazardous waste site - Clean-up activities are in progress ................... & 20.5 \\
\hline 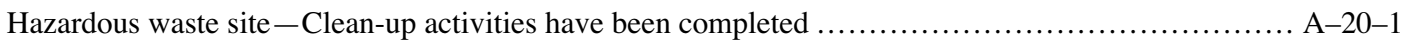 & 20.6 \\
\hline Hazardous waste site, showing direction of surface-leachate flow from site $\ldots \ldots \ldots \ldots \ldots \ldots \ldots \ldots \ldots \ldots$ A $-20-1$ & 20.2 \\
\hline 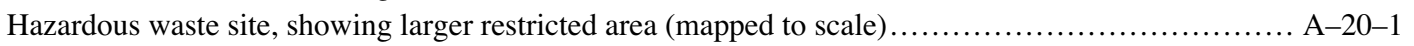 & 20.8 \\
\hline Hazardous waste site, showing smaller restricted area (mapped to scale) .......... & 20.7 \\
\hline 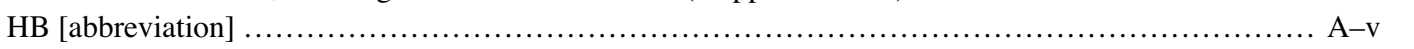 & Table 2 \\
\hline Head of raise (drawn to scale), as shown on subsurface exploration maps ............................ A-19-5 & 19.4.6 \\
\hline 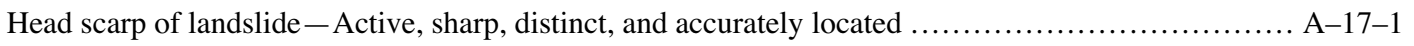 & 17.12 \\
\hline Head scarp of landslide - Inactive, subdued, indistinct, and (or) approximately located............... A-17-1 & 17.13 \\
\hline 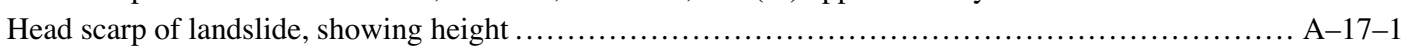 & 17.14 \\
\hline Head scarp of rotated block in landslide................ & 17.15 \\
\hline Headwall of adjoining cirques ..................................... & 13.42 \\
\hline 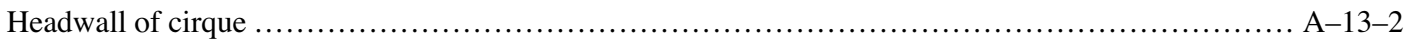 & 13.41 \\
\hline 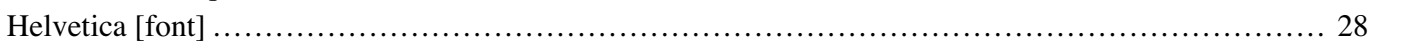 & $\mathrm{n} / \mathrm{a}$ \\
\hline HI [abbreviation] ........................... & Table 2 \\
\hline High-angle faults ................. & Sec. 2.1 \\
\hline Highway (generic) $\ldots \ldots \ldots \ldots \ldots \ldots \ldots \ldots \ldots$ & 28.1 \\
\hline Highways ................................ & Sec. 28 \\
\hline 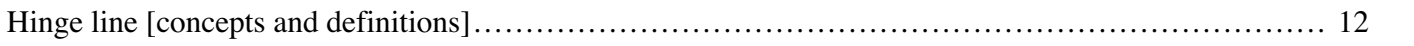 & $\mathrm{n} / \mathrm{a}$ \\
\hline 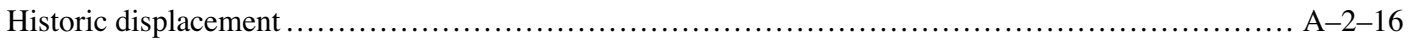 & 2.13 .1 \\
\hline Historically active debris flow, showing a sharply defined morphology ........................... A $-17-3$ & 17.56 \\
\hline Historically active landslide (mapped to scale), showing a sharply defined morphology ........ & 17.58 \\
\hline 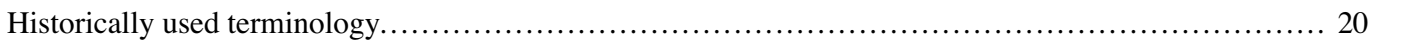 & Fig. 1 \\
\hline 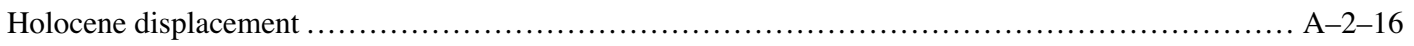 & 2.13 .2 \\
\hline 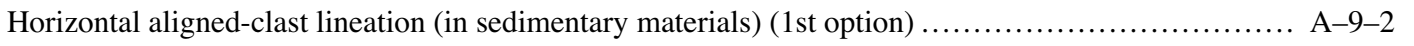 & 9.31 \\
\hline Horizontal aligned-clast lineation (in sedimentary materials) (2nd option) $\ldots \ldots \ldots \ldots \ldots \ldots \ldots \ldots \ldots \ldots \ldots \ldots \ldots \ldots \ldots \ldots \ldots$ & 9.32 \\
\hline 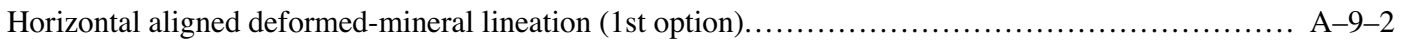 & 9.47 \\
\hline Horizontal aligned deformed-mineral lineation (2nd option)................. & 9.48 \\
\hline Horizontal aligned-grain lineation (in sedimentary materials) (1st option)........... & 9.31 \\
\hline 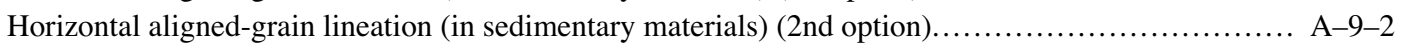 & 9.32 \\
\hline Horizontal aligned-inclusion lineation (in igneous rocks) (1st option)................ & 9.35 \\
\hline Horizontal aligned-inclusion lineation (in igneous rocks) (2nd option)... & 9.36 \\
\hline Horizontal aligned mineral-aggregate lineation (1st option) $\ldots \ldots \ldots \ldots \ldots$ & 9.43 \\
\hline Horizontal aligned mineral-aggregate lineation (2nd option) $\ldots \ldots \ldots \ldots \ldots \ldots$ & 9.44 \\
\hline 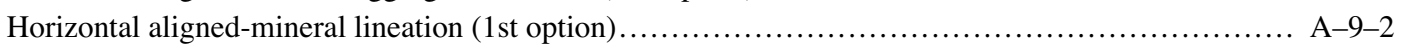 & 9.39 \\
\hline Horizontal aligned-mineral lineation (2nd option)........... & 9.40 \\
\hline Horizontal aligned-object lineation (1st option)............. & 9.27 \\
\hline Horizontal aligned-object lineation (2nd option)............... & 9.28 \\
\hline Horizontal aligned stretched-object lineation (1st option) ........ & 9.51 \\
\hline Horizontal aligned stretched-object lineation (2nd option) ..... & 9.52 \\
\hline Horizontal aligned stretched-ooid lineation (1 st option) $\ldots \ldots \ldots \ldots \ldots \ldots \ldots \ldots \ldots \ldots \ldots \ldots$ & 9.59 \\
\hline Horizontal aligned stretched-ooid lineation (2nd option) ....... & 9.60 \\
\hline Horizontal aligned stretched-pebble lineation (1st option) $\ldots \ldots \ldots \ldots \ldots \ldots \ldots \ldots$ & 9.55 \\
\hline Horizontal aligned stretched-pebble lineation ( 2 nd option) $\ldots \ldots \ldots \ldots \ldots \ldots \ldots$ & 9.56 \\
\hline
\end{tabular}


Horizontal asymmetric (S-shaped, counterclockwise sense of shear) kink-band crenulation lineation (1st option)

Horizontal asymmetric (S-shaped, counterclockwise sense of shear) kink-band crenulation lineation (2nd option)

Horizontal asymmetric (Z-shaped, clockwise sense of shear) kink-band crenulation lineation (1st

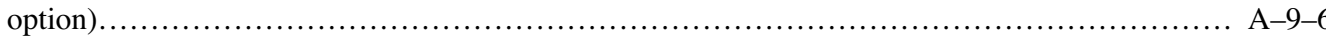

Horizontal asymmetric (Z-shaped, clockwise sense of shear) kink-band crenulation lineation (2nd option).

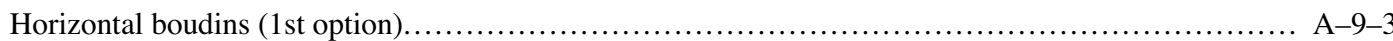

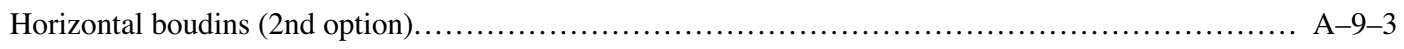

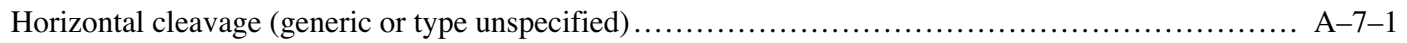

Horizontal continuous, penetrative foliation............................................ A-8 -3

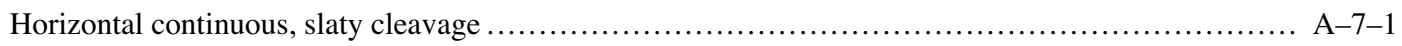

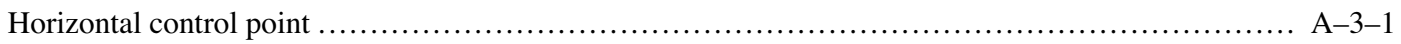

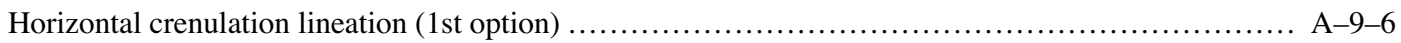

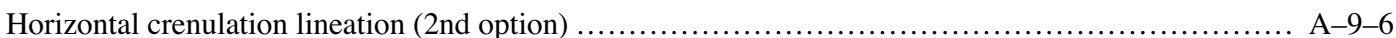

Horizontal cumulate foliation ........................................................... A

9.136

Horizontal disjunctive, asymmetric (S-shaped, counterclockwise sense of shear) crenulation cleavage.. A-7-2

8.2 .10

7.25

Horizontal disjunctive, asymmetric (S-shaped, counterclockwise sense of shear) crenulation foliation .. A-8-4

8.3 .34

Horizontal disjunctive, asymmetric (Z-shaped, clockwise sense of shear) crenulation cleavage......... A-7-2

7.31

Horizontal disjunctive, asymmetric (Z-shaped, clockwise sense of shear) crenulation foliation ......... A-8-4

8.3 .40

Horizontal disjunctive, spaced cleavage ............................................. A $-7-1$

7.13

Horizontal disjunctive, spaced foliation ............................................... A-8

8.3 .22

Horizontal disjunctive, symmetric crenulation cleavage $\ldots \ldots \ldots \ldots \ldots \ldots \ldots \ldots \ldots \ldots \ldots \ldots \ldots \ldots \ldots$ A $-7-1$

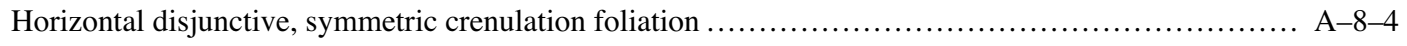

8.3 .28

Horizontal eutaxitic foliation. ......................................................... A-8 2

8.2 .19

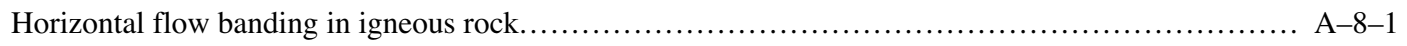

8.2 .2

Horizontal fold hinge of generic (type or orientation unspecified) small, minor fold (1st option) ........ A-9-5

Horizontal fold hinge of generic (type or orientation unspecified) small, minor fold (2nd option) ........ A-9-5

9.100

Horizontal fold hinge of small, minor anticline (1st option)................................... A-9-5

9.107

Horizontal fold hinge of small, minor anticline (2nd option)................................... A-9-5

9.108

Horizontal fold hinge of small, minor antiform (1st option) ................................... A-9-5

9.111

Horizontal fold hinge of small, minor antiform (2nd option)............................... A-9-5

9.112

Horizontal fold hinge of small, minor penecontemporaneous soft-sediment fold (1st option) ........... A-9-5

9.103

Horizontal fold hinge of small, minor penecontemporaneous soft-sediment fold (2nd option) .......... A-9-5

9.104

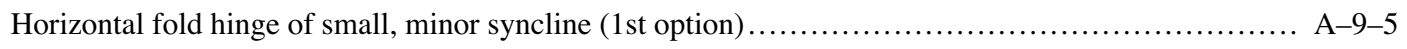

9.115

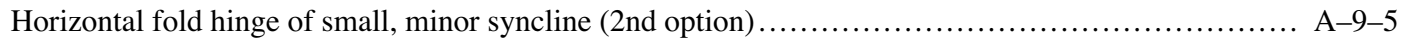

9.116

Horizontal fold hinge of small, minor synform (1st option) ............................... A-9-5

9.119

Horizontal fold hinge of small, minor synform (2nd option) ................................ A-9-5

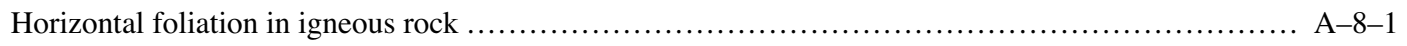

Horizontal generic (origin not known or not specified) foliation .............................. A-8-1

Horizontal generic (origin or type not known or not specified) lineation or linear structure (1st

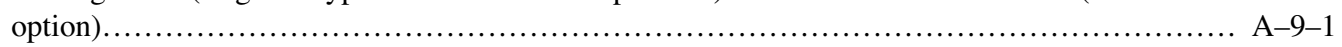

9.120

8.2 .2

8.1 .1

Horizontal generic (origin or type not known or not specified) lineation or linear structure (2nd option)....

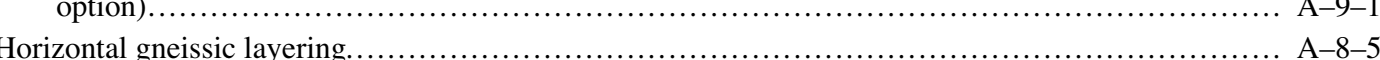

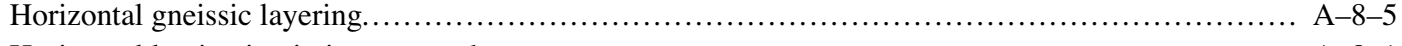

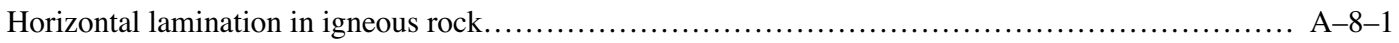

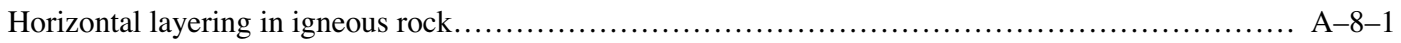




\begin{tabular}{|c|c|c|}
\hline & Page & Ref. No \\
\hline Horizontal lineation at intersection of bedding and cleavage ( 2 nd option). & A-9-4 & 9.80 \\
\hline Horizontal lineation at intersection of two cleavages (1st option) ............... & A-9-4 & 9.83 \\
\hline Horizontal lineation at intersection of two cleavages (2nd option). & A-9-4 & 9.84 \\
\hline Horizontal lineation at intersection of two foliations (1st option). & A-9-4 & 9.91 \\
\hline Horizontal lineation at intersection of two foliations (2nd option). & A-9-4 & 9.92 \\
\hline Horizontal lineation at intersection of two fractures or joints (1st option).. & A-9-4 & 9.87 \\
\hline Horizontal lineation at intersection of two fractures or joints (2nd option).. & A-9-4 & 9.88 \\
\hline Horizontal lineation at intersection of two surfaces (origin or type unspecified) (1st option). & A-9-4 & 9.95 \\
\hline Horizontal lineation at intersection of two surfaces (origin or type unspecified) (2nd option). & A-9-4 & 9.96 \\
\hline Horizontal metamorphic or tectonic foliation. & A $-8-3$ & 8.3.1 \\
\hline Horizontal metamorphic or tectonic foliation parallel to bedding & A $-8-3$ & 8.3.7 \\
\hline 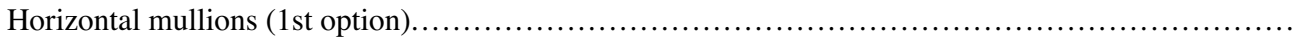 & A-9-3 & 9.67 \\
\hline Horizontal mullions (2nd option). & A-9-3 & 9.68 \\
\hline Horizontal mylonitic foliation .... & A $-8-5$ & 8.3 .55 \\
\hline Horizontal parting lineation in sedimentary materials (1st option). & A-9-1 & 9.11 \\
\hline Horizontal parting lineation in sedimentary materials (2nd option). & A-9-1 & 9.12 \\
\hline Horizontal pencil structure (1st option)............... & A-9-4 & 9.75 \\
\hline Horizontal pencil structure (2nd option).. & A-9-4 & 9.76 \\
\hline Horizontal rodding (1st option).. & A-9-3 & 9.63 \\
\hline Horizontal rodding (2nd option)... & A-9-3 & 9.64 \\
\hline Horizontal slickenline, groove, or striation on fault surface (1st option) .... & A-9-1 & 9.19 \\
\hline Horizontal slickenline, groove, or striation on fault surface (2nd option) .. & A-9-1 & 9.20 \\
\hline $\begin{array}{l}\text { Horizontal sole mark, tool mark, scour mark, flute mark, groove, or channel in sedimentary materials } \\
\text { (1st option) }\end{array}$ & A-9-1 & 9.15 \\
\hline $\begin{array}{l}\text { Horizontal sole mark, tool mark, scour mark, flute mark, groove, or channel in sedimentary materials } \\
\text { (2nd option) }\end{array}$ & A-9-1 & 9.16 \\
\hline Horizontal surface groove or striation (origin not known or not specified) (1st option) $\ldots \ldots \ldots \ldots \ldots \ldots \ldots \ldots \ldots \ldots$ & A-9-1 & 9.23 \\
\hline Horizontal surface groove or striation (origin not known or not specified) (2nd option).... & A-9-1 & 9.24 \\
\hline Horizontal symmetric minor fold hinge (1st option) & A-9-6 & 9.123 \\
\hline Horizontal symmetric minor fold hinge (2nd option)... & A-9-6 & 9.124 \\
\hline Horizontal undulatory gneissic layering................. & A- $-8-5$ & 8.3.52 \\
\hline Hornito, large ........................... & A-18-3 & 18.58 \\
\hline Hornito, small ... & A-18-3 & 18.57 \\
\hline How to use [the contents of] this standard. & .. A-ii & $\mathrm{n} / \mathrm{a}$ \\
\hline HSV [abbreviation] $\ldots \ldots \ldots \ldots \ldots \ldots \ldots \ldots \ldots \ldots \ldots \ldots \ldots$ & . A-v & Table 2 \\
\hline HSV color model .. & 26 & $\mathrm{n} / \mathrm{a}$ \\
\hline Hummock on landslide (mapped to scale). & A-17-3 & 17.50 \\
\hline Hummock on landslide (shown as point symbol when too small to outline at map scale). & A-17-3 & 17.51 \\
\hline Hummocky topography (1st option).. & A-13-2 & 13.26 \\
\hline Hummocky topography (2nd option). & A-13-2 & 13.27 \\
\hline Hummocky topography (3rd option). & A-13-2 & 13.28 \\
\hline Hydraulic conductivity............... & A-26-8 & 26.6.17 \\
\hline Hydrographic features .. & A-30-1 & Sec. 30 \\
\hline Ice-channel deposit, known transport direction (1st option)... & A-13-1 & 13.18 \\
\hline Ice-channel deposit, known transport direction (2nd option). & A-13-1 & 13.19 \\
\hline Ice-channel deposit, unknown transport direction & A-13-1 & 13.17 \\
\hline Ice-contact lava-flow margin - Identity and existence certain, location accurate $\ldots \ldots \ldots \ldots \ldots \ldots \ldots \ldots$ & A-18-3 & 18.48 \\
\hline Ice-contact lava-flow margin - Identity and existence certain, location approximate $\ldots \ldots \ldots \ldots \ldots \ldots \ldots$ & A-18-3 & 18.50 \\
\hline Ice-contact lava-flow margin - Identity and existence certain, location concealed $\ldots \ldots \ldots \ldots \ldots \ldots \ldots$ & A-18-3 & 18.52 \\
\hline Ice-contact lava-flow margin -Identity or existence questionable, location accurate $\ldots \ldots \ldots \ldots \ldots \ldots$ & A-18-3 & 18.49 \\
\hline Ice-contact lava-flow margin-Identity or existence questionable, location approximate $\ldots \ldots \ldots \ldots \ldots$ & A-18-3 & 18.51 \\
\hline Ice-contact lava-flow margin-Identity or existence questionable, location concealed $\ldots \ldots \ldots \ldots \ldots \ldots$ & A-18-3 & 18.53 \\
\hline Ice-contact slope & A-13-1 & 13.16 \\
\hline Ice-flow direction... & A-13-1 & 13.2 \\
\hline
\end{tabular}




\begin{tabular}{|c|c|}
\hline $\begin{array}{c}\text { Page } \\
\text { A-13-3 }\end{array}$ & $\begin{array}{l}\text { Ref. No. } \\
\text { Sec. } 13\end{array}$ \\
\hline ce-wedge polygon .... & 14.8 \\
\hline Ice-wedge polygons . & 14.9 \\
\hline Idaho [state location map]. & Sec. 34.1 \\
\hline Identity [concepts and definitions] . & $\mathrm{n} / \mathrm{a}$ \\
\hline 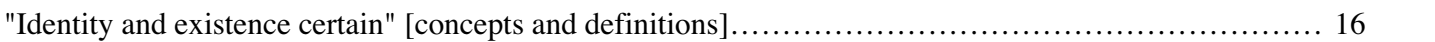 & $\mathrm{n} / \mathrm{a}$ \\
\hline "Identity certain" [concepts and definitions]................ & $\mathrm{n} / \mathrm{a}$ \\
\hline 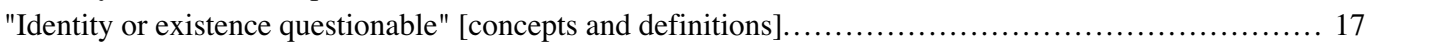 & $\mathrm{n} / \mathrm{a}$ \\
\hline 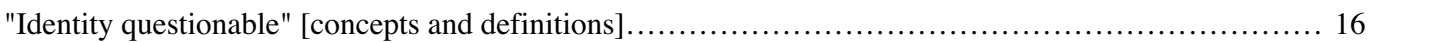 & $\mathrm{n} / \mathrm{a}$ \\
\hline 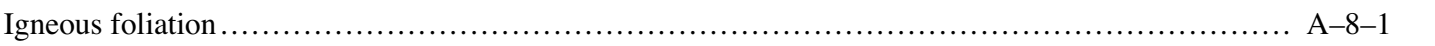 & 8.2 .2 \\
\hline Igneous layering . & 8.2.2 \\
\hline Igneous patterns (Series 300) & $\mathrm{n} / \mathrm{a}$ \\
\hline Igneous rock (1st option) ...... & 721 \\
\hline Igneous rock (2nd option). & 722 \\
\hline Igneous rock (3rd option). & 723 \\
\hline Igneous rock (4th option). & 724 \\
\hline Igneous rock (5th option). & 725 \\
\hline Igneous rock (6th option). & 726 \\
\hline Igneous rock (7th option). & 727 \\
\hline Igneous rock (8th option). & 728 \\
\hline Igneous-rock lithologic patterns. & Sec. 37.2 \\
\hline Illinois [state location map] ...... & Sec. 34.1 \\
\hline Impact craters, planetary.... & Sec. 25 \\
\hline Impact craters (terrestrial).. & Sec. 24 \\
\hline Impact features (terrestrial).. & Sec. 24 \\
\hline Implementation [of this standard]. & $\mathrm{n} / \mathrm{a}$ \\
\hline Impure coal ........................ & 659 \\
\hline Inaccessible adit (1st option).. & 19.3.12 \\
\hline Inaccessible adit (2nd option)... & 19.3.17 \\
\hline Inaccessible portal ................ & 19.3.22 \\
\hline Inaccessible tunnel entrance (1st option).... & 19.3.12 \\
\hline Inaccessible tunnel entrance (2nd option)..... & 19.3.17 \\
\hline Inaccessible vertical mine shaft, as shown on smaller scale or general-purpose maps..................... A-19-4 & 19.3.37 \\
\hline Inaccessible workings (drawn to scale), as shown on subsurface exploration maps …................ A-19-5 & 19.4.11 \\
\hline Inactive (closed) hazardous waste site.... & 20.4 \\
\hline Inactive quality-of-water site........... & 26.4.2 \\
\hline Inactive volcano on small-scale maps ............ & 18.67 \\
\hline Inches (in) to points (pts) or millimeters (mm). & Table 1 \\
\hline Incipient sliding on landslide . & 17.47 \\
\hline Incised-scarp sedimentary contact - Identity and existence certain, location accurate $\ldots \ldots \ldots \ldots \ldots \ldots \ldots$ A $-1-2$ & 1.1 .33 \\
\hline Incised-scarp sedimentary contact - Identity and existence certain, location approximate $\ldots \ldots \ldots \ldots \ldots \ldots$ A-1-2 & 1.1 .35 \\
\hline Incised-scarp sedimentary contact-Identity or existence questionable, location accurate …............ A-1-2 & 1.1 .34 \\
\hline Incised-scarp sedimentary contact -Identity or existence questionable, location approximate …....... A-1-2 & 1.1 .36 \\
\hline Inclined aligned-clast lineation (in sedimentary materials) (1st option) ....... & 9.29 \\
\hline Inclined aligned-clast lineation (in sedimentary materials) (2nd option) ...... & 9.30 \\
\hline Inclined aligned deformed-mineral lineation (1st option).... & 9.45 \\
\hline Inclined aligned deformed-mineral lineation (2nd option)................... & 9.46 \\
\hline Inclined aligned-grain lineation (in sedimentary materials) (1st option)... & 9.29 \\
\hline Inclined aligned-grain lineation (in sedimentary materials) (2nd option)... & 9.30 \\
\hline Inclined aligned-inclusion lineation (in igneous rocks) (1st option)......... & 9.33 \\
\hline Inclined aligned-inclusion lineation (in igneous rocks) (2nd option). & 9.34 \\
\hline Inclined aligned mineral-aggregate lineation (1st option) & 9.41 \\
\hline A-9-2 & 9.42 \\
\hline Inclined aligned-mineral line & 9.37 \\
\hline
\end{tabular}




\begin{tabular}{|c|c|c|}
\hline & Page & Ref. No \\
\hline Inclined aligned-mineral lineation (2nd option). & A-9-2 & 9.38 \\
\hline Inclined aligned-object lineation (1st option).... & A-9-2 & 9.25 \\
\hline Inclined aligned-object lineation (2nd option). & A-9-2 & 9.26 \\
\hline Inclined aligned stretched-object lineation (1st option). & A-9-3 & 9.49 \\
\hline Inclined aligned stretched-object lineation (2nd option). & A-9-3 & 9.50 \\
\hline Inclined aligned stretched-ooid lineation (1st option)... & A-9-3 & 9.57 \\
\hline Inclined aligned stretched-ooid lineation (2nd option). & A-9-3 & 9.58 \\
\hline Inclined aligned stretched-pebble lineation (1st option). & A-9-3 & 9.53 \\
\hline Inclined aligned stretched-pebble lineation (2nd option) $\ldots$ & A-9-3 & 9.54 \\
\hline $\begin{array}{l}\text { Inclined asymmetric (S-shaped, counterclockwise sense of shear) kink-band crenulation lineation } \\
\text { (1st option) }\end{array}$ & A-9-6 & 9.137 \\
\hline $\begin{array}{l}\text { Inclined asymmetric (S-shaped, counterclockwise sense of shear) kink-band crenulation lineation } \\
\text { (2nd option) }\end{array}$ & A-9-6 & 9.138 \\
\hline Inclined asymmetric ( $\mathrm{S}$-shaped, counterclockwise sense of shear) minor fold hinge (1st option) ... & A-9-6 & 9.125 \\
\hline Inclined asymmetric ( $\mathrm{S}$-shaped, counterclockwise sense of shear) minor fold hinge (2nd option) .... & A-9-6 & 9.126 \\
\hline 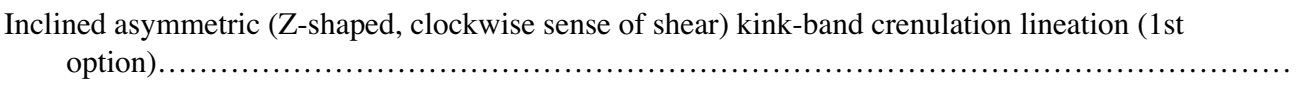 & A-9-6 & 9.141 \\
\hline Inclined asymmetric (Z-shaped, clockwise sense of shear) kink-band crenulation lineation (2nd & A-9-6 & 9.142 \\
\hline Inclined asymmetric (Z-shaped, clockwise sense of shear) minor fold hinge (1st option) ... & A-9-6 & 9.129 \\
\hline Inclined asymmetric (Z-shaped, clockwise sense of shear) minor fold hinge (2nd option). & A-9-6 & 9.130 \\
\hline 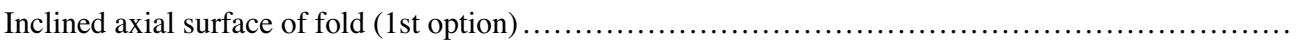 & A-5-13 & 5.10 .1 \\
\hline Inclined axial surface of fold (2nd option) ......... & A-5-13 & 5.10 .2 \\
\hline Inclined bed of economically important commodity (1st option).. & A-1-6 & 1.4.1 \\
\hline Inclined bed of economically important commodity (2nd option). & A-1-6 & 1.4 .2 \\
\hline Inclined bedding & A-6-1 & 6.2 \\
\hline Inclined bedding in crossbedded rocks & A-6-2 & 6.30 \\
\hline Inclined bedding, where top direction of beds is known from local features.. & A-6-1 & 6.13 \\
\hline 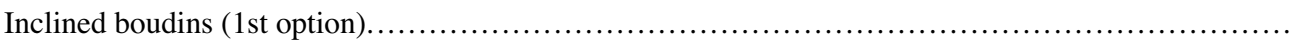 & A-9-3 & 9.69 \\
\hline Inclined boudins (2nd option).. & A-9-3 & 9.70 \\
\hline Inclined channel in sedimentary materials (1st option).. & A-9-1 & 9.13 \\
\hline Inclined channel in sedimentary materials (2nd option).. & A-9-1 & 9.14 \\
\hline Inclined clay bed (1st option) $\ldots \ldots \ldots \ldots \ldots \ldots \ldots \ldots \ldots \ldots \ldots \ldots$ & A-1-6 & 1.4 .1 \\
\hline Inclined clay bed (2nd option). & A-1-6 & 1.4 .2 \\
\hline Inclined cleavage (generic or type unspecified). & A-7-1 & 7.2 \\
\hline 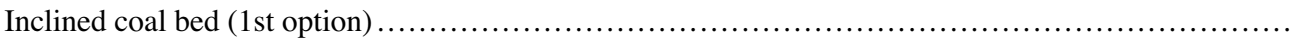 & A-1-6 & 1.4 .1 \\
\hline Inclined coal bed (2nd option) ......... & A-1-6 & 1.4 .2 \\
\hline Inclined contact (1st option) ... & A-1-6 & 1.4 .1 \\
\hline Inclined contact (2nd option) .................... & A-1-6 & 1.4 .2 \\
\hline Inclined continuous, penetrative foliation....... & A-8-3 & 8.3.17 \\
\hline Inclined continuous, slaty cleavage .................. & A-7-1 & 7.8 \\
\hline Inclined contorted bedding..$\ldots \ldots \ldots \ldots \ldots \ldots \ldots$ & A-6-2 & 6.25 \\
\hline Inclined crenulated bedding .................... & A-6-2 & 6.25 \\
\hline Inclined crenulation lineation (1st option) & A-9-6 & 9.133 \\
\hline Inclined crenulation lineation (2nd option) ......... & A-9-6 & 9.134 \\
\hline Inclined crinkled cumulate foliation ................. & A-8-2 & 8.2.17 \\
\hline Inclined crinkled eutaxitic foliation.................. & A-8-2 & 8.2 .25 \\
\hline Inclined crinkled flow banding in igneous rock... & A-8-1 & 8.2 .8 \\
\hline Inclined crinkled foliation in igneous rock...... & A-8-1 & 8.2 .8 \\
\hline Inclined crinkled lamination in igneous rock..... & A-8-1 & 8.2 .8 \\
\hline Inclined crinkled metamorphic foliation . & A-8-3 & 8.3 .14 \\
\hline Inclined crinkled tectonic foliation.. & A-8-3 & 8.3 .14 \\
\hline & A-8-1 & 8.2.11 \\
\hline & A- $-8-1$ & 8.2 .14 \\
\hline Inclined deformed cumulate foliation.. & A- $-8-2$ & 8.2.17 \\
\hline
\end{tabular}




\begin{tabular}{|c|c|c|}
\hline & Page & Ref. No. \\
\hline Inclined deformed eutaxitic foliation ............. & A-8-2 & 8.2 .25 \\
\hline Inclined deformed flow banding in igneous rock & A-8-1 & 8.2 .8 \\
\hline Inclined deformed foliation in igneous rock .......... & A-8-1 & 8.2 .8 \\
\hline Inclined deformed lamination in igneous rock ......... & A-8-1 & 8.2 .8 \\
\hline Inclined deformed metamorphic foliation............. & A-8-3 & 8.3 .14 \\
\hline Inclined deformed tectonic foliation ............... & A-8-3 & 8.3 .14 \\
\hline Inclined dike (1st option) $\ldots \ldots \ldots \ldots \ldots \ldots \ldots \ldots$ & A-1-6 & 1.4 .1 \\
\hline 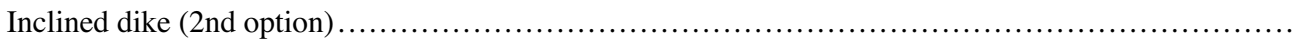 & A-1-6 & 1.4 .2 \\
\hline 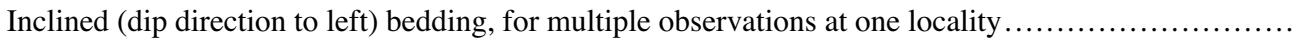 & A-6-1 & 6.7 \\
\hline 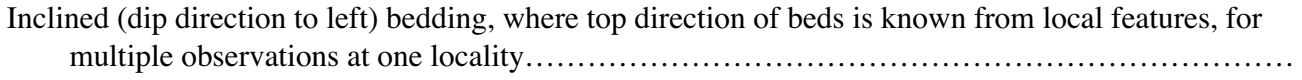 & A-6-1 & 6.18 \\
\hline Inclined (dip direction to left) cleavage (generic or type unspecified), for multiple observations at one & A-7-1 & 7.5 \\
\hline $\begin{array}{l}\text { Inclined (dip direction to left) continuous, penetrative foliation, for multiple observations at one } \\
\text { locality }\end{array}$ & A-8-3 & 8.3 .20 \\
\hline Inclined (dip direction to left) continuous, slaty cleavage, for multiple observations at one locality ...... & A-7-1 & 7.11 \\
\hline $\begin{array}{l}\text { Inclined (dip direction to left) disjunctive, asymmetric (S-shaped, counterclockwise sense of shear) } \\
\text { crenulation cleavage, for multiple observations at one locality } \ldots \ldots \ldots \ldots \ldots \ldots \ldots \ldots \ldots \ldots \ldots \ldots \ldots \ldots\end{array}$ & A-7-2 & 7.29 \\
\hline 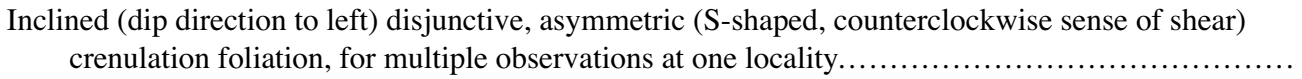 & A-8-4 & 8.3 .38 \\
\hline $\begin{array}{l}\text { Inclined (dip direction to left) disjunctive, asymmetric (Z-shaped, clockwise sense of shear) } \\
\text { crenulation cleavage, for multiple observations at one locality } \ldots \ldots \ldots \ldots \ldots \ldots \ldots \ldots \ldots \ldots \ldots \ldots \ldots \ldots\end{array}$ & A-7-2 & 7.35 \\
\hline 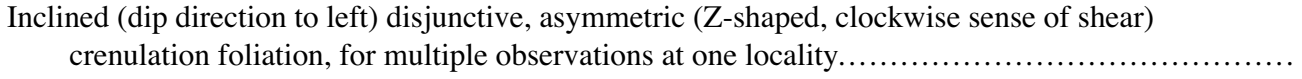 & A-8-4 & 8.3 .44 \\
\hline Inclined (dip direction to left) disjunctive, spaced cleavage, for multiple observations at one locality ... & A-7-1 & 7.17 \\
\hline Inclined (dip direction to left) disjunctive, spaced foliation, for multiple observations at one locality.... & A-8-4 & 8.3 .26 \\
\hline $\begin{array}{l}\text { Inclined (dip direction to left) disjunctive, symmetric crenulation cleavage, for multiple observations } \\
\text { at one locality }\end{array}$ & A-7-1 & 7.23 \\
\hline 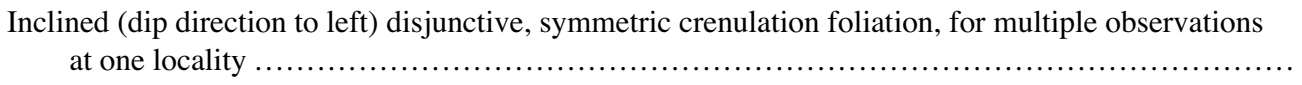 & A-8-4 & 8.3 .32 \\
\hline Inclined (dip direction to left) eutaxitic foliation, for multiple observations at one locality ............. & A-8-2 & 8.2 .23 \\
\hline Inclined (dip direction to left) flow banding in igneous rock, for multiple observations at one locality .. & A-8-1 & 8.2 .6 \\
\hline Inclined (dip direction to left) foliation in igneous rock, for multiple observations at one locality ........ & A-8-1 & 8.2 .6 \\
\hline 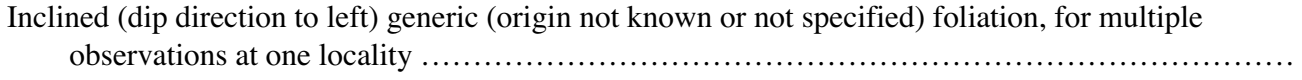 & A-8-1 & 8.1 .5 \\
\hline Inclined (dip direction to left) gneissic layering, for multiple observations at one locality .............. & A-8-5 & 8.3 .50 \\
\hline Inclined (dip direction to left) lamination in igneous rock, for multiple observations at one locality ..... & A-8-1 & 8.2 .6 \\
\hline Inclined (dip direction to left) layering in igneous rock, for multiple observations at one locality ........ & A-8-1 & 8.2 .6 \\
\hline Inclined (dip direction to left) metamorphic foliation, for multiple observations at one locality.......... & A-8-3 & 8.3 .5 \\
\hline Inclined (dip direction to left) mylonitic foliation, for multiple observations at one locality ............ & A-8-5 & 8.3 .59 \\
\hline Inclined (dip direction to left) tectonic foliation, for multiple observations at one locality .............. & A-8-3 & 8.3 .5 \\
\hline Inclined (dip direction to right) bedding, for multiple observations at one locality $\ldots \ldots \ldots \ldots \ldots \ldots \ldots \ldots$ & A-6-1 & 6.6 \\
\hline $\begin{array}{l}\text { Inclined (dip direction to right) bedding, where top direction of beds is known from local features, } \\
\text { for multiple observations at one locality } \ldots \ldots \ldots \ldots \ldots \ldots \ldots \ldots \ldots \ldots\end{array}$ & A-6-1 & 6.17 \\
\hline 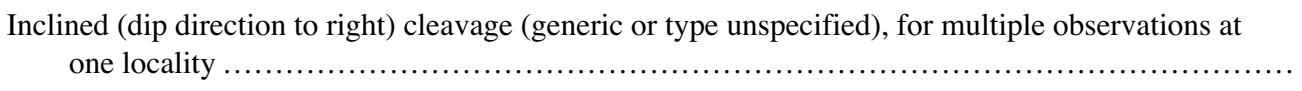 & A-7-1 & 7.4 \\
\hline 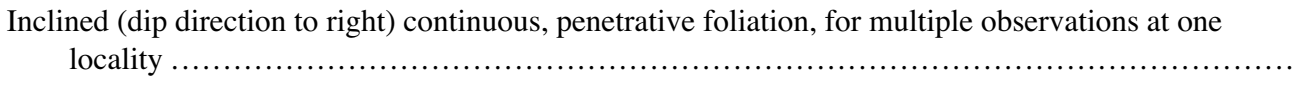 & A-8-3 & 8.3.19 \\
\hline Inclined (dip direction to right) continuous, slaty cleavage, for multiple observations at one locality .... & A-7-1 & 7.10 \\
\hline $\begin{array}{l}\text { Inclined (dip direction to right) disjunctive, asymmetric (S-shaped, counterclockwise sense of shear) } \\
\text { crenulation cleavage, for multiple observations at one locality } \ldots \ldots \ldots \ldots \ldots \ldots \ldots \ldots \ldots \ldots \ldots \ldots\end{array}$ & A-7-2 & 7.28 \\
\hline $\begin{array}{l}\text { Inclined (dip direction to right) disjunctive, asymmetric (S-shaped, counterclockwise sense of shear) } \\
\text { crenulation foliation, for multiple observations at one locality } \ldots \ldots \ldots \ldots \ldots \ldots \ldots \ldots \ldots \ldots \ldots \ldots \ldots \ldots \ldots \ldots \ldots \ldots \ldots\end{array}$ & A-8-4 & 8.3 .37 \\
\hline $\begin{array}{l}\text { Inclined (dip direction to right) disjunctive, asymmetric (Z-shaped, clockwise sense of shear) } \\
\text { crenulation cleavage, for multiple observations at one locality } \ldots \ldots \ldots \ldots \ldots \ldots \ldots \ldots \ldots \ldots \ldots \ldots \ldots \ldots \ldots \ldots\end{array}$ & A-7-2 & 7.34 \\
\hline
\end{tabular}


Inclined (dip direction to right) disjunctive, asymmetric (Z-shaped, clockwise sense of shear)

Inclined (dip direction to right) disjunctive, symmetric crenulation foliation, for multiple

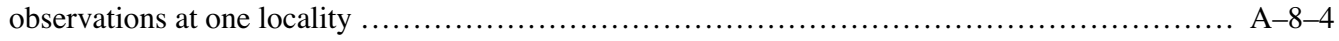

Inclined (dip direction to right) flow banding in igneous rock, for multiple observations at one

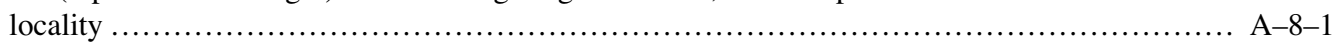

Inclined (dip direction to right) generic (origin not known or not specified) foliation, for multiple observations at one locality

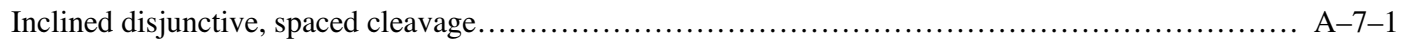

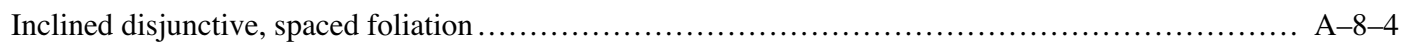

Inclined disjunctive, symmetric crenulation cleavage ....................................... A-7-1

8.3 .23

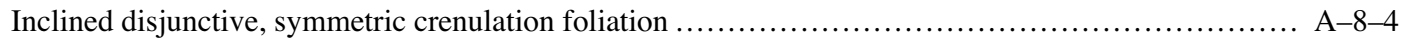

8.3 .29

Inclined drill hole for hydrocarbon exploration or exploitation, showing angle of inclination .......... A-19-6

19.5.15

Inclined drill hole for hydrocarbon exploration or exploitation, showing location of collar and projected trace and bottom of drill hole .............................................. A $-19-6$

Inclined drill hole for hydrocarbon exploration or exploitation, showing surface altitude of collar ...... A-19-6 Inclined drill hole for hydrocarbon exploration or exploitation, showing total depth of drill hole........ A-19-6 Inclined drill hole for mineral exploration, showing angle of inclination............................ A-19-4 Inclined drill hole for mineral exploration, showing location of collar and projected trace and bottom of drill hole .................................................................. A $-19-4$ Inclined drill hole for mineral exploration, showing surface altitude of collar.................... A-19-4 Inclined drill hole for mineral exploration, showing total depth of drill hole ...................... A-19-4 Inclined eutaxitic foliation........................................................... A-8

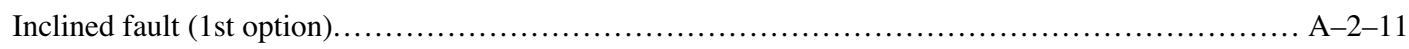

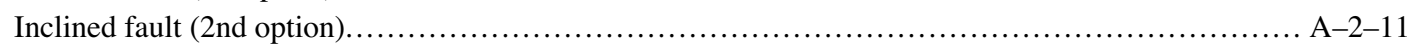

Inclined flow banding in igneous rock.................................................. A-8 -1 


\begin{tabular}{|c|c|c|}
\hline & Page & Ref. No. \\
\hline Inclined fold hinge of small, minor synform (1st option). & A-9-5 & 9.117 \\
\hline Inclined fold hinge of small, minor synform (2nd option).. & A-9-5 & 9.118 \\
\hline Inclined foliation in igneous rock ... & A-8-1 & 8.2 .3 \\
\hline Inclined foliation in igneous rock, for multiple observations at one locality. & A- $-8-1$ & 8.2 .5 \\
\hline Inclined generic (origin not known or not specified) foliation ................. & A- $-8-1$ & 8.1 .2 \\
\hline Inclined generic (origin or type not known or not specified) lineation or linear structure (1st option).. & A-9-1 & 9.3 \\
\hline Inclined generic (origin or type not known or not specified) lineation or linear structure (2nd option). & A-9-1 & 9.4 \\
\hline Inclined gneissic layering & A-8-5 & 8.3.47 \\
\hline Inclined graded bedding ... & A-6-2 & 6.27 \\
\hline Inclined groove in sedimentary materials (1st option). & A-9-1 & 9.13 \\
\hline Inclined groove in sedimentary materials (2nd option). & A-9-1 & 9.14 \\
\hline Inclined groove on fault surface (1st option) .............. & A-9-1 & 9.17 \\
\hline Inclined groove on fault surface (2nd option) & A-9-1 & 9.18 \\
\hline Inclined joint (1st option) $\ldots \ldots \ldots \ldots \ldots \ldots \ldots \ldots$ & A-4-1 & 4.2 .3 \\
\hline Inclined joint (2nd option).. & A-4-1 & 4.2.4 \\
\hline Inclined key bed (1st option).. & A-1-6 & 1.4 .1 \\
\hline Inclined key bed (2nd option).. & A-1-6 & 1.4 .2 \\
\hline Inclined lamination in igneous rock.. & $\mathrm{A}-8-1$ & 8.2 .3 \\
\hline Inclined lamination in igneous rock, for multiple observations at one locality & $\mathrm{A}-8-1$ & 8.2 .5 \\
\hline Inclined layering in igneous rock... & A-8-1 & 8.2 .3 \\
\hline Inclined layering in igneous rock, for multiple observations at one locality & $\mathrm{A}-8-1$ & 8.2 .5 \\
\hline Inclined lineation at intersection of bedding and cleavage (1st option). & A-9-4 & 9.77 \\
\hline Inclined lineation at intersection of bedding and cleavage ( 2 nd option). & A-9-4 & 9.78 \\
\hline Inclined lineation at intersection of two cleavages (1st option).. & A-9-4 & 9.81 \\
\hline Inclined lineation at intersection of two cleavages (2nd option). & A-9-4 & 9.82 \\
\hline Inclined lineation at intersection of two foliations (1st option). & A-9-4 & 9.89 \\
\hline Inclined lineation at intersection of two foliations (2nd option) .. & A-9-4 & 9.90 \\
\hline Inclined lineation at intersection of two fractures or joints (1st option)... & A-9-4 & 9.85 \\
\hline Inclined lineation at intersection of two fractures or joints (2nd option).. & A-9-4 & 9.86 \\
\hline Inclined lineation at intersection of two surfaces (origin or type unspecified) (1st option). & A-9-4 & 9.93 \\
\hline Inclined lineation at intersection of two surfaces (origin or type unspecified) (2nd option) ..... & A-9-4 & 9.94 \\
\hline Inclined metamorphic foliation... & A-8-3 & 8.3.2 \\
\hline Inclined metamorphic foliation parallel to bedding. & A-8-3 & 8.3 .8 \\
\hline Inclined metamorphic foliation parallel to overturned bedding. & A-8-3 & 8.3 .10 \\
\hline $\begin{array}{l}\text { Inclined metamorphic foliation parallel to overturned bedding, where top direction of beds is known } \\
\text { from local features }\end{array}$ & A-8-3 & 8.3.13 \\
\hline $\begin{array}{l}\text { Inclined metamorphic foliation parallel to upright bedding, where top direction of beds is known } \\
\text { from local features. }\end{array}$ & A-8-3 & 8.3.11 \\
\hline 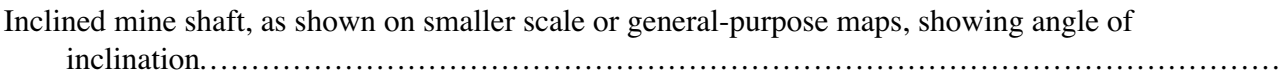 & A-19-4 & 19.3.39 \\
\hline 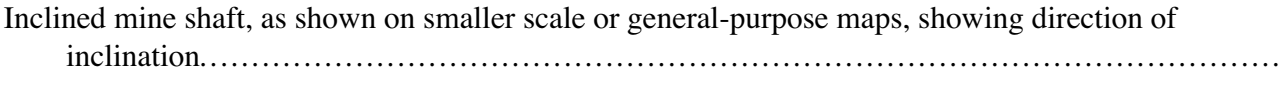 & A-19-4 & 19.3.38 \\
\hline $\begin{array}{l}\text { Inclined mine shaft at surface (drawn to scale), as shown on subsurface exploration maps, showing } \\
\text { angle of inclination }\end{array}$ & A-19-5 & 19.4.3 \\
\hline $\begin{array}{l}\text { Inclined mine shaft at surface (drawn to scale), as shown on subsurface exploration maps, showing } \\
\text { direction of inclination }\end{array}$ & A-19-5 & 19.4.2 \\
\hline Inclined mineralized stringer (1st option). & A-19-1 & 19.1.8 \\
\hline Inclined mineralized stringer (2nd option). & A-19-1 & 19.1.9 \\
\hline Inclined mullions (1st option) ............... & A-9-3 & 9.65 \\
\hline Inclined mullions (2nd option).. & A-9-3 & 9.66 \\
\hline Inclined mylonitic foliation ........... & A-8-5 & 8.3.56 \\
\hline Inclined parting lineation in sedimentary materials (1st option).. & A-9-1 & 9.9 \\
\hline Inclined parting lineation in sedimentary materials (2nd option). & A-9-1 & 9.10 \\
\hline Inclined pencil structure (1st option) $\ldots \ldots \ldots \ldots \ldots \ldots \ldots \ldots \ldots \ldots \ldots \ldots \ldots \ldots \ldots \ldots \ldots \ldots$ & A-9-4 & 9.73 \\
\hline Inclined pencil structure (2nd option).. & A-9-4 & 9.74 \\
\hline
\end{tabular}




\begin{tabular}{|c|c|c|}
\hline & Page & Ref. No. \\
\hline Inclined rodding (1st option)... & A-9-3 & 9.61 \\
\hline 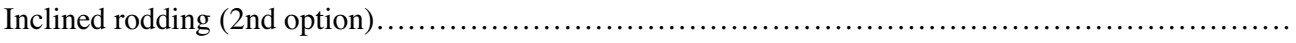 & A-9-3 & 9.62 \\
\hline Inclined scour mark in sedimentary materials (1st option).. & A-9-1 & 9.13 \\
\hline Inclined scour mark in sedimentary materials (2nd option) ... & A-9-1 & 9.14 \\
\hline Inclined slickenline on fault surface (1st option).............. & A-9-1 & 9.17 \\
\hline Inclined slickenline on fault surface (2nd option).... & A-9-1 & 9.18 \\
\hline Inclined sole mark in sedimentary materials (1st option) ...... & A-9-1 & 9.13 \\
\hline Inclined sole mark in sedimentary materials (2nd option) ........... & A-9-1 & 9.14 \\
\hline Inclined striation (origin not known or not specified) (1st option). & A-9-1 & 9.21 \\
\hline Inclined striation (origin not known or not specified) (2nd option). & A-9-1 & 9.22 \\
\hline Inclined striation on fault surface (1st option) $\ldots \ldots \ldots \ldots \ldots \ldots \ldots \ldots \ldots \ldots \ldots \ldots \ldots$ & A-9-1 & 9.17 \\
\hline Inclined striation on fault surface (2nd option). & A-9-1 & 9.18 \\
\hline Inclined surface groove (origin not known or not specified) (1st option).. & A-9-1 & 9.21 \\
\hline Inclined surface groove (origin not known or not specified) (2nd option). & A-9-1 & 9.22 \\
\hline Inclined symmetric minor fold hinge (1st option)... & A-9-6 & 9.121 \\
\hline Inclined symmetric minor fold hinge (2nd option).. & A-9-6 & 9.122 \\
\hline Inclined tectonic foliation $\ldots \ldots \ldots \ldots \ldots \ldots \ldots \ldots \ldots \ldots$ & A-8-3 & 8.3.2 \\
\hline Inclined tectonic foliation parallel to bedding. & A-8-3 & 8.3 .8 \\
\hline Inclined tectonic foliation parallel to overturned bedding & A-8-3 & 8.3 .10 \\
\hline $\begin{array}{l}\text { Inclined tectonic foliation parallel to overturned bedding, where top direction of beds is known from } \\
\text { local features }\end{array}$ & A-8-3 & 8.3.13 \\
\hline Inclined tectonic foliation parallel to upright bedding, where top direction of beds is known from & $A-8-3$ & 8.3.11 \\
\hline 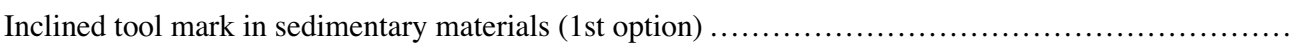 & A-9-1 & 9.13 \\
\hline Inclined tool mark in sedimentary materials (2nd option). & A-9-1 & 9.14 \\
\hline Inclined undulatory bedding .. & A-6-2 & 6.25 \\
\hline Inclined undulatory gneissic layering... & A-8-5 & 8.3 .53 \\
\hline Inclined vein (1st option) ...... & A-19-1 & 19.1.8 \\
\hline Inclined vein (2nd option) ... & A-19-1 & 19.1.9 \\
\hline Inclined veinlet (1st option)...... & A-19-1 & 19.1.8 \\
\hline Inclined veinlet (2nd option) ..... & A-19-1 & 19.1.9 \\
\hline Inclined warped bedding.............. & A-6-2 & 6.25 \\
\hline Inclined workings, as shown on subsurface exploration maps (drawn to scale).. & A-19-5 & 19.4.12 \\
\hline 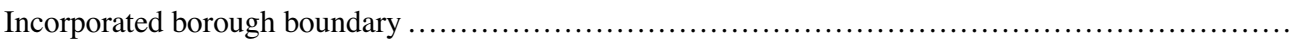 & A-29-1 & 29.5 \\
\hline Incorporated city boundary... & A-29-1 & 29.5 \\
\hline Incorporated hamlet boundary .. & A-29-1 & 29.5 \\
\hline Incorporated town boundary ... & A-29-1 & 29.5 \\
\hline Incorporated village boundary ... & A-29-1 & 29.5 \\
\hline Indefinite shoreline.. & A $-30-5$ & 30.2 .30 \\
\hline Index bathymetric contour.. & A $-30-2$ & 30.1 .27 \\
\hline Index bathymetric contour-Approximate.. & A $-30-2$ & 30.1 .28 \\
\hline Index bathymetric depression contour.. & A $-30-2$ & 30.1 .37 \\
\hline Index bathymetric rise contour (inside depression). & A-30-2 & 30.1 .38 \\
\hline Index contour on glacier or permanent snowfield... & A $-30-3$ & 30.1 .45 \\
\hline Index contour on glacier or permanent snowfield-Approximate or indefinite & A-30-3 & 30.1 .46 \\
\hline Index depression contour on glacier or permanent snowfield. & A $-30-3$ & 30.1 .49 \\
\hline Index primary bathymetric contour. & A $-30-2$ & 30.1 .21 \\
\hline Index primary bathymetric contour-Approximate & A-30-2 & 30.1 .22 \\
\hline Index primary bathymetric depression contour.. & A $-30-2$ & 30.1 .31 \\
\hline Index primary bathymetric rise contour (inside depression). & A $-30-2$ & 30.1 .32 \\
\hline 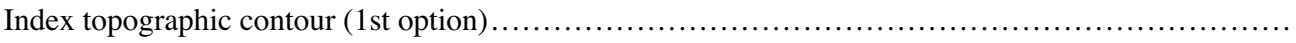 & A-30-1 & 30.1 .1 \\
\hline Index topographic contour (1st option)-Approximate or indefinite.... & A-30-1 & 30.1 .2 \\
\hline 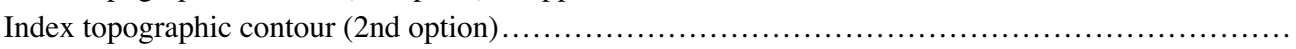 & A-30-1 & 30.1 .11 \\
\hline Index topographic contour (2nd option) $-\mathrm{A}_{\mathrm{l}}$ & A-30-1 & 30.1 .12 \\
\hline Index topographic depression contour & A $-30-1$ & 30.1 .7 \\
\hline
\end{tabular}




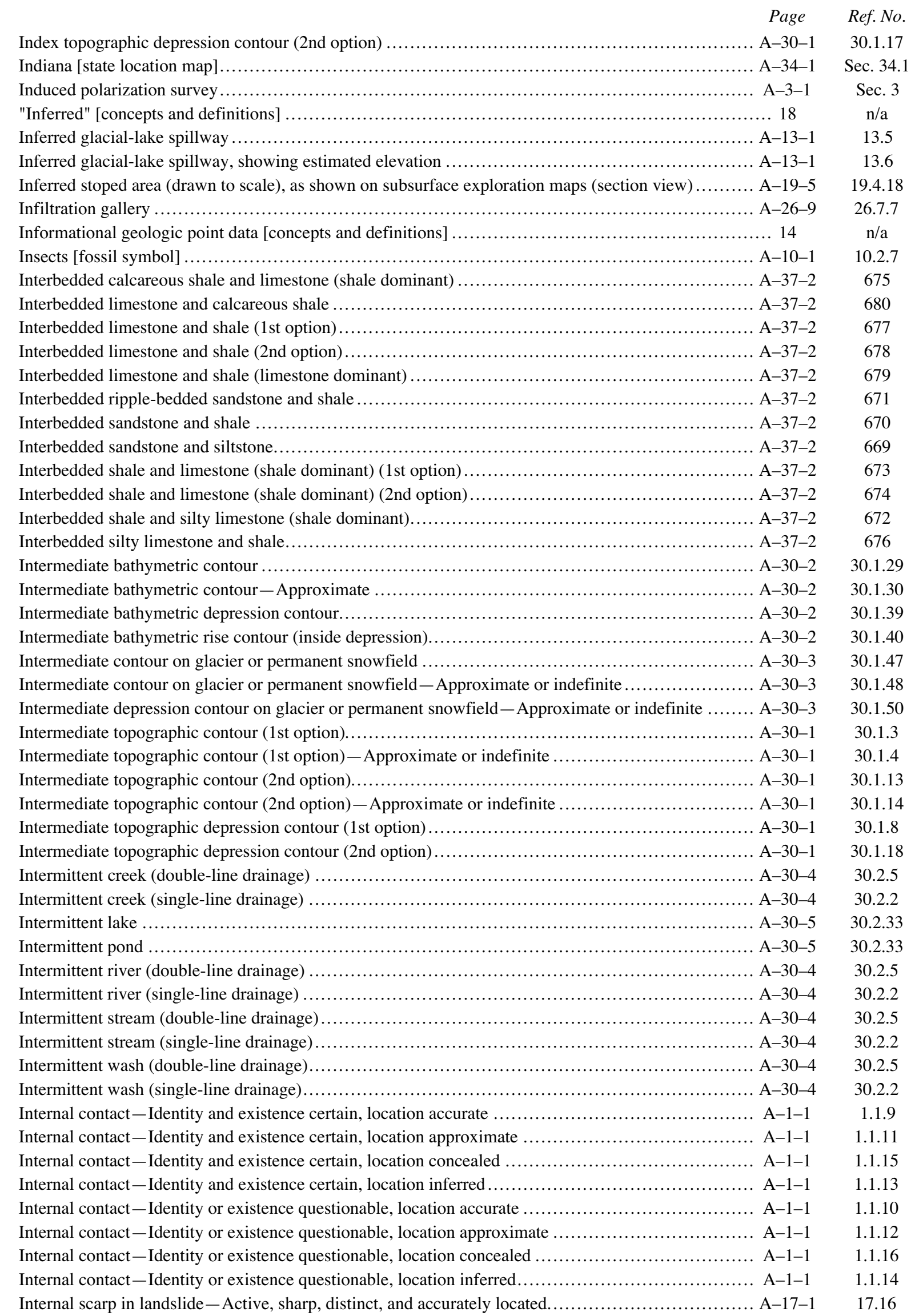




\begin{tabular}{|c|c|}
\hline Page & Ref. No. \\
\hline Internal scarp in landslide - Inactive, subdued, indistinct, and (or) approximately located............. A-17-1 & 17.17 \\
\hline 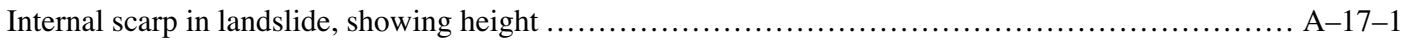 & 17.18 \\
\hline 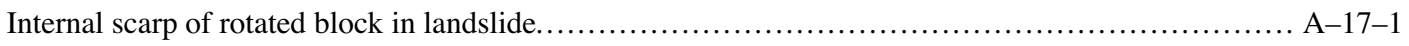 & 17.19 \\
\hline Internal thrust fault in landslide - Active, sharp, distinct, and accurately located................. & 17.22 \\
\hline Internal thrust fault in landslide - Inactive, subdued, indistinct, and (or) approximately located ........ A-17-2 & 17.23 \\
\hline 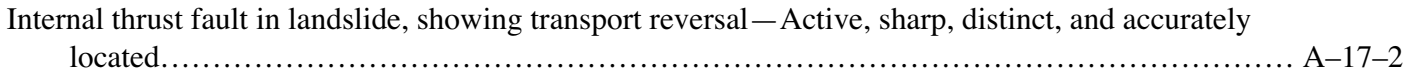 & 17.24 \\
\hline 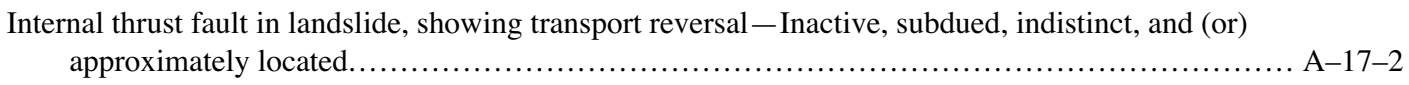 & 17.25 \\
\hline Intersection lineations & 9.77 \\
\hline Intersection of bedding and cleavage ....... & 9.77 \\
\hline Intersection of two cleavages .................. & 9.81 \\
\hline Intersection of two foliations..................... & 9.89 \\
\hline Intersection of two fractures ............. & 9.85 \\
\hline Intersection of two joints $\ldots \ldots \ldots \ldots \ldots \ldots \ldots \ldots \ldots \ldots \ldots \ldots$ & 9.85 \\
\hline Intersection of two surfaces (origin or type unspecified) $\ldots \ldots \ldots \ldots \ldots \ldots \ldots \ldots \ldots \ldots$ & 9.93 \\
\hline Intersection of workings (drawn to scale), as shown on subsurface exploration maps................ A-19-5 & 19.4 .9 \\
\hline 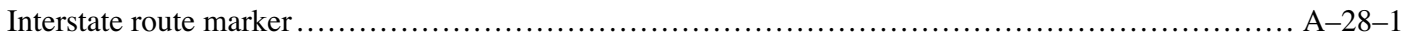 & 28.16 \\
\hline Introductory material $[$ of this standard $] \ldots \ldots \ldots \ldots \ldots$ & $\mathrm{n} / \mathrm{a}$ \\
\hline Inundated land ................................ & 30.2 .35 \\
\hline A-10-1 & 10.2 .2 \\
\hline Inverted anticline (1st option) - Identity and existence certain, location accurate $\ldots \ldots \ldots \ldots \ldots \ldots \ldots \ldots$ A-5-4 & 5.3 .33 \\
\hline Inverted anticline (1st option) - Identity and existence certain, location approximate $\ldots \ldots \ldots \ldots \ldots \ldots \ldots$ A $-5-4$ & 5.3 .35 \\
\hline Inverted anticline (1st option) - Identity and existence certain, location concealed $\ldots \ldots \ldots \ldots \ldots \ldots \ldots \ldots$ A $-5-4$ & 5.3 .39 \\
\hline 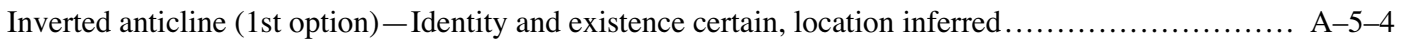 & 5.3 .37 \\
\hline Inverted anticline (1st option) - Identity or existence questionable, location accurate $\ldots \ldots \ldots \ldots \ldots \ldots$ & 5.3 .34 \\
\hline Inverted anticline (1st option) - Identity or existence questionable, location approximate ............ A-5-4 & 5.3 .36 \\
\hline Inverted anticline (1st option) - Identity or existence questionable, location concealed ............... A-5-4 & 5.3 .40 \\
\hline 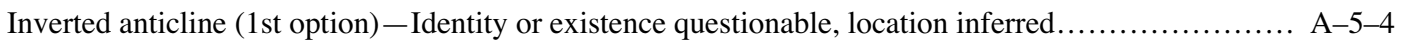 & 5.3 .38 \\
\hline Inverted anticline (2nd option) - Identity and existence certain, location accurate $\ldots \ldots \ldots \ldots \ldots \ldots \ldots \ldots$ A $-5-4$ & 5.3 .41 \\
\hline Inverted anticline (2nd option) - Identity and existence certain, location approximate $\ldots \ldots \ldots \ldots \ldots \ldots$ A $-5-4$ & 5.3 .43 \\
\hline Inverted anticline (2nd option) - Identity and existence certain, location concealed $\ldots \ldots \ldots \ldots$ & 5.3 .47 \\
\hline Inverted anticline (2nd option) - Identity and existence certain, location inferred $\ldots \ldots \ldots \ldots \ldots \ldots \ldots \ldots \ldots \ldots \ldots \ldots \ldots$ & 5.3 .45 \\
\hline Inverted anticline (2nd option) - Identity or existence questionable, location accurate $\ldots \ldots \ldots \ldots \ldots \ldots \ldots$ A $-5-4$ & 5.3 .42 \\
\hline Inverted anticline (2nd option) - Identity or existence questionable, location approximate ............. A-5-4 & 5.3 .44 \\
\hline Inverted anticline (2nd option) - Identity or existence questionable, location concealed ............... & 5.3 .48 \\
\hline Inverted anticline (2nd option) - Identity or existence questionable, location inferred................. & 5.3 .46 \\
\hline 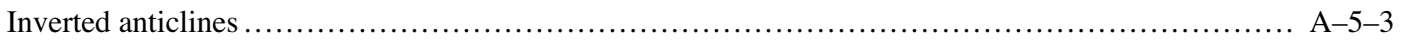 & Sec. 5.3 \\
\hline Inverted syncline (1st option) - Identity and existence certain, location accurate $\ldots \ldots \ldots \ldots \ldots$. & 5.7 .33 \\
\hline Inverted syncline (1st option) - Identity and existence certain, location approximate $\ldots \ldots \ldots \ldots \ldots \ldots \ldots \ldots \ldots$ & 5.7 .35 \\
\hline Inverted syncline (1st option) - Identity and existence certain, location concealed............... & 5.7 .39 \\
\hline Inverted syncline (1st option) - Identity and existence certain, location inferred ................ & 5.7 .37 \\
\hline Inverted syncline (1st option) - Identity or existence questionable, location accurate................ & 5.7 .34 \\
\hline Inverted syncline (1st option) - Identity or existence questionable, location approximate............. A-5-9 & 5.7 .36 \\
\hline Inverted syncline (1st option) - Identity or existence questionable, location concealed............... A-5-9 & 5.7 .40 \\
\hline Inverted syncline (1st option) - Identity or existence questionable, location inferred $\ldots \ldots \ldots \ldots \ldots \ldots \ldots$ A $-5-9$ & 5.7 .38 \\
\hline Inverted syncline (2nd option) - Identity and existence certain, location accurate $\ldots \ldots \ldots \ldots \ldots \ldots \ldots \ldots$ A $-5-9$ & 5.7 .41 \\
\hline Inverted syncline (2nd option) - Identity and existence certain, location approximate $\ldots \ldots \ldots \ldots \ldots \ldots \ldots$ A $-5-9$ & 5.7 .43 \\
\hline Inverted syncline (2nd option) - Identity and existence certain, location concealed............ & 5.7 .47 \\
\hline 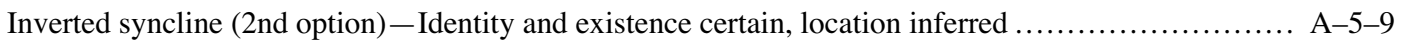 & 5.7 .45 \\
\hline Inverted syncline (2nd option) - Identity or existence questionable, location accurate $\ldots \ldots \ldots \ldots \ldots \ldots \ldots$ A $-5-9$ & 5.7 .42 \\
\hline Inverted syncline (2nd option) - Identity or existence questionable, location approximate............ A-5-9 & 5.7 .44 \\
\hline Inverted syncline (2nd option) - Identity or existence questionable, location concealed.... & 5.7 .48 \\
\hline Inverted syncline (2nd option) - Identity or existence questionable, location inferred ........ & 5.7 .46 \\
\hline 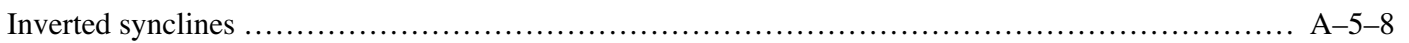 & Sec. 5.7 \\
\hline
\end{tabular}


Iowa [state location map].

Joint-Identity and existence certain, location accurate

Joint-Identity and existence certain, location approximate .

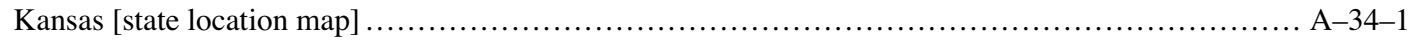

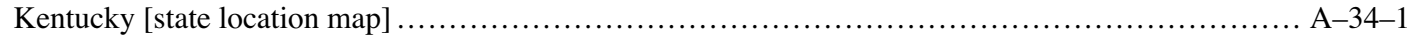

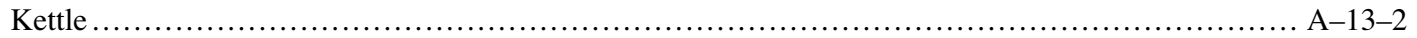

Sec. 34.1

Key bed-Identity and existence certain, location accurate

Key bed-Identity and existence certain, location approximate.

Key bed-Identity and existence certain, location concealed

A-1-3

1.2 .1

Key bed-Identity and existence certain, location inferred.

Key bed-Identity or existence questionable, location accurate

Key bed-Identity or existence questionable, location approximate

Key bed-Identity or existence questionable, location concealed....

Key bed-Identity or existence questionable, location inferred

Key bed outcrop area (2nd option)

Key bed, showing thickness and location where thickness was measured........................ A-1-6

Key beds

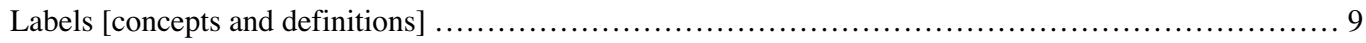

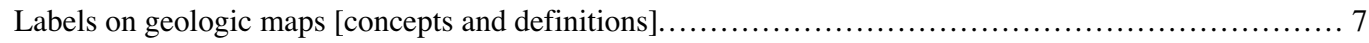

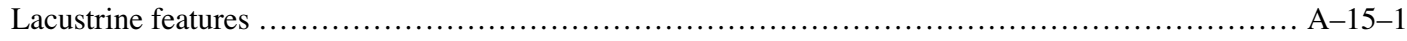

Lagging along drift (drawn to scale), as shown on subsurface exploration maps.

Lakes

Lamination in igneous rock

Lamination in igneous rock, for multiple observations at one locality .......................... A-8 -1

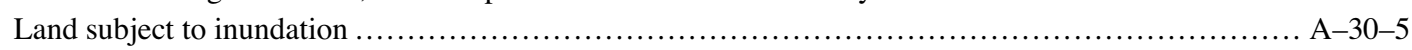

Landmark object, as shown on topographic maps or on general-purpose or smaller scale maps......... A-30-6

Landslide deposits, showing direction of downslope movement .............................. A-17-1

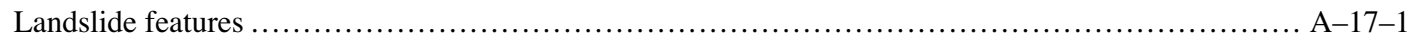

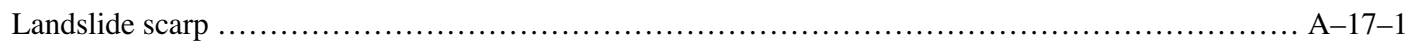

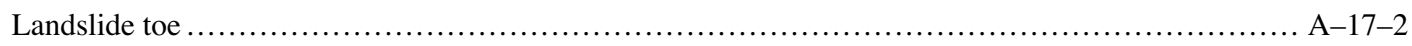

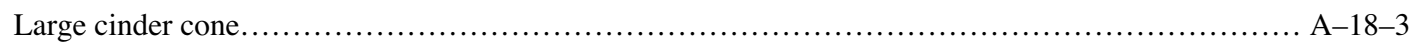

\section{$\mathrm{n} / \mathrm{a}$}

$\mathrm{n} / \mathrm{a}$

$$
\text { n/a }
$$

Sec. 15

19.4.14

Sec. 30.2

8.2.2

8.2 .5

30.2.35

30.3.7

17.11

Sec. 17

Sec. 17

Sec. 17

18.56

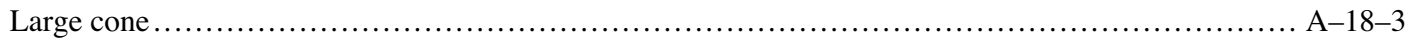

18.56

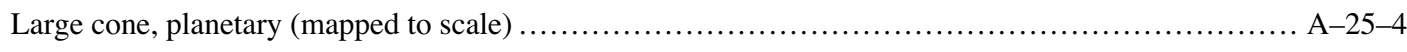

25.81

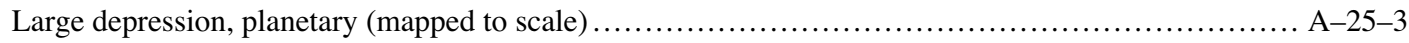

25.54

Large dome, planetary (mapped to scale). 
. A-25-4

Large endogenic crater, planetary (mapped to scale) ....................................... A-25-5

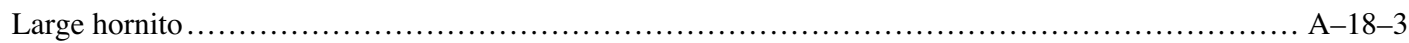

Large shield, planetary (mapped to scale) ............................................... A-25-4

Large spatter cone ................................................................... A $-18-3$

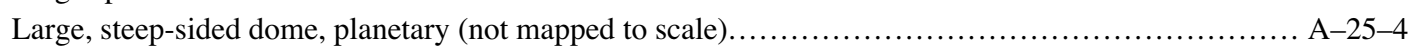

Large, steep-sided shield, planetary (not mapped to scale) ......................................... A-25-4

Large, steep-sided volcanic construct, planetary (not mapped to scale) .......................... A-25-4

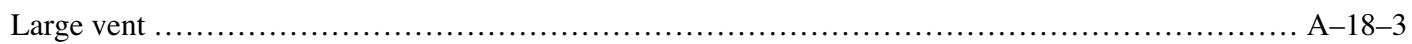

Large volcanic construct, planetary (mapped to scale) ...................................... A-25-4

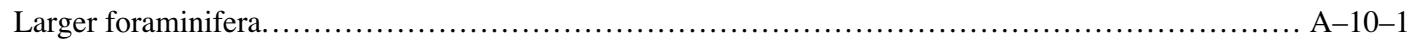

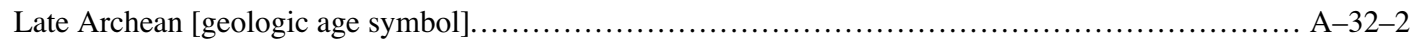

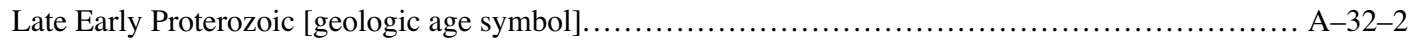

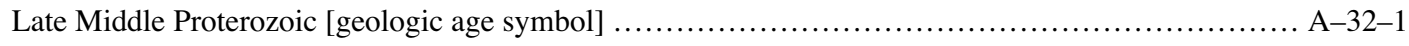

Late Proterozoic $[$ geologic age symbol] ................................................ A $-32-1$

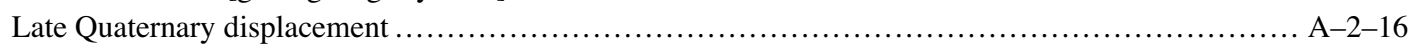

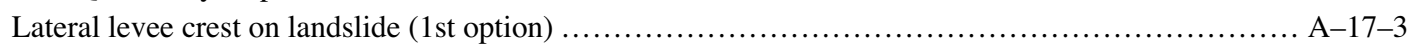

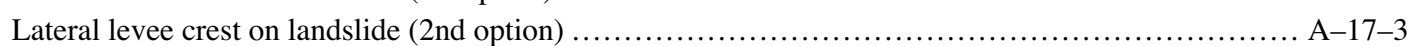

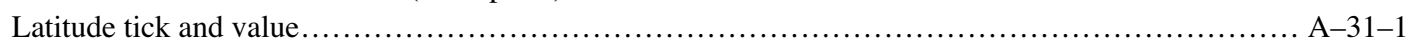

Lava-flow front-Identity and existence certain, location accurate ............................. A-18-2

Lava-flow front-Identity and existence certain, location approximate ............................ A-18-2

Lava-flow front-Identity and existence certain, location concealed ............................. A-18-2

Lava-flow front - Identity or existence questionable, location accurate ......................... A-18-2

Lava-flow front - Identity or existence questionable, location approximate ........................ A-18-2

Lava-flow front - Identity or existence questionable, location concealed ........................ A-18-2

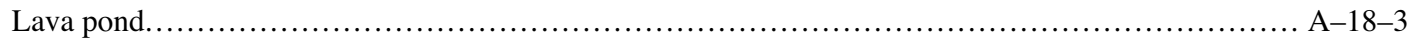

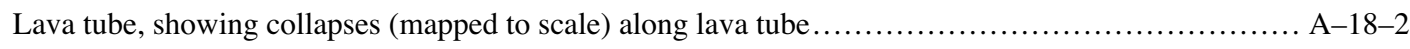

Lava tube, showing skylights (not mapped to scale) along lava tube ........................... A-18-2

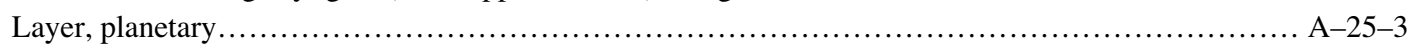

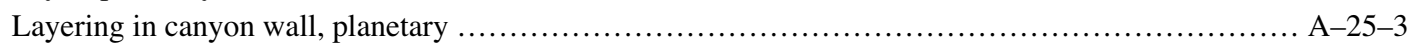

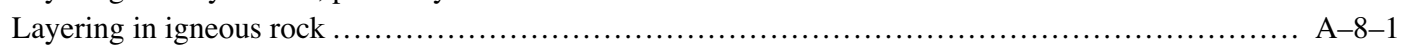

Layering in igneous rock, for multiple observations at one locality ............................. A-8-1

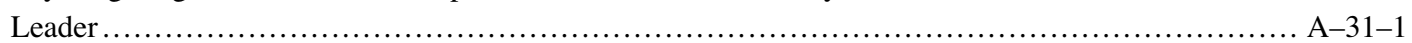

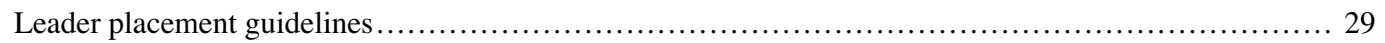

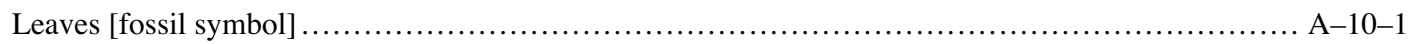

Left flank of landslide - Active, sharp, distinct, and accurately located ............................ A-17-2

Left flank of landslide -Concealed by landslide deposits or debris materials ........................ A-17-2

Left flank of landslide - Inactive, subdued, indistinct, and (or) approximately located ............... A-17-2

Left flank of landslide, showing amount of offset ...................................... A $-17-2$

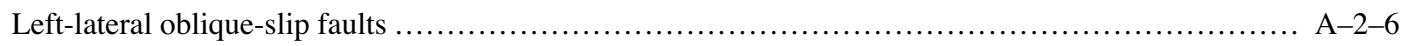

Left-lateral shear feature in landslide-Active, sharp, distinct, and accurately located ............... A-17-2

Left-lateral shear feature in landslide-Concealed by landslide deposits or debris materials ............ A-17-2

Left-lateral shear feature in landslide - Inactive, subdued, indistinct, and (or) approximately located.... A-17-2

Left-lateral shear feature in landslide, showing amount of offset............................... A-17-2

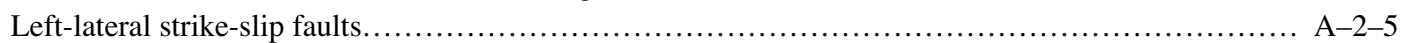

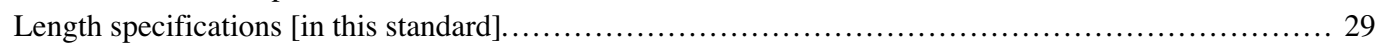

Levels of locational accuracy [concepts and definitions] ....................................... 23

Levels of scientific confidence [concepts and definitions]..................................... 16

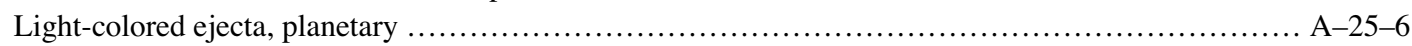

Light-duty road, composition unspecified (Class 3$)$.......................................... A-28-1

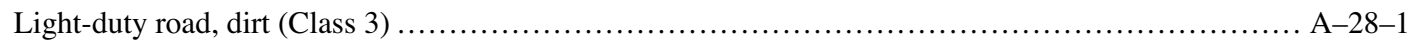

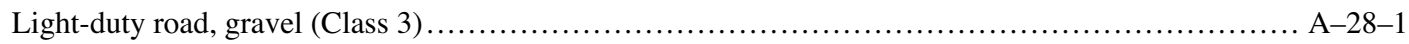

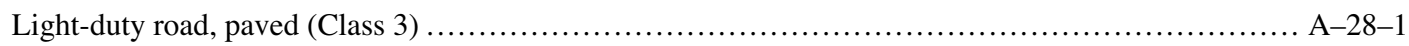

Limestone.
25.87

25.109

18.58

25.80

18.56

25.78

25.78

25.78

18.56

25.80

10.2.49

32.31

32.27

32.23

32.21

2.13 .3

17.44

17.45

31.9

18.32

18.34

18.36

18.33

18.35

18.37

18.54

18.45

18.44

25.62

25.64

8.2 .2

8.2 .5

31.11

n/a

10.2.32

17.30

17.32

17.31

17.33

Sec. 2.7

17.30

17.32

17.31

17.33

Sec. 2.6

$\mathrm{n} / \mathrm{a}$

$\mathrm{n} / \mathrm{a}$

$\mathrm{n} / \mathrm{a}$

25.119

28.12

28.11

28.10

28.9

627 


\begin{tabular}{|c|c|c|}
\hline Limestone, irregular (burrow?) fillings of saccharoidal dolomite . & $\begin{array}{c}\text { Page } \\
\text { A-37-1 }\end{array}$ & $\begin{array}{c}\text { Ref. No. } \\
\quad 631\end{array}$ \\
\hline 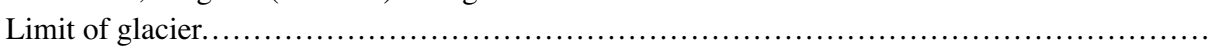 & A-13-3 & Sec. 13 \\
\hline Limit of significant glacial advance-Identity and existence certain, location accurate.. & A-13-3 & 13.58 \\
\hline Limit of significant glacial advance-Identity and existence certain, location approximate.. & A-13-3 & 13.60 \\
\hline Limit of significant glacial advance-Identity and existence certain, location concealed..... & A-13-3 & 13.62 \\
\hline Limit of significant glacial advance-Identity or existence questionable, location accurate .... & A-13-3 & 13.59 \\
\hline Limit of significant glacial advance-Identity or existence questionable, location approximate & A-13-3 & 13.61 \\
\hline Limit of significant glacial advance-Identity or existence questionable, location concealed ... & A-13-3 & 13.63 \\
\hline Limit of subsidence caused by shock - Identity and existence certain, location accurate ....... & A-21-1 & 21.16 \\
\hline Limit of subsidence caused by shock-Identity and existence certain, location concealed.. & A-21-1 & 21.22 \\
\hline Limit of subsidence caused by shock-Identity or existence certain, location approximate. & A-21-1 & 21.18 \\
\hline Limit of subsidence caused by shock-Identity or existence certain, location inferred ....... & A-21-1 & 21.20 \\
\hline Limit of subsidence caused by shock - Identity or existence questionable, location accurate..... & A-21-1 & 21.17 \\
\hline Limit of subsidence caused by shock-Identity or existence questionable, location approximate. & A-21-1 & 21.19 \\
\hline Limit of subsidence caused by shock-Identity or existence questionable, location concealed.... & A-21-1 & 21.23 \\
\hline Limit of subsidence caused by shock - Identity or existence questionable, location inferred ... & A-21-1 & 21.21 \\
\hline Limonite .. & A-37-2 & 664 \\
\hline Lim & A-37-1 & 641 \\
\hline Lim & A $-37-1$ & 641 \\
\hline oe precinitation (annual monthly daily etc) [datel_-Accurately located & A-26-8 & 26.6.1 \\
\hline Line of average precipitation (an & A-26-8 & 26.6.2 \\
\hline Line of equal chemical-constitue & A-26-8 & 26.6.11 \\
\hline [date] A nnroximately located & A-26-8 & 26.6.12 \\
\hline denth to ouven [dotal & A-26-8 & 26.6 .3 \\
\hline ifor [dotol $\Delta$ ond & A-26-8 & 26.6.4 \\
\hline Line of & A-26-8 & 26.6.3 \\
\hline al depth to bedrock [date]-Approximatel & A-26-8 & 26.6 .4 \\
\hline al depth to geologic formation [date]-Accurately located........ & A-26-8 & 26.6.3 \\
\hline Line of equal depth to geologic formation [date]-Approximately located.. & A-26-8 & 26.6 .4 \\
\hline 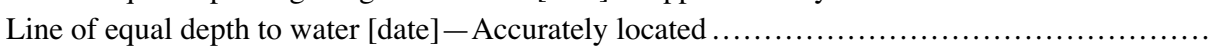 & A-26-8 & 26.6 .3 \\
\hline al depth to water [date]-Approximately located. & A-26-8 & 26.6 .4 \\
\hline Line of equal dissolved-solids concentration [date]-Accurately located....... & A-26-8 & 26.6.11 \\
\hline Line of equal dissolved-solids concentration [date]-Approximately located. & A-26-8 & 26.6.12 \\
\hline 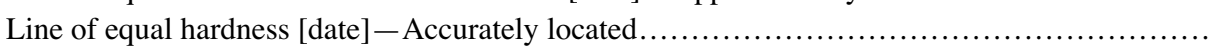 & A-26-8 & 26.6.11 \\
\hline 1 hardness [date]-Approximately located..... & A-26-8 & 26.6.12 \\
\hline al hydraulic conductivity - Accurately located ...... & A-26-8 & 26.6.17 \\
\hline Line of equal hydraulic conductivity-Approximately located. & A-26-8 & 26.6.18 \\
\hline al porosity - Accurately located ..................... & A-26-8 & 26.6.17 \\
\hline Line of equal porosity - Approximately located .................... & A-26-8 & 26.6.18 \\
\hline Line of equal precipitation (annual, monthly, daily, etc.) [date]-Accurately located... & A-26-8 & 26.6 .1 \\
\hline Line of equal precipitation (annual, monthly, daily, etc.) [date]-Approximately located.. & A-26-8 & 26.6 .2 \\
\hline 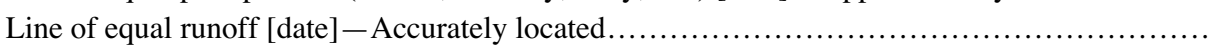 & A-26-8 & 26.6.15 \\
\hline al runoff [date]-Approximately located... & A-26-8 & 26.6.16 \\
\hline Line of equal specific conductance $[$ date $]-$ Accurately located ....... & A-26-8 & 26.6 .9 \\
\hline Line of equal specific conductance [date]-Approximately located.. & A-26-8 & 26.6.10 \\
\hline Line of equal thickness of aquifer [date]-Accurately located........ & A-26-8 & 26.6 .5 \\
\hline Line of equal thickness of aquifer [date]-Approximately located... & A-26-8 & 26.6 .6 \\
\hline Line of equal thickness of confining bed [date]-Accurately located......... & A-26-8 & 26.6 .5 \\
\hline Line of equal thickness of confining bed [date]-Approximately located..... & A-26-8 & 26.6 .6 \\
\hline Line of equal thickness of geologic formation [date]-Accurately located ......... & A-26-8 & 26.6 .5 \\
\hline Line of equal thickness of geologic formation [date]-Approximately located ...... & A-26-8 & 26.6 .6 \\
\hline Line of equal thickness of saturated material [date]-Accurately located............. & A-26-8 & 26.6 .5 \\
\hline Line of equal thickness of saturated material [date]-Approximately located.... & A-26-8 & 26.6 .6 \\
\hline 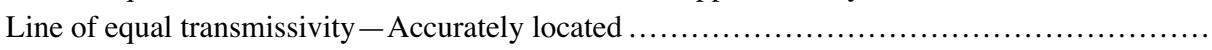 & & 26.6.17 \\
\hline
\end{tabular}




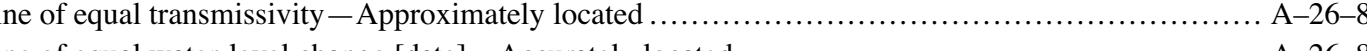

Line of equal water-level change [date]-Accurately located.................................. A-26-8

Line of equal water-level change [date] - Approximately located............................... A-26-8

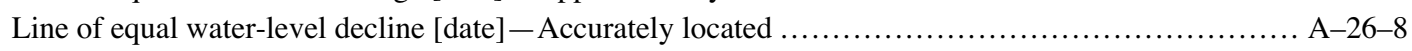

Line of equal water-level decline [date]-Approximately located ............................... A-26-8

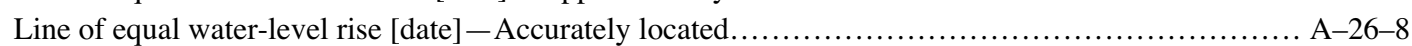

Line of equal water-level rise [date] - Approximately located.................................... A-26-8

Line of equal water temperature $[$ date $]$-Accurately located....................................... A-26-8

Line of equal water temperature $[$ date $]-$ Approximately located................................. A-26-8

Line of mean precipitation (annual, monthly, daily, etc.) [date]-Accurately located.................. A-26-8

Line of mean precipitation (annual, monthly, daily, etc.) [date]-Approximately located.............. A-26-8

Line of median precipitation (annual, monthly, daily, etc.) [date]-Accurately located ................ A-26-8

Line of median precipitation (annual, monthly, daily, etc.) [date]-Approximately located ............ A-26-8

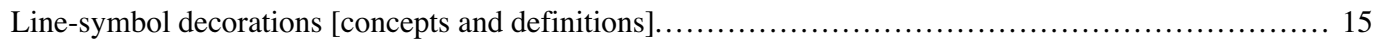

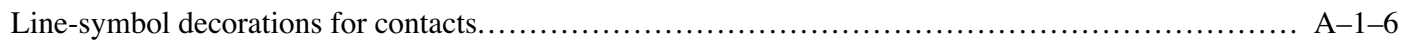

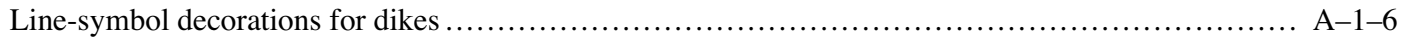

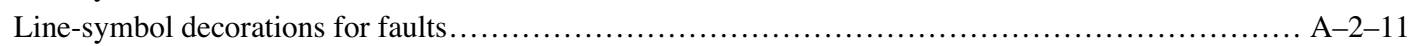

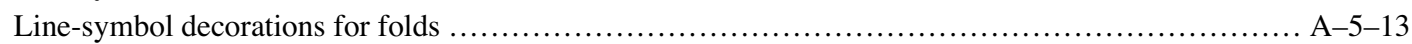

Line-symbol decorations for key beds ................................................... A-1-6

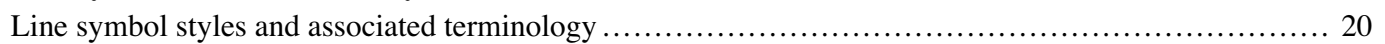

Line symbols for linear geologic features [concepts and definitions] .............................. 13

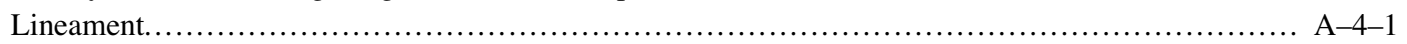

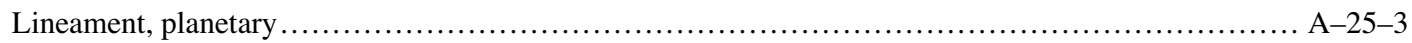

Lineament, showing name ............................................................... A

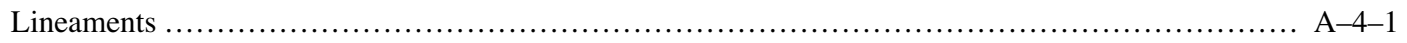

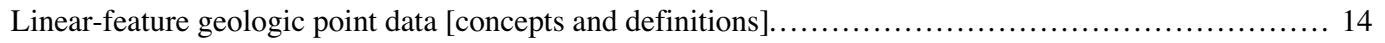

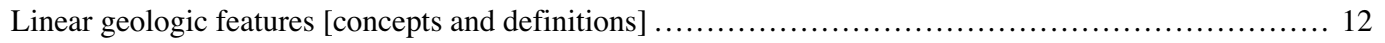

Lineation ........................................................................... A

Lineation at intersection of bedding and cleavage ............................................. A

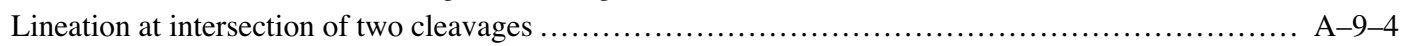

Lineation at intersection of two foliations..................................................... A

Lineation at intersection of two fractures or joints............................................ A

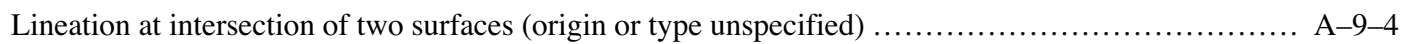

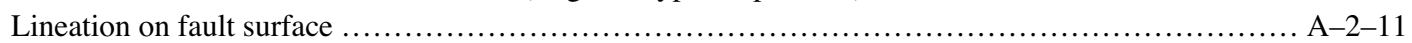

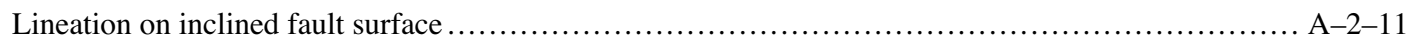

Lineation on surface of bed of economically important commodity $\ldots \ldots \ldots \ldots \ldots \ldots \ldots \ldots \ldots \ldots \ldots \ldots$ A $-1-6$

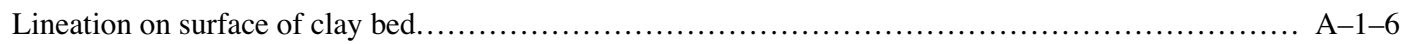

Lineation on surface of coal bed...................................................... A-1-6

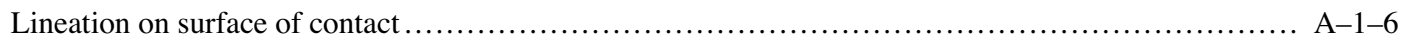

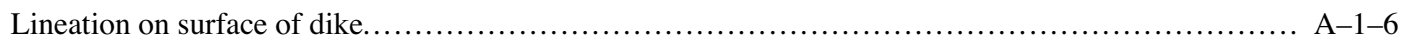

Lineation on surface of inclined bed of economically important commodity ...................... A-1-6

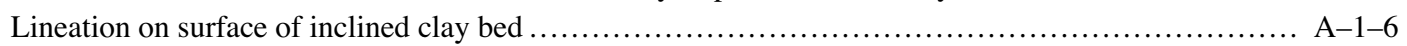

Lineation on surface of inclined coal bed ......................................................

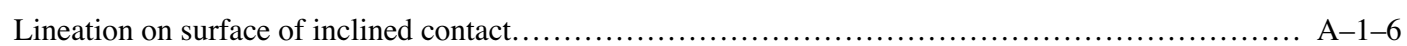

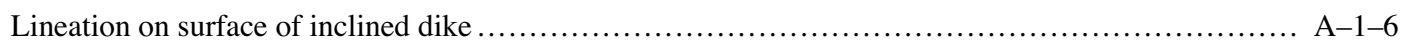

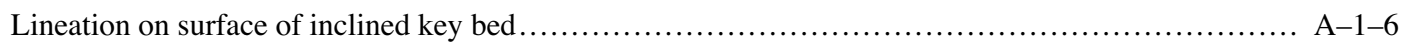

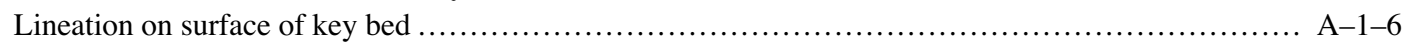

Lines in geologic map databases $[$ concepts and definitions] ..................................... 8

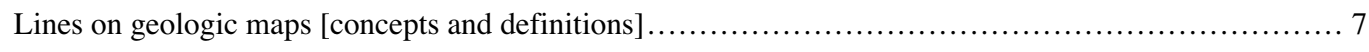

Lineweight specifications $[$ in this standard] f................................................ 29

Listric fault at head of detachment fault (sense of slip unspecified) - Identity and existence certain, location accurate

Listric fault at head of detachment fault (sense of slip unspecified) - Identity and existence certain, location approximate
26.6.18

26.6.13

26.6 .14

26.6 .13

26.6.14

26.6.13

26.6.14

26.6.7

26.6 .8

26.6.1

26.6 .2

26.6.1

26.6 .2

$\mathrm{n} / \mathrm{a}$

Sec. 1.4

Sec. 1.4

Sec. 2.11

Sec. 5.10

Sec. 1.4

Fig. 2

n/a

4.1.1

25.63

4.1 .2

Sec. 4.1

$\mathrm{n} / \mathrm{a}$

n/a

Sec. 9

9.77

9.81

9.89

9.85

9.93

2.11.12

2.11 .13

1.4 .7

1.4 .7

1.4 .7

1.4 .7

1.4 .7

1.4 .8

1.4 .8

1.4 .8

1.4 .8

1.4 .8

1.4 .8

1.4 .7

n/a

n/a

$\mathrm{n} / \mathrm{a}$ 
Listric fault at head of detachment fault (sense of slip unspecified) - Identity and existence certain, location concealed....

Listric fault at head of detachment fault (sense of slip unspecified) - Identity and existence certain, location inferred

Listric fault at head of detachment fault (sense of slip unspecified) - Identity or existence questionable, location accurate...

Listric fault at head of detachment fault (sense of slip unspecified) - Identity or existence questionable, location approximate

Listric fault at head of detachment fault (sense of slip unspecified) - Identity or existence questionable, location concealed ....

Listric fault at head of detachment fault (sense of slip unspecified)-Identity or existence questionable, location inferred

Lobate scarp, planetary .

Locality-information point data [concepts and definitions].

Locatability [concepts and definitions]

. 18

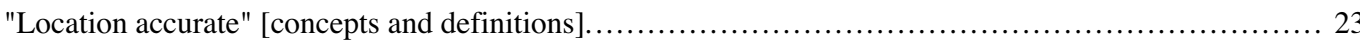

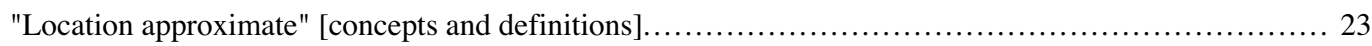

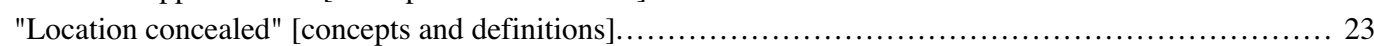

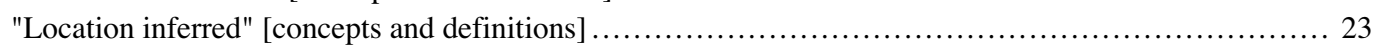

Location where contact is particularly well exposed in field .................................... A-1-6

Sec. 37

25.72

\section{$\mathrm{n} / \mathrm{a}$}

n/a

$\mathrm{n} / \mathrm{a}$

n/a

n/a

n/a

Location where thickness of bed of economically important commodity was measured................ A-1-6

1.4 .10

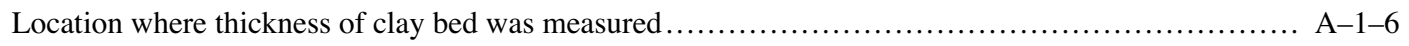

1.4 .11

Location where thickness of coal bed was measured ......................................... A $-1-6$

1.4 .11

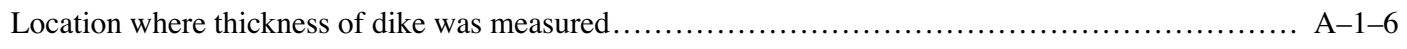

1.4 .11

Location where thickness of key bed was measured...................................... A-1-6

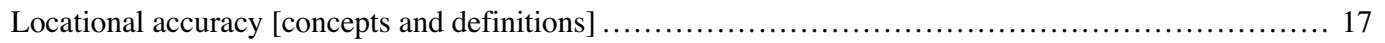

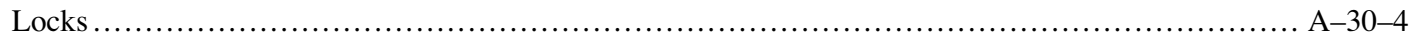

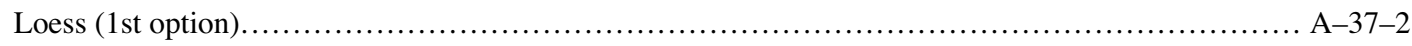

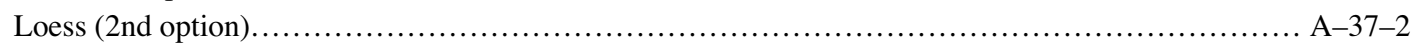

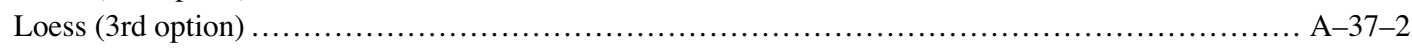

Logical steps to determine appropriate line symbol styles and associated terminology ................. 21

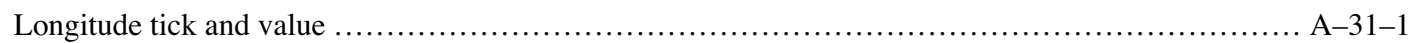

Louisiana $[$ state location map] ........................................................... A-34-1

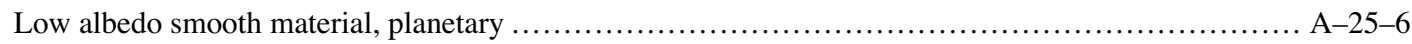

Low-angle fault (unknown or unspecified sense of slip) - Identity and existence certain, location

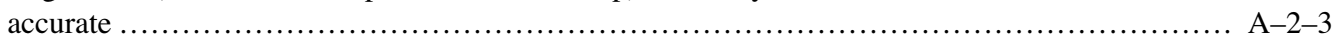

1.4 .11

1.4 .11 $\mathrm{n} / \mathrm{a}$

Low-angle fault (unknown or unspecified sense of slip) - Identity and existence certain, location approximate ....

Low-angle fault (unknown or unspecified sense of slip) - Identity and existence certain, location concealed.

Low-angle fault (unknown or unspecified sense of slip) - Identity and existence certain, location inferred.

Low-angle fault (unknown or unspecified sense of slip) - Identity or existence questionable, location accurate

Low-angle fault (unknown or unspecified sense of slip) - Identity or existence questionable, location

Low-angle fault (unknown or unspecified sense of slip) - Identity or existence questionable, location concealed

Low-angle fault (unknown or unspecified sense of slip) - Identity or existence questionable, location inferred...

Low-angle normal fault-Identity and existence certain, location accurate.......................... A-2-2

Low-angle normal fault - Identity and existence certain, location approximate.................... A-2-2

Low-angle normal fault - Identity and existence certain, location concealed....................... A-2-2 
Page Ref. No.

Low-angle normal fault-Identity or existence questionable, location accurate...................... A-2-2

2.2.10

Low-angle normal fault-Identity or existence questionable, location approximate................... A-2-2

2.2.12

Low-angle normal fault - Identity or existence questionable, location concealed................... A-2-2

2.2 .16

Low-angle normal fault-Identity or existence questionable, location inferred ..................... A-2-2

Low-flow measurement site without a gage ............................................. A $-26-5$

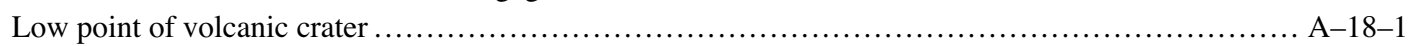

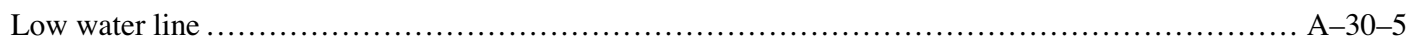

2.2 .14

26.3.16

18.7

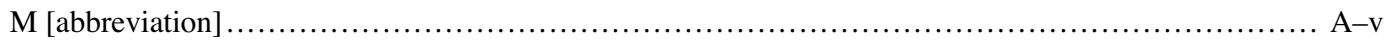

30.2 .31

Macrofossils ........................................................................... A

Table 2

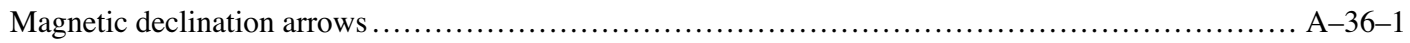

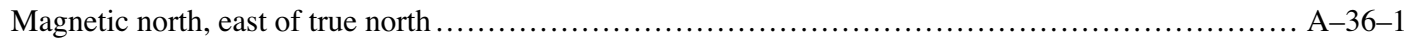

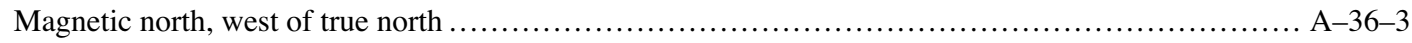

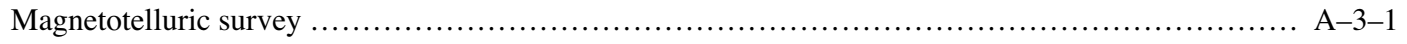

Main scarp of landslide - Active, sharp, distinct, and accurately located ........................ A-17-1

Main scarp of landslide - Inactive, subdued, indistinct, and (or) approximately located.............. A-17-1

Main scarp of landslide, showing height ...................................................... A

Main scarp of rotated block in landslide..................................................... A $17-1$

Main toe of landslide - Active, sharp, distinct, and accurately located ............................ A-17-2

Main toe of landslide - Inactive, subdued, indistinct, and (or) approximately located ................. A-17-2

Maine $[$ state location map] ............................................................ A $-34-1$

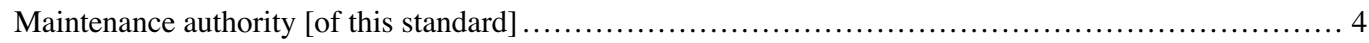

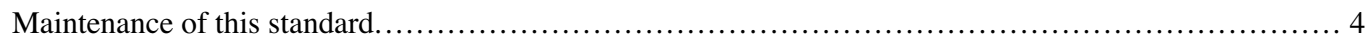

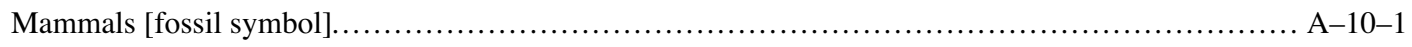

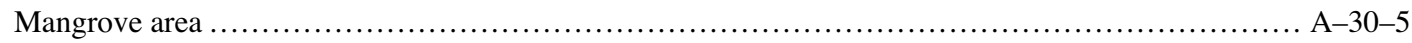

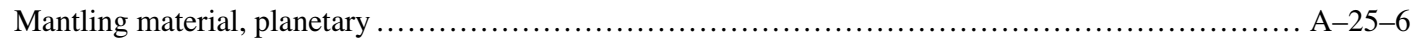

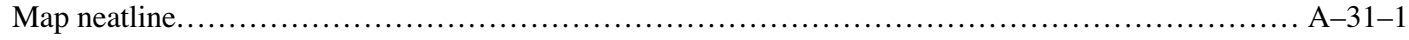

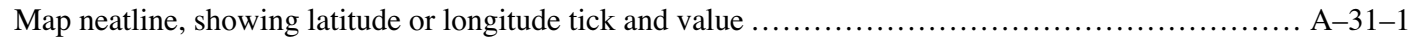

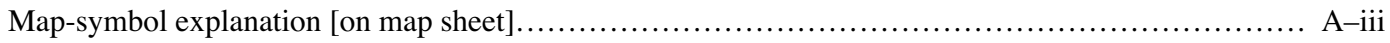

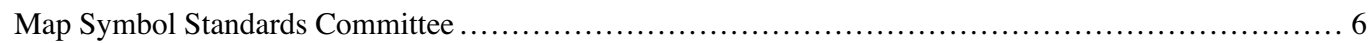

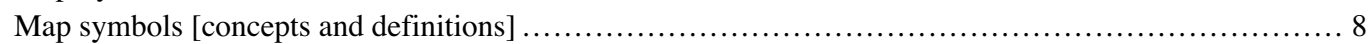

Map-unit label (add leader where necessary) ................................................... A

Map-unit label containing geologic age character (add leader where necessary) ...................... A-31-1

Map-unit labels $[$ concepts and definitions] .................................................. 9

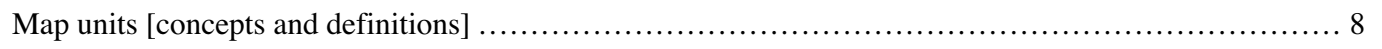

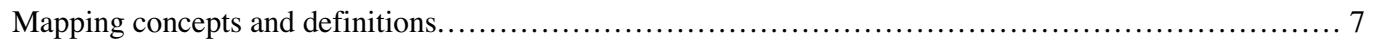

Margin of glacially scoured basin - Identity and existence certain, location accurate................. A-13-2

10.2 .1

Sec. 36

Sec. 36.1

Sec. 36.2

Sec. 3

17.12

17.13

17.14

17.15

17.20

17.21

Sec. 34.1

$\mathrm{n} / \mathrm{a}$

$\mathrm{n} / \mathrm{a}$

10.2.29

30.2.47

25.129

31.8

31.9

$\mathrm{n} / \mathrm{a}$

$\mathrm{n} / \mathrm{a}$

n/a

31.12

31.17

n/a

$\mathrm{n} / \mathrm{a}$

$\mathrm{n} / \mathrm{a}$

Margin of glacially scoured basin - Identity and existence certain, location approximate............. A-13-2

13.43

Margin of glacially scoured basin - Identity and existence certain, location concealed............... A-13-2

13.45

Margin of glacially scoured basin - Identity or existence questionable, location accurate.............. A-13-2

Margin of glacially scoured basin - Identity or existence questionable, location approximate.......... A-13-2

Margin of glacially scoured basin - Identity or existence questionable, location concealed............ A-13-2

Margin of oceanic rise - Accurately located................................................. A $22-2$

Margin of oceanic rise-Approximately located............................................... A-22-2

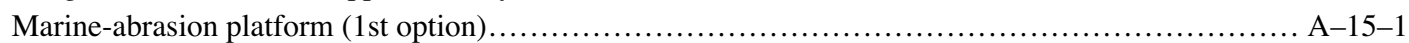

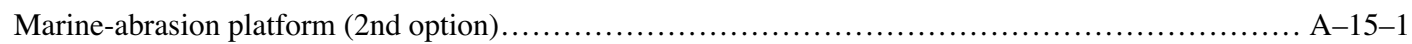

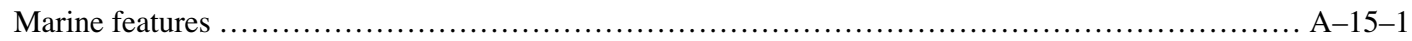

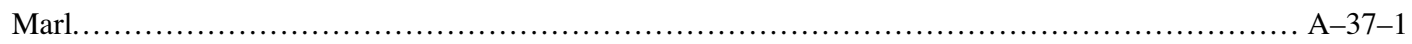

13.47

13.44

13.46

13.48

22.31

22.32

15.3

15.4

Sec. 15

623

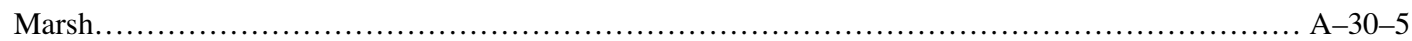

30.2 .46

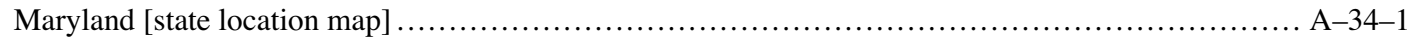

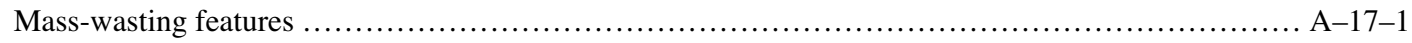

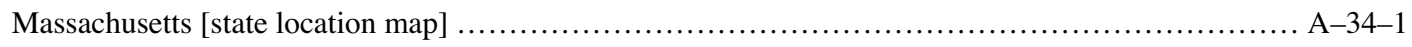

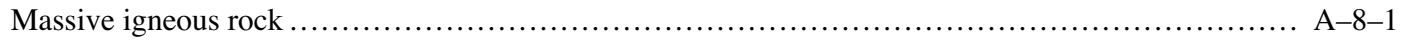

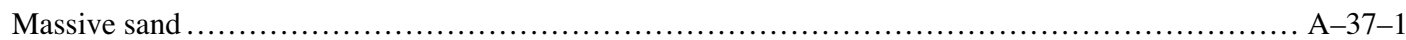

Sec. 34.1

Sec. 17

Sec. 34.1 
Massive sandstone.

Master detachment fault (sense of slip unspecified) - Identity and existence certain, location accurate.. A-2-10

Master detachment fault (sense of slip unspecified) - Identity and existence certain, location approximate

Master detachment fault (sense of slip unspecified) - Identity and existence certain, location concealed ....

Master detachment fault (sense of slip unspecified) - Identity and existence certain, location inferred .. A-2-10

Master detachment fault (sense of slip unspecified) - Identity or existence questionable, location accurate

Master detachment fault (sense of slip unspecified) - Identity or existence questionable, location approximate

Master detachment fault (sense of slip unspecified) - Identity or existence questionable, location concealed ....

Master detachment fault (sense of slip unspecified) - Identity or existence questionable, location inferred.

Maximum intensity value within closed high or closed low.

"May not be within zone of confidence" [concepts and definitions] ....

Mean declination arrows.

Mean low water line.

Measurement site without a gage.

Measurement site without a gage, equipped with a telephone or radio

Medium-sized endogenic crater (mapped to scale), planetary.

Meltwater stream

Mesa, planetary (not mapped to scale).

Mesozoic [geologic age symbol] ....

Metamorphic core complexes....

Metamorphic facies boundary

Metamorphic foliation

Metamorphic foliation parallel to bedding

Metamorphic patterns (Series 400).

Metamorphic-rock lithologic patterns

Metamorphism [lithologic pattern]....

Michigan [state location map]

Microfossils

Middle Archean [geologic age symbol]

Middle Early Proterozoic [geologic age symbol] .

Middle Middle Proterozoic [geologic age symbol].

Middle Proterozoic [geologic age symbol].

Mid-oceanic ridges

Millimeters (mm) to inches (in) or points (pts).

Mine dump (1st option).

Mine dump (2nd option)

Mine dump bench.

Mine shaft, above and below level (drawn to scale), as shown on subsurface exploration maps

Mine shaft, as shown on topographic maps or on general-purpose or smaller scale maps, showing name.

Mine shafts, as shown on subsurface exploration maps.

Mine shafts (at surface).

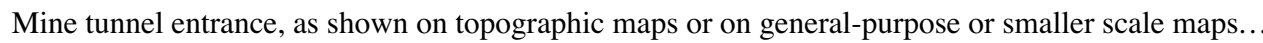

Mineral-aggregate lineation........

Mineral exploration (at surface)

Mineral lineation.

Mineral resource areas

Mineral spring used for domestic-water supply
Page

A-37-1

A-2-10

A-2-10

A-2-10

A-2-10

A-2-10

A-2-10

A-11-1

19

A-36-1

A $-30-5$

A-26-5

A-26-5

A-25-5

A-13-1

A-25-4

A-32-1

A-23-1

A-19-1

A-8-3

A-8-3

A-8-3

Ref. No.

2.10 .25

2.10.27

2.10.31

2.10.29

2.10 .28

2.10 .32

2.10.30

11.12

$\mathrm{n} / \mathrm{a}$

Sec. 36

30.2.31

26.3.13

26.3.14

25.108

13.7

25.79

32.6

Sec. 23

19.1.23

8.3 .1

8.3.4

8.3 .7

$\mathrm{n} / \mathrm{a}$

A-37-3 Sec. 37.2

A-37-3

701

A-34-1 Sec. 34.1

A-10-1 10.2.45

A-32-2 32.32

A-32-2 32.28

A-32-1 32.24

A-32-1 32.22

A-22-1 Sec. 22

Table 1

19.2.7

19.2.8

19.2 .9

19.4.4

A-19-2

A-19-5

A-30-6

30.3.6

A-19-5 Sec. 19.4

A-19-4

Sec. 19.3

30.3.3

9.41

A-9-2

A-19-3

A-9-2

Sec. 19.3

9.37

A-19-1

Sec. 19.1

26.2.6 
Mineral spring used for industrial-water supply

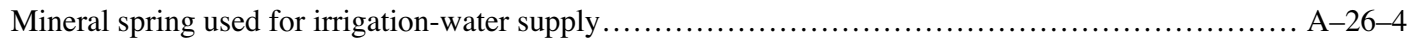

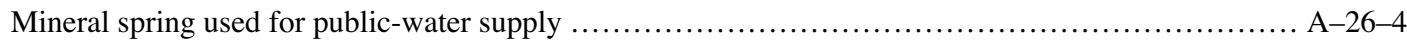

Mineral spring used for stock-water supply............................................. A-26-4

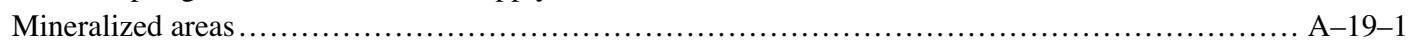

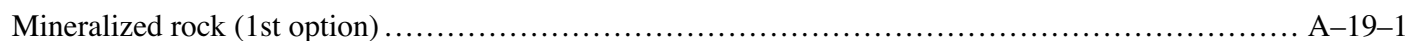

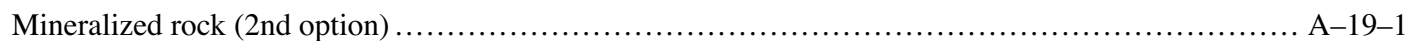

Mineralized rock, showing high level of mineralization ....................................... A-19-1

Mineralized rock, showing low level of mineralization ................................... A $-19-1$

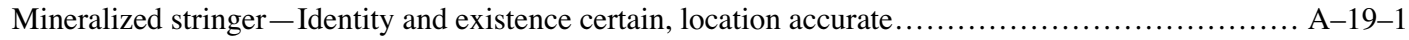

Mineralized stringer - Identity and existence certain, location approximate...................... A-19-1

Mineralized stringer - Identity and existence certain, location concealed......................... A-19-1

Mineralized stringer - Identity or existence questionable, location accurate...................... A-19-1

Mineralized stringer - Identity or existence questionable, location approximate $\ldots \ldots \ldots \ldots \ldots \ldots \ldots \ldots \ldots$ A $-19-1$

Mineralized stringer-Identity or existence questionable, location concealed....................... A-19-1

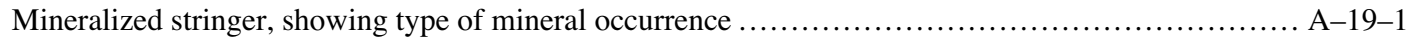

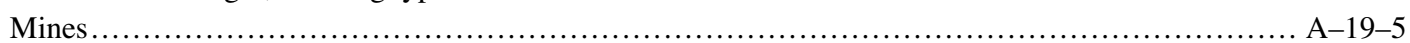

Minimum intensity value within closed high or closed low ................................... A-11-1

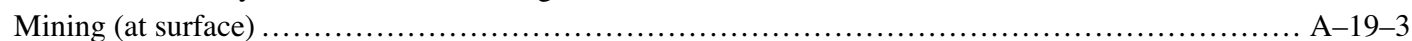

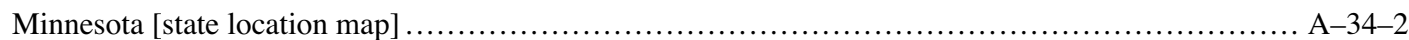

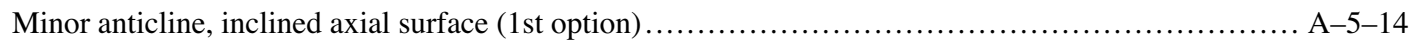

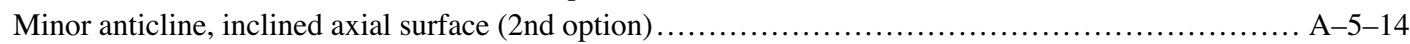

Minor anticline, vertical or near-vertical axial surface (1st option) ............................. A-5-14

Minor anticline, vertical or near-vertical axial surface (2nd option) .............................. A-5-14

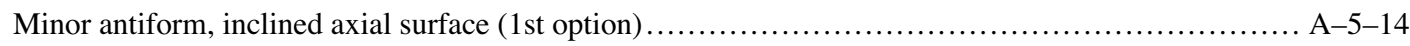

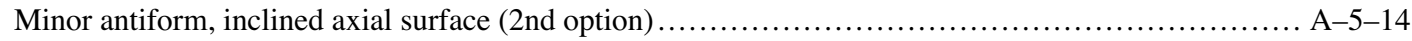

Minor antiform, vertical or near-vertical axial surface (1st option) ............................. A-5-14

Minor antiform, vertical or near-vertical axial surface (2nd option) ........................... A-5-14

Minor asymmetric anticline, inclined axial surface (1st option) .............................. A-5-14

Minor asymmetric anticline, inclined axial surface (2nd option) .................................. A $-5-14$

Minor asymmetric anticline, vertical or near-vertical axial surface (1st option)....................... A-5-14

Minor asymmetric anticline, vertical or near-vertical axial surface (2nd option)..................... A-5-14

Minor asymmetric syncline, inclined axial surface (1st option)................................ A-5-15

Minor asymmetric syncline, inclined axial surface (2nd option) ................................. A-5-15

Minor asymmetric syncline, vertical or near-vertical axial surface (1st option) ....................... A-5-15

Minor asymmetric syncline, vertical or near-vertical axial surface (2nd option) ...................... A-5-15

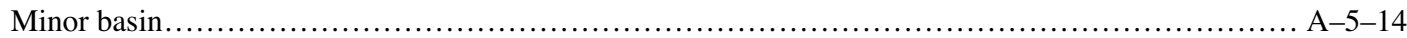

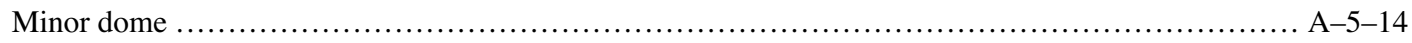

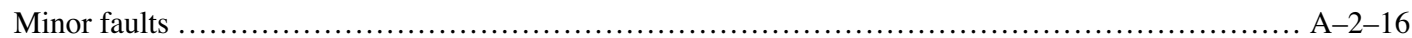

Minor fold-hinge lineations ............................................................... A-9

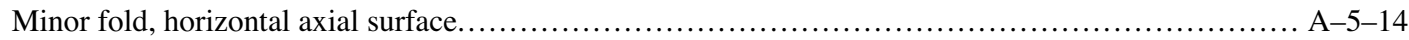

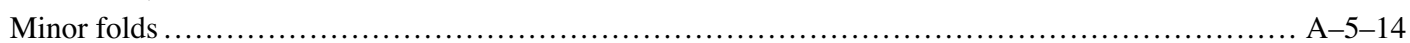

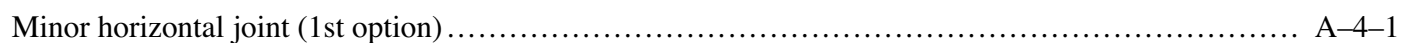

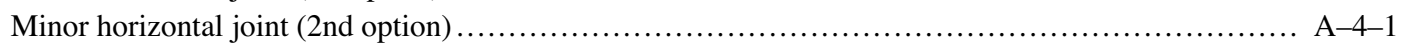

Minor inclined (dip direction to left) joint, for multiple observations at one locality (1st option) ........ A-4-1

Minor inclined (dip direction to left) joint, for multiple observations at one locality (2nd option) ....... A-4-1

Minor inclined (dip direction to right) joint, for multiple observations at one locality (1st option)....... A-4-1

Minor inclined (dip direction to right) joint, for multiple observations at one locality (2nd option)...... A-4-1

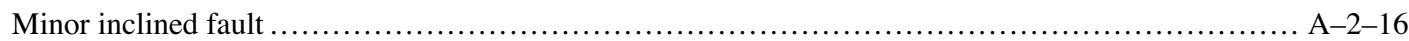

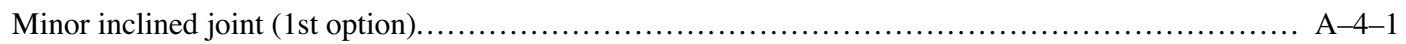

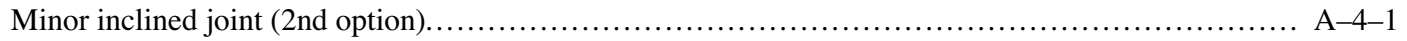

Minor inclined mineralized stringer.................................................. A $-19-1$

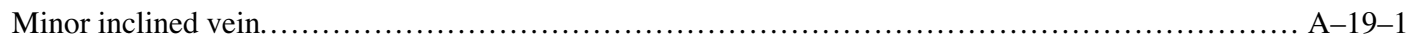

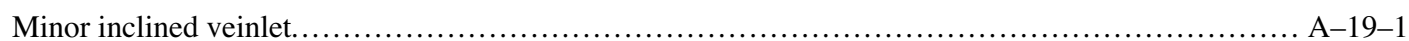

26.2 .18

26.2.14

26.2.22

26.2.10

Sec. 19.1

19.1.14

19.1.15

19.1.16

19.1.17

19.1.1

19.1.3

19.1.5

19.1.2

19.1 .4

19.1.6

19.1.7

Sec. 19.4

11.12

Sec. 19.3

Sec. 34.1

5.11 .5

5.11 .7

5.11 .4

5.11 .6

5.11 .9

5.11 .11

5.11 .8

5.11 .10

5.11 .13

5.11 .15

5.11 .12

5.11 .14

5.11 .33

5.11 .35

5.11 .32

5.11 .34

5.11 .3

5.11 .2

Sec. 2.15

9.97

5.11 .1

Sec. 5.11

4.3.1

4.3 .7

4.3 .5

4.3.11

4.3.4

4.3.10

2.15 .1

4.3 .2

4.3.8

19.1.12

19.1.12

19.1.12 
Minor inverted anticline, inclined axial surface (1st option)

Minor inverted anticline, inclined axial surface (2nd option)

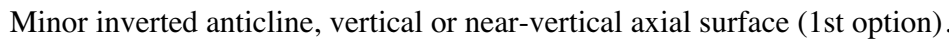

Minor inverted anticline, vertical or near-vertical axial surface (2nd option)

Minor inverted syncline, inclined axial surface (1st option).

Minor inverted syncline, inclined axial surface (2nd option)

Minor inverted syncline, vertical or near-vertical axial surface (1st option)

Minor inverted syncline, vertical or near-vertical axial surface (2nd option)

Minor joints

Minor joints, for multiple observations at one locality

Minor overturned anticline, inclined axial surface (1st option)....

Minor overturned anticline, inclined axial surface (2nd option)

Minor overturned anticline, vertical or near-vertical axial surface (1st option)

Minor overturned anticline, vertical or near-vertical axial surface (2nd option)

Minor overturned syncline, inclined axial surface (1st option).

Minor overturned syncline, inclined axial surface (2nd option)....

Minor overturned syncline, vertical or near-vertical axial surface (1st option)

Minor overturned syncline, vertical or near-vertical axial surface (2nd option).....

Minor scarp in landslide-Active, sharp, distinct, and accurately located

Minor scarp in landslide - Inactive, subdued, indistinct, and (or) approximately located

Minor scarp in landslide, showing height

Minor scarp of rotated block in landslide

Minor shear fault.

Minor syncline, inclined axial surface (1st option)

Minor syncline, inclined axial surface (2nd option)

Minor syncline, vertical or near-vertical axial surface (1st option).

Minor syncline, vertical or near-vertical axial surface (2nd option)

Minor synform, inclined axial surface (1st option)

Minor synform, inclined axial surface (2nd option)

Minor synform, vertical or near-vertical axial surface (1st option)...

Minor synform, vertical or near-vertical axial surface (2nd option)

Minor toe in landslide-Active, sharp, distinct, and accurately located

Minor toe in landslide - Inactive, subdued, indistinct, and (or) approximately located

Minor toe in landslide, showing transport reversal-Inactive, subdued, indistinct, and (or) approximately located

Minor vertical or near-vertical fault

Minor vertical or near-vertical joint (1st option)

Minor vertical or near-vertical joint (2nd option)

Minor vertical or near-vertical joint, for multiple observations at one locality (1st option)

Minor vertical or near-vertical joint, for multiple observations at one locality (2nd option).

Minor vertical or near-vertical mineralized stringer

Minor vertical or near-vertical vein

Minor vertical or near-vertical veinlet....

Miscellaneous collapse features

Miscellaneous geohydrologic features....

Miscellaneous hydrographic features

Miscellaneous map elements.

Miscellaneous patterns (Series 400)

Miscellaneous topographic features

Miscellaneous uplift features. ...

Mississippi [state location map]

Mississippian [geologic age symbol]

Missouri [state location map] ...
Page

Ref. No.

5.11 .21

5.11 .23

5.11 .20

5.11 .22

5.11 .41

5.11 .43

5.11 .40

5.11 .42

Sec. 4.3

4.3.4

5.11 .17

5.11 .19

5.11 .16

5.11 .18

5.11 .37

5.11 .39

5.11 .36

5.11 .38

17.16

17.17

17.18

17.19

2.15 .3

5.11 .25

5.11 .27

5.11 .24

5.11 .26

5.11 .29

5.11 .31

5.11 .28

5.11 .30

17.22

17.23

17.24

17.25

2.15 .2

4.3 .3

4.3 .9

4.3 .6

4.3.12

19.1.13

19.1 .13

19.1.13

Sec. 23

Sec. 26.7

Sec. 30.3

$\begin{array}{cc}\text { A-31-1 } & \text { Sec. 31 } \\ \text { Pattern Chart } & \text { n/a }\end{array}$

A-30-6 Sec. 30.3

A-23-1

A-34-2

A-32-1

A-34-2
Sec. 23

Sec. 34.1

32.14

Sec. 34.1 
Moderately inclined (between $30^{\circ}$ and $60^{\circ}$ ) bedding, as determined remotely or from aerial photographs.

Moderately overturned (between $30^{\circ}$ and $60^{\circ}$ ) bedding, as determined remotely or from aerial photographs...

Monocline (1st option) - Identity and existence certain, location accurate

Monocline (1st option) - Identity and existence certain, location approximate...................... A-5-11

Monocline (1st option) - Identity and existence certain, location concealed...................... A-5-11

Monocline (1st option) - Identity and existence certain, location inferred ...................... A-5-11

Monocline (1st option) - Identity or existence questionable, location accurate .................... A-5-11

Monocline (1st option) - Identity or existence questionable, location approximate ................ A-5-11

Monocline (1st option) - Identity or existence questionable, location concealed ................... A-5-11

Monocline (1st option) - Identity or existence questionable, location inferred ...................... A-5-11

Monocline (2nd option) - Identity and existence certain, location accurate........................ A-5-11

Monocline (2nd option) - Identity and existence certain, location approximate..................... A-5-11

Monocline (2nd option) - Identity and existence certain, location concealed....................... A-5-11

Monocline (2nd option) - Identity and existence certain, location inferred ......................... A-5-11

Monocline (2nd option) - Identity or existence questionable, location accurate ................... A-5-11

5.9 .5

5.9 .2

5.9 .4

5.9 .8

5.9 .6

5.9 .9

5.9 .11

5.9 .15

5.9 .13

Monocline (2nd option) - Identity or existence questionable, location approximate ................ A-5-11

5.9 .10

Monocline (2nd option) - Identity or existence questionable, location concealed .................. A-5-11

Monocline (2nd option) - Identity or existence questionable, location inferred.................... A-5-11

5.9 .12

5.9 .16

Monocline, anticlinal bend (1st option) - Identity and existence certain, location accurate ........... A-5-11

Monocline, anticlinal bend (1st option) - Identity and existence certain, location approximate ......... A-5-11

Monocline, anticlinal bend (1st option) - Identity and existence certain, location concealed ........... A-5-11

Monocline, anticlinal bend (1st option) - Identity and existence certain, location inferred............. A-5-11

Monocline, anticlinal bend (1st option) - Identity or existence questionable, location accurate ......... A-5-11

Monocline, anticlinal bend (1st option) - Identity or existence questionable, location approximate ...... A-5-11

Monocline, anticlinal bend (1st option) - Identity or existence questionable, location concealed ........ A-5-11

Monocline, anticlinal bend (1st option) - Identity or existence questionable, location inferred.......... A-5-11

Monocline, anticlinal bend (2nd option) - Identity and existence certain, location accurate ........... A-5-12

Monocline, anticlinal bend (2nd option) - Identity and existence certain, location approximate ........ A-5-12

Monocline, anticlinal bend (2nd option) - Identity and existence certain, location concealed .......... A-5-12

Monocline, anticlinal bend (2nd option) - Identity and existence certain, location inferred............ A-5-12

Monocline, anticlinal bend (2nd option) - Identity or existence questionable, location accurate ........ A-5-12

Monocline, anticlinal bend (2nd option) - Identity or existence questionable, location approximate .... A-5-12

Monocline, anticlinal bend (2nd option) - Identity or existence questionable, location concealed ....... A-5-12

Monocline, anticlinal bend (2nd option) - Identity or existence questionable, location inferred......... A-5-12

Monocline, synclinal bend (1st option) - Identity and existence certain, location accurate............ A-5-12

Monocline, synclinal bend (1st option) - Identity and existence certain, location approximate.......... A-5-12

Monocline, synclinal bend (1st option) - Identity and existence certain, location concealed........... A-5-12

Monocline, synclinal bend (1st option) - Identity and existence certain, location inferred ............. A-5-12

Monocline, synclinal bend (1st option) - Identity or existence questionable, location accurate .......... A-5-12

Monocline, synclinal bend (1st option) - Identity or existence questionable, location approximate ...... A-5-12

Monocline, synclinal bend (1st option) - Identity or existence questionable, location concealed ........ A-5-12

Monocline, synclinal bend (1st option) - Identity or existence questionable, location inferred .......... A-5-12

Monocline, synclinal bend (2nd option) - Identity and existence certain, location accurate............ A-5-12

Monocline, synclinal bend (2nd option) - Identity and existence certain, location approximate......... A-5-12

Monocline, synclinal bend (2nd option) - Identity and existence certain, location concealed.......... A-5-12

Monocline, synclinal bend (2nd option) - Identity and existence certain, location inferred ............. A-5-12

Monocline, synclinal bend (2nd option) - Identity or existence questionable, location accurate ........ A-5-12

Monocline, synclinal bend (2nd option) - Identity or existence questionable, location approximate ..... A-5-12

Monocline, synclinal bend (2nd option) - Identity or existence questionable, location concealed ....... A-5-12

Monocline, synclinal bend (2nd option) - Identity or existence questionable, location inferred ......... A-5-12

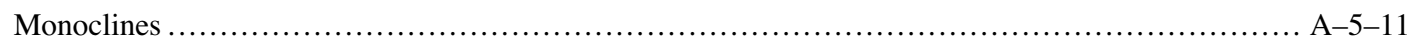

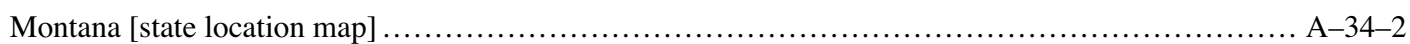

5.9 .14

5.9 .17

5.9 .19

5.9 .23

5.9 .21

5.9 .18

5.9 .20

5.9 .24

5.9 .22

5.9 .25

5.9 .27

5.9 .31

5.9 .29

5.9 .26

5.9 .28

5.9 .32

5.9 .30

5.9 .33

5.9 .35

5.9 .39

5.9 .37

5.9 .34

5.9 .36

5.9 .40

5.9 .38

5.9 .41

5.9 .43

5.9 .47

5.9 .45

5.9 .42

5.9 .44

5.9 .48

5.9 .46

Sec. 5.9

Sec. 34.1 


\begin{tabular}{|c|c|}
\hline Page & Ref. No. \\
\hline Moraine crest, asymmetrical moraine..... & 13.13 \\
\hline 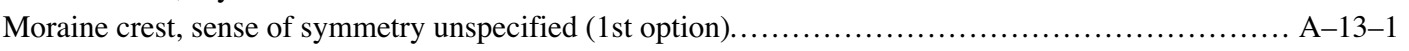 & 13.10 \\
\hline 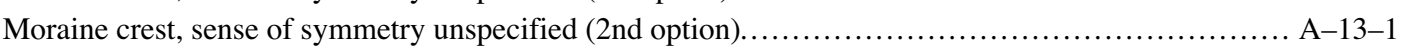 & 13.11 \\
\hline 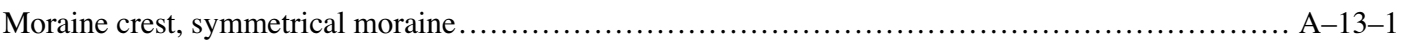 & 13.12 \\
\hline 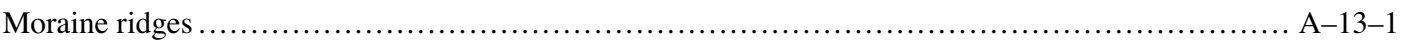 & 13.14 \\
\hline 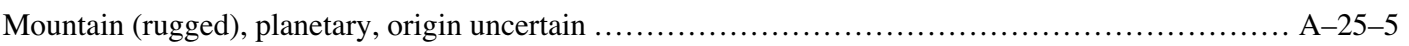 & 25.115 \\
\hline Mud pot, as shown on topographic maps or on general-purpose or smaller scale maps ................. A-30-6 & 30.3 .13 \\
\hline 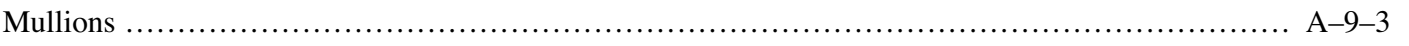 & 9.65 \\
\hline 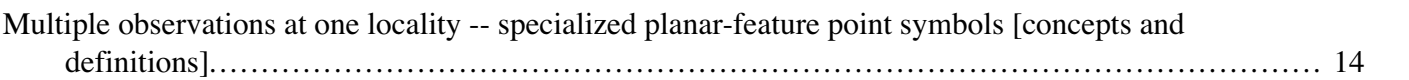 & $\mathrm{n} / \mathrm{a}$ \\
\hline Multiple vertical mine shafts, as shown on smaller scale or general-purpose maps.................... A-19-4 & 19.3.36 \\
\hline 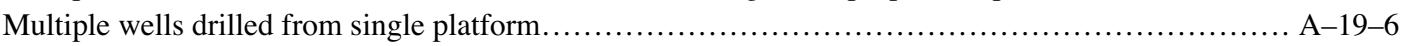 & 19.5.18 \\
\hline Municipio boundary ...................................... & 29.3 \\
\hline Mylonite zone ................................. & 2.14 .1 \\
\hline Mylonitic foliation.................................................... & 8.3 .55 \\
\hline Mylonitic foliation, for multiple observations at one locality ............ & 8.3 .58 \\
\hline Name of bed of economically important commodity .................................. & 1.4 .14 \\
\hline 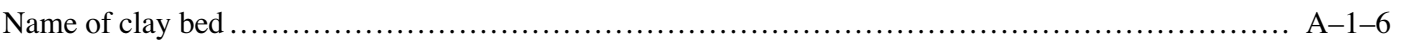 & 1.4 .13 \\
\hline Name of clinkered coal bed ........ & 1.4 .16 \\
\hline Name of clinkered coal bed area ............... & 1.4 .17 \\
\hline Name of coal bed ............................ & 1.4 .15 \\
\hline Name of depositional unit at retreatal position of stagnant ice margin... & 13.72 \\
\hline 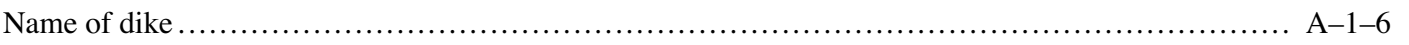 & 1.4 .18 \\
\hline Name of dike intruding fault (1st option) ....... & 1.4 .20 \\
\hline Name of dike intruding fault (2nd option) ...... & 1.4 .21 \\
\hline Name of dike of variable thickness ............. & 1.4 .19 \\
\hline Name of fault............................. & 2.11 .15 \\
\hline Name of fold .......................... & 5.10 .12 \\
\hline Name of former marine limit............ & 15.21 \\
\hline Name of former shoreline ................. & 15.21 \\
\hline Name of glaciation on glacial limit ...................... & 13.57 \\
\hline Name of glaciation on glacial terminus.................... & 13.57 \\
\hline Name of key bed.............................. & 1.4 .12 \\
\hline Name of lake ........................ & 30.2 .32 \\
\hline Name of lineament ............ & 4.1.2 \\
\hline 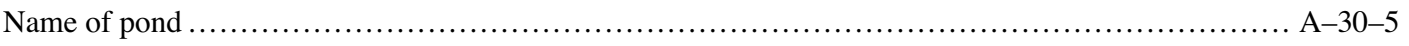 & 30.2 .32 \\
\hline Names of stratigraphic ages on chronostratigraphic-zone, chronozone, or stage boundary ............. A-31-1 & 31.24 \\
\hline Nannofossils................................................................................. A-10-1 & 10.2 .52 \\
\hline 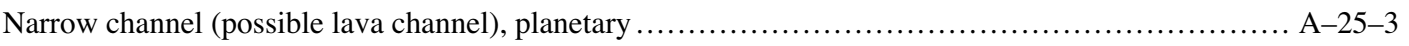 & 25.58 \\
\hline Narrow depression, planetary .................................. & 25.52 \\
\hline National boundary .................... & 29.1 \\
\hline National forest boundary ................. & 29.6 \\
\hline National grassland boundary............. & 29.6 \\
\hline National monument boundary ........ & 29.6 \\
\hline National park boundary................. & 29.6 \\
\hline National reservation boundary ........... & 29.6 \\
\hline National wilderness area boundary.... & 29.6 \\
\hline National wildlife refuge boundary ..... & 29.6 \\
\hline Natural resources ..................... & Sec. 19 \\
\hline Nautiloids........................ & 10.2 .22 \\
\hline Neatline ........................... & 31.8 \\
\hline Nebraska [state location map] ....................... & Sec. 34.1 \\
\hline Neogene $[$ geologic age symbol] $\ldots \ldots \ldots \ldots \ldots \ldots$ & 32.4 \\
\hline
\end{tabular}




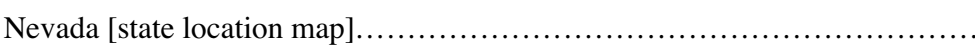

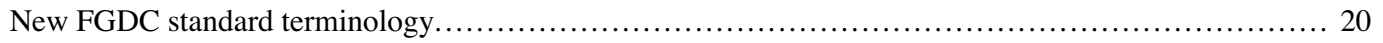

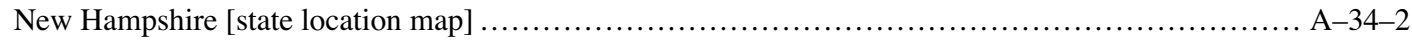

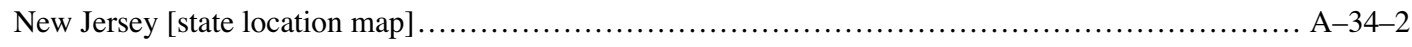

New Mexico [state location map] ................................................... A $-34-2$

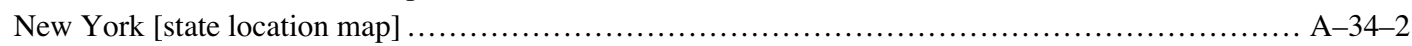

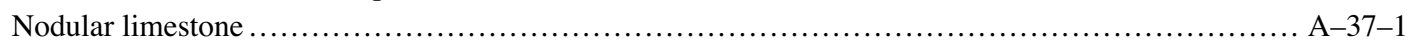

Nonflowing artesian well used for industrial-water supply .................................... A $-26-2$

Nonflowing artesian well used for irrigation-water supply .................................. A-26-2

Nonflowing artesian well used for public-water supply...................................... A $-26-3$

Nonflowing artesian well, used for domestic-water supply................................. A $-26-1$

Nonflowing artesian well, used for stock-water supply .................................... A-26-1

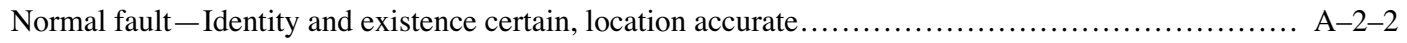

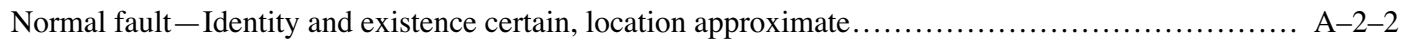

Normal fault - Identity and existence certain, location concealed.............................. A-2-2

Normal fault - Identity and existence certain, location inferred ................................. A-2-2

Normal fault -Identity or existence questionable, location accurate .............................. A-2-2

Normal fault-Identity or existence questionable, location approximate............................ A-2-2

Normal fault -Identity or existence questionable, location concealed............................. A-2-2

Normal fault-Identity or existence questionable, location inferred .............................. A-2-2

Normal fault (in cross section) ......................................................... A $-2-11$

Normal fault (on small-scale maps or figures).............................................. A $-2-11$

Normal fault, planetary - Location accurate ............................................ A $-25-1$

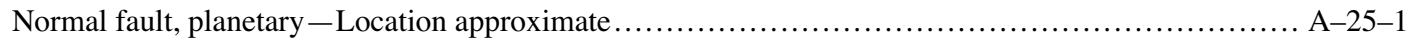

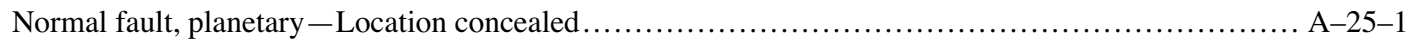

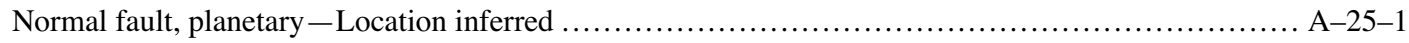

Normal faults...................................................................... A $-2-2$

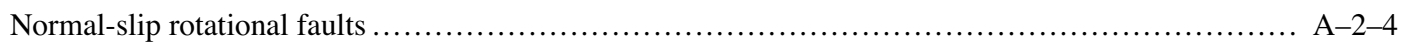

Normal-slip scissor faults................................................................. A

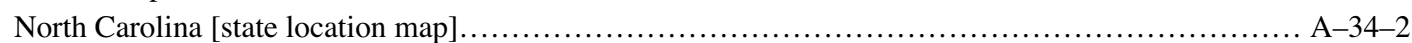

North Dakota $[$ state location map] ............................................................ A

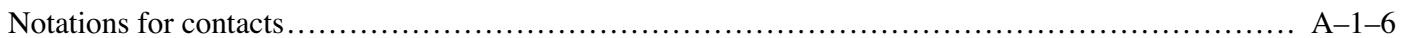

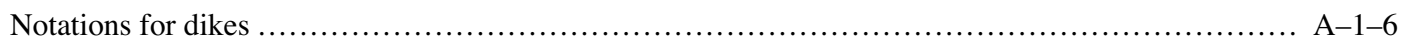

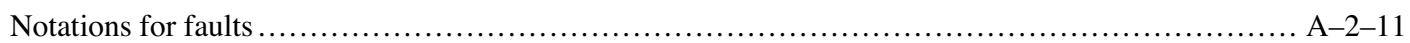

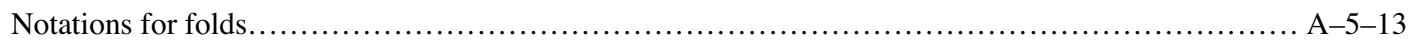

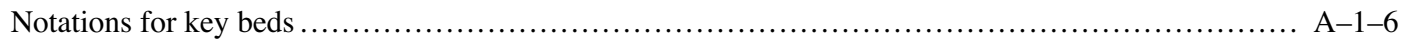

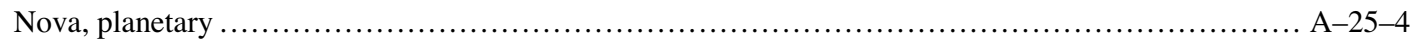

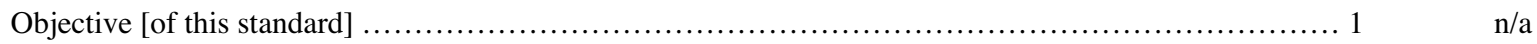

Oblique-slip fault, left-lateral offset - Identity and existence certain, location accurate ............... A-2-6 2.7 .9

Oblique-slip fault, left-lateral offset - Identity and existence certain, location approximate ............ A-2-6 2.7.11

Oblique-slip fault, left-lateral offset-Identity and existence certain, location concealed ............... A-2-6 2.7.15

Oblique-slip fault, left-lateral offset-Identity and existence certain, location inferred.................. A-2-6 2.7.13

Oblique-slip fault, left-lateral offset - Identity or existence questionable, location accurate ............. A-2-6 2.7.10

Oblique-slip fault, left-lateral offset-Identity or existence questionable, location approximate ......... A-2-6 2.7 .12

Oblique-slip fault, left-lateral offset - Identity or existence questionable, location concealed ............ A-2-6 2.7.16

Oblique-slip fault, left-lateral offset-Identity or existence questionable, location inferred.............. A-2-6 2.7 .14

Oblique-slip fault, right-lateral offset-Identity and existence certain, location accurate................ A-2-6 2.7 .1

Oblique-slip fault, right-lateral offset-Identity and existence certain, location approximate............ A-2-6 2.7 .3

Oblique-slip fault, right-lateral offset-Identity and existence certain, location concealed.............. A-2-6 2.7 .7

Oblique-slip fault, right-lateral offset - Identity and existence certain, location inferred ............... A-2-6 2.7.5

Oblique-slip fault, right-lateral offset - Identity or existence questionable, location accurate........... A-2-6 2.7 .2

Oblique-slip fault, right-lateral offset-Identity or existence questionable, location approximate........ A-2-6 2.7 .4

Oblique-slip fault, right-lateral offset-Identity or existence questionable, location concealed.......... A-2-6 2.7 .8 


\begin{tabular}{|c|c|}
\hline Page & Ref. No. \\
\hline Oblique-slip fault, right-lateral offset-Identity or existence questionable, location inferred ............ A-2-6 & 2.7.6 \\
\hline 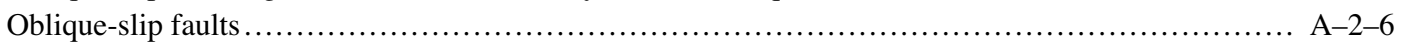 & Sec. 2.7 \\
\hline "Observable" [concepts and definitions] ....................... & $\mathrm{n} / \mathrm{a}$ \\
\hline 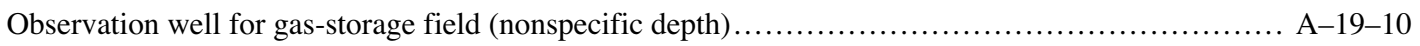 & 19.5.98 \\
\hline 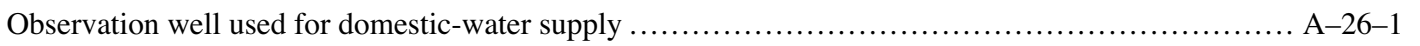 & 26.1.11 \\
\hline Observation well used for domestic-water supply, equipped with a recorder....... & 26.1 .12 \\
\hline 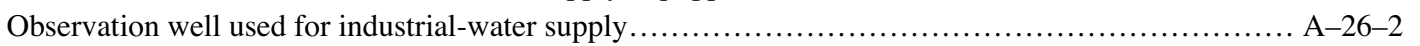 & 26.1 .38 \\
\hline Observation well used for industrial-water supply, equipped with a recorder ................ & 26.1 .39 \\
\hline 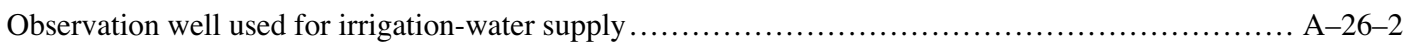 & 26.1 .29 \\
\hline Observation well used for irrigation-water supply, equipped with a recorder........................ A-26-2 & 26.1 .30 \\
\hline Observation well used for public-water supply................................. & 26.1 .47 \\
\hline Observation well used for public-water supply, equipped with a recorder ............ & 26.1 .48 \\
\hline 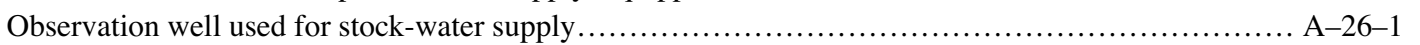 & 26.1 .20 \\
\hline Observation well used for stock-water supply, equipped with a recorder ........ & 26.1 .21 \\
\hline 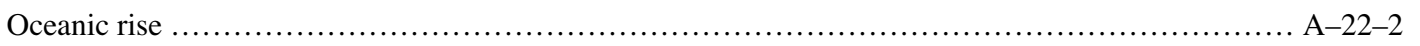 & Sec. 22 \\
\hline Ohio [state location map]............. & Sec. 34.1 \\
\hline Oil and gas field-Extent defined.......... & 19.5 .5 \\
\hline Oil and gas field-Extent not yet defined..... & 19.5.6 \\
\hline Oil and gas fields ............................. & Sec. 19.5 \\
\hline Oil and gas seep....................... & 19.5.61 \\
\hline Oil and gas show....................... & 19.5.62 \\
\hline Oil and gas well (nonspecific depth)........ & 19.5.63 \\
\hline Oil and gas wells................................. & Sec. 19.5 \\
\hline Oil field-Extent defined................ & 19.5 .1 \\
\hline Oil field-Extent not yet defined........... & 19.5.2 \\
\hline Oil fields ...................................... & Sec. 19.5 \\
\hline ... A-19-8 & 19.5.38 \\
\hline Oil shale......... & 625 \\
\hline Oil show & 19.5.39 \\
\hline Oil well, as shown on topographic maps or on general-purpose or smaller scale maps .... & 30.3 .9 \\
\hline 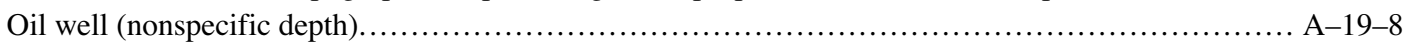 & 19.5.40 \\
\hline 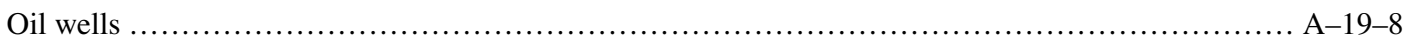 & Sec. 19.5 \\
\hline 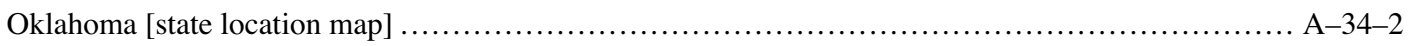 & Sec. 34.1 \\
\hline Older glacial groove, flow direction unknown - Showing general bearing ...... & 13.35 \\
\hline Older glacial groove, flow direction unknown - Showing measured bearing............ & 13.36 \\
\hline 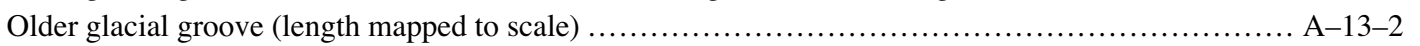 & 13.39 \\
\hline Older glacial groove (length mapped to scale), flow direction unknown ........ & 13.40 \\
\hline Older glacial groove - Showing general bearing.............................. & 13.31 \\
\hline 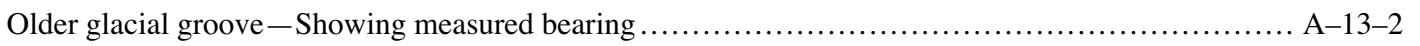 & 13.32 \\
\hline Older glacial striation, flow direction unknown - Showing general bearing ............ & 13.35 \\
\hline Older glacial striation, flow direction unknown - Showing measured bearing .... & 13.36 \\
\hline 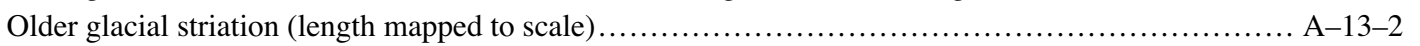 & 13.39 \\
\hline Older glacial striation (length mapped to scale), flow direction unknown................... & 13.40 \\
\hline 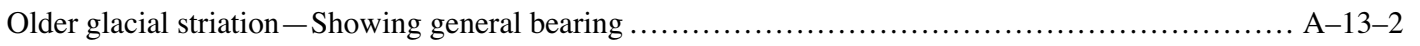 & 13.31 \\
\hline Older glacial striation - Showing measured bearing............. & 13.32 \\
\hline Oolitic dolomite......................................... & 644 \\
\hline Oolitic dolostone.................. & 644 \\
\hline Oolitic limestone............... & 635 \\
\hline Open fracture on landslide .......... & 17.34 \\
\hline 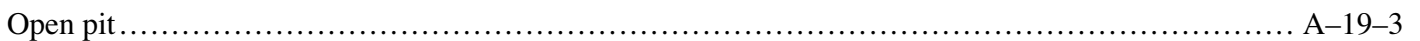 & 19.3 .4 \\
\hline 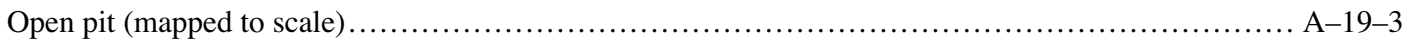 & 19.3.6 \\
\hline Open pit mine, as shown on topographic maps or on general-purpose or smaller scale maps ........... A-30-6 & 30.3 .1 \\
\hline 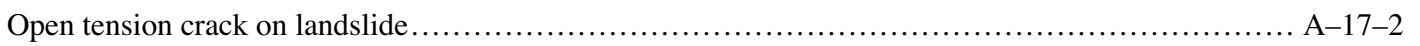 & 17.34 \\
\hline 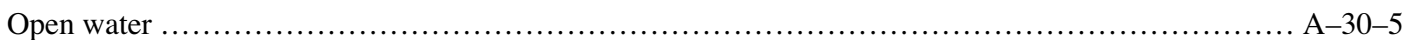 & 30.2 .29 \\
\hline
\end{tabular}




\begin{tabular}{|c|c|}
\hline Page & Ref. No. \\
\hline ange [spot color]. & Table 3 \\
\hline Ordovician [geologic age symbol] . & 32.17 \\
\hline A-37-3 & 733 \\
\hline Ore chute (drawn to scale), as shown on subsurface exploration maps. & 19.4.13 \\
\hline 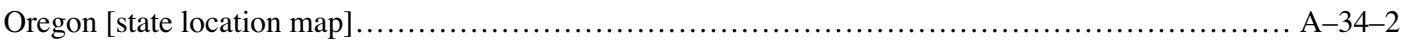 & Sec. 34.1 \\
\hline Orientation of contact [concepts and definitions]. & $\mathrm{n} / \mathrm{a}$ \\
\hline Orientation of fault [concepts and definitions] ... & $\mathrm{n} / \mathrm{a}$ \\
\hline Orientation of fold [concepts and definitions]. & $\mathrm{n} / \mathrm{a}$ \\
\hline 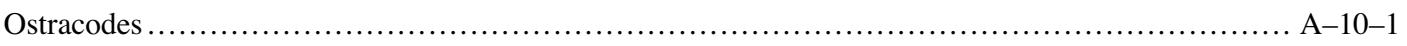 & 10.2 .53 \\
\hline Outcrop area of clay bed. & 1.2 .44 \\
\hline Outcrop area of coal bed.. & 1.2 .45 \\
\hline Outcrop area of key bed or bed of economically important commodity (1st option). & 1.2 .42 \\
\hline Outcrop area of key bed or bed of economically important commodity (2nd option). & 1.2 .43 \\
\hline Outcrop areas........... & Sec. 31 \\
\hline Outcrop point as structural control point (1st surface) & 11.22 \\
\hline Outcrop point as structural control point (2nd surface) & 11.32 \\
\hline Outcrop point as structural control point (3rd surface). & 11.42 \\
\hline Outer boundary of central mound of complex terrestrial impact crater. & 24.18 \\
\hline Outer boundary of floor of terrestrial impact crater.. & 24.17 \\
\hline Outer limit of subsidence caused by shock - Identity and existence certain, location accurate ...... & 21.16 \\
\hline Outer limit of subsidence caused by shock - Identity and existence certain, location concealed .......... A $-21-1$ & 21.22 \\
\hline Outer limit of subsidence caused by shock - Identity or existence certain, location approximate ......... A $-21-1$ & 21.18 \\
\hline Outer limit of subsidence caused by shock - Identity or existence certain, location inferred...... & 21.20 \\
\hline Outer limit of subsidence caused by shock - Identity or existence questionable, location accurate ....... A $-21-1$ & 21.17 \\
\hline Outer limit of subsidence caused by shock - Identity or existence questionable, location approximate .. A-21-1 & 21.19 \\
\hline Outer limit of subsidence caused by shock - Identity or existence questionable, location concealed ..... A $-21-1$ & 21.23 \\
\hline Outer limit of subsidence caused by shock - Identity or existence questionable, location inferred....... & 21.21 \\
\hline A-18-3 & 18.54 \\
\hline Outline of basin-Accurately located & 22.28 \\
\hline Outline of basin-Approximately located & 22.29 \\
\hline Outline of glacier .. & 30.2 .44 \\
\hline Outline of metamorphic core complex-Identity and existence certain, location accurate . & 23.1 \\
\hline Outline of metamorphic core complex-Identity and existence certain, location approximate . & 23.3 \\
\hline Outline of metamorphic core complex - Identity and existence certain, location concealed .. & 23.7 \\
\hline Outline of metamorphic core complex -Identity and existence certain, location inferred... & 23.5 \\
\hline Outline of metamorphic core complex-Identity or existence questionable, location accurate . & 23.2 \\
\hline Outline of metamorphic core complex -Identity or existence questionable, location approximate . & 23.4 \\
\hline Outline of metamorphic core complex - Identity or existence questionable, location concealed ... & 23.8 \\
\hline Outline of metamorphic core complex-Identity or existence questionable, location inferred.... & 23.6 \\
\hline Outline of permanent snowfield. & 30.2 .44 \\
\hline Outline of slip surface of landslide-Identity and existence certain, location accurate.. & 17.1 \\
\hline Outline of slip surface of landslide-Identity and existence certain, location approximate..... & 17.3 \\
\hline Outline of slip surface of landslide-Identity and existence certain, location concealed..... & 17.7 \\
\hline Outline of slip surface of landslide-Identity and existence certain, location inferred ..... & 17.5 \\
\hline Outline of slip surface of landslide-Identity or existence questionable, location accurate ........... & 17.2 \\
\hline Outline of slip surface of landslide-Identity or existence questionable, location approximate $\ldots \ldots \ldots \ldots$ A $-17-1$ & 17.4 \\
\hline Outline of slip surface of landslide-Identity or existence questionable, location concealed ....... & 17.8 \\
\hline Outline of slip surface of landslide-Identity or existence questionable, location inferred...... & 17.6 \\
\hline 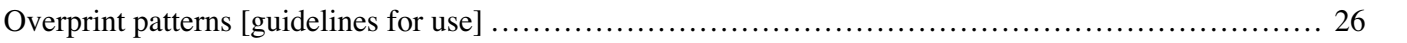 & $\mathrm{n} / \mathrm{a}$ \\
\hline Overturned anticline (1st option) - Identity and existence certain, location accurate $\ldots \ldots \ldots \ldots \ldots \ldots . . . . .$. & 5.3.17 \\
\hline Overturned anticline (1st option) - Identity and existence certain, location approximate $\ldots \ldots \ldots \ldots \ldots \ldots$ A $-5-3$ & 5.3 .19 \\
\hline Overturned anticline (1st option) - Identity and existence certain, location concealed....... & 5.3 .23 \\
\hline Overturned anticline (1st option) - Identity and existence certain, location inferred .... & 5.3.21 \\
\hline & \\
\hline
\end{tabular}




\begin{tabular}{|c|c|c|}
\hline & Page & Ref. No. \\
\hline Overturned anticline (1st option) - Identity or existence questionable, location approximate. & A-5-3 & 5.3 .20 \\
\hline Overturned anticline (1st option) - Identity or existence questionable, location concealed... & A-5-3 & 5.3 .24 \\
\hline Overturned anticline (1st option) - Identity or existence questionable, location inferred .... & A-5-3 & 5.3 .22 \\
\hline Overturned anticline (2nd option) - Identity and existence certain, location accurate.... & A-5-4 & 5.3 .25 \\
\hline Overturned anticline (2nd option)-Identity and existence certain, location approximate. & A-5-4 & 5.3 .27 \\
\hline Overturned anticline (2nd option) - Identity and existence certain, location concealed.... & A-5-4 & 5.3 .31 \\
\hline Overturned anticline (2nd option) - Identity and existence certain, location inferred .... & A-5-4 & 5.3 .29 \\
\hline Overturned anticline (2nd option) - Identity or existence questionable, location accurate.. & A-5-4 & 5.3 .26 \\
\hline Overturned anticline (2nd option) - Identity or existence questionable, location approximate... & A-5-4 & 5.3 .28 \\
\hline Overturned anticline (2nd option) - Identity or existence questionable, location concealed.... & A-5-4 & 5.3 .32 \\
\hline Overturned anticline (2nd option) - Identity or existence questionable, location inferred .... & A-5-4 & 5.3 .30 \\
\hline Overturned anticlines & A-5-3 & Sec. 5.3 \\
\hline Overturned bed of economically important commodity (1st option).. & A-1-6 & 1.4 .5 \\
\hline Overturned bed of economically important commodity (2nd option). & A-1-6 & 1.4 .6 \\
\hline Overturned bedding & A-6-1 & 6.4 \\
\hline Overturned bedding in crossbedded rocks & A-6-2 & 6.32 \\
\hline Overturned bedding, where top direction of beds is known from local features............ & A-6-1 & 6.15 \\
\hline 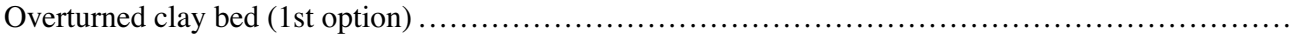 & A-1-6 & 1.4 .5 \\
\hline Overturned clay bed (2nd option) ................................... & A-1-6 & 1.4 .6 \\
\hline Overturned coal bed (1st option) ............ & A-1-6 & 1.4 .5 \\
\hline 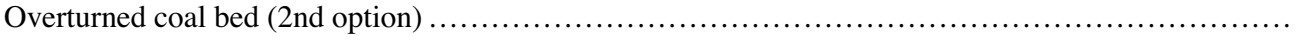 & A-1-6 & 1.4 .6 \\
\hline 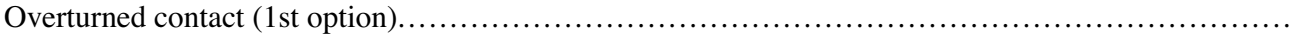 & A-1-6 & 1.4 .5 \\
\hline Overtu & A-1-6 & 1.4 .6 \\
\hline 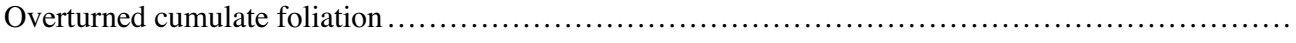 & A-8-1 & 8.2 .13 \\
\hline Overturned cumulate foliation, where top direction of layers is known from local features.......... & A-8-1 & 8.2 .16 \\
\hline 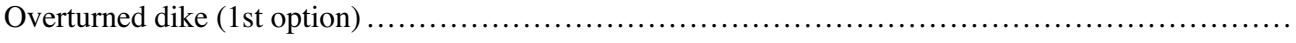 & A-1-6 & 1.4 .5 \\
\hline 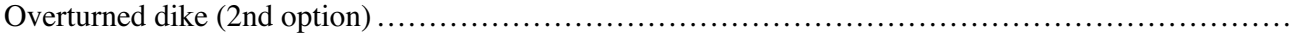 & A-1-6 & 1.4 .6 \\
\hline 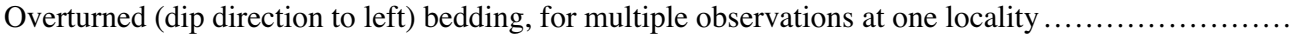 & A-6-1 & 6.10 \\
\hline $\begin{array}{l}\text { Overturned (dip direction to left) bedding, where top direction of beds is known from local features, } \\
\text { for multiple observations at one locality }\end{array}$ & A-6-1 & 6.22 \\
\hline Overturned (dip direction to right) bedding, for multiple observations at one locality $\ldots \ldots \ldots \ldots \ldots \ldots$ & A-6-1 & 6.9 \\
\hline $\begin{array}{l}\text { Overturned (dip direction to right) bedding, where top direction of beds is known from local features, } \\
\text { for multiple observations at one locality }\end{array}$ & A-6-1 & 6.21 \\
\hline Overturned graded bedding & A-6-2 & 6.29 \\
\hline Overturned key bed (1st option).. & A-1-6 & 1.4 .5 \\
\hline Overturned key bed (2nd option).. & A-1-6 & 1.4 .6 \\
\hline Overturned syncline (1st option)-Identity and existence certain, location accurate. & A-5-8 & 5.7.17 \\
\hline Overturned syncline (1st option)-Identity and existence certain, location approximate . & A-5-8 & 5.7 .19 \\
\hline Overturned syncline (1st option) - Identity and existence certain, location concealed .... & A-5-8 & 5.7 .23 \\
\hline Overturned syncline (1st option) - Identity and existence certain, location inferred.... & A-5-8 & 5.7 .21 \\
\hline Overturned syncline (1st option) - Identity or existence questionable, location accurate.. & A-5-8 & 5.7.18 \\
\hline Overturned syncline (1st option) - Identity or existence questionable, location approximate. & A-5-8 & 5.7 .20 \\
\hline Overturned syncline (1st option)-Identity or existence questionable, location concealed .... & A-5-8 & 5.7 .24 \\
\hline Overturned syncline (1st option) - Identity or existence questionable, location inferred ... & A-5-8 & 5.7 .22 \\
\hline Overturned syncline (2nd option) - Identity and existence certain, location accurate ..... & A-5-9 & 5.7 .25 \\
\hline Overturned syncline (2nd option) - Identity and existence certain, location approximate.. & A-5-9 & 5.7 .27 \\
\hline Overturned syncline (2nd option) - Identity and existence certain, location concealed ..... & A-5-9 & 5.7 .31 \\
\hline Overturned syncline (2nd option) - Identity and existence certain, location inferred...... & A-5-9 & 5.7 .29 \\
\hline Overturned syncline (2nd option) - Identity or existence questionable, location accurate ....... & A-5-9 & 5.7 .26 \\
\hline Overturned syncline (2nd option) - Identity or existence questionable, location approximate.. & A-5-9 & 5.7 .28 \\
\hline Overturned syncline (2nd option) - Identity or existence questionable, location concealed..... & A-5-9 & 5.7 .32 \\
\hline Overturned syncline (2nd option) - Identity or existence questionable, location inferred .... & A-5-9 & 5.7 .30 \\
\hline 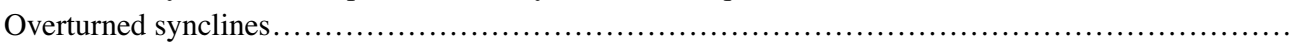 & A-5-8 & Sec. 5.7 \\
\hline Overturned thrust fault (1st option)-Identity and existence c & A-2-8 & 2.9 .1 \\
\hline Overturned thrust fault (1st option) - Identity and existence certain, loc & A-2-8 & 2.9 .3 \\
\hline
\end{tabular}




\begin{tabular}{|c|c|c|}
\hline & Page & Ref. No. \\
\hline Overturned thrust fault (1st option)-Identity and existence certain, location concealed.. & A-2-8 & 2.9 .7 \\
\hline Overturned thrust fault (1st option) - Identity and existence certain, location inferred.... & A-2-8 & 2.9 .5 \\
\hline Overturned thrust fault (1st option)-Identity or existence questionable, location accurate....... & A-2-8 & 2.9 .2 \\
\hline Overturned thrust fault (1st option) - Identity or existence questionable, location approximate... & A-2-8 & 2.9 .4 \\
\hline Overturned thrust fault (1st option) - Identity or existence questionable, location concealed...... & A-2-8 & 2.9 .8 \\
\hline Overturned thrust fault (1st option) - Identity or existence questionable, location inferred .... & A-2-8 & 2.9 .6 \\
\hline Overturned thrust fault (2nd option) - Identity and existence certain, location accurate ........... & A-2-8 & 2.9 .9 \\
\hline Overturned thrust fault (2nd option) - Identity and existence certain, location approximate...... & A-2-8 & 2.9.11 \\
\hline Overturned thrust fault (2nd option) - Identity and existence certain, location concealed........ & A-2-8 & 2.9.15 \\
\hline Overturned thrust fault (2nd option) - Identity and existence certain, location inferred.......... & A-2-8 & 2.9 .13 \\
\hline Overturned thrust fault (2nd option) - Identity or existence questionable, location accurate .............. & A-2-8 & 2.9.10 \\
\hline Overturned thrust fault (2nd option) - Identity or existence questionable, location approximate........ & A-2-8 & 2.9.12 \\
\hline Overturned thrust fault (2nd option) - Identity or existence questionable, location concealed.... & A-2-8 & 2.9.16 \\
\hline Overturned thrust fault (2nd option) - Identity or existence questionable, location inferred .... & A-2-8 & 2.9.14 \\
\hline Overturned thrust fault (3rd option) - Identity and existence certain, location accurate....... & A-2-8 & 2.9.17 \\
\hline Overturned thrust fault (3rd option) - Identity and existence certain, location approximate.. & A-2-8 & 2.9.19 \\
\hline Overturned thrust fault (3rd option) - Identity and existence certain, location concealed..... & A-2-8 & 2.9.23 \\
\hline Overturned thrust fault (3rd option) - Identity and existence certain, location inferred .... & A-2-8 & 2.9.21 \\
\hline Overturned thrust fault (3rd option)-Identity or existence questionable, location accurate . & A-2-8 & 2.9.18 \\
\hline Overturned thrust fault (3rd option)-Identity or existence questionable, location approximate . & A-2-8 & 2.9.20 \\
\hline Overturned thrust fault (3rd option) - Identity or existence questionable, location concealed ... & A-2-8 & 2.9.24 \\
\hline Overturned thrust fault (3rd option) - Identity or existence questionable, location inferred... & A-2-8 & 2.9 .22 \\
\hline 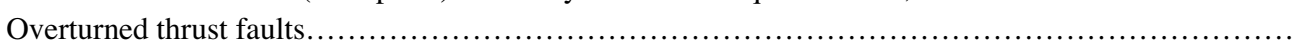 & A-2-8 & Sec. 2.9 \\
\hline Paleogene [geologic age symbol] & A-32-1 & 32.5 \\
\hline Paleontological features & A-10-1 & Sec. 10 \\
\hline Paleozoic [geologic age symbol] .. & A-32-1 & 32.10 \\
\hline Palimpsest area around complex terrestrial impact crater.. & A-24-1 & 24.20 \\
\hline 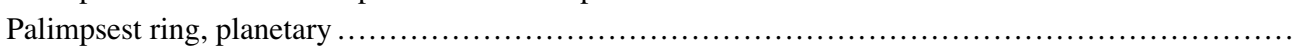 & A-25-4 & 25.93 \\
\hline Palynomorphs........ & A-10-1 & 10.2 .54 \\
\hline Pantone color [equivalents of spot colors] ... & $. . \mathrm{A}-\mathrm{v}$ & Table 3 \\
\hline 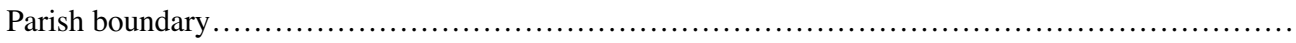 & A-29-1 & 29.3 \\
\hline Park (small) boundary & A-29-1 & 29.7 \\
\hline Partial-record low-flow measurement water gaging station (floods) $\ldots \ldots \ldots \ldots \ldots \ldots \ldots$ & A-26-5 & 26.3.11 \\
\hline Partial-record peak-flow measurement water gaging station (floods) $\ldots \ldots \ldots \ldots \ldots \ldots \ldots \ldots$ & A-26-5 & 26.3.10 \\
\hline Partial-record stage-measurement water gaging station (floods) $\ldots \ldots \ldots \ldots \ldots \ldots \ldots \ldots \ldots \ldots \ldots \ldots \ldots \ldots \ldots$ & A-26-5 & 26.3.12 \\
\hline 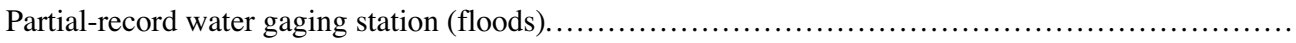 & A-26-5 & 26.3.8 \\
\hline Partial-record water gaging station (floods), equipped with a telephone or radio $\ldots \ldots \ldots \ldots \ldots \ldots \ldots \ldots$ & A-26-5 & 26.3.9 \\
\hline Parting lineation in sedimentary materials & A-9-1 & 9.9 \\
\hline Partly buried arcuate fracture, planetary..... & A-25-2 & 25.32 \\
\hline fracture, planetary .. & A-25-2 & 25.30 \\
\hline Path of gully on landslide.. & A-17-3 & 17.46 \\
\hline Pattern Chart ................ & In pocket & $\mathrm{n} / \mathrm{a}$ \\
\hline Pattern Chart explanation.... & A-38-1 & Sec. 38 \\
\hline Pattern selection guidelines ............... & 24 & $\mathrm{n} / \mathrm{a}$ \\
\hline Pattern specifications [in this standard]...... & .. 31 & $\mathrm{n} / \mathrm{a}$ \\
\hline Peak-flow measurement site without a gage. & A-26-5 & 26.3.15 \\
\hline Peat & A-37-2 & 657 \\
\hline Pelecypods........ & A-10-1 & 10.2 .24 \\
\hline Pencil structure........ & A-9-4 & 9.73 \\
\hline Penetrative foliation... & A-8-3 & 8.3.16 \\
\hline Penetrative foliation, for multiple observations at one locality. & A-8-3 & 8.3.19 \\
\hline Penetrative lineations, within tessera terrain, planetary. & A-25-3 & 25.66 \\
\hline
\end{tabular}

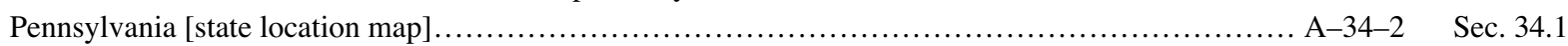




\begin{tabular}{|c|c|}
\hline Page & Ref. No. \\
\hline Pennsylvanian [geologic age symbol]. & 32.13 \\
\hline Penstock............................... & 30.2 .24 \\
\hline Perennial creek (double-line drainage) .... & 30.2 .3 \\
\hline Perennial creek (single-line drainage) ........ & 30.2 .1 \\
\hline Perennial lake ............................ & 30.2 .32 \\
\hline Perennial pond . & 30.2 .32 \\
\hline Perennial river (double-line drainage) .... & 30.2 .3 \\
\hline Perennial river (single-line drainage) ..... & 30.2 .1 \\
\hline Perennial stream (double-line drainage)... & 30.2 .3 \\
\hline Perennial stream (single-line drainage)..... & 30.2 .1 \\
\hline Periglacial features ......................... & Sec. 14 \\
\hline Periglacial patterned ground $\ldots . . . \ldots \ldots \ldots$ & 14.2 \\
\hline Periglacial patterns $($ Series 500$) \ldots$. & $\mathrm{n} / \mathrm{a}$ \\
\hline Permanent snowfield ..................... & 30.2 .44 \\
\hline Permian [geologic age symbol]........... & 32.11 \\
\hline Phosphatic-nodular rock .................. & 666 \\
\hline Pingo $\ldots \ldots \ldots \ldots \ldots \ldots \ldots$ & 14.1 \\
\hline Pipelines (oil and gas).................. & Sec. 30.3 \\
\hline Pipelines (water) .............................. & Sec. 30.2 \\
\hline Pit-crater chain (mapped to scale), planetary ................... & 25.105 \\
\hline Pit of impact crater floor, planetary (1st option) .. & 25.103 \\
\hline Pit of impact crater floor, planetary (2nd option) & 25.104 \\
\hline 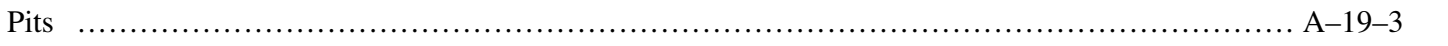 & Sec. 19.3 \\
\hline $\begin{array}{l}\text { Placement of point symbols for linear features relative to point of observation [concepts and } \\
\text { definitions }]\end{array}$ & $\mathrm{n} / \mathrm{a}$ \\
\hline $\begin{array}{l}\text { Placement of point symbols for planar features relative to point of observation }[\text { concepts and } \\
\text { definitions }]\end{array}$ & $\mathrm{n} / \mathrm{a}$ \\
\hline 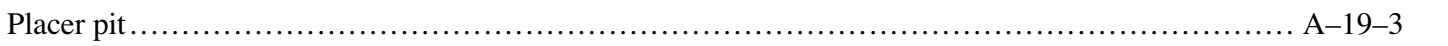 & 19.3 .2 \\
\hline 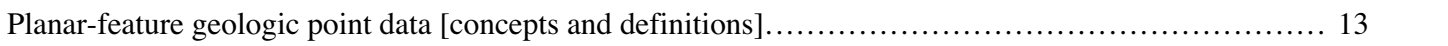 & $\mathrm{n} / \mathrm{a}$ \\
\hline 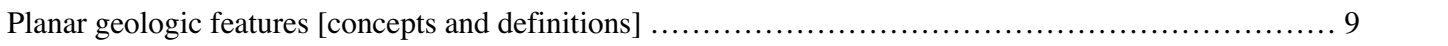 & $\mathrm{n} / \mathrm{a}$ \\
\hline 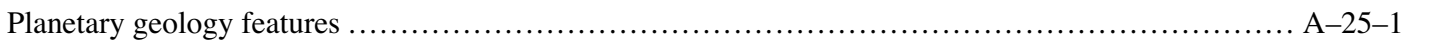 & Sec. 25 \\
\hline Planktonic foraminifera ..................................... & 10.2 .51 \\
\hline Plants $[$ fossil symbol] ...................................... & 10.2 .31 \\
\hline Plate-tectonic features......................................... & Sec. 22 \\
\hline Plugged and abandoned condensate well (nonspecific depth).... & 19.5 .75 \\
\hline Plugged and abandoned deep condensate well ..................... & 19.5 .81 \\
\hline Plugged and abandoned deep disposal well............................. & 19.5.31 \\
\hline Plugged and abandoned deep gas and condensate well $\ldots \ldots \ldots \ldots \ldots \ldots \ldots \ldots \ldots$ & 19.5.91 \\
\hline 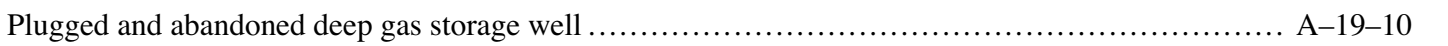 & 19.5 .97 \\
\hline Plugged and abandoned deep gas well.............................. & 19.5 .59 \\
\hline Plugged and abandoned deep observation well for gas-storage field.... & 19.5.103 \\
\hline Plugged and abandoned deep oil and gas well...................... & 19.5.71 \\
\hline 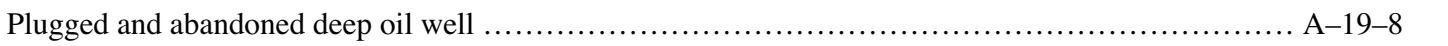 & 19.5.48 \\
\hline Plugged and abandoned deep salt-water disposal well .................... & 19.5.37 \\
\hline Plugged and abandoned disposal well (nonspecific depth) ........................... & 19.5.27 \\
\hline Plugged and abandoned gas and condensate well (nonspecific depth) ................ & 19.5 .85 \\
\hline Plugged and abandoned gas storage well (nonspecific depth) $\ldots \ldots \ldots \ldots \ldots \ldots \ldots \ldots \ldots \ldots \ldots \ldots \ldots$ A $-19-10$ & 19.5 .93 \\
\hline 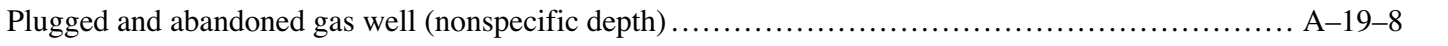 & 19.5 .53 \\
\hline Plugged and abandoned observation well for gas-storage field (nonspecific depth) ................. A-19-10 & 19.5.99 \\
\hline Plugged and abandoned oil and gas well (nonspecific depth) .................................... A $-19-9$ & 19.5 .65 \\
\hline Plugged and abandoned oil well (nonspecific depth) ........................................... A-19-8 & 19.5 .42 \\
\hline Plugged and abandoned salt-water disposal well (nonspecific depth) ............................. A-19-7 & 19.5 .33 \\
\hline Plugged and abandoned shallow condensate well.......................... & 19.5 .78 \\
\hline Plugged and abandoned shallow disposal well ............ & 19.5.29 \\
\hline Plugged and abandoned shallow gas and condensate well. & 19.5 .88 \\
\hline
\end{tabular}




\begin{tabular}{|c|c|}
\hline Page & Ref. No. \\
\hline Plugged and abandoned shallow gas storage well.......................... & 19.5 .95 \\
\hline Plugged and abandoned shallow gas well ........................................................ A-19-8 & 19.5 .56 \\
\hline Plugged and abandoned shallow observation well for gas-storage field ....... & 19.5.101 \\
\hline Plugged and abandoned shallow oil and gas well .............................. & 19.5.68 \\
\hline Plugged and abandoned shallow oil well................................. & 19.5 .45 \\
\hline Plugged and abandoned shallow salt-water disposal well............. & 19.5 .35 \\
\hline 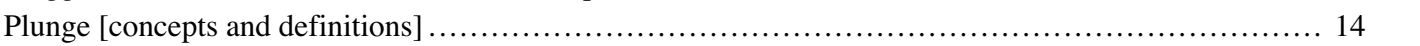 & $\mathrm{n} / \mathrm{a}$ \\
\hline 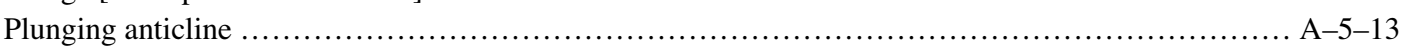 & 5.10 .5 \\
\hline Plunging syncline ...................................... & 5.10 .7 \\
\hline Plus/minus [notation on fault in cross section] ............. & 2.11 .21 \\
\hline Point features [concepts and definitions] ......................... & $\mathrm{n} / \mathrm{a}$ \\
\hline 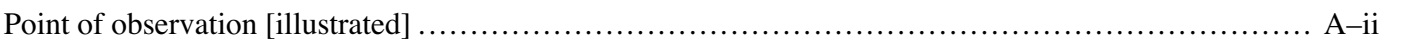 & $\mathrm{n} / \mathrm{a}$ \\
\hline Point of observation of point symbols for linear features [concepts and definitions] .................... 14 & $\mathrm{n} / \mathrm{a}$ \\
\hline Point of observation of point symbols for planar features [concepts and definitions] .................... 13 & $\mathrm{n} / \mathrm{a}$ \\
\hline 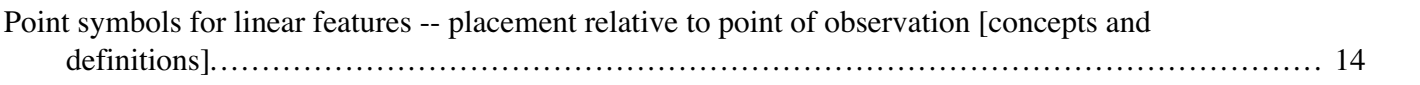 & $\mathrm{n} / \mathrm{a}$ \\
\hline 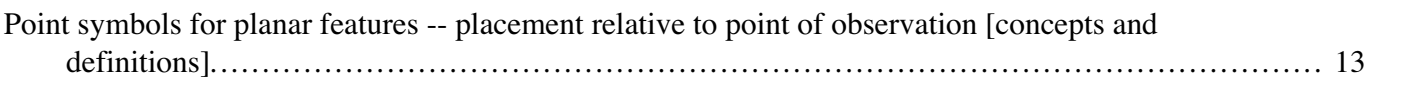 & $\mathrm{n} / \mathrm{a}$ \\
\hline 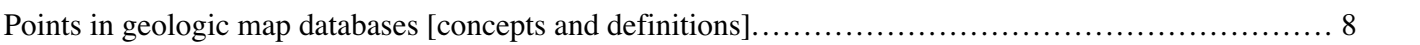 & $\mathrm{n} / \mathrm{a}$ \\
\hline 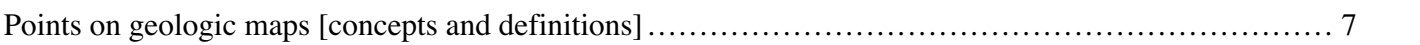 & $\mathrm{n} / \mathrm{a}$ \\
\hline 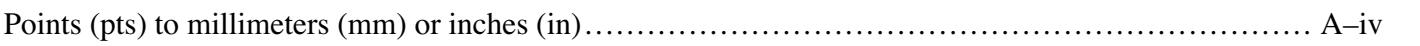 & Table 1 \\
\hline 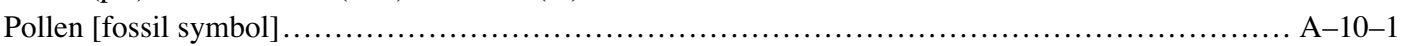 & 10.2 .58 \\
\hline Polygon, ice-wedge............. & 14.8 \\
\hline 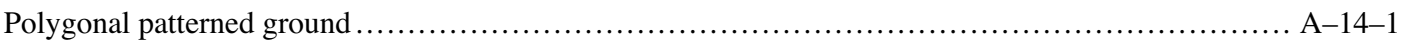 & 14.3 \\
\hline 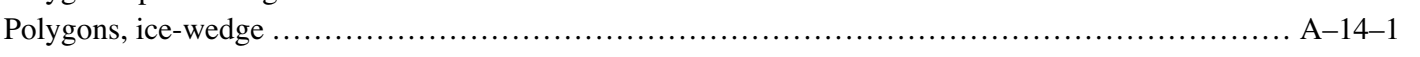 & 14.9 \\
\hline Polygons in geologic map databases [concepts and definitions] .................... & $\mathrm{n} / \mathrm{a}$ \\
\hline 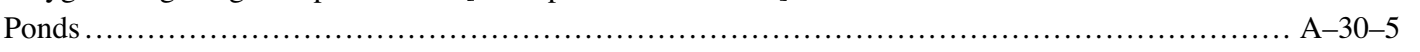 & Sec. 30.2 \\
\hline 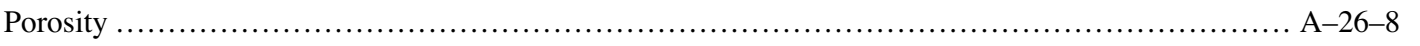 & 26.6.17 \\
\hline Porphyritic rock (1st option) .............. & 729 \\
\hline 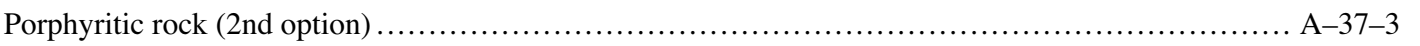 & 730 \\
\hline 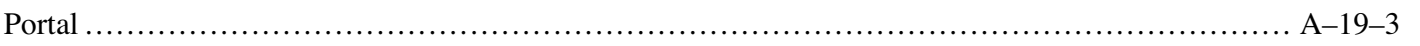 & 19.3 .19 \\
\hline Portal and open cut ....................................... & 19.3.23 \\
\hline 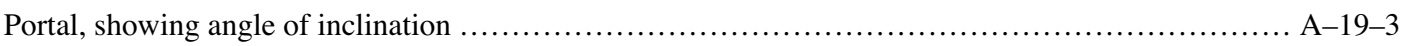 & 19.3.24 \\
\hline 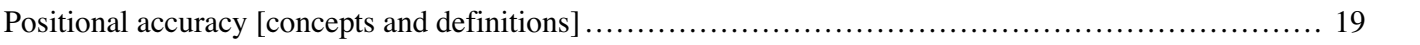 & $\mathrm{n} / \mathrm{a}$ \\
\hline 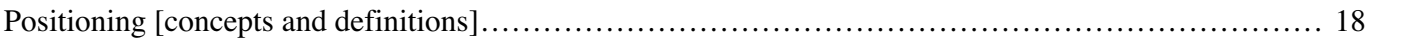 & $\mathrm{n} / \mathrm{a}$ \\
\hline 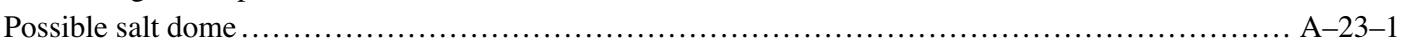 & 23.14 \\
\hline 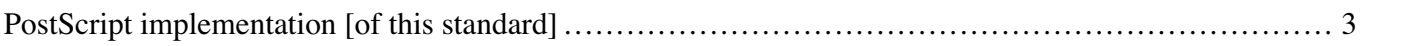 & $\mathrm{n} / \mathrm{a}$ \\
\hline $\begin{array}{l}\text { Potentiometric contour (index), showing altitude at which water level would have stood in tightly } \\
\text { cased wells [date] }- \text { Accurately located } \ldots \ldots \ldots \ldots \ldots \ldots \ldots \ldots \ldots \ldots \ldots \ldots \ldots \ldots \ldots \ldots \ldots \ldots \ldots \ldots \ldots \ldots \ldots \ldots \ldots \ldots \ldots \ldots \ldots \\
\text { A }\end{array}$ & 26.5 .13 \\
\hline $\begin{array}{l}\text { Potentiometric contour (index), showing altitude at which water level would have stood in tightly } \\
\text { cased wells [date] }- \text { Approximately located } \ldots \ldots \ldots \ldots \ldots \ldots \ldots \ldots \ldots \ldots \ldots \ldots \ldots \ldots \ldots \ldots \ldots \ldots \ldots \ldots \ldots \ldots \ldots \ldots \ldots \ldots \ldots \\
\text { A }\end{array}$ & 26.5.14 \\
\hline 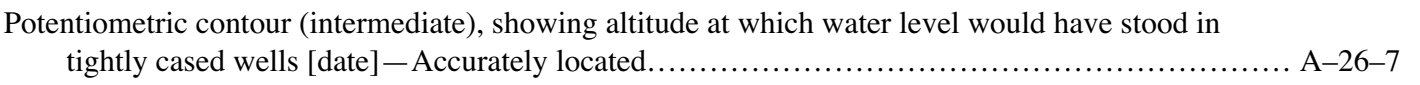 & 26.5.15 \\
\hline 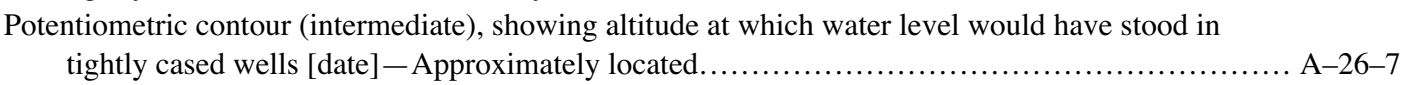 & 26.5 .16 \\
\hline Power transmission line & 30.3 .21 \\
\hline 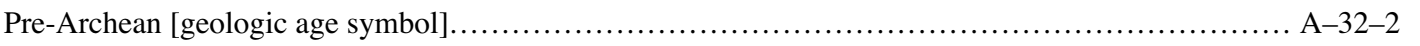 & 32.34 \\
\hline 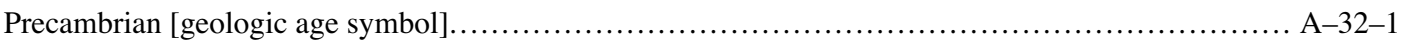 & 32.19 \\
\hline 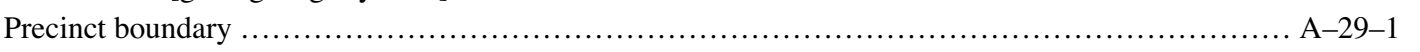 & 29.4 \\
\hline 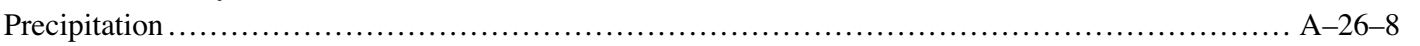 & 26.6.1 \\
\hline 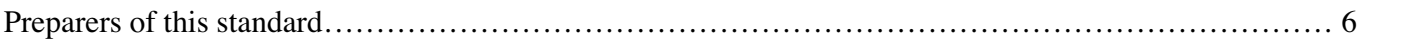 & $\mathrm{n} / \mathrm{a}$ \\
\hline Pressure ridge in landslide - Active, sharp, distinct, and accurately located $\ldots \ldots \ldots \ldots \ldots \ldots \ldots \ldots \ldots \ldots \ldots \ldots \ldots \ldots \ldots \ldots \ldots \ldots$ & 17.22 \\
\hline Pressure ridge in landslide - Inactive, subdued, indistinct, and (or) approximately located ............. A-17-2 & 17.23 \\
\hline 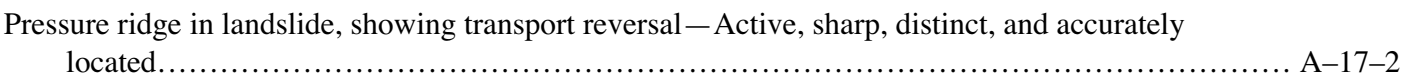 & 17.24 \\
\hline
\end{tabular}


Pressure ridge in landslide, showing transport reversal-Inactive, subdued, indistinct, and (or) approximately located.

Ref. No.

Pressure ridge on lava flow...

A-17-2 17.25

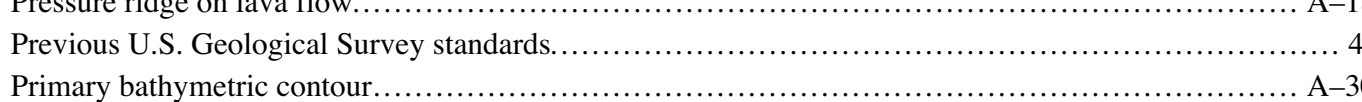

A-18-2 18.47

A-30-2

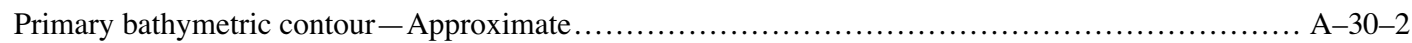

Primary bathymetric depression contour .............................................. A $-30-2$

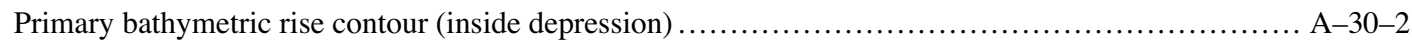

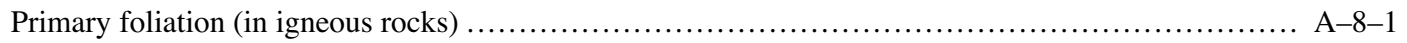

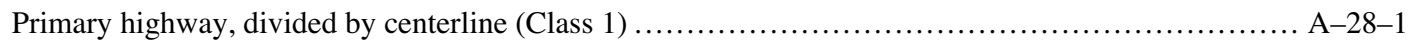

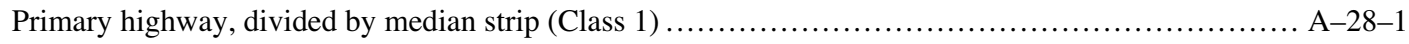

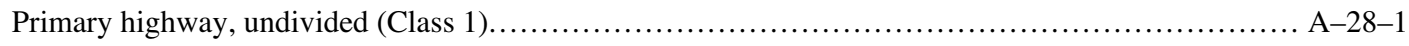

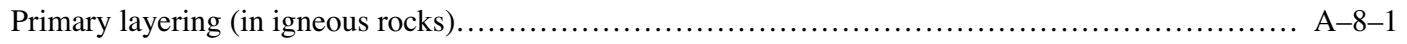

Primary terrestrial impact crater (too small to draw to scale) (1st option) ....................... A-24-1

Primary terrestrial impact crater (too small to draw to scale) (2nd option).......................... A-24-1

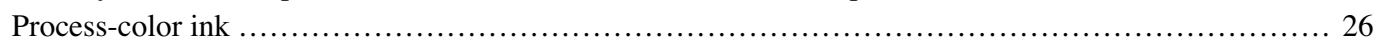

Prospect, as shown on topographic maps or on general-purpose or smaller scale maps................ A-30-6

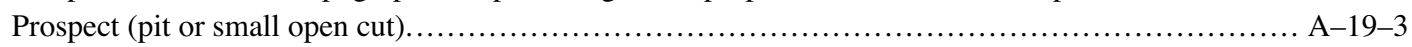

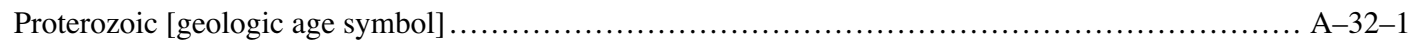

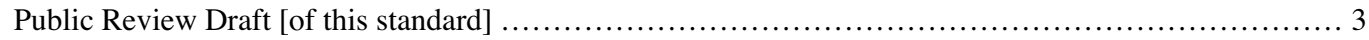

Puerto Rico $[$ location map] .......................................................... A $-34-2$

Pumping station, as shown on topographic maps or on general-purpose or smaller scale maps......... A-30-6

Purple [spot color].

A-V

Purpose of map [influencing color and pattern selection].....

Quality-of-water site, type of measurement unspecified ................................... A-26-6

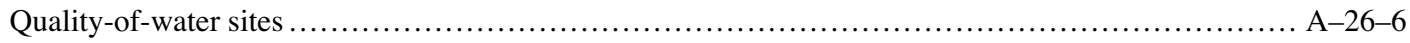

Quarry ............................................................................ A-19-3

Quarry, as shown on topographic maps or on general-purpose or smaller scale maps................ A-30-6

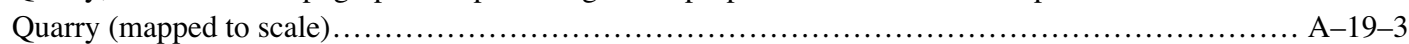

Sec. 34.1

30.3.15

Table 3

$\mathrm{n} / \mathrm{a}$

Quartz .......................................................................... A $-37-3$

Quartzite ......................................................................... A $-37-3$

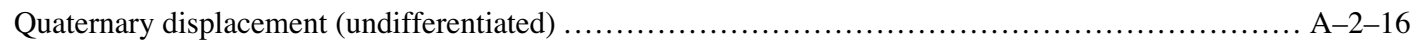

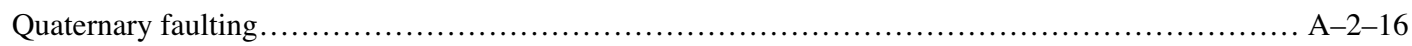

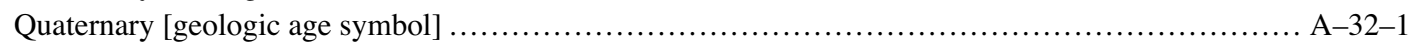

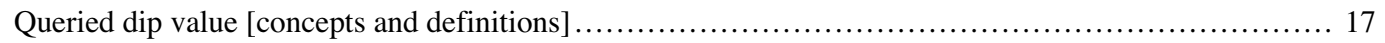

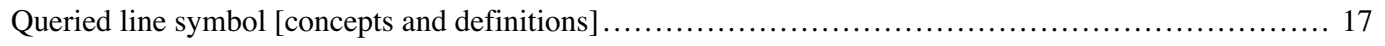

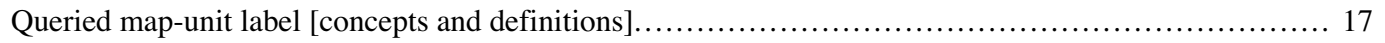

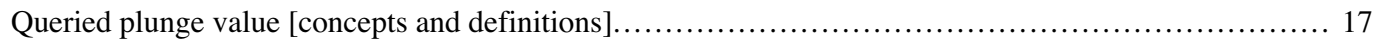

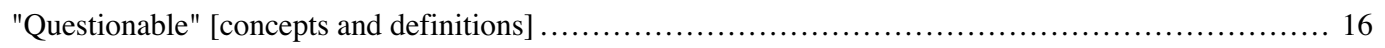

26.4.1

Sec. 26.4

19.3.4

30.3 .1

19.3.6

732

702

2.13.4

Sec. 2.13

32.2

$\mathrm{n} / \mathrm{a}$

n/a

$\mathrm{n} / \mathrm{a}$

$\mathrm{n} / \mathrm{a}$

$\mathrm{n} / \mathrm{a}$

$\mathrm{R}$ [abbreviation]

A-V

Radial fracture, planetary (associated with coronae) ................................................ A

Table 2

Radially grooved ejecta (schematic), planetary .......................................... A $-25-3$

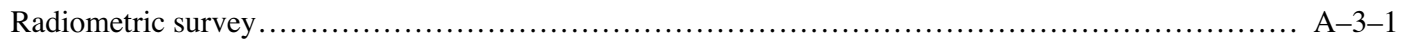

Sec. 3

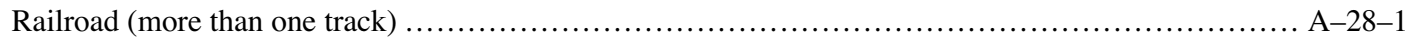

28.20

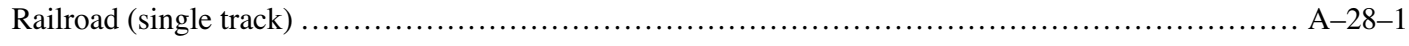

28.19

Raise (drawn to scale), as shown on subsurface exploration maps ............................ A-19-5

19.4 .8

Raise extending through level (drawn to scale), as shown on subsurface exploration maps ............ A-19-5

19.4 .7

Raised rim of impact crater, planetary, showing visible ejecta blanket .......................... A-25-5

25.96

Raised rim of larger impact crater, planetary ........................................... A $-25-5$

25.94

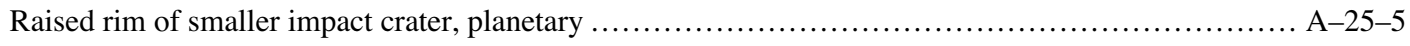

25.95

Range label 


\begin{tabular}{|c|c|}
\hline Page & Ref. No. \\
\hline 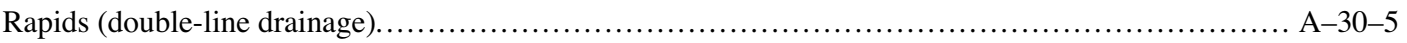 & \\
\hline apids (single-line drainage)..... & 30.2 .27 \\
\hline 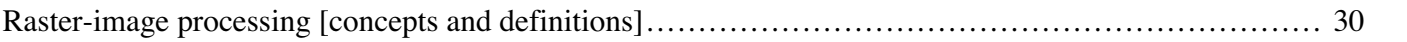 & $\mathrm{n} / \mathrm{a}$ \\
\hline 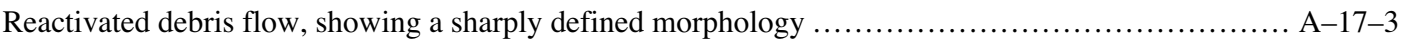 & 17.56 \\
\hline Reactivated landslide (mapped to scale), showing a sharply defined morphology $\ldots \ldots \ldots \ldots \ldots \ldots \ldots \ldots \ldots \ldots \ldots \ldots \ldots \ldots$ & 17.58 \\
\hline 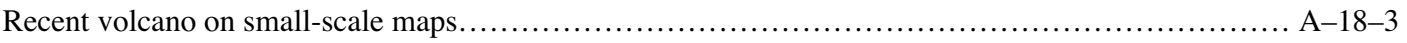 & 18.65 \\
\hline 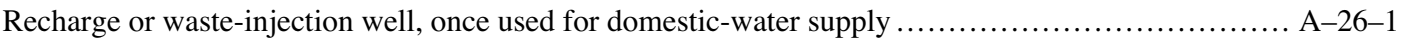 & 26.1.10 \\
\hline 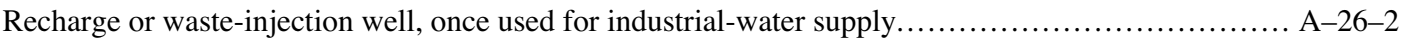 & 26.1.37 \\
\hline 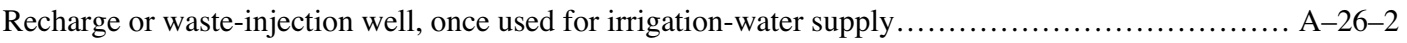 & 26.1.28 \\
\hline 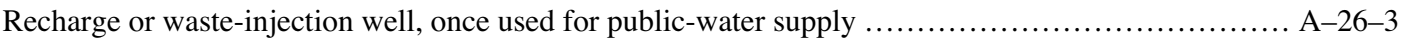 & 26.1.46 \\
\hline Recharge or waste-injection well, once used for stock-water supply & 26.1 . \\
\hline 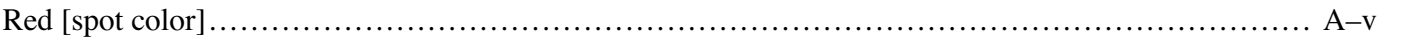 & Tabl \\
\hline References.. & $\mathrm{n} / \mathrm{a}$ \\
\hline Regional fracture, $\mathrm{p}$ & 25.29 \\
\hline Related standards [to this standard]. & $\mathrm{n} / \mathrm{a}$ \\
\hline
\end{tabular}

Relation of new FGDC standard terminology to historically used terminology and to traditional line

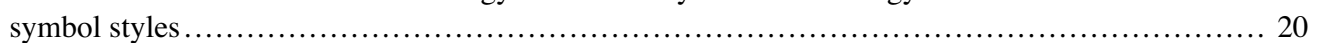

Relation [of this standard] to previous U.S. Geological Survey standards ............................. 4

Relative age of intrusive or extrusive units where known (Y, younger; O, older).................... A-1-6

Relict landslide (mapped to scale), showing a weak morphology .............................. A-17-3

Reptiles $[$ fossil symbol] ............................................................ A $-10-1$

Reservoir (uncovered) with man-made shoreline $\ldots \ldots \ldots \ldots \ldots \ldots \ldots \ldots \ldots \ldots \ldots \ldots \ldots \ldots \ldots \ldots \ldots$ A $-30,5$

Reservoir with natural shoreline ................................................... A $-30-5$

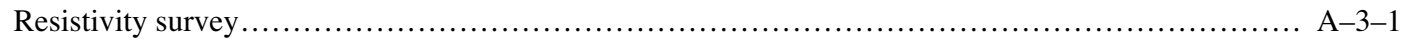

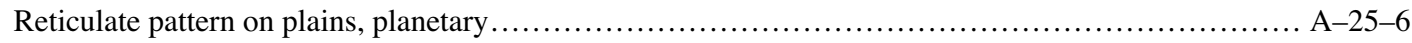

Retreatal position of stagnant ice margin - Identity and existence certain, location accurate ........... A-13-3

Retreatal position of stagnant ice margin - Identity and existence certain, location approximate ........ A-13-3

Retreatal position of stagnant ice margin - Identity and existence certain, location concealed .......... A-13-3

Retreatal position of stagnant ice margin - Identity and existence certain, location inferred ............ A-13-3

Retreatal position of stagnant ice margin - Identity or existence questionable, location accurate ........ A-13-3

Retreatal position of stagnant ice margin - Identity or existence questionable, location approximate .... A-13-3

Retreatal position of stagnant ice margin - Identity or existence questionable, location concealed ....... A-13-3

Retreatal position of stagnant ice margin - Identity or existence questionable, location inferred......... A-13-3

Retreatal position of stagnant ice margin, showing name of depositional unit........................ A-13-3

Reverse fault-Identity and existence certain, location accurate .............................. A-2-3

A $-2-3$

Reverse fault - Identity and existence certain, location approximate $\ldots \ldots \ldots \ldots \ldots \ldots \ldots \ldots \ldots \ldots \ldots \ldots \ldots \ldots \ldots \ldots \ldots \ldots \ldots$

Reverse fault - Identity and existence certain, location concealed $\ldots \ldots \ldots \ldots \ldots \ldots \ldots \ldots \ldots \ldots \ldots \ldots \ldots \ldots \ldots \ldots \ldots \ldots \ldots \ldots$

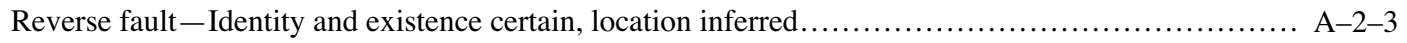

Reverse fault-Identity or existence questionable, location accurate $\ldots \ldots \ldots \ldots \ldots \ldots \ldots \ldots \ldots \ldots \ldots \ldots$ A $-2-3$

Reverse fault -Identity or existence questionable, location approximate $\ldots \ldots \ldots \ldots \ldots \ldots \ldots \ldots \ldots \ldots \ldots \ldots \ldots \ldots \ldots \ldots \ldots$

Reverse fault-Identity or existence questionable, location concealed ........................... A-2-3

Fig. 1

$\mathrm{n} / \mathrm{a}$

1.4 .9

17.61

10.2 .30

30.2 .39

30.2 .36

Sec. 3

25.131

13.64

13.66

13.70

13.68

13.65

13.67

13.71

13.69

13.72

2.4 .1

Reverse fault-Identity or existence questionable, location inferred.............................. A $-2-3$

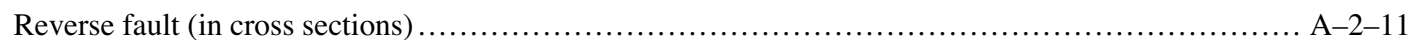

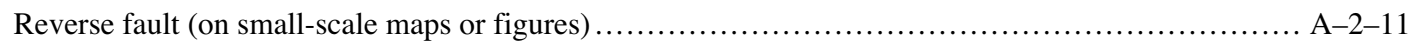

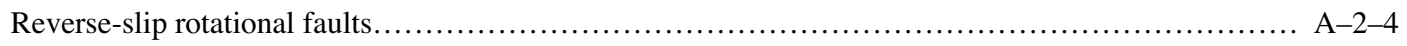

Sec. 2.4

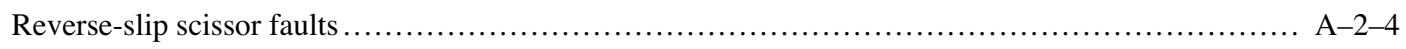

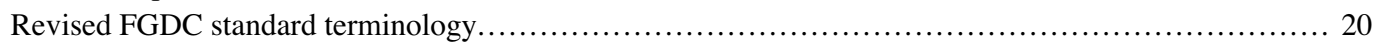

Sec. 2.5

Sec. 2.5

Fig. 1

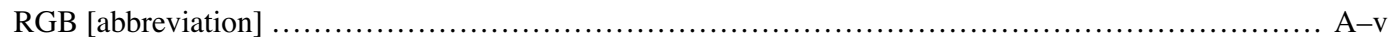

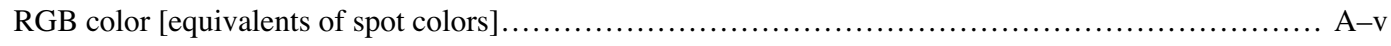

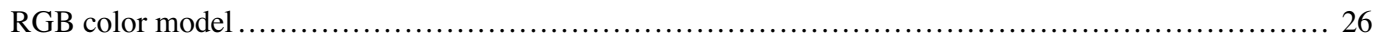

Table 2

Table 3

$\mathrm{n} / \mathrm{a}$

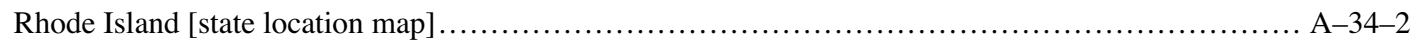

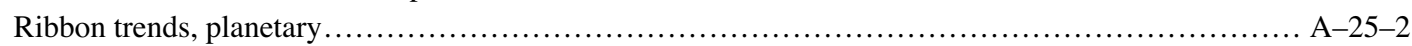

Sec. 34.1

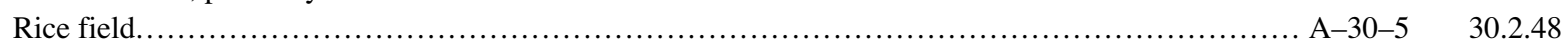




\begin{tabular}{|c|c|}
\hline Page & Ref. No. \\
\hline Ridge belt, planetary .. & 25.39 \\
\hline 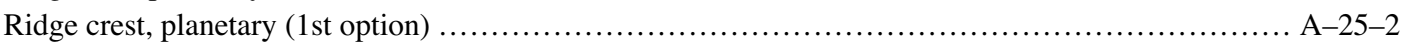 & 25.41 \\
\hline Ridge crest, planetary (1st option), showing abrupt termination of ridge....... & 25.43 \\
\hline 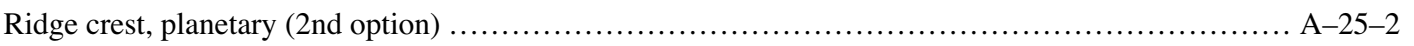 & 25.42 \\
\hline Ridge crest, planetary (2nd option), showing abrupt termination of ridge................ & 25.44 \\
\hline 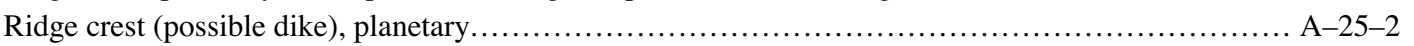 & 25.45 \\
\hline Ridges in landslides ...................... & Sec. 17 \\
\hline Ridges on beach.......................... & 15.2 \\
\hline 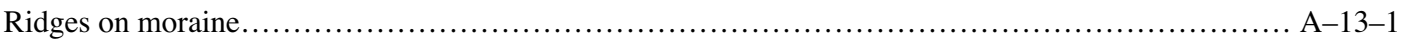 & 13.14 \\
\hline Right flank of landslide-Active, sharp, distinct, and accurately located ... & 17.26 \\
\hline Right flank of landslide - Concealed by landslide deposits or debris materials ....................... A-17-2 & 17.28 \\
\hline Right flank of landslide - Inactive, subdued, indistinct, and (or) approximately located............... A-17-2 & 17.27 \\
\hline Right flank of landslide, showing amount of offset............................................. A $-17-2$ & 17.29 \\
\hline 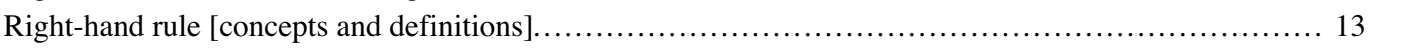 & $\mathrm{n} / \mathrm{a}$ \\
\hline 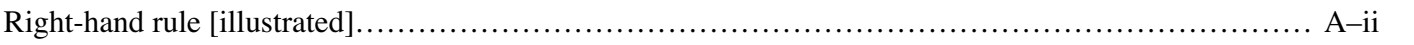 & $\mathrm{n} / \mathrm{a}$ \\
\hline 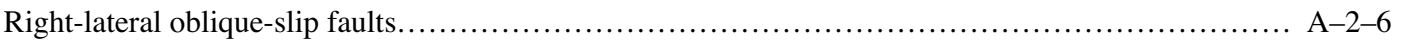 & Sec. 2.7 \\
\hline Right-lateral shear feature in landslide - Active, sharp, distinct, and accurately located................ A-17-2 & 17.26 \\
\hline Right-lateral shear feature in landslide - Concealed by landslide deposits or debris materials........... A-17-2 & 17.28 \\
\hline Right-lateral shear feature in landslide-Inactive, subdued, indistinct, and (or) approximately located.. A-17-2 & 17.27 \\
\hline Right-lateral shear feature in landslide, showing amount of offset .............................. A-17-2 & 17.29 \\
\hline 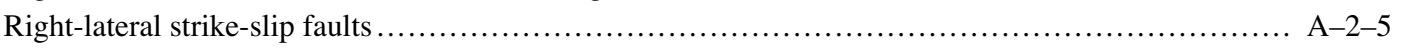 & Sec. 2.6 \\
\hline Rim around closed depression of eolian origin in bedrock - Accurately located..................... A-16-1 & 16.4 \\
\hline Rim around closed depression of eolian origin in bedrock - Approximately located.................. A-16-1 & 16.5 \\
\hline 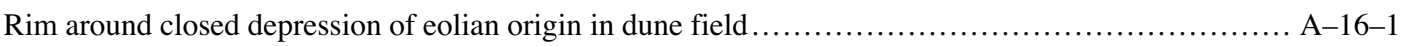 & 16.3 \\
\hline Rim crest, formed by shock or sand blowouts - Identity and existence certain, location accurate........ A-21-2 & 21.24 \\
\hline Rim crest, formed by shock or sand blowouts - Identity and existence certain, location concealed....... A-21-2 & 21.28 \\
\hline Rim crest, formed by shock or sand blowouts - Identity or existence certain, location approximate..... A-21-2 & 21.26 \\
\hline Rim crest, formed by shock or sand blowouts - Identity or existence questionable, location accurate.... A-21-2 & 21.25 \\
\hline 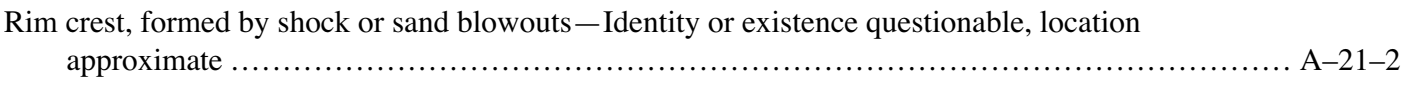 & 21.27 \\
\hline 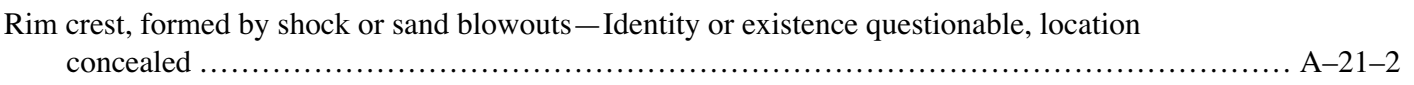 & 21.29 \\
\hline Rim of volcanic crater - Identity and existence certain, location accurate $\ldots \ldots \ldots \ldots \ldots \ldots \ldots \ldots \ldots \ldots$ A-18 -1 & 18.1 \\
\hline Rim of volcanic crater - Identity and existence certain, location concealed $\ldots \ldots \ldots \ldots \ldots \ldots \ldots \ldots \ldots \ldots \ldots \ldots \ldots \ldots \ldots$ & 18.5 \\
\hline Rim of volcanic crater-Identity or existence certain, location approximate $\ldots \ldots \ldots \ldots \ldots \ldots \ldots \ldots \ldots \ldots \ldots \ldots \ldots \ldots \ldots$ & 18.3 \\
\hline Rim of volcanic crater-Identity or existence questionable, location accurate .................. & 18.2 \\
\hline Rim of volcanic crater - Identity or existence questionable, location approximate ................... A-18-1 & 18.4 \\
\hline Rim of volcanic crater - Identity or existence questionable, location concealed ..................... A-18-1 & 18.6 \\
\hline Rim of volcanic crater, showing low point of crater................................................ A & 18.7 \\
\hline Rimless impact crater, planetary...................... & 25.98 \\
\hline "RIP" [concepts and definitions] .................... & $\mathrm{n} / \mathrm{a}$ \\
\hline Ripple-bedded sand ................. & 611 \\
\hline Ripple-bedded sandstone .......... & 611 \\
\hline Ripple-bedded subgraywacke...... & 656 \\
\hline River mileage marker ......................... & 30.2 .4 \\
\hline Rivers............................ & Sec. 30.2 \\
\hline Road (generic) .................. & 28.2 \\
\hline Roads ............................ & Sec. 28 \\
\hline 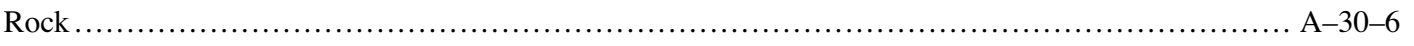 & 30.3 .16 \\
\hline Rock slide, consisting of a relatively intact mass of displaced materials ...... & 17.62 \\
\hline 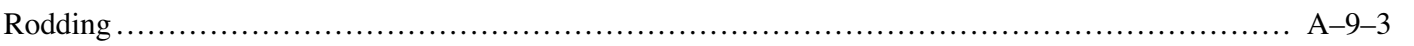 & 9.61 \\
\hline Rootless vent area on lava flow................................. & 18.60 \\
\hline 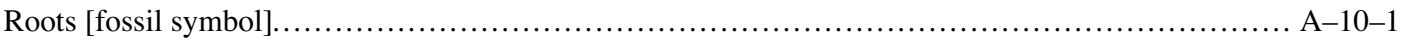 & 10.2 .33 \\
\hline Rotational fault, normal-slip offset - Identity and existence certain, location accurate........... & 2.5 .9 \\
\hline Rotational fault, normal-slip offset-Identity and existence certain, location approximate... & 2.5.11 \\
\hline
\end{tabular}




\begin{tabular}{|c|c|c|}
\hline & Page & Ref. No. \\
\hline tational fault, normal-slip offset-Identity and existence certain, location concealed.. & A-2-4 & 2.5.15 \\
\hline tational fault, normal-slip offset-Identity and existence certain, location inferred ....... & A-2-4 & 2.5 .13 \\
\hline otational fault, normal-slip offset-Identity or existence questionable, location accurate ..... & A $-2-4$ & 2.5 .10 \\
\hline otational fault, normal-slip offset-Identity or existence questionable, location approximate .... & A $-2-4$ & 2.5 .12 \\
\hline otational fault, normal-slip offset-Identity or existence questionable, location concealed ... & A-2-4 & 2.5.16 \\
\hline otational fault, normal-slip offset-Identity or existence questionable, location inferred.... & A-2-4 & 2.5 .14 \\
\hline otational fault, reverse-slip offset-Identity and existence certain, location accurate $\ldots \ldots \ldots \ldots$ & A-2-4 & 2.5 .1 \\
\hline otational fault, reverse-slip offset-Identity and existence certain, location approximate .... & A-2-4 & 2.5.3 \\
\hline otational fault, reverse-slip offset-Identity and existence certain, location concealed .... & A-2-4 & 2.5 .7 \\
\hline otational fault, reverse-slip offset-Identity and existence certain, location inferred....... & A-2-4 & 2.5 .5 \\
\hline otational fault, reverse-slip offset-Identity or existence questionable, location accurate $\ldots \ldots \ldots$ & A-2-4 & 2.5.2 \\
\hline otational fault, reverse-slip offset-Identity or existence questionable, location approximate. & A-2-4 & 2.5 .4 \\
\hline otational fault, reverse-slip offset-Identity or existence questionable, location concealed ... & A-2-4 & 2.5 .8 \\
\hline otational fault, reverse-slip offset-Identity or existence questionable, location inferred... & A-2-4 & 2.5 .6 \\
\hline Rotational faults . & A-2-4 & Sec. 2.5 \\
\hline Rotational landslide, consisting of a relatively intact mass of displaced materials. & A-17-3 & 17.62 \\
\hline Ruir & A-30-6 & 30.3 .20 \\
\hline Runoff. & A-26-8 & 26.6.15 \\
\hline on landslide. & A-17-3 & 17.48 \\
\hline Sag pond on landslide (ma & A-17-3 & 17.49 \\
\hline Salt & A-37-2 & 668 \\
\hline Salt and (or) shale diapirs. & A-23-1 & 23.15 \\
\hline Salt dome & A-23-1 & 23.13 \\
\hline Salt flat... & A-30-5 & 30.2 .41 \\
\hline Salt-water disposal well (n & A-19-7 & 19.5.32 \\
\hline Sample loc & A-31-1 & 31.21 \\
\hline Sand pit . & A-19-3 & 19.3.2 \\
\hline Sand pit, as shown on topographic maps or on general-purpose or smaller scale maps. & A-30-6 & 30.3.2 \\
\hline Sandy do & A $-37-2$ & 645 \\
\hline Sandy & A-37-2 & 645 \\
\hline Sandy & A-37-1 & 636 \\
\hline Sandy & A-37-1 & 619 \\
\hline Sans-se & .. A-ii & $\mathrm{n} / \mathrm{a}$ \\
\hline Saturated & A-26-8 & 26.6.5 \\
\hline Scale calculati & A-35-6 & Sec. 35 \\
\hline Scales.. & A-35-1 & Sec. 35 \\
\hline Scarp at top of ice-contact slope.... & A-13-1 & 13.15 \\
\hline $\begin{array}{l}\text { Scarp on detachment fault (sense of slip unspecified) (1st option) - Identity and existence certain, } \\
\text { location accurate }\end{array}$ & & 2.12 .69 \\
\hline $\begin{array}{l}\text { Scarp on detachment fault (sense of slip unspecified) (1st option) - Identity and existence certain, } \\
\text { location approximate }\end{array}$ & A $-2-15$ & 2.12 .71 \\
\hline $\begin{array}{l}\text { Scarp on detachment fault (sense of slip unspecified) (1st option) - Identity or existence } \\
\text { questionable, location accurate }\end{array}$ & & 2.12 .70 \\
\hline $\begin{array}{l}\text { Scarp on detachment fault (sense of slip unspecified) (1st option) - Identity or existence } \\
\text { questionable, location approximate }\end{array}$ & A-2-15 & 2.12 .72 \\
\hline $\begin{array}{l}\text { Scarp on detachment fault (sense of slip unspecified) (2nd option) - Identity and existence certain, } \\
\text { location accurate }\end{array}$ & A-2- & 2.12 .73 \\
\hline $\begin{array}{l}\text { Scarp on detachment fault (sense of slip unspecified) (2nd option) - Identity and existence certain, } \\
\text { location approximate }\end{array}$ & A-2-15 & 2.12 .75 \\
\hline $\begin{array}{l}\text { Scarp on detachment fault (sense of slip unspecified) (2nd option) - Identity or existence } \\
\text { questionable, location accurate }\end{array}$ & A-2-15 & 2.12 .74 \\
\hline & & \\
\hline
\end{tabular}


Scarp on detachment fault (sense of slip unspecified) (3rd option) - Identity and existence certain, location accurate...

Scarp on detachment fault (sense of slip unspecified) (3rd option) - Identity and existence certain, location approximate

carp on detachment fault (sense of slip unspecified) (3rd option) - Identity or existence

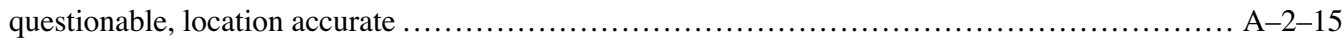

Scarp on detachment fault (sense of slip unspecified) (3rd option) - Identity or existence questionable, location approximate

Scarp on dune crest, caused by slip A-16-1

Scarp on fault-Identity and existence certain, location accurate ............................... A-2-12

Scarp on fault-Identity and existence certain, location approximate ............................. A-2-12

Scarp on fault-Identity or existence questionable, location accurate ............................. A $-2-12$

Scarp on fault-Identity or existence questionable, location approximate ........................ A-2-12

Scarp on listric fault at head of detachment fault (sense of slip unspecified) - Identity and existence

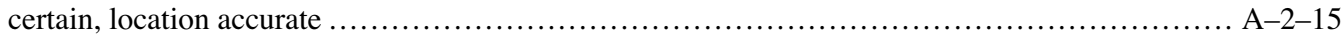

Scarp on listric fault at head of detachment fault (sense of slip unspecified) - Identity and existence

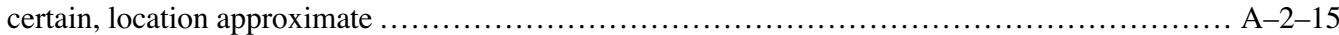

Scarp on listric fault at head of detachment fault (sense of slip unspecified) - Identity or existence questionable, location accurate ....................................................... A $-2-15$

Scarp on listric fault at head of detachment fault (sense of slip unspecified) - Identity or existence

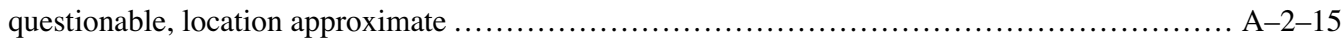

Scarp on low-angle fault (unknown or unspecified sense of slip) - Identity and existence certain, location accurate.

Scarp on low-angle fault (unknown or unspecified sense of slip) - Identity and existence certain, location approximate

Scarp on low-angle fault (unknown or unspecified sense of slip) - Identity or existence questionable, location accurate

Scarp on low-angle fault (unknown or unspecified sense of slip)-Identity or existence

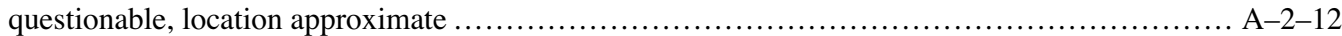

Scarp on low-angle normal fault-Identity and existence certain, location accurate ................. A-2-12

Scarp on low-angle normal fault-Identity and existence certain, location approximate ................ A-2-12

Scarp on low-angle normal fault-Identity or existence questionable, location accurate ................ A-2-12

Scarp on low-angle normal fault-Identity or existence questionable, location approximate ............ A-2-12

Scarp on master detachment fault (sense of slip unspecified) - Identity and existence certain,

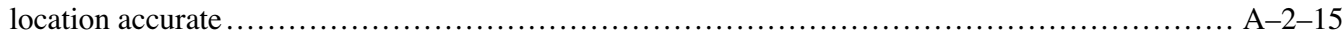

Scarp on master detachment fault (sense of slip unspecified) - Identity and existence certain,

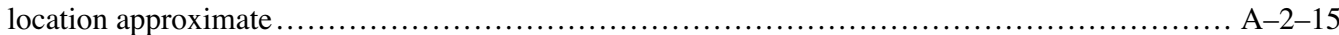

Scarp on master detachment fault (sense of slip unspecified) - Identity or existence questionable,

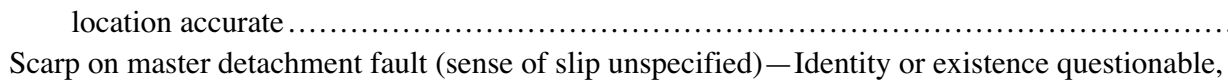

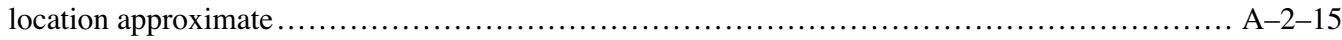

Scarp on normal fault - Identity and existence certain, location accurate ........................ A $-2-12$

Scarp on normal fault -Identity and existence certain, location approximate ...................... A-2-12

Scarp on normal fault - Identity or existence questionable, location accurate ...................... A $-2-12$

Scarp on normal fault - Identity or existence questionable, location approximate ................... A $-2-12$

Scarp on oblique-slip fault, left-lateral offset-Identity and existence certain, location accurate......... A-2-13

Scarp on oblique-slip fault, left-lateral offset-Identity and existence certain, location approximate..... A-2-13

Scarp on oblique-slip fault, left-lateral offset-Identity or existence questionable, location accurate.... A-2-13

Scarp on oblique-slip fault, left-lateral offset-Identity or existence questionable, location

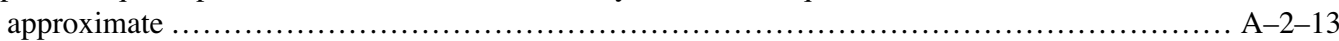

Scarp on oblique-slip fault, right-lateral offset-Identity and existence certain, location accurate ....... A-2-13

Scarp on oblique-slip fault, right-lateral offset - Identity and existence certain, location approximate ... A-2-13

Scarp on oblique-slip fault, right-lateral offset-Identity or existence questionable, location accurate... A-2-13

Scarp on oblique-slip fault, right-lateral offset-Identity or existence questionable, location 
Page Ref. No,

Scarp on overturned thrust fault (1st option) - Identity and existence certain, location approximate ..... A-2-14

Scarp on overturned thrust fault (1st option) - Identity or existence questionable, location accurate ..... A-2-14

Scarp on overturned thrust fault (1st option) - Identity or existence questionable, location

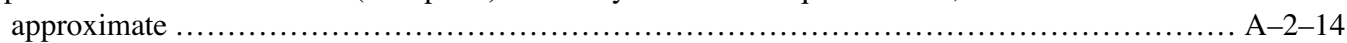

2.12 .60

Scarp on overturned thrust fault (2nd option) - Identity and existence certain, location accurate ........ A-2-14

2.12 .61

Scarp on overturned thrust fault (2nd option) - Identity and existence certain, location approximate .... A-2-14

2.12 .63

Scarp on overturned thrust fault (2nd option) - Identity or existence questionable, location accurate .... A-2-14

2.12 .62

Scarp on overturned thrust fault (2nd option) - Identity or existence questionable, location

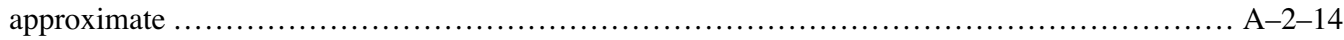

2.12 .64

Scarp on overturned thrust fault (3rd option) - Identity and existence certain, location accurate........ A-2-14

Scarp on overturned thrust fault (3rd option) - Identity and existence certain, location approximate..... A-2-14

Scarp on overturned thrust fault (3rd option) - Identity or existence questionable, location accurate..... A-2-14

Scarp on overturned thrust fault (3rd option) - Identity or existence questionable, location

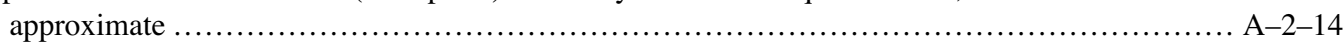

Scarp on reverse fault-Identity and existence certain, location accurate .......................... A-2-12

Scarp on reverse fault-Identity and existence certain, location approximate ..................... A-2-12

Scarp on reverse fault-Identity or existence questionable, location accurate...................... A-2-12

Scarp on reverse fault-Identity or existence questionable, location approximate.................... A-2-12

Scarp on rotational fault, normal-slip offset-Identity and existence certain, location accurate ......... A-2-13

Scarp on rotational fault, normal-slip offset-Identity and existence certain, location approximate ..... A-2-13

Scarp on rotational fault, normal-slip offset-Identity or existence questionable, location accurate ..... A-2-13

Scarp on rotational fault, normal-slip offset-Identity or existence questionable, location

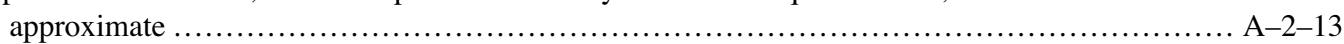

Scarp on rotational fault, reverse-slip offset-Identity and existence certain, location accurate ......... A-2-13

Scarp on rotational fault, reverse-slip offset-Identity and existence certain, location approximate ...... A-2-13

Scarp on rotational fault, reverse-slip offset-Identity or existence questionable, location accurate..... A-2-13

Scarp on rotational fault, reverse-slip offset-Identity or existence questionable, location

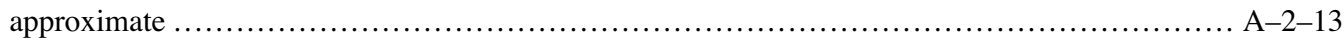

Scarp on scissor fault, normal-slip offset-Identity and existence certain, location accurate........... A-2-13

Scarp on scissor fault, normal-slip offset-Identity and existence certain, location approximate......... A-2-13

Scarp on scissor fault, normal-slip offset-Identity or existence questionable, location accurate ........ A-2-13

Scarp on scissor fault, normal-slip offset-Identity or existence questionable, location approximate .... A-2-13

Scarp on scissor fault, reverse-slip offset-Identity and existence certain, location accurate ............ A-2-13

Scarp on scissor fault, reverse-slip offset-Identity and existence certain, location approximate ........ A-2-13

Scarp on scissor fault, reverse-slip offset-Identity or existence questionable, location accurate ........ A-2-13

Scarp on scissor fault, reverse-slip offset-Identity or existence questionable, location approximate .... A-2-13

Scarp on strike-slip fault, left-lateral offset-Identity and existence certain, location accurate......... A-2-13

Scarp on strike-slip fault, left-lateral offset-Identity and existence certain, location approximate ...... A-2-13

Scarp on strike-slip fault, left-lateral offset-Identity or existence questionable, location accurate...... A-2-13

Scarp on strike-slip fault, left-lateral offset-Identity or existence questionable, location approximate.. A-2-13

Scarp on strike-slip fault, right-lateral offset-Identity and existence certain, location accurate ........ A-2-13

Scarp on strike-slip fault, right-lateral offset-Identity and existence certain, location approximate ..... A-2-13

Scarp on strike-slip fault, right-lateral offset-Identity or existence questionable, location accurate ..... A-2-13

Scarp on strike-slip fault, right-lateral offset-Identity or existence questionable, location

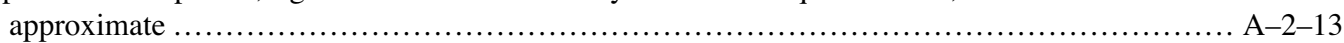

Scarp on thrust fault (1st option) - Identity and existence certain, location accurate .................. A-2-14

Scarp on thrust fault (1st option) - Identity and existence certain, location approximate ............... A-2-14

Scarp on thrust fault (1st option) - Identity or existence questionable, location accurate.............. A-2-14

Scarp on thrust fault (1st option) - Identity or existence questionable, location approximate........... A-2-14

Scarp on thrust fault (2nd option) - Identity and existence certain, location accurate ................. A-2-14

Scarp on thrust fault (2nd option) - Identity and existence certain, location approximate ............. A-2-14

Scarp on thrust fault (2nd option) - Identity or existence questionable, location accurate............. A-2-14

Scarp on thrust fault (2nd option) - Identity or existence questionable, location approximate.......... A-2-14

Scarp on thrust fault (3rd option) - Identity and existence certain, location accurate................. A-2-14

Scarp on thrust fault (3rd option) - Identity and existence certain, location approximate............. A-2-14 


\begin{tabular}{|c|c|}
\hline & \\
\hline arp on thrust fault (3rd option) - Identity or existence questionable, location accurate $\ldots \ldots \ldots \ldots \ldots \ldots$ A $-2-14$ & \\
\hline arp on thrust fault (3rd option) - Identity or existence questionable, location approximate ............. A-2-14 & 2.12 .56 \\
\hline . A-25-4 & 25.71 \\
\hline . A-2-12 & Sec. 2.12 \\
\hline carps on fluvial terraces.. & Sec. 12 \\
\hline carps on landslides........ & Sec. 17 \\
\hline scarps on sedimentary contacts.... & Sec. 1.1 \\
\hline A-37-3 & 705 \\
\hline hist and gneiss.. & 707 \\
\hline chistose granite.. & 704 \\
\hline 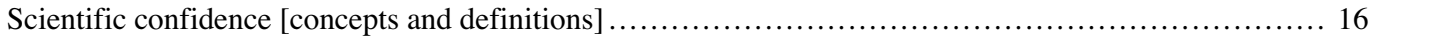 & $\mathrm{n} / \mathrm{a}$ \\
\hline ientific confidence and locational accuracy of geologic features [concepts and definitions] .............. 15 & $\mathrm{n} / \mathrm{a}$ \\
\hline issor fault, normal-slip offset-Identity and existence certain, location accurate …................ A & 2.5 .9 \\
\hline issor fault, normal-slip offset-Identity and existence certain, location approximate $\ldots \ldots \ldots \ldots \ldots \ldots \ldots$ A $-2-4$ & 2.5 .11 \\
\hline issor fault, normal-slip offset-Identity and existence certain, location concealed ....................... & 2.5.15 \\
\hline issor fault, normal-slip offset-Identity and existence certain, location inferred.................... A & 2.5 .13 \\
\hline issor fault, normal-slip offset -Identity or existence questionable, location accurate …............. A $\mathrm{A}-2-4$ & 2.5.10 \\
\hline issor fault, normal-slip offset-Identity or existence questionable, location approximate .. & 5.12 \\
\hline issor fault, normal-slip offset-Identity or existence questionable, location concealed .... & 2.5 .16 \\
\hline ault, normal-slip offset-Identity or existence questionable, location inferred........ & 2.5 .14 \\
\hline lip offset-Identity and existence certain, location accurate .................. & 5.1 \\
\hline Scissor fault, reverse-slip offset-Identity and existence certain, location approximate ..................... & 2.5.3 \\
\hline issor fault, reverse-slip offset-Identity and existence certain, location concealed $\ldots \ldots \ldots \ldots \ldots \ldots \ldots$ & 2.5.7 \\
\hline istence certain, location inferred............. & 2.5 .5 \\
\hline Scissor fault, reverse-slip offset-Identity or existence questionable, location accurate $\ldots \ldots \ldots \ldots \ldots$ & 2.5.2 \\
\hline lip offset-Identity or existence questionable, location approximate ............ & 2.5.4 \\
\hline tence questionable, location concealed.... & 2.5 .8 \\
\hline reverse-sip oftset-Identity or existence questionabie, location interred.. & 2.5.6 \\
\hline A-2-4 & Sec. 2.5 \\
\hline 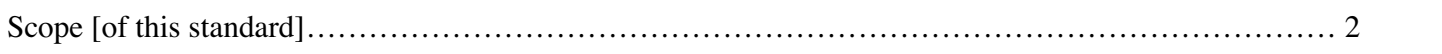 & $\mathrm{n} / \mathrm{a}$ \\
\hline A-9-1 & 9.13 \\
\hline Seams & 22.36 \\
\hline rigin (shown as point symbol when too small to outline at map scale) .......... & 22.38 \\
\hline - & 22.37 \\
\hline in as point symbol when too small to o & 22.39 \\
\hline (2. & 25.122 \\
\hline ctonism)...... & Sec. 8.3 \\
\hline A-28 & 28.7 \\
\hline iss 2$)$... & 28.8 \\
\hline A-28 & 28.6 \\
\hline A-2: & 25.99 \\
\hline aw to scale) (1st option)...... & 24.2 \\
\hline ct crater (too small to draw to scale) (2nd op & 24.4 \\
\hline & 31.5 \\
\hline A-31-1 & 31.6 \\
\hline A $-31-1$ & 31.7 \\
\hline A-12-1 & 12.7 \\
\hline A-12-1 & 12.8 \\
\hline$\ldots \ldots \ldots \ldots \ldots \ldots \ldots \ldots \ldots \ldots \ldots$ & 12.6 \\
\hline ....... Pattern Chart & $\mathrm{n} / \mathrm{a}$ \\
\hline$\ldots$ A-37-1 & Sec. 37.1 \\
\hline A-17-3 & 17.48 \\
\hline ......... A-3-1 & Sec. 3 \\
\hline erif font [illustrated] . & $\mathrm{n} / \mathrm{a}$ \\
\hline
\end{tabular}




\begin{tabular}{|c|c|}
\hline Page & Ref. No. \\
\hline Serpentinite... & 710 \\
\hline Shallow condensate well.. & 19.5.76 \\
\hline Shallow core................ & 19.5 .8 \\
\hline Shallow disposal well.. & 19.5.28 \\
\hline Shallow dry hole .................. & 19.5.21 \\
\hline Shallow gas and condensate well.. & 19.5.86 \\
\hline Shallow gas storage well ................. & 19.5.94 \\
\hline Shallow gas well........... & 19.5.54 \\
\hline Shallow junked hole..................... & 19.5.24 \\
\hline Shallow, linear depression, planetary ............ & 25.55 \\
\hline Shallow, linear valley, planetary ............. & 25.55 \\
\hline Shallow, narrow channel, planetary............. & 25.55 \\
\hline Shallow observation well for gas-storage field..... & 19.5.100 \\
\hline Shallow oil and gas well.......................... & 19.5.66 \\
\hline Shallow oil well............ & 19.5.43 \\
\hline Shallow salt-water disposal well & 19.5.34 \\
\hline Shaly dolomite .................... & 647 \\
\hline Shaly dolostone ...... & 647 \\
\hline Shaly limestone ....... & 638 \\
\hline Shaly sandstone............ & 612 \\
\hline Shaly silt ................... & 616 \\
\hline Sharp groove, planetary.......... & 25.48 \\
\hline Shear features in landslides ............ & Sec. 17 \\
\hline Shear zone ............................... & 2.14 .1 \\
\hline Shear zones [concepts and definitions] ...... & $\mathrm{n} / \mathrm{a}$ \\
\hline Sheared rock within fault .................... & 2.14 .2 \\
\hline Sheath folds (antiformal) ... & Sec. 5.4 \\
\hline Sheath folds (synformal)............ & Sec. 5.8 \\
\hline .. A-30-6 & 30.3 .19 \\
\hline Shoreline .................. & 30.2 .29 \\
\hline Shoreline, aggradational .... & Sec. 15 \\
\hline Shoreline cliff - Identity and existence certain, location accurate ................ & 15.22 \\
\hline Shoreline cliff -Identity and existence certain, location approximate .............. & 15.24 \\
\hline Shoreline cliff-Identity or existence questionable, location accurate............... & 15.23 \\
\hline Shoreline cliff -Identity or existence questionable, location approximate $\ldots \ldots \ldots \ldots \ldots$ & 15.25 \\
\hline 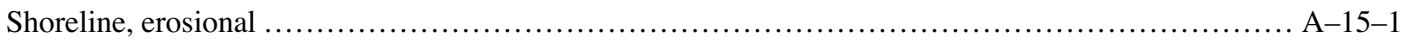 & Sec. 15 \\
\hline Shoreline, former $\ldots \ldots \ldots \ldots \ldots \ldots \ldots \ldots \ldots \ldots \ldots$ & Sec. 15 \\
\hline Shut-in water well ............. & 26.1 .4 \\
\hline Siderite ......................... & 665 \\
\hline Significant glacial advance........ & Sec. 13 \\
\hline Silicoflagellates ................ & 10.2 .60 \\
\hline .. A-37-1 & 616 \\
\hline Siltstone.................. & 616 \\
\hline Silty dolomite ................... & 646 \\
\hline Silty dolostone ...... & 646 \\
\hline Silty limestone ....... & 637 \\
\hline Silty shale $. . . . . . . . \ldots \ldots \ldots \ldots . .$. & 619 \\
\hline Silurian [geologic age symbol] ............... & 32.16 \\
\hline 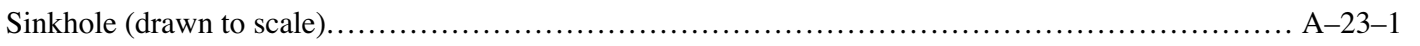 & 23.10 \\
\hline Sinkhole, formed by shock - Identity and existence certain, location accurate ...................... A-21-2 & 21.30 \\
\hline Sinkhole, formed by shock - Identity or existence certain, location approximate $\ldots \ldots \ldots \ldots \ldots \ldots \ldots \ldots$ A $-21-2$ & 21.32 \\
\hline Sinkhole, formed by shock - Identity or existence certain, location concealed $\ldots \ldots \ldots \ldots \ldots \ldots \ldots \ldots \ldots$ A $-21-2$ & 21.34 \\
\hline Sinkhole, formed by shock - Identity or existence questionable, location accurate................... A-21-2 & 21.31 \\
\hline Sinkhole, formed by shock - Identity or existence questionable, location approximate................. A-21-2 & 21.33 \\
\hline
\end{tabular}




\begin{tabular}{|c|c|}
\hline $\begin{array}{c}\text { Page } \\
\text { Sinkhole, formed by shock }- \text { Identity or existence questionable, location concealed.................... A-21-2 }\end{array}$ & $\begin{array}{l}\text { Ref. No. } \\
21.35\end{array}$ \\
\hline Sinkhole (too small to draw to scale) & 23.9 \\
\hline Siphon & 30.2 .23 \\
\hline Size of map-unit areas [influencing color and pattern selection].. & $\mathrm{n} / \mathrm{a}$ \\
\hline Skylights (not mapped to scale) along lava tube .................... & 18.44 \\
\hline 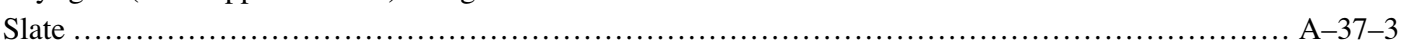 & 703 \\
\hline Slaty cleavage.. & 7.7 \\
\hline Slaty cleavage, for multiple observations at one locality. & 7.10 \\
\hline Slickenline on fault surface ............................... & 9.17 \\
\hline Slide material, planetary ...... & 25.117 \\
\hline Slip surface of landslide .. & 17.9 \\
\hline Sluice gate .................. & 30.2 .14 \\
\hline Slump, consisting of a relatively intact mass of displaced materials. & 17.62 \\
\hline 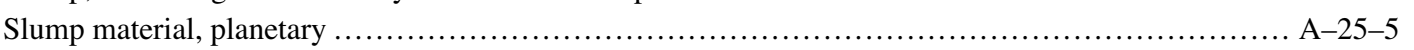 & 25.117 \\
\hline Small cinder cone............ & 18.55 \\
\hline Small cone ........... & 18.55 \\
\hline Small dome, planetary (not mapped to scale).. & 25.77 \\
\hline Small endogenic crater (mapped to scale), planetary ... & 25.107 \\
\hline Small endogenic crater, planetary .......................... & 25.106 \\
\hline Small hornito .......................... & 18.57 \\
\hline Small, minor anticline, inclined axial surface (1st option)......... & 5.11 .5 \\
\hline Small, minor anticline, inclined axial surface (2nd option)......... & 5.11 .7 \\
\hline Small, minor anticline, vertical or near-vertical axial surface (1st option) .... & 5.11 .4 \\
\hline Small, minor anticline, vertical or near-vertical axial surface (2nd option) ....... & 5.11 .6 \\
\hline 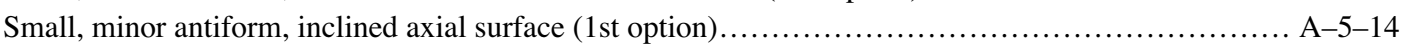 & 5.11 .9 \\
\hline 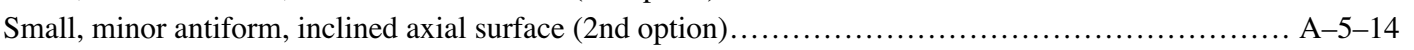 & 5.11 .11 \\
\hline Small, minor antiform, vertical or near-vertical axial surface (1st option) .......................... A-5-14 & 5.11 .8 \\
\hline Small, minor antiform, vertical or near-vertical axial surface (2nd option) ....... & 5.11 .10 \\
\hline Small, minor asymmetric anticline, inclined axial surface (1st option) ......... & 5.11 .13 \\
\hline Small, minor asymmetric anticline, inclined axial surface (2nd option) .... & 5.11 .15 \\
\hline Small, minor asymmetric anticline, vertical or near-v & 5.11 .12 \\
\hline Small, minor asymmetric anticline, vertical or near-vertical axial surface (2nd option)...... & 5.11 .14 \\
\hline Small, minor asymmetric syncline, inclined axial surface (1st option) ............ & 5.11 .33 \\
\hline Small, minor asymmetric synclin & 5.11 .35 \\
\hline Small, minor asymmetric syncline, vertical or near-ve & 5.11 .32 \\
\hline Small, minor asymmetric syncline, vertical or near-vertical axial surface (2nd option).. & 5.11 .34 \\
\hline Small, minor basin. & 5.11 .3 \\
\hline Small, minor dome ... & 5.11 .2 \\
\hline Small, minor faults ..... & Sec. 2.15 \\
\hline Small, minor fold-hinge lineations ........... & 9.97 \\
\hline Small, minor fold, horizontal axial surface & 5.11 .1 \\
\hline Small, minor folds ........................... & Sec. 5.11 \\
\hline Small, minor horizontal joint (1st option)... & 4.3 .1 \\
\hline 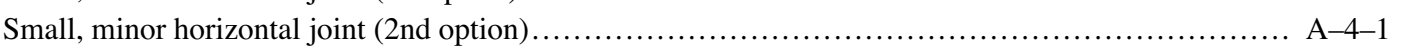 & 4.3 .7 \\
\hline $\begin{array}{l}\text { Small, minor inclined (dip direction to left) joint, for multiple observations at one locality } \\
\text { (1st option) }\end{array}$ & 4.3.5 \\
\hline $\begin{array}{l}\text { Small, minor inclined (dip direction to left) joint, for multiple observations at one locality (2nd } \\
\text { option) }\end{array}$ & 4.3.11 \\
\hline $\begin{array}{l}\text { Small, minor inclined (dip direction to right) joint, for multiple observations at one locality (1st } \\
\text { option) }\end{array}$ & 4.3.4 \\
\hline $\begin{array}{l}\text { Small, minor inclined (dip direction to right) joint, for multiple observations at one locality (2nd } \\
\text { option) }\end{array}$ & 4.3.10 \\
\hline mall, minor inclined fault & 2.15.1 \\
\hline …....... A-4-1 & 4.3.2 \\
\hline Small, minor inclined joint (2nd option) ................. & 4.3 .8 \\
\hline
\end{tabular}


Page
Pagen

Small, minor inclined mineralized stringer $\ldots \ldots \ldots \ldots \ldots \ldots \ldots \ldots \ldots \ldots \ldots \ldots \ldots \ldots \ldots \ldots \ldots \ldots \ldots$ A $-19-1$

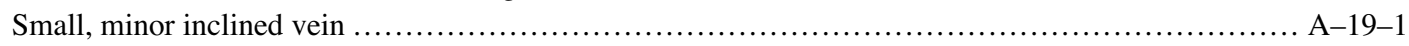

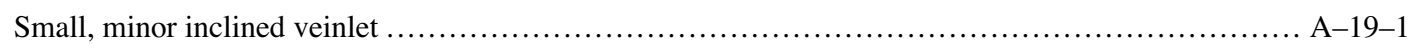

Small, minor inverted anticline, inclined axial surface (1st option) ............................. A-5-14

Small, minor inverted anticline, inclined axial surface (2nd option) ............................ A-5-14

Small, minor inverted anticline, vertical or near-vertical axial surface (1st option)................. A-5-14

Small, minor inverted anticline, vertical or near-vertical axial surface (2nd option)................... A-5-14

Small, minor inverted syncline, inclined axial surface (1st option) .............................. A-5-15

Small, minor inverted syncline, inclined axial surface (2nd option).............................. A-5-15

Small, minor inverted syncline, vertical or near-vertical axial surface (1st option) ................... A-5-15

Small, minor inverted syncline, vertical or near-vertical axial surface (2nd option) .................. A-5-15

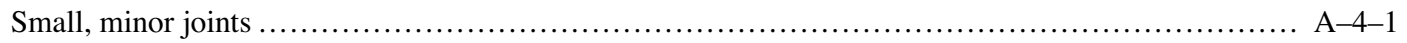

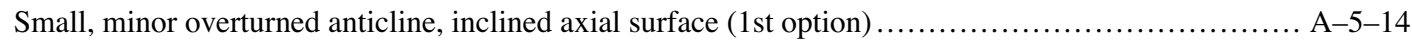

Small, minor overturned anticline, inclined axial surface (2nd option) ........................ A-5-14

Small, minor overturned anticline, vertical or near-vertical axial surface (1st option)................ A-5-14

Small, minor overturned anticline, vertical or near-vertical axial surface (2nd option)................. A-5-14

Small, minor overturned syncline, inclined axial surface (1st option) . . . . . . . . . . . . . . . . . . . . .

Small, minor overturned syncline, inclined axial surface (2nd option) ......................... A-5-15

Small, minor overturned syncline, vertical or near-vertical axial surface (1st option)................ A-5-15

Small, minor overturned syncline, vertical or near-vertical axial surface (2nd option) ................. A-5-15

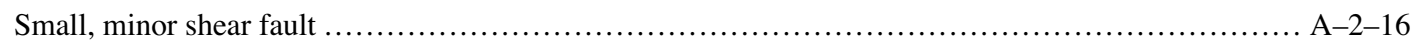

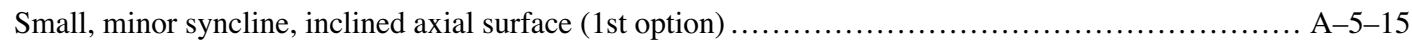

Small, minor syncline, inclined axial surface (2nd option) . ..................................... A -5

Small, minor syncline, vertical or near-vertical axial surface (1st option)........................ A-5-15

Small, minor syncline, vertical or near-vertical axial surface (2nd option)...................... A-5-15

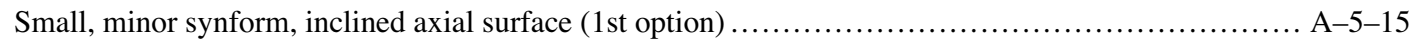

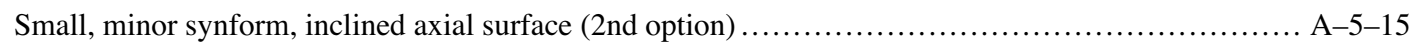

Small, minor synform, vertical or near-vertical axial surface (1st option)....................... A-5-15

Small, minor synform, vertical or near-vertical axial surface (2nd option)....................... A-5-15

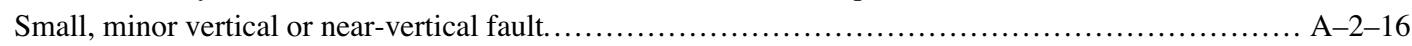

Small, minor vertical or near-vertical joint (1st option) .......................................... A-4

Small, minor vertical or near-vertical joint (2nd option) ......................................... A

Small, minor vertical or near-vertical joint, for multiple observations at one locality (1st option) ....... A-4-1

Small, minor vertical or near-vertical joint, for multiple observations at one locality (2nd option) ...... A-4-1

Small, minor vertical or near-vertical mineralized stringer.................................... A-19-1

Small, minor vertical or near-vertical vein ................................................ A-19-1

Small, minor vertical or near-vertical veinlet............................................. A-19-1

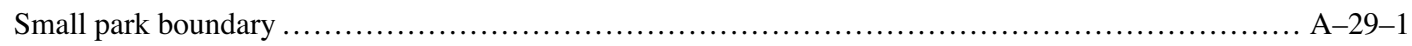

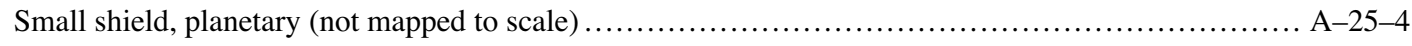

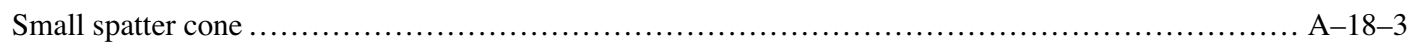

Small tholi, planetary (mapped to scale) .............................................. A-25-4

Small tholi, planetary (not mapped to scale) ............................................ A-25-4

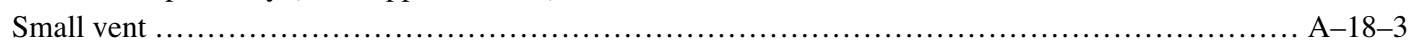

Small volcanic construct, planetary (not mapped to scale) ...................................... A-25-4

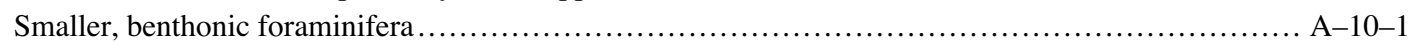

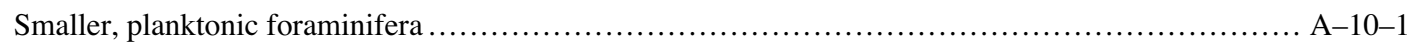

Snow-survey course, equipped with a recorder......................................... A-27-1

Snow-survey course, equipped with a telephone or radio................................... A $-27-1$

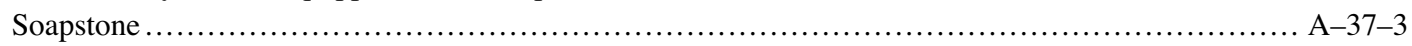

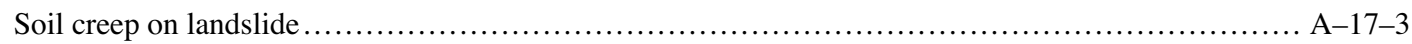

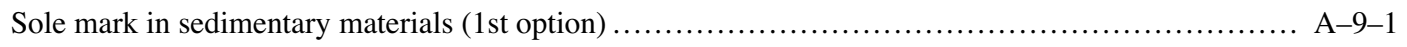

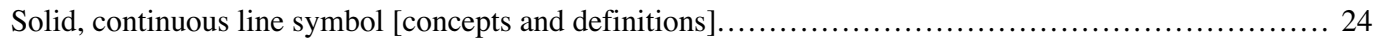

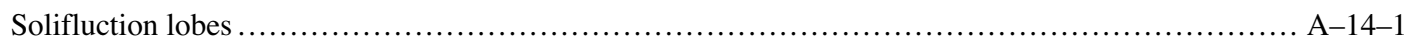

Sorted circles.
Ref. No.

19.1 .12

19.1 .12

19.1 .12

5.11 .21

5.11 .23

5.11 .20

5.11 .22

5.11 .41

5.11 .43

5.11 .40

5.11 .42

Sec. 4.3

5.11 .17

5.11 .19

5.11 .16

5.11 .18

5.11 .37

5.11 .39

5.11 .36

5.11 .38

2.15 .3

5.11 .25

5.11 .27

5.11 .24

5.11 .26

5.11.29

5.11 .31

5.11 .28

5.11 .30

2.15 .2

4.3 .3

4.3 .9

4.3.6

4.3.12

19.1 .13

19.1.13

19.1.13

29.7

25.77

18.55

25.90

25.89

18.55

25.77

10.2 .50

10.2.51

27.4

27.3

710

17.47

9.13

n/a

14.7

14.4 


\begin{tabular}{|c|c|}
\hline South Carolina [state location map]... & $\begin{array}{l}\text { Ref. No. } \\
\text { Sec. } 34.1\end{array}$ \\
\hline South Dakota [state location map] ....... & Sec. 34.1 \\
\hline Souvenir $[$ font $] \ldots \ldots \ldots \ldots \ldots \ldots \ldots \ldots \ldots$ & $\mathrm{n} / \mathrm{a}$ \\
\hline Spaced cleavage ......... & 7.13 \\
\hline Spaced foliation.......... & 8.3.22 \\
\hline Spatter cone, large.... & 18.56 \\
\hline Spatter cone, small .. & 18.55 \\
\hline Spatter rampart................... & 18.59 \\
\hline 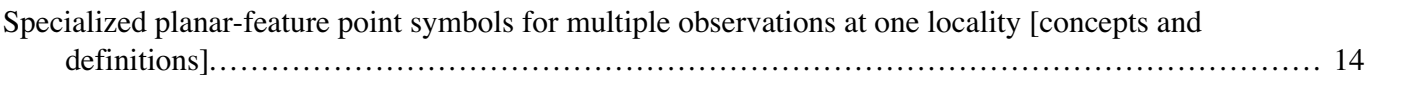 & $\mathrm{n} / \mathrm{a}$ \\
\hline pecific conductance & 26.6.9 \\
\hline pecifying color for line symbols ... & $\mathrm{n} / \mathrm{a}$ \\
\hline pecifying color for map-unit areas... & $\mathrm{n} / \mathrm{a}$ \\
\hline pecifying color for point symbols.... & $\mathrm{n} / \mathrm{a}$ \\
\hline pecifying positional accuracy with the zone of confidence [concepts and definitions] $\ldots \ldots \ldots \ldots$ & $\mathrm{n} / \mathrm{a}$ \\
\hline Spicules & 10.2 .61 \\
\hline Spillway, glacial lake & 13.3 \\
\hline 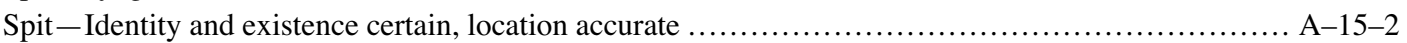 & 15.26 \\
\hline Spit-Identity and existence certain, location approximate & 15.28 \\
\hline Spit-Identity or existence questionable, location accurate & 15.27 \\
\hline Spit-Identity or existence questionable, location approximate & 15.29 \\
\hline Splotch, planetary & 25.130 \\
\hline Sponges $[$ fossil symbol] & 10.2.25 \\
\hline Spores $[$ fossil symbol] & 10.2 .58 \\
\hline Spot color specifications used in this standard and their equivalent colors in other color models ......... A-v & Table 3 \\
\hline Spot colors [in this standard] & Table 3 \\
\hline Spreading axes & Sec. 22 \\
\hline Spring, as shown on topographic maps or on general-purpose or smaller scale maps $\ldots \ldots \ldots \ldots \ldots \ldots \ldots \ldots \ldots \ldots$ & 30.3 .12 \\
\hline Spring on landslide & 17.48 \\
\hline Spring, type of use unspecified & 26.2.1 \\
\hline Spring used for collection of water-quality data... & 26.2 .3 \\
\hline Spring used for domestic-water supply ............... & 26.2.4 \\
\hline Spring used for industrial-water supply .... & 26.2.16 \\
\hline Spring used for irrigation-water supply.... & 26.2.12 \\
\hline Spring used for public-water supply ........ & 26.2.20 \\
\hline Spring used for stock-water supply ....... & 26.2 .8 \\
\hline . A-26-4 & Sec. 26.2 \\
\hline S-shaped (counterclockwise sense of shear) crenulation cleavage. & 7.25 \\
\hline -shaped (counterclockwise sense of shear) crenulation cleavage, for multiple observations at one & 7.28 \\
\hline S-shaped (counterclockwise sense of shear) crenulation foliation.. & 8.3.34 \\
\hline S-shaped (counterclockwise sense of shear) crenulation foliation, for multiple observations at one & 8.3.37 \\
\hline -shaped (counterclockwise sense of shear) kink-band crenulation lineation......................... & 9.137 \\
\hline -shaped (counterclockwise sense of shear) minor fold hinge .................. & 9.125 \\
\hline 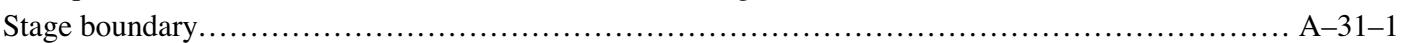 & 31.23 \\
\hline tage-measurement site without a gage .. & 26.3.17 \\
\hline tagnant ice margin ... & Sec. 13 \\
\hline tandards development procedures . & $\mathrm{n} / \mathrm{a}$ \\
\hline tate boundary ........................ & 29.2 \\
\hline tate forest boundary...... & 29.6 \\
\hline State grassland boundary .. & 29.6 \\
\hline tate location maps.......... & Sec. 34 \\
\hline State monument bound & 29.6 \\
\hline State park boundary ................... & 29.6 \\
\hline
\end{tabular}




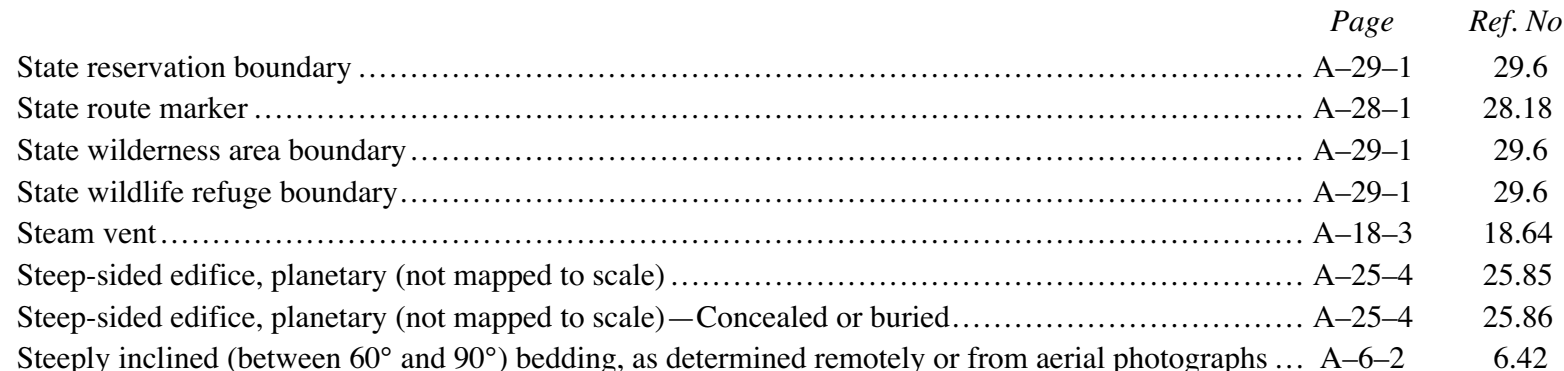

Steeply overturned (between $60^{\circ}$ and $90^{\circ}$ ) bedding, as determined remotely or from aerial

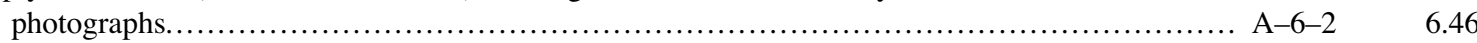

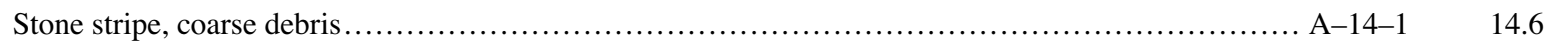

Stone stripe, fine debris ......................................................... A $-14-1 \quad 14.5$

Stoped area (drawn to scale), as shown on subsurface exploration maps (section view)................ A-19-5 19.4 .17

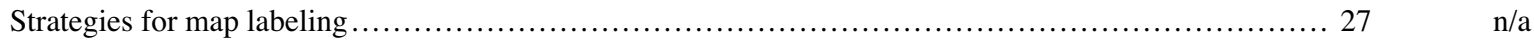

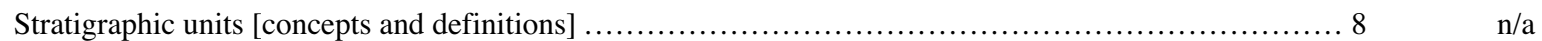

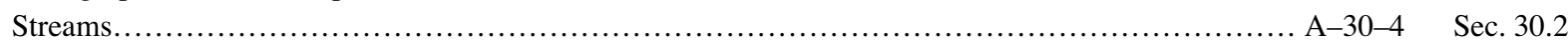

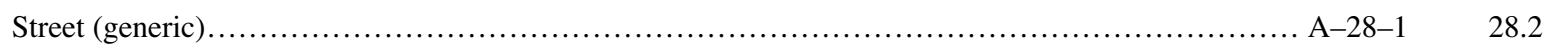

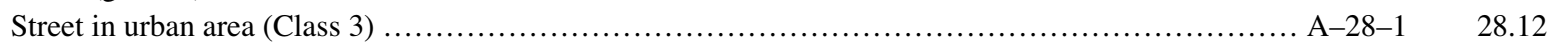

Streets ............................................................................ A $-28-1 \quad$ Sec. 28

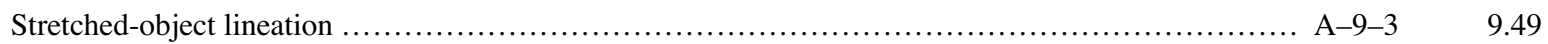

Stretched-ooid lineation ................................................................. A-9-3 9.57

Stretched-pebble lineation ............................................................. A-9-3 9.53

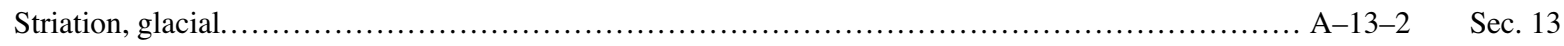

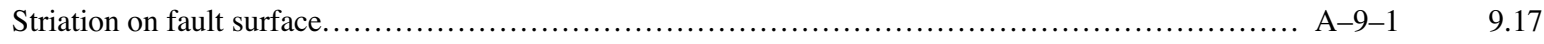

Striation (origin not known or not specified) $\ldots \ldots \ldots \ldots \ldots \ldots \ldots \ldots \ldots \ldots \ldots \ldots \ldots \ldots \ldots \ldots \ldots \ldots \ldots$ A $-9-1 \quad 9.21$

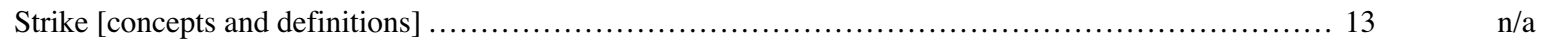

Strike-slip fault (in cross section) (1st option: A, away from observer; T, toward observer) ............. A-2-11 2.11 .20

Strike-slip fault (in cross section) (2nd option: minus, away from observer; plus, toward observer)...... A-2-11 2.11 .21

Strike-slip fault, left-lateral offset - Identity and existence certain, location accurate................. A-2-5 2.6 .9

Strike-slip fault, left-lateral offset-Identity and existence certain, location approximate................ A-2-5 2.6 .11

Strike-slip fault, left-lateral offset-Identity and existence certain, location concealed................. A-2-5 2.6.15

Strike-slip fault, left-lateral offset - Identity and existence certain, location inferred ................... A-2-5 2.6.13

Strike-slip fault, left-lateral offset-Identity or existence questionable, location accurate............... A-2-5 2.6 .10

Strike-slip fault, left-lateral offset -Identity or existence questionable, location approximate........... A-2-5 2.6 .12

Strike-slip fault, left-lateral offset -Identity or existence questionable, location concealed............. A-2-5 2.6.16

Strike-slip fault, left-lateral offset -Identity or existence questionable, location inferred .............. A-2-5 2.6.14

Strike-slip fault, planetary, left-lateral offset -Location accurate ............................. A-25-1 25.17

Strike-slip fault, planetary, left-lateral offset-Location approximate.......................... A-25-1 25.18

Strike-slip fault, planetary, left-lateral offset - Location concealed .............................. A-25-1 25.20

Strike-slip fault, planetary, left-lateral offset-Location inferred .................................. A-25-1 25.19

Strike-slip fault, planetary, right-lateral offset-Location accurate ................................. A-25-1 25.13

Strike-slip fault, planetary, right-lateral offset-Location approximate ................................ A-25-1 25.14

Strike-slip fault, planetary, right-lateral offset-Location concealed ................................... A-25-1 25.16

Strike-slip fault, planetary, right-lateral offset-Location inferred.................................... A-25-1 25.15

Strike-slip fault, right-lateral offset - Identity and existence certain, location accurate ................ A-2-5 2.6 .1

Strike-slip fault, right-lateral offset-Identity and existence certain, location approximate .............. A-2-5 2.6 .3

Strike-slip fault, right-lateral offset - Identity and existence certain, location concealed ................ A-2-5 2.6 .7

Strike-slip fault, right-lateral offset - Identity and existence certain, location inferred................. A-2-5 2.6 .5

Strike-slip fault, right-lateral offset - Identity or existence questionable, location accurate ............ A-2-5 2.6 .2

Strike-slip fault, right-lateral offset-Identity or existence questionable, location approximate......... A-2-5 2.6 .4

Strike-slip fault, right-lateral offset - Identity or existence questionable, location concealed............ A-2-5 2.6 .8

Strike-slip fault, right-lateral offset - Identity or existence questionable, location inferred............. A-2-5 2.6 .6

Strike-slip faults ..................................................................... A $-2-5 \quad$ Sec. 2.6

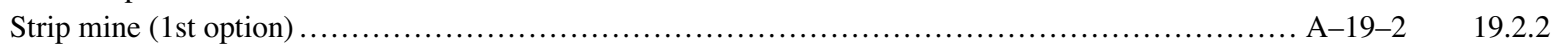




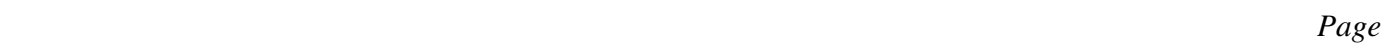

Strip mine (2nd option) .

A-19-2

Stromatolites

A-10-1

Stromatoporoids ....

A-10-1

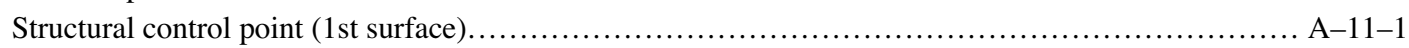

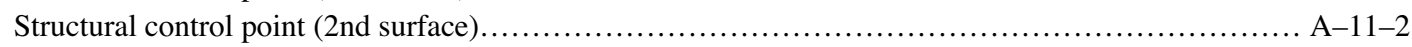

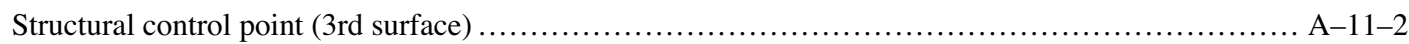

Structure contour, 1 st surface (index) - Accurately located ..................................... A-11-1

Structure contour, 1st surface (index) - Approximately located ................................ A-11-1

Structure contour, 1 st surface (index), showing datum..................................... A $-11-1$

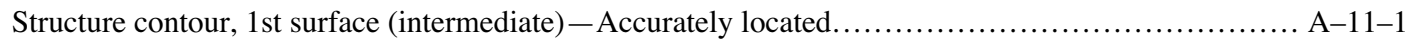

Structure contour, 1 st surface (intermediate) - Approximately located.......................... A-11-1

Structure contour, 2nd surface (index) - Accurately located ................................ A-11-2

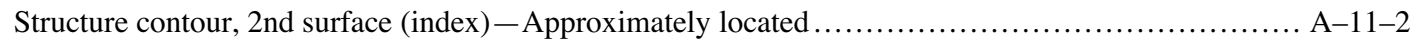

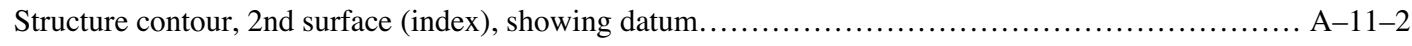

Structure contour, 2nd surface (intermediate) - Accurately located............................ A-11-2

Structure contour, 2nd surface (intermediate) - Approximately located............................ A-11-2

Structure contour, 3rd surface (index) - Accurately located................................. A-11-2

Structure contour, 3rd surface (index) - Approximately located.............................. A-11-2

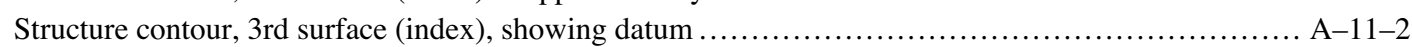

Structure contour, 3rd surface (intermediate) - Accurately located ............................... A-11-2

Structure contour, 3rd surface (intermediate) - Approximately located ............................ A-11-2

Structure contour around closed area of lower values, 1st surface (index) - Accurately located......... A-11-1

Structure contour around closed area of lower values, 1st surface (index)-Approximately located..... A-11-1

Structure contour around closed area of lower values, 1st surface (intermediate) - Accurately located .. A-11-1

Structure contour around closed area of lower values, 1st surface (intermediate)-Approximately located.

Structure contour around closed area of lower values, 2nd surface (index) - Accurately located......... A-11-2

Structure contour around closed area of lower values, 2nd surface (index) - Approximately located..... A-11-2

Structure contour around closed area of lower values, 2nd surface (intermediate)-Accurately located.

A-11-2

Structure contour around closed area of lower values, 2nd surface (intermediate) - Approximately

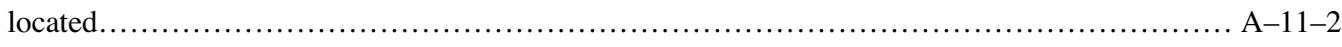

Structure contour around closed area of lower values, 3rd surface (index) - Accurately located ........ A-11-2

Structure contour around closed area of lower values, 3rd surface (index) - Approximately located ..... A-11-2

Structure contour around closed area of lower values, 3rd surface (intermediate)-Accurately located.. A-11-2

Structure contour around closed area of lower values, 3rd surface (intermediate) - Approximately located.

Structure contour (index), as shown on hydrologic maps, showing altitude of top or base of, or horizon within, stratigraphic unit, aquifer, or confining bed-Accurately located ............... A-26-7

Structure contour (index), as shown on hydrologic maps, showing altitude of top or base of, or horizon within, stratigraphic unit, aquifer, or confining bed-Approximately located............ A-26-7

Structure contour (intermediate), as shown on hydrologic maps, showing altitude of top or base of, or horizon within, stratigraphic unit, aquifer, or confining bed-Accurately located............... A-26-7

Structure contour (intermediate), as shown on hydrologic maps, showing altitude of top or base of, or horizon within, stratigraphic unit, aquifer, or confining bed-Approximately located.......... A-26-7

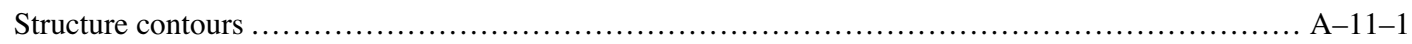

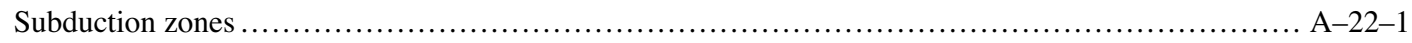

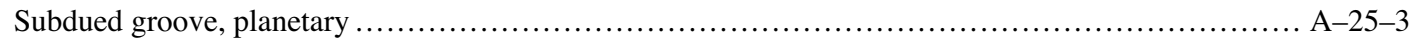

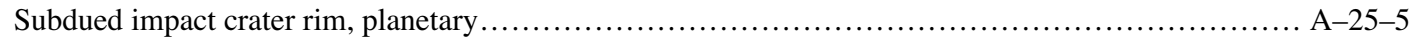

Subgraywacke ................................................................ A $-37-2$

Subsidence caused by shock - Identity and existence certain, location accurate ................... A-21-1

Subsidence caused by shock - Identity and existence certain, location concealed ..................... A-21-1

Subsidence caused by shock - Identity or existence certain, location approximate................... A-21-1

Subsidence caused by shock - Identity or existence certain, location inferred ....................... A-21-1

Subsidence caused by shock - Identity or existence questionable, location accurate .................. A-21-1 
Page Ref. No.

Subsidence caused by shock - Identity or existence questionable, location approximate............. A-21-1

Subsidence caused by shock - Identity or existence questionable, location concealed ................. A-21-1

21.23

Subsidence caused by shock - Identity or existence questionable, location inferred ................. A-21-1

Subsurface workings, as shown on subsurface exploration maps.............................. A-19-5

Subsurface workings, projected to surface (1st option) ..................................... A-19-2

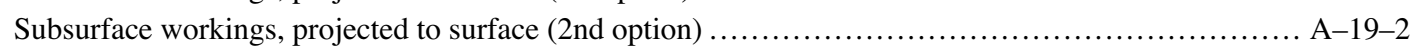

Subsurface workings, projected to surface (3rd option) ......................................... A-19-2

Subsurface workings, projected to surface (4th option) ........................................ A-19-2

Subsurface workings, projected to surface (5th option) ......................................... A-19-2

Subsurface workings, projected to surface (6th option) ..................................... A-19-2

21.21

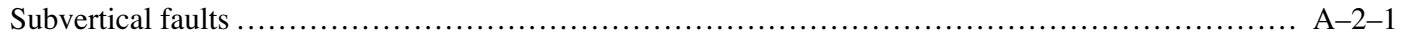

Suggested map-unit colors for plutonic rocks .......................................... A $-33-1$

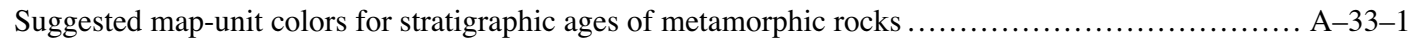

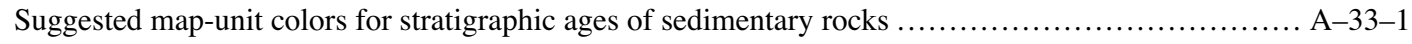

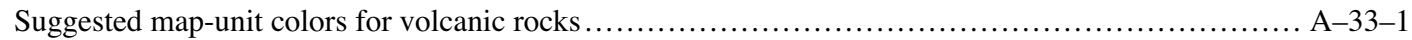

Suggested ranges of map-unit colors for volcanic and plutonic rocks and for stratigraphic ages of

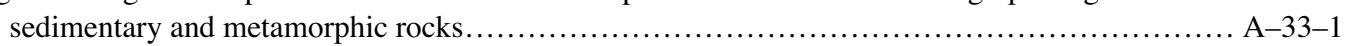

Superficial crater material having weak radar backscatter coefficient, planetary...................... A-25-6

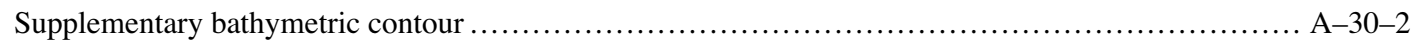

Supplementary bathymetric contour-Approximate ........................................ A-30-2

Sec. 19.4

19.2.10

19.2.11

19.2.12

19.2.13

19.2.14

19.2.15

Sec. 2.1

Sec. 33.1

Sec. 33.2

Sec. 33.2

Sec. 33.1

Supplementary bathymetric depression contour....................................... A $-30-2$

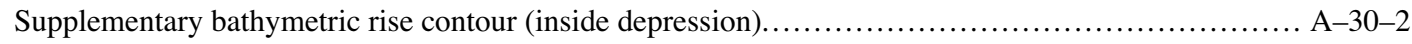

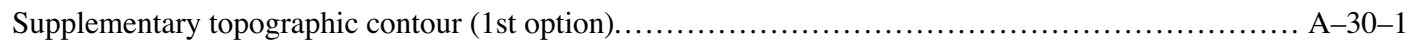

Supplementary topographic contour (1st option) - Approximate or indefinite $\ldots \ldots \ldots \ldots \ldots \ldots \ldots \ldots \ldots$ A $-30-1$

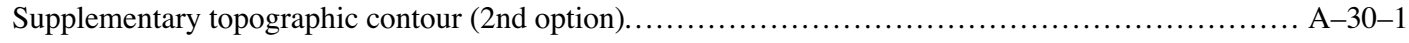

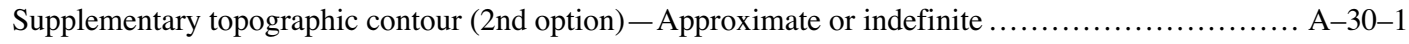

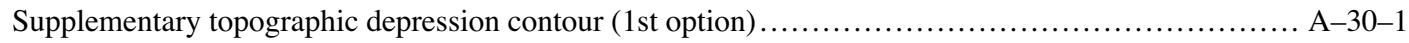

Supplementary topographic depression contour (2nd option) ................................. A-30-1

Surface grooves (origin not known or not specified) . ................................... A-9-1

Sec. 33

25.133

30.1 .25

30.1 .26

30.1 .35

30.1 .36

30.1 .5

30.1 .6

30.1 .15

30.1 .16

30.1 .9

30.1 .19

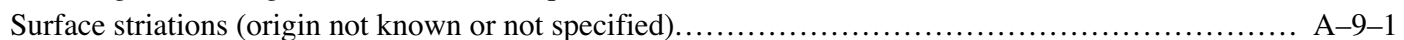

9.21

9.21

Surface trace of active deep-seismofocal zone-Accurately located................................ A-22-1 22.7

Surface trace of active deep-seismofocal zone-Approximately located.............................. A-22-1 22.8

Surface trace of active deep-seismofocal zone, showing fore-arc sediments.......................... A-22-1 22.9

Surface trace of active subduction zone-Accurately located..................................... A-22-1 22.7

Surface trace of active subduction zone-Approximately located.................................. A-22-1 22.8

Surface trace of active subduction zone, showing fore-arc sediments ......................... A $-22-1 \quad 22.9$

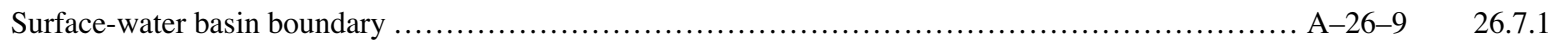

Surface-water subbasin boundary ..................................................... A $-26-9 \quad 26.7 .2$

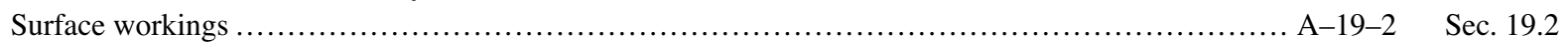

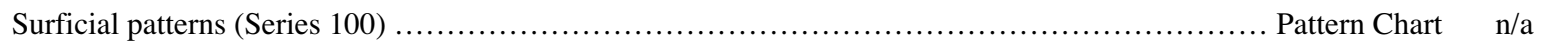

Survey station ......................................................................... A $-3-1 \quad 3.3 .5$

Suspended condensate well (nonspecific depth) ............................................... A-19-9 19.5.74

Suspended deep condensate well ............................................................. A-19-9 19.5 .80

Suspended deep gas and condensate well ............................................... A $-19-10 \quad 19.5 .90$

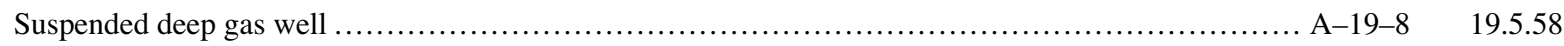

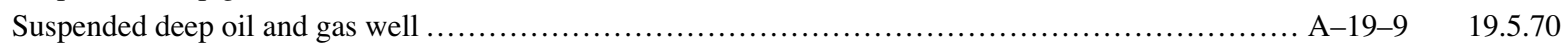

Suspended deep oil well .............................................................. A $-19-8 \quad 19.5 .47$

Suspended gas and condensate well (nonspecific depth)...................................... A-19-10 19.5.84

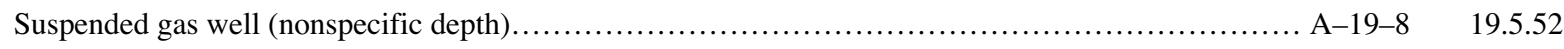

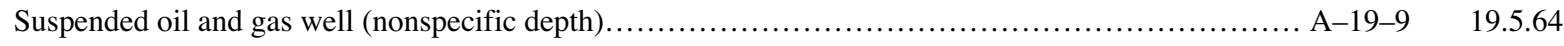

Suspended oil well (nonspecific depth)................................................... A-19-8 19.5 .41

Suspended shallow condensate well .......................................................... A-19-9 19.5.77

Suspended shallow gas and condensate well ............................................... A $-19-10 \quad 19.5 .87$

Suspended shallow gas well............................................................ A-19-8 19.5 .55 


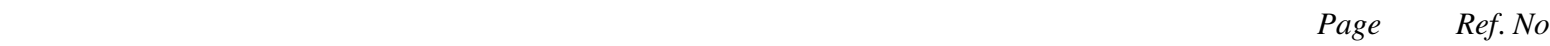

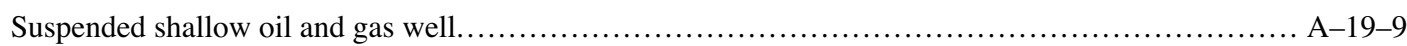

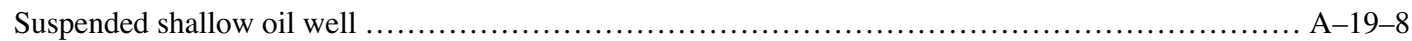

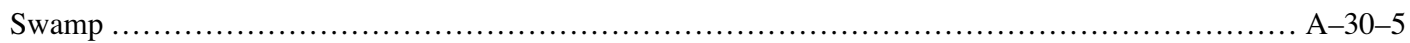

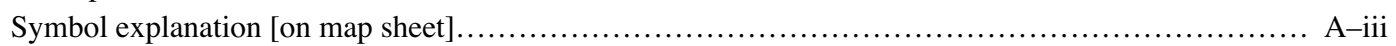

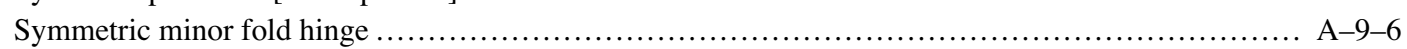

Synclinal linear depression on landslide........................................................ A

Synclinal soft-sediment fold on landslide ................................................... A

19.5.67

19.5 .44

30.2 .46

Syncline (1st option) - Identity and existence certain, location accurate ......................... A-5-6

$\mathrm{n} / \mathrm{a}$

9.121

17.42

17.42

Syncline (1st option) - Identity and existence certain, location approximate ....................... A-5-6

5.5 .1

Syncline (1st option) - Identity and existence certain, location concealed $\ldots \ldots \ldots \ldots \ldots \ldots \ldots \ldots \ldots \ldots \ldots$ A $-5-6$

5.5 .3

Syncline (1st option) - Identity and existence certain, location inferred .................................

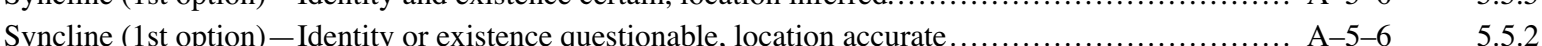

Syncline (1st option) - Identity or existence questionable, location approximate ...................... A-5-6 5.5 .4

Syncline (1st option) - Identity or existence questionable, location concealed .................... A-5-6 5.5.8

Syncline (1st option) - Identity or existence questionable, location inferred ...................... A-5-6 5.5.6

Syncline (2nd option) - Identity and existence certain, location accurate ...................... A $-5-6 \quad 5.5 .9$

Syncline (2nd option) - Identity and existence certain, location approximate ........................ A-5-6 5.5.11

Syncline (2nd option) - Identity and existence certain, location concealed .......................... A-5-6 5.5.15

Syncline (2nd option) - Identity and existence certain, location inferred........................... A-5-6 5.5.13

Syncline (2nd option) - Identity or existence questionable, location accurate ....................... A-5-6 5.5 .10

Syncline (2nd option) - Identity or existence questionable, location approximate.................... A-5-6 5.5 .12

Syncline (2nd option) - Identity or existence questionable, location concealed.................... A-5-6 5.5.16

Syncline (2nd option) - Identity or existence questionable, location inferred .................... A-5-6 5.5.14

Synclines.......................................................................... A-5-6 Sec. 5.5

Synform (1st option) - Identity and existence certain, location accurate ........................ A-5-7 5.6 .1

Synform (1st option) - Identity and existence certain, location approximate ..................... A-5-7 5.6.3

Synform (1st option) - Identity and existence certain, location concealed ...................... A-5-7 5.6.7

Synform (1st option) - Identity and existence certain, location inferred......................... A-5-7 5.6 .5

Synform (1st option) - Identity or existence questionable, location accurate........................ A-5-7 5.6 .2

Synform (1st option) - Identity or existence questionable, location approximate..................... A-5-7 5.6 .4

Synform (1st option) - Identity or existence questionable, location concealed...................... A-5-7 5.6 .8

Synform (1st option) - Identity or existence questionable, location inferred ......................... A-5-7 5.6 .6

Synform (2nd option) - Identity and existence certain, location accurate .......................... A-5-7 5.6 .9

Synform (2nd option) - Identity and existence certain, location approximate ....................... A-5-7 5.6 .11

Synform (2nd option) - Identity and existence certain, location concealed ...................... A-5-7 5.6.15

Synform (2nd option) - Identity and existence certain, location inferred........................... A-5-7 5.6 .13

Synform (2nd option) - Identity or existence questionable, location accurate....................... A-5-7 5.6.10

Synform (2nd option) -Identity or existence questionable, location approximate................... A-5-7 5.6.12

Synform (2nd option) - Identity or existence questionable, location concealed.................... A-5-7 5.6.16

Synform (2nd option) - Identity or existence questionable, location inferred . ................... A-5-7 5.6.14

Synformal sheath fold (1st option) - Identity and existence certain, location accurate............... A-5-10 5.8.1

Synformal sheath fold (1st option) - Identity and existence certain, location approximate.............. A-5-10 5.8.3

Synformal sheath fold (1st option) - Identity and existence certain, location concealed................ A-5-10 5.8.7

Synformal sheath fold (1st option) - Identity and existence certain, location inferred .................. A-5-10 5.8.5

Synformal sheath fold (1st option) - Identity or existence questionable, location accurate.............. A-5-10 5.8.2

Synformal sheath fold (1st option) - Identity or existence questionable, location approximate.......... A-5-10 5.8.4

Synformal sheath fold (1st option) - Identity or existence questionable, location concealed............. A-5-10 5.8.8

Synformal sheath fold (1st option) - Identity or existence questionable, location inferred ............... A-5-10 5.8.6

Synformal sheath fold (2nd option) - Identity and existence certain, location accurate................ A-5-10 5.8.9

Synformal sheath fold (2nd option) - Identity and existence certain, location approximate............. A-5-10 5.8.11

Synformal sheath fold (2nd option) - Identity and existence certain, location concealed.............. A-5-10 5.8.15

Synformal sheath fold (2nd option) - Identity and existence certain, location inferred ............... A-5-10 5.8.13

Synformal sheath fold (2nd option) - Identity or existence questionable, location accurate............ A-5-10 5.8.10

Synformal sheath fold (2nd option) - Identity or existence questionable, location approximate......... A-5-10 5.8.12 


\begin{tabular}{|c|c|}
\hline Page & Ref. No. \\
\hline Synformal sheath fold (2nd option) - Identity or existence questionable, location concealed........... A-5-10 & 5.8 .16 \\
\hline ynformal sheath fold (2nd option) - Identity or existence questionable, location inferred ............ A-5-10 & 5.8 .14 \\
\hline 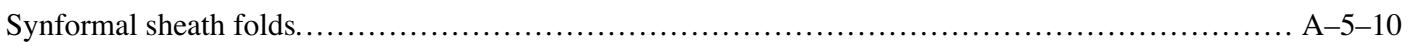 & Sec. 5.8 \\
\hline Synforms................ & Sec. 5.6 \\
\hline $\mathrm{T}$ [abbreviation].. & Table 2 \\
\hline A-19-2 & 19.2.6 \\
\hline Tailings pond.. & 30.2 .43 \\
\hline A-37-3 & 710 \\
\hline TBI [abbreviation]. & Table 2 \\
\hline Technical specifications used in the preparation of this standard.... & $\mathrm{n} / \mathrm{a}$ \\
\hline 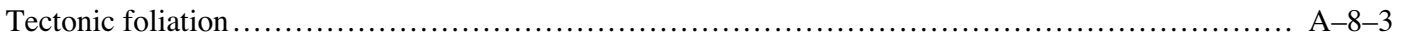 & 8.3 .1 \\
\hline Tectonic foliation, for multiple observations at one locality............ & 8.3 .4 \\
\hline Tectonic foliation parallel to bedding............................... & 8.3 .7 \\
\hline Telephone line...$\ldots \ldots \ldots \ldots \ldots \ldots \ldots \ldots \ldots \ldots \ldots \ldots$ & 30.3 .22 \\
\hline Tennessee $[$ state location map] ............. & Sec. 34.1 \\
\hline Tension crack on landslide (1st option) ..... & 17.35 \\
\hline Tension crack on landslide (2nd option) .... & 17.36 \\
\hline Tension crack on landslide (3rd option) .......... & 17.37 \\
\hline Tension fracture on landslide (1st option)......... & 17.35 \\
\hline Tension fracture on landslide (2nd option).... & 17.36 \\
\hline Tension fracture on landslide (3rd option) ................... & 17.37 \\
\hline Terminus of glacier............................ & Sec. 13 \\
\hline 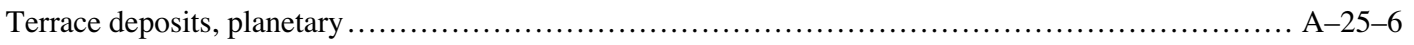 & 25.120 \\
\hline Terrestrial impact crater with raised rim - Identity and existence certain, location accurate ............ A-24-1 & 24.11 \\
\hline Terrestrial impact crater with raised rim-Identity or existence certain, location approximate .......... A-24-1 & 24.13 \\
\hline Terrestrial impact crater with raised rim - Identity or existence certain, location concealed ............ A-24-1 & 24.15 \\
\hline Terrestrial impact crater with raised rim - Identity or existence questionable, location accurate ......... A-24-1 & 24.12 \\
\hline Terrestrial impact crater with raised rim-Identity or existence questionable, location approximate ..... A-24-1 & 24.14 \\
\hline Terrestrial impact crater with raised rim-Identity or existence questionable, location concealed ........ A-24-1 & 24.16 \\
\hline Terrestrial impact crater without raised rim - Identity and existence certain, location accurate .......... A-24-1 & 24.5 \\
\hline Terrestrial impact crater without raised rim - Identity and existence certain, location concealed ........ A-24-1 & 24.9 \\
\hline Terrestrial impact crater without raised rim - Identity or existence certain, location approximate ....... A-24-1 & 24.7 \\
\hline Terrestrial impact crater without raised rim-Identity or existence questionable, location accurate ...... A-24-1 & 24.6 \\
\hline errestrial impact crater without raised rim - Identity or existence questionable, location & \\
\hline A-24-1 & 24.8 \\
\hline Terrestrial impact crater without raised rim - Identity or existence questionable, location concealed .... A-24-1 & 24.10 \\
\hline 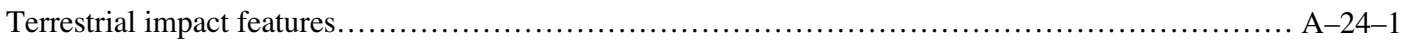 & Sec. 24 \\
\hline Terrestrial palimpsest area....... & 24.19 \\
\hline Territory boundary ............. & 29.2 \\
\hline Tertiary [geologic age symbol]. & 32.3 \\
\hline Test hole for well used for domestic-water supply........... & 26.1 .15 \\
\hline Test hole for well used for industrial-water supply ................... & 26.1 .42 \\
\hline 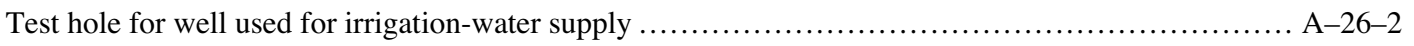 & 26.1 .33 \\
\hline 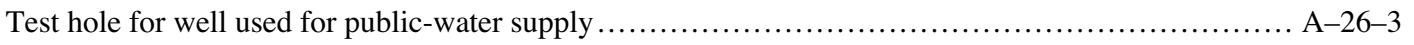 & 26.1.51 \\
\hline Test hole for well used for stock-water supply ..................... & 26.1 .24 \\
\hline 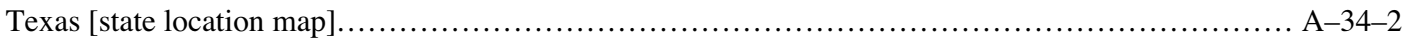 & Sec. 34.1 \\
\hline Thermal area $\ldots \ldots \ldots \ldots \ldots \ldots \ldots \ldots \ldots \ldots \ldots . \ldots \ldots \ldots$ & 18.61 \\
\hline Thermal spring ......... & 18.62 \\
\hline Thermal spring, as shown on topographic maps or on general-purpose or smaller scale maps........... & 30.3 .13 \\
\hline 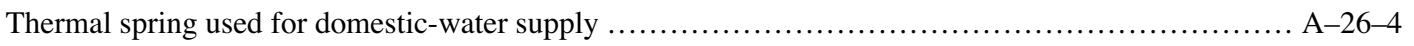 & 26.2 .5 \\
\hline Thermal spring used for industrial-water supply ............ & 26.2.17 \\
\hline Thermal spring used for irrigation-water supply .............. & 26.2 .13 \\
\hline Thermal spring used for public-water supply......... & 26.2 .21 \\
\hline
\end{tabular}




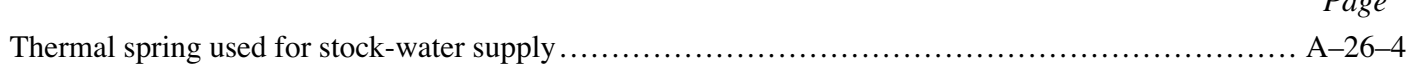

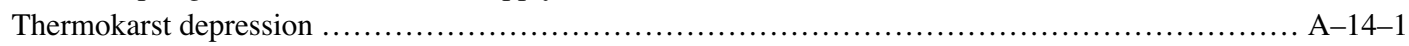

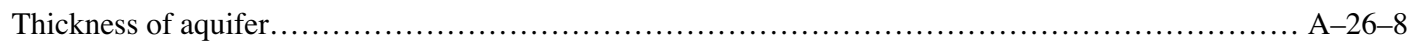

Thickness of bed of economically important commodity and location where thickness was measured... A-1-6

Thickness of clay bed and location where thickness was measured ............................. A-1-6

Thickness of coal bed and location where thickness was measured ............................... A-1-6

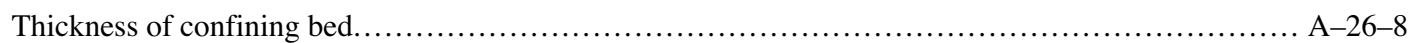

Thickness of dike and location where thickness was measured .................................. A-1-6

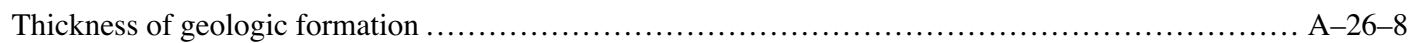

Thickness of key bed and location where thickness was measured............................... A-1-6

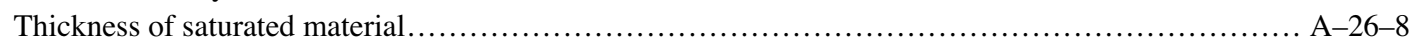

Thrust fault (1st option) - Identity and existence certain, location accurate ...................... A-2-7

Thrust fault (1st option) - Identity and existence certain, location approximate ..................... A-2-7

Thrust fault (1st option) - Identity and existence certain, location concealed ..................... A-2-7

Thrust fault (1st option) - Identity and existence certain, location inferred ....................... A-2-7

Thrust fault (1st option) - Identity or existence questionable, location accurate .................... A-2-7

Thrust fault (1st option) - Identity or existence questionable, location approximate .................. A-2-7

Thrust fault (1st option) - Identity or existence questionable, location concealed ..................... A-2-7

Thrust fault (1st option) - Identity or existence questionable, location inferred....................... A-2-7

Thrust fault (2nd option) - Identity and existence certain, location accurate . ....................... A-2-7

Thrust fault (2nd option) - Identity and existence certain, location approximate ................... A-2-7

Thrust fault (2nd option) - Identity and existence certain, location concealed .................... A-2-7

Thrust fault (2nd option) - Identity and existence certain, location inferred ....................... A-2-7

Thrust fault (2nd option) - Identity or existence questionable, location accurate .................... A-2-7

Thrust fault (2nd option) - Identity or existence questionable, location approximate ................ A-2-7

Thrust fault (2nd option) - Identity or existence questionable, location concealed ................. A-2-7

Thrust fault (2nd option) - Identity or existence questionable, location inferred..................... A-2-7

Thrust fault (3rd option) - Identity and existence certain, location accurate ...................... A-2-7

Thrust fault (3rd option) - Identity and existence certain, location approximate .................... A-2-7

Thrust fault (3rd option) - Identity and existence certain, location concealed ...................... A-2-7

Thrust fault (3rd option) - Identity and existence certain, location inferred ......................... A-2-7

Thrust fault (3rd option) - Identity or existence questionable, location accurate...................... A-2-7

Thrust fault (3rd option) - Identity or existence questionable, location approximate.................. A-2-7

Thrust fault (3rd option) - Identity or existence questionable, location concealed.................... A-2-7

Thrust fault (3rd option) - Identity or existence questionable, location inferred ..................... A-2-7

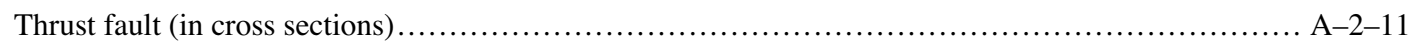

Thrust fault (on small-scale maps or figures) ............................................. A $-2-11$

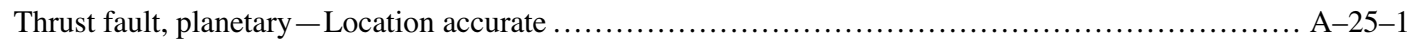

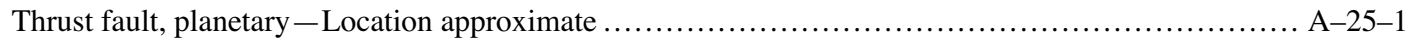

Thrust fault, planetary - Location concealed ............................................... A $-25-1$

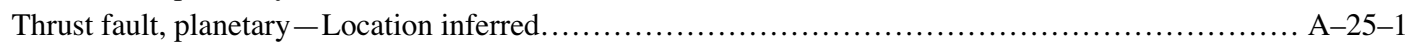

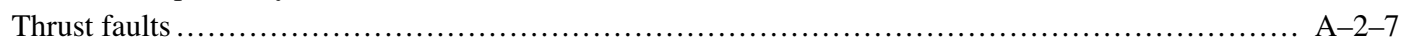

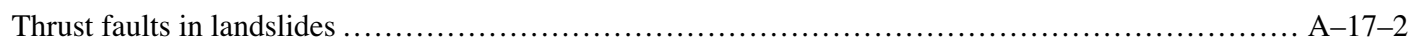

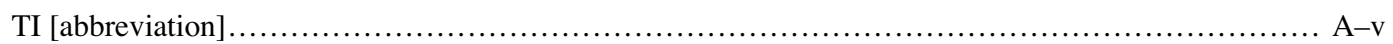

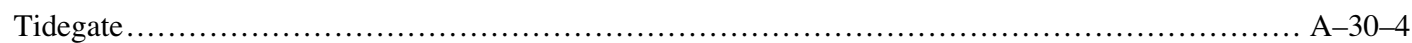

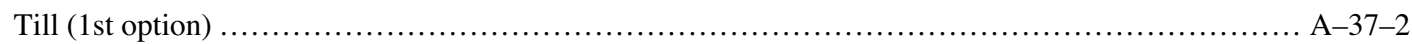

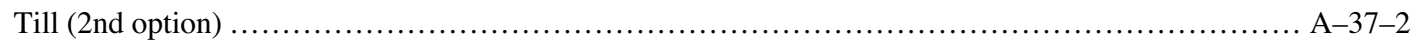

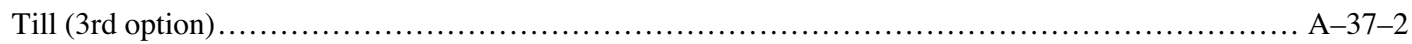

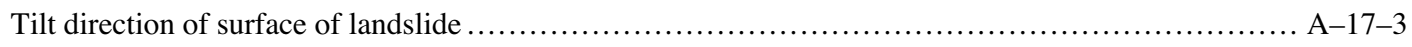

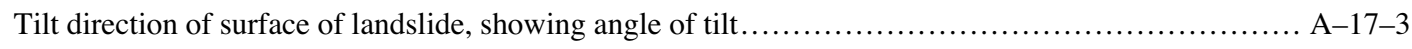

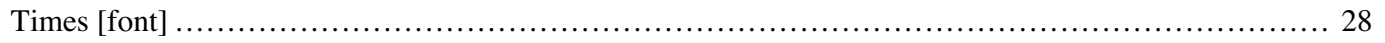

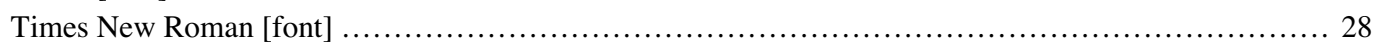

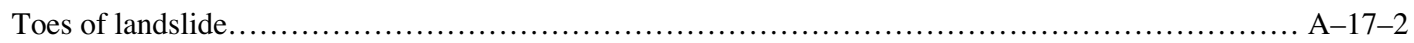

Tool mark in sedimentary materials.....
Ref. No.

26.2 .9

14.11

26.6 .5

1.4 .11

1.4 .11

1.4.11

26.6.5

1.4 .11

26.6.5

1.4 .11

26.6.5

2.8 .1

2.8 .3

2.8 .7

2.8 .5

2.8 .2

2.8 .4

2.8 .8

2.8 .6

2.8 .9

2.8 .11

2.8 .15

2.8 .13

2.8 .10

2.8 .12

2.8 .16

2.8 .14

2.8 .17

2.8 .19

2.8 .23

2.8 .21

2.8 .18

2.8 .20

2.8.24

2.8.22

2.11 .17

2.11.24

25.21

25.22

25.24

25.23

Sec. 2.8

Sec. 17

Table 2

30.2.13

681

682

683

17.52

17.53

$\mathrm{n} / \mathrm{a}$

n/a

Sec. 17

9.13 
Topographic contours.

Topographic depression contours (1st option), showing tick spacing of adjacent contours..............

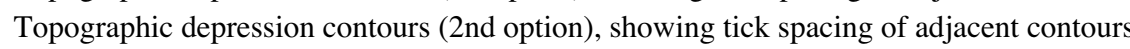

Topographic features.

Toreva block, consisting of a relatively intact mass of displaced materials

Town boundary

Township and range line-Definite

Township and range line-Location approximate

Township boundary

Township label.

Trace fossils

Tracks [fossil symbol]

Traditional line symbol styles.

Trail.....

Transform faults

Transmissivity

Transportation features

Trench (drawn to scale).

Trench (generalized trace)

Trenches

Trend of glacier

Trend of permanent snowfield.

Triassic [geologic age symbol]

Trilobites

Trough line [concepts and definitions]

Trough line of fold

Trough, planetary

Tuffaceous rock.

Tunnel (drawn to scale), as shown on subsurface exploration maps

Tunnel entrance (1st option)

Tunnel entrance (1st option), showing angle of inclination. .

Tunnel entrance (2nd option)

Tunnel entrance (2nd option), showing angle of inclination.

Type of rock [influencing color and pattern selection]

Type size [pertaining to map labeling]

Type specifications [in this standard]...

Type style [pertaining to map labeling]

U/D [notation on fault showing local normal offset]

U/D [notation on fault showing local reverse offset] ....

Unconformable contact - Identity and existence certain, location accurate

Unconformable contact-Identity and existence certain, location approximate

Unconformable contact-Identity and existence certain, location concealed

Unconformable contact-Identity and existence certain, location inferred

Unconformable contact-Identity or existence questionable, location accurate.....

Unconformable contact-Identity or existence questionable, location approximate

Unconformable contact-Identity or existence questionable, location concealed.

Unconformable contact-Identity or existence questionable, location inferred....

Underclay....

Underground aqueduct.

Underground gas pipeline

Underground oil pipeline

Underground or submerged water pipeline

Underwater aqueduct.
Page

A-30-1

A-30-1

A-30-1

A-30-1

A-17-3

A-29-1

A-31-1

A-31-1

A-29-1

A-31-1

A-10-1

A-10-1

.. 20

A-28-1

A-22-1

A-26-8

A-28-1

A-19-3

A-19-3

A-22-2

A-30-5

A-30-5

A-32-1

A-10-1

.. 12

A-5-13

A-25-3

A-37-3

A-19-5

A-19-3

A-19-3

A-19-3

A-19-3

.. 25

.. 28

.. 29

. 28

A-2-11

A-2-11

A-1-2

A-1-2

A-1-2

A-1-2

A-1-2

A-1-2

A-1-2

A-1-2

A-37-2

A-30-4

A-30-6

A-30-6

A-30-4

A-30-4
Ref. No.

Sec. 30.1

30.1 .10

30.1 .20

Sec. 30

17.62

29.4

31.1

31.2

29.4

31.3

10.2.41

10.2.44

Fig. 1

28.15

Sec. 22

26.6.17

Sec. 28

19.3.8

19.3.7

Sec. 22

30.2.45

30.2 .45

32.9

10.2 .8

$\mathrm{n} / \mathrm{a}$

5.10 .11

25.52

711

19.4.9

19.3.9

19.3 .13

19.3.14

19.3.18

$\mathrm{n} / \mathrm{a}$

$\mathrm{n} / \mathrm{a}$

$\mathrm{n} / \mathrm{a}$

$\mathrm{n} / \mathrm{a}$

2.11.2

2.11 .3

1.1.25

1.1.27

1.1.31

1.1 .29

1.1 .26

1.1 .28

1.1 .32

1.1 .30

660

30.2.18

30.3.23

30.3.23

30.2.20

30.2 .18 


\begin{tabular}{|c|c|}
\hline Page & Ref. No. \\
\hline Undulatory bedding & 6.25 \\
\hline Undulatory gneissic layering.. & 8.3 .52 \\
\hline Unimproved road (Class 4)................ & 28.13 \\
\hline 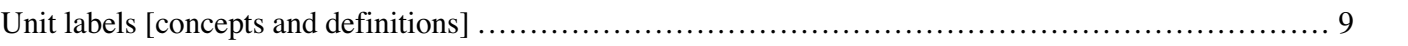 & $\mathrm{n} / \mathrm{a}$ \\
\hline Units for lineweights, lengths, and distances [in this standard] .............. & $\mathrm{n} / \mathrm{a}$ \\
\hline 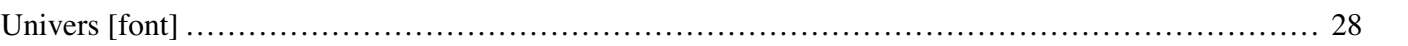 & $\mathrm{n} / \mathrm{a}$ \\
\hline Unsurveyed shoreline ............... & 30.2 .30 \\
\hline Unused spring .................... & 26.2 .2 \\
\hline Unused water well........ & 26.1 .2 \\
\hline Updates to this standard . & $\mathrm{n} / \mathrm{a}$ \\
\hline Uplift, local, intensely disturbed...... & 23.12 \\
\hline Use of patterns ...................... & $\mathrm{n} / \mathrm{a}$ \\
\hline U.S. route marker.................... & 28.17 \\
\hline U.S. Virgin Islands [location map]....... & Sec. 34.1 \\
\hline 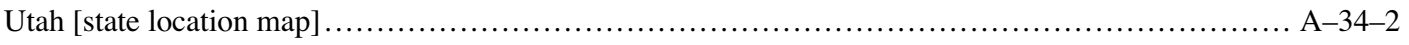 & Sec. 34.1 \\
\hline Vein-Identity and existence certain, location accurate ........ & 19.1.1 \\
\hline Vein-Identity and existence certain, location approximate ......... & 19.1.3 \\
\hline Vein-Identity and existence certain, location concealed ............. & 19.1 .5 \\
\hline Vein-Identity or existence questionable, location accurate $\ldots \ldots \ldots \ldots \ldots . . . .$. & 19.1.2 \\
\hline Vein-Identity or existence questionable, location approximate ........... & 19.1.4 \\
\hline Vein-Identity or existence questionable, location concealed $\ldots \ldots \ldots \ldots \ldots \ldots$ & 19.1.6 \\
\hline 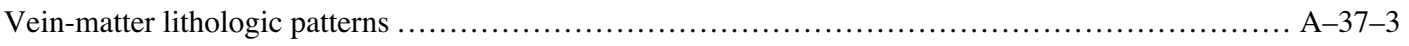 & Sec. 37.2 \\
\hline Vein, showing type of mineral occurrence ....................... & 19.1.7 \\
\hline Veinlet-Identity and existence certain, location accurate .......... & 19.1.1 \\
\hline Veinlet-Identity and existence certain, location approximate ...... & 19.1.3 \\
\hline Veinlet-Identity and existence certain, location concealed ......... & 19.1 .5 \\
\hline Veinlet-Identity or existence questionable, location accurate $\ldots \ldots \ldots \ldots \ldots$ & 19.1.2 \\
\hline Veinlet-Identity or existence questionable, location approximate $\ldots \ldots \ldots \ldots \ldots \ldots \ldots \ldots$ & 19.1.4 \\
\hline 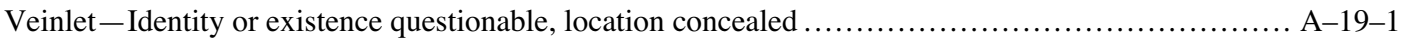 & 19.1.6 \\
\hline Veinlet, showing type of mineral occurrence..................... & 19.1.7 \\
\hline 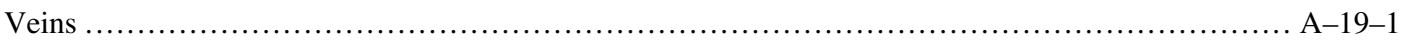 & Sec. 19.1 \\
\hline Vent, large ....................................... & 18.56 \\
\hline Vent, small....................... & 18.55 \\
\hline Vermont $[$ state location map] ....................... & Sec. 34.1 \\
\hline Vertebrates............................................... & 10.2.26 \\
\hline 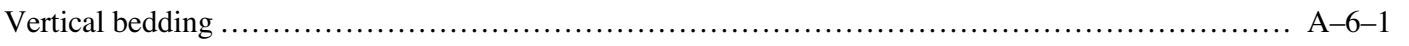 & 6.3 \\
\hline 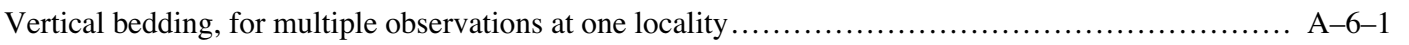 & 6.8 \\
\hline Vertical bedding, where top direction of beds is known from local features................. & 6.14 \\
\hline 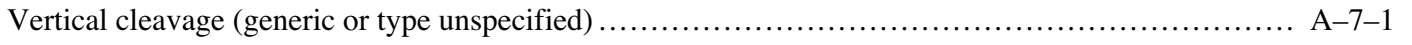 & 7.3 \\
\hline Vertical cleavage (generic or type unspecified), for multiple observations at one locality............. A-7-1 & 7.6 \\
\hline 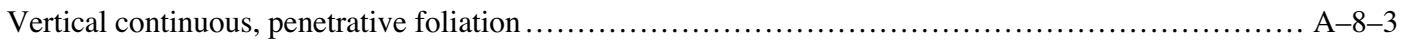 & 8.3.18 \\
\hline Vertical continuous, penetrative foliation, for multiple observations at one locality $\ldots \ldots \ldots \ldots \ldots \ldots \ldots$ A $-8-3$ & 8.3 .21 \\
\hline 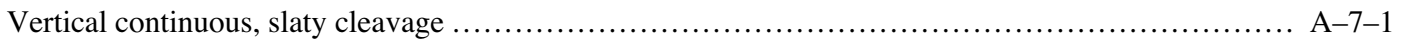 & 7.9 \\
\hline Vertical continuous, slaty cleavage, for multiple observations at one locality $\ldots \ldots \ldots \ldots \ldots \ldots \ldots \ldots \ldots \ldots \ldots \ldots \ldots \ldots \ldots$ & 7.12 \\
\hline 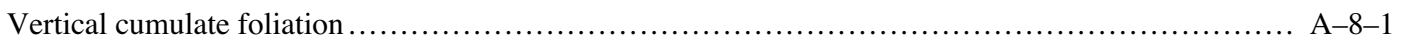 & 8.2 .12 \\
\hline Vertical cumulate foliation, where top direction of layers is known from local features ............... A-8-1 & 8.2 .15 \\
\hline 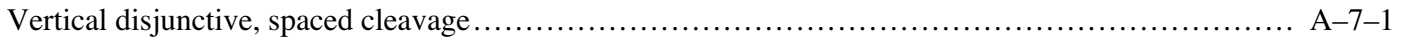 & 7.15 \\
\hline Vertical disjunctive, spaced cleavage, for multiple observations at one locality .... & 7.18 \\
\hline 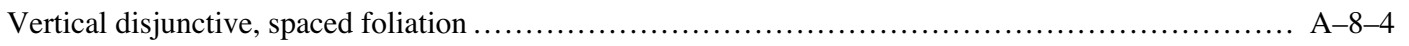 & 8.3 .24 \\
\hline Vertical disjunctive, spaced foliation, for multiple observations at one locality $\ldots \ldots \ldots \ldots \ldots \ldots \ldots \ldots \ldots$ A- $8-4$ & 8.3 .27 \\
\hline 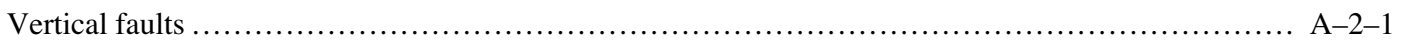 & Sec. 2.1 \\
\hline 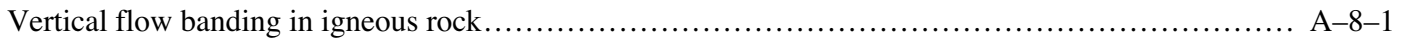 & 8.2 .4 \\
\hline Vertical flow banding in igneous rock, for multiple observations at one locality . & 8.2 .7 \\
\hline
\end{tabular}


Vertical foliation in igneous rock....................................................... A $-8-1$

Vertical foliation in igneous rock, for multiple observations at one locality ......................... A-8-1

8.2 .7

Vertical generic (origin not known or not specified) foliation

A-8-1

8.1 .3

Vertical generic (origin not known or not specified) foliation, for multiple observations at one

$$
\text { locality }
$$

Vertical lamination in igneous rock.....

Vertical lamination in igneous rock, for multiple observations at one locality

Vertical layering in igneous rock.

Vertical layering in igneous rock, for multiple observations at one locality ........................ A-8-1

Vertical metamorphic foliation

Vertical metamorphic foliation, for multiple observations at one locality $\ldots \ldots \ldots \ldots \ldots \ldots \ldots \ldots \ldots \ldots . \mathrm{A}-8-3$

Vertical metamorphic foliation parallel to bedding....

Vertical metamorphic foliation parallel to bedding, where top direction of beds is known from local features.

Vertical mine shaft, as shown on smaller scale or general-purpose maps ....................... A-19-4

19.3.35

Vertical mine shaft at surface (drawn to scale), as shown on subsurface exploration maps.............. A-19-5

Vertical or near-vertical axial surface of fold (1st option)....

A-5-13

Vertical or near-vertical axial surface of fold (2nd option)

Vertical or near-vertical bed of economically important commodity (1st option).................... A-1-6

5.10 .4

Vertical or near-vertical bed of economically important commodity (2nd option)................... A-1-6

Vertical or near-vertical bedding, as determined remotely or from aerial photographs................ A-6-2

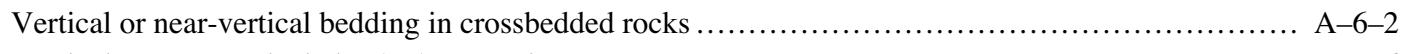

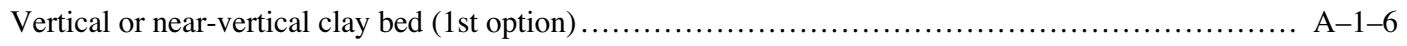

6.31

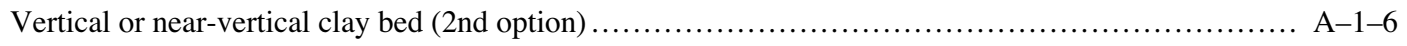

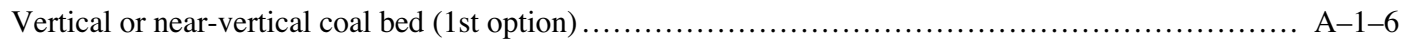

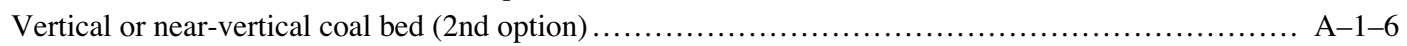

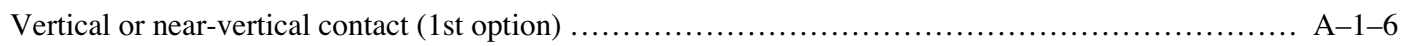

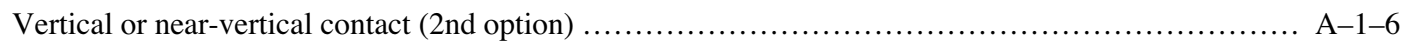

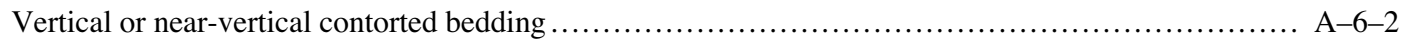

1.4 .4

6.26

Vertical or near-vertical crenulated bedding .............................................. A-6-2

6.26

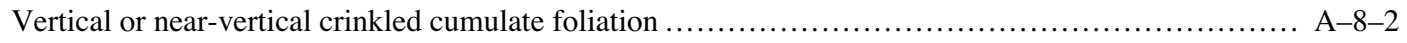

8.2 .18

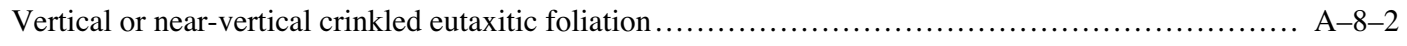

8.2 .26

Vertical or near-vertical crinkled flow banding in igneous rock $\ldots \ldots \ldots \ldots \ldots \ldots \ldots \ldots \ldots \ldots \ldots \ldots \ldots$ A-8 -1

8.2 .9

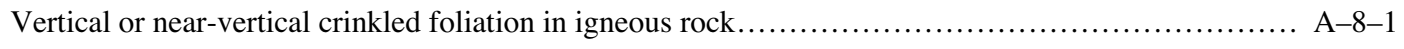

8.2 .9

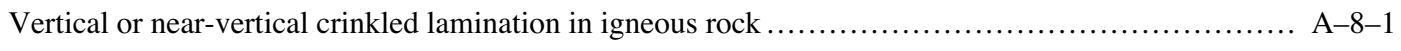

8.2 .9

Vertical or near-vertical crinkled metamorphic foliation $\ldots \ldots \ldots \ldots \ldots \ldots \ldots \ldots \ldots \ldots \ldots \ldots \ldots \ldots \ldots \ldots \ldots \ldots \ldots \ldots \ldots \ldots$

8.3 .15

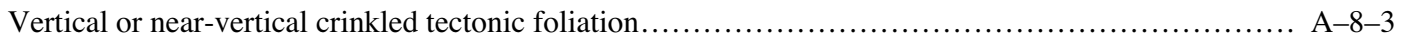

8.3 .15

Vertical or near-vertical deformed cumulate foliation...................................... A-8 2

8.2 .18

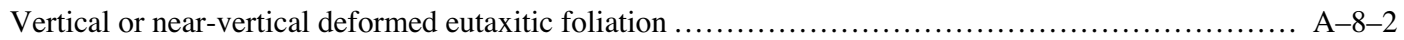

8.2 .26

Vertical or near-vertical deformed flow banding in igneous rock ............................. A-8 -1

8.2 .9

Vertical or near-vertical deformed foliation in igneous rock ................................... A-8 -1

8.2 .9

Vertical or near-vertical deformed lamination in igneous rock ................................. A-8 -1

8.2 .9

Vertical or near-vertical deformed metamorphic foliation................................... A-8 -3

8.3 .15

Vertical or near-vertical deformed tectonic foliation ......................................... A

8.3 .15

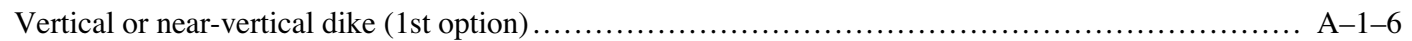

1.4 .3

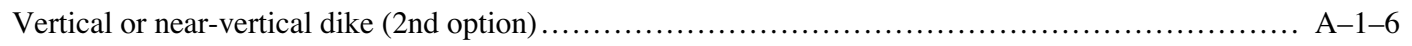

Vertical or near-vertical disjunctive, asymmetric (S-shaped, counterclockwise sense of shear) crenulation cleavage.

Vertical or near-vertical disjunctive, asymmetric (S-shaped, counterclockwise sense of shear) crenulation cleavage, for multiple observations at one locality

Vertical or near-vertical disjunctive, asymmetric (Z-shaped, clockwise sense of shear) crenulation cleavage.

Vertical or near-vertical disjunctive, asymmetric (Z-shaped, clockwise sense of shear) crenulation cleavage, for multiple observations at one locality .... 
Vertical or near-vertical disjunctive, asymmetric (Z-shaped, clockwise sense of shear) crenulation foliation....

Vertical or near-vertical disjunctive, asymmetric (Z-shaped, clockwise sense of shear) crenulation foliation, for multiple observations at one locality.....

ertical or near-vertical disjunctive, asymmetric (S-shaped, counterclockwise sense of shear) crenulation foliation.

Vertical or near-vertical disjunctive, asymmetric (S-shaped, counterclockwise sense of shear) crenulation foliation, for multiple observations at one locality.

Vertical or near-vertical disjunctive, symmetric crenulation cleavage, for multiple observations at one locality

Vertical or near-vertical disjunctive, symmetric crenulation foliation

Vertical or near-vertical disjunctive, symmetric crenulation foliation, for multiple observations at one locality

Vertical or near-vertical eutaxitic foliation.

Vertical or near-vertical eutaxitic foliation, for multiple observations at one locality $\ldots . \ldots . . . . .2$

8.2 .21

Vertical or near-vertical eutaxitic foliation, for multiple observations at one locality .................. A $-8-2$

8.2 .24

Vertical or near-vertical fault (1st option)

Vertical or near-vertical fault (2nd option)

Vertical or near-vertical generic (origin or type not known or not specified) lineation or linear structure (1st option)

Vertical or near-vertical generic (origin or type not known or not specified) lineation or linear structure (2nd option)

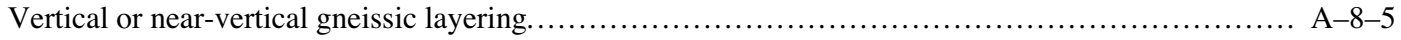

Vertical or near-vertical gneissic layering, for multiple observations at one locality ................. A-8-5

Vertical or near-vertical graded bedding

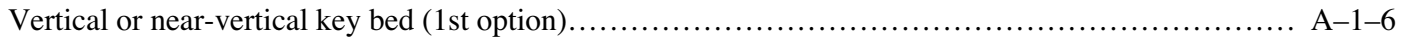

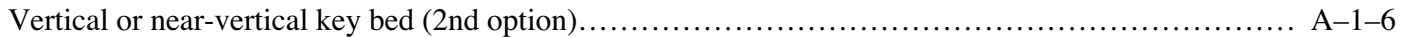

Vertical or near-vertical mineralized stringer (1st option) .................................... A-19-1

Vertical or near-vertical mineralized stringer (2nd option) . . . . . . . .

Vertical or near-vertical mylonitic foliation

Vertical or near-vertical mylonitic foliation, for multiple observations at one locality ................. A-8-5

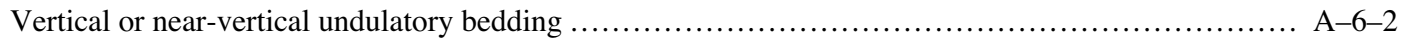

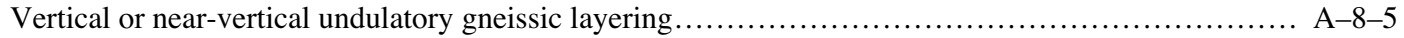

8.3.54

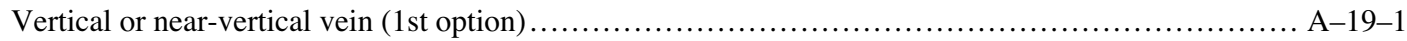

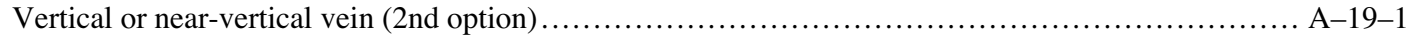

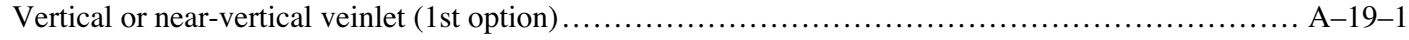

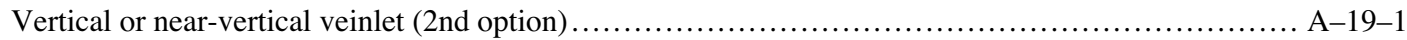

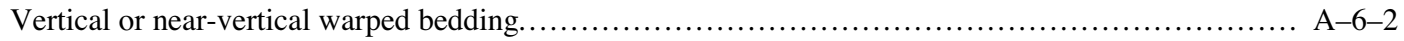

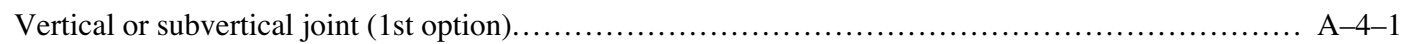

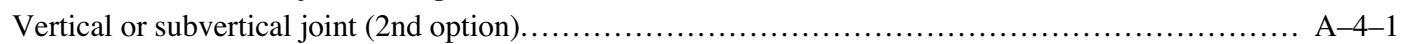

19.1.10

19.1.11

19.1.10

19.1.11

6.26

4.2 .5

4.2 .6

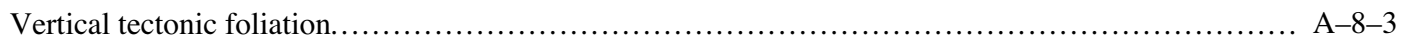

8.3 .3

Vertical tectonic foliation, for multiple observations at one locality ............................ A $-8-3$

8.3.6

Vertical tectonic foliation parallel to bedding

Vertical tectonic foliation parallel to bedding, where top direction of beds is known from local features....

Vertical (top direction to left) bedding, where top direction of beds is known from local features, for multiple observations at one locality

Vertical (top direction to right) bedding, where top direction of beds is known from local features, for multiple observations at one locality .............................................. A $-6-1$

Very small dome, planetary (not mapped to scale) ........................................... A $-25-4$

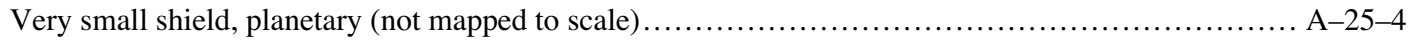

Very small tholi, planetary (not mapped to scale)........................................ A-25-4

Very small volcanic construct, planetary (not mapped to scale) .............................. A-25-4

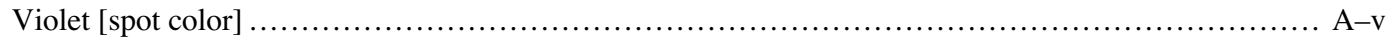

Table 3

Virginia [state location map]. 


\begin{tabular}{|c|c|}
\hline Page & Ref. No. \\
\hline Vitrophyre ............. & 731 \\
\hline Volcanic agglomerate....... & 715 \\
\hline Volcanic breccia ............ & 715 \\
\hline 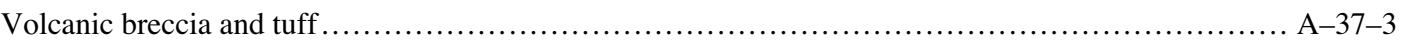 & 714 \\
\hline Volcanic crater-Identity and existence certain, location accurate............. & 18.1 \\
\hline Volcanic crater-Identity and existence certain, location concealed............ & 18.5 \\
\hline 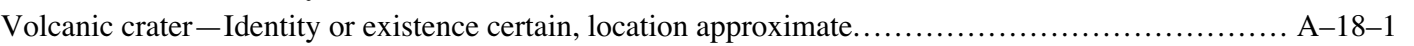 & 18.3 \\
\hline 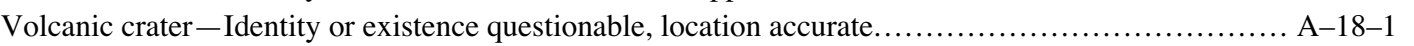 & 18.2 \\
\hline 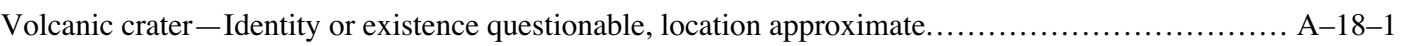 & 18.4 \\
\hline Volcanic crater-Identity or existence questionable, location concealed.......................... A-18-1 & 18.6 \\
\hline Volcanic crater, showing low point of crater ............................ & 18.7 \\
\hline Volcanic edifice - Accurately located .................... & 22.33 \\
\hline Volcanic edifice-Approximately located ................ & 22.34 \\
\hline Volcanic features ................................. & Sec. 18 \\
\hline Volcanic fissure..................................... & 18.41 \\
\hline Volcanic fissure where lava has been emitted $\ldots \ldots \ldots \ldots \ldots \ldots \ldots \ldots \ldots$ & 18.43 \\
\hline 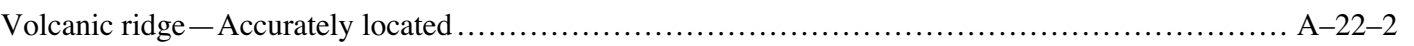 & 22.33 \\
\hline Volcanic ridge - Approximately located ......................... & 22.34 \\
\hline Volcano, planetary, having summit crater......................... & 25.112 \\
\hline Volcano, planetary, without summit crater....................... & 25.113 \\
\hline Warped bedding .... & 6.25 \\
\hline 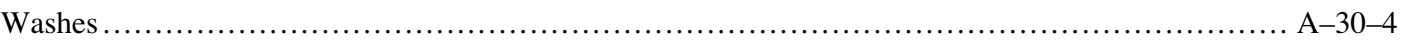 & Sec. 30.2 \\
\hline 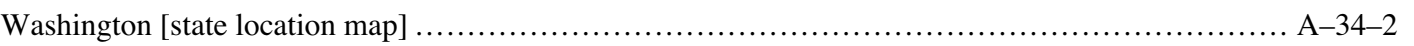 & Sec. 34.1 \\
\hline 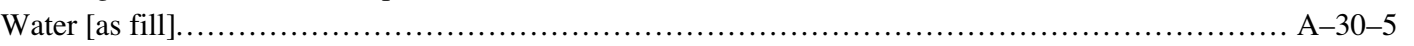 & 30.2 .29 \\
\hline 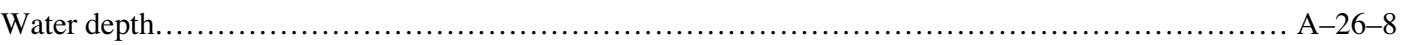 & 26.6 .3 \\
\hline 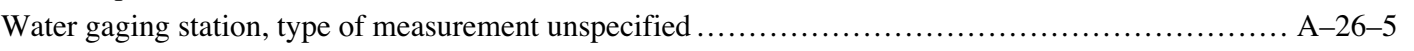 & 26.3 .1 \\
\hline 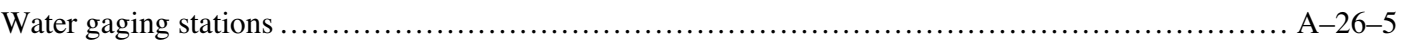 & Sec. 26.3 \\
\hline 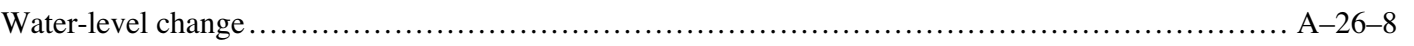 & 26.6.13 \\
\hline 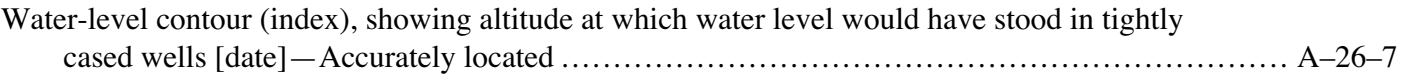 & 26.5 .13 \\
\hline $\begin{array}{l}\text { Water-level contour (index), showing altitude at which water level would have stood in tightly } \\
\text { cased wells }[\text { date }]-\text { Approximately located } \ldots \ldots \ldots \ldots \ldots \ldots \ldots \ldots \ldots \ldots \ldots \ldots \ldots \ldots \ldots \ldots \ldots \ldots \ldots \ldots \ldots \ldots\end{array}$ & 26.5.14 \\
\hline 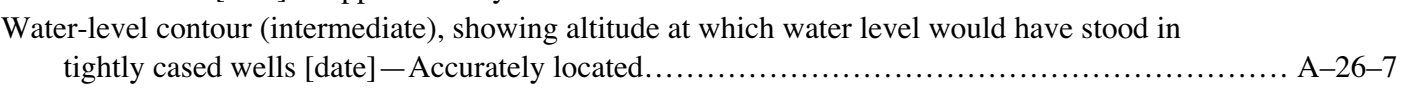 & 26.5.15 \\
\hline $\begin{array}{l}\text { Water-level contour (intermediate), showing altitude at which water level would have stood in } \\
\text { tightly cased wells [date] }- \text { Approximately located } \ldots \ldots \ldots \ldots \ldots \ldots \ldots \ldots \ldots \ldots \ldots \ldots \ldots \ldots \ldots \ldots \ldots \ldots\end{array}$ & 26.5.16 \\
\hline 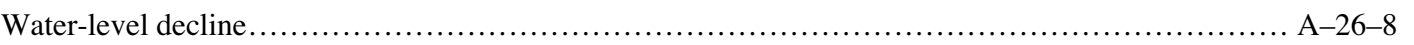 & 26.6 .13 \\
\hline 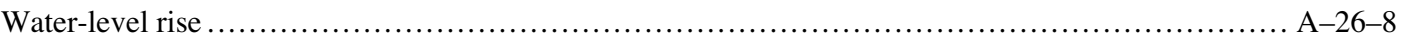 & 26.6 .13 \\
\hline $\begin{array}{l}\text { Water-quality-zone contour (index), showing altitude of top or base of, or horizon within, [type of] } \\
\quad \text { water-quality zone or water in aquifer [date] - Accurately located............................. A-26-7 }\end{array}$ & 26.5.17 \\
\hline 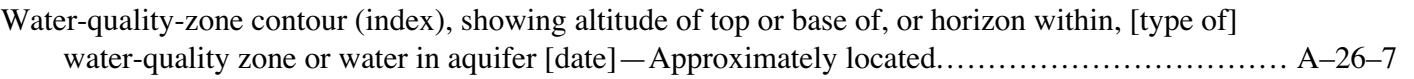 & 26.5 .18 \\
\hline $\begin{array}{l}\text { Water-quality-zone contour (intermediate), showing altitude of top or base of, or horizon within, } \\
\text { [type of] water-quality zone or water in aquifer [date] - Accurately located } \ldots \ldots \ldots \ldots \ldots \ldots \ldots \ldots \ldots \ldots \ldots\end{array}$ & 26.5.19 \\
\hline $\begin{array}{l}\text { Water-quality-zone contour (intermediate), showing altitude of top or base of, or horizon within, } \\
\text { [type of] water-quality zone or water in aquifer [date] - Approximately located ................ A-26-7 }\end{array}$ & 26.5.20 \\
\hline Water-table contour (index), showing altitude of unconfined water table [date]-Accurately located.... A-26-7 & 26.5 .9 \\
\hline 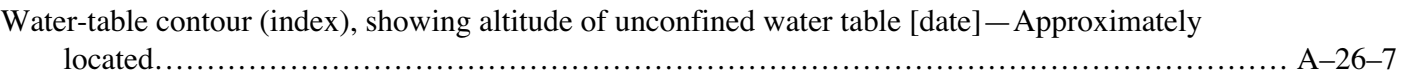 & 26.5 .10 \\
\hline 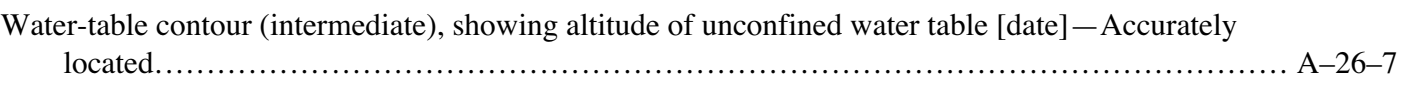 & 26.5 .11 \\
\hline 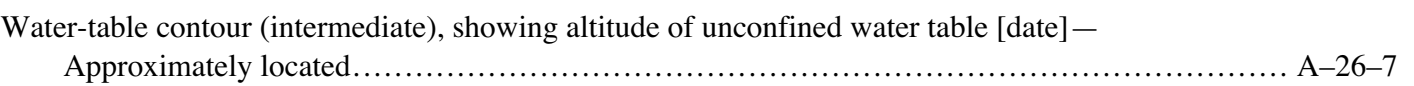 & 26.5 .12 \\
\hline Water temperature ................................. & 26.6 .7 \\
\hline
\end{tabular}


Water well, as shown on topographic maps or on general-purpose or smaller scale maps................

Water well, type unpecified..................... A-30-6

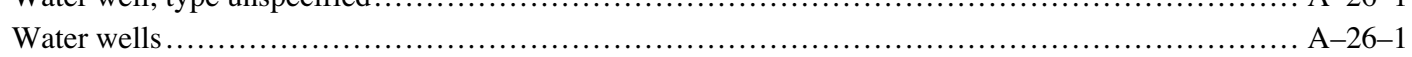

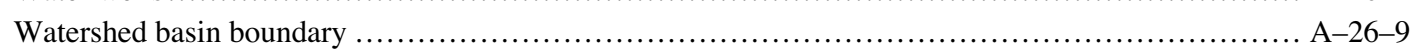

Watershed subbasin boundary ......................................................... A-26-9

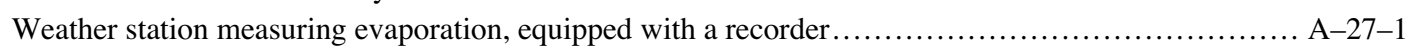

Weather station measuring evaporation, equipped with a telephone or radio ........................ A-27-1

Weather station measuring humidity, equipped with a recorder ................................ A-27-1

Weather station measuring humidity, equipped with a telephone or radio .......................... A-27-1

Weather station measuring precipitation, equipped with a recorder.............................. A-27-1

Weather station measuring precipitation, equipped with a telephone or radio ....................... A-27-1

Weather station measuring solar radiation, equipped with a recorder............................ A-27-1

Weather station measuring solar radiation, equipped with a telephone or radio .................... A $-27-1$

Weather station measuring temperature, equipped with a recorder............................. A-27-1

Weather station measuring temperature, equipped with a telephone or radio....................... A-27-1

Weather station measuring wind velocity, equipped with a recorder............................ A $-27-1$

Weather station measuring wind velocity, equipped with a telephone or radio....................... A $-27-1$

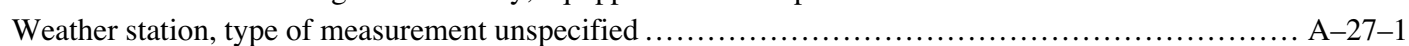

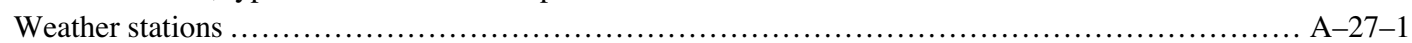

"Web-safe" color [concepts and definitions] ................................................... 30

Well location for hydrocarbon exploration or exploitation ..................................... A-19-6

Well used for collection of water data ......................................................... A-26-1

Well used for domestic-water supply .................................................. A $-26-1$

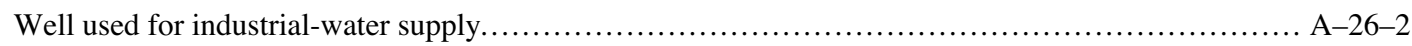

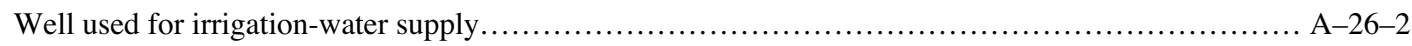

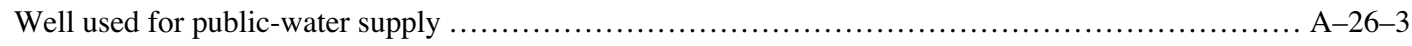

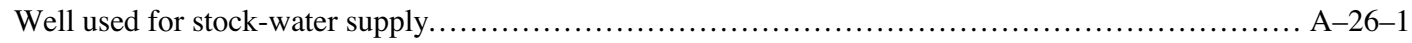

Wells drilled for hydrocarbon exploration or exploitation................................... A-19-6

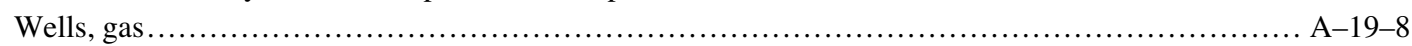

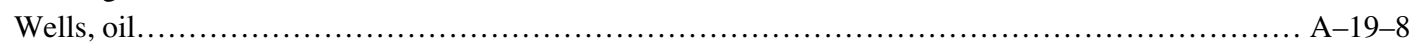

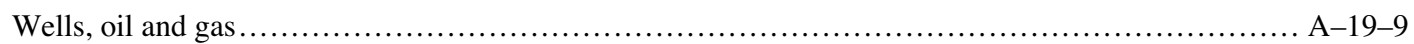

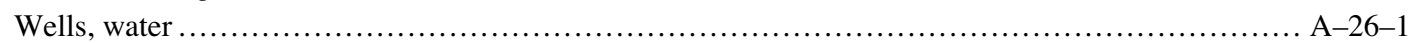

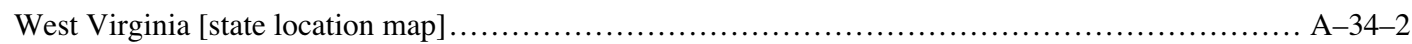

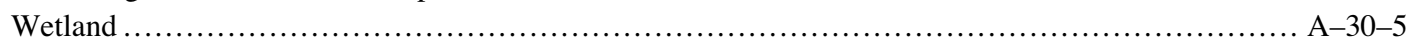

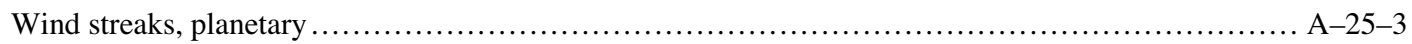

Windmill, as shown on topographic maps or on general-purpose or smaller scale maps............... A-30-6

Winze (drawn to scale), as shown on subsurface exploration maps ............................... A-19-5

Winze extending through level (drawn to scale), as shown on subsurface exploration maps............ A-19-5

Wisconsin $[$ state location map] ...................................................... A $-34-2$

"Within zone of confidence" [concepts and definitions] ........................................ 19

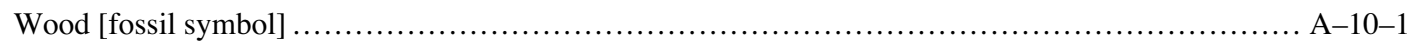

Workings (at surface, or projected to surface) ................................................... A

Workings (drawn to scale), as shown on subsurface exploration maps ........................... A-19-5

Workings (subsurface), as shown on subsurface exploration maps............................. A-19-5

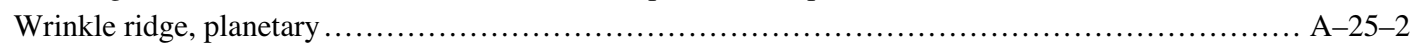

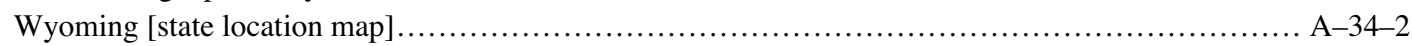

Y [abbreviation]

$\mathrm{A}-\mathrm{V}$

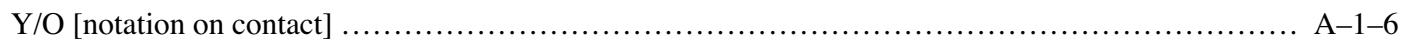

Younger glacial groove - Showing general bearing .......................................... A-13-2

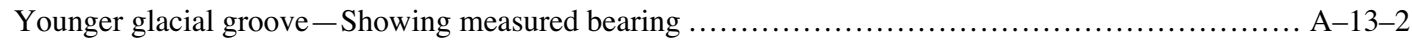

Younger glacial groove, flow direction unknown - Showing general bearing.................... A-13-2

Younger glacial groove, flow direction unknown - Showing measured bearing .................. A $-13-2$

Younger glacial groove (length mapped to scale).
Ref. No.
30.3 .10

26.1 .1

Sec. 26.1

26.7 .1

26.7 .2

27.8

27.7

27.12

27.11

27.6

27.5

27.14

27.13

27.10

27.9

27.16

27.15

27.1

Sec. 27

$\mathrm{n} / \mathrm{a}$

19.5.10

26.1.6

26.1.7

26.1.34

26.1.25

26.1 .43

26.1.16

Sec. 19.5

Sec. 19.5

Sec. 19.5

Sec. 19.5

Sec. 26.1

Sec. 34.1

30.2 .46

25.68

30.3 .8

19.4 .6

19.4.7

Sec. 34.1

$\mathrm{n} / \mathrm{a}$

10.2.34

Sec. 19.2

19.4.10

Sec. 19.4

25.37

Sec. 34.1

Table 2

1.4 .9

13.29

13.30

13.33

13.34

13.37 


\begin{tabular}{|c|c|}
\hline Page & Ref. No. \\
\hline Younger glacial groove (length mapped to scale), flow direction unknown . & 13.38 \\
\hline Younger glacial striation - Showing general bearing........................ & 13.29 \\
\hline 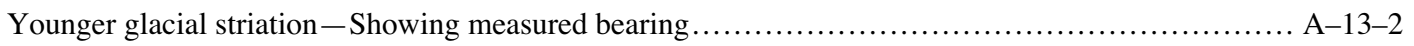 & 13.30 \\
\hline 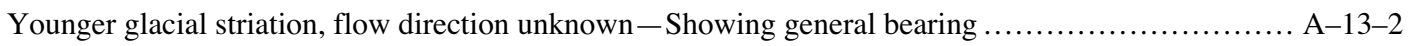 & 13.33 \\
\hline Younger glacial striation, flow direction unknown - Showing measured bearing..................... A-13-2 & 13.34 \\
\hline 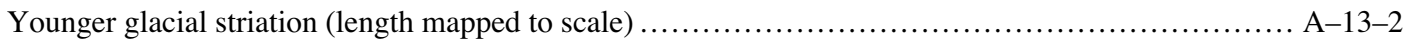 & 13.37 \\
\hline Younger glacial striation (length mapped to scale), flow direction unknown............... & 13.38 \\
\hline Zeolitic rock. & 716 \\
\hline Zone of altered rock (1st option) .. & 19.1.14 \\
\hline Zone of altered rock (2nd option) ............................ & 19.1.15 \\
\hline Zone of altered rock, showing high level of mineralization ................... & 19.1.16 \\
\hline Zone of altered rock, showing low level of mineralization ..................... & 19.1.17 \\
\hline 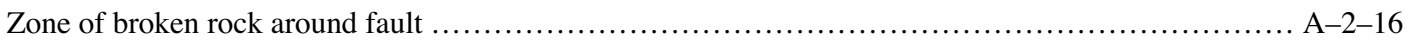 & 2.14 .4 \\
\hline Zone of broken rock within fault ............................... & 2.14 .3 \\
\hline 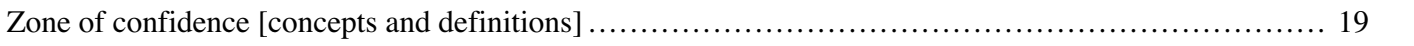 & $\mathrm{n} / \mathrm{a}$ \\
\hline 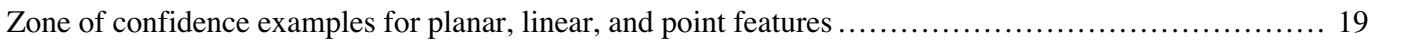 & Fig. 3 \\
\hline 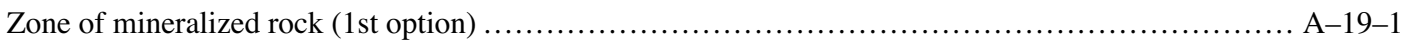 & 19.1.14 \\
\hline 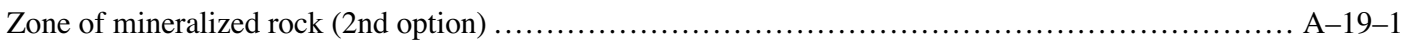 & 19.1.15 \\
\hline Zone of mineralized rock, showing high level of mineralization .................................. A-19-1 & 19.1.16 \\
\hline Zone of mineralized rock, showing low level of mineralization $\ldots \ldots \ldots \ldots \ldots \ldots \ldots \ldots \ldots \ldots \ldots \ldots \ldots \ldots \ldots \ldots \ldots \ldots \ldots \ldots$ & 19.1.17 \\
\hline 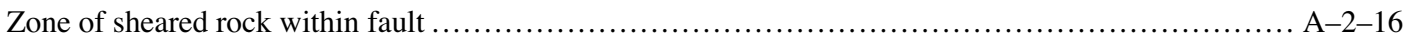 & 2.14 .2 \\
\hline 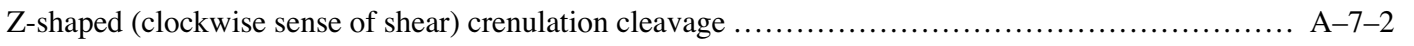 & 7.31 \\
\hline Z-shaped (clockwise sense of shear) crenulation cleavage, for multiple observations at one locality ..... A-7-2 & 7.34 \\
\hline Z-shaped (clockwise sense of shear) crenulation foliation........................................ A-8 -4 & 8.3 .40 \\
\hline Z-shaped (clockwise sense of shear) crenulation foliation, for multiple observations at one locality ..... A-8-4 & 8.3 .43 \\
\hline 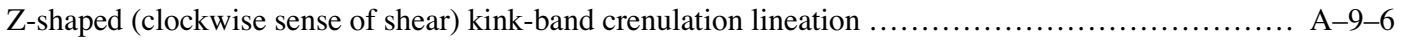 & 9.141 \\
\hline Z-shaped (clockwise sense of shear) minor fold hinge ................. & 9.129 \\
\hline
\end{tabular}


CMYK Color Chart

[CMY $(K=0)$ value below color box; generic lookup-table symbol number in upper left-hand corner of color box]
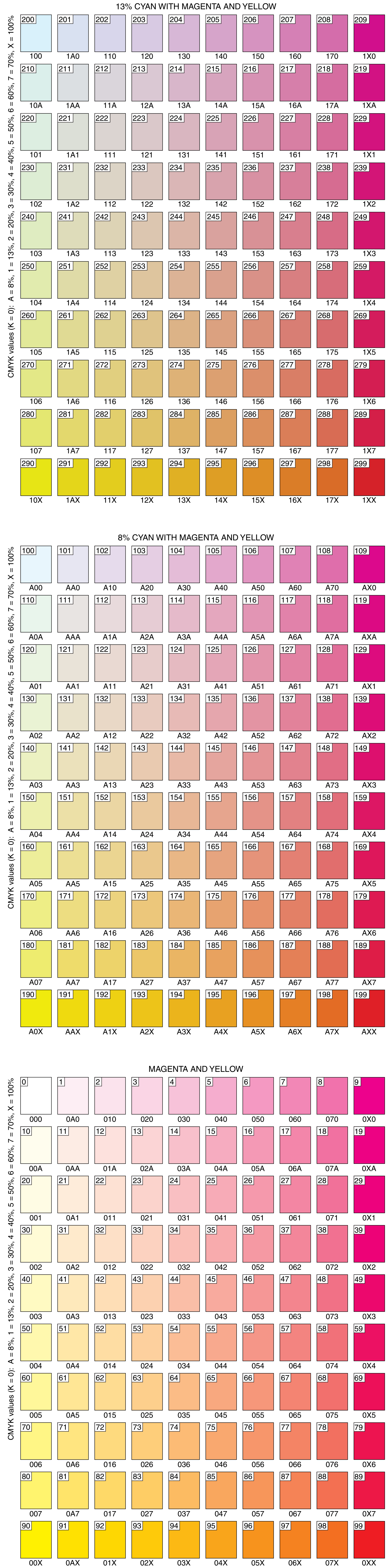
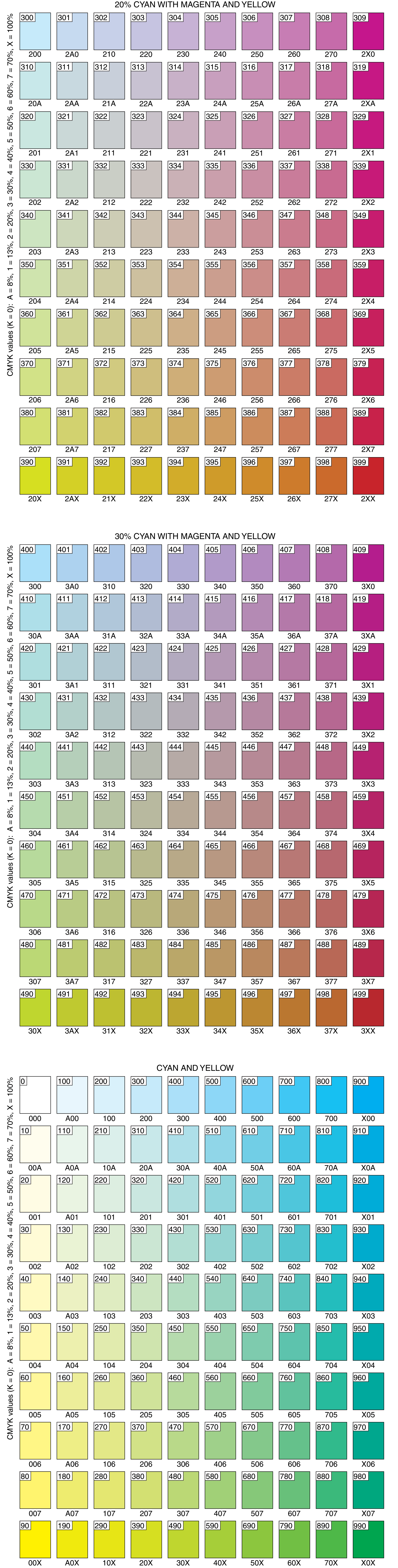
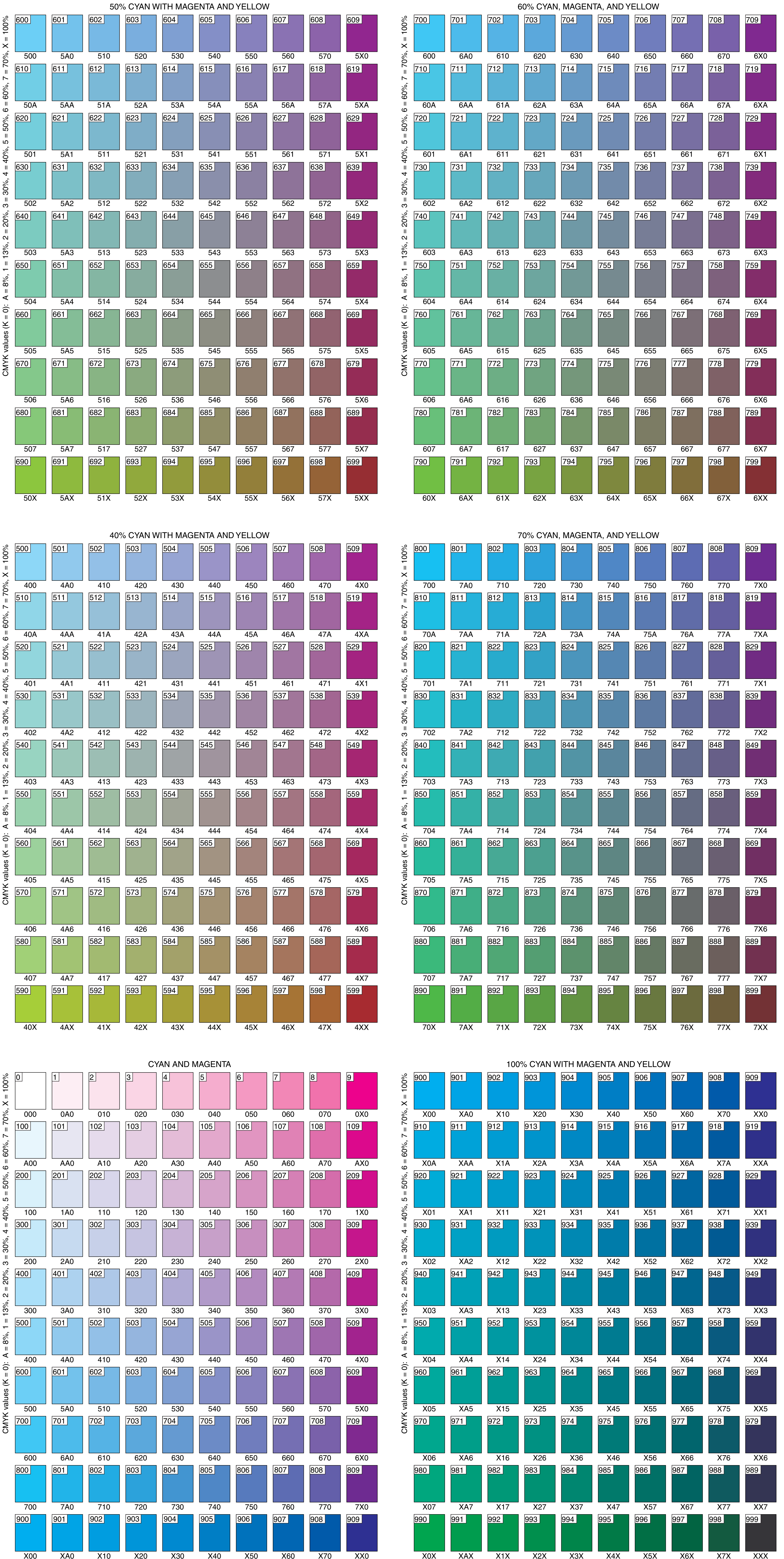

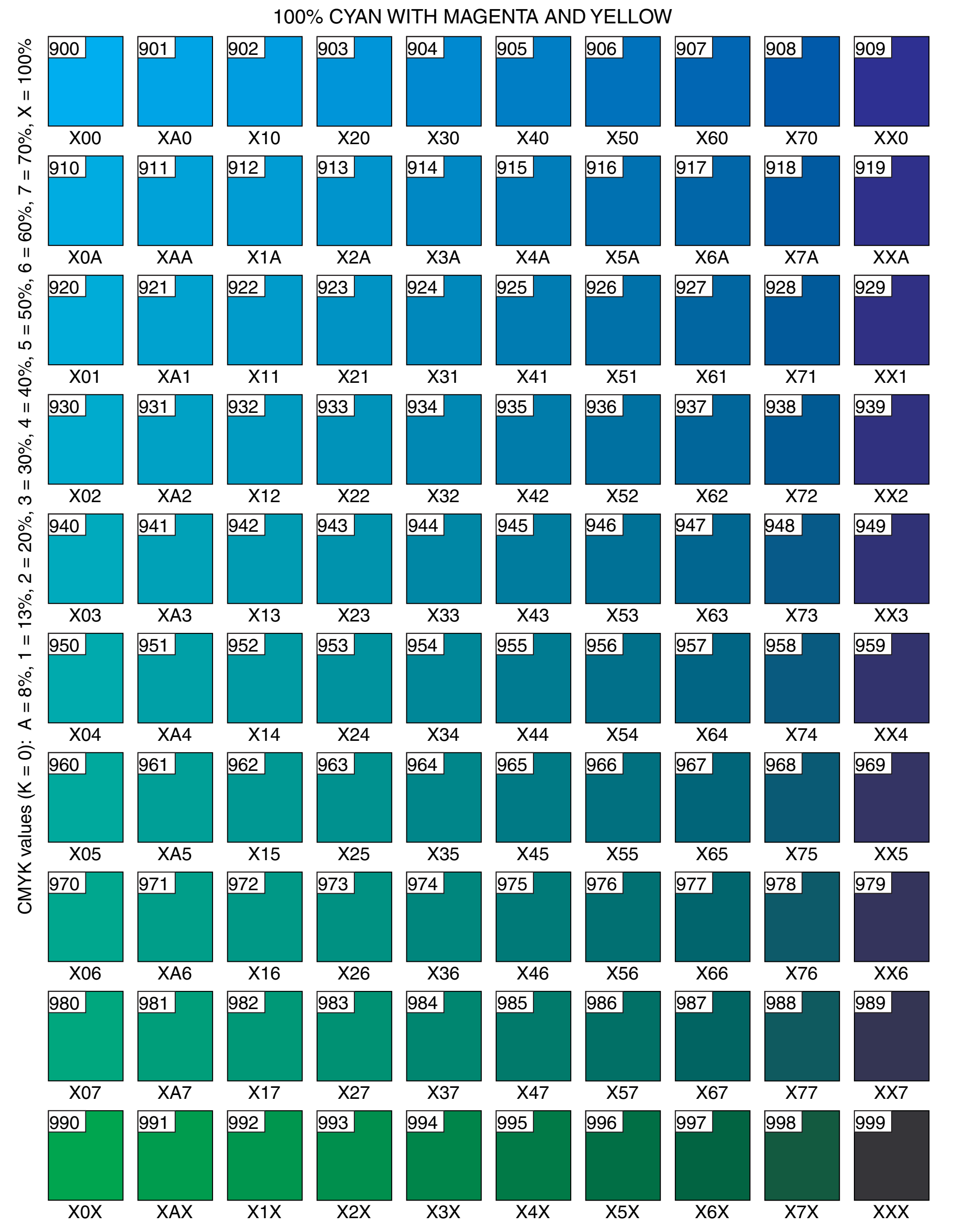



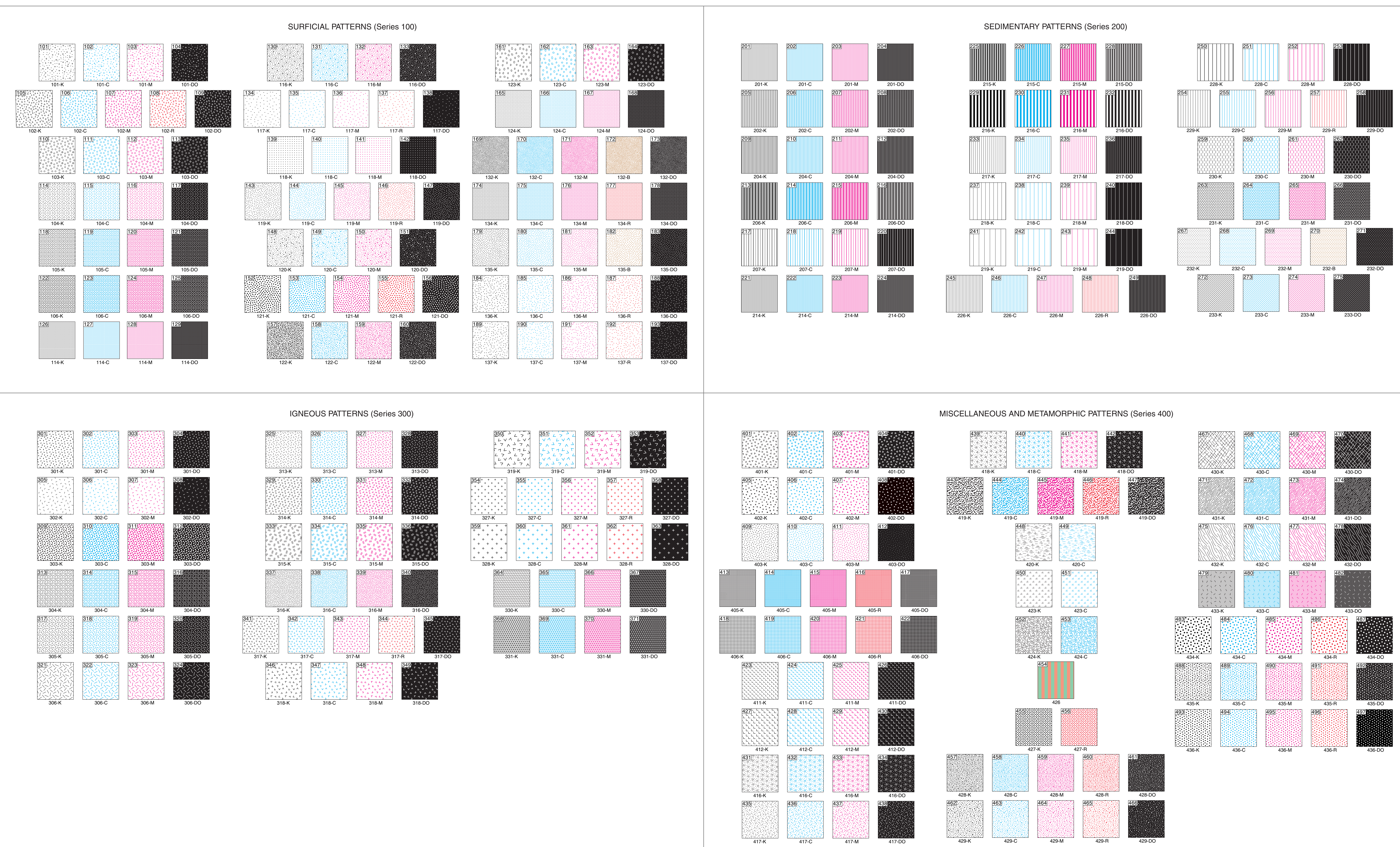

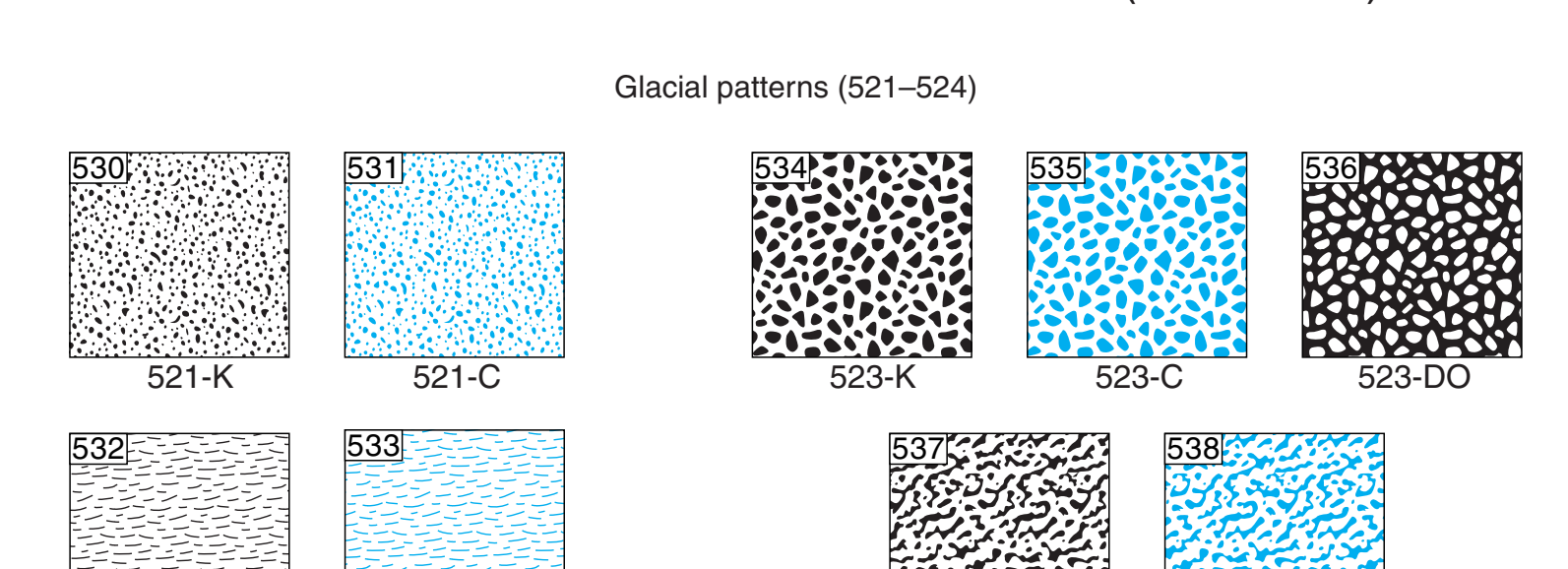

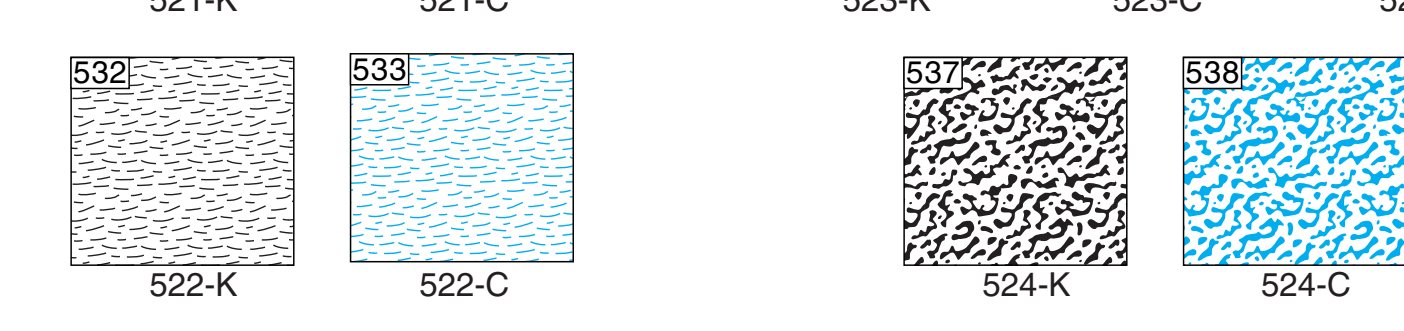

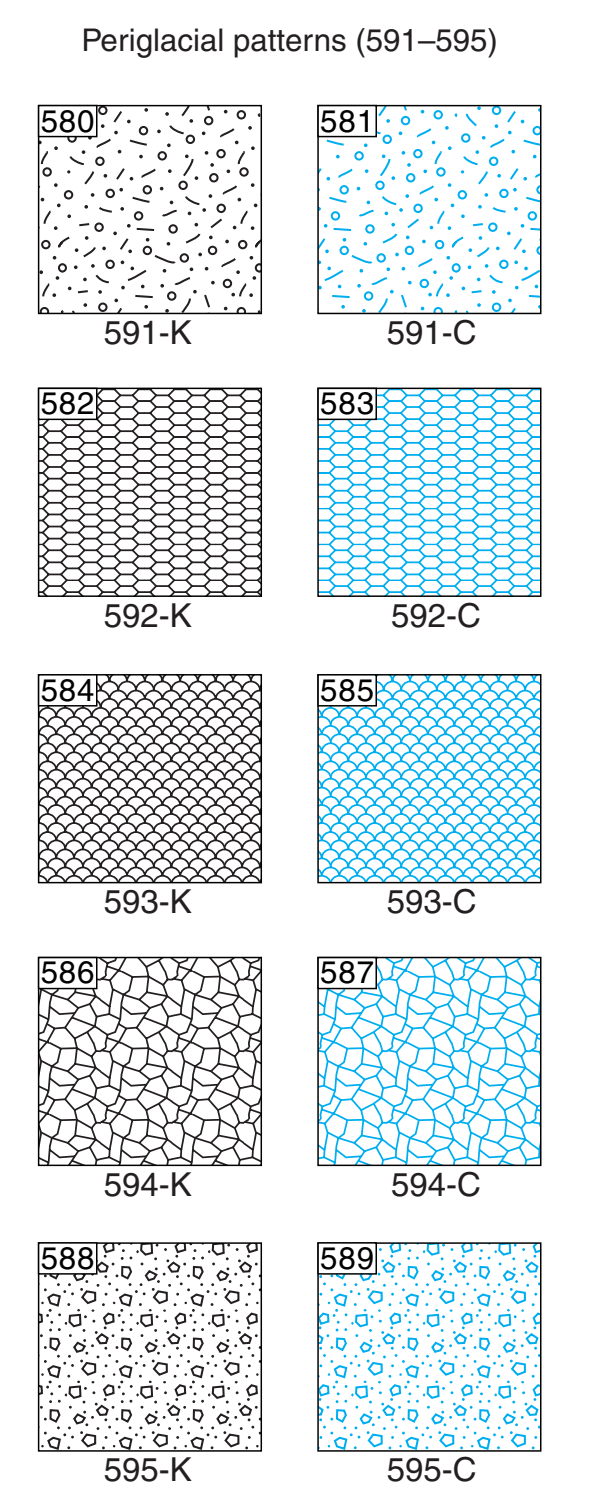

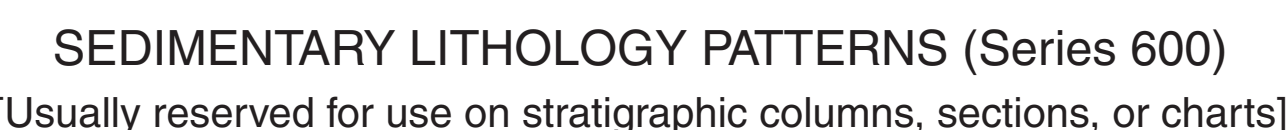

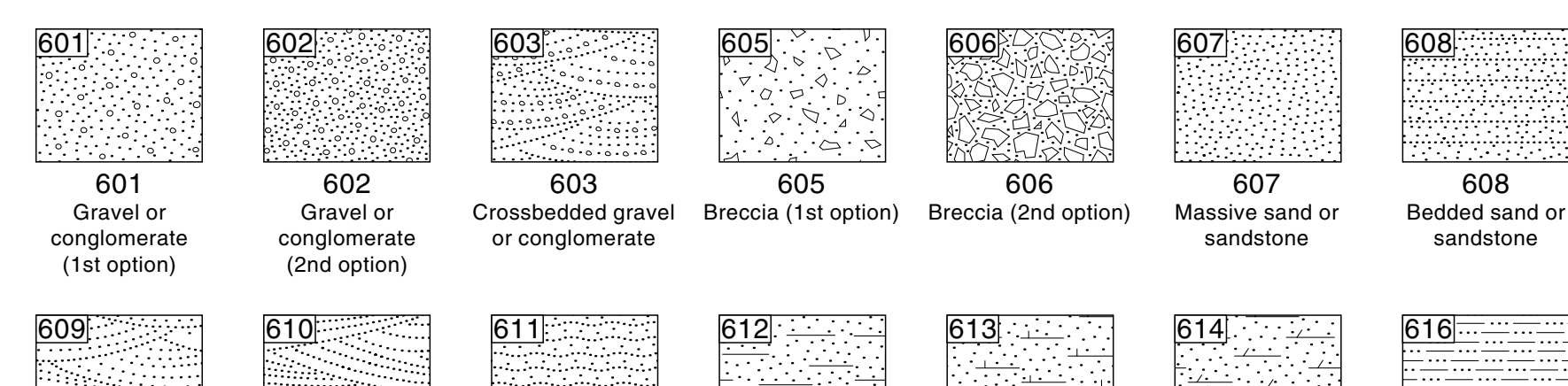

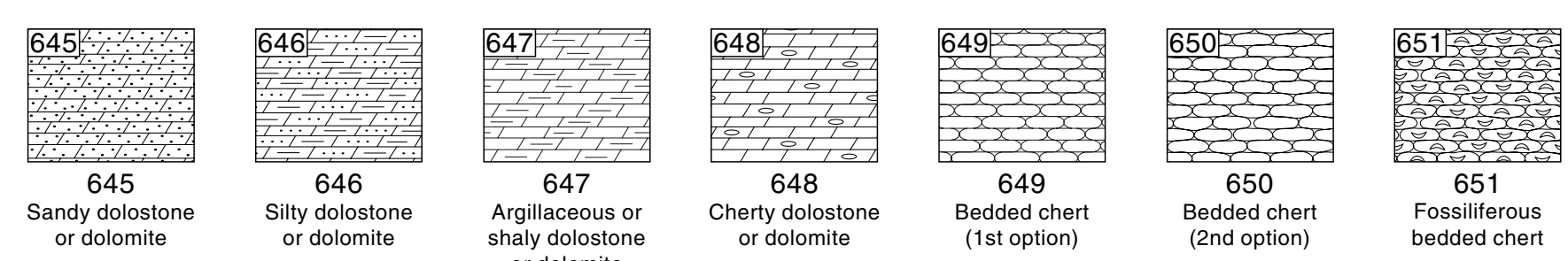

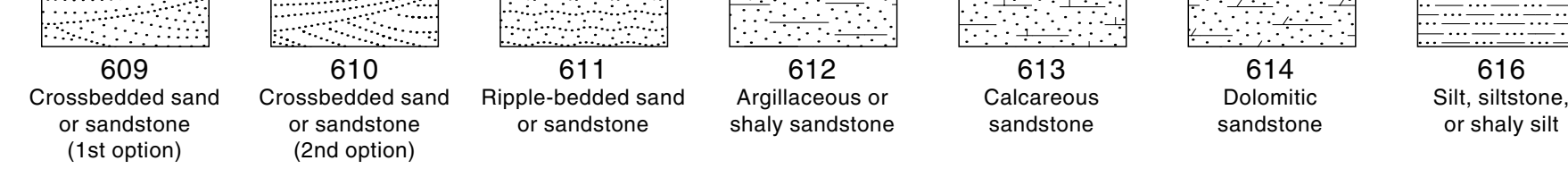

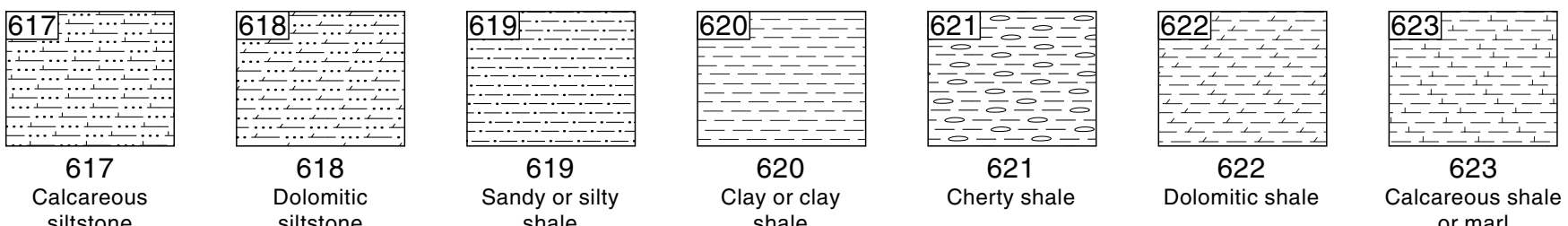

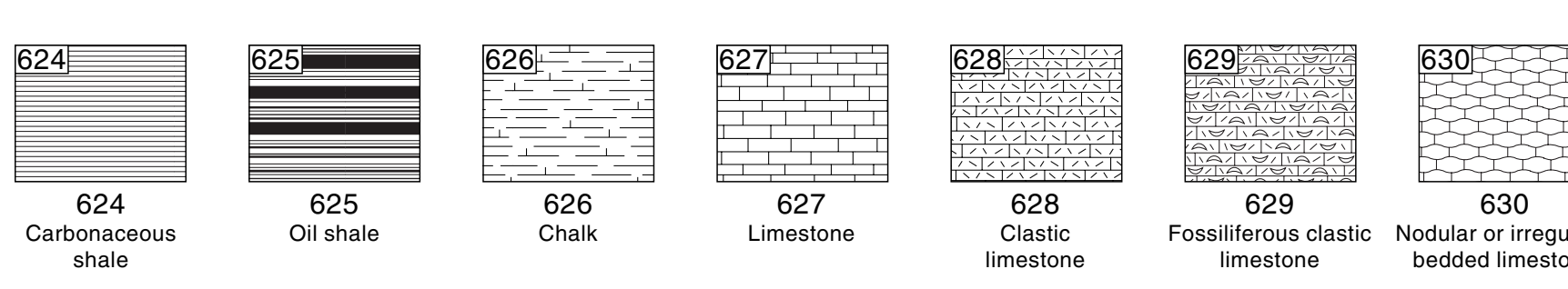

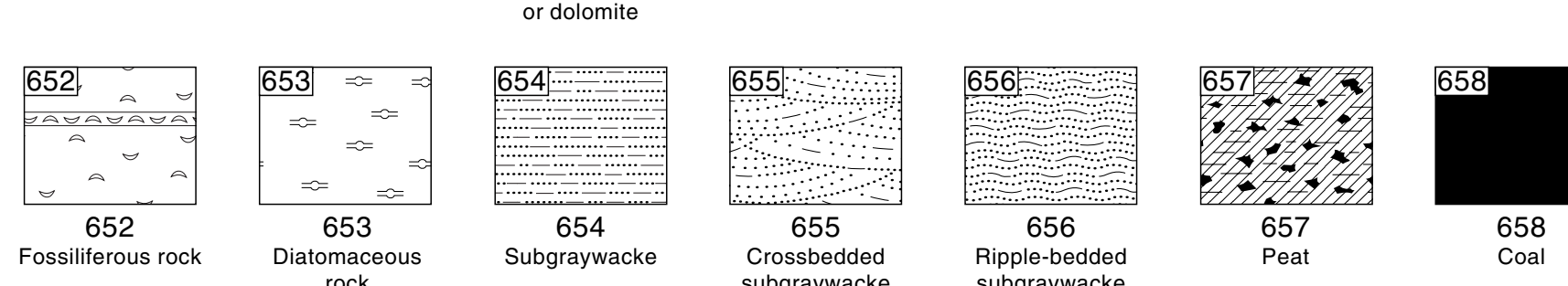

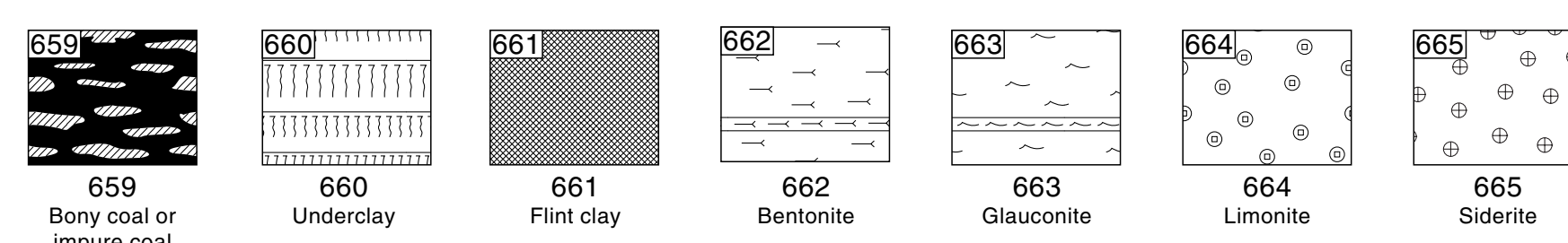

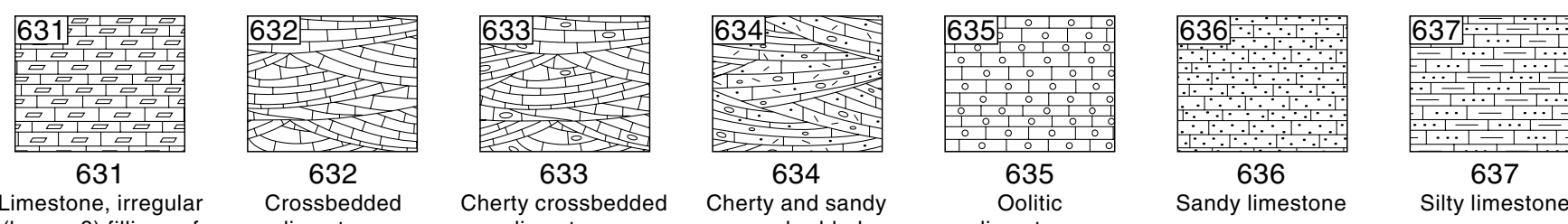

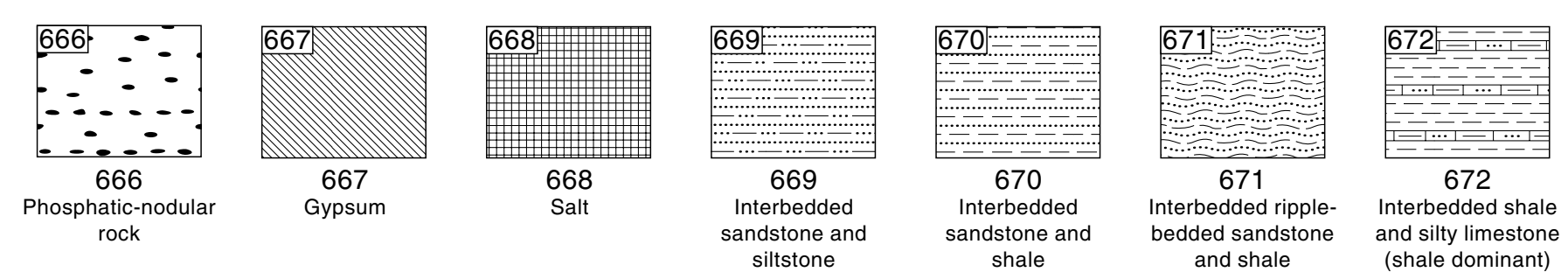

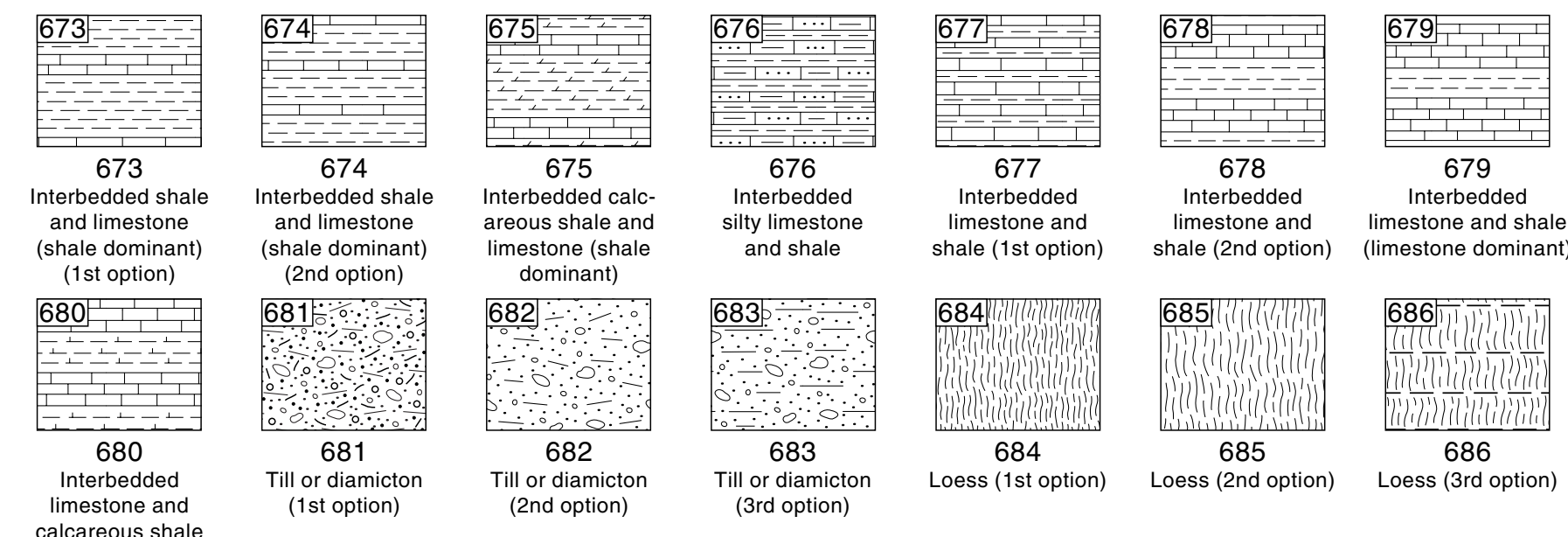

METAMORPHIC, G GNEOUS, AND VEIN-MATTER LTTHOLOGY PATTERNS (Series 700)

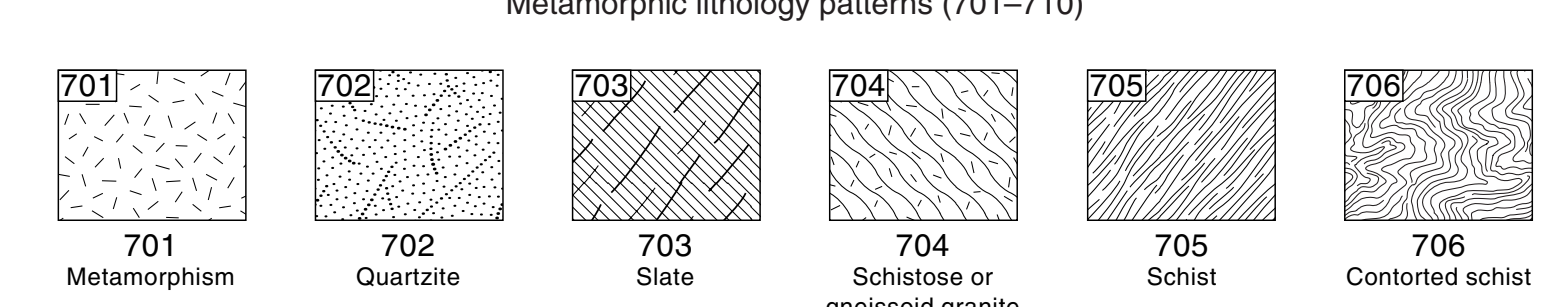

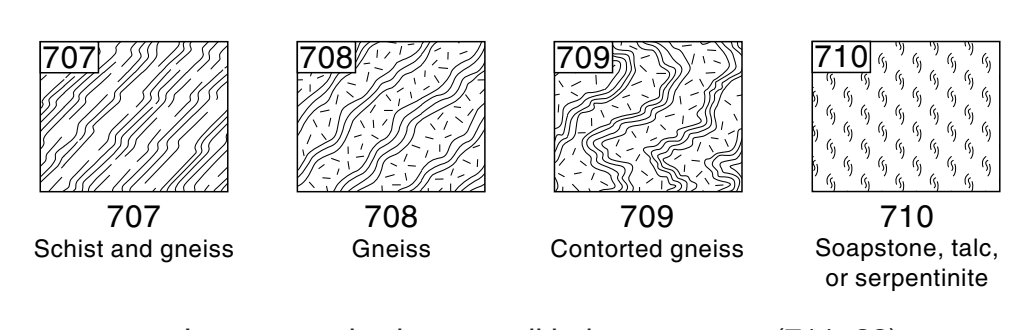

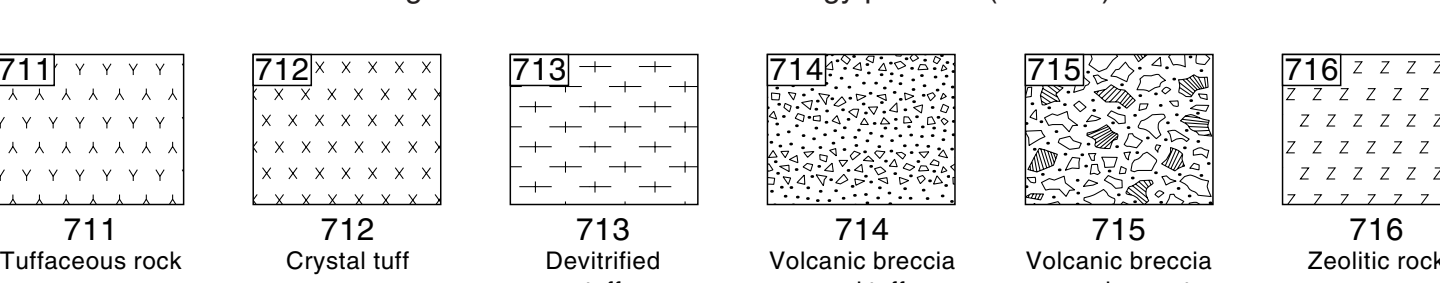

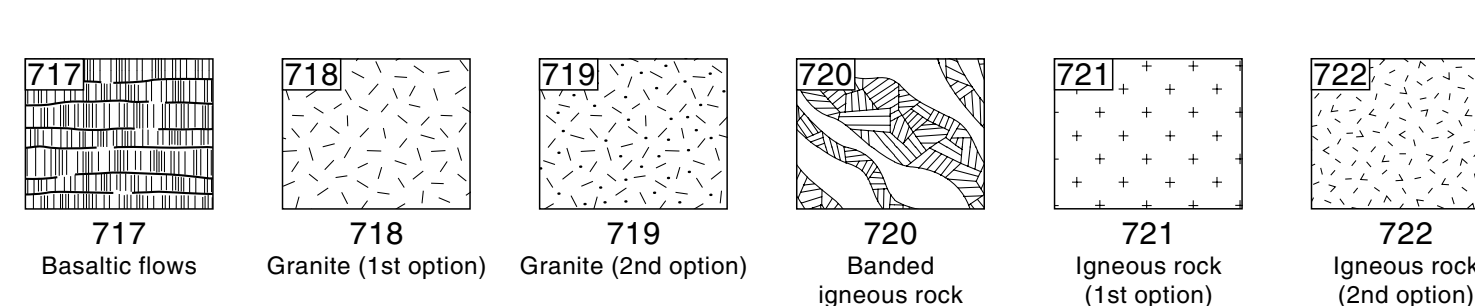

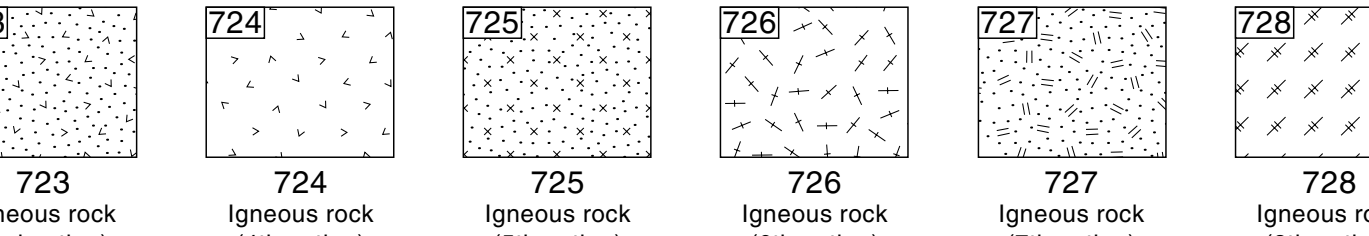

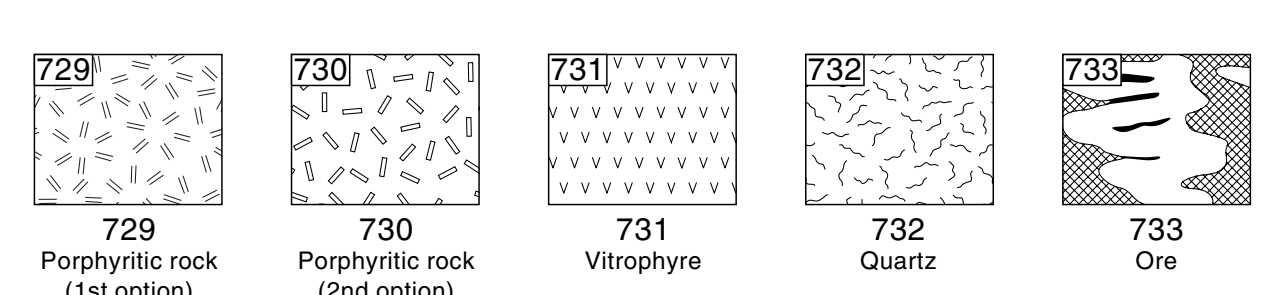

